\title{
Rational Ligand Design for U(VI) and Pu(IV)*
}

by

Géza Szigethy

B.A. (Princeton University), 2004

A dissertation submitted in partial satisfaction of the

requirements for the degree

of Doctor of Philosophy

in

Chemistry

in the

Graduate Division

of the

University of California, Berkeley

Committee in charge:

Professor Kenneth N. Raymond, Chair

Professor Richard A. Andersen

Professor Garrison Sposito

Fall 2009

* This research and the ALS are supported by the Director, Office of Science, Office of Basic Energy Sciences (OBES), and the OBES Division of Chemical Sciences, Geosciences, and Biosciences of the U.S. Department of Energy at LBNL under Contract No. DE-AC0205CH11231. 


\title{
Rational Ligand Design for U(VI) and Pu(IV)
}

\author{
by
}

Géza Szigethy

B.A. (Princeton University), 2004

A dissertation submitted in partial satisfaction of the

requirements for the degree

of Doctor of Philosophy

in

Chemistry

in the

Graduate Division

of the

University of California, Berkeley

Committee in charge:

Professor Kenneth N. Raymond, Chair

Professor Richard A. Andersen

Professor Garrison Sposito

Fall 2009 
The dissertation of Géza Szigethy is approved:

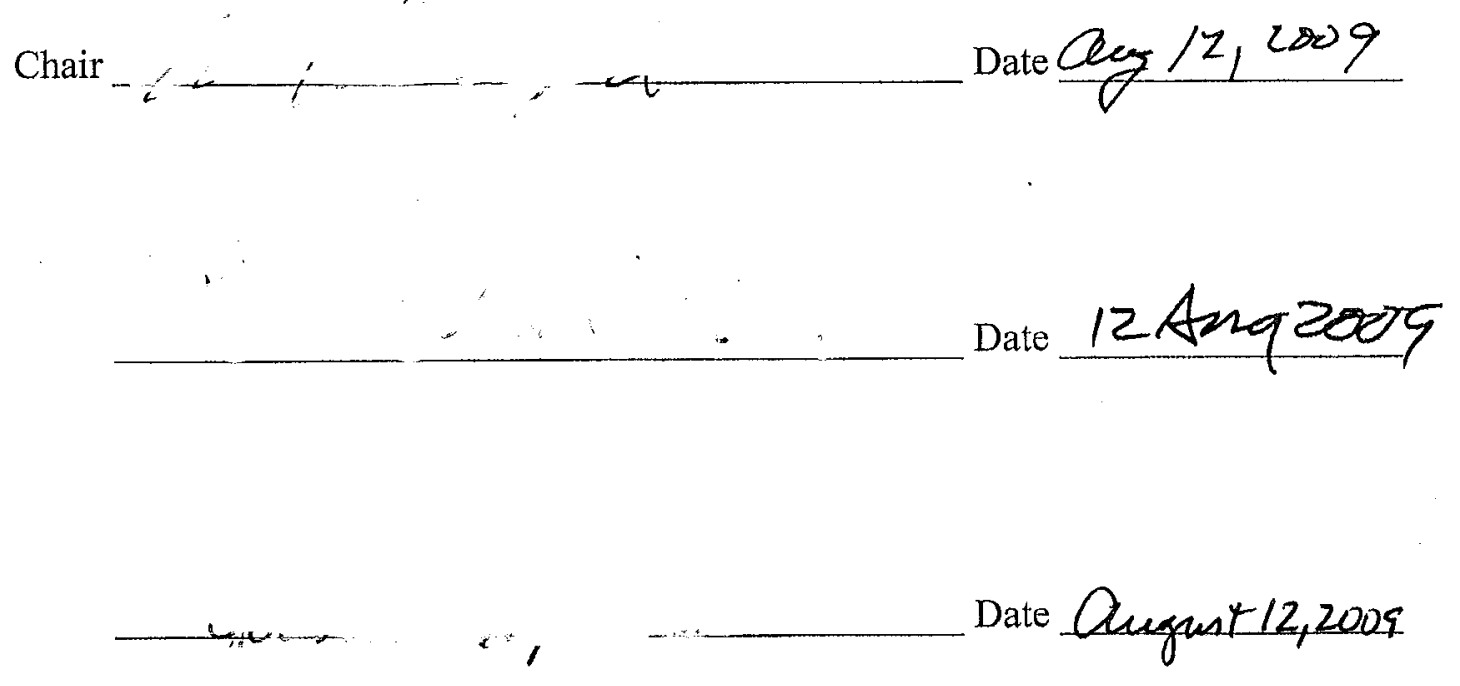

University of California, Berkeley

Fall 2009 
Rational Ligand Design for U(VI) and Pu(IV)

Copyright $@ 2009$

Géza Szigethy 


\author{
Abstract \\ Rational Ligand Design for U(VI) and Pu(IV) \\ by \\ Géza Szigethy \\ Doctor of Philosophy in Chemistry \\ University of California, Berkeley \\ Professor Kenneth N. Raymond, Chair
}

Nuclear power is an attractive alternative to hydrocarbon-based energy production at a time when moving away from carbon-producing processes is widely accepted as a significant developmental need. Hence, the radioactive actinide power sources for this industry are necessarily becoming more widespread, which is accompanied by the increased risk of exposure to both biological and environmental systems. This, in turn, requires the development of technology designed to remove such radioactive threats efficiently and selectively from contaminated material, whether that be contained nuclear waste streams or the human body. Raymond and coworkers (University of California, Berkeley) have for decades investigated the interaction of biologically-inspired, hard Lewis-base ligands with high-valent, early-actinide cations. It has been established that such ligands bind strongly to the hard Lewis-acidic early actinides, and many polybidentate ligands have been developed and shown to be effective chelators of actinide contaminants in vivo.

Work reported herein explores the effect of ligand geometry on the linear U(IV) dioxo dication (uranyl, $\mathrm{UO}_{2}{ }^{2+}$ ). The goal is to utilize rational ligand design to develop 
ligands that exhibit shape selectivity towards linear dioxo cations and provides thermodynamically favorable binding interactions. The uranyl complexes with a series of tetradentate 3-hydroxy-pyridin-2-one (3,2-HOPO) ligands were studied in both the crystalline state as well as in solution. Despite significant geometric differences, the uranyl affinities of these ligands vary only slightly but are better than DTPA, the only FDA-approved chelation therapy for actinide contamination.

The terepthalamide (TAM) moiety was combined into tris-bidentate ligands with 1,2and 3,2-HOPO moieties were combined into hexadentate ligands whose structural preferences and solution thermodynamics were measured with the uranyl cation. In addition to achieving coordinative saturation, these ligands exhibited increased uranyl affinity compared to bis-Me-3,2-HOPO ligands. This result is due in part to their increased denticity, but is primarily the result of the presence of the TAM moiety.

In an effort to explore the relatively unexplored coordination chemistry of $\mathrm{Pu}$ (IV) with bidentate moieties, a series of $\mathrm{Pu}(\mathrm{IV})$ complexes were also crystallized using bidentate hydroxypyridinone and hydroxypyrone ligands. The geometries of these complexes are compared to that of the analogous Ce(IV) complexes. While in some cases these showed the expected structural similarities, some ligand systems led to significant coordination changes, A series of crystal structure analyses with $\mathrm{Ce}(\mathrm{IV})$ indicated that these differences are most likely the result of crystallization condition differences and solvent inclusion effects. 


\section{Acknowledgements}

First and foremost I would like to thank my advisor, Professor Ken Raymond. Throughout my studies he has been supportive of the pursuit of my own interests as they apply to the research in his group; this has been the case from the first time we met and I requested to work on the subject of this dissertation. As my research progressed, he allowed those interests to naturally lead me along various avenues of investigation as he encouraged me to become a more well-rounded scientist and fill in the gaps my approach naturally created. I am also immensely grateful for his support of my life outside of the lab, helping to ensure that I was not just well-rounded in science, but in life as well.

I have by no means worked in a vacuum these past five years, and I have been assisted and encouraged by a large number of people in various fields without whom this research project would not have progressed as it has. Firstly, I want to thank Dr. David Shuh of Lawrence Berkeley National Labs (LBNL). He has been a constant source of encouragement and significant assistance in the workings of LBNL and has ensured that much of my work with $\mathrm{Pu}(\mathrm{IV})$ and $\mathrm{U}(\mathrm{VI})$ at the National Lab proceeded with acceptable speed and efficiency. He, too, has supported my life outside the lab, which is of course also greatly appreciated.

Throughout my time in the Raymond group I have found myself very interested in Xray crystallography, in which I have been assisted by several experts: Drs. Fred Hollander, Allen Oliver, and Antonio DiPasquale of UC Berkeley and Dr. Simon Teat of the Advanced Light Source at LBNL. These four, in addition to Ken, have taught me to evaluate, collect, and refine x-ray crystallographic data and have encouraged my constant development in the subject, for which I thank them. 
Many people within the Raymond group have also been of great assistance to me, starting with Prof. Anne Gorden and Dr. John Gorden, who introduced me to the Raymond group, its research, and lab practice in general. They also introduced me to working with radioactive isotopes and instructed me on how to do so safely. Many thanks are deserved by Dr. Jide Xu, whose synthetic expertise I have inquired upon many times, and who has never been too busy to knock about crazy synthesis ideas and suggest ways to actually make them possible. I greatly appreciate the assistance of Dr. Rebecca Abergel, Trisha Hoette, and Dr. Brandi Schottel for passing on their combined wisdom on solution titrations and their patience with my many questions and requests for help interpreting results.

My time in the Raymond group has been made much more enjoyable due to the company of many of my lab mates. Dr. Mike Pluth and Trisha Hoette have been great company and provided many a sounding board for a variety of subjects. Drs. Anthony D'Aleo, Evan Moore, and Michael Seitz have also shared their friendship as well as their knowledge with me. I could not list everyone else without forgetting many people, so I will settle with saying that I appreciate my interactions with all my labmates and groupmates, and I thank them for their support and friendship throughout my time in Berkeley.

I also want to thank my family for their support of my efforts in graduate school. And finally I want to thank my wife Dora for all her support throughout my doctoral research. She has been incredibly patient and phenomenal in helping us survive the distance over these five years. She has sacrificed much in support of me, my efforts here, and our marriage, for which I am eternally grateful.

- THANK YOU ALL! 


\section{Table of Contents}

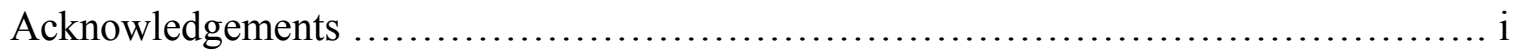

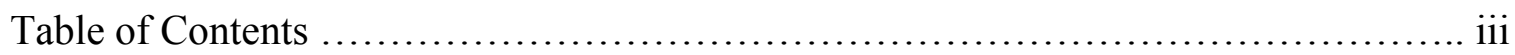

List of Figures, Tables, and Schemes .......................................... vii

Chapter 1: Introduction..............................................................1 1

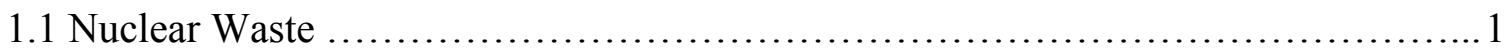

1.2 Treatment of Radioactive Waste: The Selectivity Problem .......................4

1.3 Actinides in Biology; Chelation Therapy .................................... 9

1.4 Rational Ligand Design: Siderophore-Inspired Sequestering Agents .............. 12

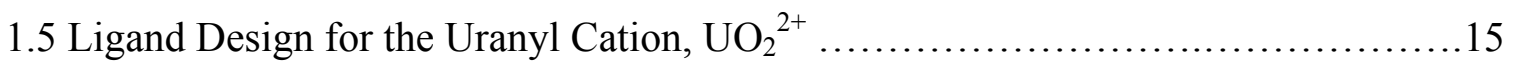

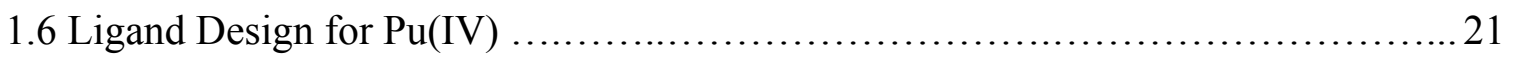

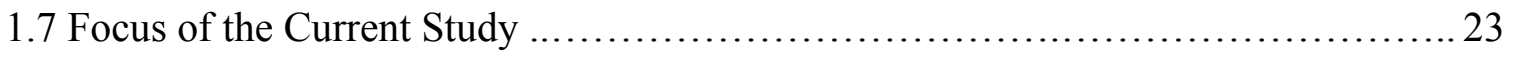

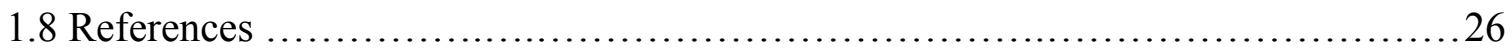

Chapter 2: Design, Structure, and Solution Thermodynamics of $\mathrm{UO}_{2}(\mathrm{Bis}-\mathrm{Me}-3,2-$

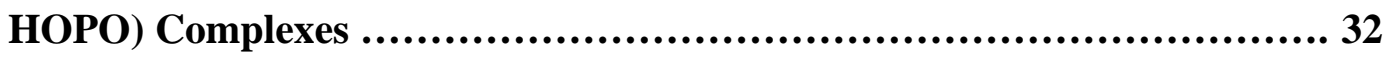

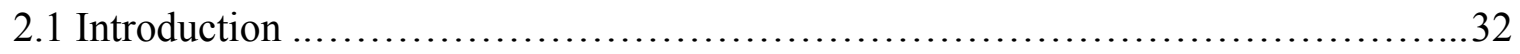

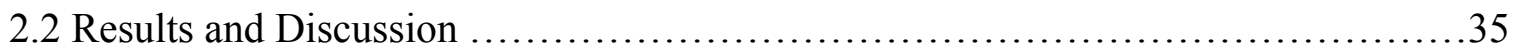

2.2.1 Tetradentate Ligand Design and Synthesis ...........................35

2.2.2 Synthesis and Structural Comparison of $\mathrm{UO}_{2}{ }^{2+}$ Complexes................38

2.2.3 Soluble Tetradentate Ligand Design and Synthesis .....................55

2.2.4 Ligand Substitution Effects on $\mathrm{UO}_{2}{ }^{2+}$ Complexes ......................6. 62 
2.2.5 Solution Thermodynamics

2.2.6 Substituted $m$-Xylene-Me-3,2-HOPO Ligands: Synthesis and Structure ...85

2.3 Conclusions and Future Directions............................................ 92

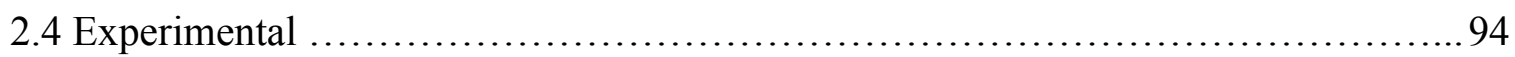

2.4.1 Synthesis of Backbone Diamines ......................................94

2.4.2 Synthesis of Benzyl-Protected bis-Me-3,2-HOPO Ligands ...............104

2.4.3 Benzyl-Deprotection of bis-Me-3,2-HOPO Ligands .....................114

2.4.4 Synthesis/Crystallization Techniques for $\mathrm{UO}_{2}{ }^{2+}$ Complexes ..............122

2.4.5 X-ray Diffraction Data Collection ................................. 128

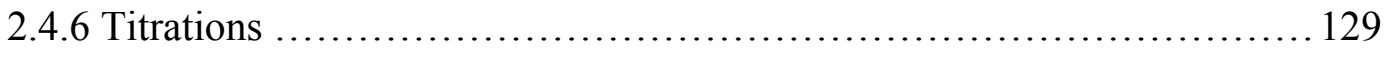

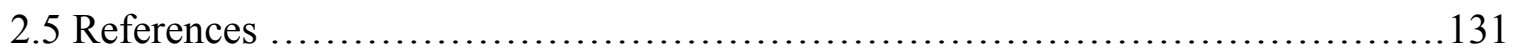

Chapter 3: Design, Structure, and Solution Thermodynamics of

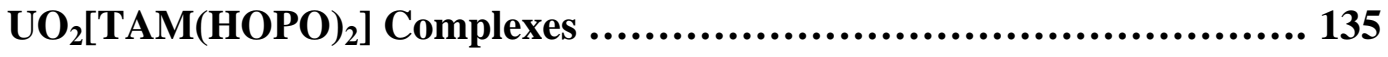

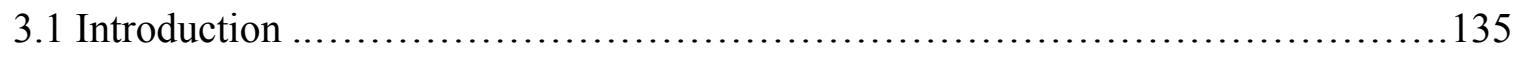

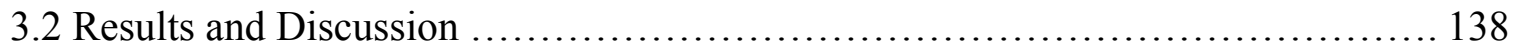

3.2.1 Hexadentate Ligand Design and Synthesis .......................... 138

3.2.2 Synthesis and Structural Comparison of $\mathrm{UO}_{2}{ }^{2+}$ Complexes ...............142

3.2.3 Soluble Hexadentate Ligand Design and Synthesis ......................155

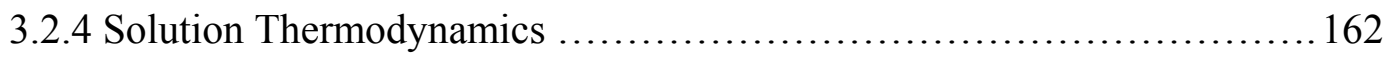

3.3 Conclusions and Future Directions.......................................... 175

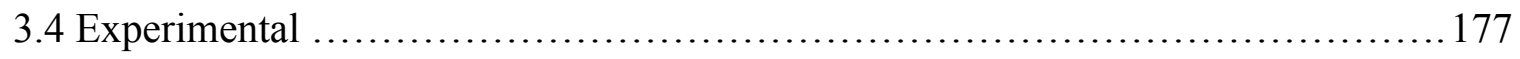

3.4.1 Synthesis of Benzyl-Protected TAM(HOPO) $)_{\mathbf{n}}$ Ligands ................ 177 
3.4.2 Synthesis of Benzyl-Protected PEG-TAM(HOPO) $\mathbf{n}$ Ligands 184

3.4.3 Benzyl Deprotection of TAM(HOPO $)_{n}$ and PEG-TAM(HOPO $)_{n}$

Ligands 195

3.4.4. Synthesis/Crystallization Techniques for Uranyl Complexes 200

3.4.5 X-ray Diffraction Data Collection 203

3.4.6 Titrations 203

3.5 References .205

\section{Chapter 4: Pu(IV) Coordination Chemistry with HOPO and Hydroxypyrone}

Ligands............................................................207

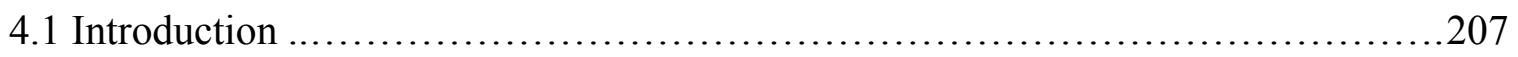

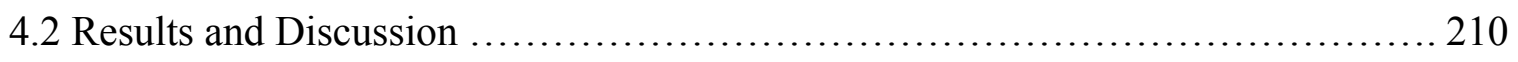

4.2.1 The Pu(IV)-1,2-Hydroxypyridinone Complex ........................... 211

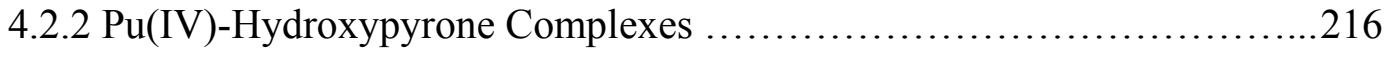

4.2.3 Additional Ce(IV)-Hydroxypyrone Complexes ........................225

4.2.4 Linear Octadentate $\mathrm{TAM}_{4}$ and $\mathrm{TAM}_{2} \mathrm{HOPO}_{2}$ Ligands ...................231

4.3 Conclusions and Future Directions.......................................... 237

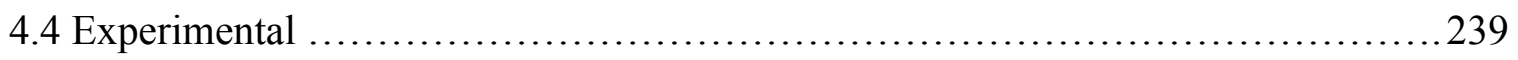

4.4.1 Pu(IV) Stock Solution Preparation .................................. 240

4.4.2 Bidentate Ligand Synthesis .......................................241

4.4.3 Synthesis of Ce-Kojate/Maltol Complexes ............................242

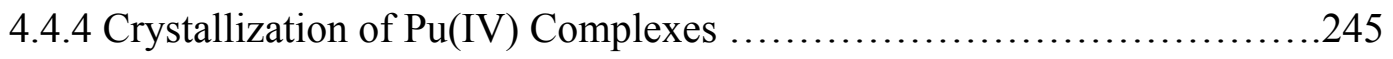

4.4.5 Synthesis of Octadentate $\mathrm{TAM}_{4}$ and $\mathrm{TAM}_{2} \mathrm{HOPO}_{2}$ Ligands .............246 


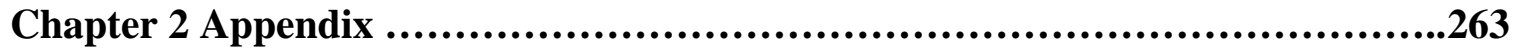

A2.1 Crystallographic Refinement Details.........................................263

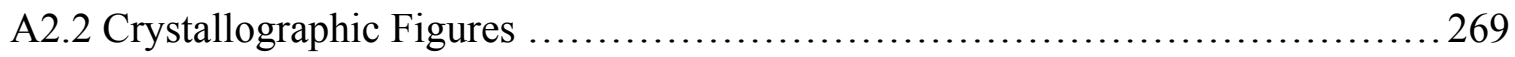

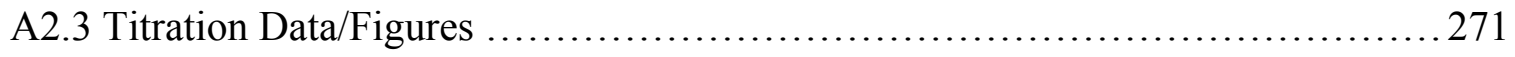

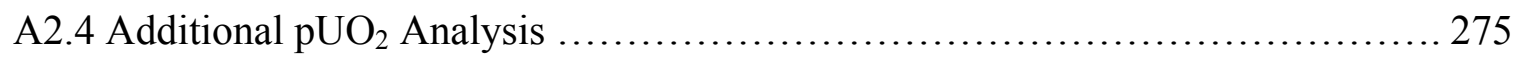

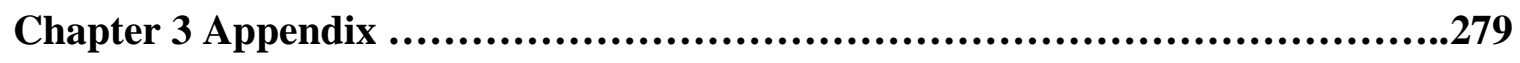

A3.1 Crystallographic Refinement Details.......................................2279

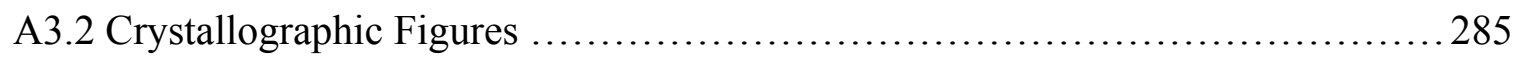

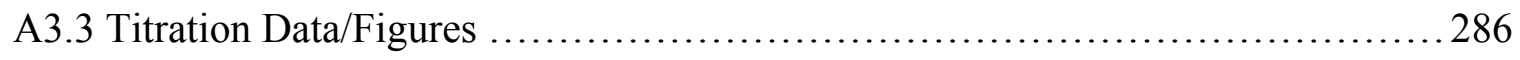

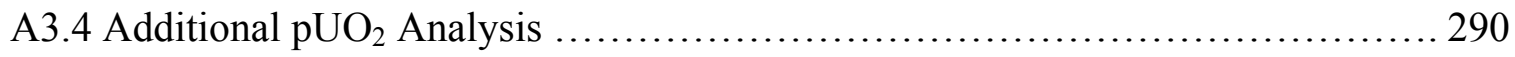

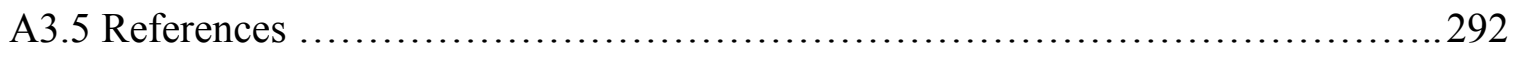

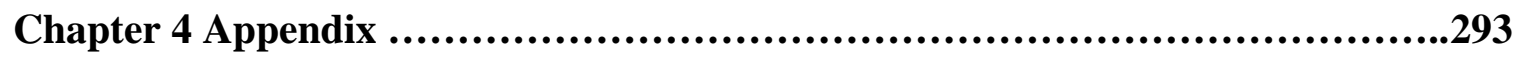

A4.1 Crystallographic Refinement Details ....................................... 293

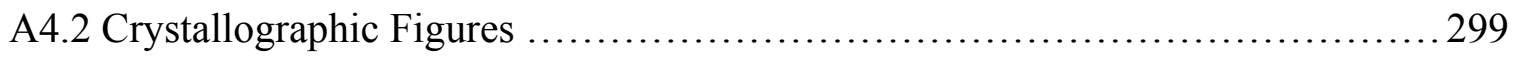

A4.3 Competition Batch Titration Methodology ................................... 301

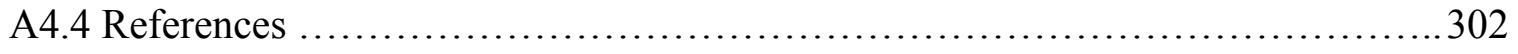




\section{List of Figures, Tables, and Schemes}

\section{Chapter 1}

Figure 1-1. Extractants used in PUREX-based nuclear fuel separations ................. 6

Figure 1-2. N- and S-coordinating extractants for $\mathrm{An} / \mathrm{Ln}(\mathrm{III})$ separations $\ldots \ldots \ldots \ldots \ldots \ldots 8$

Figure 1-3. Cavitands designed for $\mathrm{An} / \mathrm{Ln}(\mathrm{III})$ separations $\ldots \ldots \ldots \ldots \ldots \ldots \ldots \ldots \ldots \ldots$

Figure 1-4. Polyaminoacetic acids used in current chelation therapies ................. 12

Figure 1-5. Catechol and hydroxamate: typical chelating moieties in siderophore ligands

Figure 1-6. Synthetic siderophore moiety analogs used in Raymond group ligands ......13

Figure 1-7. The effect of amide linkage on binding moiety $\mathrm{pK}_{\mathrm{a}} \ldots \ldots \ldots \ldots \ldots \ldots \ldots \ldots \ldots$

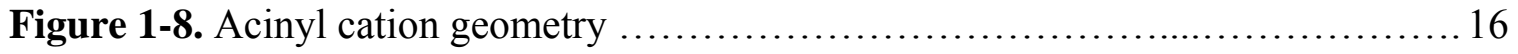

Figure 1-9. Cation-cation interaction observed with $\mathrm{NpO}_{2}{ }^{+}$and unobserved with $\mathrm{UO}_{2}{ }^{2+}$

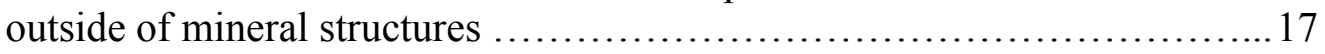

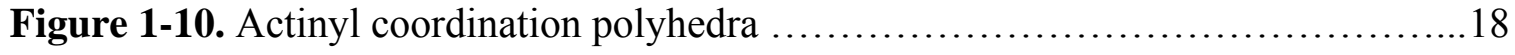

Figure 1-11. Stereognostic, tripodal ligands for uranyl chelation ......................19

Figure 1-12. General coordination mode of $\mathrm{UO}_{2}{ }^{2+}$ with catecholate-type ligands .......20

Figure 1-13. $\left[\mathrm{M}(\text { catechol })_{4}\right]^{4-}(\mathrm{M}=\mathrm{Th}, \mathrm{Ce}, \mathrm{U})$ complex geometry reproduced from Sofen et al. and trigonal coordination geometry $\ldots \ldots \ldots \ldots \ldots \ldots \ldots \ldots \ldots . . \ldots 22$

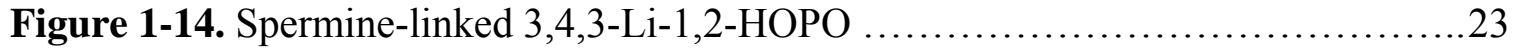

Figure 1-15. Me-3,2-HOPO ligands used in $\mathrm{UO}_{2}{ }^{2+}$ and $\mathrm{Pu}(\mathrm{IV})$ structural studies .......23

\section{Chapter 2}

Figure 2-1. Me-3,2-HOPO ligands used in previous $\mathrm{UO}_{2}{ }^{2+}$ structural studies 32

Figure 2-2. Crystal structures of $\mathrm{UO}_{2}(2-1)(\mathrm{DMF})$ (left) and $\mathrm{UO}_{2}(2-4)(\mathrm{DMSO})$ (right) from Xu et al. 
Figure 2-3. Rigidly-linked bis-Me-3,2-HOPO ligands synthesized for structural studies with the uranyl cation 36

Figure 2-4. Speculated structure of uranyl coordination polymer with 2-10 40

Figure 2-5. Top and side views of X-ray diffraction structures of uranyl complexes with (a) 2-2, (b) 2-6, (c) 2-7, (d) 2-8, (e) 2-13, (f) 2-14, (g) 2-15, (h) 2-16........42

Figure 2-6. Equatorial $\mathrm{O}-\mathrm{U}-\mathrm{O}$ bond angle designations for $\mathrm{UO}_{2}(2-1)(\mathrm{DMF})$ and $\mathrm{UO}_{2}$ (bis-Me-3,2-HOPO)(solv) tabulated in Table 2-3

Figure 2-7. Conformational metrics used to compared $\mathrm{UO}_{2}$ (bis-Me-3,2-HOPO) structures

Figure 2-8. Top and side views of the 2-16·DMSO crystal structure 54

Figure 2-9. PEG-functionalized bis-Me-3,2-HOPO ligands 2-17 through 2-23 .58

Figure 2-10. Top and side views of X-ray diffraction structures of uranyl complexes with alkyl-substituted bis-Me-3,2-HOPO ligands

Figure 2-11. Intramolecular steric interference and amide torsion angle in $\left[\mathrm{UO}_{2}(2-37)\right]_{2}$ complexes .....

Figure 2-12. Relative energy calculations for rotation of an acetamide substituent about the $\mathrm{N}_{\text {amide }}-\mathrm{C}_{\text {thiophene }}$ bond without and with ortho thioethyl substitution 68

Figure 2-13. Relative energy calculations for rotation of an acetamide substituent about the $\mathrm{N}_{\text {amide }}-\mathrm{C}_{\text {thiophene }}$ bond in the presence of an ortho acetamide in the absence and presence of 2,5-dithioethyl substitution 70

Figure 2-14. Proposed hydrogen bond stabilization in (a) HOPO-amide ligands, (b) bis-HOPO ligands with short linkers, and (c) HOPO ligands containing solubilizing PEG-amide groups next to linking amides ................. 76

Figure 2-15. Proposed speciation behavior of the uranyl-(2-21) complex in solution ...80

Figure 2-16. Proposed speciation behavior of $\mathrm{UO}_{2}(2-1)_{\mathrm{n}}$ in solution .81

Figure 2-17. 2-position functionalization in $m$-xy-Me-3,2-HOPO ligands .86

Figure 2-18. Top and side views of the $\mathrm{X}$-ray diffraction structures of uranyl complexes with 2-substituted $m$-xy-Me-3,2-HOPO ligands .88

Figure 2-19. The conformational metric Tors. .89 
Table 2-1. Crystallographic parameters for $\mathrm{UO}_{2}$ (bis-Me-3,2-HOPO) complexes

Table 2-2. Equatorial U-O bond distances in $\mathrm{UO}_{2}$ (bis-Me-3,2-HOPO) crystal structures

Table 2-3. O-U-O angles in the uranyl coordination plane in $\mathrm{UO}_{2}$ (bis-Me-3,2-HOPO) crystal structures 46

Table 2-4. Conformational parameters measured from $\mathrm{UO}_{2}$ (bis-Me-3,2-HOPO) crystal structures.

Table 2-5. Crystallographic parameters for 2-16·DMSO 54

Table 2-6. Crystallographic parameters for uranyl complexes with 2-37 and 2-38 .....66

Table 2-7. U-O bond lengths and conformational parameters from $\left[\mathrm{UO}_{2}(2-37)(\mathrm{DMSO})\right]_{2}$ and $\mathrm{UO}_{2}(2-38)(\mathrm{DMSO})$ structures .72

Table 2-8. $\mathrm{pK}_{\mathrm{a}}$ values of bis-Me-3,2-HOPO ligands ........................... 74

Table 2-9. $\log \beta_{m l h}$ values for uranyl titrations with Me-3,2-HOPO ligands ........... 77

Table 2-10. Calculated $\mathrm{pUO}_{2}$ values for Me-3,2-HOPO ligands $\ldots \ldots \ldots \ldots \ldots \ldots \ldots . \ldots 2$

Table 2-11. Crystallographic parameters for $\mathrm{UO}_{2}(2-39)$ and $\mathrm{UO}_{2}(2-40)(\mathrm{DMSO}) \ldots \ldots . .89$

Table 2-12. Equatorial U-O bond lengths and conformational parameters in $\mathrm{UO}_{2}(m-\mathrm{xy}-$ Me-3,2-HOPO) complexes $\mathrm{UO}_{2}(2-15)(\mathrm{DMF}), \mathrm{UO}_{2}(2-39)$, and $\mathrm{UO}_{2}(2-$ 40)(DMSO) 89

Scheme 2-1. Synthesis of bis-Me-3,2-HOPO ligands 2-6 through 2-16 ................ 38

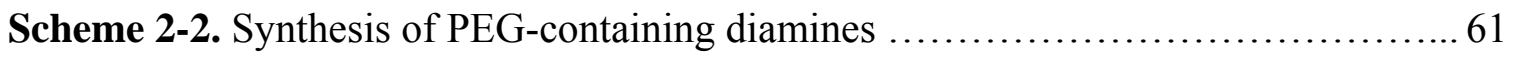

Scheme 2-3. Syntheses of PEG-functionalized bis-Me-3,2-HOPO ligands 2-17 through 2-23 61

Scheme 2-4. Synthesis of alkyl-substituted bis-Me-3,2-HOPO ligands 2-37 and 2-38 ...63

Scheme 2-5. Synthesis of substituted $m$-xy-Me-3,2-HOPO ligands 2-39 and 2-40 .87 


\section{Chapter 3}

Figure 3-1. Expanded porphyrin Hexaphyrin and its structures with $\mathrm{UO}_{2}{ }^{2+}$ and $\mathrm{NpO}_{2}{ }^{+}$ from Sessler et al.

Figure 3-2. Hydroxypyridinone (Me-3,2- and 1,2-HOPO) and catechol-analog (CAM, TAM) chelating moieties

Figure 3-3. Macrocyclic and linear ligands developed by Nabeshima et al. ........... 138

Figure 3-4. Solvent accessible area in $\mathrm{UO}_{2}$ (bis-Me-3,2-HOPO) complexes ............139

Figure 3-5. General design for $\mathrm{TAM}(\mathrm{HOPO})_{2}$ ligands and initial synthesis targets .... 140

Figure 3-6. Top and side views of X-ray diffraction structures of uranyl complexes with

$\mathrm{TAM}(\mathrm{HOPO})_{2}$ ligands

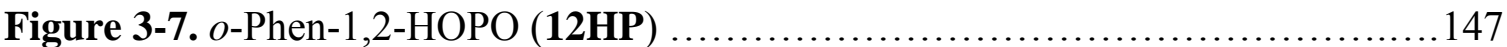

Figure 3-8. Top and side views of X-ray diffraction structures of $\mathrm{UO}_{2}(12 \mathrm{HP})(\mathrm{DMSO})$

Figure 3-9. Schematic of equatorial U-O bond lengths and binding pocket layout in uranyl complexes with TAM(HOPO $)_{2}$ and bis-Me-3,2-HOPO ligands as referred to in Tables 3-2 and 3-3 and Figure 3-10

Figure 3-10. Uranyl binding pockets in the $\mathrm{UO}_{2}\left[\mathrm{TAM}(\mathrm{HOPO})_{2}\right]$ crystal structures ....151

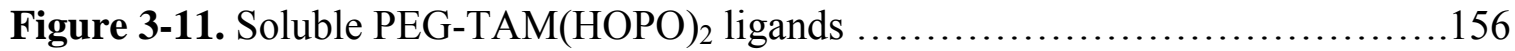

Figure 3-12. Mixed tetradentate ligands Pr-TAM-2Li-Me-3,2-HOPO (3-22) and PEG-Pr-2Li-Me-3,2-HOPO (3-23)

Figure 3-13. Spectrophotometric reversibility plots of uranyl titrations with TAM(2Li1,2-HOPO) $)_{2}, \mathbf{3 - 1}$ 166

Figure 3-14. Hypothesized deprotonation and TAM rotation in uranyl complex formationwith $\mathrm{TAM}(\mathrm{HOPO})_{2}$ ligands

Figure 3-15. Proposed speciation in uranyl titrations with ligands 3-22 and 3-23 169

Figure 3-16. Representative speciation diagrams for uranyl complexes at standard $\mathrm{pUO}_{2}$ conditions of $\left[\mathrm{UO}_{2}{ }^{2+}\right]=1 \mu \mathrm{M},[\mathrm{L}]=10 \mu \mathrm{M}$

Table 3-1. Crystallographic parameters for $\mathrm{UO}_{2}\left[\mathrm{TAM}(\mathrm{HOPO})_{2}\right]$ and $\mathrm{UO}_{2}$ (o-phen-1,2HOPO) complexes 
Table 3-2. Equatorial U-O bond lengths in $\mathrm{UO}_{2}\left[\mathrm{TAM}(\mathrm{HOPO})_{2}\right]$ and analogous $\mathrm{UO}_{2}$ (bis-HOPO) complexes determined by X-ray crystallography and labeled according to Figure 3-9 148

Table 3-3. In-plane O-U-O bite angles in $\mathrm{UO}_{2}\left[\mathrm{TAM}(\mathrm{HOPO})_{2}\right]$ complexes determined by X-ray crystallography 152

Table 3-4. Interplanar angles in $\mathrm{UO}_{2}\left[\mathrm{TAM}(\mathrm{HOPO})_{2}\right]$ complex crystal structures 153

Table 3-5. $\mathrm{pK}_{\mathrm{a}}$ values for TAM-containing ligands 163

Table 3-6. $\log \beta_{m l h}$ and $\mathrm{pUO}_{2}$ values for TAM-containing ligands 170

Scheme 3-1. Synthesis of hexadentate TAM(HOPO $)_{2}$ ligands 3-1 through 3-5 142

Scheme 3-2. Synthesis of PEG-TAM(HOPO $)_{2}$ ligands 3-12 through 3-15 158

Scheme 3-3. Synthesis of ligands 3-22 and 3-23 161

\section{Chapter 4}

Figure 4-1. High-efficiency Raymond group ligands for $\mathrm{Pu}(\mathrm{IV})$ decorporation 208

Figure 4-2. Crystal structure of $\mathrm{Pu}(5 \mathrm{LiO}-\mathrm{Me}-3,2-\mathrm{HOPO})_{2}$ 209

Figure 4-3. N-hydroxy-pyridin-2-one, 1,2-HOPO, 4-1

Figure 4-4. X-ray diffraction structure of the $\mathrm{Pu}(\mathrm{IV})-1,2-\mathrm{HOPO}$ mixed salt complex $\mathrm{Pu}(4-1)_{4} \cdot \mathrm{Pu}(4-1)_{3}\left(\mathrm{H}_{2} \mathrm{O}\right)_{2} \cdot \mathrm{ClO}_{4}$

Figure 4-5. Crystal structure of $\mathrm{Ce}(\mathbf{4 - 1})_{4}$

Figure 4-6. Ideal coordination polyhedra: Square antiprism $\left(\mathrm{D}_{4 \mathrm{~d}}\right)$, bicapped trigonal prism $\left(\mathrm{C}_{2 \mathrm{v}}\right)$, and trigonal dodecahedron $\left(\mathrm{D}_{2 \mathrm{~d}}\right)$

Figure 4-7. 3-Hydroxy-pyran-4-one ligands

Figure 4-8. Top and side views of crystal structures of $\mathrm{Pu} / \mathrm{Ce}(4-2 / 4-3)_{4}$ complexes along with schematics of their coordination polyhedra ................. 218

Figure 4-9. Schematic of the ligand disorder in the $\mathrm{Pu}(4-3)_{4}$ crystal structure .220

Figure 4-10. Coordination polyhedra of the $\mathrm{Pu}(4-2)_{4}$ and $\mathrm{Pu}(4-3)_{4}$ complexes with spanned edges indicated by arrows whose heads point towards the methyl 
substituents

Figure 4-11. Hoard and Silverton edge notation of trigonal dodecahedral geometry (left) and coordination polyhedra of the $\mathrm{Ce}(\mathbf{4 - 2})_{4}$ (middle) and $\mathrm{Ce}(4-3)_{4}$ (right) complexes

Figure 4-12. Hydroxypyrone ligands used in exploring Ce(IV) coordination chemistry 225

Figure 4-13. Top and side views of $\mathrm{CeL}_{4}$ complexes with hydroxypyrone ligands .....228

Figure 4-14. Edge notation for $\mathrm{D}_{2 \mathrm{~d}}$ and $\mathrm{D}_{4 \mathrm{~d}}$ coordination polyhedra used in Table 4-9 230

Figure 4-15. Design strategy of linear tetrakis-bidentate ligands for coordinative saturation of $f$-element cations

Figure 4-16. Possible coordination modes for the TAM moiety upon mono and bisdeprotonation

Figure 4-17. (a) Evolution of the luminescence spectrum of $\mathrm{Yb}(\mathbf{4 - 1 7 g})$ upon addition of DTPA; (b) Competition titration $\log / \log$ plot for $\mathrm{Yb}(\mathbf{4 - 1 7} \mathbf{g})$ against DTPA at varying $\mathrm{pH}$ 237

Table 4-1. Crystallographic parameters for the Pu(IV)-1,2-HOPO structure 212

Table 4-2. Shape measure values for $\mathrm{Pu} / \mathrm{Ce}(\mathrm{IV})$ complexes with 4-1 215

Table 4-3. Crystallographic parameters for $\mathrm{Pu} / \mathrm{Ce}(4-2 / 4-3)_{4}$ complexes 217

Table 4-4. Calculated $\mathrm{Pu}-\mathrm{O}$ distances in the $\mathrm{Pu}(4-3)_{4}$ crystal structure for each disordered ligand group

Table 4-5. $\mathrm{Pu} / \mathrm{Ce}-\mathrm{O}$ bond distances and bite angles from the $\mathrm{Pu} / \mathrm{Ce}(\mathbf{4}-\mathbf{2} / \mathbf{4}-3)_{4}$ crystal structures 222

Table 4-6. Shape measure (S) values for $\mathrm{Pu} / \mathrm{Ce}(4-2 / 4-3)_{4}$ complexes 223

Table 4-7. Crystallographic parameters for Ce(IV)-hydroxypyrone crystal structures 226

Table 4-8. Shape measure (S) values for Ce(IV)-hydroxypyrone complexes in Figure $4-13$ 229

Table 4-9. MM3 calculation results on the $\mathrm{Ce}(\mathbf{4 - 2 / 4 - 3})_{4}$ structures .229

Scheme 4-1. Synthesis of $\mathrm{TAM}_{4}$ and $\mathrm{TAM}_{2} \mathrm{HOPO}_{2}$ ligands .233 


\section{Chapter 2 Appendix}

Figure A2-1. Polymeric chain in the crystal structure of $\mathrm{UO}_{2}(2-2) \ldots \ldots \ldots \ldots \ldots \ldots . . . . . . . .269$

Figure A2-2. Polymeric chain in the crystal structure of $\mathrm{UO}_{2}(2-14) \ldots \ldots \ldots \ldots \ldots \ldots . . . . . . . .269$

Figure A2-3. Asymmetric unit in the crystal structure of $\mathrm{UO}_{2}(\mathbf{2 - 1 6})(\mathrm{DMSO}) \ldots \ldots \ldots . .270$

Figure A2-4. Asymmetric unit in the dimeric crystal structure of $\left[\mathrm{UO}_{2}(2-37)(\mathrm{DMSO})\right]_{2}$

Figure A2-5. Spectrophotometric titration reversibility curves for uranyl titrations with

(a) 2-1, (b) 2-17, (c) 2-18, (d) 2-19, (e) 2-20, (f) 2-21, (g) 2-22, (h) 2-23 ...271

Figure A2-6. Acid to base UV-visible titration curves for uranyl titrations with (a) 2-1, (b) 2-17, (c) 2-18, (d) 2-19, (e) 2-20, (f) 2-21, (g) 2-22, (h) 2-23

Figure A2-7. Strong acid UV-visible titration curves for uranyl titrations with (a) 2-1,

(b) 2-17, (c) 2-18, (d) 2-19, (e) 2-20, (f) 2-21, (g) 2-22, (h) 2-23

Figure A2-8. Calculated speciation diagrams for complexation of $\mathrm{UO}_{2}{ }^{2+}$ at $[\mathrm{M}]=1 \mu \mathrm{M}$, $[\mathrm{L}]=10 \mu \mathrm{M}$ with Me-3,2-HOPO ligands

Figure A2-9. Species distribution for uranyl in the presence of seven bis-Me-3,2-

HOPO ligands

Table A2-1. Calculated $\mathrm{pUO}_{2}{ }^{*}$ value for Me-3,2-HOPO ligands .276

Table A2-2. Percentage of complexes $\mathrm{UO}_{2}{ }^{2+}$ in the presence of multiple bis-Me-3,2-HOPO ligands 278

\section{Chapter 3 Appendix}

Figure A3-1. Asymmetric unit in the crystal structure of $\mathrm{UO}_{2}[\mathrm{TAM}(2 \mathrm{Li}-\mathrm{Me}-3,2-$

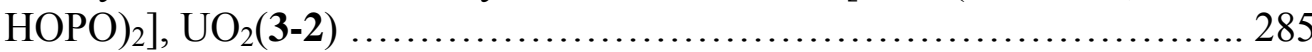

Figure A3-2. Asymmetric unit in the crystal structure of $\mathrm{UO}_{2}[\mathrm{TAM}(o-p h e n-M e-3,2-$

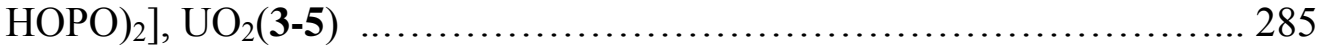

Figure A3-3. Acid to base UV-visible titration curves of TAM-containing ligands ....286

Figure A3-4. Spectrophotometric titration reversibility curves of TAM-containing ligands 287 
Figure A3-5. Spectrophotometric titration reversibility curves for uranyl titrations with

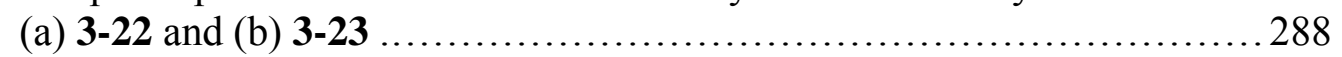

Figure A3-6. Acid to base UV-visible titration curves for uranyl titrations with (a) 3-1, (b) 3-12, (c) 3-13, (d) 3-14, (e) 3-15, (f) 3-22, (g) 3-23 ................. 288

Figure A3-7. Strong acid UV-visible titrations curves for uranyl titrations with (a) 3-22

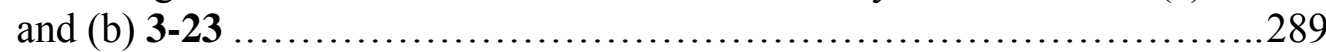

Figure A3-8. Calculated speciation diagrams for complexation of $\mathrm{UO}_{2}{ }^{2+}$ at $[\mathrm{M}]=1 \mu \mathrm{M}$, $[\mathrm{L}]=10 \mu \mathrm{M}$ with TAM-containing ligands ............................. 289

Table A3-1. Calculated $\mathrm{pUO}_{2}{ }^{*}$ value for TAM-containing ligands .................. 290

Table A3-2. Percentage of complexed $\mathrm{UO}_{2}{ }^{2+}$ in the presence of multiple

$\mathrm{TAM}(\mathrm{HOPO})_{2}$ ligands 292

\section{Chapter 4 Appendix}

Figure A4-1. Crystallographic refinement of disordered solvent pocket in the crystal

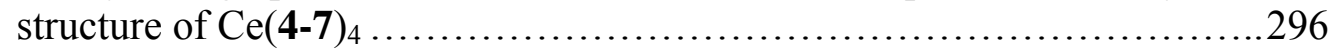

Figure A4-2. Asymmetric unit in the crystal structure of $\mathrm{Ce}(4-2)_{4} \ldots \ldots \ldots \ldots \ldots \ldots . . \ldots 299$

Figure A4-3. Asymmetric unit in the crystal structure of $\mathrm{Ce}(4-3)_{4} \ldots \ldots \ldots \ldots \ldots \ldots \ldots . \ldots 300$

Figure A4-4. Asymmetric unit in the crystal structure of $\mathrm{Pu}(4-2)_{4} \ldots \ldots \ldots \ldots \ldots \ldots . \ldots . \ldots 300$

Figure A4-5. Asymmetric unit in the crystal structure of $\mathrm{Ce}(4-6)_{4} \ldots \ldots \ldots \ldots \ldots \ldots . \ldots 300$

Figure A4-6. Experimental schematic of 3-layer crystallization technique utilized with $\mathrm{Ce}(\mathrm{IV})$-hydroxypyron complexes 


\section{Chapter 1: Introduction}

\subsection{Nuclear Waste}

Highly radioactive and fissile actinides - both naturally occurring and synthesized were first utilized at significant levels upon the discovery of plutonium in 1941. This was followed by the implementation of the Manhattan Project, whose goal was the development of fissile elements and their technology towards the fabrication of thermonuclear devices. These efforts ultimately culminated in the detonation of two nuclear bombs fueled by ${ }^{235} \mathrm{U}$ and ${ }^{239} \mathrm{Pu}$ over Nagasaki and Hiroshima, Japan, respectively, ending the war with Japan in 1945. The subsequent developments in nuclear technology have led to the widespread use of radioactive elements in applications such as clinical radiography, pharmaceutical tracer studies, and cancer radiation therapy. Actinide-specific applications include ${ }^{238} \mathrm{Pu}$ radioisotope thermoelectric generators that power satellites and space probes, and ${ }^{241} \mathrm{Am}$ sources in conventional smoke detectors. However, by far the most visible and widespread uses of nuclear technology are their applications in thermonuclear weapons and civilian and military nuclear power generation; nuclear power alone accounted for $20 \%$ of the electrical output in the United States in 2003, second only to coal. ${ }^{1}$ These applications typically utilize the fissile isotopes ${ }^{235} \mathrm{U}$ and ${ }^{239} \mathrm{Pu}$ which undergo fission after slow neutron capture to produce a mixture of fission products, more neutrons, and heat which is used for power generation.

The element uranium is the more easily obtained of the two common fissile fuel materials mentioned above because it is the heaviest naturally-occurring element and can be found in several uranium-containing ores such as uraninite, carnotite, autunite and many others (uranium wt\% of 88,53 , and 54 respectively). ${ }^{2}$ Such ores are found all over 
the world with several reserves across the western United States, and can be mined using deep shaft and shallow pit mining as well as in situ leaching. ${ }^{1,3}$ Unfortunately for nuclear applications, the desired ${ }^{235} \mathrm{U}$ only occurs in an abundance of $0.7 \%$ of all naturally occurring uranium, requiring a large scale and labor intensive process of isotope separation following the chemical separation processes of ore milling and refinement to attain the required enrichment levels of ${ }^{235} \mathrm{U}$ (3\% to greater than $90 \%$, depending on the application). ${ }^{1}$ In contrast, ${ }^{239} \mathrm{Pu}$ is generated by neutron bombardment of the $99.3 \%$ abundant ${ }^{238} \mathrm{U}$ followed by beta decay according to Equation 1-1 below. ${ }^{4}$ This process does not require the lengthy isotopic separation of uranium enrichment, since it is achieved by a series of chemical separations from the parent uranium, fission daughter and neutron capture byproducts. Whether isolating desired actinides from ores or transmutation targets, these separations nonetheless generate significant amounts of radioactive byproduct waste that can threaten the cleanliness of soil and groundwater supplies of surrounding communities and ecosystems.

$$
{ }^{238} \mathrm{U}+n \longrightarrow{ }^{239} \mathrm{U} \stackrel{\beta^{-}}{\longrightarrow}{ }^{239} \mathrm{~Np} \stackrel{\beta^{-}}{\longrightarrow}{ }^{239} \mathrm{Pu} \quad \text { Eq. } 1-1
$$

In nuclear power generation applications, the uranium or plutonium oxides are typically packed into pellets that are housed in larger modular assemblies for use in nuclear reactors. After their effective lifetime, these fuel assemblies are held in storage pools to allow for the decay of short-lived isotopes before they are interred in long-term geological repositories in the "once-through" fuel cycle employed by the United States. In France and some other countries, these fuel assemblies are disassembled and the nuclear fuel is dissolved and reprocessed to separate the fission product contaminants from the reusable fissile elements. Typical spent uranium fuel rods from light water 
reactors contain $95.6 \%$ uranium, $0.9 \%$ plutonium, and 3.5\% fission products that range in composition from light transition metals to heavy alkali earth and lanthanide elements, ${ }^{5}$ necessitating lengthy and large-scale separation procedures for such complicated mixtures of elements. In 2000, the United States alone had an inventory of spent nuclear fuel of 42,000 metric tons, which was already three times the projected 2014 inventory of France or Korea. ${ }^{5}$ Alarmingly, these numbers do not even include military or ore mining waste. Such large quantities of nuclear waste require well characterized methods by which the waste can be stored and the surrounding environment and communities protected from the deleterious effects of waste exposure.

The expected operational lifetime of nuclear geological repositories is 10,000 years, during which time the isotopic and elemental makeup of spent fuel and other radioactive waste can change dramatically due to the varying half-lives of actinides and their fission daughters. For example, the initial activity at discharge of spent uranium fuel may be due in the largest part to ${ }^{239} \mathrm{~Np}\left(\mathrm{t}_{1 / 2}=2.34 \mathrm{~d}\right)$, while after 10 years the primary source of activity are several isotopes of cesium $\left({ }^{124} \mathrm{Cs} \mathrm{t}_{1 / 2}=2.01 \mathrm{yr}\right) .{ }^{6}$ The safe storage of nuclear materials over this lifetime requires the development of inert, well-characterized waste forms that can withstand possible erosion, corrosion, and radiation damage, and from which the radioactive elements cannot leech into ground water present at the geological site; current research on such materials focuses on the use of vitrification of waste actinides in glasses or ceramics. ${ }^{7}$ However, because the radiation type, half-lives, and chemical forms of radioactive waste vary depending on the source material, its operational environment, and its particular isotopic composition, such efficient storage materials require that the nuclear material contained within them be separated into 
fractions of similar chemical behavior and radioactive decay methods and half-lives. This separation, in turn, requires very specific reprocessing and separation technologies.

\subsection{Treatment of Radioactive Waste: The Selectivity Problem}

The challenges of chemically separating natural ore and nuclear waste solutions into appropriate fractions and physical forms can be illustrated by the nuclear waste forms found at the Savannah River Site (SRS) in Georgia. Here, amounts of acidic, highactivity waste were treated with large excesses of sodium hydroxide and are currently being stored in 48 carbon steel tanks. Physical forms of the stored waste include salt cake, sludge, and solution supernatant, with elements in the tank ranging from trace actinides to sodium in multi-molar concentrations. The primary sources of radioactivity in each tank and physical phase therein vary due to the chemical composition in each phase, with the primary activity in sludge arising from ${ }^{90} \mathrm{Sr}\left(9.8 \times 10^{7} \mathrm{Ci}, \mathrm{t}_{1 / 2}=28.9 \mathrm{yr}\right)$ $\left(1 \mathrm{Ci}=3.7 \times 10^{10} \mathrm{dpm}\right)$, while that in the salt cake and supernatant is estimated to be from ${ }^{137} \mathrm{Cs}\left(8.9 \times 10^{7} \mathrm{Ci}, \mathrm{t}_{1 / 2}=30.0 \mathrm{yr}\right)$. Reprocessing this waste is a complex physical and chemical challenge in which the most significant sources of radiation need to be removed from the bulk wastes to facilitate efficient storage and/or reprocessing applicaitons. ${ }^{8}$

Proposed methods for achieving efficient separations of SRS waste have focused on sorption of high valent $\mathrm{U}, \mathrm{Pu}$, and $\mathrm{Sr}$ into filterable sorbants such as monosodium titanate, followed by precipitation of the high activity Cs using sodium tetraphenylborate, leaving behind low-activity supernatants for evaporative treatments. However, these strategies suffer from the unknown chemical makeup of each tank's waste, which raises difficulties in controlling the radioactivity levels in the filtered sorbants and also results 
in unanticipated amounts of flammable benzene byproducts during Cs precipitation. As a result, these processes have been abandoned in favor of solvent extraction methods that are still under development. ${ }^{8}$

The initial purification of mined uranium from ores is of similar complexity because of the chemical variety of uranium ores. Complicated element mixtures are also encountered upon spent nuclear fuel reprocessing. Initial methods for plutonium separation from these mixtures focused on precipitation methods using $\mathrm{BiPO}_{4}$ to separate plutonium from uranium. These precipitation methods have also been abandoned because of the need for batch processes, large quantities of wastes, and because the uranium is discarded and not isolated for reuse. ${ }^{6,9}$

Current separation processes focus on liquid extractions, which utilize organic extractants dissolved in a water-immiscible organic phase (typically kerosene) which complex desired aqueous metal ions with high efficiency and draw them into the organic phase. The phases naturally separate and can be partitioned in a continual process, allowing for high throughput processes. Many recent reviews have outlined the wide variety of solvent extraction systems that have been and are still being developed. ${ }^{9-12}$

The most common liquid extraction process yet developed, and the one upon which most of the more recent extraction processes are based, is the PUREX process (Plutonium Uranium Reduction EXtraction). The extractant employed in this process is tributylphosphate (TBP, Figure 1-1); a 30\% solution of TBP in kerosene binds selectively via the hard Lewis basic oxygen atom to the high-valent actinides $\mathrm{U}(\mathrm{VI})$ and $\mathrm{Pu}(\mathrm{IV})$ dissolved in 3- to 4-molar nitric acid, drawing them into the aliphatic phase. $\mathrm{U}(\mathrm{VI})$ and $\mathrm{Pu}(\mathrm{IV})$ are selectively bound according to principles of hard/soft acid/base preferences, ${ }^{13}$ 
while other $f$-element ions in their lower oxidation states and other transition metal and main group elements remain in the aqueous phase. The rich redox chemistry of Pu allows selective chemical reduction of $\mathrm{Pu}(\mathrm{IV})$ to $\mathrm{Pu}(\mathrm{III})$ which is poorly bound by TBP and can be back-extracted into nitric acid solution, leaving only the U(VI)-TBP complex in the organic solution. Subsequent stripping and scrubbing washes of both the Pu-rich aqueous and U-rich organic layers result in efficient, high-yield isolation of plutonium and uranium which can be reapplied towards power generation needs. Modifications of the PUREX process have been ongoing for over 50 years, but most of the new processes still utilize mono- or poly-dentate oxygen-donating extractants (Figure 1-1).

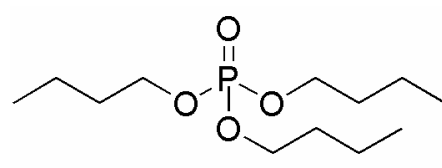

TBP

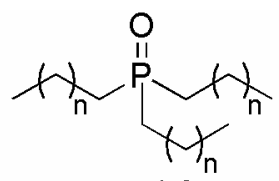

$\mathrm{n}=4-6$

TRPO<smiles>CCCCN(CC(C)C)C(=O)CP(=O)(CC(C)C)c1ccccc1</smiles>

CMPO

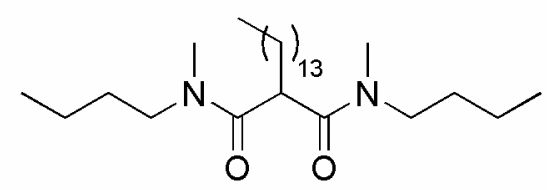

DMDBTDMA<smiles>NC(=O)CP(=O)(c1ccccc1)c1ccccc1</smiles>

Diphenylcarbamoylmethyl phosphine oxide

Figure 1-1. Extractants used in PUREX-based nuclear fuel separations.

The efficiency of the PUREX and related processes relies on the preference of actinides to bind to hard Lewis bases in their higher oxidation states. However, the similar behavior of many lanthanide/actinide pairs makes efficient separation between $f$ elements (actinide/lanthanide or actinide/actinide) a difficult challenge in separations science. The hard oxygen donors in the PUREX processes do not adequately address this challenge because all $f$-elements are typically characterized as exhibiting hard Lewis acidity ${ }^{14}$ and thus interact strongly with the hard Lewis basic extractants in Figure 1-1. 
Early processes developed to address this challenge depend on tetraalkylammonium salts and diethylenetriaminepentaacetic acid (DTPA) to achieve satisfactory An/Ln(III) separations. ${ }^{9,10,15}$ However, current work in An/Ln(III) extractants focuses on exploiting the increased covalency of An(III) cations over their Ln(III) analogs due to the greater radial extension of the actinide $5 f$ orbitals in comparison to the lanthanide $4 f$ orbitals. Evidence for this difference in chemical bond covalency was observed very early on in ion exchange studies by Diamond and Seaborg ${ }^{16}$ and more recently using actinide crystalline halide formation enthalpies, ${ }^{17}$ actinide-chalcogen crystal structure bond lengths, ${ }^{18,19}$ and theoretical studies on $\mathrm{M}-\mathrm{N}$ bond orders in $\mathrm{U} / \mathrm{Nd}(\mathrm{III})-\mathrm{Cp}{ }^{*}$ complexes. ${ }^{20}$ Electron paramagnetic resonance and electron-nuclear double resonance studies have even shown $5 f$ participation in the plutonium complexes with the hard Lewis basic $\mathrm{CO}_{2}{ }^{-}$ complexant. $^{21}$

Taking advantage of the $5 f$ covalency in actinide cations the above studies establish has led to several developments in extraction technology geared towards efficient $\mathrm{An} / \mathrm{Ln}(\mathrm{III})$ separations, for which the reader is referred to a recent review. ${ }^{12}$ Ligands for An/Ln(III) separations are typically polydentate and utilize softer (more electronically polarizable) coordinating groups such as pyridines and dithiophosphinic acids (Figure 12). These ligands typically coordinate the $f$-elements more weakly than oxygen donors, but they exhibit very good selectivity for the An(III) cations due to the participation of their $5 f$ electronic orbitals in ligand bonding. The higher denticity of the nitrogencontaining ligands in Figure 1-2 also increases separation efficiency due to the assistance of the chelate effect and allows for preorganization of binding moieties to complement their target cations, further increasing extraction efficiencies. ${ }^{12}$ 


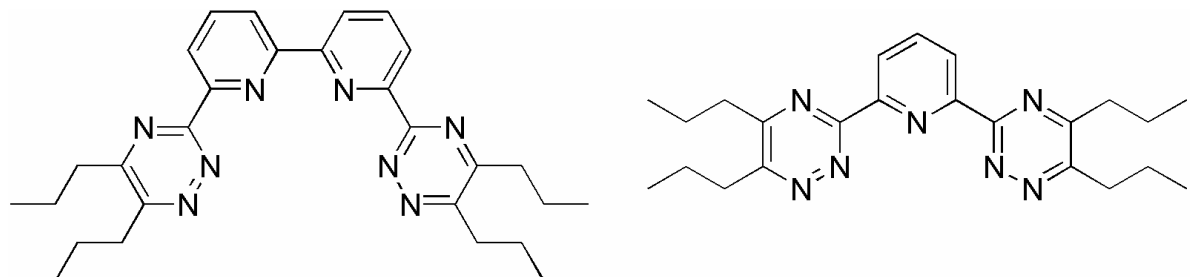

Figure 1-2. N- and S-coordinating extractants for An/Ln(III) separations.
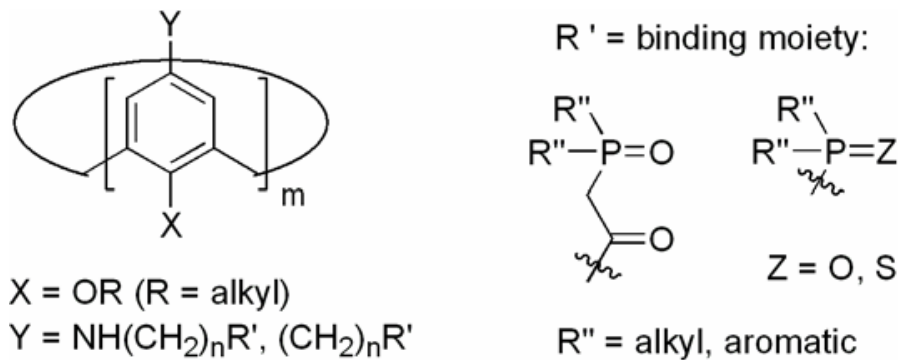

Figure 1-3. Cavitands designed for $\mathrm{An} / \mathrm{Ln}(\mathrm{III})$ separations.

Higher denticity and preorganization are also the inspiration behind the development of cavitands (Figure 1-3) as potential extractants for $\mathrm{An} / \mathrm{Ln}$ (III) separations. These capshaped molecules allow for easy functional group variation that enables tailoring of solubility, rigidity, and ligand donor characteristics. This variability allows the preorganization of the cavitand to be coupled with other extraction technologies that utilize binding moieties such as CMPO and dithiophosphinic acids. Of particular interest to this report is that the introduction of rigidity into the extractant and preorganization of the molecule for cation binding has been demonstrated to increase the selectivity and efficiency of cavitands for An/Ln(III) separations. ${ }^{12,22}$ Development of selectivity within the $f$-block series presents the most complex challenge to separations technology to date and clearly demonstrates the need for fine-tuning the cation selectivity of extractants to be used in nuclear waste separations and fuel reprocessing. 


\subsection{Actinides in Biology; Chelation Therapy}

Since geological interment on very long time scales is the current method of choice for nuclear waste storage, the threat of biological/human contamination by leaching of actinides and their fission daughters is of great concern. ${ }^{23-25}$ Whether actinide intoxication results from leaking stored waste or during handling of waste and/or ore tailings, nontoxic decorporation agents are needed to address the unique health threat these elements pose. While low efficiency extractants may be tolerated in industrial actinide reprocessing, biological actinide decontamination requires ligands that display both high selectivity and high affinity for target ion removal. Reviews by Andersen and Durbin on chelation therapy for metal intoxication and therapeutic drug design highlight the several aspects important to the design of ion-specific decorporation agents. ${ }^{26,27}$ Practical clinical considerations require that the chelating agents exhibit low toxicity and rapid binding kinetics. Much like in extractions, however, the aspect of utmost importance in drug design is the drug's affinity and selectivity for target ions.

Maximizing ligand affinity necessarily requires optimizing the thermodynamic benefit of target cation binding, driving the equilibrium in Equation 1-2 towards $M_{x} L_{y}$ complex formation and free energy maximization described by Equation 1-3. As in $\mathrm{An} / \mathrm{Ln}(\mathrm{III})$ separations, optimization relies upon matching the hardness/softness between ligand and target ion, utilizing either strong ionic or covalent interactions according to the target metal. Higher denticity ligands also lead to increasing ion affinity by utilizing the chelate effect by which thermodynamic stability is increased through increasing the number of metal interactions per ligand; this also lowers the entropy term in the free energy equation by requiring the desolvation of fewer ligands upon metal coordination, although 
significant enthalpy gain can be achieved if polydentate coordination reduces the repulsive energy of similarly charged moieties on the chelating ligand. ${ }^{26}$

$$
\begin{gathered}
x M+y L \stackrel{\beta_{x y}}{\rightleftharpoons} M_{x} L_{y} \quad \beta_{x y}=\frac{\left[M_{x} L_{y}\right]}{[M]^{x}[L]^{y}} \\
\Delta G=\Delta H-T \Delta S=-R T \ln \beta_{x y}
\end{gathered}
$$

A second crucial aspect of chelator design is the selectivity of the ligand for the target ion, because the metal intoxication is presumably a minor component in the biological system when compared to the alkali, alkali earth, and transition metals in the system. These other ions may serve crucial functions in the normal operation of proteins and intercellular signaling, resulting in toxic side effects in the event of non-specific ion removal. Selectivity optimization requires knowledge of the preferred coordination modes of the target element which are then applied towards the design of ligands that complement this behavior. Electronic considerations include, as for metal affinity, the choice of hard or soft coordinating atoms. Important geometric considerations include preferred coordination number, geometry, and ionic radius agreement between the metal and ligand. The positive effect of favorable ligand geometry coupled with the chelating effect of polydentate ligands can be seen in the relative An/Ln(III) separating ability of polypyridine ligands in nuclear separations, ${ }^{12}$ but is of utmost importance in biological media where the avoidance of essential element chelation can determine a drug's clinical implementation.

Unlike transition and main group element intoxication, for which the primary health threat is the metal's chemical toxicity, actinide contamination carries with it the added risk of radiation damage and tumor development following acute and chronic exposures 
respectively. Studies on mice have shown that actinide residence in blood plasma after injection drops significantly in the first 100 hours after injection, and also that actinides distribute themselves throughout the body very differently depending on the element. ${ }^{27}$ For example, approximately $90 \%$ of injected $\mathrm{Pu}(\mathrm{IV})$ deposits in the skeleton and soft tissue but remains in the circulating plasma longer than $\mathrm{U}, \mathrm{Np}$, and Am, while only about $35 \%$ of injected U(VI) deposits in the body, split primarily between the skeleton and kidney. ${ }^{28}$ Thus, actinide chelators must be administered shortly after exposure and must display rapid binding kinetics in order to remove the actinides before they leave the blood plasma and become associated with organ matrices. However, if the actinides do associate within the organism, the chelating agent must bind with sufficient affinity to affect the removal of the actinides from both relatively weak, non-specific interactions seen between $\mathrm{U}(\mathrm{VI})$ and proteins to strong inorganic interactions in bone. ${ }^{29}$

Current chelation therapies for actinide intoxication rely on the Ca and Zn salts of the polyaminopolycarboxylic acid diethylenetriaminepentaacetic acid (DTPA), whose closely-related cousin ethylenediaminetetraacetic acid (EDTA) has been used in the chelation therapy of transition metals (Figure 1-4). ${ }^{30,31}$ Both EDTA and DTPA demonstrate high formation constants with most actinides, with typical log $\mathrm{K}_{\mathrm{ML}}\left(\mathrm{K}_{\mathrm{ML}}=\right.$ $[\mathrm{ML}] /[\mathrm{M}][\mathrm{L}])$ values of 23-30, but DTPA has the advantage of being an octadentate ligand $\left(\mathrm{N}_{3} \mathrm{O}_{5}\right.$ coordination) that complements the high coordination numbers of the $f$ elements. Their drawbacks include the need for frequent, large dosage intravenous administrations, coupled with their toxic tendencies to remove essential minerals from the body. This, coupled with the low efficiency of polyaminocarboxylic acids towards high 
valent early actinide removal, illustrates the need for future development for actinideselective chelator design. ${ }^{30}$

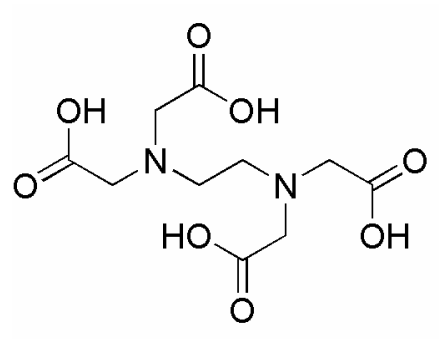

EDTA

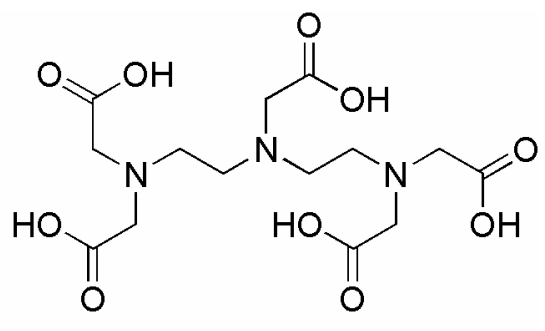

DTPA

Figure 1-4. Polyaminoacetic acids used in current chelation therapies.

\subsection{Rational Ligand Design: Siderophore-Inspired Sequestering Agents}

The unique coordination modes and biological chemistry of the actinides encourages a strategy of rational ligand design geared towards selective actinide chelation. The theory of rational design is discussed fully in a recent review ${ }^{32}$ and reconciles the typical chemical behavior of target ions with an appropriate ligand scaffold design. Pertinent considerations include ion size and typical coordination number, proton versus metal affinity of binding moieties at applicable $\mathrm{pH}$, hard/soft complement between ligand and metal ion, and ligand chelating ability.

The similarities between $\mathrm{Pu}(\mathrm{IV})$ and $\mathrm{Fe}(\mathrm{III})$ such as their charge per ionic radius ratio (4.2 and 4.6 e/ $\AA$ respectively), their rapid formation of insoluble hydroxides, and the binding of $\mathrm{Pu}(\mathrm{IV})$ by $\mathrm{Fe}(\mathrm{III})$-specific proteins in vivo prompted the Raymond group to pursue actinide ligand design by utilizing Fe(III)-binding moieties typically seen in nature. ${ }^{33}$ This biomimetic approach has focused for decades on the application of binding groups found in siderophores (bacterial ferric ion-specific chelators) in rationallydesigned ligands for targeted metal sequestration. ${ }^{34}$ Siderophores typically employ poly- 
bidentate, hard Lewis basic ligands typically of the catechol and/or hydroxamate forms (Figure 1-5). Both of these moieties require deprotonation to facilitate metal binding, making complex formation pH-dependent, but the high charge densities on $\mathrm{Fe}(\mathrm{III})$ and the high-valent early actinides typically encourage strong complex formation even at physiological $\mathrm{pH}^{33}$<smiles>Oc1ccccc1O</smiles>

Catachol<smiles>[14CH3]C(=O)NO</smiles>

Hydroxamate

Figure 1-5. Catechol and hydroxamate: typical chelating moieties in siderophore ligands.<smiles>[R]NC(=O)c1cccc(O)c1O</smiles>

Catecholamide

(CAM)<smiles>[R]NC(=O)c1cc(S(=O)(=O)O)cc(O)c1O</smiles>

$\operatorname{CAM}(\mathbf{S})$<smiles>[R]NC(=O)c1ccc(C(=O)O)c(O)c1O</smiles>

$\operatorname{CAM}(\mathbf{C})$<smiles>[R]NC(=O)c1ccc(C(=O)N(C)C)c(O)c1O</smiles>

Terephthalamide

(TAM)

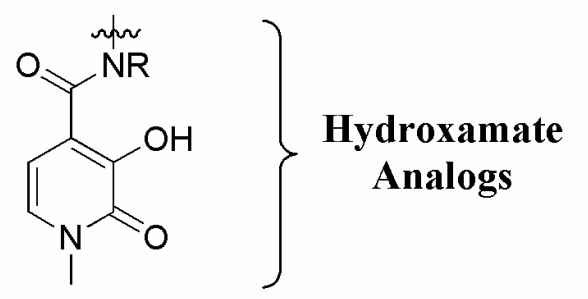

\section{1,2-HOPO Me-3,2-HOPO}

Figure 1-6. Synthetic siderophore moiety analogs used in Raymond group ligands.

In order to expand upon siderophore ligand design, the Raymond group has developed a library of binding moieties that include substituted catecholamides (CAM) and terephthalamides (TAM) as catechol analogs and hydroxypyridinones (HOPO) as analogs to hydroxamic acids (Figure 1-6). ${ }^{28,30,32,33,35}$ These moieties bind metal ions in a bidentate fashion via hard Lewis basic oxygens, making them ideal as strong chelators towards the f-elements. The introduction of the amide moiety ortho to the phenolic 
oxygen serves both as a point through which the ligand can be attached to a ligand scaffold, and also lowers the ligand $\mathrm{pK}_{\mathrm{a}}$ due to the electron withdrawing properties of the amide substituent. If the amide is primary, it also stabilizes the deprotonated and metal-

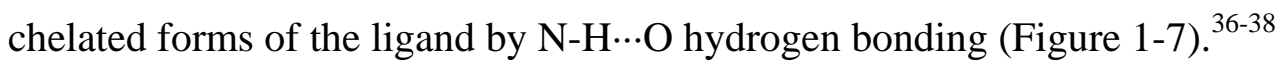
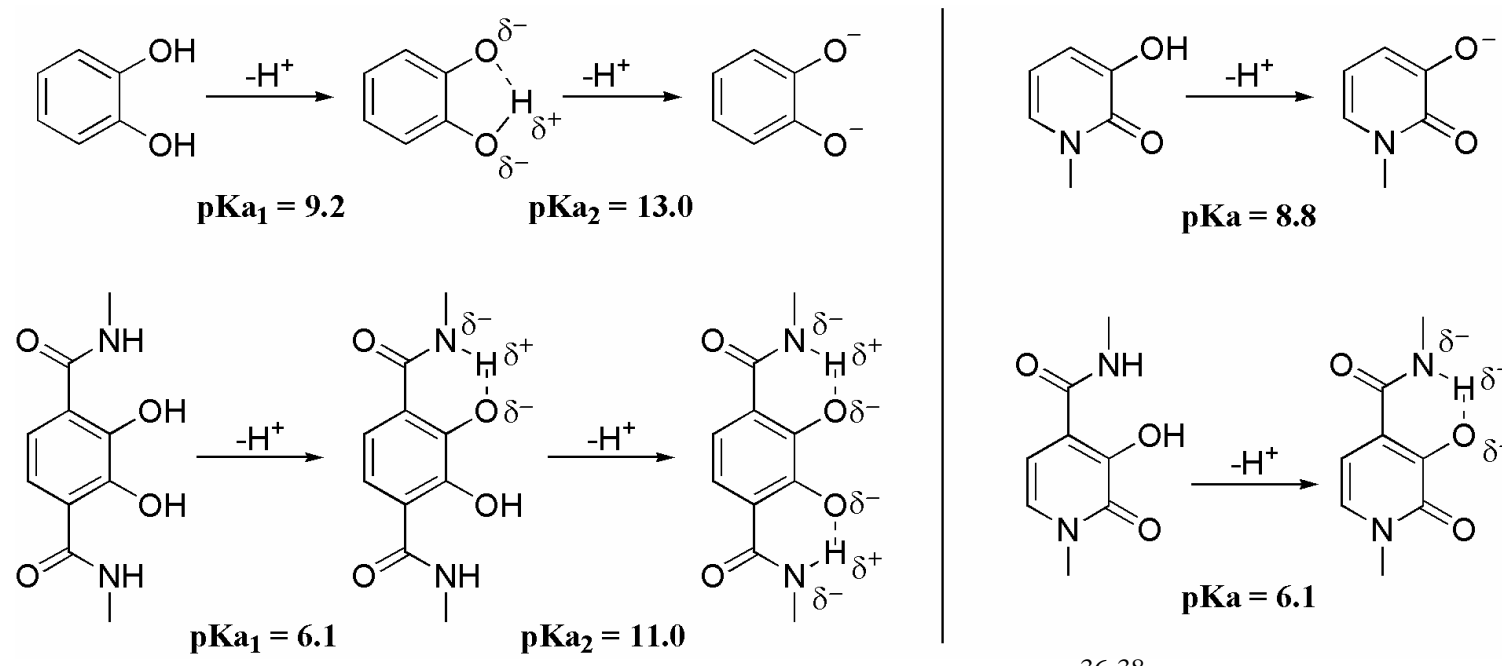

Figure 1-7. The effect of amide linkage on binding moiety $\mathrm{pK}_{\mathrm{a}} \cdot{ }^{36-38}$

The variety of siderophore-inspired binding moieties introduces a great deal of flexibility in ligand design for actinide sequestration, taking into consideration ligand geometry, potential steric hindrances, and proton affinity. Several polybidentate ligand geometries are accessible by coupling the binding moieties in Figure 1-6 to linear, triand tetrapodal backbone scaffolds, and are discussed in detail in a recent review. ${ }^{28} \mathrm{~A}$ large number of these ligands geometries have also been investigated in vivo to determine their efficacy in removing actinides and their toxicity to the infected organism..$^{28,30,35,39}$ The ligands display significant toxicity variations which range from very mild to severe; it is currently unclear what makes these ligand toxic because the difference between innocent and toxic effects is sometimes as drastic as the use of a different binding moiety and other times as minute as changing the linker lengths by one methylene unit. 
Importantly, however, many of the ligands investigated display effective removal of actinide contamination if applied immediately after actinide administration.

Trends in actinide removal efficacy can be tied in many cases to the choice of backbone geometry, but in the history of in vivo studies on actinide removal, there has been little structural investigation as to why such trends exist. Examples of these mysteries include why tetradentate ligands with 4 to 5 atom inter-group spacings show superior $\mathrm{Pu}(\mathrm{IV})$ chelating ability compared to their 3 and 6 atom inter-group analogs, why linear octadentate ligands typically outperform their $\mathrm{H}$-shaped analogs, and why the 4 and 5 atom intergroup spacing in tetradentate ligands seems to be optimal for U(VI) removal. ${ }^{30}$ Understanding such questions requires a detailed inspection of the coordination chemistry between the target ion and the multidentate ligand. Such a study would provide useful insight on the rational design of next-generation polybidentate ligands.

\subsection{Ligand Design for the Uranyl Cation, $\mathrm{UO}_{2}{ }^{2+}$}

The strongly electrophilic nature of early actinides in their highest oxidation states $\left(5+\right.$ and $6+$ ) results in the formation of dioxo cations of the general form $\mathrm{AnO}_{2}{ }^{\mathrm{n}+}$ (actinyl, $\mathrm{An}=\mathrm{U}, \mathrm{Np}, \mathrm{Pu} ; \mathrm{n}=1,2)^{14}$ which are particularly relevant targets for selective extraction because $\mathrm{Np}$ and $\mathrm{U}$ in biological and mildly oxidizing media are typically found in their $\mathrm{Np}(\mathrm{V})$ and $\mathrm{U}(\mathrm{VI})$ actinyl forms (Figure 1-8). Unlike transition metal dioxo species, actinyl cation geometry is strictly linear and no examples of typical uranyl or neptunyl complexes exist in which the dioxo moiety deviates more than a couple degrees from linearity. This persistent topology provides both a challenge and opportunity in the design 
of selective chelating moieties targeting $\mathrm{U}$ and $\mathrm{Np}$; the linear dioxo ion represents an especially unique target in biological and industrial systems allowing for geometric selectivity in addition to the typical electronic selectivity criteria used in chelation therapy, ${ }^{26}$ but the design strategies for selective $f$-element chelation (e.g. high denticity ligands designed for spherical cations) do not apply and require a new approach in chelator design. The unique actinyl geometry is responsible for lower polyaminopolycarboxylic acid $\log \mathrm{K}_{\mathrm{ML}}$ values for $\mathrm{Np}(\mathrm{V})$ and $\mathrm{U}(\mathrm{VI})$ which are 7.2 and 11, and 11 and 16 for EDTA and DTPA respectively; this weaker association results from the incomplete use of the six or eight chelating atoms in EDTA or DTPA, only three of which are able to make binding interactions with any one actinyl cations. Thus, the development of actinyl-specific chelators is needed in the medical community, and current work on the subject understandably focuses on the more ubiquitous uranium(VI) dioxo cation (uranyl, $\mathrm{UO}_{2}{ }^{2+}$ ).

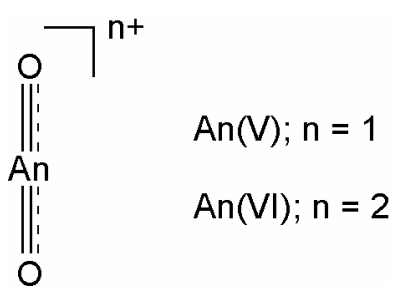

Figure 1-8. Actinyl cation geometry.

The nature of the bond between uranium and the terminal oxygen atom in the uranyl cation has been the subject of debate for decades, ${ }^{40-43}$ and the reader is directed to Denning's comprehensive review for a discussion thereof. ${ }^{44}$ Due to uranium $5 f-6 p$ hybridization that makes the low lying core electrons available for additional bonding to the oxygen $2 p_{x, y}$ orbitals, the $\mathrm{U}-\mathrm{O}$ bond order is approximately 3 because of delocalization of uranium core electrons and oxygen lone pair electrons into molecular 
orbitals in the actinyl cation. As a consequence, the uranyl oxo atoms are notoriously chemically inert; they exchange in water with a half-life greater than 40,000 hours, and typically do not partake in significant bonding interactions with Lewis acids. ${ }^{14,45}$ In contrast, the neptunium(V) dioxo cation (neptunyl, $\mathrm{NpO}_{2}{ }^{+}$) experiences a slightly lower overall $\mathrm{Np}-\mathrm{O}$ bond order ${ }^{46}$ and the terminal oxo moieties are observed to behave more classically as hard Lewis bases and are even known to participate in cation-cation interactions in crystal structures of discrete molecular $\mathrm{NpO}_{2}{ }^{+}$species as illustrated in Figure $1-9{ }^{47}$ and in polycationic species with trivalent cations (e.g. Fe, In, Sc) ${ }^{48}$

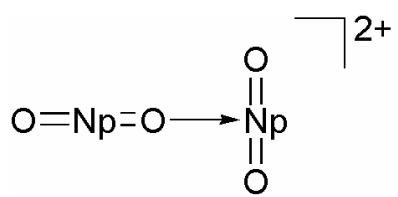

Figure 1-9. Cation-cation interaction observed with $\mathrm{NpO}_{2}{ }^{+}$and unobserved with $\mathrm{UO}_{2}{ }^{2+}$ outside of mineral structures.

The presence of the generally inert oxo moieties in actinyl cations relegates the coordination chemistry of actinyls to a coordination plane perpendicular to the $\mathrm{An}=\mathrm{O}$ vectors. ${ }^{14}$ The large ionic radius of uranium allows for a variety of energetically accessible actinyl coordination polyhedra that are typically hexagonal-bipyramidal, pentagonal-bipyramidal, octahedral or trigonal-bipyramidal $(6,5,4$, and 3 atoms in the actinyl coordination plane respectively, Figure 1-10). Only the equatorially coordinating ligands are dynamic in these polyhedra, so the coordination modes can be more simply classified as hexagonal-, pentagonal-, square- and trigonal-planar in nature respectively. The widely accepted explanation for population of the actinyl coordination plane is that these interactions are primarily electrostatic in nature and are greatly influenced by steric effects. ${ }^{14}$ Thus, the coordinatively crowded hexagonal bipyramidal polyhedra are observed in the presence of bidentate ligands with small bite angles [e.g. $\mathrm{UO}_{2}\left(\mathrm{NO}_{3}\right)_{3}{ }^{-}$or 
$\mathrm{UO}_{2}\left(\mathrm{CH}_{3} \mathrm{CO}_{2}\right)_{3}{ }^{-}$; bite angle $\left.=52^{\circ}\right],{ }^{49,50}$ trigonal bipyramidal and octahedral observed only with exceptionally sterically bulky ligands (e.g. $\mathrm{UO}_{2}\left[\mathrm{~N}\left(\mathrm{SiMe}_{3}\right)_{2}\right]_{3} ; \mathrm{UO}_{2}\left[\mathrm{PhC}\left(\mathrm{NSiMe}_{3}\right)_{2}\right]_{2}$, respectively) $)^{51,52}$, and pentagonal bipyramidal describing the rest, including many monodentate and bidentate ligands of moderate bite angle [e.g. $\mathrm{UO}_{2}(\mathrm{DMSO})_{5}{ }^{2+}$, $\mathrm{UO}_{2}$ (tropolonato $)_{2}\left(\text { py); bite angle }=64^{\circ}\right]^{53,54}$
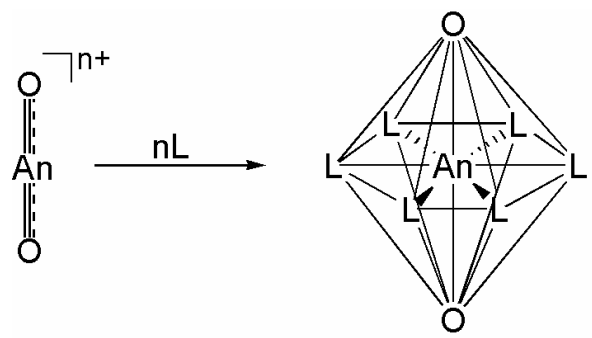

Hexagonal Bipyramidal

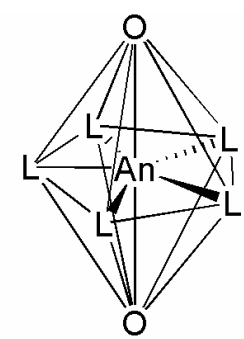

Pentagonal Bipyramidal

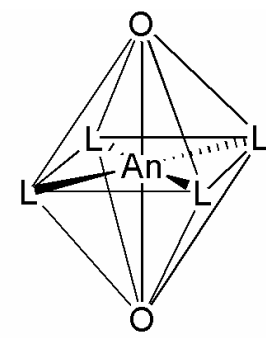

Octahedral

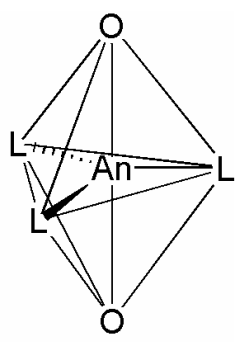

Trigonal Bipyramidal

Figure 1-10. Actinyl coordination polyhedra. Actinyl bond order is reduced for clarity.

Actinyls, like other $f$-elements, display a preference to bind with hard Lewis bases over softer donors. This is illustrated by the significant formation constants of the actinyl cations with the carbonate dianion $\left(\mathrm{CO}_{3}{ }^{2-}\right)$, the complexes of which are important in the geological transport of solubilized early actinides and are the predominant forms of oceanic uranium. ${ }^{23,24}$ Complexes with the more polarizable nitrogen, sulfur, or phosphorous coordinating atoms are typically weaker and only form stable complexes when these atoms are integrated into multidentate ligands or are auxiliary to harder oxygen donor atoms. The instability in aqueous media of the macrocyclic $\mathrm{AnO}_{2}([18]$ Crown-6) $(\mathrm{An}=\mathrm{Np}, \mathrm{U})$ complexes demonstrate that even oxygen atoms can be poor donor atoms to the actinyls when not in carbonyl or phenolate forms. ${ }^{55,56}$ However, if properly situated in polydentate scaffolds, even "soft" thioether sulfur atoms have been shown to coordinate to the uranyl cation, demonstrating that poor donor atoms can be utilized in an auxiliary fashion. ${ }^{57,58}$ Such ligand considerations focus exclusively 
on equatorial coordination modes without considering the oxo atoms as possible contributors to Lewis acid/base interaction. However, the uranyl oxo moiety does interact with crown-ether-stabilized alkali metal cations $\mathrm{Li}, \mathrm{Na}, \mathrm{K}$, and $\mathrm{Cs}$ in the crystalline phase, ${ }^{51,59,60}$ with relative O-M bond lengths indicating that uranyl oxo interactions with hard Lewis acids are potentially worth including in actinyl-selective ligands.

Prior work in the Raymond group has attempted to introduce stereognostic, or shape selective coordination modes in actinyl-specific ligands. These attempts centered on the design of tripodal ligands, the apex of which contained a protonated amine cap with the proton acting as the intended hard Lewis acid appropriately situated for actinyl oxo interactions $^{61,62}$ (Figure 1-11). These ligands demonstrated good uranyl complex formation, although sometimes oligomeric in nature, with asymmetric $\mathrm{U}=\mathrm{O}$ bond stretching properties supporting the hypothesis that a hydrogen-bonding interaction is present in the complex.
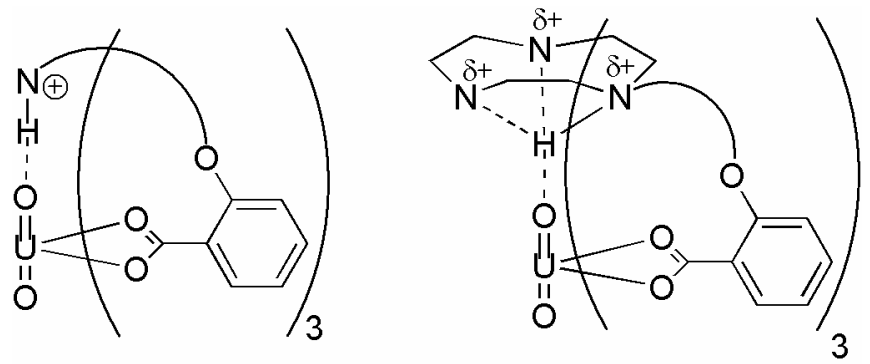

Figure 1-11. Stereognostic, tripodal ligands for uranyl chelation.

Intramolecular interactions of uranyl oxo atoms and Lewis acids have also been investigated by Arnold et. al. by utilizing polypyrrole fold-over macrocyclic ligands. ${ }^{63-65}$ The proximity of a protonated or $3 d$ metal-bound coordination pocket provides Lewis acidic interactions to one uranyl oxo atom at a minimum $\mathrm{M}_{3 d^{-}} \mathrm{O}$ bond length of $2.0 \AA$. This strong interaction and the hydrogen bonding seen in previous Raymond group 
ligands illustrate the potential for stereognostic interactions to play a role in actinyl ligand design.

Despite these promising results, uranyl coordination studies have typically focused on the actinyl coordination plane as the primary target for selective ligand design. Uranyl complex structural studies were carried out with the bidentate catecholate/hydroxamate analogs $1,2-\mathrm{HOPO}$ and $\mathrm{Me}-3,2-\mathrm{HOPO}$ to yield neutral $\mathrm{UO}_{2} \mathrm{~L}_{2}$ (solv.) complexes. ${ }^{57,66}$ These structures adopted the general structure shown in Figure 1-12, in which the deprotonated chelates bind on four points of a pentagon in the uranyl coordination plane, with the fifth coordination site occupied by solvent. This general structure persists even when the phenolate oxygen is replaced by a thiol group, ${ }^{57}$ or when the chelating bite angle is reduced from approximately $66^{\circ}$ to $64^{\circ}$ by using the tropolonate anion,,$^{54}$ indicating that such a coordination mode is relatively insensitive to moderate steric perturbations, ligand geometry, and charge density of the chelating unit.

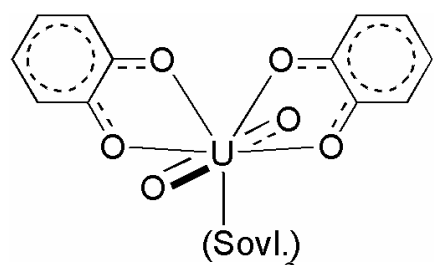

Figure 1-12. General coordination mode of $\mathrm{UO}_{2}{ }^{2+}$ with catecholate-type ligands.

Using these structural trends, many poly-bidentate ligands have been designed and subsequent biological uranyl decorporation studies indicated that linear bis-bidentate ligands utilizing the 1,5-pentane and diethyl ether linkers remove uranium well, whether the ligand utilizes the Me-3,2-HOPO, CAM(C), or CAM(S) moieties. ${ }^{35,39}$ However, linear octadentate 1,2- or Me-3,2-HOPO ligands also demonstrate a good ability to remove uranyl even once it has associated with bone and soft tissues. ${ }^{30}$ This juxtaposition of significantly different form with comparable function reveals the gap in understanding 
that still persists in the design of actinyl-specific chelators and encourages further exploration on the interaction of uranyl with siderophore-inspired ligands.

\subsection{Ligand Design for Pu(IV)}

With the exception of rare natural nuclear reactors, ${ }^{67}$ plutonium is exclusively manmade, and is a relatively abundant actinide species in nuclear fuel applications. Because of its widespread use it is an important target in the development of selective extractants in biphasic and biological environments, in which it typically adopts the $4+$ oxidation state. While $\mathrm{Pu}(\mathrm{V})$ and $\mathrm{Pu}(\mathrm{VI})$ adopt the linear actinyl geometry discussed above, $\mathrm{Pu}(\mathrm{IV})$ is a spherical cation like the lanthanides and late-actinides which makes ligand design for $\mathrm{Pu}(\mathrm{IV})$ a particularly challenging task that requires a detailed study of its coordination compounds to identify what separates its behavior from that of the other spherical $f$ elements.

As with most $f$-elements, the relatively low radial extension of $f$-orbitals in $\mathrm{Pu}(\mathrm{IV})$ does not impose strong bond directionality upon its coordination complexes. This, coupled with its relatively large ionic radius, affords $\mathrm{Pu}(\mathrm{IV})$ a great deal of flexibility in its coordination number and geometry, with an impressive twelve coordinating atoms observed in the $\mathrm{Pu}\left(\mathrm{NO}_{3}\right)_{6}{ }^{2-}$ complex. $^{68}$ Important to Raymond group ligand design, however, is that $\mathrm{Pu}(\mathrm{IV})$ typically forms octacoordinate complexes with bidentate, oxygen-donating ligands. ${ }^{69,70}$ The applicability of this behavior to siderophore-inspired ligands was corroborated in early structural studies of the $\left[\mathrm{Ce}(\text { catechol })_{4}\right]^{4-}$ complex $\left(\mathrm{Ce}^{4+}\right.$ is a generally accepted $\mathrm{Pu}^{4+}$ analog) which was shown to be octacoordinate by 
crystallography, with the oxygen atoms coordinating on the vertices of a trigonal dodecahedron (Figure 1-13). ${ }^{33}$
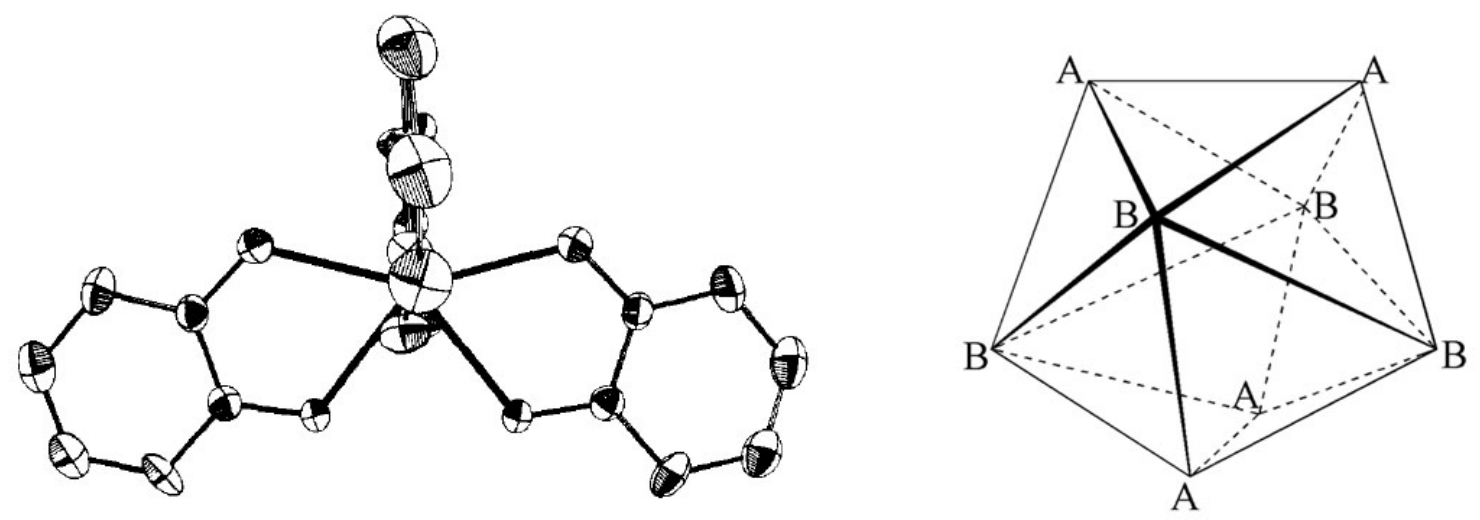

Figure 1-13. $\left[\mathrm{M}(\text { catechol })_{4}\right]^{4-}(\mathrm{M}=\mathrm{Th}, \mathrm{Ce}, \mathrm{U})$ complex geometry reproduced from Sofen et al. $^{71}$ and trigonal dodecahedral coordination geometry.

The trigonal dodecahedron ( $\mathrm{D}_{2 \mathrm{~d}}$ coordination geometry), is one of three eightcoordinate polyhedra identified by Kepert, the other two of which are the square antiprism $\left(\mathrm{D}_{4 \mathrm{~d}}\right)$ and bicapped trigonal prism $\left(\mathrm{C}_{2 \mathrm{v}}\right){ }^{72}$ Because only small angular differences exist between the three standard eight-coordinate polyhedra, and because of the coordinative flexibility of the actinides, the change in the coordination polyhedron that occurs upon the use of an asymmetric chelating moiety such as the 1,2- or Me-3,2HOPO moiety cannot be predicted and requires detailed structural investigation.

Due to its higher dynamic coordination number than actinyl cations, the Raymond group and its collaborators have focused on the use of octadentate ligands in Pu(IV) decorporation studies. ${ }^{28}$ To date, the spermine-linked tetrakis-1,2-HOPO ligand 3,4,3Li1,2-HOPO (Figure 1-14) has shown the best ability to remove injected Pu(IV). ${ }^{30}$ Because of the low acidity of the 1,2-HOPO moiety, this ligand forms octadentate Pu(IV) chelates at physiological $\mathrm{pH}$. However, no structural information exists on the geometry of the ligand about the $\mathrm{Pu}(\mathrm{IV})$ ion, without which rational modifications to the ligand are very 
difficult to make, leaving ligand design up to a guess-and-check process. Because as of June 15 there were only 45 plutonium structures in the Cambridge Crystallographic Database, it is essential that more structural information be gathered on plutonium complexes - especially those with siderophore-inspired chelates - to provide a knowledge base upon which to develop future actinide-specific chelating agents.

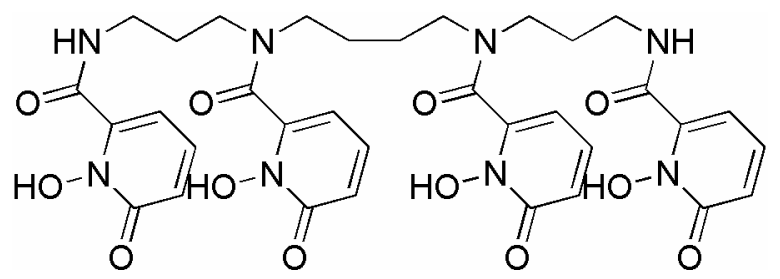

Figure 1-14. Spermine-linked 3,4,3-Li-1,2-HOPO.

\subsection{Focus of the Current Study}

In a desire to better understand the detailed coordination chemistry of actinides with the synthetic siderophore analogs in Figure 1-6, synthesis and single crystal structural analysis of actinide coordination compounds has been a long-standing goal of the Raymond group. Two recent structural studies detailed the coordination chemistry of tetradentate Me-3,2-HOPO ligands about $\mathrm{U}(\mathrm{VI})$ (uranyl, $\mathrm{UO}_{2}{ }^{2+}$ ) and $\mathrm{Pu}(\mathrm{IV}){ }^{66,73,74}$ The $\mathrm{U}(\mathrm{VI})$ complexes were those with parent bidentate and linearly-linked, tetradentate bisMe-3,2-HOPO ligands, while that with Pu(IV) was with the tetradentate, ethyl-ether bridged 5LiO-Me-3,2-HOPO ligand (Figure 1-15).<smiles>CCCNC(=O)c1ccn(C)c(=O)c1O</smiles>

Pr-Me-3,2-HOPO<smiles></smiles>

nLi-Me-3,2-HOPO<smiles>Cn1ccc(C(=O)NCCOCCNC(=O)c2ccn(C)c(=O)c2O)c(O)c1=O</smiles>

5LiO-Me-3,2-HOPO

Figure 1-15. Me-3,2-HOPO ligands used in $\mathrm{UO}_{2}{ }^{2+}$ and $\mathrm{Pu}(\mathrm{IV})$ structural studies. 
From the $\mathrm{U}(\mathrm{VI})$ complexes an unconstrained ligand geometry about the uranyl cation was established. Although a tetradentate ligand coordination mode is unsurprisingly maintained in all the complexes similar to that illustrated in Figure 1-12, linker length and flexibility have a dramatic effect on the geometry of the resultant coordination complex. ${ }^{66}$ However, the uranyl affinities of these ligands have not been measured, leaving the question of how the observed geometric differences affect formation constant unanswered. Additionally, the uranyl complexes with bis-HOPO ligands utilizing rigid backbones have never been studied, leaving open the question as to whether uranyl affinity can be further optimized by pre-organizing the ligand into a more favorable conformation than that offered by the nLi-Me-3,2-HOPO ligands.

The recent structural study on the $\mathrm{Pu}(\mathrm{IV})$ complex with the tetradentate 5LiO-Me-3,2HOPO ligand demonstrated that $\mathrm{Pu}(\mathrm{IV})$ seems to preferentially form eight-coordinate complexes with these ligands, a result that was predicted by and consistent with the analogous Ce(IV) crystal structures with the same ligand. ${ }^{73,74}$ As of 2005, however, this was the only $\mathrm{Pu}(\mathrm{IV})$ structure that utilized binding moieties in Figure 1-6, so the untethered, "relaxed" geometric targets for ligand design were as yet unexplored. These, in turn, would help provide direction for future $\mathrm{Pu}(\mathrm{IV})$-specific chelator design.

The focus of this dissertation is to further explore the coordination chemistry of $\mathrm{UO}_{2}{ }^{2+}$ and $\mathrm{Pu}(\mathrm{IV})$ with ligands incorporating siderophore-inspired binding moieties. Efforts with the former will focus on the effects of introducing rigidity to tetradentate and hexadentate ligands, while work with the latter will be focused on elucidating the unconstrained, "relaxed" coordination modes of spherical actinides with catecholate and hydroxamate analogs. Because structural information on coordination complexes is so 
important to the goal of selective ligand design, very specific structural studies must be performed to explore, challenge, and ultimately understand the tolerances of actinide cations for specific binding groups and their structural modifications. Additionally, thermodynamic measurements must be performed to determine the effect of structural perturbations in solution.

Chapter 2 discusses the design and synthesis of rigidly-linked tetradentate bis-Me3,2-HOPO ligands, and their complexes with the uranyl cation will be structurally compared. Solution thermodynamic measurements will be explored and select rigid ligands will be compared against the previously-reported, linearly-linked bis-Me-3,2HOPO ligands.

Chapter 3 details the design and synthesis of tris-bidentate $\mathrm{TAM}(\mathrm{HOPO})_{2}$ ligands designed to saturate the coordination plane of the uranyl cation. Structural comparisons of the resulting uranyl complexes will be made. The synthesis of and solution phase thermodynamic measurements with water-soluble versions of these ligands will be discussed. These results are compared to the tetradentate bis-Me-3,2-HOPO ligands discussed in Chapter 2.

Chapter 4 describes the synthesis of and crystallographic comparison between three $\mathrm{Pu}(\mathrm{IV})$ complexes with the 1,2-HOPO moiety and its structural analogs the 2hydroxypyrones and their Ce(IV) analogs. To further explore the coordination chemistry of the Ce(IV)-2-hydroxypyrone, a series of $\mathrm{CeL}_{4}$ crystal structures $(\mathrm{L}=$ maltol or kojic acid derivatives) were synthesized and their coordination geometries compared. Using the trends in $\mathrm{Pu}(\mathrm{IV})$ and $\mathrm{Ce}(\mathrm{IV})$ coordination geometries seen in their crystal structures, a new class of tetrakis-bidentate $\mathrm{TAM}_{4}$ and $\mathrm{TAM}_{2} \mathrm{HOPO}_{2}$ ligands were synthesized which 
are undergoing studies on their ability to sensitize metal-centered lanthanide(III) luminescence in a collaborative effort within the Raymond group.

\subsection{References}

(1) Murray, R. L. Understanding Radioactive Waste; $5^{\text {th }}$ ed.; Battelle Press: Columbus, OH, 2003.

(2) Emsley, J. The Elements; $3^{\text {rd }}$ ed.; Clarendon Press: Oxford, 1998.

(3) Myasoedov, B. F.; Drozhko, E. G. In Chemical Separation Technologies and Related Methods of Nuclear Waste Management; Choppin, G. R., Khankhasayev, M. K., Eds.; Kluwer Academic Publishers: Dordrecht, 1999, p 209-246.

(4) Grenthe, I.; Drozdzynski, J.; Fujino, T.; Buck, E. C.; Albrecht-Schmitt, T. E.; Wolf, S. F. In The Chemistry of the Actinide and Transactinide Elements; $3^{\text {rd }}$ ed.; Morss, L. R., Edelstein, N. M., Fuger, J., Eds.; Springer: Dordrecht, The Netherlands, 2006; Vol. 1, p 253-698.

(5) "Disposition of High-Level Waste and Spent Nuclear Fuel; The Continuing Societal and Technical Challenges,” National Research Council, 2001.

(6) Clark, D. L.; Hecker, S. S.; Jarvinen, G. D.; Neu, M. P. In The Chemistry of the Actinide and Transactinide Elements; $3^{\text {rd }}$ ed.; Morss, L. R., Edelstein, N. M., Fuger, J., Eds.; Springer: Dordrecht, The Netherlands, 2006; Vol. 2, p 813-1264.

(7) Lumpkin, G. R. In Elements; Mineralogical Society of America: Chantilly, VA, 2006; Vol. 2, p 365-372.

(8) “Alternatives for High-Level Waste Salt Processing at the Savannah River Site," National Research Council, 2000. 
(9) Choppin, G. R. In Chemical Separation Technologies and Related Methods of Nuclear Waste Management; Choppin, G. R., Khankhasayev, M. K., Eds.; Kluwer Academic Publishers: Dordrecht, 1999, p 1-16.

(10) Mathur, J. N.; Murali, M. S.; Nash, K. L. Solv. Extr. Ion Exch. 2001, 19, 357-390.

(11) Paiva, A. P.; Malik, P. J. Radioanal. Nucl. Chem. 2004, 261, 485-496.

(12) Dam, H. H.; Reinhoudt, D. N.; Verboom, W. Chem. Soc. Rev. 2007, 36, 367-377.

(13) Pearson, R. G. Coord. Chem. Rev. 1990, 100, 403-425.

(14) Greenwood, N. N.; Earnshaw, A. Chemistry of the Elements; Pergamon Press: New York, 1997.

(15) Nash, K. L. In Actinides; May, I., Alvares, R., Bryan, N., Eds.; RSC Publishing: Manchester, UK, 2005, p 465-472.

(16) Diamond, R. M.; Street, K., Jr.; Seaborg, G. T. J. Am. Chem. Soc. 1954, 76, 14611469.

(17) Ionova, G.; Madic, C.; Guillaumont, R. Radiochimica Acta 1997, 78, 83-90.

(18) Gaunt, A. J.; Reilly, S. D.; Enriquez, A. E.; Scott, B. L.; Ibers, J. A.; Sekar, P.; Ingram, K. I. M.; Kaltsoyannis, N.; Neu, M. P. Inorg. Chem. 2008, 47, 29-41.

(19) Jensen, M. P.; Bond, A. H. J. Am. Chem. Soc. 2003, 124, 9870-9877.

(20) Roger, M.; Belkhiri, L.; Arliguie, T.; Thuéry, P.; Boucekkine, A.; Ephritikhine, M. Organometallics 2008, 27, 33-42.

(21) Bhide, M. K.; Kadam, R. M.; Babu, Y.; Natarajan, V.; Sastry, M. D. Chem. Phys. Lett. 2000, 332, 98-104.

(22) Boerrigter, H.; Tomasberger, T.; Verboom, W.; Reinhoudt, D. N. Eur. J. Org. Chem. 1999, 665-674. 
(23) Clark, D. L.; Hobart, D. E.; Neu, M. P. Chem. Rev. 1995, 95, 25-48.

(24) Klinkhammer, G. P.; Palmer, M. R. Geochim. Cosmochim. Acta 1991, 55, 17991806.

(25) Bodvarsson, G. S.; Boyle, W.; Patterson, R.; Williams, D. Journal of Contaminant Hydrology 1999, 38, 3-24.

(26) Andersen, O. Chem. Rev. 1999, 99, 2683-2710.

(27) Durbin, P. W. In The Chemistry of the Actinide and Transactinide Elements; $3^{\text {rd }}$ ed.; Morss, L. R., Edelstein, N. M., Fuger, J., Eds.; Springer: Dordrecht, The Netherlands, 2006; Vol. 5, p 3339-3440.

(28) Gorden, A. E. V.; Xu, J.; Raymond, K. N. Chem. Rev. 2003, 103, 4207-4282.

(29) Van Horn, J. D.; Huang, H. Coord. Chem. Rev. 2006, 250, 765-775.

(30) Durbin, P. W. Health Phys. 2008, 95, 465-492.

(31) Fukuda, S. Current Med. Chem. 2005, 12, 2765-2770.

(32) Ansoborlo, É.; Amekraz, B.; Moulin, C.; Moulin, V.; Taran, F.; Bailly, T.; Burgada, R.; Hengé-Napoli, M.-H.; Jeanson, A.; Den Auwer, C.; Bonin, L.; Moisy, P. C. R. Chemie 2007, 10, 1010-1019.

(33) Raymond, K. N.; Freeman, G. E.; Kappel, M. J. Inorg. Chim. Acta. 1984, 94, 193204.

(34) Raymond, K. N. Coord. Chem. Rev. 1990, 105, 135-153.

(35) Durbin, P. W.; Kullgren, B.; Raymond, K. N. Rad. Prot. Dosim. 1998, 79, 433443.

(36) Xu, J.; Radkov, E.; Ziegler, M.; Raymond, K. N. Inorg. Chem. 2000, 39, 41564164. 
(37) Martell, A. E.; Smith, R. M. Critical Stability Constants; Plenum: New York, 1977.

(38) Garrett, T. M.; Miller, P. W.; Raymond, K. N. Inorg. Chem. 1989, 28, 128-133.

(39) Durbin, P. W.; Kullgren, B.; Ebbe, S. N.; Xu, J.; Raymond, K. N. Health Phys. 2000, 78, 511-521.

(40) Wadt, W. R. J. Am. Chem. Soc. 1981, 103, 6053-6057.

(41) Tatsumi, K.; Hoffman, R. Inorg. Chem. 1980, 19, 2656-2658.

(42) Dyall, K. G. Mol. Phys. 1999, 96, 511-518.

(43) Walch, P. F.; Ellis, D. E. J. Chem. Phys. 1976, 65, 2387-2392.

(44) Denning, R. G. In Structure and Bonding; Springer-Verlag: Berlin, 1992; Vol. 79, p 215-276.

(45) Gordon, G.; Taube, H. J. Inorg. Nucl. Chem. 1961, 19, 189-191.

(46) Shamov, G. A.; Schreckenbach, G. J. Phys. Chem. A 2005, 109, 10961-10974.

(47) Forbes, T. Z.; Burns, P. C. J. Solid Sate Chem. 2009, 182, 43-48.

(48) Yoshida, Z.; Johnson, S. G.; Kimura, T.; Krsul, J. R. In The Chemistry of the Actinide and Transactinide Elements; $3^{\text {rd }}$ ed.; Morss, L. R., Edelstein, N. M., Fuger, J., Eds.; Springer: Dordrecht, The Netherlands, 2006; Vol. 2, p 699-812.

(49) Rammo, N. N.; Hamid, K. R.; Ibrahim, T. K. J. Alloys. Compd. 1994, 210, 319324.

(50) Templeton, D. H.; Zalkin, A.; Ruben, H.; Templeton, L. K. Acta Cryst. C 1985, C41, 1439-1441.

(51) Burns, C. J.; Clark, D. L.; Donohoe, R. J.; Duval, P. B.; Scott, B. L.; Tait, C. D. Inorg. Chem. 2000, 3924. 
(52) Sarsfield, M. J.; Helliwell, M.; Raftery, J. Inorg. Chem. 2004, 43, 3170-3179.

(53) Deshayes, L.; Keller, N.; Lance, M.; Nierlich, M.; Vigner, D. Acta Cryst. C. 1992, C41, 2209-2211.

(54) Degetto, S.; Marangoni, G.; Bombieri, G.; Forsellini, E.; Baracoo, L.; Gaziani, R. J. Chem. Soc. Dalton Trans. 1974, 1933-1939.

(55) Clark, D. L.; Keogh, D. W.; Palmer, P. D.; Scott, B. L.; Tait, C. D. Angew. Chem. Int. Ed. 1998, 37, 164-166.

(56) Thuéry, P.; Keller, N.; Lance, M.; Vigner, J. D.; Nierlich, M. New J. Chem. 1995, 19, 619.

(57) Casellato, U.; Vigato, P. A.; Tamburini, S.; Graziani, R.; Vidali, M. Inorg. Chim. Acta. 1983, 72, 141-147.

(58) Casellato, U.; Sitran, S.; Tamburini, S.; Vigato, P. A.; Graziani, R. Inorg. Chim. Acta. 1986, 114, 111-117.

(59) Danis, J. A.; Lin, M. R.; Scott, B. L.; Eichhorn, B. W.; Runde, W. H. Inorg. Chem. 2001, 40, 3389-3394.

(60) Thuéry, P.; Masci, B. J. Chem. Soc. Dalton Trans. 2003, 2411-2417.

(61) Franczyk, T. S.; Czerwinski, K. R.; Raymond, K. N. J. Am. Chem. Soc. 1992, $114,8138-8146$.

(62) Walton, P. H.; Raymond, K. N. Inorg. Chim. Acta. 1995, 240, 593-601.

(63) Arnold, P. L.; Blake, A. J.; Wilson, C.; Love, J. B. Inorg. Chem. 2004, 43, 8206.

(64) Arnold, P. L.; Patel, D.; Blake, A. J.; Wilson, C.; Love, J. B. J. Am. Chem. Soc. 2006, 128, 9610-9611. 
(65) Berard, J. J.; Schreckenback, G.; Arnold, P. L.; Patel, D.; Love, J. B. Inorg. Chem. 2008, 47, 11583-11592.

(66) Xu, J.; Raymond, K. N. Inorg. Chem. 1999, 38, 308-315.

(67) Sheng, Z. Z.; Kuroda, P. K. Nature 1984, 312, 535-536.

(68) Matonic, J. H.; Neu, M. P.; Enriquez, A. E.; Paine, R. T.; Scott, B. L. J. Chem. Soc. Dalton Trans. 2002, 2328-2332.

(69) Grigoriev, M. S.; Krot, N. N.; Bessonov, A. A.; Lyssenko, K. A. Acta Cryst. E 2006, E62, m2889-m2890.

(70) Brown, D.; Whittaker, B.; Tacon, J. J. Chem. Soc. Dalton Trans. 1975, 34-39.

(71) Sofen, S. R.; Abu-Dari, K.; Freyberg, D. P.; Raymond, K. N. J. Am. Chem. Soc. 1978, 100, 7882-7887.

(72) Kepert, D. L. Inorganic Stereochemistry; Springer-Verlag: New York, 1982.

(73) Gorden, A. E. V.; Shuh, D. K.; Tiedemann, B. E. F.; Wilson, R. E.; Xu, J.; Raymond, K. N. Chem. Eur. J. 2005, 11, 2842-2848.

(74) Gorden, A. E. V.; Shuh, D. K.; Tiedemann, B. E. F.; Wilson, R. E.; Xu, J.; Raymond, K. N. Chem. Eur. J. 2007, 13, 378. 


\section{Chapter 2:}

\section{Design, Structure, and Solution Thermodynamics of $\mathrm{UO}_{2}$ (Bis-Me-3,2-HOPO) Complexes}

\subsection{Introduction}

The most recent work by Raymond and co-workers on uranyl coordination chemistry utilized the propyl-substituted catechol and hydroxamate analog 3-hydroxy-1-methyl-1Hpyridin-2-one (Pr-Me-3,2-HOPO, 2-1) and its linearly-linked, tetradentate bis-Me-3,2HOPO ligands (nLi-Me-3,2-HOPO, 2-2, 2-3, 2-4, 2-5; "Li" stands for "linear, n = number of methylene units in backbone spacer; Figure 2-1). ${ }^{1}$ Crystal structure analysis of the uranyl complexes with $\mathbf{2 - 1}, \mathbf{2 - 3}, \mathbf{2 - 4}$ and 2-5 shows that the coordination complexes with mono- and bis-bidentate complexes adopt $\mathrm{UO}_{2}(\mathbf{2 - 1})_{2}$ (solv.) and $\mathrm{UO}_{2}(\mathbf{2 - 3} / \mathbf{2 - 4} / \mathbf{2}-$ 5)(solv.) structures, respectively. In each case the ligands coordinate in a plane perpendicular to the oxo moieties at four points of a pentagon, with the fifth equatorial coordination position occupied by a solvent molecule (Figure 2-2).<smiles>CCCNC(=O)c1ccn(C)c(=O)c1O</smiles>

Pr-Me-3,2-HOPO, 2-1<smiles></smiles>

nLi-Me-3,2-HOPO $\mathrm{n}=2:$ 2Li-Me-3,2-HOPO, 2-2

$\mathrm{n}=3:$ 3Li-Me-3,2-HOPO, 2-3

$\mathrm{n}=4:$ 4Li-Me-3,2-HOPO, 2-4

$\mathrm{n}=5$ : 5Li-Me-3,2-HOPO, 2-5

Figure 2-1. Me-3,2-HOPO ligands used in previous $\mathrm{UO}_{2}{ }^{2+}$ structural studies.

The untethered $\mathrm{UO}_{2}(\mathbf{2 - 1})_{2}(\mathrm{DMF})$ complex provides what can be considered the "relaxed," or ideal geometry for Me-3,2-HOPO coordination about the uranyl cation, as this structure is not influenced by the geometric requirements imposed by a linker between the two chelating moieties. This structure is characterized by HOPO ring 
coordination such that they are very nearly co-planar with each other and perpendicular to the $\mathrm{U}=\mathrm{O}$ vector. The $\mathrm{HOPO}$ moieties coordinate in a head-to-toe fashion that consequently cannot be maintained upon tethering the amide moieties to a common linker, but this planar coordination mode will be from hereon considered as the target geometry for further structural comparison.
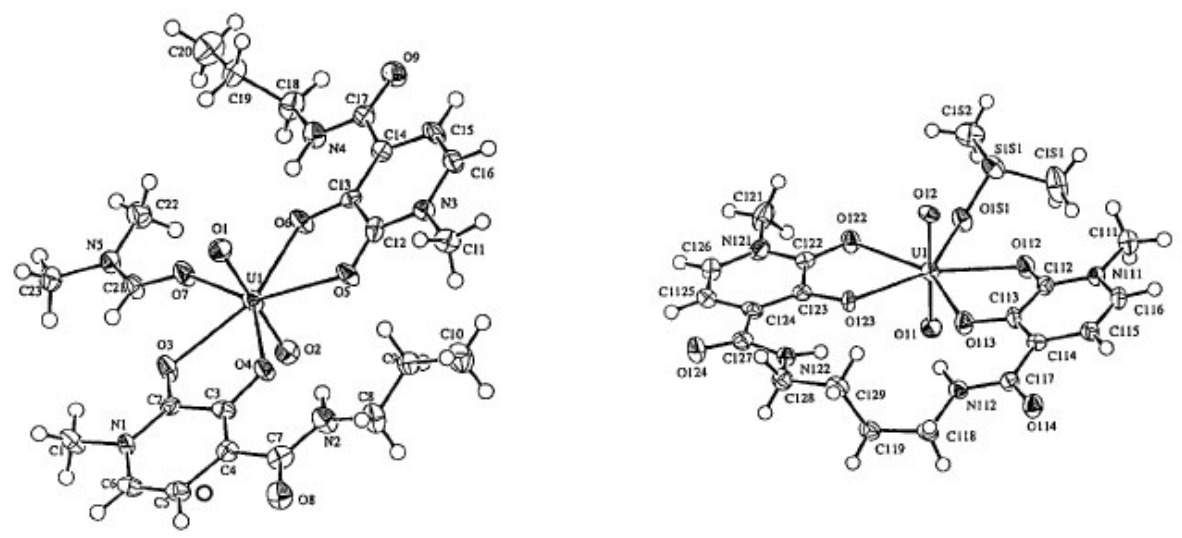

Figure 2-2. Crystal structures of $\mathrm{UO}_{2}(\mathbf{2 - 1})_{2}(\mathrm{DMF})$ (left) and $\mathrm{UO}_{2}(\mathbf{2 - 4})(\mathrm{DMSO})$ (right) from Xu et al. ${ }^{1}$

A co-planar coordination mode is typical of poly-bidentate uranyl coordination complexes with a wide variety of planar chelating moieties such as nitrate, ${ }^{2}$ tropolonate, ${ }^{3}$ salicylates, ${ }^{4}$ acetylacetonate, ${ }^{5}$ 3-hydroxy-pyran-4-ones ${ }^{6}$ and parent 1-hydroxypyridin-2one (1,2-HOPO), ${ }^{7}$ the latter two of which are structural analogs to the Me-3,2-HOPO moiety. Uranyl complexes with 3-hydroxy-pyran-4-ones, 1,2-HOPO and 2-1 share the general form $\mathrm{UO}_{2} \mathrm{~L}_{2}$ (solv) ( $\mathrm{L}=$ bidentate moiety) which results in pentagonal planar coordination perpendicular to the $\mathrm{U}=\mathrm{O}$ vector with ligand bite angles of $66-67^{\circ}$. Thus, the unconstrained structure of the $\mathrm{UO}_{2}(\mathbf{2 - 1})_{2}(\mathrm{DMF})$ complex is very consistent with complexes of structurally analogous chelating moieties and provides an apparently wellcorroborated coordination geometry target for further ligand design. 
In the crystal structures of uranyl with $\mathbf{2 - 3 , 2 - 4}$, and $\mathbf{2 - 5}$ the most immediate structural consequence of linker inclusion is a variable amount of distortion of the HOPO moieties out of co-planarity and perpendicularity to the $\mathrm{U}=\mathrm{O}$ vectors. Because the degree of deviation is highly dependent on the length of the linker, the effect is presumed to be caused solely by the geometric requirements of the linear linker as the HOPO moieties attempt to conform to the pentagonal planar coordination mode illustrated in the parent $\mathrm{UO}_{2}(\mathbf{2 - 1})(\mathrm{DMF})$ complex. It was determined that the $4 \mathrm{Li}-\mathrm{Me}-3,2-\mathrm{HOPO}$ ligand in the $\mathrm{UO}_{2}(\mathbf{2}-4)(\mathrm{DMSO})$ complex provided the closest approach to the untethered geometry of $\mathrm{UO}_{2}(\mathbf{2 - 1})(\mathrm{DMF})$, while ${ }^{1} \mathrm{H}$ NMR analysis of the amide proton shift (indicating favorable hydrogen bond between amide protons and deprotonated phenolate oxgyen) suggested that the ethyl linker in $\mathbf{2 - 2}$ provided the best arrangement for intramolecular hydrogen bonding.

One informative extension of the previous geometric study would involve the substitution of rigid linkers for the linear ones already explored. Since complexes with linkers of 2 to 5 methylene spacers have been shown to coordinate the uranyl cation, such rigidity may introduce a degree of ligand preorganization that would benefit actinyl selectivity, as the linear linkers in $\mathbf{2 - 2}$ through $\mathbf{2 - 5}$ could admittedly allow effective chelation to both spherical and non-spherical cations alike, with unknown ion size selectivity. To date there has also been no quantification of the relative affinity of HOPO ligands for the uranyl cation in solution. Thermodynamic measurement of this affinity would provide a much-needed comparison to the trends seen in the solid state and would provide invaluable information on further ligand design efforts. Finally, attempts have not been made to combine the in-plane chelation mode of bis-bidentate siderophore 
ligands with out-of-plane functionalization that could be used as a stereognostic function that provides Lewis acidity for interaction with the Lewis basic oxo substituents. Amidate ligands of similar geometry have been previously developed in the Raymond group and their complexes with transition metal oxo and dioxo species have been studied, ${ }^{8,9}$ but were never explored with the uranyl cation.

This chapter will address efforts to expand the knowledge of the uranyl-bis-Me-3,2HOPO coordination behavior. The work described explores the effect of rigid linkers on the uranyl complex geometries, the effect of linker geometry and length on uranyl affinity as measured by solution thermodynamics, and efforts towards introducing out-of-plane substituents onto in-plane coordinating bis-Me-3,2-HOPO moieties.

\subsection{Results and Discussion}

\subsubsection{Tetradentate Ligand Design and Synthesis}

In order to incorporate backbone rigidity into bis-Me-3,2-HOPO ligands for structure/affinity studies, aromatic moieties were used as linkers. Phenyl groups are particularly versatile in such an application because their chemistry is well-understood and a wide variety of convenient precursors are available for purchase or are readily synthesized. Such substitutional variety allows the incorporation of a phenyl group into linkers that provide the same number of carbons between amide nitrogens as those previously explored in the Raymond group. Additionally, the number and arrangement of aromatic rings in the backbone allows significant variation in the geometry and relative flexibility of the bis-Me-3,2-HOPO ligand. 
A series of bis-Me-3,2-HOPO ligands containing aromatic linkers were designed and are illustrated in Figure 2-3 below. The linkers were chosen to provide a wide variety in ligand geometry and rigidity (via the absence/presence of flexible methylene spacers). Like the linear linkers previously studied, the number of carbon atoms in the linker between the amide nitrogen (from hereon referred to as the value " $n$ ") was also allowed to vary between 2 to 5 , providing a rough resemblance to the ligand scope explored by $\mathrm{Xu}$ et al. ${ }^{1}$<smiles>[R][Y]([H])(C)NC(=O)c1ccn(C)c(=O)c1O</smiles><smiles>[Y]c1cscc1C(=O)c1cscn1</smiles><smiles>CC(C)(C)c1ccccc1C(N)=O</smiles><smiles>CC(C)(C)c1ccccc1C(C)(C)C</smiles>

3,4-Thiophene $o$-Phenylene (thio), 2-6 (o-phen), 2-7<smiles>Cc1cccc(C(C)(C)C)c1</smiles>

$m$-Phenylene (m-phen), 2-9

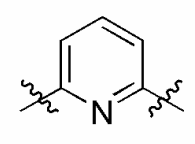

2,6-Pyridine (py), 2-10<smiles>CC(C)(C)C1c2cccc3cccc(c23)C1(C)C(F)(F)F</smiles>

1,8-Naphthalene (naphth), 2-11

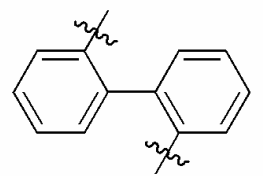

2,2'-Biphenyl

(biph), 2-12

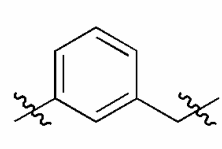

$m$-Toluene (m-tol), 2-13

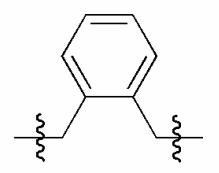

$o$-Xylene (o-xy), 2-14

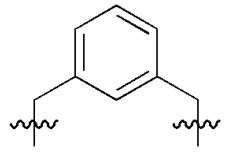

$m$-Xylene (m-xy), 2-15

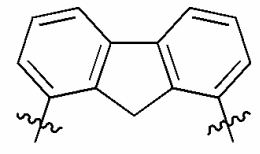

1,8-Fluorene (fluo), 2-16

Figure 2-3. Rigidly-linked bis-Me-3,2-HOPO ligands synthesized for structural studies with the uranyl cation. Backbone abbreviations are indicated and amide attachment points are indicated by wavy lines.

Between ligands of similar $n$ values, the differences between the ligand geometries range from subtle angular differences in very rigid scaffolds as in thio-Me-3,2-HOPO (2-6) and $\boldsymbol{o - p h e n - M e - 3 , 2 - H O P O ~ ( 2 - 7 ) ~ t o ~ l a r g e ~ d i f f e r e n c e s ~ i n ~ b a c k b o n e ~ f l e x i b i l i t y ~ a s ~ i n ~}$ m-xy-Me-3,2-HOPO (2-15) and fluo-Me-3,2-HOPO (2-16). Between $m$-phen-Me-3,2HOPO (2-9) and py-Me-3,2-HOPO (2-10) the only difference is the introduction of the 
pyridine nitrogen, which was expected to act as a hydrogen bond acceptor in the chelated complex.

A ligand design strategy utilizing the program HostDesigner was also employed to determine promising ligand linker geometries. ${ }^{10,11}$ The 4Li-Me-3,2-HOPO ligand was used as a model for these calculations because it provided a coordination geometry about the uranyl ligand that most closely approached that in $\mathrm{UO}_{2}(\mathbf{2 - 1})_{2}(\mathrm{DMF})$. Using the $\mathrm{UO}_{2}(\mathbf{2}-\mathbf{4})(\mathrm{DMSO})$ complex geometry as a starting point, possible linkers between two amide attachment points were calculated. Of the calculated ligand geometries, however, only the 1,8-diaminofluorene linker was reasonably synthetically accessible, giving rise to the target ligand 2-16.

Ligands 2-6 through 2-16 were all synthesized by amide coupling between the linker diamine and the benzyl-protected Me-3,2-HOPO moiety as illustrated in Scheme 2-1. In ligands containing at least one aryl amine, the amide coupling utilized the acid chloride of the benzyl-protected Me-3,2-HOPO acid, while the primary diamine precursors for $\boldsymbol{o}$ and $m$-xy-Me-3,2-HOPO (2-14 and 2-15) were coupled using the thiazoline-activated, benzyl-protected Me-3,2-HOPO moiety. ${ }^{12}$ Benzyl-protected ligand precursors 2-6(Bn) 2

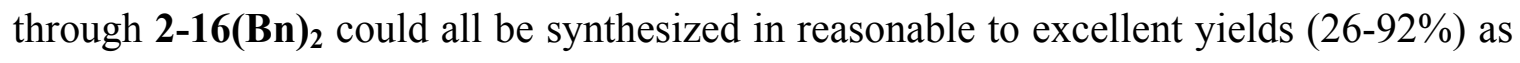
crystalline solids that were isolated cleanly by a combination of silica gel column chromatography and/or crystallization. These compounds range in color from white to brown, with darker colors typical of more highly conjugated ligands. Subsequent benzyl deprotection in strongly acidic, aqueous conditions was performed cleanly at room temperature over three days to afford the target ligands cleanly as white or beige, amorphous solids. The deprotected ligands in their protonated forms are generally 
insoluble in organic solvents except for DMF and DMSO, but deprotonation significantly increases their solubility in organic and aqueous media.

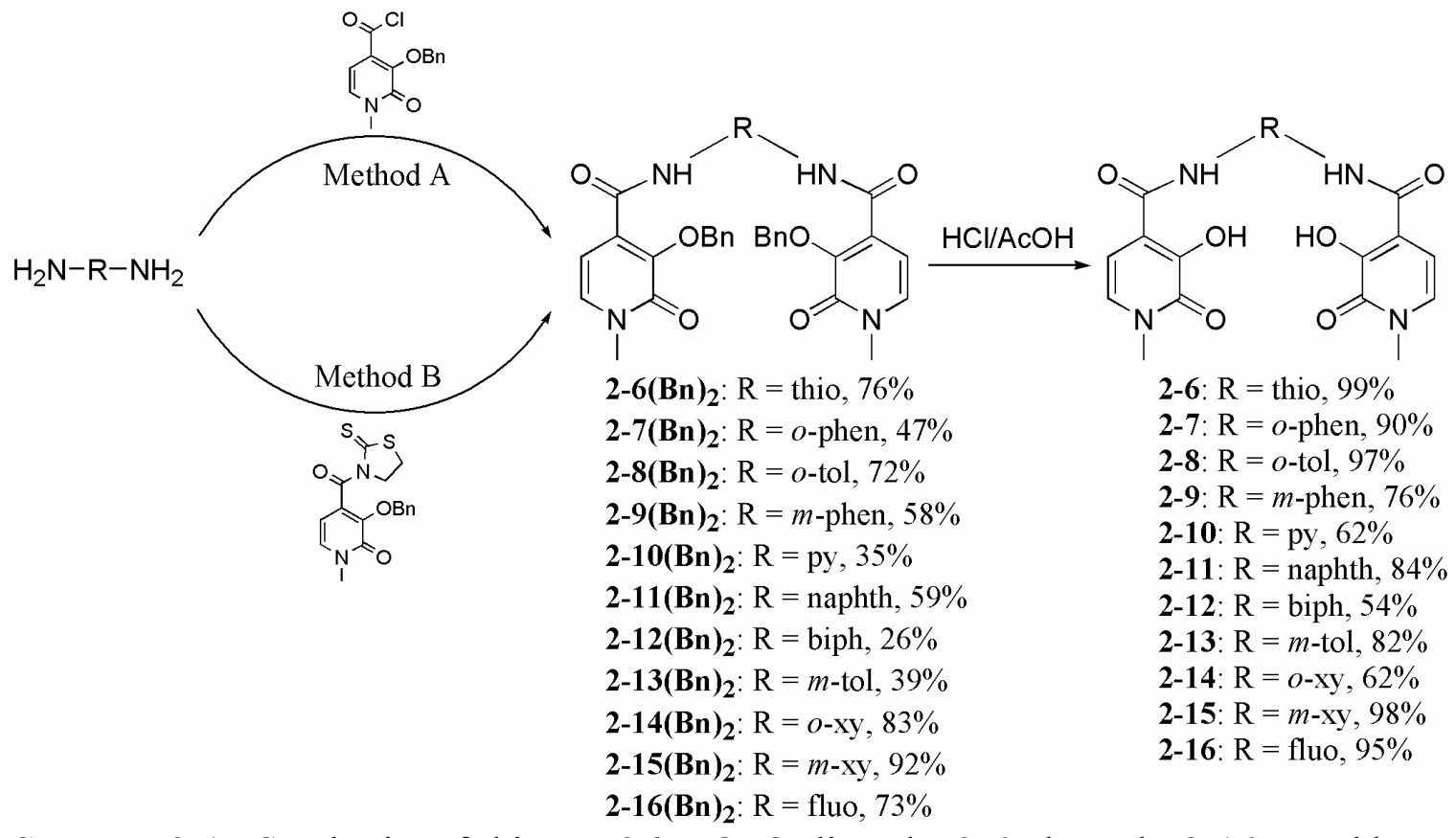

Scheme 2-1. Synthesis of bis-Me-3,2-HOPO ligands 2-6 through 2-16. Backbone abbreviations correspond to those listed in Figure 2-3.

\subsubsection{Synthesis and Structural Comparison of Uranyl Complexes}

Most uranyl complexes with bis-Me-3,2-HOPO ligands could be isolated following one of two general procedures. The first method consisted of refluxing the bis-Me-3,2$\mathrm{HOPO}$ ligand with uranyl nitrate and $\mathrm{Et}_{3} \mathrm{~N}$ or methanolic $\mathrm{KOH}$ to deprotonate the $\mathrm{HOPO}$ moiety, resulting in an orange or red powder which could be filtered. These powders were dried under vacuum over $\mathrm{P}_{2} \mathrm{O}_{5}$, but because the uranyl complexes are typically hydroscopic, their solids were often isolated as their polysolvates following this method. The second method involved dissolving the bis-Me-3,2-HOPO ligand, uranyl nitrate, and $\mathrm{Et}_{3} \mathrm{~N}$ in DMF or DMSO, making a typically dark red, homogeneous solution of the uranyl complex. Diffusion of a volatile organic solvent into this solution at room temperature or $4{ }^{\circ} \mathrm{C}$ yielded the uranyl complex in its crystalline form, which was isolated by filtration 
and dried by aspiration overnight, again often leading to solvates of the uranyl complexes. Uranyl complexes isolated as powders ranged in color from orange to red, and in some cases brown (with 2-6), but their final hue is solvent-dependent, with the complexes typically dark red in the presence of DMSO or DMF, and lighter red or orange in the presence of water or methanol. It is known from previous work with $\mathrm{UO}_{2}$ (bis-Me3,2-HOPO) complexes that the uranyl coordination plane is not saturated by the bis-Me3,2-HOPO ligands, ${ }^{1}$ and so this color change is most assuredly caused by variable coordination at the fifth equatorial coordination site at the uranium center, modifying the LMCT transition. This behavior also explains the general inability to isolate the uranyl complex without some form of solvent, whether in crystalline or amorphous phases.

While uranyl complexation reactions with most of the bis-Me-3,2-HOPO ligands in Figure 2-3 led to the formation of $\mathrm{UO}_{2} \mathrm{~L}$ (solv.) complexes (where $\mathrm{L}$ is the tetradentate, bis-Me-3,2-HOPO ligand), reactions with ligands 2-9, 2-10, 2-11, and 2-12 never lead to clean isolation of characterizable complexes. Following either of the two general methods described above, these ligands caused red or orange solids to very quickly precipitate out of solution, in temperature ranges of $25-140{ }^{\circ} \mathrm{C}$, even in DMSO. The resultant solids were typically only sparingly soluble in hot DMSO, and MALDI mass spectrometry of the precipitated solids suggests the presence of poorly-defined polymeric/oligomeric material, although clean NMR or mass spectrometry spectra were never obtained. Due to their low solubilities in DMSO and their propensity to form rapidly at elevated temperatures, these products are hypothesized to be coordination polymers. The incomplete saturation of the uranyl coordination plane typical of HOPO moieties allows 
for a great deal of feasible coordinative variation about the uranyl cation, making the polymeric structure impossible to guess.

The reason for this behavior is understandable if the geometry of the aromatic linker is investigated more closely. In ligands $\mathbf{2 - 9}, \mathbf{2 - 1 0}$ and $\mathbf{2 - 1 1}$, the linkers are completely rigid species, providing very little flexibility to the ligand geometry; ligands 2-9 and 2-10 hold the HOPO moieties far away from each other while 2-11 holds them very close. In such instances, the ligands are apparently incapable of distorting enough to enable mononuclear species formation and must each bind to more than one uranyl cation, leading to a polymeric mixture of products. Although ligand 2-12 contains some degree of rotational freedom about its center bond, it seems that it, too, is more inclined to bind two independent uranyl cations rather than one, again leading to polymeric material. A proposed schematic for such polymeric material is illustrated in Figure 2-4, although the coordination modes are purely speculative. Most important to the current study, however, is that these ligand geometries in 2-9 through 2-12 serve to define the boundaries of bisMe-3,2-HOPO ligand capability to form mononuclear species.

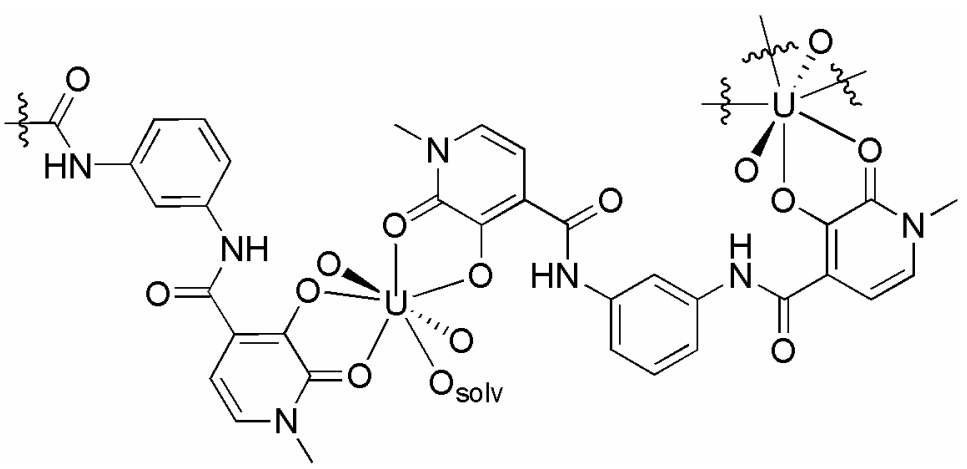

Figure 2-4. Speculated structure of uranyl coordination polymer with 2-10.

Crystals of the $\mathrm{UO}_{2}$ (bis-Me-3,2-HOPO) complexes were grown in order to perform X-ray crystallographic measurements and compare their coordination geometries. Crystallization attempts were also performed with ligand 2-2 because prior attempts did 
not result in crystals of sufficient quality for X-ray diffraction studies. In some instances, the uranyl complexes were isolated as X-ray quality crystals from their reaction solutions. When the complexes were isolated first as amorphous solids, however, crystals were readily grown by dissolving the solids in DMF, DMSO, or a mixture thereof, followed by vapor diffusion of a volatile organic solvent (typically $\mathrm{MeOH}$ or $\mathrm{Et}_{2} \mathrm{O}$ ) at room temperature or $4{ }^{\circ} \mathrm{C}$. The highly insoluble $\mathrm{UO}_{2}(\mathbf{2 - 1 6})$ complex could only be crystallized by slow cooling of a near-boiling DMSO solution, and only at the highest temperatures did X-ray quality crystals form; at temperatures below ca. $140{ }^{\circ} \mathrm{C}$ polycrystalline or amorphous materials quickly precipitated.

Crystalline uranyl complexes exhibited a variety of crystal habits ranging from blocks to needles to thin plates $10 \mu \mathrm{m}$ wide at their thickest point. In some cases the crystals were so small and poorly diffracting that very high intensity synchrotron radiation was required to collect data sufficient for crystallographic characterization. Because the uranyl center must coordinate a fifth atom to achieve coordinative saturation, the solvent system was of utmost importance in the crystallization process, with DMSO generally yielding the best and most consistent results. As a result of this coordinative variability, crystal color ranged from orange to deep red, with solvent inclusions common in the crystal lattice. Crystal structures of the $\mathrm{UO}_{2}$ (bis-Me-3,2-HOPO) complexes are shown in Figure 2-5 and their crystallographic parameters are listed in Table 2-1. 


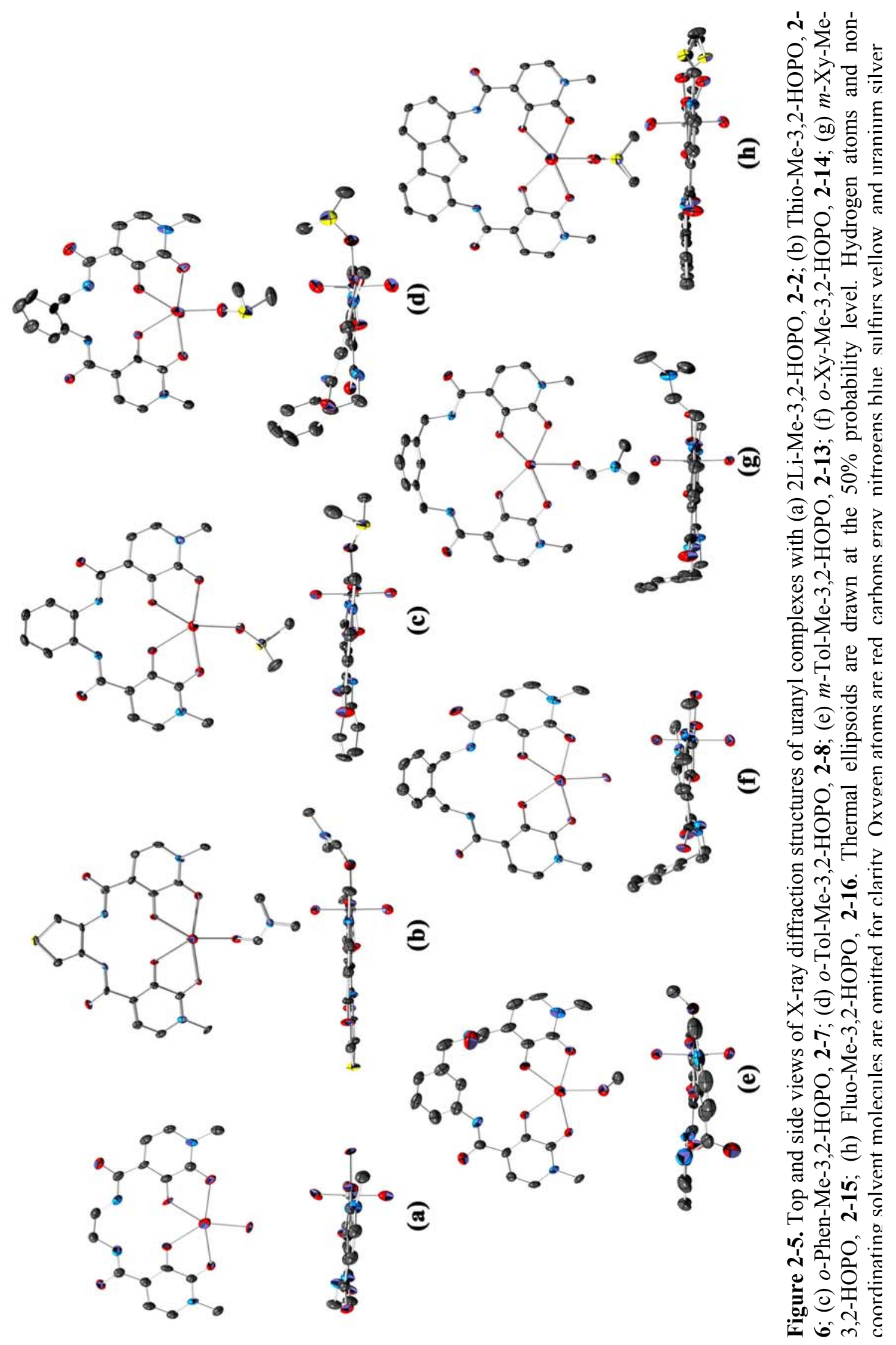


Table 2-1. Crystallographic parameters for $\mathrm{UO}_{2}$ (bis-Me-3,2-HOPO) complexes.

\begin{tabular}{|c|c|c|c|c|c|c|c|c|}
\hline Compound & $\mathrm{UO}_{2}(2-2)$ & $\begin{array}{c}\mathrm{UO}_{2}(2-6) \\
(\mathrm{DMF})\end{array}$ & $\begin{array}{l}\mathrm{UO}_{2}(2-7) \\
\text { (DMSO) }\end{array}$ & $\begin{array}{l}\mathrm{UO}_{2}(2-8) \\
\text { (DMSO) }\end{array}$ & $\begin{array}{c}\mathrm{UO}_{2}(2-13) \\
(\mathrm{MeOH})\end{array}$ & $\mathrm{UO}_{2}(2-14)$ & $\begin{array}{c}\mathrm{UO}_{2}(2-15) \\
\text { (DMF) }\end{array}$ & $\begin{array}{c}\mathrm{UO}_{2}(2-16) \\
(\mathrm{DMSO})\end{array}$ \\
\hline Formula & $\mathrm{C}_{18} \mathrm{H}_{16} \mathrm{~N}_{4} \mathrm{O}_{8} \mathrm{U}$ & $\mathrm{C}_{21} \mathrm{H}_{21} \mathrm{~N}_{5} \mathrm{O}_{9} \mathrm{SU}$ & $\mathrm{C}_{22} \mathrm{H}_{22} \mathrm{~N}_{4} \mathrm{O}_{9} \mathrm{SU}$ & $\mathrm{C}_{23} \mathrm{H}_{24} \mathrm{~N}_{4} \mathrm{O}_{9} \mathrm{SU}$ & $\begin{array}{c}\mathrm{C}_{22} \mathrm{H}_{22} \mathrm{~N}_{4} \mathrm{O}_{9} \mathrm{U} \cdot \\
\mathrm{C}_{2} \mathrm{H}_{6} \mathrm{OS} \\
\end{array}$ & $\begin{array}{c}\mathrm{C}_{22} \mathrm{H}_{20} \mathrm{~N}_{4} \mathrm{O}_{8} \mathrm{U} \cdot \\
\mathrm{CH}_{4} \mathrm{O} \\
\end{array}$ & $\mathrm{C}_{25} \mathrm{H}_{27} \mathrm{~N}_{5} \mathrm{O}_{9} \mathrm{U}$ & $\begin{array}{c}\mathrm{C}_{29} \mathrm{H}_{26} \mathrm{~N}_{4} \mathrm{O}_{9} \mathrm{SU} \cdot \\
1.6 \mathrm{H}_{2} \mathrm{O}\end{array}$ \\
\hline MW & 630.36 & 757.52 & 756.53 & 770.55 & 802.59 & 738.49 & 779.55 & 870.15 \\
\hline $\mathbf{T}[\mathbf{K}]$ & $223(2)$ & $156(2)$ & $161(2)$ & $193(2)$ & $165(2)$ & $155(2)$ & $180(2)$ & $173(2)$ \\
\hline Cryst. system & Monoclinic & Orthorhombic & Monoclinic & Triclinic & Triclinic & Monoclinic & Triclinic & Orthorhombic \\
\hline $\begin{array}{l}\text { Space group } \\
\end{array}$ & $\mathrm{P} 2_{1} / \mathrm{c}$ & $\mathrm{Pna}_{1}$ & $\mathrm{P} 2_{1} / \mathrm{n}$ & P-1 & P-1 & $\mathrm{P} 2_{1} / \mathrm{c}$ & P-1 & Pnma \\
\hline Appearance & Plate & Plate & Prism & Plate & Wedge & Prism & Plate & Rhombohedron \\
\hline Color & Red & Red & Red & Red & Orange & Red & Red & Red \\
\hline$a[\AA ̊]$ & $8.282(2)$ & $7.1702(9)$ & $13.7333(7)$ & $7.0126(5)$ & $8.8897(18)$ & $9.0217(5)$ & $10.7826(16)$ & $10.724(3)$ \\
\hline$b[\AA]$ & $15.468(3)$ & $16.166(2)$ & $13.6987(7)$ & $13.3648(9)$ & $13.317(3)$ & $15.2874(9)$ & $11.6388(17)$ & $15.738(4)$ \\
\hline$c[\AA]$ & $14.523(3)$ & $20.461(3)$ & $13.8370(7)$ & $27.8747(19)$ & $13.577(3)$ & $17.1567(10)$ & $11.6533(17)$ & $17.310(4)$ \\
\hline$\alpha\left[^{\circ}\right]$ & 90 & 90 & 90 & $92.8340(10)$ & $64.277(3)$ & 90 & $109.684(2)$ & 90 \\
\hline$\beta\left[^{\circ}\right]$ & $100.380(8)$ & 90 & $112.0530(10)$ & $96.215(2)$ & $75.544(3)$ & $93.8610(10)$ & $94.916(2)$ & 90 \\
\hline$\gamma\left[^{\circ}\right]$ & 90 & 90 & 90 & $100.443(2)$ & $71.472(3)$ & 90 & $104.023(2)$ & 90 \\
\hline $\mathbf{V}\left[\AA^{3}\right]$ & $1829.9(7)$ & $2371.7(5)$ & $2412.7(2)$ & $2547.7(3)$ & $1361.2(5)$ & $2360.9(2)$ & $1313.4(3)$ & $2921.7(12)$ \\
\hline $\mathbf{Z}$ & 2 & 4 & 4 & 4 & 2 & 4 & 2 & 4 \\
\hline$\rho_{\text {calcd }}\left[\mathrm{g} \mathrm{cm}^{-3}\right]$ & 2.288 & 2.121 & 2.083 & 2.009 & 1.958 & 2.078 & 1.971 & 1.978 \\
\hline$\mu_{\text {palcd }}\left[\mathrm{mm}^{-1}\right]$ & 4.821 & 6.995 & 6.875 & 3.590 & 6.102 & 6.938 & 6.243 & 3.150 \\
\hline$\theta_{\min }, \theta_{\max },\left[^{\circ}\right]$ & $2.12,30.14$ & $2.52,26.42$ & $1.78,26.37$ & $1.61,31.21$ & $1.68,24.44$ & $1.79,26.38$ & $1.89,26.44$ & $1.91,33.64$ \\
\hline $\begin{array}{c}\text { Total } \\
\text { reflections }\end{array}$ & 14287 & 12701 & 13455 & 33111 & 7628 & 13261 & 8514 & 16117 \\
\hline Data/restr./ param. & $4135 / 78 / 264$ & $4534 / 31 / 338$ & $4876 / 0 / 338$ & $12370 / 28 / 708$ & $4443 / 25 / 392$ & $4764 / 0 / 337$ & $5216 / 0 / 365$ & $4361 / 36 / 228$ \\
\hline$F(000)$ & 1184 & 1448 & 1448 & 1480 & 776 & 1416 & 752 & 1683 \\
\hline $\mathbf{T}_{\min } / \mathbf{T}_{\max }$ & 0.798 & 0.596 & 0.288 & 0.875 & 0.689 & 0.654 & 0.655 & 0.905 \\
\hline Cryst. size $\left[\mathrm{mm}^{3}\right]$ & $\begin{array}{c}0.06 \times 0.04 \times \\
0.01 \\
\end{array}$ & $\begin{array}{c}0.12 \times 0.06 \times \\
0.03 \\
\end{array}$ & $\begin{array}{c}0.35 \times 0.09 \times \\
0.06 \\
\end{array}$ & $\begin{array}{c}0.07 \times 0.06 \times \\
0.03 \\
\end{array}$ & $\begin{array}{c}0.15 \times 0.08 \times \\
0.06 \\
\end{array}$ & $\begin{array}{c}0.20 \times 0.15 \times \\
0.11 \\
\end{array}$ & $\begin{array}{c}0.11 \times 0.09 \times \\
0.03 \\
\end{array}$ & $\begin{array}{c}0.05 \times 0.03 \times \\
0.02 \\
\end{array}$ \\
\hline $\mathbf{R}_{1}[I>2 \sigma(I)]^{a}$ & 0.0576 & 0.0419 & 0.0256 & 0.0449 & 0.0403 & 0.0336 & 0.0292 & 0.0438 \\
\hline $\mathrm{wR}_{2}(\text { all data })^{a}$ & 0.1366 & 0.1035 & 0.0688 & 0.1166 & 0.0956 & 0.0779 & 0.0706 & 0.1241 \\
\hline GOF $^{a}$ & 1.151 & 0.976 & 1.039 & 1.108 & 1.020 & 1.333 & 0.997 & 1.143 \\
\hline
\end{tabular}

${ }^{a} \mathrm{R}_{1}=\Sigma|| \mathrm{F}_{\mathrm{o}}|-| \mathrm{F}_{\mathrm{c}}|/ \Sigma| \mathrm{F}_{\mathrm{o}} \mid ; \mathrm{wR}_{2}=\left[\Sigma\left[w\left(\mathrm{~F}_{\mathrm{o}}^{2}-\mathrm{F}_{\mathrm{c}}{ }^{2}\right)^{2}\right] / \Sigma\left[w\left(\mathrm{~F}_{\mathrm{o}}{ }^{2}\right)^{2}\right]\right]^{1 / 2} ; \mathrm{GOF}=\left[\Sigma w\left(\left|\mathrm{~F}_{\mathrm{o}}\right|-\left|\mathrm{F}_{\mathrm{c}}\right|\right)^{2} /(n-m)\right]^{1 / 2}$ 
In a result consistent with prior studies, ${ }^{1}$ the bis-Me-3,2-HOPO ligands in Figure 2-5 bind the uranyl in a tetradentate, mononuclear fashion with the two HOPO moieties chelating the uranyl at four points of a pentagon perpendicular to the $\mathrm{O}=\mathrm{U}=\mathrm{O}$ vector, resulting in an overall pentagonal bipyramidal coordination polyhedron about the uranium. The fifth equatorial coordination site is typically occupied by a molecule of solvent from the crystallization solution. In most cases this is DMF or DMSO, although the $\mathrm{UO}_{2}(\mathbf{2 - 1 3})$ complex contains a coordinated methanol. The two complexes $\mathrm{UO}_{2}(\mathbf{2 - 2})$ and $\mathrm{UO}_{2}(\mathbf{2 - 1 4})$ appear in Figure 2-5 to be coordinated by a water molecule, but in fact the coordinating oxygen is an amide oxygen from another $\mathrm{UO}_{2}$ (bis-Me-3,2-HOPO) moiety in the crystal, resulting in one-dimensional coordination polymer chains in the crystal lattice (shown in Appendix). Because these complexes are moderately soluble in DMSO and DMF, the polymeric structure must be a strictly solid state phenomenon, with the monomeric $\mathrm{UO}_{2}(\mathbf{2 - 2})$ and $\mathrm{UO}_{2}(\mathbf{2 - 1 4})$ complexes freely solubilized in solution. Cursory inspection of the crystal structures reveals that the degree of co-planarity of the HOPO moieties varies depending on the linker used, which is consistent with the $\mathrm{UO}_{2}(\mathbf{2 - 3} / \mathbf{2 - 4} / \mathbf{2}$ 5) structures. In all cases the uranyl cation remains linear, with $U=\mathrm{O}_{\text {oxo }}$ bonds that vary a maximum of $0.02 \AA$ intermolecularly and average a typical $\mathrm{U}=\mathrm{O}_{\text {oxo }}$ distance of $1.78 \AA$.

Table 2-2 compares the equatorial U-O bond distances in the $\mathrm{UO}_{2}$ (bis-Me-3,2HOPO) complexes against those in the untethered $\mathrm{UO}_{2}(\mathbf{2 - 1})_{2}(\mathrm{DMF})$ complex previously reported. ${ }^{1}$ Despite the variety of backbone geometries in each of these complexes, the U$\mathrm{O}$ bonds are surprisingly constant, with maximum variations within each bond type of 0.06-0.07 $\AA$. This range is comparable even to the $0.05 \AA$ intramolecular bond distance variation between the $\mathrm{U}-\mathrm{O}_{\text {amide }}$ bonds in the $\mathrm{UO}_{2}(\mathbf{2}-1)_{2}(\mathrm{DMF})$ complex. Thus, the $\mathrm{U}-\mathrm{O}$ 
bond variations between ligands of differing backbone geometries cannot be considered overly significant and suggest that the bond strengths with the uranyl cation must be similar, despite the change in ligand geometry and linker lengths.

Table 2-2. Equatorial U-O bond distances in $\mathrm{UO}_{2}$ (bis-Me-3,2-HOPO) crystal structures

\begin{tabular}{|c|c|c|}
\hline Complex & U-O(amide), $\AA$ & U-O(phenolate), $\AA$ \\
\hline $\mathrm{UO}_{2}(\mathbf{2 - 1})_{2}{ }^{[1]}$ & $2.457(5), 2.407(5)$ & $2.329(5), 2.329(5)$ \\
\hline $\mathrm{UO}_{2}(\mathbf{2 - 2})$ & $2.471(8), 2.442(8)$ & $2.301(7), 2.383(7)$ \\
\hline $\mathrm{UO}_{2}(\mathbf{2 - 6})(\mathrm{DMF})$ & $2.437(7), 2.431(6)$ & $2.337(7), 2.350(7)$ \\
\hline $\mathrm{UO}_{2}(\mathbf{2 - 7})(\mathrm{DMSO})$ & $2.446(3), 2.458(3)$ & $2.349(3), 2.330(3)$ \\
\hline $\mathrm{UO}_{2}(\mathbf{2 - 8})(\mathrm{DMSO})^{a}$ & $2.464(4), 2.450(4) ;$ & $2.306(4), 2.362(4) ;$ \\
\hline $\mathrm{UO}_{2}(\mathbf{2 - 1 3})(\mathrm{MeOH})$ & $2.447(5), 2.477(4)$ & $2.319(6), 2.374(5)$ \\
\hline $\mathrm{UO}_{2}(\mathbf{2 - 1 4})$ & $2.461(5), 2.417(5)$ & $2.320(5), 2.341(5)$ \\
\hline $\mathrm{UO}_{2}(\mathbf{2 - 1 5})(\mathrm{DMF})$ & $2.416(3), 2.432(4)$ & $2.364(4), 2.331(4)$ \\
\hline $\mathrm{UO}_{2}(\mathbf{2 - 1 6})(\mathrm{DMSO})^{b}$ & $2.433(3)$ & $2.357(3), 2.353(3)$ \\
\hline
\end{tabular}

${ }^{a}$ The crystal contained two unique uranyl complexes.

${ }^{b}$ Each half of the molecule is related crystallographically to the other half.

The crystal structures in Figure 2-5 exhibit a significant amount of variation in the coordination plane O-U-O bond angles, which are numbered according to Figure 2-6 and are compared against those in the "unconstrained" $\mathrm{UO}_{2}(\mathbf{2 - 1})_{2}(\mathrm{DMF})$ structure in Table 23 below. Angles $\sigma_{2}$ and $\sigma_{5}$ correspond to the bite angles of the HOPO moieties, and are constant at $c a .66 \pm 2^{\circ}$, indicating that the coordination mode of the Me-3,2-HOPO moiety does not vary significantly despite the variation in $n$ and ligand geometry. $\sigma_{1}$ is the $\mathrm{O}_{\text {phenolate- }}-\mathrm{U}-\mathrm{O}_{\text {phenolate }}$ bond angle, which can be considered an overall "ligand bite" angle and is in large part dictated by the linker geometry and how closely it holds the HOPO moieties; $\sigma_{1}$ increases with increasing $n$, consistent with HOPO moieties being held farther apart. The reverse trend is seen in the angles to either side of the coordinated solvent oxygen $\left(\sigma_{3}\right.$ and $\left.\sigma_{4}\right)$. The sum of $\sigma_{3}$ and $\sigma_{4}$ is thus a measure of the "solvent accessibility" of the uranyl cation, which increases as ligand bite angle decreases. 

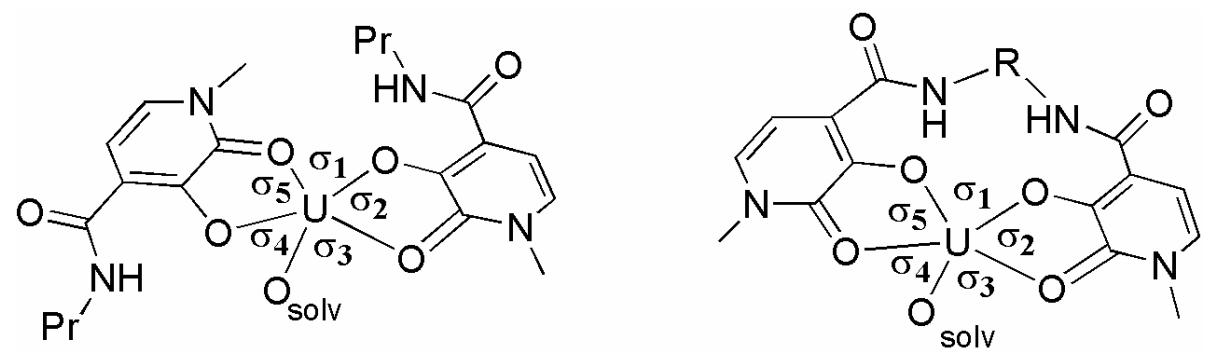

Figure 2-6. Equatorial O-U-O bond angle designations for $\mathrm{UO}_{2}(\mathbf{2 - 1})_{2}(\mathrm{DMF})$ (left) and $\mathrm{UO}_{2}$ (bis-Me-3,2-HOPO)(solv) (right), tabulated in Table 2-3. Uranyl oxo atoms are removed for clarity.

Table 2-3. O-U-O angles in the uranyl coordination plane in $\mathrm{UO}_{2}$ (bis-Me-3,2-HOPO) crystal structures. Angle designations correspond to those in Figure 2-6.

\begin{tabular}{|c|c|c|c|c|c|c|c|}
\hline Complex & $n^{a}$ & $\sigma_{1},\left[^{\circ}\right]$ & $\sigma_{2},\left[^{\circ}\right]$ & $\sigma_{3},\left[^{\circ}\right]$ & $\sigma_{4},\left[^{\circ}\right]$ & $\sigma_{5},\left[^{\circ}\right]$ & $\sigma_{3}+\sigma_{4},[\circ]$ \\
\hline $\mathrm{UO}_{2}(\mathbf{2 - 1})_{2}^{[1]}$ & -- & $76.8(2)$ & $66.6(2)$ & $76.1(2)$ & $74.2(2)$ & $66.4(2)$ & $150.3(2)$ \\
\hline $\mathrm{UO}_{2}(\mathbf{2 - 2})$ & 2 & $66.3(3)$ & $65.2(3)$ & $76.6(3)$ & $87.9(3)$ & $64.8(3)$ & 164.1(3) \\
\hline $\mathrm{UO}_{2}(\mathbf{2 - 6})(\mathrm{DMF})$ & 2 & $65.2(2)$ & $65.0(3)$ & $81.7(3)$ & $82.1(2)$ & $66.2(2)$ & $163.7(3)$ \\
\hline $\mathrm{UO}_{2}(2-7)(\mathrm{DMSO})$ & 2 & $65.7(1)$ & $65.83(9)$ & $84.5(1)$ & $78.8(1)$ & $65.9(1)$ & $162.8(1)$ \\
\hline $\mathrm{UO}_{2}(\mathbf{2 - 8})(\mathrm{DMSO})^{b}$ & 3 & $\begin{array}{l}66.6(2) ; \\
66.4(2)\end{array}$ & $\begin{array}{l}65.6(2) ; \\
65.2(2)\end{array}$ & $\begin{array}{l}77.2(2) ; \\
84.5(2)\end{array}$ & $\begin{array}{l}85.0(2) \\
79.9(2)\end{array}$ & $\begin{array}{l}66.0(2) ; \\
65.8(2)\end{array}$ & $\begin{array}{l}\text { 162.1(2); } \\
163.3(2)\end{array}$ \\
\hline $\mathrm{UO}_{2}(\mathbf{2 - 1 3})(\mathrm{MeOH})$ & 4 & $75.1(2)$ & $67.0(2)$ & $74.1(2)$ & $78.3(2)$ & $65.6(2)$ & $152.3(2)$ \\
\hline $\mathrm{UO}_{2}(\mathbf{2 - 1 4})$ & 4 & $73.7(1)$ & $65.8(1)$ & $75.7(1)$ & $78.7(1)$ & $66.8(1)$ & $153.4(1)$ \\
\hline $\mathrm{UO}_{2}(\mathbf{2 - 1 5})(\mathrm{DMF})$ & 5 & $92.8(1)$ & $66.2(1)$ & $67.4(1)$ & $67.9(1)$ & $66.0(1)$ & $134.9(1)$ \\
\hline $\mathrm{UO}_{2}(\mathbf{2 - 1 6})(\mathrm{DMSO})$ & 5 & $94.1(1)$ & $65.51(9)$ & $67.73(7)$ & $67.73(7)$ & $65.51(9)$ & $134.7(1)$ \\
\hline
\end{tabular}

${ }^{a}$ Number of carbons between amide nitrogens in linker.

${ }^{b}$ Crystal contained two unique uranyl complexes.

In addition to the variable "ligand bite" angle about the uranyl cation, each ligand clearly exhibits a different amount of flexibility; some ligands appear completely planar, while others bend either the HOPO moieties or the aromatic linker out of the uranyl coordination plane. In order to better compare and quantify the relative distortions exhibited in these structures, a series of metrics was developed that are illustrated in Figure 2-7. The first, $\theta$, is the angular deviation between the two HOPO moieties as defined by the least squares plane containing the HOPO ring nitrogen and the five ring carbons, and is in effect a measure of pucker or ruffle in the ligand geometry. A low $\theta$ value suggests the ligand lies flat about the uranyl coordination plane, although a low $\theta$ value could be caused by HOPO moieties bending out of the uranyl coordination plane while remaining nearly parallel to each other. Because the unconstrained $\mathrm{UO}_{2}(2-$ 
1) $)_{2}(\mathrm{DMF})$ complex is a very nearly flat complex, high $\theta$ values are most likely a sign that the backbone geometric constraints are not complementary to the uranyl coordination preferences.
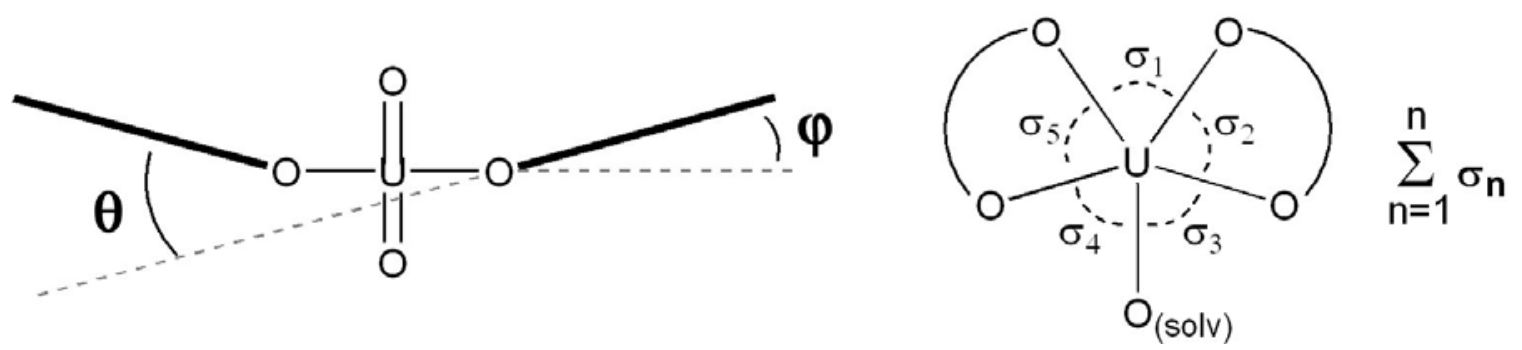

Figure 2-7. Conformational metrics used to compare the $\mathrm{UO}_{2}$ (bis-Me-3,2-HOPO) structures.

A second evaluated metric is $\varphi$, which represents the angular deviation between the least-squares plane of each HOPO ring and the uranyl coordination plane as defined by the least squares plane that includes the five equatorially coordinating oxygen atoms. As with $\theta$, a low $\varphi$ value is consistent with a planar coordination mode of the HOPO moiety, while a high $\varphi$ indicates ligand ruffling and by extension, a disagreement between the ligand geometry and uranyl coordination preferences.

A third metric, $\Sigma \sigma_{\mathrm{n}}$, corresponds to the total equatorial angle sum about the uranyl cation. In perfectly planar uranyl coordination, each oxygen would coordinate perpendicular to both $\mathrm{U}=\mathrm{O}_{\text {oxo }}$ vectors, with the sum $\left(\Sigma \sigma_{\mathrm{n}}\right)$ of angles $\sigma_{1}$ through $\sigma_{5}$ (Figure 2-6) being $360^{\circ}$. If the coordination about the uranyl plane becomes crowded due to ligand geometry or steric hindrance, the coordinating oxygens can move out of the ideal uranyl coordination plane, and thus the total angle sum about the uranyl cation would necessarily be greater than $360^{\circ}$, with larger values indicating a more strained structure. Large differences in $\Sigma$ are not expected because the coordination environment is not solely dictated by the bis-Me-3,2-HOPO ligand geometry; the fifth coordination site is 
occupied without exception by a separate molecule, usually solvent. This molecule is free to bind in a method dictated by yet free from the steric constraints of the bis-Me-3,2HOPO ligand, giving rise to only small differences in $\Sigma \sigma_{\mathrm{n}}$. Because the current study attempts to find a rigid ligand that best complements the uranyl coordination geometry, structures in which the metrics above approach their minimum possible values $\left(0^{\circ}\right.$ for $\theta$ and $\varphi$, and $360^{\circ}$ for $\Sigma \sigma_{\mathrm{n}}$ ) will be evaluated to be the best ligands for further study.

Table 2-4. Conformational parameters measured from $\mathrm{UO}_{2}$ (bis-Me-3,2-HOPO) crystal structures. Parameters are explained schematically in Figure 2-7.

\begin{tabular}{|c|c|c|c|c|}
\hline Complex & $\mathbf{n}^{\boldsymbol{a}}$ & $\boldsymbol{\theta},\left[^{\circ}\right]$ & $\boldsymbol{\varphi},\left[^{\circ}\right]$ & $\boldsymbol{\Sigma} \boldsymbol{\sigma}_{\mathbf{n}},\left[^{\circ}\right]$ \\
\hline $\mathrm{UO}_{2}(\mathbf{2 - 1})_{2}{ }^{[1]}$ & -- & $8.71(9)$ & $6.00(7), 2.90(4)$ & $360.1(4)$ \\
\hline $\mathrm{UO}_{2}(\mathbf{2 - 2})$ & 2 & $1.6(7)$ & $9.1(5), 10.1(5)$ & $360.8(7)$ \\
\hline $\mathrm{UO}_{2}(\mathbf{2 - 6})(\mathrm{DMF})$ & 2 & $5.8(4)$ & $2.8(4), 7.1(3)$ & $360.2(5)$ \\
\hline $\mathrm{UO}_{2}(\mathbf{2 - 7})(\mathrm{DMSO})$ & 2 & $9.43(5)$ & $6.35(3), 12.59(4)$ & $360.7(2)$ \\
\hline $\mathrm{UO}_{2}(\mathbf{2 - 8})(\mathrm{DMSO})^{b}$ & 3 & $22.0(1) ;$ & $11.10(8), 14.4(1) ;$ & $360.4(4) ;$ \\
\hline $\mathrm{UO}_{2}(\mathbf{2 - 1 3})(\mathrm{MeOH})$ & 4 & $10.5(5)$ & $0.7(4), 10.0(4)$ & $361.8(4)$ \\
\hline $\mathrm{UO}_{2}(\mathbf{2 - 1 4})$ & 4 & $21.4(2)$ & $8.4(2), 14.1(2)$ & $360.7(4)$ \\
\hline $\mathrm{UO}_{2}(\mathbf{2 - 1 5})(\mathrm{DMF})$ & 5 & $5.6(3)$ & $1.4(2), 6.0(2)$ & $360.3(2)$ \\
\hline $\mathrm{UO}_{2}(\mathbf{2 - 1 6})(\mathrm{DMSO})^{c}$ & 5 & $8.9(2)$ & $5.7(2)$ & $360.6(2)$ \\
\hline
\end{tabular}

${ }^{a}$ Number of carbons between amide nitrogens in linker.

${ }^{b}$ The crystal contains two unique uranyl complexes.

${ }^{c}$ The two HOPO moieties are crystallographically identical, giving rise to only one $\phi$ value.

The conformational parameter values for the structures in Figure 2-5 are listed in Table 2-4 along with $n$, the number of carbons between amide nitrogens (an approximate measure in linker length). These values are compared against those of the $\mathrm{UO}_{2}(2-$ 1) $)_{2}(\mathrm{DMF})$ structure. Despite being unconstrained by a linker, $\mathrm{UO}_{2}(\mathbf{2 - 1})_{2}(\mathrm{DMF})$ displays larger than expected $\theta$ and $\varphi$ values, indicating that small deviations do not necessarily represent a poor geometric agreement between ligand geometry and uranyl coordination preferences. As expected, however, the total equatorial angle sum $\Sigma$ is ideal within error. Because the uranyl complexes with $\mathbf{2 - 3}, \mathbf{2 - 4}$, and $\mathbf{2 - 5}$ are reported to have $\theta$ values of $38.86^{\circ}, 9.68^{\circ}$, and $13.40^{\circ}$ respectively, ${ }^{1}$ and because ligand $2-4$ exhibited the closest approach to the overall geometry seen in the $\mathrm{UO}_{2}(2-1)_{2}(\mathrm{DMF})$ complex in the previous 
study, deviations in $\theta$ and $\varphi$ greater than $10^{\circ}$ were taken as indications that the ligand geometries are significantly warped about the uranyl cation.

$\mathrm{UO}_{2}(\mathbf{2 - 2})$ : Comparison of the one-dimensional polymeric structure of $\mathrm{UO}_{2}(\mathbf{2 - 2})(n=$ 2) to that of $\mathrm{UO}_{2}(\mathbf{2 - 1})_{2}(\mathrm{DMF})$ reveals that the consequence of 2-2 ligand geometry is a very co-planar coordination mode about the uranyl cation. The $\theta$ value is artificially low because unlike every other $\mathrm{UO}_{2}$ (bis-Me-3,2-HOPO) structure evaluated here or elsewhere, the HOPO planes bend in opposite directions, resulting in an almost parallel arrangement of the HOPO moieties. Thus, the more appropriate value to inspect is $\varphi$, which still adopts comparable values to those in the $\mathrm{UO}_{2}(\mathbf{2 - 1})_{2}(\mathrm{DMF})$ complex. The $2 \mathrm{Li}$ backbone in $\mathbf{2 - 2}$ is able to adopt the staggered gauche conformation typical of alkane chains, and the amide moieties are still able to maintain a hydrogen bonding interaction between the amide proton and the HOPO phenolate (average N--O distance: $2.66 \AA$ ), so there are no outward signs of unreasonable ligand distortions to dissuade further investigation of this complex in solution phase measurements.

$\mathrm{UO}_{2}(2-6)(\mathrm{DMF})$ and $\mathrm{UO}_{2}(2-7)(\mathrm{DMSO})$ : The completely rigid and conjugated $n=2$ ligands 2-6 and 2-7, adopt strikingly planar arrangements about the uranyl cation. Unsurprisingly, the observed values for $\theta, \varphi$, and $\Sigma \sigma_{\mathrm{n}}$ are quite low in both complexes. However, the observed values do vary significantly from each other, despite their relatively low values, which is surprising considering 2-6 and 2-7 are both fully conjugated and should have similarly inflexible ligand geometries. Geometrically, ligands 2-6 and 2-7 differ primarily in the angle of attachment between the amide substituents on the aromatic linker ring; ortho substitution angles on a thiophene are ideally $72^{\circ}$, while those for a phenyl ring are $60^{\circ}$. The $12^{\circ}$ difference is expected to be the 
cause for the greater planarity in the $\mathrm{UO}_{2}(\mathbf{2 - 6})(\mathrm{DMF})$ because the wider thiophene substitution angle separates the amide moieties farther apart than on the phenyl linker in 2-7. This angular difference results in a increase of $0.07 \AA$ in the $\mathrm{N}_{\text {amide }}-\mathrm{N}_{\text {amide }}$ distance in the $\mathrm{UO}_{2}(\mathbf{2}-7)(\mathrm{DMSO})$ and $\mathrm{UO}_{2}(\mathbf{2 - 6})(\mathrm{DMF})$ structures. Upon mononuclear uranyl coordination, intramolecular $\mathrm{N}-\mathrm{H}^{\cdots} \mathrm{O}$ hydrogen bonding is maintained between the linking amides and the Me-3,2-HOPO phenolate oxygens, requiring in turn that the protons on the linking amides point towards each other; the smaller $\mathrm{N}_{\text {amide }}-\mathrm{N}_{\text {amide }}$ distance in 2-7 results in a more sterically crowded conformation than that in $\mathbf{2 - 6}$. The result of this steric crowding causes the $\mathrm{N}_{\text {amide }}-\mathrm{C}_{\text {backbone }}$ torsion angle in the $\mathrm{UO}_{2}(2-$ 7)(DMSO) complex to adopt values of $148^{\circ}$ and $157^{\circ}$ to relieve close approach of the amide protons. For comparison, the same torsion angles in $\mathrm{UO}_{2}(\mathbf{2 - 6})(\mathrm{DMF})$ are $172^{\circ}$ and $176^{\circ}$ (much closer to the ideal $180^{\circ}$ ) because the amide moieties are held farther apart by the thiophene ring of 2-6 than the phenyl ring of 2-7. Despite these small differences, however, both 2-6 and 2-7 exhibit promising geometries and were examined in subsequent studies.

$\mathrm{UO}_{2}(\mathbf{2 - 8})(\mathrm{DMSO})$ : The $\mathrm{UO}_{2}(\mathbf{2 - 8})(\mathrm{DMSO})$ comlex $(n=3)$ displays the highest $\theta, \varphi$, and $\Sigma \sigma_{\mathrm{n}}$ values in Table 2-4. The most obvious structural consequence of this backbone choice is the significant deviation of the linker ring from co-planarity with the uranyl coordination plane. This distortion is necessary to bring the Me-3-2-HOPO moieties into binding positions with the uranyl cation, but is not completely successful because the HOPO moieties themselves are also significantly twisted out of the uranyl coordination plane. It was determined that although this ligand was physically capable of binding the 
uranyl cation in a mononuclear fashion, it could only do so upon considerable ligand distortion, and this backbone architecture was not investigated further.

$\mathrm{UO}_{2}(\mathbf{2 - 1 3})(\mathrm{MeOH})$ : Although the difference between ligands $\mathbf{2 - 8}$ and $\mathbf{2 - 1 3}$ is only a substitutional shift of the methylene spacer from ortho to para in the aromatic linker, the $\mathrm{UO}_{2}(\mathbf{2 - 1 3})(\mathrm{MeOH})$ complex $(n=4)$ displayed far lower $\theta$, $\varphi$, and $\Sigma \sigma_{\mathrm{n}}$ values than $\mathrm{UO}_{2}(\mathbf{2}$ 8)(DMSO), even exhibiting the lowest $\varphi$ value of $0.7^{\circ}$. However, careful investigation of the crystal structure indicates that in order for the ligand to bind in the observed mononuclear fashion, one linking amide rotates $88^{\circ}$ out of plane from the HOPO to which it is attached. It has been well-established that hydrogen-bonding between the deprotonated phenolate oxygen and the amide proton stabilizes the deprotonated and metal-coordinated forms of Raymond group ligands containing amides ortho to coordinating phenolate oxygens, ${ }^{13}$ and the strength of this interaction in Me-3,2-HOPO moieties (measured by downfield ${ }^{1} \mathrm{H}$ NMR shift) has been shown to vary with linker length in previous $\mathrm{UO}_{2}$ (bis-Me-3,2-HOPO) complexes. ${ }^{1}$ Although the twisted amide proton is hydrogen-bonded to an amide from another molecule in the crystal structure $(\mathrm{N}$ -O distance of $3.06 \AA)$, it is apparent that backbone geometry in $\mathrm{UO}_{2}(\mathbf{2 - 1 3})(\mathrm{MeOH})$ requires that this hydrogen bonding interaction be broken to enable mononuclear uranyl binding. The ${ }^{1} \mathrm{H}$ NMR signal for the benzylic amide in $\mathrm{UO}_{2}(\mathbf{2}-\mathbf{1 3})(\mathrm{MeOH})$ is also ca. 4 ppm lower than that for the aromatic amide proton (and $c a .2$ ppm lower than the benzylic amide proton in $\mathrm{UO}_{2}(\mathbf{2 - 8})(\mathrm{DMSO})$, suggesting that the twisted conformation observed in Figure 2-5 is maintained in solution. This amide twist necessarily represents a high-energy conformation (stabilized in the solid state by intermolecular hydrogen bonding), so the $m$-toluene linker geometry in $\mathbf{2 - 1 3}$ was not pursued in further studies. 
$\mathrm{UO}_{2}$ (2-14): The one-dimensional polymeric $\mathrm{UO}_{2}(\mathbf{2}-14)$ complex structure $(n=4)$ exhibited some of the largest $\theta$ and $\varphi$ values in Table 2-4 and also required the linker to bend significantly out of the uranyl plane. No significant $\pi$-stacking was observed between HOPO rings or the xylene backbone, so this poor geometric agreement between the ligand and the uranyl cation was assumed to be characteristic of the complex, and so the $o$-xylene linker in $\mathbf{2 - 1 4}$ was also not pursued in further studies.

$\mathrm{UO}_{2}(2-15)(D M F)$ : The aromatic backbone in the $\mathrm{UO}_{2}(\mathbf{2}-15)(\mathrm{DMF})$ complex $(n=5)$ was also observed to bend dramatically out of the HOPO and uranyl coordination planes, but the complex also exhibits arguably the most favorable combination of $\theta, \varphi$, and $\Sigma \sigma_{n}$ values in Table 2-4. Ligands 2-14 and 2-15 both contain two methylene spacers between the aromatic linker ring and the amide nitrogens, which are obviously responsible for the ligands' respective abilities to bind the uranyl in a mononuclear fashion despite their large $n$ values. Some weak intermolecular $\pi$-stacking interactions in the crystal lattice between HOPO moieties (3.4 $\AA$ interplane distance) may exist, but no such interaction exists with the backbone phenyl ring, again suggesting that - like 2-14 - the ligand distortion observed is native to the uranyl complex and not solely a result of crystal packing effects. Although the need for ligand distortion in 2-14 and 2-15 to facilitate mononuclear chelation is rather significant in each complex, the resultant co-planar arrangement of the HOPO moieties in 2-15 about the uranyl cation encouraged the further study of the $m$-xylene linker geometry both because of its favorable geometric agreements as well as for a point for comparison against the ligands with $n=2$, for which the values in Table 2-4 were also very favorable. By geometric evaluation, the $m$-xylene linker in 2-15 is also more appropriate in uranyl complexes than the $5 \mathrm{Li}$ linker in $\mathbf{2 - 5}$, for 
which a $\theta$ value of $13.4^{\circ}$ was observed. Whether the less severe distortion about the uranyl with 2-15 is a result of decreased degrees of freedom compared to 2-5 or because gauche interactions and the alkyl torsion angles they require are absent in 2-15 is unclear. No matter the reason, however, 2-15 will provide a very good comparison to $\mathbf{2 - 5}$ in further thermodynamic studies.

$U_{2}(2-16)(D M S O)$ : There can be little argument that the low $\theta, \varphi$, and $\Sigma \sigma_{\mathrm{n}}$ values of the $\mathrm{UO}_{2}(\mathbf{2}-15)(\mathrm{DMF})$ complex are made possible by the flexibility of the linker. Surprisingly, however, the uranyl complex $\mathrm{UO}_{2}(\mathbf{2 - 1 6})(\mathrm{DMSO})$ with the completely rigid 1,8-fluorene linker $(n=5)$ also exhibits very low $\theta, \varphi$, and $\Sigma \sigma_{\mathrm{n}}$ values, which is intriguing considering it contains none of the flexibility exhibited in 2-15. Additionally, the equatorial $\mathrm{O}_{\text {phenolate }}-\mathrm{U}-\mathrm{O}_{\text {phenolate }}$ bond angle $\left(\sigma_{1}\right.$, Figure 2-6) in the $\mathrm{UO}_{2}(\mathbf{2}-\mathbf{1 6})(\mathrm{DMSO})$ complex is only one degree higher than that in $\mathrm{UO}_{2}(\mathbf{2 - 1 5})(\mathrm{DMF})$. This, compared with the values in Table 2-4, suggests that ligand 2-16 presents a conformation that is complementary to the uranyl coordination geometry. Therefore, the fluorene backbone is a very attractive scaffold geometry for further actinyl coordination applications.

The rigidity of 2-16 coupled with the favorable conformational parameter measures in $\mathrm{UO}_{2}(\mathbf{2}-\mathbf{1 6})(\mathrm{DMSO})$ suggests that 2-16 may be pre-organized for chelation to the uranyl cation. This is corroborated by the similarity between the ligand geometry in Figure 2$5(\mathrm{~h})$ and that in a crystal structure of uncomplexed 2-16 grown out of DMSO- $d_{6}$ during NMR characterization. This uncomplexed crystal structure is shown in Figure 2-8 and its crystallographic parameters are listed in Table 2-5. The crystal structure contains a DMSO molecule to which the ligand is hydrogen bound through both its phenolic functionalities, with $\mathrm{O}_{\mathrm{DMSO}}-\mathrm{O}_{\text {phenol }}$ distances of 2.63 and $2.68 \AA$. Because of this 
hydrogen bonding interaction above the plane of the HOPO planes, there is an unsurprising $43^{\circ}$ deviation between the two HOPO ring planes, but visual inspection makes it clear that the overall ligand geometry is similar to that seen in the $\mathrm{UO}_{2}(2-$ 16)(DMSO) complex. The observed interaction with the DMSO molecule suggests this ligand arrangement is solvent-dependent, but that 2-16 can adopt such a conformation makes it a very interesting scaffold for actinyl ligand design.

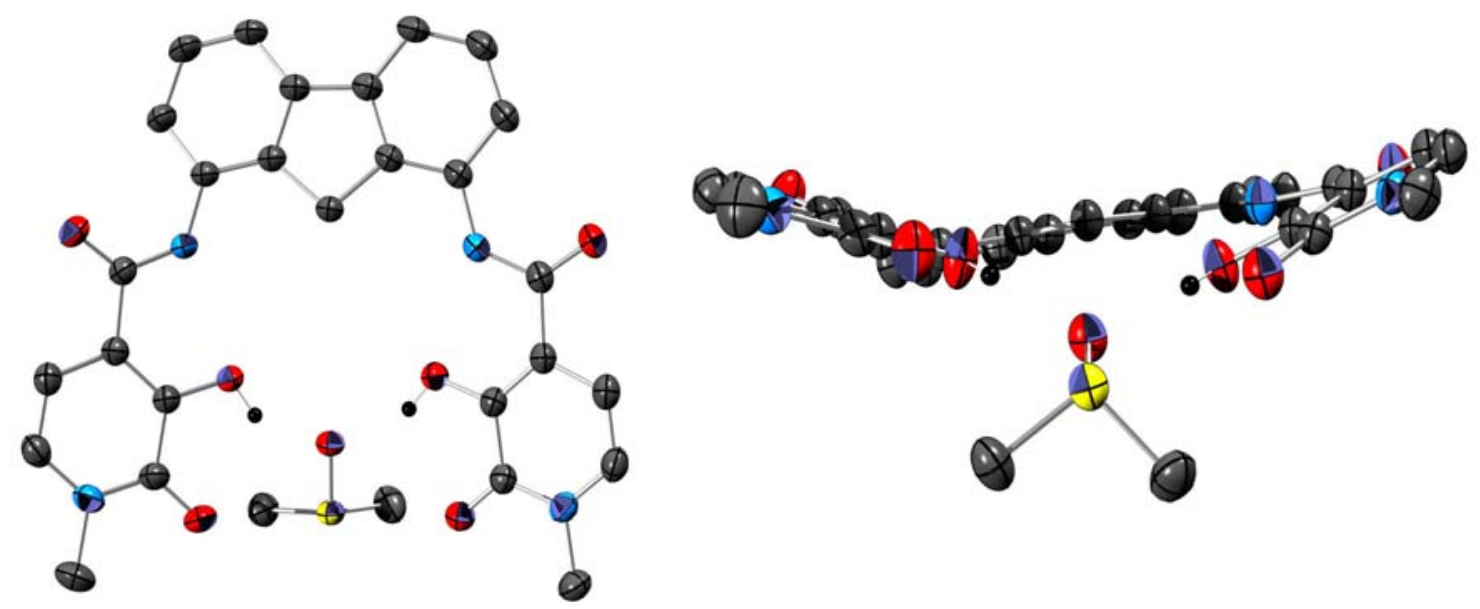

Figure 2-8. Top and side views of the 2-16. DMSO crystal structure. Thermal ellipsoids are drawn at the $50 \%$ probability level. Hydrogen atoms are omitted for clarity except for phenolate hydrogens. Oxygen atoms are red, carbons gray, nitrogens blue, sulfur yellow, and hydrogens are black.

Table 2-5. Crystallographic parameters for 2-16. DMSO.

\begin{tabular}{|c|c|c|c|}
\hline Formula & $\begin{array}{c}\mathrm{C}_{27} \mathrm{H}_{22} \mathrm{~N}_{4} \mathrm{O}_{6} \\
\mathrm{C}_{2} \mathrm{H}_{6} \mathrm{OS}\end{array}$ & $\begin{array}{c}\text { Data/ restr./ } \\
\text { param. }\end{array}$ & $8182 / 15 / 482$ \\
\hline MW & 576.61 & $\mathbf{T}[\mathbf{K}]$ & 193(2) \\
\hline Crystal system & Monoclinic & $\rho_{\text {calcd }}\left[\mathrm{g} \mathrm{cm}^{-3}\right]$ & 1.428 \\
\hline Space group & $\mathrm{P} 22_{1} / \mathrm{n}$ & $\mu_{\text {palcd }}\left[\mathrm{mm}^{-1}\right]$ & 0.218 \\
\hline Appearance & Parallelepiped & $\theta_{\min }, \theta_{\max },\left[^{\circ}\right]$ & $2.14,33.67$ \\
\hline Color & Yellow & Total reflections & 39273 \\
\hline$a[\AA]$ & $10.9305(9)$ & $\mathbf{Z}$ & 4 \\
\hline$b[\AA]$ & $20.7752(16)$ & $F(000)$ & 1208 \\
\hline$c[\AA \AA]$ & $12.9772(10)$ & $\mathbf{T}_{\min } / \mathbf{T}_{\max }$ & 0.985 \\
\hline$\alpha\left[^{\circ}\right]$ & 90 & Cryst. size $\left[\mathrm{mm}^{3}\right]$ & $0.11 \times 0.07 \times 0.04$ \\
\hline$\beta\left[^{\circ}\right]$ & $114.494(2)$ & $\mathrm{R}_{1}[I>2 \sigma(I)]^{a}$ & 0.0503 \\
\hline$\gamma\left[^{\circ}\right]$ & 90 & $\mathrm{wR}_{2}{\text { (all data })^{a}}^{a}$ & 0.1506 \\
\hline $\mathbf{V}\left[\AA^{3}\right]$ & $2681.7(4)$ & $\mathbf{G O F}^{a}$ & 1.151 \\
\hline
\end{tabular}

By the comparison discussed above, the ligands utilizing the 2Li-, 3,4-thiophene-, ophenylene-, $\alpha, \alpha^{\prime}-m$-xylene-,and 1,8-fluorene-linkers (2-2, 2-6, 2-7, 2-15, 2-16) were 
chosen as linker geometries for further study in solution thermodynamic measurements. Interestingly, reasonable coordination geometries were displayed only by ligands with $n$ $=2$ or 5 . Only one of four ligands with $n=3(\mathbf{2 - 8})$ was able to form mononuclear, isolatable complexes with the uranyl cation, while the two $n=4$ ligands that formed mononuclear uranyl complexes (2-13, 2-14) displayed significantly distorted geometries. Thus, it seems that linkers of intermediate length have very stringent requirements on their geometries to enable mononuclear complex formation, while those with $n=2$ may be of more appropriate geometry in their uncoordinated forms, and those with $n=5$ must possess appropriate flexibility (in the case of 2-15) or be pre-organized for chelation (as with 2-16) to enable mononuclear uranyl coordination without unreasonable ligand or coordination geometry distortion. Because the effect of linear linker length in bis-Me-3,2HOPO ligands on uranyl affinity has also never been investigated, ligands 2-3 through 25 were also studied via solution thermodynamic measurements.

\subsubsection{Soluble Tetradentate Ligand Design and Synthesis}

In order to perform thermodynamic measurements with bis-Me-3,2-HOPO ligands using aqueous thermodynamic measurements, the ligands must be soluble in water at concentrations of at least 5-50 $\mu \mathrm{M}$ to enable UV-Visible absorption measurements, and at $200-500 \mu \mathrm{M}$ for potentiometric measurements. Additionally, the uranyl complexes with these ligands must be equally soluble at all stages of protonation/complexation. Unfortunately, none of the bis-Me-3,2-HOPO ligands reported above are sufficiently soluble to enable such measurements, so new ligands that incorporated water-solubilizing substituents were needed. The substituents could not be introduced on the Me-3,2-HOPO moiety because that would affect the electronic structure of the chelating moiety we are 
intending to characterize. Thus, the solubilizing group must necessarily be introduced onto the ligand linker while maintaining ligand geometries as similar to those used in crystallographic studies as possible.

The solubilizing group chosen was the methyl-protected triethyleneglycol moiety (3,6,9-trioxa-decane) referred to as PEG. The advantages of this solubilizing group are that it carries no charge and thus does not change the charge state of the resultant ligands, and its low reactivity and moderate length lend themselves to standard synthetic reaction and chromatographic procedures. In addition, this group is widely utilized in the literature for just these purposes, so much of the preliminary synthetic procedures to incorporate this group onto a variety of molecules are already established. This allows the same solubilizing group to be introduced in a variety of ways onto a variety of linker geometries, introducing as little variation across the ligands as possible.

The biggest concern of using the PEG moiety is the steric bulk it could potentially introduce, because the thermodynamic measurements with uranyl are intended to probe the effect of sterics arrangements and ligand geometry on the formation constants with bis-Me-3,2-HOPO ligands. This requires that the solubilized ligands very closely approach the geometry of their less soluble analogs used in crystallographic studies. In addition, any steric bulk introduced by the PEG should not affect the proton affinity of the ligand, as this change would inevitably affect the uranyl affinity of the ligands. Unfortunately, such an effect is unavoidable with the 3,4-thiophen- and o-phenylenelinked bis-Me-3,2-HOPO ligands because they are conjugated through their linkers, and thus any substitution on the aromatic backbones must necessarily result in an electronic effect on the HOPO moieties. While such a perturbation is unavoidable, such problems 
should ideally not exist with the linearly-linked $\mathrm{nLi}$ - and the $\alpha, \alpha^{\prime}-m$-xylene linkers because the linkers are not electronically conjugated to the HOPO moieties.

With these considerations in mind, the PEG-functionalized bis-Me-3,2-HOPO ligands shown in Figure 2-9 were designed. Conspicuously absent from the ligand structures in Figure 2-9 is the 1,8-fluorene backbone. Ligand 2-16 displayed outstanding geometric agreement with uranyl coordination geometry and also showed a degree of preorganization in its unbound structure, making it an excellent candidate for uranyl affinity studies. Unfortunately, the tremendous insolubility imparted on bis-Me-3,2HOPO ligands by the fluorene backbone cannot be undone by solubilizing group substitution; the 1,8-diamine substitution on the fluorene is already a multi-step procedure made possible by very severe reaction conditions that many solubilizing groups would not survive and which would lower the isolated yields of completed ligand below practical limits. ${ }^{14}$ Additionally, the natural electrophillic substitution behavior of fluorene (and its structural analog dibenzofuran) does not lend itself towards 1,8substitution, making a PEG-substituted 1,8-diaminofluorene a synthetically impractical ligand.

Although ligands 2-2 through 2-5 are already significantly more soluble than the fluorene-linked 2-16, the PEG-nLi-Me-3,2-HOPO ligands 2-17 through 2-20 are significantly more soluble due to inclusion of a PEG-amide next to one of the linking HOPO amides. This positioning contradicts the design considerations above, as this position will most assuredly affect the geometry and/or the electronics of the resultant ligand. However, because substitution on the 2Li- linker cannot avoid such influence, the PEG moiety was introduced at the same position on the $3 \mathrm{Li}-, 4 \mathrm{Li}-$, and $5 \mathrm{Li}-$ linkers to 
make the comparisons at least internally consistent. In this manner, it can be assumed that whatever effect the PEG-amide substituent has on the resultant metal-ligand interactions, it is similar in all the PEG-nLi-Me-3,2-HOPO ligands.

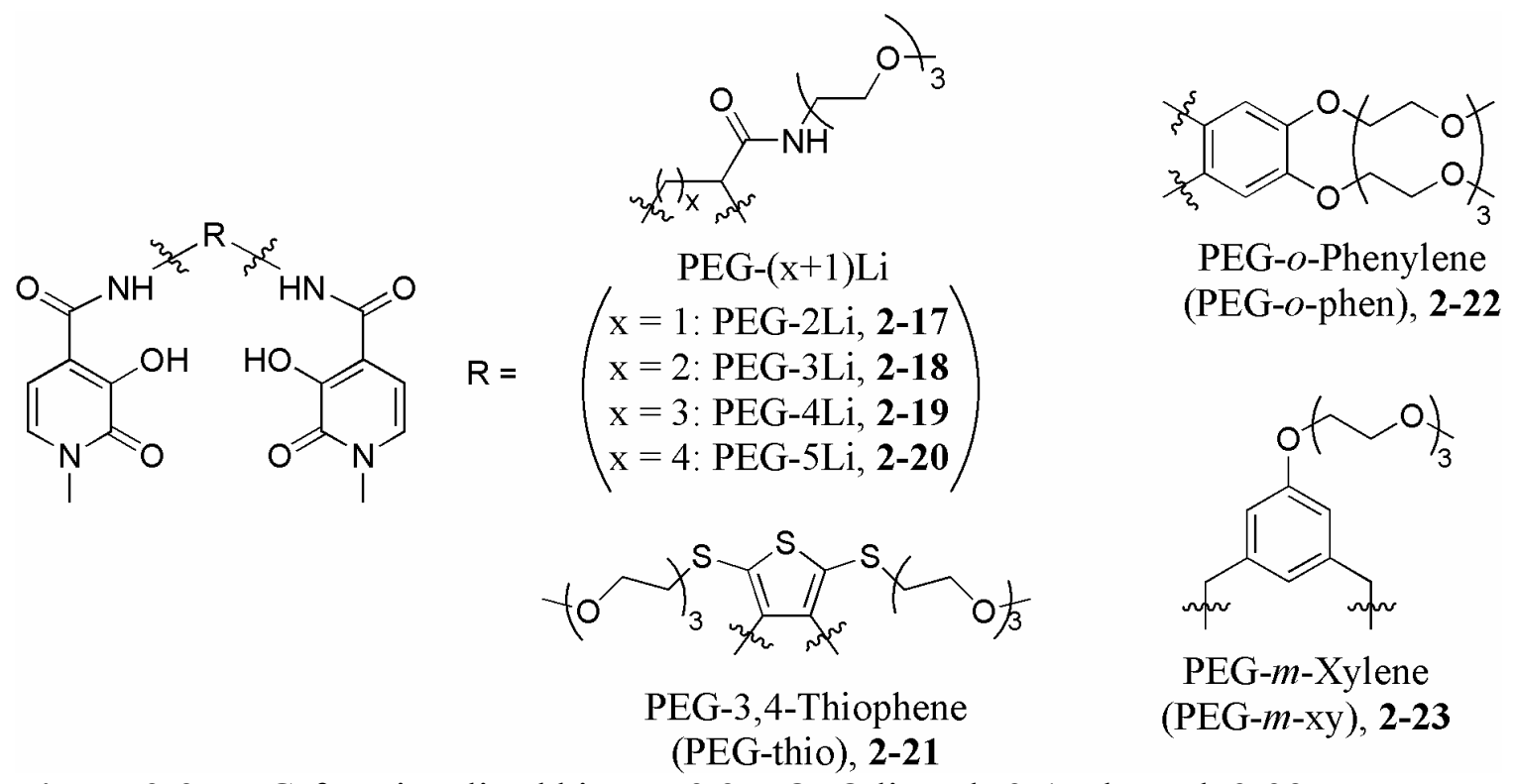

Figure 2-9. PEG-functionalized bis-Me-3,2-HOPO ligands 2-17 through 2-23.

In addition to the possible electronic effects of PEG substitution on the thiophene ring in 2-21, a significant steric influence is unfortunately unavoidable because the only position available for substitution on the thiophene ring are the 2- and 5-positions ortho to the amide substituent. It is possible that substitution here will cause steric interference with the amide oxygens upon uranyl coordination. Such steric crowding on polysubstituted thiophene rings containing thioamides has been observed, causing out-ofplane rotation of phenyl and thioamide substitutents, ${ }^{15}$ but how such steric considerations will affects the structure of a uranyl complex with bis-Me-3,2-HOPO ligands is unknown. In addition, without the inclusion of solubilizing groups, ligand 2-6 is tremendously insoluble, so a sacrifice in geometric consistency between unsubstituted and substituted ligands 2-6 and 2-21 must be accepted to facilitate the desired thermodynamic 
measurements. Other conjugated 5-member rings such as pyrroles and furans were investigated as structural alternatives to the thiophene linkers (with pyrroles able to support solubilizing groups on the ring nitrogen and furans capable of substitution reactions to form pyrroles), but neither of these options were synthetically feasible using the amide-coupled bis-Me-3,2-HOPO ligand geometry investigated here. In contrast, however, PEG substitution on the phenyl ring in $\mathbf{2 - 2 2}$ is expected to have little steric influence on the geometry of the resultant uranyl complexes because they are meta to the amide moiety, unlike the ortho substitutions in $\mathbf{2 - 2 1}$.

Substitution on the $\alpha, \alpha^{\prime}-m$-xylene backbone in 2-23 contains perhaps the most ideal PEG substitution position of the ligands in Figure 2-9. Firstly, the PEG moiety is located four carbons from the linking amide nitrogens, ensuring minimal steric effects. Secondly, the PEG group is electronically isolated from the HOPO moieties due to the presence of the methylene spacers between the phenyl ring and the HOPO amides. Thus, it is expected that the behavior of ligand $\mathbf{2 - 2 3}$ in solution will most closely resemble that of 215 than any of the other PEG-substituted ligands will to their less soluble structural analogs.

The syntheses of the diamine precursors for ligands 2-17 through 2-21 and 2-23 are illustrated in Scheme 2-2; the linking diamine for 2-22 was synthesized following literature procedures. ${ }^{16}$ The subsequent amide coupling and deprotection of the PEGsubstituted bis-Me-3,2-HOPO ligands proceeded in a similar fashion to their unsubstituted analogs and is illustrated in Scheme 2-3. The Boc-protected precursors to 2-24(Boc $)_{2}$ through $\left.\mathbf{2 - 2 7 ( B o c )}\right)_{2}$ were purchased from commercial sources as either a racemic mixture or the enantiopure (L) form. Because the exact chirality is not of interest 
in this study, the handedness of the isolated ligands or their intermediates was never verified after a reaction step and was assumed to have been conserved throughout the reaction steps; stereochemistry is omitted from graphics for clarity. The PEG-nLidiamines 2-24 through 2-27 were generated from compounds 2-24(Boc) $)_{2}$ through 227(Boc) $)_{2}$ using TFA deprotection and were not isolated before use in amide coupling reactions. Diamine 2-34 was similarly never isolated and was used in subsequent reactions without purification. Amide coupling of Me-3,2-HOPO moieties to the diamine backbones proceeded as before through either the thaizoline-activated or acid chloride of benzyl-protected Me-3,2-HOPO. Reaction of deprotected PEG-nLi diamines 2-25 through 2-27 with the thiazoline-activated moiety proceeded typically in low yields and was found to be ineffective with the PEG-2Li diamine 2-24. Successful amide coupling

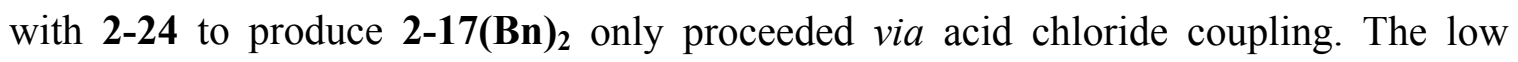
yields observed with the PEG-nLi linkers are most likely the result of steric hindrance introduced by the bulk of the PEG moiety which affects the reactivity of the amine geminal to the PEG-amide substituent. The PEG moiety could feasibly also provide hydrogen bonding opportunities for the deprotected amines, deactivating them towards amide coupling. 

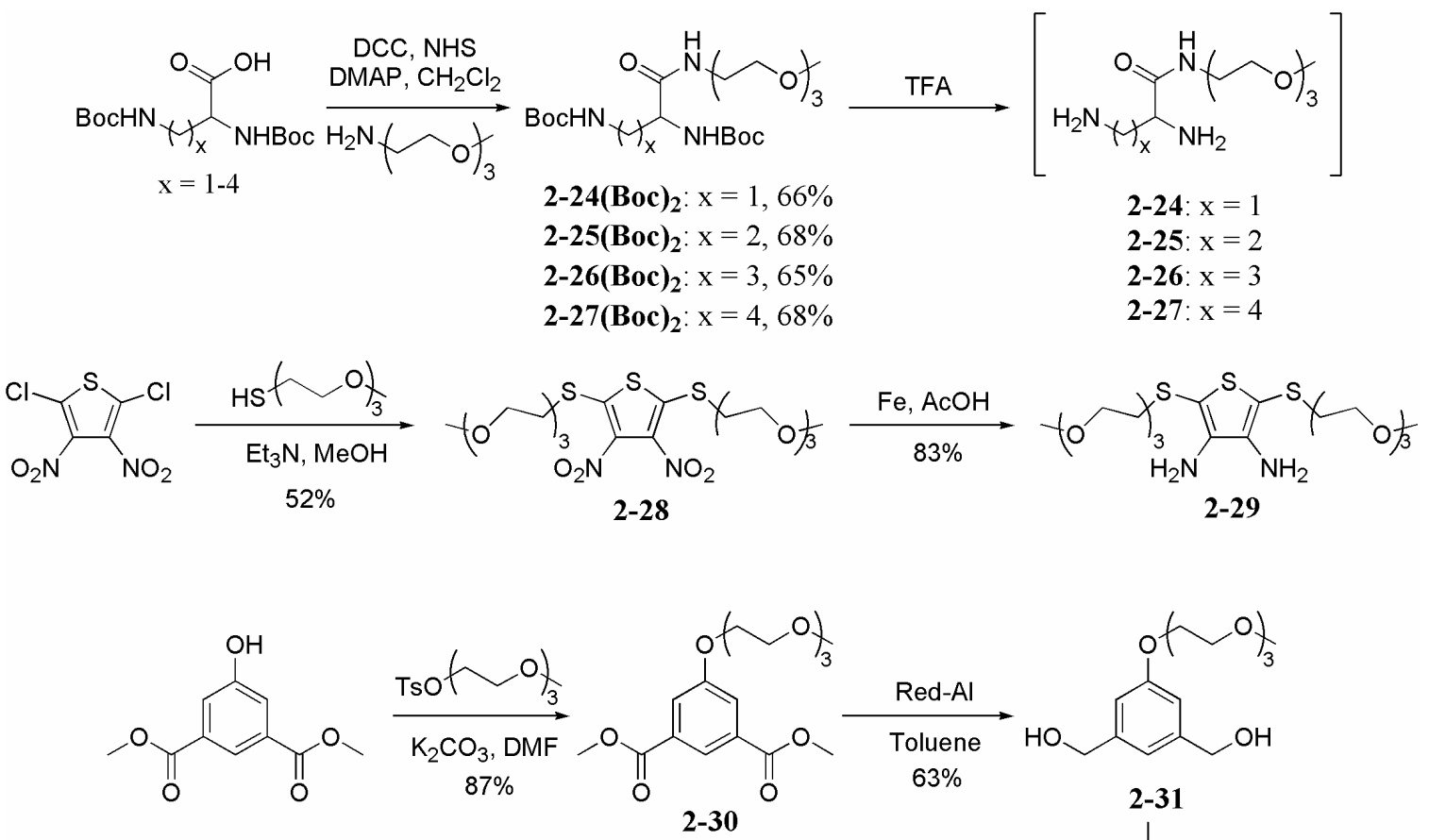

$-30$<smiles>CCOCCOc1cc(CN)cc(COCCOCCO)c1</smiles>

2-34

2-33

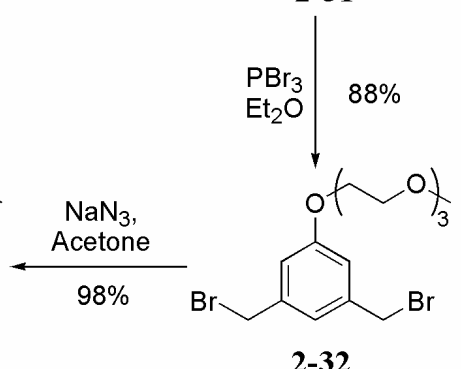

Scheme 2-2. Syntheses of PEG-containing diamines. Compounds in brackets were used in subsequent reactions without isolation or characterization.

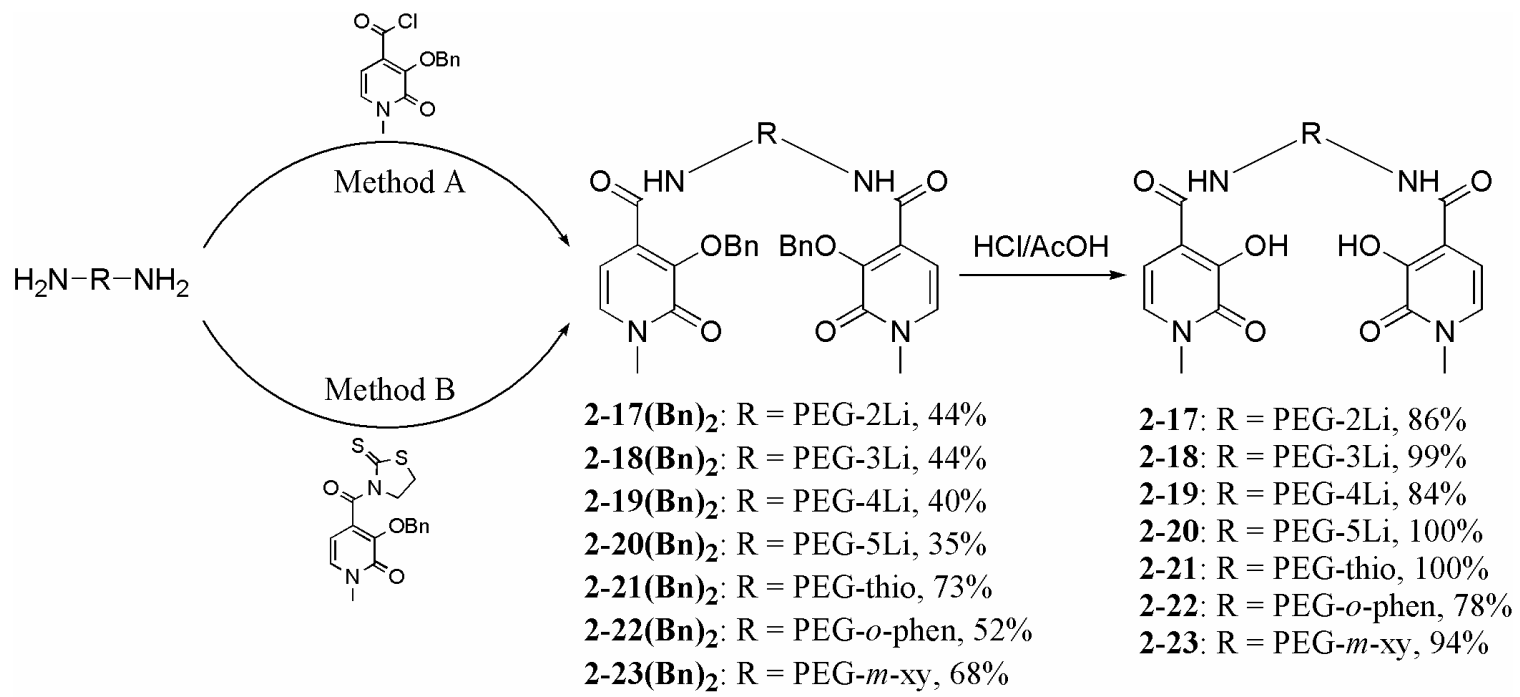

Scheme 2-3. Syntheses of PEG-functionalized bis-Me-3,2-HOPO ligands 2-17 through 2-23. 
Benzyl deprotection of the bis-Me-3,2-HOPO ligands in Scheme 2-3 proceeded cleanly via aqueous acidic deprotection. Isolation could not be achieved using standard precipitation methods because of the ligands' increased aqueous and organic solubilities, so rigorous co-evaporation with $\mathrm{MeOH}$ and $\mathrm{CHCl}_{3}$ was employed to remove residual acids and benzyl alcohol from the reaction mixture. The isolated ligands were typically beige solids, the exceptions being $\mathbf{2 - 2 2}$ which was a yellow solid, and 2-21 which was a dark brown, tacky oil. All PEG-substituted ligands exhibited sufficient aqueous solubility to enable solution thermodynamic measurements on their free and uranyl-complexed forms.

\subsubsection{Ligand Substitution Effects on Uranyl Complexes}

The significance of the steric effect of the PEG moiety is evident in the amide coupling difficulties encountered in the synthesis of PEG-nLi-Me-3,2-HOPO 2-17 through 2-20. However, because this same substitution strategy was employed on all the PEG-nLi-Me-3,2-HOPO ligands, reasonable comparisons can still be carried out within the series, but no such structurally analogous series exists for the PEG-o-phen- and PEGthio-Me-3,2-HOPO ligands $\mathbf{2 - 2 1}$ and $\mathbf{2 - 2 2}$. Thus, the effect of the PEG moiety on the resultant complex geometries was of concern. Because of the electronic conjugation through the 3,4-thiophene- and o-phenylene-linked ligands, PEG substitution in 2-21 and 2-22 was bound to necessarily affect the HOPO ring electronics (evidenced by the strong color of these ligands compared to their unsubstituted analogs). These considerations encouraged structural investigation of the effect of PEG-substitution on the geometry of the uranyl complexes with $\mathbf{2 - 2 1}$ and $\mathbf{2 - 2 2}$. 

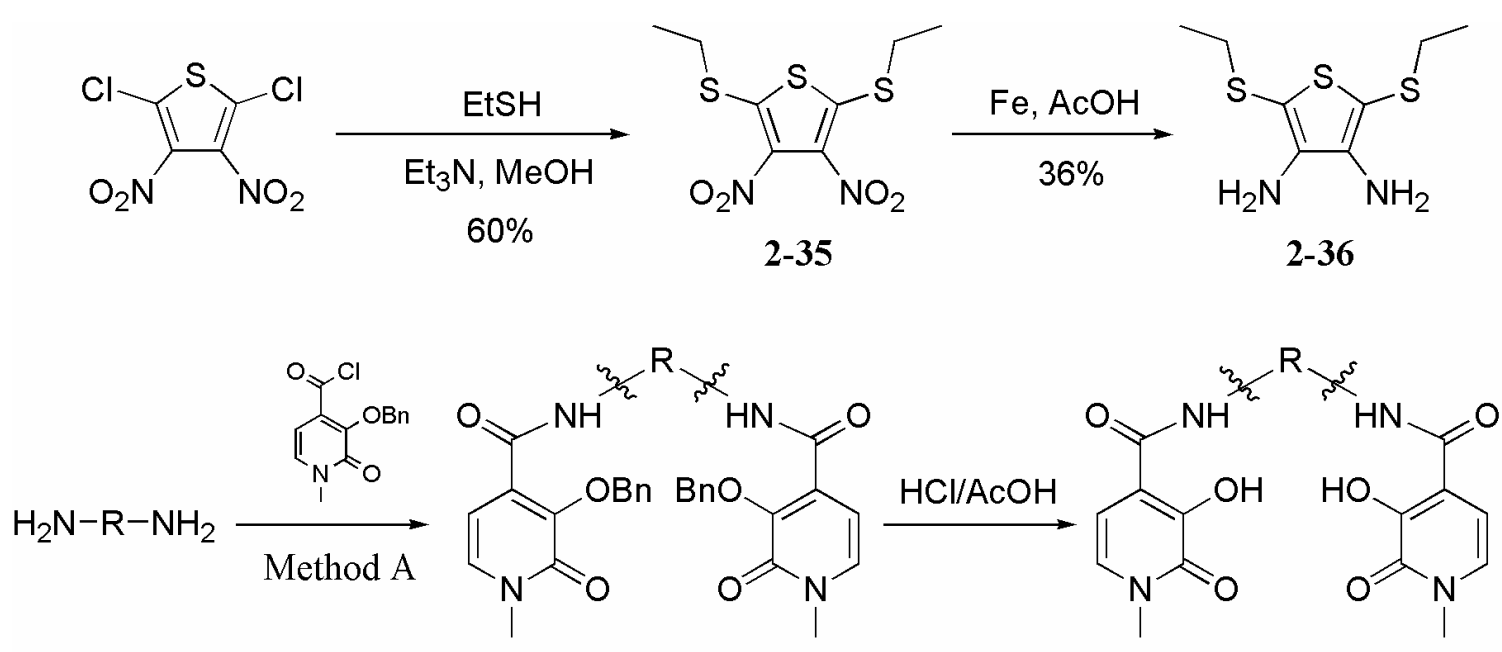

$$
\begin{array}{ll}
\mathbf{2 - 3 7}(\mathbf{B n})_{2}: \mathrm{R}=\text { Et-thio, } 66 \% & \mathbf{2 - 3 7}: \mathrm{R}=\text { Et-thio, } 74 \% \\
\mathbf{2 - 3 8}(\mathbf{B n})_{2}: \mathrm{R}=\text { Pr- } o \text {-phen, } 70 \% & \mathbf{2 - 3 8}: \mathrm{R}=\text { Pr-o-phen, } \mathbf{8 5} \%
\end{array}
$$

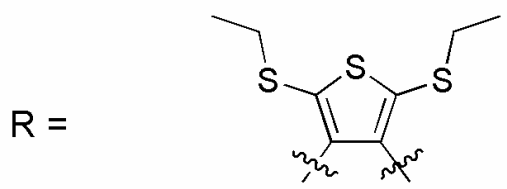

Et-3,4-Thiophene (Et-thio)<smiles>CCCOc1cc(C)c(C)cc1OCCC</smiles>

Pr-o-Phenylene (Pr-o-phen)

Scheme 2-4. Synthesis of alkyl-substituted bis-Me-3,2-HOPO ligands 2-37 and 2-38.

X-ray structures are the best method by which to gain the desired structural knowledge, but PEG functionalities typically do not facilitate single crystal growth. The ligands Et-thio-Me-3,2-HOPO (2-37) and Pr-o-phen-Me-3,2-HOPO (2-38) in Scheme 2-4 were synthesized to provide both the electronic and pertinent steric effects of the PEGsubstituents on ligands $\mathbf{2 - 2 1}$ and $\mathbf{2 - 2 2}$, while their short alkyl chains make them more suited for crystallization attempts. The diamine precursor for $\mathbf{2 - 3 8 ( B n})_{2}$ was synthesized following literature procedure, ${ }^{17}$ while that for $\left.\mathbf{2 - 3 7 ( B n}\right)_{2}$ was synthesized as shown in Scheme 2-4. Synthesis of ligands 2-37 and 2-38 proceeded via acid chloride amide coupling followed by acidic benzyl deprotection used for many bis-Me-3,2-HOPO ligands described above. The only difficulty in the syntheses described in Scheme 2-4 was the reduction of $\mathbf{2 - 3 5}$ using powdered iron. A procedure similar to that described by 
Erker ${ }^{18}$ was used to make diamine 2-29, but the $36 \%$ yield of 2-36 was much lower than the $83 \%$ yield of $\mathbf{2 - 2 9}$. This drop in yield was most likely caused by the long reaction time used in the reduction reaction, which was made necessary by the low solubility of 235. Higher yields can most likely be afforded by either using more solvent to encourage complete dissolution of $\mathbf{2 - 3 5}$ or by using lower reaction temperatures. This reaction was not repeated because the isolated yield was sufficient to proceed in the synthesis of 237(Bn) 2 . Ligands 2-37 and 2-38 were isolated as beige and yellow solids, respectively.

The $\mathrm{UO}_{2}(\mathbf{2 - 3 7 / 2 - 3 8})$ complexes were isolated by crystallization from crude reaction solutions in DMSO. Layering $\mathrm{MeOH}$ on top of a deep red, crude $\mathrm{UO}_{2}(\mathbf{2 - 3 7})$ solution in DMSO at $4{ }^{\circ} \mathrm{C}$ led to the crystallization of two different crystal habits, both of which were X-ray quality and from which the two crystal structures with 2-37 were determined. One crystal habit was stable upon removal from the solvent, while the other desolvated rapidly upon removal from solution. $\mathrm{MeOH}$ diffusion into a crude DMSO solution of $\mathrm{UO}_{2}(\mathbf{2 - 3 7})$ at room temperature also led to deep red, X-ray quality crystals that were very stable outside of solution. The X-ray diffraction structures of these three crystalline species are shown in Figure 2-10, and their crystallographic parameters are listed in Table 2-6. 

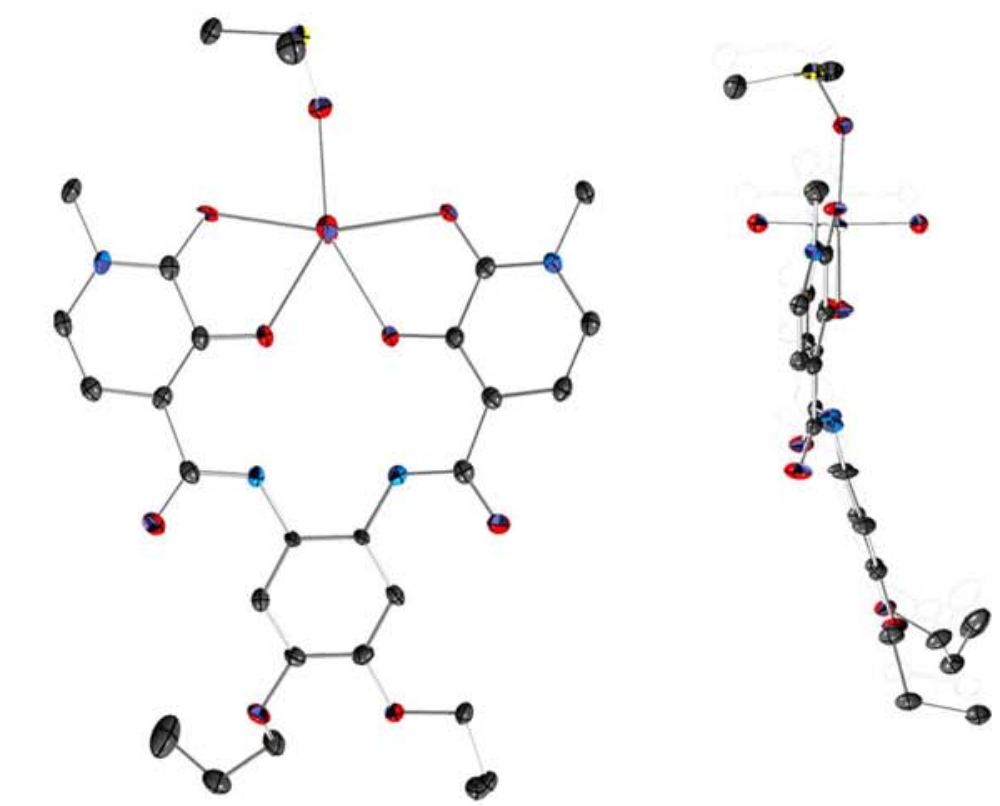

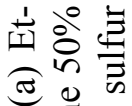

官

品 语

0 잉

○․ㅡ.

ํ. 흏

iे का

$\sum_{i}^{0} \overline{0}$

ํㅡㄹ

过

丞

ते

ह0 을

돌 बำ

के के

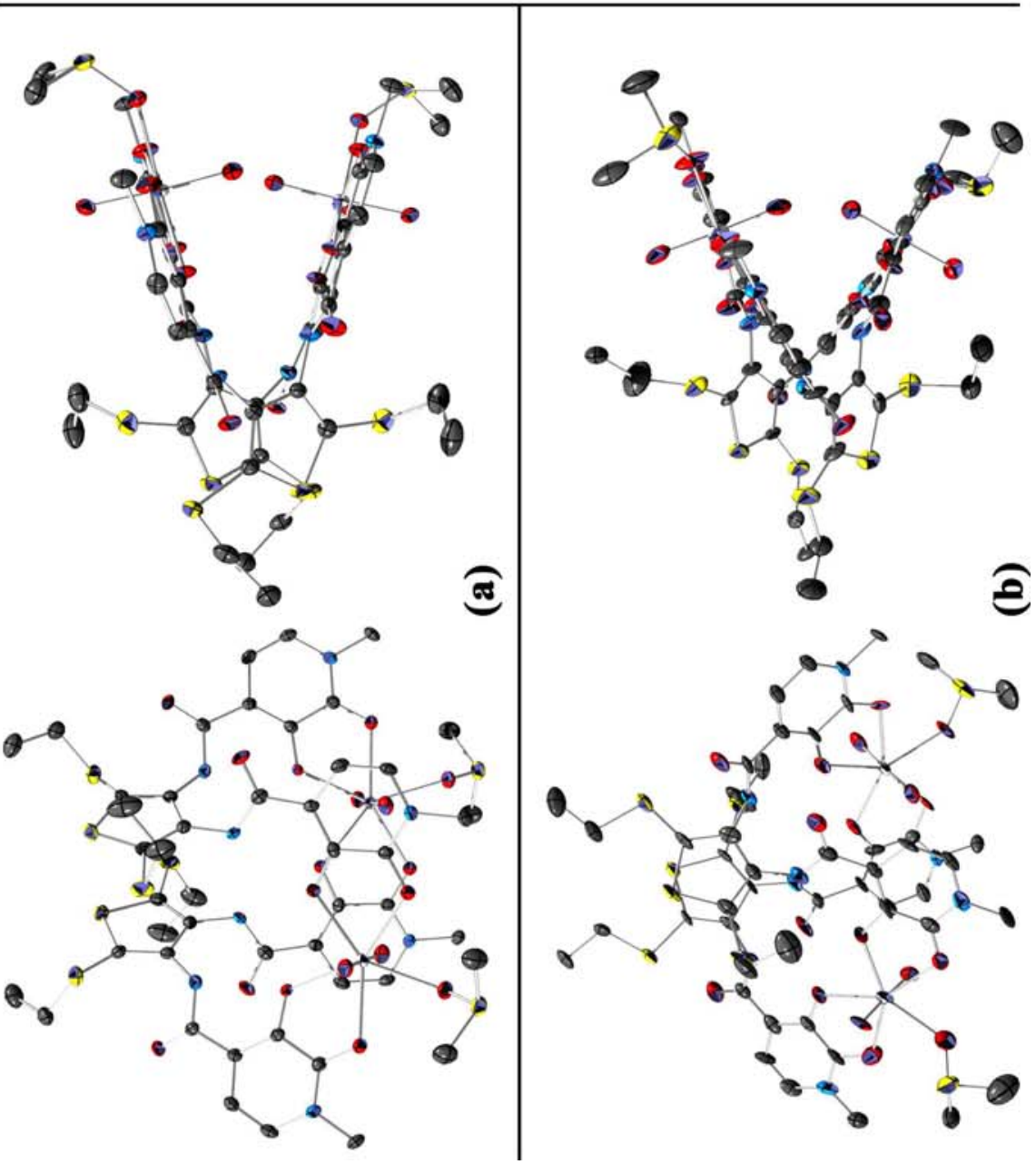

x

홀

خํ.

궁

4.

语

总

त क

की 00

웡 언

त

乌

赵事

文记

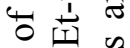

完全

舟

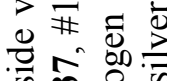

क की

盯贻总

응 वे ग्ञ

인

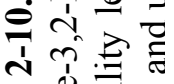

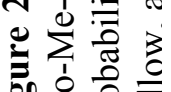

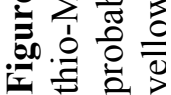


Table 2-6. Crystallographic parameters for uranyl complexes with 2-37 and 2-38.

\begin{tabular}{|c|c|c|c|}
\hline & $\begin{array}{c}{\left[\mathrm{UO}_{2}(2-37)(\mathrm{DMSO})\right]_{2},} \\
\# 1\end{array}$ & $\begin{array}{c}{\left[\mathrm{UO}_{2}(2-37)(\mathrm{DMSO})\right]_{2},} \\
\# 2\end{array}$ & $\mathrm{UO}_{2}(2-38)(\mathrm{DMSO})$ \\
\hline Formula & $\mathrm{C}_{48} \mathrm{H}_{56} \mathrm{~N}_{8} \mathrm{O}_{18} \mathrm{~S}_{8} \mathrm{U}_{2}$ & $\begin{array}{c}\mathrm{C}_{48} \mathrm{H}_{56} \mathrm{~N}_{8} \mathrm{O}_{18} \mathrm{~S}_{8} \mathrm{U}_{2} \\
\cdot 5.33\left(\mathrm{CH}_{4} \mathrm{O}\right)\end{array}$ & $\begin{array}{c}\mathrm{C}_{28} \mathrm{H}_{34} \mathrm{~N}_{4} \mathrm{O}_{11} \mathrm{SU} \\
.1 / 4 \mathrm{H}_{2} \mathrm{O}\end{array}$ \\
\hline MW & 1765.55 & 1936.35 & 877.19 \\
\hline $\mathbf{T}[\mathbf{K}]$ & $135(2)$ & $143(2)$ & $115(2)$ \\
\hline Crystal system & Orthorhombic & Triclinic & Orthorhombic \\
\hline Space group & Fdd2 & P-1 & Pbca \\
\hline Appearance & Block & Plate & Plate \\
\hline Color & Red & Orange & Red \\
\hline$a[\AA]]$ & $20.2557(8)$ & $12.472(5)$ & $13.451(2)$ \\
\hline$b[\AA]$ & $44.6259(18)$ & $16.603(7)$ & $15.839(2)$ \\
\hline$c[\AA]]$ & $13.5050(6)$ & $18.674(7)$ & $28.445(4)$ \\
\hline$\alpha\left[^{\circ}\right]$ & 90 & $101.808(6)$ & 90 \\
\hline$\beta\left[^{\circ}\right]$ & 90 & $90.613(6)$ & 90 \\
\hline$\gamma\left[^{\circ}\right]$ & 90 & $109.875(6)$ & 90 \\
\hline$V\left[\AA^{3}\right]$ & $12207.6(9)$ & $3546(2)$ & $6060.3(16)$ \\
\hline $\mathbf{Z}$ & 8 & 2 & 8 \\
\hline$\rho_{\text {calcd }}\left[\mathrm{g} \mathrm{cm}^{-3}\right]$ & 1.921 & 1.813 & 1.923 \\
\hline$\mu_{\text {palcd }}\left[\mathrm{mm}^{-1}\right]$ & 5.648 & 4.875 & 5.494 \\
\hline$\theta_{\min }, \theta_{\max },\left[^{\circ}\right]$ & $1.83,25.36$ & $2.54,25.54$ & $3.29,26.36$ \\
\hline Total reflections & 48794 & 24740 & 27837 \\
\hline Data/ restr./ param. & $5286 / 3 / 400$ & $12742 / 146 / 913$ & $5978 / 0 / 410$ \\
\hline$F(000)$ & 6848 & 1904 & 3428 \\
\hline $\mathbf{T}_{\min } / \mathbf{T}_{\max }$ & 0.540 & 0.522 & 0.324 \\
\hline Cryst. size $\left[\mathrm{mm}^{3}\right]$ & $0.19 \times 0.17 \times 0.05$ & $0.26 \times 0.15 \times 0.08$ & $0.35 \times 0.10 \times 0.05$ \\
\hline $\mathrm{R}_{1}[I>2 \sigma(I)]^{a}$ & 0.0140 & 0.0794 & 0.0304 \\
\hline $\mathrm{wR}_{2}\left(\right.$ all data) ${ }^{a}$ & 0.0310 & 0.2271 & 0.0738 \\
\hline GOF $^{a}$ & 1.019 & 1.034 & 1.040 \\
\hline
\end{tabular}

The biggest surprise in the uranyl structures in Figure 2-10 is that the uranyl complexes with 2-37 are dimers of two $\mathrm{UO}_{2}(\mathbf{2 - 3 7})(\mathrm{DMSO})$ units, with each ligand coordinating two metal centers. The uranyl centers exhibit the expected pentagonal bipyramidal coordination geometry with four equatorial oxygens provided by two different 2-37 ligands with the fifth coordinating oxygen provided by a DMSO molecule. The only significant difference between the crystal structures in the two different crystal habits of the uranyl-2-37 complex is that one was devoid of solvent inclusions (structure \#1), while the other included 5.33 MeOH molecules in the unit cell (structure \#2) which was the cause of the rapid desolvation out of solution. The $\left[\mathrm{UO}_{2}(\mathbf{2 - 3 7})\right]_{2}$ complex in structure \#1 also straddles a crystallographic 2-fold axis, making only one uranyl cation and one 2-37 ligand crystallographically unique. The dimeric nature of this complex is 
maintained in mass spectrometric measurement; the mononuclear form is also seen in the mass spectrum, but this is most likely present as a decomposition of the dimer under the measurement conditions. The most striking difference between the dimeric $\left[\mathrm{UO}_{2}(\mathbf{2 - 3 7})\right]_{2}$ structures and the monomeric $\mathrm{UO}_{2}(\mathbf{2 - 6})(\mathrm{DMF})$ complex is the torsion angle of the amides with respect to the thiophene ring; in $\mathrm{UO}_{2}(\mathbf{2 - 6})(\mathrm{DMF})$ the amides are nearly co-planar with the thiophene ring $\left(176^{\circ}\right.$ and $171^{\circ}$ torsion), while in $\left[\mathrm{UO}_{2}(\mathbf{2 - 3 7})\right]_{2}$ they obviously deviate significantly from co-planarity. The amide proton is still in an appropriate position to facilitate strong intramolecular hydrogen bonding to the deprotonated HOPO phenolate oxygens, with N--O bond distances of $2.66-2.80 \AA$ compared to $2.62 \AA$ in $\mathrm{UO}_{2}(\mathbf{2 - 6})(\mathrm{DMF})$.

It was assumed that the reason for the dimeric structure is the potential close contact between the linking amide oxygens and the thioalkyl sulfur atoms, causing the amide twist observed (Figure 2-11). The distance between amide oxygens and sulfur atoms on the same sides of the thiophene linkers range between $2.90 \AA$ and $5.01 \AA$, depending on the degree of amide twist observed. The minimum value of $2.90 \AA$ is less than the sum of the sulfur and oxygen Van der Waals radii of these atoms $(3.3 \AA)$, illustrating that close approaches in this position are capable of existing. Although close contacts may indeed form, twisting the amide into planarity with the thiophene will bring the sulfur and oxygen atoms into closer proximity, leading to even higher ligand strain energies.

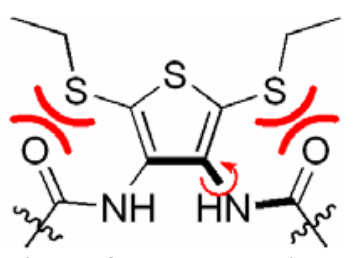

Figure 2-11. Intramolecular steric interference and amide torsion angle in $\left[\mathrm{UO}_{2}(\mathbf{2 - 3 7})\right]_{2}$ complexes. 
The steric influence of substitution at the 2- and 5-positions of the thiophene backbone was investigated further by molecular dynamics calculations in the CAChe software suite using PM5 parameters. Using simplified models of the thiophene backbone and with only one amide substituent, the $\mathrm{N}_{\text {amide }}-\mathrm{C}_{\text {thiophene }}$ torsion angle was rotated through a full $360^{\circ}$ rotation at $5^{\circ}$ intervals, relaxing the geometry at each step to convergence. For these calculations, $0^{\circ}$ corresponded to the amide moiety completely coplanar with the thiophene ring and in the conformation seen in the $\mathrm{UO}_{2}(\mathbf{2}-\mathbf{6})(\mathrm{DMF})$ crystal structure (mononuclear type coordination). This calculation was performed in the absence and presence of a thioethyl substituent ortho to the amide moiety; the results for both calculations are shown in Figure 2-12.
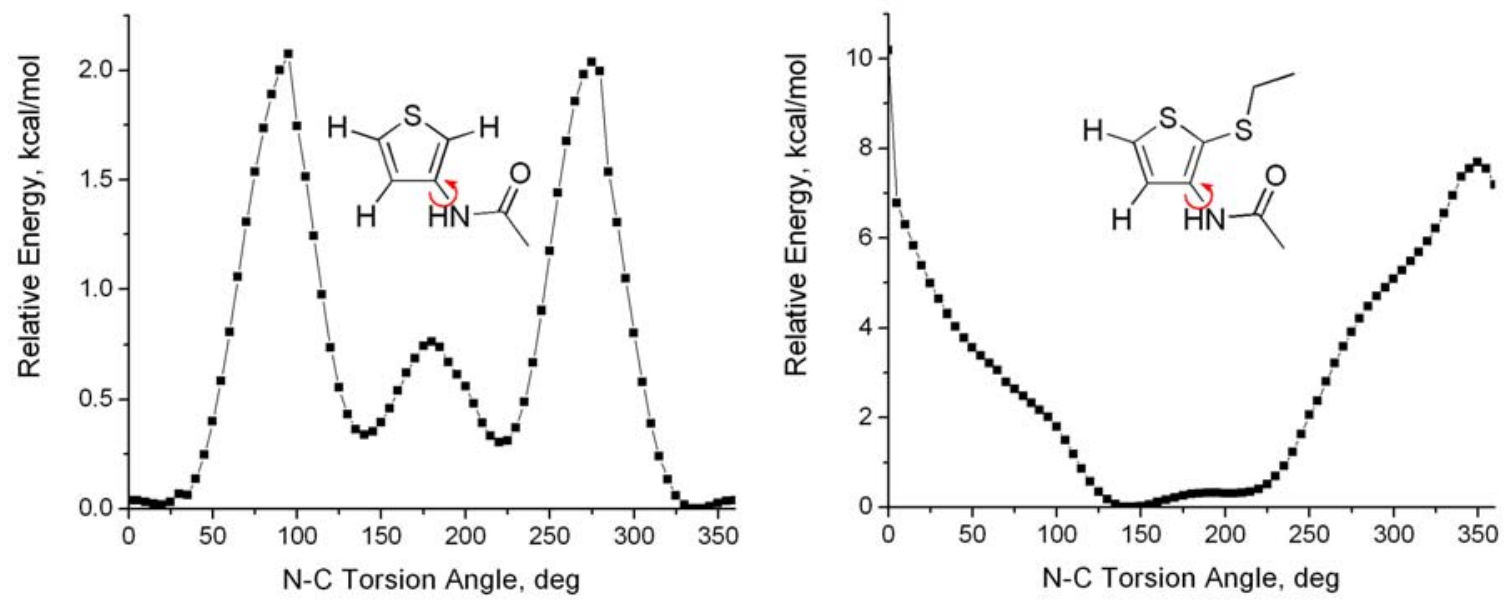

Figure 2-12. Relative energy calculations for rotation of an acetamide substituent about the $\mathrm{N}_{\text {amide }}-\mathrm{C}_{\text {thiophene }}$ bond without (left) and with (right) ortho thioethyl substitution.

Calculations on the unsubstituted thiophene display energetic lows near $0^{\circ}$ and highs near $90^{\circ}$ and $270^{\circ}$, corresponding to when the amide moiety is in and out of conjugation with the thiophene ring, respectively (Figure 2-12, left). The energy difference between these two extremes is $c a .2 \mathrm{kcal} / \mathrm{mol}$ and corresponds well to other such reported values. ${ }^{19}$ Introduction of the thioalkyl group close to the amide substituent changes the energy profile significantly (Figure 2-12, right), with the highest relative energy occurring near 
$0^{\circ}$ and the lowest near $180^{\circ}$, with a larger energy difference than in the unsubstituted model. The high relative energy at $0^{\circ}$ is a result of the unfavorable close approach between the amide oxygen and the thioethyl sulfur atoms, while the lowest energy values at $180^{\circ}$ result from both the absence of this steric interaction as well as a favorable hydrogen-bonding interaction between the amide proton and the thioethyl sulfur. Most significant to the current study, however, is that the energy maxima do not occur at $90^{\circ}$ and $270^{\circ}$, indicating that amide torsion out of the aromatic plane is favored in the presence of a substituent at the 2-position of the thiophene.

As a better representation of what is occurring in the $\left[\mathrm{UO}_{2}(\mathbf{2 - 3 7})(\mathrm{DMSO})\right]_{2}$ complex, the relative energies of rotation about a $\mathrm{N}_{\text {amide }}-\mathrm{C}_{\text {thiophene }}$ bond were calculated when both the 3- and 4-positions of the thiophene are substituted with acetamides. The incremental amide rotation described above was performed in the absence and presence of thioethyl substituents at the 2- and 5-positions of the thiophene rings to simulate ligands $\mathbf{2 - 6}$ and 237 respectively. Only one amide was constrained to the reported torsion angles, while the other was allowed to refine freely. The results of these calculations are illustrated in Figure 2-13. Energy minimizations at each step are complicated by hydrogen bonding and steric interactions between the two amides, and sharp drops in relative energy upon incremental amide rotation are a consequence of significant rearrangement of the neighboring amide, typically facilitating a new hydrogen-bonding interaction. Thus the relative energies at the sharp, highest energy peaks should not be considered at face value, with more attention paid towards overall energetic trends. 

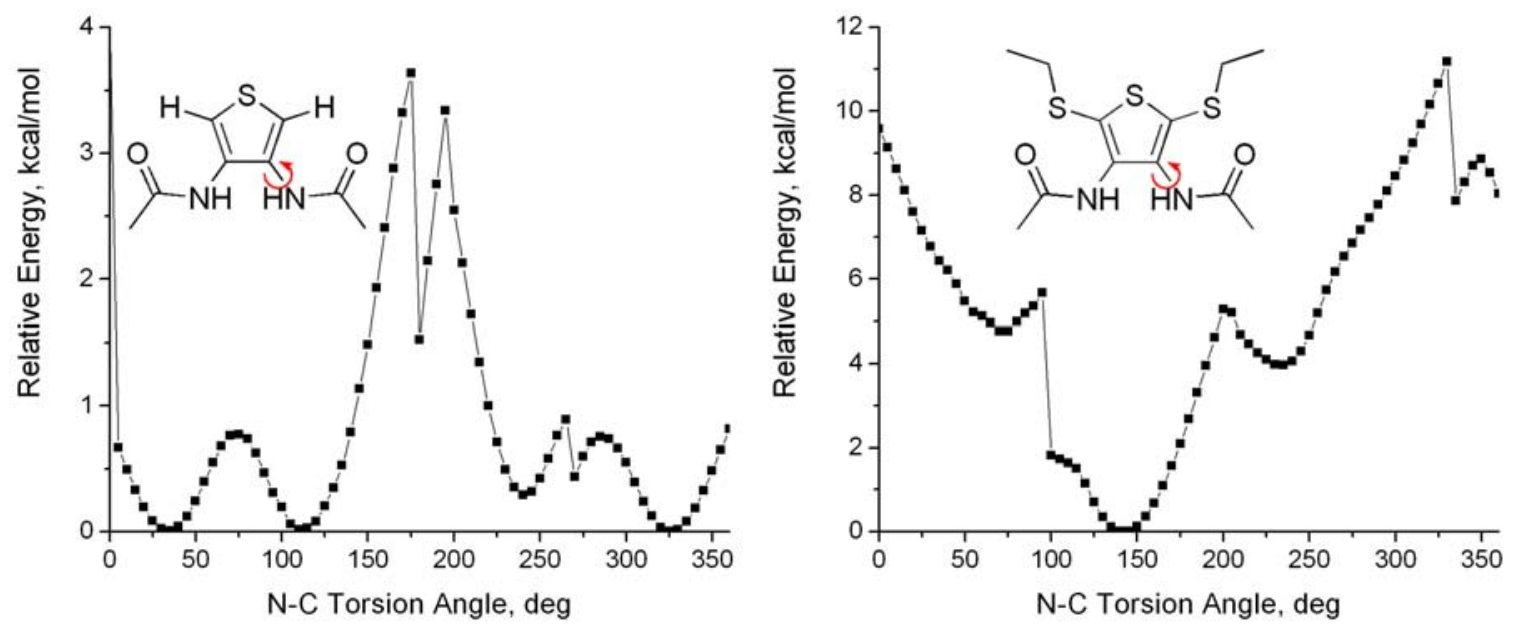

Figure 2-13. Relative energy calculations for rotation of an acetamide substituent about the $\mathrm{N}_{\text {amide }}-\mathrm{C}_{\text {thiophene }}$ bond in the presence of an ortho acetamide in the absence (left) and presence (right) of 2,5-dithioethyl substitution.

Calculations on the unsubstituted bis-amide substrate (Figure 2-13, left) do not achieve their highest relative energies at $90^{\circ}$ and $270^{\circ}$ as in the 2,5-unsubtituted monoamide (Figure 2-12, left), although those angles still correspond to local maxima. Upon rotation of the amide moiety to place its oxygen atom towards the neighboring amide (rotation approaching $100^{\circ}$ ), a favorable hydrogen-bonding interaction with the neighboring amide proton is established. Upon further rotation, however, steric interference raises the relative energy to its highest values. This indicates that the orthoamide alone has a significant effect on the backbone geometry, and explains the very small deviations from planarity seen in the $\mathrm{UO}_{2}(\mathbf{2 - 6})(\mathrm{DMF})$ crystal structure.

Calculations on the 2,5-disubstituted bis-amide (Figure 2-13, right) reveal again that the bis-thioethyl substitution has a great effect on relative energies, displaying global maximum when the amide moiety is in plane with the thiophene and in the conformation seen in the $\mathrm{UO}_{2}(\mathbf{2 - 6})(\mathrm{DMF})$ structure (zero torsion angle). This explains why the uranyl complex with 2-37 cannot adopt the geometry observed with 2-6. As with the asymmetrically substituted model, the global energy minimum occurs near $150^{\circ}$ due to 
favorable $\mathrm{N}-\mathrm{H} \cdots \mathrm{S}$ hydrogen bonding, but such an arrangement is not one that promotes uranyl chelation, and it is thus unsurprising this rotation is not observed in the crystal structures in Figure 2-10. Interestingly, however, there are local energy minima at $70^{\circ}$ and $235^{\circ}$ at which the amide group is significantly twisted out of conjugation with the thiophene ring. These values correspond very well to the $\mathrm{N}_{\text {amide }}-\mathrm{C}_{\text {thiophene }}$ bond torsion angles exhibited in the $\left[\mathrm{UO}_{2}(\mathbf{2 - 3 7})(\mathrm{DMSO})\right]_{2}$ crystal structures which are $65^{\circ}$ and $245^{\circ}$ for structure $\# 1$, and are $\left(59^{\circ}, 239^{\circ}\right)$ and $\left(57^{\circ}, 246^{\circ}\right)$ for structure $\# 2$.

These theoretical studies confirm that the steric contribution of the two thioethyl substituents on the thiophene backbone in 2-37 is the cause of significant $\mathrm{N}_{\text {amide }}-\mathrm{C}_{\text {thiophene }}$ bond torsion and thus the dimeric $\left[\mathrm{UO}_{2}(\mathbf{2 - 3 7})(\mathrm{DMSO})\right]_{2}$ crystal structure. In addition, the theoretical energetic minima correspond well to the torsion angles seen in the solid state. Returning to ligand 2-21, the PEG moiety will most assuredly introduce a larger steric influence than the thioethyl substituent in $\mathbf{2 - 3 7}$, so in the solution thermodynamic measurements described later, it is assumed that $\mathbf{2 - 2 1}$ forms dimeric uranyl complexes in a similar fashion to that seen in the $\left[\mathrm{UO}_{2}(\mathbf{2 - 3 7})(\mathrm{DMSO})\right]_{2}$ crystal structures.

In contrast to both $\left[\mathrm{UO}_{2}(\mathbf{2 - 3 7})(\mathrm{DMSO})\right]_{2}$ structures, the $\mathrm{UO}_{2}(\mathbf{2 - 3 8})(\mathrm{DMSO})$ crystal structure displays the expected mononuclear speciation, with a pentagonal planar uranyl coordination geometry provided on four points by $\mathbf{2 - 3 8}$ and on the fifth equatorial coordination site by a DMSO oxygen. Significantly, the propoxy substituents are situated away from the amide linkers, so it can be assumed that PEG substitution in 2-22 will not impart a significant steric influence on the uranyl complex geometry.

Equatorial U-O bond lengths and conformational analysis results for the crystal structures in Figure 2-10 are listed in Table 2-7. It can be seen that the U-O bond 
distances in the $\mathrm{UO}_{2}(\mathbf{2 - 3 8})(\mathrm{DMSO})$ complex are essentially the same as those in $\mathrm{UO}_{2}(\mathbf{2}$ 7)(DMSO). As in the $\mathrm{UO}_{2}(\mathbf{2}-7)(\mathrm{DMSO})$ structure, the amide bonds in $\mathrm{UO}_{2}(\mathbf{2 - 3 8})(\mathrm{DMSO})$ undergo a small degree of $\mathrm{C}_{\text {ring }}-\mathrm{N}_{\text {amide }}$ torsion $\left(156^{\circ}\right.$ and $\left.167^{\circ}\right)$ presumably to relieve a potential close contact between the amide protons. The $\theta$ and $\varphi$ values are marginally higher than in $\mathrm{UO}_{2}(2-7)(\mathrm{DMSO})$, but the overall structure of the complex suggests that the PEG substitution in $\mathbf{2 - 3 8}$ makes no significant impact on the uranyl complex geometry compared to $\mathbf{2 - 7}$.

Table 2-7. U-O bond lengths and conformational parameters from $\left[\mathrm{UO}_{2}(\mathbf{2 - 3 7})(\mathrm{DMSO})\right]_{2}$ and $\mathrm{UO}_{2}(\mathbf{2}-\mathbf{3 8})(\mathrm{DMSO})$ structures.

\begin{tabular}{|c|c|c|c|c|c|}
\hline Complex & $\theta,\left[^{\circ}\right]$ & $\varphi,\left[^{\circ}\right]$ & $\Sigma \sigma_{\mathrm{n}},\left[^{\circ}\right]$ & $\begin{array}{c}\text { U-O(amide), } \\
{[\AA \AA} \\
{[\AA]}\end{array}$ & $\begin{array}{c}\text { U-O(phenol), } \\
{[\AA \AA]}\end{array}$ \\
\hline $\begin{array}{c}{\left[\mathrm{UO}_{2}(\mathbf{2 - 3 7})\right.} \\
(\mathrm{DMSO})]_{2}, \# 1\end{array}$ & $12.3(2)$ & $3.8(1), 8.7(1)$ & $360.5(2)$ & $2.359(2), 2.442(2)$ & $2.360(2), 2.400(2)$ \\
\hline $\begin{array}{c}{\left[\mathrm{UO}_{2}(\mathbf{2 - 3 7})\right.} \\
(\mathrm{DMSO})]_{2}, \# 2 \\
\end{array}$ & $\begin{array}{c}11.0(9), \\
11.8(8)\end{array}$ & $\begin{array}{c}3.7(8), 6.1(6), \\
7.1(6), 8.7(8)\end{array}$ & $\begin{array}{l}360.1(7), \\
360.2(8) \\
\end{array}$ & $\begin{array}{c}\text { 2.417(10), } 2.418(11), \\
2.442(9), 2.472(10)\end{array}$ & $\begin{array}{c}2.337(9), 2.338(9), \\
2.365(10), 2.381(10)\end{array}$ \\
\hline $\begin{array}{c}\mathrm{UO}_{2}(\mathbf{2 - 3 8}) \\
(\mathrm{DMSO})\end{array}$ & $21.70(7)$ & $10.67(5), 12.41(5)$ & $360.3(2)$ & $2.421(3), 2.459(3)$ & $2.338(3), 2.342(3)$ \\
\hline
\end{tabular}

The conformational parameters in the two $\left[\mathrm{UO}_{2}(\mathbf{2 - 3 7})(\mathrm{DMSO})\right]_{2}$ complexes are comparable to those in the $\mathrm{UO}_{2}(\mathbf{2 - 6})(\mathrm{DMF})$ structure and are generally below or near the $10^{\circ}$ limit set on their interplanar angles. This indicates that reasonable coordination geometries are maintained despite the change in coordination behavior. The equatorial U$\mathrm{O}$ bond distances, however, are more intriguing: in $\left[\mathrm{UO}_{2}(\mathbf{2 - 3 7})(\mathrm{DMSO})\right]_{2}$ structure $\# 2$, the $\mathrm{U}-\mathrm{O}$ bonds are similar to those in all the other $\mathrm{UO}_{2}$-bis-Me-3,2-HOPO complexes investigated here, with the $\mathrm{U}-\mathrm{O}_{\text {amide }}$ bonds longer than the $\mathrm{U}-\mathrm{O}_{\text {phenol }}$ bonds. $\mathrm{In}\left[\mathrm{UO}_{2}(2\right.$ 37)(DMSO) $]_{2}$ structure \#1, however, one Me-3,2-HOPO moiety reverses this trend, with the $\mathrm{U}-\mathrm{O}_{\text {amide }}$ bond shorter than the $\mathrm{U}-\mathrm{O}_{\text {phenol }}$ bond (2.36 $\AA, 2.40 \AA$ respectively). Why such a reversal of relative bond lengths occurs in this structure and not in structure \#2 (both grown from the same vial) is unclear, but can be assumed to be a solid state 
phenomenon and one that cannot be compared directly to the mononuclear, unsubstituted $\mathrm{UO}_{2}(\mathbf{2 - 6})(\mathrm{DMF})$ complex.

\subsubsection{Solution Thermodynamics}

In the presence of dissolved metal ion and protonated ligand, a $\mathrm{pH}$-dependent metalligand complex of general formula $\mathrm{M}_{m} \mathrm{~L}_{l} \mathrm{H}_{h}$ forms according to the equilibrium shown in Equation 2-1. The relative amount of each species in solution is determined by Equation $2-2$, the rearrangement of which provides the standard formation constant notation of $\log$ $\beta_{m l h}$ (Equation 2-3). The $\log \beta_{m l h}$ value describes a cumulative formation constant, but a stepwise formation constant $\log \mathrm{K}$ can be calculated from $\log \beta_{m l h}$ values using Equation 2-4. When considering protonation constants, the stepwise formation constants are commonly reported as $-\log \mathrm{K}\left(\mathrm{pK}_{\mathrm{a}}\right)$, which are dissociation constants (i.e. the first $\mathrm{pK}_{\mathrm{a}}$ value corresponds to the last proton association).

$$
\begin{gathered}
m \mathrm{M}^{\mathrm{a}+}+l \mathrm{~L}^{\mathrm{b}-}+h \mathrm{H}^{+} \stackrel{\beta_{m l h}}{=} \mathrm{M}_{m} \mathrm{~L}_{l} \mathrm{H}_{h}^{(m \mathrm{a}-l \mathrm{~b}+h)^{+}} \\
{\left[\mathrm{M}_{m} \mathrm{~L}_{l} \mathrm{H}_{h}\right]=\beta_{m l h}[\mathrm{M}]^{m}[\mathrm{~L}]^{l}[\mathrm{H}]^{h}} \\
\log \beta_{m l h}=\log \left(\frac{\left[\mathrm{M}_{m} \mathrm{~L}_{l} \mathrm{H}_{h}\right]}{\left[\mathrm{M}^{m}[\mathrm{~L}]^{l}[\mathrm{H}]^{h}\right.}\right) \\
\log \mathrm{K}_{01 n}=\log \left(\frac{\left[\mathrm{LH}_{n}\right]}{\left[\mathrm{LH}_{n-1}\right][\mathrm{H}]}\right)=\log \left(\frac{\beta_{01 n}}{\beta_{01(n-1)}}\right)=\log \beta_{01 n}-\log \beta_{01(n-1)}
\end{gathered}
$$

A series of potentiometric and spectrophotometric titrations were carried out to determine the affinity of the bis-Me-3,2-HOPO ligands for proton and the uranyl cation. Protonation constants of the PEG-functionalized ligands 2-17 through 2-23 were carried out using potentiometric titrations with $c a$. 150-200 $\mu \mathrm{M}$ ligand concentration in $0.1 \mathrm{M} \mathrm{KCl}$ solution with a starting concentration of 5\% DMSO. The 
DMSO was required for consistency with later uranyl titrations in which the neutral uranyl complexes displayed poor solubility even at very low concentrations. The data from at least three independent titrations, each consisting of one forward ( $\mathrm{pH} 3$ to $\mathrm{pH}$ 10) and one reverse $(\mathrm{pH} 10$ to $\mathrm{pH} 3)$ titration were combined to give the $\mathrm{pK}_{\mathrm{a}}$ values listed in Table 2-8. Refinement of the forward and backward runs of each titration separately resulted in similar values, so were refined together to provide better fit statistics. As a point of comparison to the values determined for the PEG-nLi-Me-3,2HOPO ligands 2-17 through 2-20, the protonation constants for the $2 \mathrm{Li}$ - and $4 \mathrm{Li}-\mathrm{Me}-$ 3,2-HOPO ligands (2-2 and 2-4) were measured by spectrophotometric titrations at ca. $50 \mu \mathrm{M}$ ligand concentrations, again in $0.1 \mathrm{M} \mathrm{KCl}$ and $5 \%$ DMSO. Spectrophotometry was required due to the lower solubility of these ligands as compared to their PEG-containing analogs. Data from three independent spectrophotometric titrations were again combined to give the $\mathrm{pK}_{\mathrm{a}}$ values reported, but with independent refinement performed on the forward and backward titration runs.

Table 2-8. $\mathrm{pK}_{\mathrm{a}}$ values of bis-Me-3,2-HOPO ligands.

\begin{tabular}{|c|c|c|c|c|}
\hline Ligand & $\boldsymbol{n}$ & $\mathbf{p K}_{\mathrm{a} 1}$ & $\mathbf{p K}_{\mathbf{a} 2}$ & $\mathbf{\Sigma p K}_{\mathbf{a}}$ \\
\hline Pr-Me-3,2-HOPO, 2-1 & -- & $6.12^{112]}$ & -- & -- \\
\hline 2Li-Me-3,2-HOPO, 2-2 & 2 & $5.82(3)$ & $6.68(3)$ & $12.50(4)$ \\
\hline 4Li-Me-3,2-HOPO, 2-4 & 4 & $6.01(1)$ & $7.02(4)$ & $13.03(4)$ \\
\hline PEG-2Li-Me-3,2-HOPO, 2-17 & 2 & $5.10(6)$ & $6.45(1)$ & $11.55(6)$ \\
\hline PEG-3Li-Me-3,2-HOPO, 2-18 & 3 & $5.37(2)$ & $6.72(2)$ & $12.09(3)$ \\
\hline PEG-4Li-Me-3,2-HOPO, 2-19 & 4 & $5.25(4)$ & $6.60(3)$ & $11.85(5)$ \\
\hline PEG-5Li-Me-3,2-HOPO, 2-20 & 5 & $5.52(7)$ & $6.75(2)$ & $12.27(7)$ \\
\hline PEG-thio-Me-3,2-HOPO, 2-21 & 2 & $4.91(8)$ & $6.22(1)$ & $11.13(8)$ \\
\hline PEG- $o$-phen-Me-3,2-HOPO, 2-22 & 2 & $5.09(3)$ & $6.29(1)$ & $11.38(3)$ \\
\hline PEG- $m$-xy-Me-3,2-HOPO, 2-23 & 5 & $5.70(6)$ & $6.75(2)$ & $12.45(6)$ \\
\hline
\end{tabular}

Table 2-8 also lists the sum of the $\mathrm{pK}_{\mathrm{a}}$ values $\left(\sum \mathrm{pK}_{\mathrm{a}}\right)$, which corresponds to the log $\beta_{012}$ formation constant and is a general measure of how acidic the ligand is, with lower $\Sigma \mathrm{pK}_{\mathrm{a}}$ values indicating a more acidic ligand. Because metal complexation with Raymond 
group ligands requires the deprotonation of the binding moiety, the more acidic a ligand is, the better it can bind metal cations at lower acidities, assuming the coordinating moieties display similar structural and electronic behavior.

The first trend visible in Table 2-8 is that linearly-linked ligands with shorter linkers (smaller $n$ ) have lower $\Sigma \mathrm{pK}_{\mathrm{a}}$ values than those with longer linkers. This trend is seen in the PEG-nLi-Me-3,2-HOPO ligands 2-17 through $\mathbf{2 - 2 0}\left(\Delta \Sigma \mathrm{pK}_{\mathrm{a}} \approx 0.7\right)$ as well as between the 2Li- and 4Li-Me-3,2-HOPO ligands $\mathbf{2 - 2}$ and $\mathbf{2 - 4}\left(\Delta \Sigma \mathrm{pK}_{\mathrm{a}} \approx 0.5\right)$. The second trend is that substitution of the PEG moiety to the linear linkers also lowers the $\Sigma \mathrm{pK}_{\mathrm{a}}$ compared to the structurally analogous non-functionalized ligands, as evidenced by comparing the 2Li- versus PEG-2Li-Me-3,2-HOPO ligands (2-2 vs. 2-17) and their $n=4$ analogs (2-4 vs. 2-19). This effect is most prominent in a lowering of the $\mathrm{pK}_{\mathrm{a} 1}$ value $\left(\Delta \mathrm{pK}_{\mathrm{a} 1, \max } \approx\right.$ $0.7)$, but also affects the $\mathrm{pK}_{\mathrm{a} 2}$ values $\left(\Delta \mathrm{pK}_{\mathrm{a} 2, \max } \approx 0.4\right)$. The drop in $\Sigma \mathrm{pK}_{\mathrm{a}}$ values upon introduction of the PEG substituent to the backbone linker $(\Delta \Sigma \mathrm{pK} / \mathrm{a} / \mathrm{PEG} \approx 1.1)$ is more significant than that accompanying incremental shortening of the linker lengths (average $\left.\Delta \Sigma \mathrm{pK}_{\mathrm{a}} / n \approx 0.23\right)$. Because the PEG moiety is not expected to have a significant inductive effect on the HOPO moiety electronics, and because the ligand moieties in each ligand are identical, the lowering of $\mathrm{pK}_{\mathrm{a}}$ values in both trends is most likely a result of increased stabilization of the HOPO anion upon deprotonation.

Raymond group ligands containing primary amides ortho to phenolic oxygens are stabilized upon deprotonation by intramolecular hydrogen bonding as shown in Figure 214(a), ${ }^{13}$ but the trends in $\Delta \Sigma \mathrm{pK}_{\mathrm{a}}$ described above indicate that additional hydrogen bond interactions occur with smaller linker lengths and in the presence of the PEG solubilizing moiety. The drop in $\mathrm{pK}_{\mathrm{a} 1}$ associated with shortened linker length can be explained by an 
increased proximity of the second amide group to the initially deprotonated phenolate group as shown in Figure 2-14(b). Such an interaction explains the steady drop in $\mathrm{pK}_{\mathrm{a} 1}$ as linker length decreases, both in the PEG-functionalized ligands and their nonfunctionalized versions.

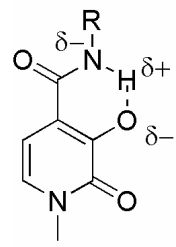

(a)

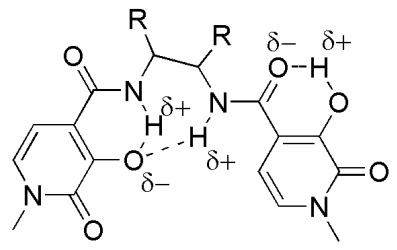

(b)

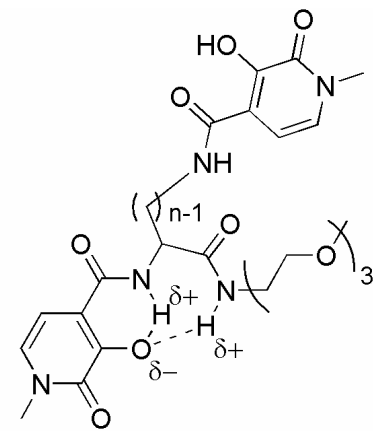

(c)

Figure 2-14. Proposed hydrogen bond stabilization in (a) HOPO-amide ligands, (b) bisHOPO ligands with short linkers, and (c) HOPO ligands containing solubilizing PEGamide groups next to linking amides.

The drop in $\mathrm{pK}_{\mathrm{a} 1}$ upon PEG-functionalization to linearly-linked bis-Me-3,2-HOPO ligands can be described in a similar manner; the PEG-functionalized ligands have one additional amide group in close proximity to one of the HOPO phenolate oxygens, independent of linker length. This group may provide hydrogen bonding interactions to the deprotonated HOPO phenolate as illustrated in Figure 2-14(c), explaining the systematic drop in $\mathrm{pK}_{\mathrm{a}}$ observed upon inclusion of the PEG-amide solubilizing group. Although such an effect on ligand $\mathrm{pK}_{\mathrm{a}}$ was undesirable, the inclusion of the PEG-amide group systematically across the PEG-nLi-Me-3,2-HOPO ligands 2-17 through 2-20 allows for the effect of PEG substitution to be considered constant, allowing the effect of linker length and geometry to be the prominent trend across the PEG-nLi-Me-3,2-HOPO ligand series. 
The stability of the uranyl complexes with ligands 2-17 through 2-20 was evaluated by performing spectrophotometric titrations. These were carried out again with a starting DMSO concentration of $c a .5 \%$ to assist in the solvation of the neutral uranyl complexes. The uranyl complexes were found to be much less soluble than the protonated bis-Me3,2-HOPO ligands and made uranyl titrations with the unsubstituted ligands 2-2 through 2-5 impossible even at $5 \mu \mathrm{M}$ concentrations. In addition to 2-17 through 2-23, the parent bidentate Pr-Me-3,2-HOPO ligand 2-1 was found to be sufficiently soluble for spectrophotometric titrations. Metal-to-ligand ratios used in the titrations were those observed in the crystal structures of the uranyl complexes with unsubstituted Me-3,2HOPO ligands, namely 1:1 for bis-Me-3,2-HOPO ligands and 1:2 for Pr-Me-3,2-HOPO. These ratios were controlled by careful addition of a ligand solution in DMSO of known concentration and a standardized aqueous uranyl solution to the titration vessel.

Table 2-9. $\log \beta_{m l h}$ values for uranyl titrations with Me-3,2-HOPO ligands.

\begin{tabular}{|c|c|c|c|c|c|c|c|c|}
\hline Ligand & $\log \boldsymbol{\beta}_{11-2}$ & $\log \beta_{11-1}$ & $\log \beta_{110}$ & $\log \beta_{12-1}$ & $\log \beta_{120}$ & $\log \beta_{22-1}$ & $\log \beta_{22-1}$ & $\log \boldsymbol{\beta}_{\mathbf{2 2 0}}$ \\
\hline $\mathbf{2 - 1}^{a}$ & $-2.50(6)$ & - & $10.64(3)$ & $11.48(4)$ & $17.91(2)$ & - & - & - \\
\hline $\mathbf{2 - 1 7}$ & - & $6.30(8)$ & $12.5(1)$ & - & - & - & - & - \\
\hline $\mathbf{2 - 1 8}$ & - & $5.86(6)$ & $12.6(1)$ & - & - & - & - & - \\
\hline $\mathbf{2 - 1 9}$ & - & $6.97(6)$ & $13.9(1)$ & - & - & - & - & - \\
\hline $\mathbf{2 - 2 0}$ & - & $5.64(4)$ & $13.42(7)$ & - & - & - & - & - \\
\hline $\mathbf{2 - 2 1}^{\boldsymbol{a}}$ & - & - & - & - & - & $15.74(5)$ & $22.47(8)$ & $28.1(1)$ \\
\hline $\mathbf{2 - 2 2}^{2-2}$ & - & $6.6(1)$ & $13.04(1)$ & - & - & - & - & - \\
\hline $\mathbf{2 - 2 3}^{\boldsymbol{a}}$ & - & $5.38(5)$ & $12.89(1)$ & - & - & - & - & - \\
\hline
\end{tabular}

${ }^{a}$ Only forward (acid to base) titration data was used due to observed irreversibility

Three independent titrations, with each titration consisting of a forward (acid to base) and reverse (base to acid) titration, were measured between 2.4 and 11.0 except where reversibility analysis indicated a point in the titrations beyond which the complexes underwent an irreversible chemical change. The data for each titration direction was analyzed separately, and reversibility was evaluated by comparing the values $\mathrm{V}^{*} \mathrm{~A}$ 
(volume*Absorbance) for the forward and backward directions at two separate wavelengths. Because the uranyl complexes formed at low $\mathrm{pH}$, two strong acid titrations $(\mathrm{pH} 3.0$ to 1.6) were carried out for each ligand. The data from the titrations were combined to yield the formation constants listed in Table 2-9.

Most uranyl titrations were reversible through the highest $\mathrm{pH}$ ranges of the titrations (typically $\mathrm{pH}$ 11.0-11.4), indicating that no unforeseen chemical changes occur in the metal-ligand complex that fundamentally change the chemical properties of the ligand or complex in solution. However, titrations with fully-conjugated ligands 2-6 and 2-7 exhibited terrible reversibility when titrations were taken to $\mathrm{pH}$ 11.0. It was found, however, that if the titrations were carried out only up to $\mathrm{pH} 8.5$ and 9.0 respectively, that reversibility was again observed in the titrations. The cause of this reversibility is unknown, but corresponds roughly to additional deprotonation or continued hydroxide introduction to form a $\mathrm{UO}_{2} \mathrm{LH}_{-2}$ species (where $\mathrm{H}_{-1}$ represents hydroxide coordination or incremental complex deprotonation). Such a species can be refined upon in uranyl titrations with 2-17 through 2-19, but to maintain consistency across the various bis-Me3,2-HOPO ligands, titration data were truncated before the onset of the $\mathrm{UO}_{2} \mathrm{LH}_{-2}$ product. For most ligands, this data truncation occurred at about $\mathrm{pH}$ 9.0. Reversibility analyses are provided in the Appendix along with UV-visible titration spectra and speciation diagrams for uranyl complex formation with each of the Me-3,2-HOPO ligands measured.

At low $\mathrm{pH}$ the uranyl titrations with all Me-3,2-HOPO ligands displayed a rapid increase in intensity indicating deprotonation of the ligand and complexation of the uranyl cation. For tetradentate ligands with the exception of 2-21, the complex formed was refined as a $\mathrm{UO}_{2} \mathrm{~L}$ complex. The simultaneous deprotonation is expected because of 
the similarity in $\mathrm{pK}_{\mathrm{a} 1}$ and $\mathrm{pK}_{\mathrm{a} 2}$ values of these ligands and because metal chelation will drive deprotonation of the ligand at lower $\mathrm{pH}$. The UV-visible spectra change again around neutral $\mathrm{pH}$ to what was refined as a $\mathrm{UO}_{2} \mathrm{~L}(\mathrm{OH})$ species. This partial hydrolysis occurs at very mildly basic conditions because it does not require the displacement of a ligand; the fifth equatorial coordination position on the uranyl is known to be occupied by solvent in bis-Me-3,2-HOPO complexes, and thus hydroxide coordination need not overcome a chelate effect of the ligand. The formation of a partial hydrolysis complex $\mathrm{UO}_{2} \mathrm{~L}(\mathrm{OH})$ is also seen in the aqueous solution thermodynamics of the uranyldesferrioxamine $\mathrm{B}$ (DFO) complex, which was observed to form a $\mathrm{UO}_{2}(\mathrm{DFO})(\mathrm{OH})$ complex starting at $c a$. $\mathrm{pH} 7(\log \beta 11-1=22.8)$, indicting that the uranyl center is not sufficiently complexed by the ligand to exclude solvent-dependent coordination. ${ }^{20}$ Because DFO is a hexadentate ligand designed for Fe(III) chelation, it is unclear if formation of $\mathrm{UO}_{2}(\mathrm{DFO})(\mathrm{OH})$ requires a displacement of an otherwise-coordinated ligand, but with the tetradentate bis-Me-3,2-HOPO ligands this hydrolysis is most assuredly a coordination of hydroxide (or deprotonation of coordinated water), and not a displacement of coordinated ligand.

The dimeric crystal structure of the $\left[\mathrm{UO}_{2}(\mathbf{2 - 3 7})\right]_{2}$ complex required the refinement of dimeric uranyl complexes with $\mathbf{2 - 2 1}$ in solution. Strong acid titration data refinement did not support a model consistent with the formation of $\mathrm{UO}_{2}(\mathbf{2 - 2 1}) \mathrm{H}_{0 / 1}$ monomer units preceding dimer formation, so direct dimer formation at low $\mathrm{pH}$ was assumed. This behavior suggests that the substitution on the thiophene ligand does not allow mononuclear complex formation even at the low concentrations used, which the amide torsion angle calculations would lead us to expect. In the solid state the uranyl dimer with 
2-21 exhibited two coordinated DMSO molecules, so it was assumed the complex could undergo two partial hydrolysis events to form first a $\left[\mathrm{UO}_{2}(\mathbf{2 - 2 1})\right]_{2}(\mathrm{solv})(\mathrm{OH})$ complex, then a $\left[\mathrm{UO}_{2}(\mathbf{2 - 2 1})(\mathrm{OH})\right]_{2}$ dimer (Figure 2-15). The onset of irreversibility (practically at $\mathrm{pH}$ 8.0) coincided with hydrolysis/deprotonation beyond the formation of the dihydroxide. This behavior is consistent with the irreversibility of the $\mathrm{UO}_{2}(\mathbf{2 - 2 2})$ complex titrations in which irreversibility was observed upon hydrolysis/deprotonation beyond the formation of the $\mathrm{UO}_{2}(\mathbf{2 - 2 2})(\mathrm{OH})$ species.

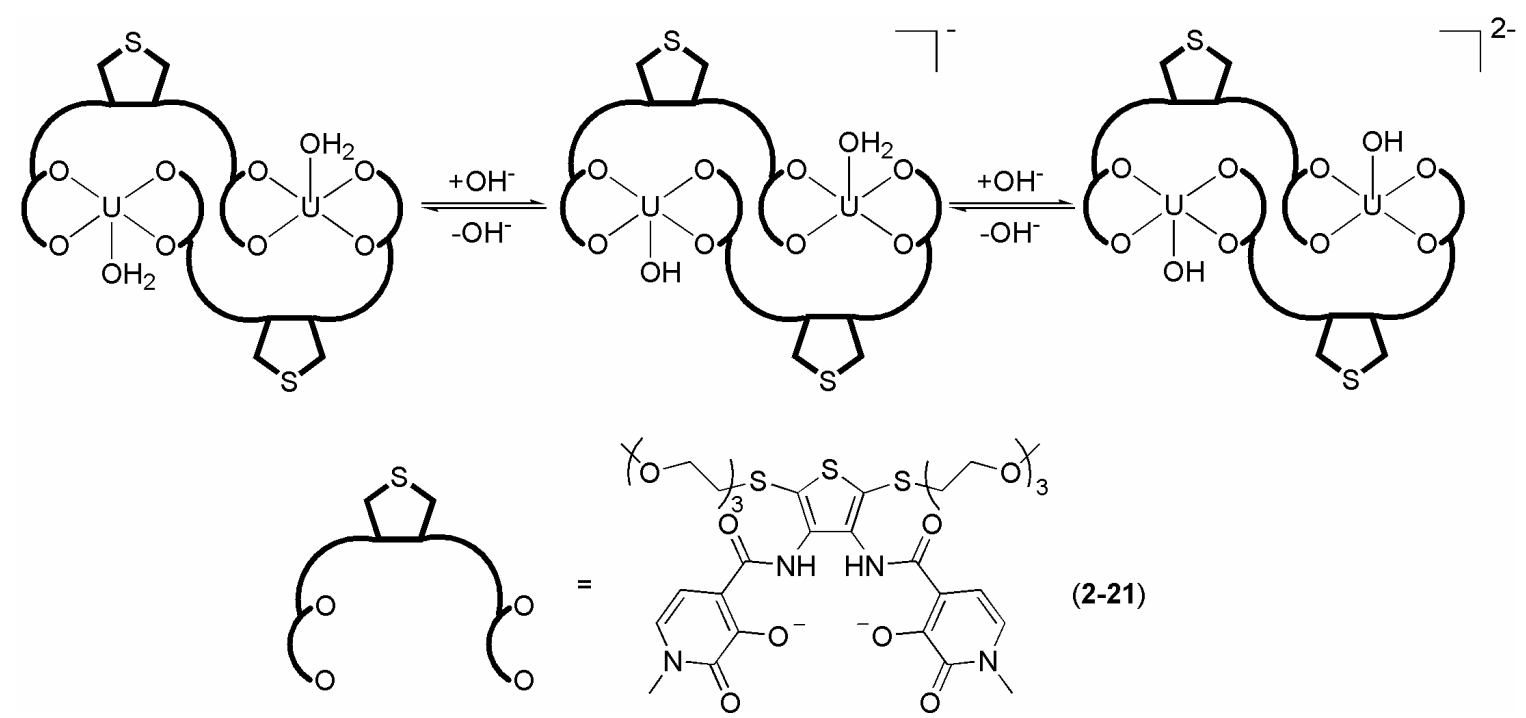

Figure 2-15. Proposed speciation behavior of the uranyl-(2-21) complex in solution. Uranyl oxo atoms are omitted for clarity.

The speciation of uranyl complexes with $\mathbf{2 - 1}$ in solution are necessarily very different from that with bis-Me-3,2-HOPO ligands, as Table 2-9 indicates. Because coordination of 2-1 requires only one deprotonation, 2-1 binds at very low $\mathrm{pH}$ to first form a $\mathrm{UO}_{2}$ (21)(solv $)_{\mathrm{x}}$ complex which is the major species until $\mathrm{pH} 5.5$, when the $\mathrm{UO}_{2}(\mathbf{2 - 1})_{2}(\mathrm{solv})$ complex observed in crystal structure analysis becomes the dominant species. ${ }^{1}$ This complex first undergoes the expected partial hydrolysis to form $\mathrm{UO}_{2}(\mathbf{2 - 1})(\mathrm{OH})$, then experiences one more hydrolysis below $\mathrm{pH} 10$. This last hydrolysis product could be refined either as a $\mathrm{UO}_{2}(\mathbf{2 - 1}) \mathrm{H}_{-2}$ or $\mathrm{UO}_{2}(\mathbf{2 - 1})_{2} \mathrm{H}_{-2}$ species, with near negligible changes in 
the other formation constants. The former corresponds to displacement of one ligand upon coordination of another hydroxide, while the latter corresponds to either deprotonation of a coordinated ligand or the introduction of hydroxide to the uranyl coordination plane without ligand displacement. Since Me-3,2-HOPO moieties does not have a particularly acidic proton after the phenolic proton that is removed in the initial metal chelation event, it is assumed that additional hydroxide coordination is occurring at high $\mathrm{pH}$. However, such introduction would crowd the uranyl coordination plane, and the low concentrations and 1:2 $\mathrm{UO}_{2}: \mathbf{2}-\mathbf{1}$ ratios used in the titrations lead us to believe that ligand displacement to form a $\mathrm{UO}_{2}(\mathbf{2 - 1})(\mathrm{OH})_{2}$ species at high $\mathrm{pH}$ is the more likely speciation in these titrations (Figure 2-16)
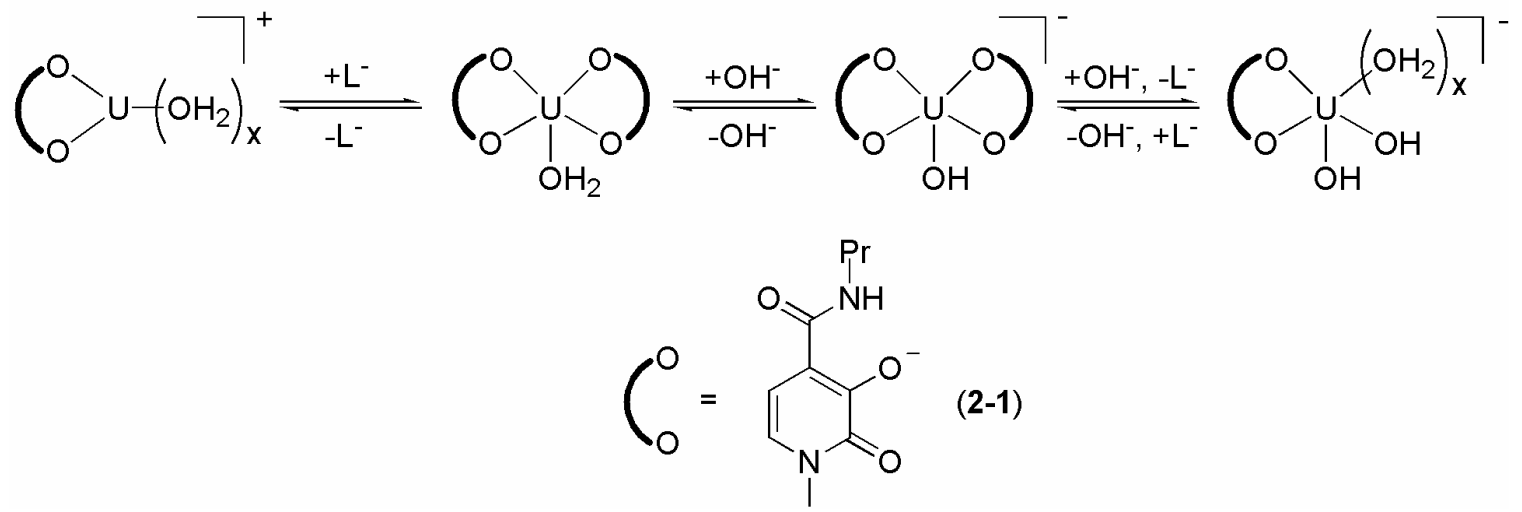

Figure 2-16. Proposed speciation behavior of $\mathrm{UO}_{2}(\mathbf{2 - 1})_{\mathrm{n}}$ in solution. Uranyl oxo atoms are omitted for clarity.

Because the uranyl formation constants in Table 2-9 are species dependent, with each ligand displaying different $\mathrm{pK}_{\mathrm{a}}$ values and complexation behavior, a species-independent method was needed to compare the overall uranyl affinity for these ligands to ultimately assess the effect of changing ligand geometry on uranyl affinity. A metric commonly used in the Raymond group for this purpose is that of $\mathrm{pM}$ (in this case $\mathrm{pUO}_{2}$ ), where $\mathrm{pUO}_{2}=-\log \left[\mathrm{UO}_{2}{ }^{2+}\right.$ free. " $\mathrm{UO}_{2}{ }^{2+}$ free" refers to solvated uranyl ion free of complexation by ligand or hydroxide. Just as with $\mathrm{pH}$, the higher the $\mathrm{pUO}_{2}$, the smaller the concentration 
of uncomplexed uranyl in solution and the greater the ligand affinity. $\mathrm{pUO}_{2}$ is calculated using standard conditions of $\left[\mathrm{UO}_{2}{ }^{2+}\right]=1 \mu \mathrm{M}$ and $[\mathrm{L}]=10 \mu \mathrm{M}(\mathrm{L}: \mathrm{M}=10)$, and thus the minimum $\mathrm{pUO}_{2}$ value is 6.0 , at which no metal complexation occurs. While typically reported at physiological $\mathrm{pH}$, once the protonation and uranyl formation constants for each ligand are known, the $\mathrm{pUO}_{2}$ can be calculated at any $\mathrm{pH} ; \mathrm{pUO}_{2}$ values at $\mathrm{pH} 2.5,7.4$ and 8.5 (low, physiological, and titration upper limit $\mathrm{pH}$ values) are listed for each Me3,2-HOPO ligand in Table 2-10. No matter the ligand, however, $\mathrm{pUO}_{2}$ is expected to rise upon increasing $\mathrm{pH}$ because of the increased concentrations of uranyl hydrolysis, which will lower the $\left[\mathrm{UO}_{2}{ }^{2+}\right.$ free $]$ independent of ligand identity. Thus, one must compare $\mathrm{pUO}_{2}$ values at different $\mathrm{pH}$ carefully with this effect in mind. Further discussion on $\mathrm{pUO}_{2}$ calculations and additional analysis is presented in the Appendix.

Table 2-10. Calculated $\mathrm{pUO}_{2}$ values for Me-3,2-HOPO ligands.

\begin{tabular}{|c|c|c|c|}
\hline \multirow{2}{*}{ Ligand } & \multicolumn{3}{|c|}{$\mathrm{pUO}_{2}{ }^{2}$} \\
\hline & pH 2.5 & pH 7.4 & pH 8.5 \\
\hline $2-1$ & $7.98(3)$ & $14.70(4)$ & $15.96(3)$ \\
\hline 2-17 & $7.0(1)$ & $14.63(8)$ & $15.75(8)$ \\
\hline $2-18$ & $6.6(1)$ & $14.24(7)$ & $15.34(6)$ \\
\hline 2-19 & $8.0(1)$ & $15.39(7)$ & 16.43(9) \\
\hline $2-20$ & 7.1(1) & 14.44(6) & $15.16(4)$ \\
\hline $2-21$ & $6.01(1)$ & $13.39(3)$ & $14.48(2)$ \\
\hline $2-22$ & $7.62(4)$ & $14.97(9)$ & 16.1(1) \\
\hline $2-23$ & $6.55(6)$ & $14.00(3)$ & $14.87(5)$ \\
\hline
\end{tabular}

The $\mathrm{pUO}_{2}$ values in Table 2-10 reveal that the uranyl affinity of bis-HOPO ligands varies by a measurable amount with changes in their ligand geometry. However, the affinities do not follow gradual trends of the sort seen in ligand $\mathrm{pK}_{\mathrm{a}}$ values in Table 2-8, where linker length and degree of conjugation affected ligand proton affinity in an incremental fashion. Quite the contrary, small changes in linker length and geometry cause large changes in $\mathrm{pUO}_{2}$, with no noticeable correlation with the physical metrics measured from their crystal structures. Common to all ligands, however, is a dramatic 
rise in $\mathrm{pUO}_{2}(c a .7$ log units) between $\mathrm{pH} 2.5$ and $\mathrm{pH}$ 7.4. It is not possible to credit this increase entirely to an rise in ligand affinity, because uranyl hydrolysis becomes more important at neutral $\mathrm{pH}$ compared to very low $\mathrm{pH}$. However, Me-3,2-HOPO ligands require deprotonation for metal chelation, and so it is reasonable to suspect that the majority of this rise in $\mathrm{pUO}_{2}$ comes from a change in ligand affinity upon complete deprotonation at higher $\mathrm{pH}$.

Focusing on the PEG-nLi-Me-3,2-HOPO ligands 2-17 through 2-20, there is no affinity trend upon incremental increase in linker length at any $\mathrm{pH}$. The ligand $\mathrm{pK}_{\mathrm{a}}$ has been shown to decrease with shortening of the linker, so uranyl affinity independent of ligand geometry would suggest that $\mathbf{2 - 1 7}$ should bind most strongly to the uranyl cation, but this is not the case; 2-17 displays the second highest affinity, with the 4Li- ligand 219 displaying the strongest uranyl affinity both at low and high $\mathrm{pH}$. 2-19 has a higher $\mathrm{pK}_{\mathrm{a}}$ than 2-17 and 2-18, so the observed uranyl affinity must be due to geometry effects. This high affinity is consistent with the relatively relaxed geometry observed in the $\mathrm{UO}_{2}$ (24)(DMSO) crystal structure as well as with the superior ability for 2-4 to chelate actinides in vivo. ${ }^{1,21}$

Of the rigidly linked ligands $\mathbf{2 - 2 1}$ through $\mathbf{2 - 2 3}, \mathbf{2 - 2 1}$ has the lowest $\mathrm{pK}_{\mathrm{a}}$ of all ligands measured here, and yet displays the poorest uranyl affinity at all $\mathrm{pH}$. While at low $\mathrm{pH}$ this may be due to the need to form dimeric uranyl complexes to achieve stability, the low $\mathrm{pUO}_{2}$ at higher $\mathrm{pH}$ must be a geometric effect. The "ligand bite" angle in the $\left[\mathrm{UO}_{2}(\mathbf{2}-\right.$ 37)(DMSO) $]_{2}$ structure (\#1) is $14^{\circ}$ wider than that in the $\mathrm{UO}_{2}(\mathbf{2}-\mathbf{6})(\mathrm{DMF})$ structure, indicating that the chelating moieties are not as constrained about the uranyl coordination plane in the dimeric compared to monomeric complexes. Nonetheless, this coordinative 
relaxation cannot overcome the fundamental uranyl chelating inferiority of 2-21 compared to other bis-Me-3,2-HOPO moieties. Whether this would be the case for a less sterically constrained ligand linked through a furan or pyrone that could bind in a mononuclear fashion remains to be explored.

The $o$-phenylene linked ligand $\mathbf{2 - 2 2}$ shows the most favorable $\mathrm{pUO}_{2}$ of the rigidlylinked ligands, nearing that of $\mathbf{2 - 1 9}$. As minor $\mathrm{pK}_{\mathrm{a}}$ changes are apparently not a large factor in uranyl affinity, this affinity reveals the favorable geometric agreement between the ligand and the uranyl coordination preferences. The $\mathrm{pUO}_{2}$ of $\mathbf{2 - 2 2}$ is also higher than that of 2-17 (both $n=2$ ), indicating that ligand rigidity and preorganization are favorable attributes in uranyl-specific ligands. In contrast to $\mathbf{2 - 2 2}$, the $\mathrm{pUO}_{2}$ of $\mathbf{2 - 2 3}$ is significantly lower, revealing that despite the favorable conformational parameters $\theta, \varphi$, and $\Sigma \sigma_{\mathrm{n}}$ seen in the $\mathrm{UO}_{2}(\mathbf{2 - 1 5})(\mathrm{DMF})$ structure, the $\alpha, \alpha^{\prime}-m$-xylene linker does not provide a very good geometric agreement compared to ligands containing shorter or differently-constrained linkers.

Of particular interest is the high affinity of 2-1 for the uranyl cation, which rivals that of 2-19 at $\mathrm{pH} 2.5$ and $\mathbf{2 - 1 7}$ at high $\mathrm{pH}$. One must consider that at very low and very high $\mathrm{pH}$ values the uranyl-(2-1) complexes are $\mathrm{UO}_{2}(\mathbf{2}-\mathbf{1})$ complexes and are not coordinatively saturated. In addition, the coordination of one equivalent of 2-1 to uranyl at low $\mathrm{pH}$ requires only a single deprotonation event, while for bis-Me-3,2-HOPO moieties it requires two, making it easier to form a chelate at lower $\mathrm{pH}$. This, combined with the ability to lose one chelating moiety at high $\mathrm{pH}$ to produce multiple partially hydrolyzed species maintains a relatively low $\left[\mathrm{UO}_{2}{ }^{2+}\right.$ free $]$ in the $\mathrm{pH}$ range measured, and thus the high $\mathrm{pUO}_{2}$. However, this incomplete coordination allows the chelation of other binding 
moieties to the uranyl center, and the effect of these other ligands on the coordination of 2-1 to the uranyl center cannot be determined from the measurements described above.

\subsubsection{Substituted $m$-Xylene-Me-3,2-HOPO Ligands: Synthesis and Structure}

As discussed in Chapter 1, a few research groups including our own have attempted to develop actinyl ligands that incorporate a Lewis acidic moiety that is properly situated to encourage interaction with the very mildly Lewis basic uranyl oxo atoms. Previous Raymond group attempts have involved ligand designs that place protonated amines or amide protons in close vicinity to the uranyl oxo moieties, ${ }^{22,23}$ while Arnold and coworkers have developed fold-over macrocycles that place Lewis acidic transition metals in close proximity to one uranyl oxo moiety, thus breaking the chemical symmetry of the uranyl oxo atoms. ${ }^{24,25}$ Such shape-selective ligands could be used both to access new uranyl chemistry as well as pave the way towards highly selective actinyl ligands.

The design principles utilized for bis-Me-3,2-HOPO ligand development described above have focused solely on the equatorial binding modes of the uranyl cation, the considerations for which are chemically and geometrically orthogonal to developing a Lewis-acid/base interaction with the uranyl oxo atoms. Focusing on geometric considerations, interaction with the oxo atoms requires placement of a Lewis acid out of the uranyl coordination plane, which is almost mutually exclusive with the efforts to design preorganized, planar ligands for equatorial coordination. Of the linker geometries explored in the bis-Me-3,2-HOPO ligands above, only the $\alpha, \alpha^{\prime}-m$-xylene linker geometry employed in 2-15 couples favorable equatorial coordination behavior with a ligand geometry that presents a significant deviation from the uranyl coordination plane; the outof-plane bend of the phenyl ring in the $m$-xylene linker provides a place at which possible 
Lewis-acidic moieties could be tethered. Significantly, the $m$-xylene linker is also synthetically amenable to functionalization at the 2-position between the linker methylene groups, where an appropriately designed Lewis-acid functional group could be incorporated into the ligand (Figure 2-17).<smiles>[R]c1c(CNC(=O)c2ccn(C)c(=O)c2O)cccc1CNC(=O)c1ccn(C)c(=O)c1O</smiles>

Figure 2-17. 2-position functionalization in $m$-xy-Me-3,2-HOPO ligands.

In the $\mathrm{UO}_{2}(\mathbf{2 - 1 5})(\mathrm{DMF})$ crystal structure the backbone carbon between the benzylic substituents is $6 \AA$ away from the closest intramolecular uranyl oxo moiety, so a reasonably large substituent is needed in order to interact closely with the uranyl oxo atom if linked to the ligand at the 2-position. However, before designing a linker of appropriate size, it is necessary to investigate how substitution at the 2-position on the $m$ xylene backbone will affect the resultant uranyl complex geometry. It was suspected that substitution at this position could to some degree disrupt the hydrogen bonding between the amide protons and the HOPO phenolate oxygens that are responsible for increasing metal affinity in these ligands. Thus, to probe the effects of simple substitution at the $m$ xylene 2-position, the two substituted $m$-xy-Me-3,2-HOPO ligands 2-OH-5-Me-m-xyMe-3,2-HOPO (2-39) and 2-OMe-5Me-m-xy-Me-3,2-HOPO (2-40) were synthesized according to Scheme 2-5. The hydroxyl and methoxy substituents at the 2-position of the aromatic linker were chosen for their synthetic accessibility, and also because they simulate single-atom attachment at this position; attachment to the aryl ring using carbon or nitrogen linkers could introduce the added steric bulk of hydrogen atoms into this potentially crowded position. The hydroxyl- and methoxy-bearing linkers were chosen to 
probe the difference in structural influence between hydrogen bond accepting/donating (hydroxyl) and purely hydrogen-bond accepting (methoxy) functionalities.

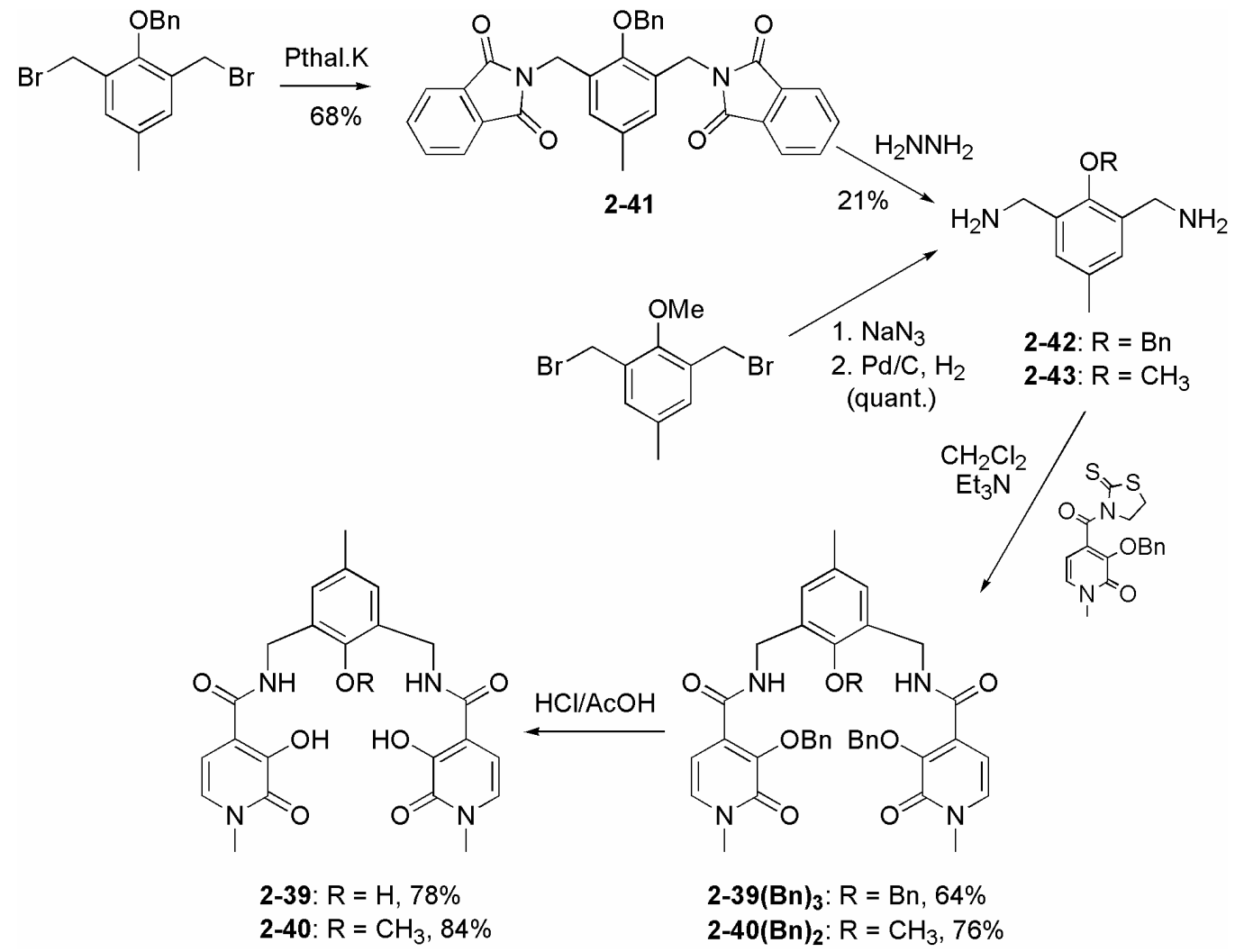

Scheme 2-5. Synthesis of substituted $m$-xy-Me-3,2-HOPO ligands 2-39 and 2-40.

The 1:1 uranyl complexes with $\mathbf{2 - 3 9}$ and $\mathbf{2 - 4 0}$ were synthesized in methods similar to those for the other uranyl-bis-Me-3,2-HOPO complexes, yielding the expected red complexes that are rather insoluble in most organic solvents. Crystals suitable for X-ray diffraction were grown out of DMSO solutions of the complex using standard vapor diffusion techniques. These crystal structures are shown in Figure 2-18 and their crystallographic parameters are listed in Table 2-11. As with the other bis-Me-3,2-HOPO ligands, 2-39 and 2-40 bind the uranyl cation at four points in the equatorial coordination plane, with the fifth coordination site occupied by solvent. In the $\mathrm{UO}_{2}(\mathbf{2 - 3 9})$ structure the fifth coordination site is occupied by the amide oxygen of another uranyl complex, which again is a solid state phenomenon also seen in the $\mathrm{UO}_{2}(\mathbf{2 - 2})$ and $\mathrm{UO}_{2}(\mathbf{2 - 1 4})$ structures. 


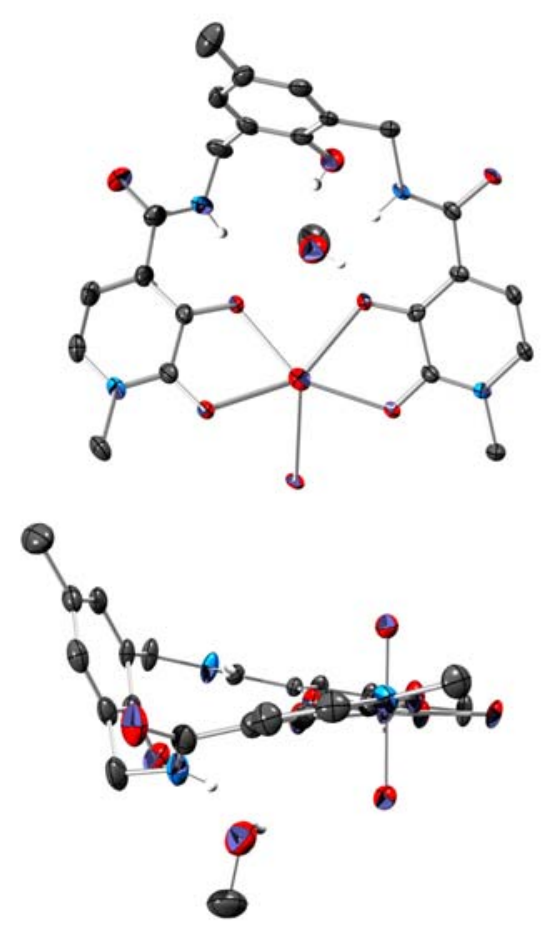

(a)

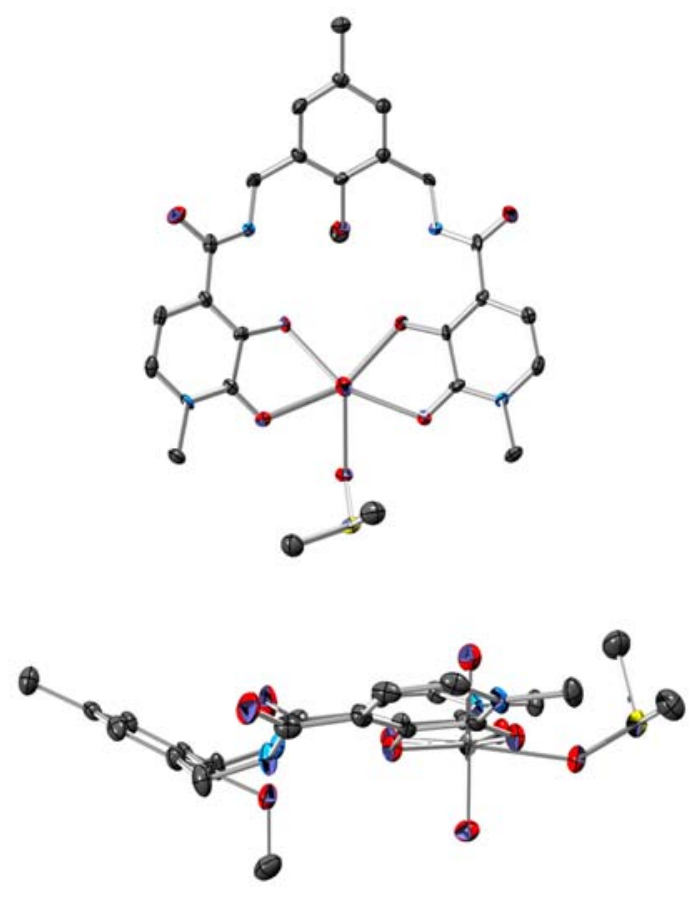

(b)

Figure 2-18. Top and side views of $X$-ray diffraction structures of uranyl complexes with 2-substituted $m$-xy-Me-3,2-HOPO ligands: (a) 2-OH-5-Me- $m$-xy-Me-3,2-HOPO, 2-39; (b) 2-OMe-5-Me- $m$-xy-Me-3,2-HOPO, 2-40. Thermal ellipsoids are drawn at the $50 \%$ probability level. Hydrogen atoms are omitted for clarity except for the hydrogenbonding protons in $\mathrm{UO}_{2}(\mathbf{2 - 3 9})$. A water molecule was omitted from the structure of $\mathrm{UO}_{2}$ (2-40)(DMSO) because it was not involved in the intraligand hydrogen bonding network. Oxygen atoms are red, carbons gray, nitrogens blue, sulfur yellow, and uranium is silver.

One obvious characteristic of complexes in Figure 2-18 is that the orientation of the backbone aryl ring is markedly different than that seen in the unsubstituted $\mathrm{UO}_{2}(2-$ 15)(DMF) complex. To better compare the structures in Figure 2-18 with the $\mathrm{UO}_{2}(2-$ 15)(DMF) structure, equatorial U-O bond lengths and conformational parameters $\theta, \varphi$, and $\Sigma \sigma_{\mathrm{n}}$ for $m$-xy-bis-Me-3,2-HOPO complexes are listed in Table 2-12. Also listed is a fourth conformational parameter Tors., which measures the $\mathrm{C}_{\text {aryl }}-\mathrm{C}_{\text {benzylic }}$ bond torsion in the ligand backbone (Figure 2-19). A linker ring co-planar with the rest of the ligand would exhibit Tors. $=180^{\circ}$, while a perdendicular ring would exhibit Tors. $=90^{\circ}$. 
Table 2-11. Crystallographic parameters for $\mathrm{UO}_{2}(\mathbf{2 - 3 9})$ and $\mathrm{UO}_{2}(\mathbf{2 - 4 0})(\mathrm{DMSO})$.

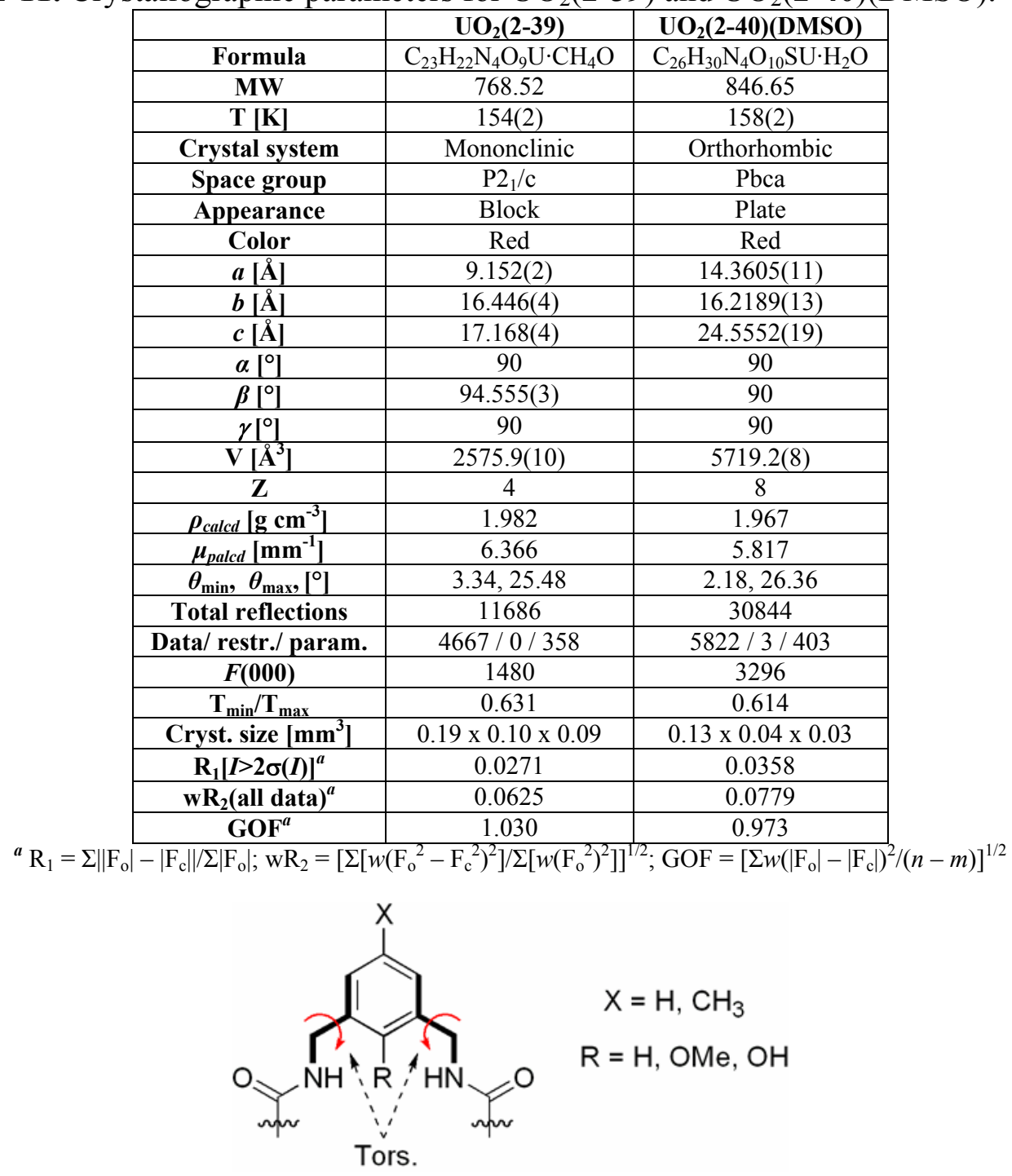

Figure 2-19. The conformational metric Tors.

Table 2-12. Equatorial U-O bond lengths and conformational parameters in $\mathrm{UO}_{2}(m-\mathrm{xy}-$ Me-3,2-HOPO) complexes $\mathrm{UO}_{2}(\mathbf{2 - 1 5})(\mathrm{DMF}), \mathrm{UO}_{2}$ (2-39), and $\mathrm{UO}_{2}$ (2-40)(DMSO).

\begin{tabular}{|c|c|c|c|c|c|c|}
\hline Complex & $\boldsymbol{\theta},\left[^{\circ}\right]$ & $\varphi,\left[^{\circ}\right]$ & $\Sigma \sigma_{n},\left[^{\circ}\right]$ & Tors., $\left[^{\circ}\right]$ & $\begin{array}{c}\text { U-O(amide), } \\
{[\AA \AA]}\end{array}$ & $\begin{array}{c}\text { U-O(phenol), } \\
{[\AA \AA]}\end{array}$ \\
\hline $\mathrm{UO}_{2}(\mathbf{2 - 1 5})(\mathrm{DMF})$ & $5.6(3)$ & $\begin{array}{l}1.4(2), \\
6.0(2) \\
\end{array}$ & $360.3(2)$ & $\begin{array}{c}93.7(6), \\
97.7(7) \\
\end{array}$ & $\begin{array}{l}2.427(3) \\
2.429(3) \\
\end{array}$ & $\begin{array}{l}2.357(3) \\
2.353(3) \\
\end{array}$ \\
\hline $\mathrm{UO}_{2}(\mathbf{2 - 3 9})$ & $19.7(2)$ & $\begin{array}{l}2.9(2), \\
16.9(2)\end{array}$ & $361.6(2)$ & $\begin{array}{l}88.5(6), \\
122.8(5)\end{array}$ & $\begin{array}{l}2.418(3), \\
2.458(3)\end{array}$ & $\begin{array}{l}2.392(3), \\
2.438(3)\end{array}$ \\
\hline $\mathrm{UO}_{2}(\mathbf{2}-\mathbf{4 0})(\mathrm{DMSO})$ & $33.5(1)$ & $\begin{array}{l}14.2(1), \\
20.0(1)\end{array}$ & $362.0(2)$ & $\begin{array}{l}130.6(5), \\
131.6(6)\end{array}$ & $\begin{array}{l}2.443(4), \\
2.462(4)\end{array}$ & $\begin{array}{l}2.361(4), \\
2.362(4)\end{array}$ \\
\hline
\end{tabular}

The equatorial $\mathrm{U}-\mathrm{O}$ bond lengths in the $\mathrm{UO}_{2}(\mathbf{2 - 3 9})$ and $\mathrm{UO}_{2}(\mathbf{2 - 4 0})(\mathrm{DMSO})$ complexes do not differ significantly from those of the parent $\mathrm{UO}_{2}(\mathbf{2}-\mathbf{1 5})(\mathrm{DMF})$ structure, but the conformational parameters do so drastically. With both 2-39 and 2-40 the $\theta, \varphi$, 
and $\Sigma \sigma_{\mathrm{n}}$ values are significantly higher than with $\mathbf{2 - 1 5}$. These values reflect the more ruffled ligand conformation in the uranyl complexes with the substituted $m$-xylene ligands that results in a slightly more crowded coordination environment about the uranyl center. The Tors. values, however, are the most illuminating values in Table 2-12: while the unsubstituted ligand 2-15 bends the aryl ring nearly perpendicular to the uranyl coordination and HOPO ring planes, the substituted ligands bend the aryl ring as much as $40^{\circ}$ less. Ligands incorporating an $\alpha, \alpha^{\prime}-m$-xylene linker have only rotation about the benzylic methylene group available for geometric relaxation, which is what the Tors. parameter measures. However, the degree to which the aryl ring bends out of the plane of the HOPO moieties is directly responsible for how close the HOPO moieties can approach, resulting in the relatively strained geometries seen in the $\mathrm{UO}_{2}(\mathbf{2 - 3 9})$ and $\mathrm{UO}_{2}(\mathbf{2}-\mathbf{4 0})(\mathrm{DMSO})$ structures.

The larger Tors. values in $\mathrm{UO}_{2}(\mathbf{2 - 3 9})$ and $\mathrm{UO}_{2}(\mathbf{2 - 4 0})(\mathrm{DMSO})$ may explain the other conformational parameters, but they are in turn caused by the hydrogen bond interactions between the hydroxyl or methoxy substituents in the linker aryl groups in 2-39 and 2-40 and the $\mathrm{HOPO}$ amide protons. The $\mathrm{UO}_{2}(\mathbf{2}-\mathbf{4 0})(\mathrm{DMSO})$ structure exhibits a very symmetric hydrogen-bonding interaction in which the methoxy oxygen lies $2.82 \AA$ and $2.86 \AA$ from the linking amide nitrogens, which themselves are $2.78 \AA$ and $2.76 \AA$ away from the HOPO phenolate oxygens respectively. Thus, it is obviously a strong intramolecular hydrogen bonding network that is responsible for the distorted molecular structure of the resultant uranyl complex. In contrast, the hydroxyl substituent in the $\mathrm{UO}_{2}(\mathbf{2 - 3 9})$ structure is both a hydrogen bond donor and acceptor; the crystal structure contains a methanol inclusion in close approach to the hydrogen bonding pocket. The 
methanol oxygen accepts a hydrogen bond from the backbone hydroxyl oxygen and one amide nitrogen (2.69 $\AA$ and $3.38 \AA$ respectively). The amide nitrogens maintain hydrogen bonding distances from the HOPO phenolates $(2.78 \AA$ and $2.83 \AA$ ), and one amide nitrogen is $2.81 \AA$ from the backbone aryl hydroxyl oxygen (Figure 2-18). Thus, the observed distortion in the $\mathrm{UO}_{2}(\mathbf{2 - 3 9})$ structure is also caused by intermolecular hydrogen bonding interactions caused by the inclusion of the hydroxyl moiety on the backbone. While it is obvious that the methanol inclusion plays some role in the observed effects in the solid state structure, the methanol-independent intramolecular hydrogen bonding indicates that the hydroxyl substituent is the cause of the observed coordinative distortion from planarity.

From the structural investigations above we conclude that substitution at the 2position of an $\alpha, \alpha^{\prime}-m$-xylene linker between HOPO moieties cannot be performed using either hydrogen bond accepting or donating attachments because these unavoidably interact with the amide protons of the HOPO moieties, which in turn significantly disrupt the coordination geometry about the uranyl cation. The thermodynamic effect of this substitution and subsequent geometry change could not be examined using solution thermodynamics because the aqueous solubilities of ligands 2-39 and 2-40 were not sufficient for such measurements. The structures in Figure 2-18 do, however, emphasize the importance of performing detailed structural analysis of ligand variations, as seemingly small structural changes can cause significant changes in the resultant metal complexes. 


\subsection{Conclusions and Future Directions}

Eleven bis-Me-3,2-HOPO ligands were synthesized that incorporate aromatic rings of various geometry and connectivity into their linkers. Their uranyl complexes were isolated and crystallized and the effect of ligand geometry on the resultant complex geometry were assessed. Significantly, metallation reactions with four of the new bis-Me3,2-HOPO ligands failed to produce monomeric results, effectively defining the limits of geometric flexibility in the uranyl-bis-Me-3,2-HOPO interactions. Ligands with 3,4thiophene-, $o$-phenylene-, and $\alpha, \alpha^{\prime}-m$-xylene-linkers displayed the most favorable agreement between the ligand geometry and the uranyl coordination preferences as compared to the $\mathrm{UO}_{2}(\mathrm{Pr}-\mathrm{Me}-3,2-\mathrm{HOPO})_{2}(\mathrm{DMF})$ structure and were selected for solution thermodynamic measurements.

Seven PEG-substituted bis-Me-3,2-HOPO ligands were synthesized, including four analogs to the $2 \mathrm{Li}$ - through $5 \mathrm{Li}-\mathrm{Me}-3,2-\mathrm{HOPO}$ ligands previously investigated. The steric effects of the PEG-moiety substitutions on the resultant geometry of the uranyl complexes with these ligands were investigated by synthesizing alky-substituted ligands 2-37 and 2-38 and examining their crystal structures. While the substitution on the $o$ phenylene backbone was seen to have only minor effects on the complex, substitution onto the 3,4-thiophene backbone was shown to completely change the coordination modes of the ligand.

Solution thermodynamic measurements demonstrated that linker length and geometry have a measurable effect on the proton affinity of bis-Me-3,2-HOPO ligands, but that uranyl affinity of these ligands does not correlate with the proton affinity trends. Significantly, the PEG-4Li-Me-3,2-HOPO ligand 2-19 was shown to bind most strongly 
to the uranyl cation, which supports earlier results on in vivo chelation and uranyl complex geometry. The $o$-phenylene linker in 2-22 imparted the second highest uranyl affinity, rivaling that of $\mathbf{2 - 1 9}$, and significantly higher than that of 2Li-linked 2-17, indicating the benefit of preorganization and rigidity in uranyl affinity.

Two $m$-xylene-bis-Me-3,2-HOPO ligands substituted at the 2-position of the aryl linker were synthesized to explore the effect of introducing an out-of-plane functionality for future stereognostic actinyl recognition applications. It was assessed from crystal structure analysis that utilizing an oxygen linkage to the aryl ring significantly disrupts the hydrogen-bonding network independent of whether the oxygen bearing moiety displays both hydrogen bond accepting and/or donating capabilities. The effect of this geometric disruption on the solution thermodynamics of these ligands would be of great interest for future study, as backbone substitution remains one of the few ways in which a moiety that interacts with the uranyl oxo atoms can be introduced to a ligand designed to bind to the equatorial uranyl coordination plane.

One aspect the titrations performed and the $\mathrm{pUO}_{2}$ values reported do not address is the matter of selectivity. In most applications associated with biological removal of actinides, high binding constants are necessary, but high selectivity is also desirable if an administered drug is to be effective. Further development of the bis-Me-3,2-HOPO ligands described above will require titration measurements against biologically relevant ions such as $\mathrm{Ca}^{2+}$ and $\mathrm{Zn}^{2+}$, as well as other actinides; it may be that while the uranyl affinity of the ligands explored here may be in many ways comparable, their selectivities for actinyl cations over other non-f-element species may be significantly different. 


\subsection{Experimental}

General. Unless otherwise noted, all chemicals and solvents were purchased from commercial sources and used as received or synthesized using literature procedures. Solvents indicated as "dry" were made so by passing them through anhydrous alumina columns or by storage over molecular sieves. The syntheses of Me-3,2-HOPO-(Bn)$\mathrm{COOH}$ and its 2-mercaptothiazoline activated analog are described in an earlier Raymond group publications. ${ }^{12}$ All reactions brought to reflux were done so with an efficient condenser attached to the reaction flask. NMR spectra were collected using Bruker AMX-400 and AM-400 spectrometers $\left({ }^{1} \mathrm{H} 400 \mathrm{MHz},{ }^{13} \mathrm{C} 100 \mathrm{MHz}\right)$ in $\mathrm{CDCl}_{3}$ unless

otherwise noted. ${ }^{1} \mathrm{H}$ ( or ${ }^{13} \mathrm{C}$ ) NMR resonances are reported in ppm relative to the solvent resonances, taken as $7.26(77.23)$ for $\mathrm{CDCl}_{3}$ and 2.50 (39.51) for DMSO- $d_{6}$. Mass spectrometry and elemental analyses were performed at the Microanalytical Facility, College of Chemistry, University of California, Berkeley. Melting points are uncorrected. Elemental analyses are reported in a "calculated (found)" format. Reactions were monitored by TLC on 60 mesh $\mathrm{F}_{254}$ silica gel from EMD Chemicals, Inc. Silica gel chromatography was performed on EcoChrom Silica (32-63 D $60 \AA$ ) and reported $\mathrm{R}_{\mathrm{f}}$ are those corresponding to the solvent used for chromatographic elution unless otherwise noted. Organic solutions were dried using anhydrous sodium sulfate and solvents were removed on a rotary evaporator or under high vacuum on a Schlenk line. Yields indicate the amount of isolated compound and reactions are un-optimized.

\subsubsection{Synthesis of Backbone Diamines}

1,8-Diaminofluorene. A solution of 1,8-diaminofluoreneone ${ }^{14}(122 \mathrm{mg}, 0.580$ $\mathrm{mmol}), 80 \%$ hydrazine monohydrate $(0.25 \mathrm{~mL}, 4.01 \mathrm{mmol})$, and $\mathrm{KOH}(0.215 \mathrm{~g}, 3.83$ 
mmol) in $3 \mathrm{~mL}$ of diethylene gloycol was heated to $120{ }^{\circ} \mathrm{C}$ with a reflux condenser attached. The dark orange solution was stirred for 2 hours. The condenser was removed and the solution was heated to $200{ }^{\circ} \mathrm{C}$ to boil off excess hydrazine and water. The condenser was reattached and the solution was stirred for 5 hours at $200{ }^{\circ} \mathrm{C}$ and then overnight at $100{ }^{\circ} \mathrm{C}$. The solution was cooled to room temperature, diluted with $5 \mathrm{~mL}$ of water, neutralized with $6 \mathrm{M} \mathrm{HCl}$, and diluted with water to a final volume of $25 \mathrm{~mL}$. This solution was extracted with $\mathrm{CH}_{2} \mathrm{Cl}_{2}(3 \times 25 \mathrm{~mL})$, the combined organics were dried and the solvent was removed. The residual yellow/orange oil was dissolved in $2 \mathrm{~mL}$ of $\mathrm{CH}_{2} \mathrm{Cl}_{2}$ and eluted on a silica column with EtOAc. Fractions with $\mathrm{R}_{\mathrm{f}}=0.29$ were collected, dried and the solvent was removed to yield $75 \mathrm{mg}(66 \%)$ of a light orange solid. $\mathrm{C}_{13} \mathrm{H}_{12} \mathrm{~N}_{2}$ : C: 79.56(79.23); H: 6.16(6.26); N: 14.27(13.98). ${ }^{1} \mathrm{H}$ NMR: $\delta 3.45$ (s, $\left.\mathrm{CH}_{2}, 2 \mathrm{H}\right), \delta 3.75\left(\mathrm{br}, \mathrm{s}, \mathrm{NH}_{2}, 4 \mathrm{H}\right), \delta 6.68(\mathrm{~d}$, arom $H, \mathrm{~J}=7.2 \mathrm{~Hz}, 2 \mathrm{H}), \delta$ 7.21-7.28 (m, arom $H, 4 \mathrm{H}) .{ }^{13} \mathrm{C}$ NMR: $\delta 31.17,111.45,113.72,127.11,128.43,142.76,143.33$. MS $(\mathrm{FAB}+): \mathrm{m} / \mathrm{z} 196(\mathrm{MH}+) . \mathrm{MP}: 152-154^{\circ} \mathrm{C}$.

PEG-2Li-(NHBoc) $)_{2}$, 2-24(Boc) $)_{2}$. A mixture of Boc-(Boc)-L-DAP-OH (0.900 g, 2.96 mmol), NHS (0.340 g, $2.95 \mathrm{mmol})$, and a catalytic amount of DMAP in $50 \mathrm{~mL}$ of $\mathrm{CH}_{2} \mathrm{Cl}_{2}$ was stirred under argon while being cooled in an ice bath. DCC $(0.611 \mathrm{~g}, 2.95 \mathrm{mmol})$ was added and the resultant suspension was stirred in the ice bath for four hours. A solution of 3,6,9-trioxa-1-aminodecane ${ }^{26}(0.486 \mathrm{~g}, 2.98 \mathrm{mmol})$ in $10 \mathrm{~mL}$ of $\mathrm{CH}_{2} \mathrm{Cl}_{2}$ was added and the solution was allowed to warm to room temperature overnight. The solution was cooled in an ice bath and filtered. The filtrate was washed with $1 \mathrm{M} \mathrm{HCl}(2 \times 25 \mathrm{~mL}), 1$ $\mathrm{M} \mathrm{NaOH}$ in $20 \%$ sat. brine $(2 \times 25 \mathrm{~mL})$, sat. brine, dried with $\mathrm{Na}_{2} \mathrm{SO}_{4}$ and the solvent was removed under vacuum. The residue was dissolved in $20 \mathrm{~mL}$ EtOAc and eluted on a 
silica column with the same, collecting fractions with $\mathrm{R}_{\mathrm{f}}=0.11$, which yielded $0.878 \mathrm{~g}$ of a colorless oil after drying and solvent removal, which solidifies upon standing (66\%). ${ }^{1} \mathrm{H}$ NMR: $\delta 1.35$ (s, $\left.\mathrm{CH}_{3}, 18 \mathrm{H}\right), \delta 3.29\left(\mathrm{~s}, \mathrm{CH}_{3}, 3 \mathrm{H}\right), \delta 3.34-3.37\left(\mathrm{~m}, \mathrm{CH}_{2}, 4 \mathrm{H}\right), \delta 3.45-3.48$ (m, $\mathrm{CH}_{2}, 4 \mathrm{H}$ ), $\delta 3.54-3.57\left(\mathrm{~m}, \mathrm{CH}_{2}, 6 \mathrm{H}\right), \delta 4.13$ (quartet, $\mathrm{CH}, \mathrm{J}=4.4 \mathrm{~Hz}, 1 \mathrm{H}$ ), $\delta 5.39$ (s, br, $\mathrm{N} H, 1 \mathrm{H}), \delta 5.86(\mathrm{~d}, \mathrm{~N} H, \mathrm{~J}=6.0 \mathrm{~Hz}, 1 \mathrm{H}), \delta 6.97(\mathrm{~s}, \mathrm{br}, \mathrm{N} H, 1 \mathrm{H}) .{ }^{13} \mathrm{C}$ NMR: $\delta 28.31$, $28.34,39.26,42.54,55.52,58.96,69.58,70.27,70.41,70.49,71.88,79.57,79.93,156.00$, 156.94, 170.65. $\mathrm{C}_{20} \mathrm{H}_{39} \mathrm{~N}_{3} \mathrm{O}_{8}:$ C: 53.44 (53.46); H: 8.74 (8.74); N: 9.35 (9.36). MS (FAB+): m/z 450.28 (MH+), $472.26(\mathrm{MNa}+)$.

PEG-3Li-(NHBoc) $)_{2}$, 2-25(Boc) $)_{2}$. This compound was synthesized in an analogous manner to that for $\mathbf{2 - 2 4 ( B o c )})_{2}$, using Boc-(Boc)-L/D-DAB-OH as a starting material. Colorless oil that solidifies upon standing, $68 \%$. Eluent: EtOAc, $\mathrm{R}_{\mathrm{f}}=0.10 . \mathrm{C}_{21} \mathrm{H}_{41} \mathrm{~N}_{3} \mathrm{O}_{8}$ : C: 54.41 (54.66); H: 8.91 (9.16); N: 9.06 (9.00). ${ }^{1} \mathrm{H}$ NMR: $\delta 1.42$ (s, $\left.\mathrm{CH}_{3}, 18 \mathrm{H}\right), \delta 1.71$ $1.76\left(\mathrm{~m}, \mathrm{CH}_{2}, 1 \mathrm{H}\right), \delta 1.86-1.91\left(\mathrm{~m}, \mathrm{CH}_{2}, 1 \mathrm{H}\right), \delta 3.00-3.02\left(\mathrm{~m}, \mathrm{CH}_{2}, 1 \mathrm{H}\right), \delta 3.38\left(\mathrm{~s}, \mathrm{CH}_{3}+\right.$ $\left.\mathrm{CH}_{2}, 4 \mathrm{H}\right), \delta 3.42-3.45\left(\mathrm{~m}, \mathrm{CH}_{2}, 2 \mathrm{H}\right), \delta 3.56\left(\mathrm{t}, \mathrm{CH}_{2}, \mathrm{~J}=4.0 \mathrm{~Hz}, 4 \mathrm{H}\right), \delta 3.62-3.66(\mathrm{~m}$, $\mathrm{CH}_{2}, 10 \mathrm{H}$ ), $\delta 4.14$ (quartet, $\left.\mathrm{CH}, \mathrm{J}=6.8 \mathrm{~Hz}, 1 \mathrm{H}\right), \delta 5.19$ (s, br, $\left.\mathrm{NH}, 1 \mathrm{H}\right), \delta 5.47$ (s, br, $\mathrm{NH}$, $1 \mathrm{H}), \delta 6.99$ (s, NH, 1H). ${ }^{13} \mathrm{C}$ NMR: $\delta 28.52,28.62,34.46,37.01,39.55,52.01,59.18$, $69.70,70.40,70.60,70.71,72.09,79.50,79.98,155.86,156.58,171.86 . \mathrm{MS}(\mathrm{FAB}+): \mathrm{m} / \mathrm{z}$ $464.5(\mathrm{MH}+)$.

PEG-4Li-(NHBoc) 2, 2-26(Boc) 2. This compound was synthesized in an analogous manner to that for 2-24(Boc) $)_{2}$, using Boc-(Boc)-L-Orn-OH as a starting material. Colorless oil, 65\%. Eluent: EtOAc, $\mathrm{R}_{\mathrm{f}}=0.12 . \mathrm{C}_{22} \mathrm{H}_{43} \mathrm{~N}_{3} \mathrm{O}_{8}$ : C: 55.33 (54.96); H: 9.07 (9.38); H: 8.80 (8.67). ${ }^{1} \mathrm{H}$ NMR: $\delta 1.41$ (s, $\left.\mathrm{CH}_{3}, 18 \mathrm{H}\right), \delta$ 1.49-1.61 (m, $\left.\mathrm{CH}_{2}, 3 \mathrm{H}\right), \delta 1.76-$ $1.82\left(\mathrm{~m}, \mathrm{CH}_{2}, 1 \mathrm{H}\right), \delta 3.04-3.11\left(\mathrm{~m}, \mathrm{CH}_{2}, 1 \mathrm{H}\right), \delta 3.18\left(\mathrm{~s}, \mathrm{br}, \mathrm{CH}_{2}, 1 \mathrm{H}\right), \delta 3.36\left(\mathrm{~s}, \mathrm{CH}_{3}\right.$, 
$3 \mathrm{H}), \delta 3.41\left(\mathrm{t}, \mathrm{CH}_{2}, \mathrm{~J}=4.8 \mathrm{~Hz}, 2 \mathrm{H}\right), \delta 3.51-3.56\left(\mathrm{~m}, \mathrm{CH}_{2}, 4 \mathrm{H}\right), \delta 3.60-3.64\left(\mathrm{~m}, \mathrm{CH}_{2}, 6 \mathrm{H}\right)$, $\delta 4.14(\mathrm{~s}, \mathrm{br}, \mathrm{CH}, 1 \mathrm{H}), \delta 4.79$ (s, br, NH,1H), $\delta 5.31(\mathrm{~d}, \mathrm{NH}, \mathrm{J}=7.6 \mathrm{~Hz}, 1 \mathrm{H}), \delta 6.76(\mathrm{~s}$, br, $\mathrm{N} H, 1 \mathrm{H}) .{ }^{13} \mathrm{C}$ NMR: $\delta 26.34,28.50,28.59,30.60,39.40,39.87,53.84,59.13,69.77$, 70.40, 70.57, 70.67, 72.07, 79.27, 79.84, 155.75, 156.34, 172.27. MS (FAB+): m/z 478 $(\mathrm{MH}+)$.

PEG-5Li-(NHBoc) $)_{2}$, 2-27(Boc $)_{2}$. This compound was synthesized in an analogous manner to that for $\mathbf{2 - 2 4 ( B o c )}$, using Boc-(Boc)-L-Lys-OH as a starting material. Colorless oil, 68\%. Eluent: EtOAc, $\mathrm{R}_{\mathrm{f}}=0.22 . \mathrm{C}_{23} \mathrm{H}_{45} \mathrm{~N}_{3} \mathrm{O}_{8}: \mathrm{C}: 56.19$ (55.83); H: 9.23 (9.58); N: 8.55 (8.48). ${ }^{1} \mathrm{H}$ NMR: $\delta 1.33-1.52\left(\mathrm{~m}, \mathrm{CH}_{2}+\mathrm{CH}_{3}, 22 \mathrm{H}\right), \delta$ 1.58-1.62 (m, $\mathrm{CH}$, 1H), $\delta$ 1.76-1.85 (m, CH, 1H), $\delta 3.09$ (quartet, $\left.\mathrm{CH}_{2}, \mathrm{~J}=6.0 \mathrm{H}, 2 \mathrm{H}\right), \delta 3.37\left(\mathrm{~s}, \mathrm{CH}_{3}, 3 \mathrm{H}\right), \delta$ 4.43 (quartet, $\left.\mathrm{CH}_{2}, \mathrm{~J}=5.2 \mathrm{~Hz}, 2 \mathrm{H}\right), \delta 3.53-3.57\left(\mathrm{~m}, \mathrm{CH}_{2}, 4 \mathrm{H}\right), \delta 3.62-3.65\left(\mathrm{~m}, \mathrm{CH}_{2}, 6 \mathrm{H}\right)$, $\delta 4.07$ (quartet, br, $\mathrm{CH}, \mathrm{J}=5.2 \mathrm{~Hz}, 1 \mathrm{H}), \delta 4.46(\mathrm{~s}, \mathrm{br}, \mathrm{NH}, 1 \mathrm{H}), \delta 5.27$ (s, br, NH,1H), $\delta$ 6.60 (s, br, NH, 1H). ${ }^{13} \mathrm{C}$ NMR: $\delta 22.80,28.53,28.62,29.81,32.74,39.37,40.23,54.55$, $59.17,69.81,70.41,70.61,70.68,72.10,79.21,79.96,155.82,156.28,172.24 . \mathrm{MS}$ (FAB+): $\mathrm{m} / \mathrm{z} 492.5(\mathrm{MH}+)$.

PEG-nLi-diamines, (2-24 through 2-27). Diamines 2-24 through 2-27 were generated in situ by stirring 0.25-0.50 mmol of their Boc-protected precursors 2-24(Boc) $)_{2}$ through 2-27(Boc $)_{2}$ in 3-5 mL of TFA for five hours, followed by removal of the acid under vacuum and co-evaporation of the residue with $\mathrm{CH}_{2} \mathrm{Cl}_{2}(3 \times 5 \mathrm{~mL})$. This crude diamine was not purified or characterized before use in subsequent HOPO-coupling reactions. An excess of $\mathrm{Et}_{3} \mathrm{~N}$ was added to the reaction flask to compensate for any TFA no removed under workup of the acidic deprotection. 
2,5-Bis-[1-thio-(3,6,9-trioxa-decane)]-3,4-dinitro-thiophene, 2-28. A solution of 2,5-dichloro-3,4-dinitrothiophene ${ }^{27}(2.00 \mathrm{~g}, 8.23 \mathrm{mmol})$, 3,6,9-trioxa-1-decanethiol ${ }^{28}$ (3.26 g, $18.1 \mathrm{mmol})$, and $\mathrm{Et}_{3} \mathrm{~N}(2.55 \mathrm{~mL}, 18.3 \mathrm{mmol})$ in $100 \mathrm{~mL}$ of $\mathrm{MeOH}$ was stirred at room temperature for 1 day, turning a deep red in the process. The solvent was removed under vacuum, the residue dissolved in $100 \mathrm{~mL} \mathrm{CH}_{2} \mathrm{Cl}_{2}$, and the solution was washed with $1 \mathrm{M} \mathrm{HCl}(2 \times 50 \mathrm{~mL})$, saturated brine, then dried and the solvent was removed under vacuum. The resultant dark brown oil was dissolved in $12 \mathrm{~mL}$ of EtOAc and purified on a silica gel column, eluting with EtOAc $\left(\mathrm{R}_{\mathrm{f}}=0.13\right)$, yielding a red oil after solvent removal (2.26 g, 52\%). $\mathrm{C}_{18} \mathrm{H}_{30} \mathrm{~N}_{2} \mathrm{O}_{10} \mathrm{~S}_{3:} \mathrm{C}: 40.74$ (40.97); H: 5.70 (5.99); N: 5.28 (5.28); S: 18.13 (18.14). ${ }^{1} \mathrm{H}$ NMR: $\delta 3.21\left(\mathrm{t}, \mathrm{CH}_{2}, \mathrm{~J}=6.0 \mathrm{~Hz}, 4 \mathrm{H}\right), \delta 3.37\left(\mathrm{~s}, \mathrm{CH}_{3}, 6 \mathrm{H}\right), \delta$ 3.53-3.55 (m, $\left.\mathrm{CH}_{2}, 4 \mathrm{H}\right), \delta 3.62-3.67\left(\mathrm{~m}, \mathrm{CH}_{2}, 12 \mathrm{H}\right), \delta 3.79\left(\mathrm{t}, \mathrm{CH}_{2}, \mathrm{~J}=6.0 \mathrm{~Hz}, 4 \mathrm{H}\right) .{ }^{13} \mathrm{C}$ NMR: $\delta 36.72,59.27,69.09,70.73,70.79,70.90,72.08,141.29$ (one thiophene carbon is not detected). $\mathrm{MS}(\mathrm{FAB}+)$ : m/z $531(\mathrm{MH}+), 485\left(\left[\mathrm{M}-\mathrm{CH}_{3} \mathrm{O}\left(\mathrm{CH}_{2}\right)_{2}\right]+\right)$.

2,5- Bis-[1-thio-(3,6,9-trioxa-decane)]-3,4-diaminothiophene, 2-29. Iron powder (325 mesh $3.50 \mathrm{~g}, 62.6 \mathrm{mmol})$ was stirred for 1 hour in a solution of 2-28 (2.21 g, 4.17 $\mathrm{mmol}$ ) in $20 \mathrm{~mL}$ of $\mathrm{AcOH}$ and $2 \mathrm{~mL}$ of water at $70{ }^{\circ} \mathrm{C}$. The reaction mixture was poured into $500 \mathrm{~mL}$ of water and extracted with $\mathrm{CH}_{2} \mathrm{Cl}_{2}(2 \times 200 \mathrm{~mL})$. The combined organics were dried and the solvent was removed under vacuum. Dissolution of the resultant oil in $5 \mathrm{~mL}$ of $5 \% \mathrm{MeOH}$ in EtOAc and purification on a silica gel column with the same yielded a brown oil after solvent removal $(1.63 \mathrm{~g}, 83 \%) . \mathrm{R}_{\mathrm{f}}(10 \% \mathrm{MeOH}$ in EtOAc $)=$ 0.41. $\mathrm{C}_{18} \mathrm{H}_{34} \mathrm{~N}_{2} \mathrm{O}_{6} \mathrm{~S}_{3}$ : C: 45.93 (45.82); H: 7.28 (7.37); N: 5.95 (5.60); S: 20.44 (20.18). ${ }^{1} \mathrm{H}$ NMR: $\delta 3.38$ (s, $\left.\mathrm{CH}_{3}+\mathrm{NH}_{2}, 10 \mathrm{H}\right), \delta 3.54-3.64$ (m, $\left.\mathrm{CH}_{2}, 24 \mathrm{H}\right) .{ }^{13} \mathrm{C}$ NMR: $\delta 24.45$, 37.56, 59.21, 69.28, 70.42, 70.70, 72.15, 106.18, 141.91. MS (FAB+): m/z 470 (MH+). 
5-[1-0xo-(3,6,9-trioxa-decane)]-isophthalic acid dimethyl ester, 2-30. A mixture of 5-hydroxy-isophthalic acid dimethyl ester (3.50 g, $16.6 \mathrm{mmol}), 1$-( $p$-tolylsulfonyl)-3,6,9trioxa-decane ${ }^{28}(5.83 \mathrm{~g}, 18.3 \mathrm{mmol})$, and $\mathrm{K}_{2} \mathrm{CO}_{3}(4.62 \mathrm{~g}, 33.4 \mathrm{mmol})$ in $50 \mathrm{~mL}$ of DMF was stirred at $120{ }^{\circ} \mathrm{C}$ for eight hours. The mixture was cooled to room temperature, poured into $200 \mathrm{~mL}$ of water and extracted with EtOAc $(3 \times 100 \mathrm{~mL})$. The combined organics were washed with saturated brine, dried and the solvent was removed. The resultant oil was dissolved in $15 \mathrm{~mL}$ of EtOAc and eluted on a silica gel column with the same. Fractions with $\mathrm{R}_{\mathrm{f}}=0.37$ were collected, dried and the solvent removed to yield $5.14 \mathrm{~g}$ of a colorless oil (87\%). $\mathrm{C}_{17} \mathrm{H}_{24} \mathrm{O}_{8}: \mathrm{C}: 57.30$ (57.18); H: 6.79 (6.87). ${ }^{1} \mathrm{H}$ NMR: $\delta$ $3.36\left(\mathrm{~s}, \mathrm{CH}_{3}, 3 \mathrm{H}\right), \delta 3.52-3.54\left(\mathrm{~m}, \mathrm{CH}_{2}, 2 \mathrm{H}\right), \delta 3.63-3.69\left(\mathrm{~m}, \mathrm{CH}_{2}, 4 \mathrm{H}\right), \delta 3.72-3.74(\mathrm{~m}$, $\left.\mathrm{CH}_{2}, 2 \mathrm{H}\right), \delta 3.87\left(\mathrm{t}, \mathrm{CH}_{2}, \mathrm{~J}=4.8 \mathrm{~Hz}, 2 \mathrm{H}\right), \delta 3.92\left(\mathrm{~s}, \mathrm{CH}_{3}, 6 \mathrm{H}\right), \delta 4.20\left(\mathrm{t}, \mathrm{CH}_{2}, \mathrm{~J}=4.8 \mathrm{~Hz}\right.$, $2 \mathrm{H}), \delta 7.75(\mathrm{~d}$, arom. $H, \mathrm{~J}=1.2 \mathrm{~Hz}, 2 \mathrm{H}), \delta 8.26(\mathrm{t}$, arom. $H, 1.2 \mathrm{~Hz}, 1 \mathrm{H}) .{ }^{13} \mathrm{C} \mathrm{NMR}: \delta$ $52.60,59.23,68.19,69.69,70.76,70.83,71.07,72.08,120.09,123.28,131.87,159.04$, 166.27. $\mathrm{MS}(\mathrm{FAB}+): \mathrm{m} / \mathrm{z} 357(\mathrm{MH}+)$.

5-[1-oxo-(3,6,9-trioxa-decane)]- $\alpha, \alpha^{\prime}$-dihydroxy-m-xylene, 2-31. A solution of 2-30 $(3.56 \mathrm{~g}, 10.0 \mathrm{mmol})$ in $30 \mathrm{~mL}$ of dry toluene was stirred in an ice bath under nitrogen. A $65 \mathrm{wt} \%$ solution of Red-Al in toluene (Aldrich, $7.50 \mathrm{~mL}, 25.0 \mathrm{mmol}$ ) was added via syringe and the resultant yellow solution was allowed to stir overnight at room temperature. The reaction was quenched by slow addition of $50 \mathrm{~mL}$ of water and stirring until the yellow color disappears. The layers were separated and the aqueous layer was extracted with recycling $\mathrm{CHCl}_{3}(10 \times 100 \mathrm{~mL})$. The combined organics were washed with saturated brine, dried and the solvent was removed. The residue was purified on a silica gel column using $5 \% \mathrm{MeOH}$ in $\mathrm{CHCl}_{3}$ as eluent $\left(\mathrm{R}_{\mathrm{f}}=0.09\right)$, to yield $1.92 \mathrm{~g}$ of a 
colorless oil as a partial hydrate $(63 \%) . \mathrm{C}_{15} \mathrm{H}_{24} \mathrm{O}_{6} \cdot 1 / 3 \mathrm{H}_{2} \mathrm{O}: \mathrm{C}: 58.81$ (58.73); $\mathrm{H}: 8.12$ (8.36). ${ }^{1} \mathrm{H}$ NMR: $\delta 2.50(\mathrm{~s}, \mathrm{br}, \mathrm{OH}, 2 \mathrm{H}), \delta 3.36\left(\mathrm{~s}, \mathrm{CH}_{3}, 3 \mathrm{H}\right), \delta 3.52-3.54\left(\mathrm{~m}, \mathrm{CH}_{2}, 2 \mathrm{H}\right), \delta$ 3.62-3.67 (m, $\left.\mathrm{CH}_{2}, 4 \mathrm{H}\right), \delta 3.70-3.72\left(\mathrm{~m}, \mathrm{CH}_{2}, 2 \mathrm{H}\right), \delta 3.82\left(\mathrm{t}, \mathrm{CH}_{2}, \mathrm{~J}=4.8 \mathrm{~Hz}, 2 \mathrm{H}\right), \delta 4.10$ (t, $\left.\mathrm{CH}_{2}, \mathrm{~J}=4.8 \mathrm{~Hz}, 2 \mathrm{H}\right), \delta 4.59$ (s, benzyl $\left.H, 4 \mathrm{H}\right), \delta 6.81$ (s, arom. $\left.H, 2 \mathrm{H}\right), \delta 6.88(\mathrm{~s}$, arom. $H, 1 \mathrm{H}) .{ }^{13} \mathrm{C}$ NMR: $\delta 59.21,65.11,67.64,69.96,70.67,70.83,70.95,72.07,112.34$, 117.81, 143.02, 159.34. MS (FAB+): m/z $307([\mathrm{M}-\mathrm{OH}] \mathrm{Na}+)$.

5-[1-oxo-(3,6,9-trioxa-decane)]-a, $\boldsymbol{\alpha}^{\prime}$-dibromo-m-xylene, 2-32. While cooling in an ice bath under nitrogen, $\mathrm{PBr}_{3}(1.35 \mathrm{~mL}, 14.2 \mathrm{mmol})$ in $30 \mathrm{~mL}$ of $\mathrm{Et}_{2} \mathrm{O}$ was added to a solution of $\mathbf{2 - 3 1 \cdot 1 / 3 \mathbf { H } _ { 2 }} \mathrm{O}(1.89 \mathrm{~g}, 6.17 \mathrm{mmol})$ in $40 \mathrm{~mL}$ of $\mathrm{Et}_{2} \mathrm{O}$. The reaction was stirred for 1 hour cold, allowed to warm to room temperature overnight, and then poured onto $100 \mathrm{~g}$ of ice. The layers were separated and the aqueous layers were extracted with EtOAc $(2 \times 50 \mathrm{~mL})$. The combined organic layers were washed with saturated $\mathrm{NaHCO}_{3}$, water, saturated brine, dried and the solvent was removed. The residue was purified by elution on a silica gel column with $\mathrm{Et}_{2} \mathrm{O}\left(\mathrm{R}_{\mathrm{f}}=0.46\right)$, yielding $2.31 \mathrm{~g}$ of a colorless oil (88\%). $\mathrm{C}_{15} \mathrm{H}_{22} \mathrm{Br}_{2} \mathrm{O}_{4}$ : C: 42.48 (42.43); $\mathrm{H}: 5.20$ (5.42). ${ }^{1} \mathrm{H}$ NMR: $\delta 3.38\left(\mathrm{~s}, \mathrm{CH}_{3}, 3 \mathrm{H}\right), \delta$ 3.54-3.56 (m, $\left.\mathrm{CH}_{2}, 2 \mathrm{H}\right), \delta 3.64-3.56\left(\mathrm{~m}, \mathrm{CH}_{2}, 4 \mathrm{H}\right), \delta 3.72-3.75\left(\mathrm{~m}, \mathrm{CH}_{2}, 2 \mathrm{H}\right), \delta 3.85(\mathrm{t}$, $\left.\mathrm{CH}_{2}, \mathrm{~J}=4.8 \mathrm{~Hz}, 2 \mathrm{H}\right), \delta 4.14\left(\mathrm{t}, \mathrm{CH}_{2}, \mathrm{~J}=4.8 \mathrm{~Hz}, 2 \mathrm{H}\right), \delta 4.42(\mathrm{~s}$, benzyl $H, 4 \mathrm{H}), \delta 6.88(\mathrm{~d}$, arom. $H, \mathrm{~J}=1.2 \mathrm{~Hz}, 2 \mathrm{H}), \delta 6.99$ (s, arom. $H, 1 \mathrm{H}) .{ }^{13} \mathrm{C}$ NMR: $\delta 33.06,59.26,67.81$, $69.79,70.78,70.85,71.05,72.10,115.55,122.15,139.74,159.37 . \mathrm{MS}(\mathrm{FAB}+): \mathrm{m} / \mathrm{z} 427$ $(\mathrm{MH}+)$.

5-[1-oxo-(3,6,9-trioxa-decane)]-a, $\alpha^{\prime}$-bis-azido-m-xylene, 2-33. A mixture of 2-32 $(1.61 \mathrm{~g}, 3.77 \mathrm{mmol})$ and $\mathrm{NaN}_{3}(1.22 \mathrm{~g}, 18.8 \mathrm{mmol})$ in $50 \mathrm{~mL}$ of acetone was refluxed overnight, cooled, and filtered. After removal of solvent, the residual oil was dissolved in 
$\mathrm{Et}_{2} \mathrm{O}$ and filtered again to remove residual salts, and the solvent removed under vacuum to yield $1.30 \mathrm{~g}$ of a pale yellow oil $(98 \%) . \mathrm{C}_{15} \mathrm{H}_{22} \mathrm{~N}_{6} \mathrm{O}_{4}$ : C: 51.42 (51.51); $\mathrm{H}: 6.33$ (6.58); N: 23.99 (23.66). ${ }^{1} \mathrm{H}$ NMR: $\delta 3.37$ (s, $\left.\mathrm{CH}_{3}, 3 \mathrm{H}\right), \delta 3.53-3.56\left(\mathrm{~m}, \mathrm{CH}_{2}, 2 \mathrm{H}\right), \delta 3.64-3.66$ (m, $\left.\mathrm{CH}_{2}, 4 \mathrm{H}\right), \delta 3.68-3.69\left(\mathrm{~m}, \mathrm{CH}_{2}, 2 \mathrm{H}\right), \delta 3.86\left(\mathrm{t}, \mathrm{CH}_{2}, \mathrm{~J}=4.8 \mathrm{~Hz}, 2 \mathrm{H}\right), \delta 4.15\left(\mathrm{t}, \mathrm{CH}_{2}, \mathrm{~J}\right.$ $=4.8 \mathrm{~Hz}, 2 \mathrm{H}), \delta 4.31(\mathrm{~s}$, benzyl $H, 4 \mathrm{H}), \delta 6.84$ (s, arom. $H, 3 \mathrm{H}) .{ }^{13} \mathrm{C}$ NMR: $\delta 54.66$, $59.22,67.80,69.80,70.76,70.84,71.02,72.10,114.30,120.24,137.67,159.70 . \mathrm{MS}$ $(\mathrm{FAB}+): 323\left(\mathrm{M}-\mathrm{N}_{2}+\right)$.

5-[1-oxo-(3,6,9-trioxa-decane)]- $\alpha, \alpha^{\prime}$-diamino-m-xylene, 2-34. A mixture of 2-33 $(0.720 \mathrm{~g}, 2.05 \mathrm{mmol})$ and $5 \% \mathrm{Pd} / \mathrm{C}$ (wet, $0.194 \mathrm{~g}$ ) was stirred in $12 \mathrm{~mL}$ of $\mathrm{MeOH}$ at room temperature under 500 psi of $\mathrm{H}_{2}$ overnight in a Parr bomb. The reaction solution was filtered through a pad of celite, which was washed with $\mathrm{MeOH}(2 \times 10 \mathrm{~mL})$, and the solvent was removed from the combined filtrates to yield a colorless, oily residue that was used without characterization or further purification in the next step of HOPO ligand synthesis.

2,5-Bis-ethylsulfanyl-3,4-dinitro-thiophene, 2-35. A solution of 2,5-dichloro-3,4dinitrothiophene $^{27}(1.50 \mathrm{~g}, 6.17 \mathrm{mmol})$, ethanethiol $(1.0 \mathrm{~mL}, 13 \mathrm{mmol})$, and $\mathrm{Et}_{3} \mathrm{~N}$ (1.9 $\mathrm{mL}, 14 \mathrm{mmol}$ ) in $50 \mathrm{~mL}$ of $\mathrm{MeOH}$ was stirred at room temperature for 2 hours, causing precipitates to form. The solvent and excess EtSH were removed under vacuum, the residue was dissolved in $100 \mathrm{~mL}$ of $\mathrm{CH}_{2} \mathrm{Cl}_{2}$, and the solution was washed with $0.5 \mathrm{M} \mathrm{HCl}$ $(2 \times 25 \mathrm{~mL})$, saturated brine, then dried and the solvent was removed under vacuum. The resultant residue was recrystallized from hot acetone and in two crops yielded $1.09 \mathrm{~g}$ of yellow flakes, $60 \%$. $\mathrm{C}_{8} \mathrm{H}_{10} \mathrm{~N}_{2} \mathrm{O}_{4} \mathrm{~S}_{3}$ : C: 32.64 (32.95); H: 3.42 (3.30); N: 9.52 (9.20); S: 32.68 (32.49). ${ }^{1} \mathrm{H}$ NMR: $\delta 1.43$ (t, $\mathrm{CH}_{3}, \mathrm{~J}=7.2 \mathrm{~Hz}, 6 \mathrm{H}$ ), $\delta 3.04$ (quartet, $\mathrm{CH}_{2}, \mathrm{~J}=7.2 \mathrm{~Hz}$, 
$4 \mathrm{H}) .{ }^{13} \mathrm{C}$ NMR: $\delta 14.08,30.95,141.45$ (one thiophene carbon is not detected). MS $(\mathrm{FAB}+): \mathrm{m} / \mathrm{z} 294(\mathrm{MH}+) . \mathrm{MP}: 134-136^{\circ} \mathrm{C}$.

2,5-Bis-ethylsulfanyl-3,4-diamino-thiophene, 2-36. A solution of 2-35 (1.00 g, 3.41 mmol) in $50 \mathrm{~mL}$ of $10: 1 \mathrm{AcOH} / \mathrm{H}_{2} \mathrm{O}$ was stirred at $100{ }^{\circ} \mathrm{C}$. Iron powder $(-325$ mesh, 2.86 g, $51.1 \mathrm{mmol}$ ) was added to the solution. The solution quickly turned red then brown and was accompanied by bubble formation. The suspension was stirred one hour, poured into $500 \mathrm{~mL}$ water and extracted with $\mathrm{CH}_{2} \mathrm{Cl}_{2}(3 \times 100 \mathrm{~mL})$. The combined organics were washed with sat. brine, dried and the solvent was removed under vacuum. The residue was dissolved in $5 \mathrm{~mL} \mathrm{CH} \mathrm{CH}_{2} \mathrm{Cl}_{2}$ and eluted on a silica column with the same. Brown fractions with $\mathrm{R}_{\mathrm{f}}=0.12$ were collected, dried, and the solvent was removed under vacuum to yield $0.290 \mathrm{~g}$ of a brown oil, $36 \% . \mathrm{C}_{8} \mathrm{H}_{14} \mathrm{~N}_{2} \mathrm{~S}_{3}$ : C: 40.99 (41.18); H: 6.02 (5.96), N: 11.95 (11.75); S: 41.04 (40.84). ${ }^{1} \mathrm{H}$ NMR: $\delta 1.23$ (t, $\left.\mathrm{CH}_{3}, \mathrm{~J}=7.2 \mathrm{~Hz}, 6 \mathrm{H}\right), \delta$ 2.65 (quartet, $\mathrm{CH}_{2}, \mathrm{~J}=7.2 \mathrm{~Hz}, 4 \mathrm{H}$ ), $\delta 3.79$ (s, br, $\mathrm{NH}_{2}, 4 \mathrm{H}$ ). ${ }^{13} \mathrm{C}$ NMR: $\delta$ 15.24, 32.20, 107.92, 140.80. MS (FAB+): m/z $234(\mathrm{MH}+)$.

2-Benzyloxy-5-methyl-a, $\alpha^{\prime}$-bis-phthalimido-m-xylene, 2-41. A mixture of 2benzyloxy-1,3-bis-bromomethyl-5-methyl-benzene $\mathrm{e}^{29}(1.41 \mathrm{~g}, 3.67 \mathrm{mmol})$ and potassium phthalimide (1.36 g, $7.36 \mathrm{mmol})$ in $30 \mathrm{~mL}$ of DMF was stirred at $120{ }^{\circ} \mathrm{C}$ overnight. Once cooled to room temperature, $50 \mathrm{~mL}$ of water was added. The precipitated solids were filtered off on a Büchner funnel, washed with water and dried under vacuum. This solid was recrystallized from $\mathrm{CHCl}_{3}$ in three crops to yield $1.29 \mathrm{~g}$ of a white solid that analysis showed to be the hemihydrate, $67 \%$. $\mathrm{C}_{32} \mathrm{H}_{24} \mathrm{~N}_{2} \mathrm{O}_{5} \cdot \frac{1}{2} \mathrm{H}_{2} \mathrm{O}$ : C: 73.13 (73.25); $\mathrm{H}: 4.79$ (4.65); N: 5.33 (5.27). ${ }^{1} \mathrm{H}$ NMR: $\delta 2.14\left(\mathrm{~s}, \mathrm{CH}_{3}, 3 \mathrm{H}\right), \delta 4.93$ (s, $\left.\mathrm{CH}_{2}, 4 \mathrm{H}\right), \delta 5.28(\mathrm{~s}$, benzyl $H, 2 \mathrm{H}), \delta 6.86(\mathrm{~s}$, arom. $H, 2 \mathrm{H}), \delta 7.36(\mathrm{t}$, arom. $H, \mathrm{~J}=7.2 \mathrm{~Hz}, 1 \mathrm{H}), \delta 7.42(\mathrm{t}$, 
arom. $H, \mathrm{~J}=7.2 \mathrm{~Hz}, 2 \mathrm{H}), \delta 7.64(\mathrm{~d}$, arom. $H, \mathrm{~J}=7.2 \mathrm{~Hz}, 2 \mathrm{H}), \delta 7.76(\mathrm{dd}$, arom. $H, \mathrm{~J}=$ 5.6, $3.2 \mathrm{~Hz}, 4 \mathrm{H}), \delta 7.88(\mathrm{dd}$, arom. $H, \mathrm{~J}=5.6,3.2 \mathrm{~Hz}, 4 \mathrm{H}) .{ }^{13} \mathrm{C}$ NMR: $\delta 21.25,36.51$, $75.96,123.64,128.20,128.27,128.35,128.78,129.83,132.33,134.26,168.44 . \mathrm{MS}$ (FAB+): $517(\mathrm{MH}+) . \mathrm{MP}: 254-256^{\circ} \mathrm{C}$.

2-Benzyloxy-5-methyl- $\alpha, \boldsymbol{\alpha}^{\prime}$-diamino-m-xylene, 2-42. To a suspension of 241.1/2 $\mathrm{H}_{2} \mathrm{O}(1.00 \mathrm{~g}, 1.90 \mathrm{mmol})$ in $10 \mathrm{~mL}$ of $2: 1 \mathrm{EtOH} /$ toluene was added $\mathrm{H}_{2} \mathrm{NNH}_{2} \cdot \mathrm{H}_{2} \mathrm{O}$ $(0.40 \mathrm{ml}, 8.3 \mathrm{mmol})$ of, and the mixture was refluxed under nitrogen for two days. The suspension was cooled to room temperature and the solids were filtered off and redissolved in $100 \mathrm{~g}$ of $40 \%$ aqueous $\mathrm{NaOH}$. This mixture was extracted with 4 x $50 \mathrm{~mL}$ of $\mathrm{CHCl}_{3}$. The combined organic extracts were washed with $50 \mathrm{~mL}$ of water, dried with $\mathrm{Na}_{2} \mathrm{SO}_{4}$ and the solvent was removed under vacuum to yield $105 \mathrm{mg}$ of an oil, $c a 21 \%$. ${ }^{1} \mathrm{H}$ NMR: $\delta 1.61$ (s, NH$\left.H_{2}, 4 \mathrm{H}\right), \delta 2.31\left(\mathrm{~s}, \mathrm{CH}_{3}, 3 \mathrm{H}\right), \delta 3.82$ (s, $\left.\mathrm{CH}_{2}, 4 \mathrm{H}\right), \delta 4.84$ (s, benzyl $H, 2 \mathrm{H}$ ), $\delta 7.03$ (s, arom. $H, 2 \mathrm{H}$ ), $\delta$ 7.31-7.45 (m, arom. $H, 5 \mathrm{H}) .{ }^{13} \mathrm{C}$ NMR: $\delta 20.95,76.13$, $127.88,128.08,128.26,128.33,128.69,134.33,136.48,137.21,152.43$. This material was used in HOPO coupling reactions without further characterization.

2-Methoxy-5-methyl-a, $\alpha^{\prime}$-diamino-m-xylene, 2-43. A mixture of 2-methoxy-5methyl- $\alpha, \alpha^{\prime}$-dibromo- $m$-xylene ${ }^{30}(1.00 \mathrm{~g}, 3.25 \mathrm{mmol})$ and $\mathrm{NaN}_{3}(1.06 \mathrm{~g}, 16.3 \mathrm{mmol})$ was stirred in acetone at reflux for three hours. After cooling to room temperature the mixture was filtered, and the filtrate solvent was removed under vacuum. . The resultant oil was dissolved in $10 \mathrm{~mL}$ of $\mathrm{MeOH}$, and 5\% $\mathrm{Pd} / \mathrm{C}$ (wet, $0.10 \mathrm{~g}$ ) was added and the mixture was stirred under 500 psi of $\mathrm{H}_{2}$ overnight. After filtration through celite followed by solvent removal, a colorless oil was isolated in quantitative yield. ${ }^{1} \mathrm{H}$ NMR: $\delta 1.55\left(\mathrm{~s}, \mathrm{br}, \mathrm{NH}_{2}\right.$, $4 \mathrm{H}), \delta 2.30\left(\mathrm{~s}, \mathrm{CH}_{3}, 3 \mathrm{H}\right), \delta 3.77\left(\mathrm{~s}, \mathrm{CH}_{3}, 3 \mathrm{H}\right), \delta 3.84\left(\mathrm{~s}, \mathrm{CH}_{2}, 4 \mathrm{H}\right), \delta 7.02$ (s, arom. $H$, 
$2 \mathrm{H})$. This material was used in HOPO coupling reactions without further purification or characterization.

\subsubsection{Synthesis of Benzyl-Protected bis-Me-3,2-HOPO Ligands}

General: The diamines used in the HOPO-coupling reactions were either purchased and used as received or synthesized by literature procedures or as described above.

Method A: A suspension of Me-3,2-HOPO-(Bn)-COOH (1 equivalent, 2-10 mmol) in 30-60 mL of dry toluene and 3-5 drops of DMF was stirred at room temperature under nitrogen. Oxalyl chloride (1.5 equivalents) was introduced into the suspension via syringe, causing foaming and resulting in a yellow, homogenous solution. This solution was stirred at room temperature for a minimum of four hours, then the solvent and residual oxalyl chloride were removed under vacuum, followed by co-evaporation with $\mathrm{CH}_{2} \mathrm{Cl}_{2}$. The residue was held under vacuum for several hours and the resultant yellow oil was then dissolved in $50-100 \mathrm{~mL}$ of dry $\mathrm{CH}_{2} \mathrm{Cl}_{2}$. To this solution was added via cannula a solution of diamine ( $1 / 2$ equivalent) and $\mathrm{Et}_{3} \mathrm{~N}$ ( 1 equivalent) in $25-50 \mathrm{~mL}$ of dry $\mathrm{CH}_{2} \mathrm{Cl}_{2}$, and the solution was stirred overnight. The solution was washed with $1 \mathrm{M} \mathrm{HCl}$ $(2 \times 25-50 \mathrm{~mL})$, then saturated brine $(25 \mathrm{~mL})$, dried with $\mathrm{Na}_{2} \mathrm{SO}_{4}$ and the solvent was removed under vacuum. The residue was re-dissolved in a minimum amount of $\mathrm{CH}_{2} \mathrm{Cl}_{2}$ and purified by silica gel chromatography. For compounds 2-6(Bn $)_{2}$ through 2-16(Bn $)_{2}$, chromatography was followed by re-dissolving the isolated material in $\mathrm{CH}_{2} \mathrm{Cl}_{2}$ and layering with $\mathrm{Et}_{2} \mathrm{O}$, yielding microcrystalline solids which were dried under vacuum.

Method B: A solution of diamine (1 equivalent, 1-5 mmol, primary amines only) was stirred over night in 50-100 $\mathrm{mL}$ of $\mathrm{CH}_{2} \mathrm{Cl}_{2}$ with Me-3,2-HOPO-Thiaz(Bn) and $\mathrm{Et}_{3} \mathrm{~N}$ (two equivalents each) or until the yellow color of the thiaz disappeared. The solution 
was washed with $1 \mathrm{M} \mathrm{HCl}(2 \times 25-50 \mathrm{~mL}), 1 \mathrm{M} \mathrm{NaOH}(3 \times 25-50 \mathrm{~mL})$, saturated brine $(25 \mathrm{~mL})$, dried and the solvent was removed under vacuum. Purification of the compound proceeded as described for Method A.

3,4-Thio-Me-3,2-HOPO(Bn) 2, 2-6(Bn) 2. Method A; eluent: 4\% $\mathrm{MeOH}$ in $\mathrm{CH}_{2} \mathrm{Cl}_{2}$, $\mathrm{R}_{\mathrm{f}}=0.21$. Light brown, amorphous solid, $76 \% . \mathrm{C}_{32} \mathrm{H}_{28} \mathrm{~N}_{4} \mathrm{O}_{6} \mathrm{~S}: \mathrm{C}: 64.42$ (64.04); H: 4.73 (4.76); N: 9.39 (9.42); S: 5.37 (5.25). ${ }^{1} \mathrm{H}$ NMR: $\delta 3.56$ (s, $\mathrm{CH}_{3}, 6 \mathrm{H}$ ), $\delta 5.35$ (s, benzyl $H$, 4H), $\delta 6.66(\mathrm{~d}, \mathrm{HOPO} H, \mathrm{~J}=7.2 \mathrm{~Hz}, 2 \mathrm{H}), \delta 7.07(\mathrm{~d}, \mathrm{HOPO} H, \mathrm{~J}=7.2 \mathrm{~Hz}, 2 \mathrm{H}), \delta 7.12-$ $7.17(\mathrm{~m}$, arom. $H, 6 \mathrm{H}), \delta 7.26(\mathrm{~s}, \mathrm{CH}, 2 \mathrm{H}), \delta 7.28-7.30(\mathrm{~m}$, arom. $H, 4 \mathrm{H}), \delta 9.67(\mathrm{~s}, \mathrm{~N} H$, 2H). ${ }^{13} \mathrm{C}$ NMR: $\delta 37.80,74.85,104.70,113.88,128.56,128.61,128.70,129.13,130.20$, 132.31, 135.84, 146.30, 159.38, 161.64. MS (FAB+): m/z 597 (MH+). MP: 193-195 ${ }^{\circ} \mathrm{C}$.

o-Phen-Me,3,2-HOPO(Bn) 2, 2-7(Bn)2. Method A; no chromatography needed. Beige crystals, 47\%. $\mathrm{C}_{34} \mathrm{H}_{30} \mathrm{~N}_{4} \mathrm{O}_{6}$ : C: 69.14 (68.89); H: 5.12(5.13); N: 9.49 (9.29). ${ }^{1} \mathrm{H}$ NMR: $\delta 3.60$ (s, $\left.\mathrm{CH}_{3}, 6 \mathrm{H}\right), \delta 5.42$ (s, benzyl $\left.H, 4 \mathrm{H}\right), \delta 6.70(\mathrm{~d}, \mathrm{HOPO} H, \mathrm{~J}=7.2 \mathrm{~Hz}, 2 \mathrm{H}$ ), $\delta 7.10(\mathrm{~d}, \mathrm{HOPO} H, \mathrm{~J}=7.2 \mathrm{~Hz}, 2 \mathrm{H}), \delta 7.14-7.34(\mathrm{~m}$, arom. $H, 14 \mathrm{H}), \delta 9.61(\mathrm{~s}, \mathrm{~N} H, 2 \mathrm{H})$. ${ }^{13} \mathrm{C}$ NMR: $\delta 37.93,74.81,105.05,125.08,126.46,128.74,128.90,129.35,130.27$, 130.88, 132.34, 135.95, 146.33, 159.61, 162.35. MS (FAB+): m/z $591(\mathrm{MH}+)$. MP: 197$199^{\circ} \mathrm{C}$.

o-Tol-Me,3,2-HOPO(Bn $\left.)_{2}, \mathbf{2 - 8 ( B n}\right)_{2}$. Method A; eluent: $2 \% \mathrm{MeOH}$ in $\mathrm{CH}_{2} \mathrm{Cl}_{2}, \mathrm{R}_{\mathrm{f}}=$ 0.22. Feathery, white crystals of the hemihydrate, $72 \%$. $\mathrm{C}_{35} \mathrm{H}_{32} \mathrm{~N}_{4} \mathrm{O}_{6} \cdot{ }^{1 / 2} \mathrm{H}_{2} \mathrm{O}: \mathrm{C}: 68.50$ (68.55); H: 5.42 (5.55); N: 9.13 (9.09). ${ }^{1} \mathrm{H}$ NMR: $\delta 3.57$ (s, $\left.\mathrm{CH}_{3}, 3 \mathrm{H}\right), \delta 3.62$ (s, $\mathrm{CH}_{3}$, $3 \mathrm{H}), \delta 5.33$ (s, benzyl $H, 2 \mathrm{H}), \delta 5.45$ (s, benzyl $H, 2 \mathrm{H}), \delta 6.65-6.70(\mathrm{~m}, \mathrm{HOPO} H, 2 \mathrm{H}), \delta$ 7.05-7.35 (m, arom $H+\mathrm{HOPO} H, 14 \mathrm{H}), \delta 7.56(\mathrm{~d}$, arom $H, \mathrm{~J}=8.0 \mathrm{~Hz}, 1 \mathrm{H}), \delta 8.39(\mathrm{~s}$, $\mathrm{N} H, 1 \mathrm{H}), \delta 9.89(\mathrm{~s}, \mathrm{~N} H, 1 \mathrm{H}) .{ }^{13} \mathrm{C}$ NMR: $\delta 37.92,37.99,40.10,74.68,74.83,105.09$, 
$105.22,124.76,126.23,128.50,128.74,128.87,128.91,129.17,129.21,130.00,132.14$, $132.21,132.49,135.35,136.13,136.38,146.22,146.58,159.70,159.79,162.77,163.66$. MS (FAB+): m/z $605(\mathrm{MH}+)$. MP: $169-171^{\circ} \mathrm{C}$.

m-Phen-Me-3,2-HOPO(Bn) $)_{2}$ 2-9(Bn) 2 . Method A; eluent: $8 \% \mathrm{MeOH}$ in $\mathrm{CH}_{2} \mathrm{Cl}_{2}, \mathrm{R}_{\mathrm{f}}$ $=0.30$. Colorless crystals, $58 \% . \mathrm{C}_{34} \mathrm{H}_{30} \mathrm{~N}_{4} \mathrm{O}_{6}: \mathrm{C}: 69.14$ (68.83); H: 5.12 (5.07); N: 9.48 (9.39). ${ }^{1} \mathrm{H}$ NMR (DMSO- $d_{6}$ ): $\delta 3.53\left(\mathrm{~s}, \mathrm{CH}_{3}, 6 \mathrm{H}\right), \delta 5.23$ (s, benzyl $\left.H, 4 \mathrm{H}\right), \delta 6.32(\mathrm{~d}$, HOPO $H, \mathrm{~J}=6.8 \mathrm{~Hz}, 2 \mathrm{H}), \delta$ 7.22-7.31 (m, arom. $H, 9 \mathrm{H}), \delta 7.38-7.40$ (m, arom. $H, 4 \mathrm{H}), \delta$ $7.96(\mathrm{~d}, \mathrm{HOPO} H, \mathrm{~J}=6.8 \mathrm{~Hz}, 2 \mathrm{H}), \delta 8.11$ (s, arom. $H, 1 \mathrm{H}$ ); (amide protons not visible). ${ }^{13} \mathrm{C}$ (DMSO- $\left.d_{6}\right): \delta 36.94,73.13,102.83,111.15,115.27,127.92,128.13,128.20,134.40$, 136.95, 138.76, 143.60, 162.90. MS (FAB+): m/z 591.2 (MH+). MP: $115-117^{\circ} \mathrm{C}$.

Py-Me-3,2-HOPO(Bn) 2, 2-10(Bn)2. Method A; eluent: 4\% $\mathrm{MeOH}$ in $\mathrm{CH}_{2} \mathrm{Cl}_{2}, \mathrm{R}_{\mathrm{f}}=$ 0.31 (EtOAc) Off-white crystals, 35\%. $\mathrm{C}_{33} \mathrm{H}_{29} \mathrm{~N}_{5} \mathrm{O}_{6}: \mathrm{C}: 67.00$ (66.65); $\mathrm{H}: 4.94$ (5.28); N: 11,84 (11.77). ${ }^{1} \mathrm{H}$ NMR: $\delta 3.64$ (s, CH3, 6H), $\delta 5.43$ (s, benzyl $\left.H, 4 \mathrm{H}\right), \delta 6.81$ (d, HOPO $H, \mathrm{~J}=7.2 \mathrm{~Hz}, 2 \mathrm{H}$ ), $\delta 7.17(\mathrm{~d}, \mathrm{HOPO} H, \mathrm{~J}=7.2 \mathrm{~Hz}, 2 \mathrm{H}) \delta 7.19-7.23$ (m, arom. $H, 6 \mathrm{H}), \delta$ 7.34-7.36 (m, arom $H, 4 \mathrm{H}), \delta 7.72(\mathrm{t}$, arom. $H, \mathrm{~J}=8.0 \mathrm{~Hz}, 1 \mathrm{H}), \delta 7.98(\mathrm{~d}$, arom. $H, \mathrm{~J}=$ $8.0 \mathrm{~Hz}, 2 \mathrm{H}), \delta 10.22(\mathrm{~s}, \mathrm{~N} H, 2 \mathrm{H}) .{ }^{13} \mathrm{C}$ NMR: $\delta 38.01,75.24,104.56,110.66,128.78$, $129.11,129.79,130.73,132.55,135.37,140.76,149.50,159.70,161.68$. MS (FAB+): $\mathrm{m} / \mathrm{z} 592(\mathrm{MH}+)$. MP: $165-167^{\circ} \mathrm{C}$.

1,8-Napth-Me-3,2-HOPO(Bn)2, 2-11(Bn)2. Method A; eluent: 4\% $\mathrm{MeOH}$ in $\mathrm{CH}_{2} \mathrm{Cl}_{2}, \mathrm{R}_{\mathrm{f}}=0.35$. Light brown crystals, 59\%. $\mathrm{C}_{38} \mathrm{H}_{32} \mathrm{~N}_{4} \mathrm{O}_{6}: \mathrm{C}: 71.24$ (71.12); H: 5.03 (4.98); N: 8.74 (8.73). ${ }^{1} \mathrm{H}$ NMR: $\delta 3.57$ (s, $\left.\mathrm{CH}_{3}, 6 \mathrm{H}\right), \delta 5.19$ (s, benzyl $\left.H, 4 \mathrm{H}\right), \delta 6.72(\mathrm{~d}$, HOPO $H, \mathrm{~J}=7.2 \mathrm{~Hz}, 2 \mathrm{H}), \delta$ 7.04-7.16 (m, arom. $H, 12 \mathrm{H}), \delta 7.38(\mathrm{~m}$, arom. $H, 4 \mathrm{H}), \delta$ $7.74(\mathrm{~d}, \mathrm{HOPO} H, \mathrm{~J}=7.6 \mathrm{~Hz}, 2 \mathrm{H}), \delta 9.98(\mathrm{~s}, \mathrm{~N} H, 2 \mathrm{H}) .{ }^{13} \mathrm{C} \mathrm{NMR}: \delta 37.77,75.09,123.11$, 
$123.05,125.05,125.55,127.81,128.78,129.23,130.87,131.82,132.51,135.79,136.04$ 145.58, 159.31, 162.33. MS (FAB+): m/z 641.3 (MH+). MP: 221-222 ${ }^{\circ} \mathrm{C}$.

Biph-Me-3,2-HOPO(Bn) 2, 2-12(Bn) 2. Method A; eluent: 4\% $\mathrm{MeOH}$ in $\mathrm{CH}_{2} \mathrm{Cl}_{2}, \mathrm{R}_{\mathrm{f}}$ $=0.35$. Fluffy, white microcrystalline solid, $26 \% . \mathrm{C}_{40} \mathrm{H}_{34} \mathrm{~N}_{4} \mathrm{O}_{6}:$ C: 72.06 (71.71); H: 5.14 (5.21); N: 8.40 (8.18). ${ }^{1} \mathrm{H}$ NMR (DMSO- $\left.d_{6}\right): \delta 3.42\left(\mathrm{~s}, \mathrm{CH}_{3}, 6 \mathrm{H}\right), \delta 5.01(\mathrm{dd}, \mathrm{J}=11.2$, $21.6 \mathrm{~Hz}$, benzyl $H, 4 \mathrm{H}), \delta 6.13(\mathrm{~d}, \mathrm{~J}=7.2 \mathrm{~Hz}, \mathrm{HOPO} H, 2 \mathrm{H}), \delta$ 7.15-7.24 (m, arom. $H$, 14H), $\delta$ 7.39-7.44 (m, arom. $H, 4 \mathrm{H}), \delta 7.909$ (dd, J = $8 \mathrm{~Hz}, \mathrm{HOPO} H, 2 \mathrm{H}), \delta 9.669$ (s, $\mathrm{N} H, 2 \mathrm{H}) .{ }^{13} \mathrm{C}$ NMR (DMSO- $\left.d_{6}\right): \delta 36.93,72.56,102.65,123.73,125.24,128.14,128.55$, 130.54, 131.15, 133.71, 135.35, 136.06, 143.82, 158.47, 161.90. MS (FAB): m/z 667.3 (MH+). MP: $123-125^{\circ} \mathrm{C}$.

m-Tol-Me-3,2-HOPO(Bn) 2, 2-13(Bn)2. Method A; eluent: 4\% $\mathrm{MeOH} / \mathrm{CH}_{2} \mathrm{Cl}_{2}, \mathrm{R}_{\mathrm{f}}=$ 0.33. White, microcrystalline solid, 39\%. $\mathrm{C}_{35} \mathrm{H}_{32} \mathrm{~N}_{4} \mathrm{O}_{6}$ : C: 69.52 (69.22); $\mathrm{H}: 5.33$ (5.43); N: 9.27 (9.04). ${ }^{1} \mathrm{H}$ NMR: $\delta 3.60\left(\mathrm{~s}, \mathrm{CH}_{3}, 3 \mathrm{H}\right), \delta 3.63\left(\mathrm{~s}, \mathrm{CH}_{3}, 3 \mathrm{H}\right), \delta 4.31\left(\mathrm{~d}, \mathrm{CH}_{2}, \mathrm{~J}=5.6\right.$ $\mathrm{Hz}, 2 \mathrm{H}), \delta 5.29(\mathrm{~s}$, benzyl $H, 2 \mathrm{H}), \delta 5.48(\mathrm{~s}$, benzyl $H, 2 \mathrm{H}), \delta 6.84(\mathrm{~d}, \mathrm{HOPO} H, \mathrm{~J}=7.2$ $\mathrm{Hz}, 2 \mathrm{H}), \delta 6.90(\mathrm{~d}, \mathrm{HOPO} H, \mathrm{~J}=7.2 \mathrm{~Hz}, 2 \mathrm{H}), \delta$ 7.13-7.24 (m, arom. $H, 9 \mathrm{H}), \delta$ 7.30-7.35 (m, arom. $H, 3 \mathrm{H}), \delta$ 7.44-7.46 (m, arom. $H, 2 \mathrm{H}), \delta 8.22(\mathrm{t}, \mathrm{NH}, \mathrm{J}=5.1 \mathrm{~Hz}, 1 \mathrm{H}), \delta 9.94(\mathrm{~s}$, $\mathrm{N} H, 1 \mathrm{H}) .{ }^{13} \mathrm{C}$ NMR: $\delta 37.95,38.01,74.82,75.94,104.93,105.16,119.15,119.43$, $124.07,128.86,128.95,129.23,129.36,129.46,129.67,130.30,130.60,132.36,132.42$, $135.94,138.07,138.62,146.71,146.85,159.70,159.70,159.79,161.04,163.21 . \mathrm{MS}$ $(\mathrm{FAB}+): \mathrm{m} / \mathrm{z} 604(\mathrm{MH}+) . \mathrm{MP}: 134-136^{\circ} \mathrm{C}$.

o-Xy-Me-3,2-HOPO(Bn) 2 , 2-14(Bn) 2 . Method B; eluent: $4 \% \mathrm{MeOH}$ in $\mathrm{CH}_{2} \mathrm{Cl}_{2}, \mathrm{R}_{\mathrm{f}}=$ 0.14. Feathery, white crystals, $83 \%$. $\mathrm{C}_{36} \mathrm{H}_{34} \mathrm{~N}_{4} \mathrm{O}_{6}:$ C: 69.89 (69.61); H: 5.54 ( 5.71); N: 9.06 (8.93). ${ }^{1} \mathrm{H}$ NMR: $\delta 3.58\left(\mathrm{~s}, \mathrm{CH}_{3}, 6 \mathrm{H}\right), \delta 4.39\left(\mathrm{~d}, \mathrm{CH}_{2}, \mathrm{~J}=16.4 \mathrm{~Hz}, 4 \mathrm{H}\right), \delta 5.28(\mathrm{~s}$, 
benzyl $H, 4 \mathrm{H}), \delta 6.75(\mathrm{~d}, \mathrm{HOPO} H, \mathrm{~J}=7.2 \mathrm{~Hz}, 2 \mathrm{H}), \delta$ 7.09-7.12 (m, arom. $H, 4 \mathrm{H}), \delta$ 7.17-7.26 (m, arom. $H+\mathrm{HOPO} H, 12 \mathrm{H}), \delta 8.19(\mathrm{t}, \mathrm{N} H, \mathrm{~J}=5.2 \mathrm{~Hz}, 2 \mathrm{H}) .{ }^{13} \mathrm{C}$ NMR: $\delta$ $15.48,37.92,41.11,74.79,105.15,128.27,128.87,128.92,129.19,129.56,130.58$, 132.25, 135.85, 136.04, 146.60, 159.73, 163.22. MS (FAB+): m/z 619 (MH+). MP: 160$161^{\circ} \mathrm{C}$.

m-Xy-Me-3,2-HOPO(Bn) 2, 2-15(Bn) 2 . Method B; eluent: 8\% MeOH in $\mathrm{CH}_{2} \mathrm{Cl}_{2}, \mathrm{R}_{\mathrm{f}}$ $=0.30$. Off-white crystals, $92 \% . \mathrm{C}_{36} \mathrm{H}_{34} \mathrm{~N}_{4} \mathrm{O}_{6}: \mathrm{C}: 69.89$ (69.56); H: 5.54 (5.67); N: 9.86 (8.97). ${ }^{1} \mathrm{H}$ NMR (DMSO- $\left.d_{6}\right): \delta 3.50\left(\mathrm{~s}, \mathrm{CH}_{3}, 6 \mathrm{H}\right), \delta 4.34\left(\mathrm{~d}, \mathrm{CH}_{2}, \mathrm{~J}=6.0 \mathrm{~Hz}, 4 \mathrm{H}\right), \delta 5.18$ (s, benzyl $H, 4 \mathrm{H}), \delta 6.28(\mathrm{~d}, \mathrm{HOPO} H, \mathrm{~J}=7.2 \mathrm{~Hz}, 2 \mathrm{H}), \delta$ 7.13-7.18 (m, arom. $H, 4 \mathrm{H}), \delta$ 7.28-7.34 (m, arom. $H, 10 \mathrm{H}), \delta 7.52(\mathrm{~d}, \mathrm{HOPO} H, \mathrm{~J}=7.2 \mathrm{~Hz}, 2 \mathrm{H}), \delta 8.73(\mathrm{t}, \mathrm{N} H, \mathrm{~J}=6.0$ Hz). ${ }^{13} \mathrm{C}$ NMR (DMSO- $\left.d_{6}\right): \delta 36.91,42.39,72.89,103.03,125.78,126.24,127.99$, $128.19,128.33,133.74,134.03,136.79,138.89,143.86,158.77,163.91 . \mathrm{MS}(\mathrm{FAB}+)$ : m/z $619.3(\mathrm{MH}+)$. MP: $135-136{ }^{\circ} \mathrm{C}$.

Fluo-Me-3,2-HOPO(Bn) 2, 2-16(Bn) 2 . Method A; eluent: 4\% $\mathrm{MeOH}$ in $\mathrm{CH}_{2} \mathrm{Cl}_{2}, \mathrm{R}_{\mathrm{f}}=$ 0.12. Pale yellow powder as the hemihydrate, $73 \%$. $\mathrm{C}_{41} \mathrm{H}_{34} \mathrm{~N}_{4} \mathrm{O}_{6} \cdot 1 / 2 \mathrm{H}_{2} \mathrm{O}$ : C: 71.60 (71.29); H: 5.13 (5.02); N: 8.15 (8.13). ${ }^{1} \mathrm{H}$ NMR: $\delta 2.77$ (s, $\left.\mathrm{CH}_{2}, 2 \mathrm{H}\right), \delta 3.67$ (s, $\mathrm{CH}_{3}$, $6 \mathrm{H}), \delta 5.02(\mathrm{~s}$, benzyl $H, 4 \mathrm{H}), \delta 6.91(\mathrm{~d}, \mathrm{HOPO} H, \mathrm{~J}=7.2 \mathrm{~Hz}, 2 \mathrm{H}), \delta 7.02(\mathrm{~m}$, arom. $H$, $6 \mathrm{H}), \delta 7.22(\mathrm{~d}, \mathrm{HOPO} H, \mathrm{~J}=7.2 \mathrm{~Hz}, 2 \mathrm{H}), \delta 7.33-7.39(\mathrm{~m}$, arom. $H, 6 \mathrm{H}), \delta 7.56(\mathrm{~d}$, arom. $H, \mathrm{~J}=7.6 \mathrm{~Hz}, 2 \mathrm{H}), \delta 7.87$ (d, arom. $H, \mathrm{~J}=8.0 \mathrm{~Hz}, 2 \mathrm{H}), \delta 9.72(\mathrm{~s}, \mathrm{~N} H, 2 \mathrm{H}) .{ }^{13} \mathrm{C} \mathrm{NMR}: \delta$ $32.10,38.04,75.39,104.86,117.05,119.98,128.18,128.79,129.07,129.50,130.99$, $132.63,132.85,134.06,135.85,142.46,146.59,159.65,161.22 . \mathrm{MS}(\mathrm{FAB}+): \mathrm{m} / \mathrm{z} 679$ $(\mathrm{MH}+)$. MP: Upon heating, the compound melted very gradually starting at about $120^{\circ} \mathrm{C}$, fully melting only around $180^{\circ} \mathrm{C}$. 
PEG-2Li-Me-3,2-HOPO(Bn), 2-17(Bn)2. Method A; eluent: 5\% $\mathrm{MeOH}$ in $\mathrm{CH}_{2} \mathrm{Cl}_{2}$, $\mathrm{R}_{\mathrm{f}}=0.15$. Colorless, tacky residue as the monohydrate, $44 \% . \mathrm{C}_{38} \mathrm{H}_{45} \mathrm{~N}_{5} \mathrm{O}_{10} \cdot \mathrm{H}_{2} \mathrm{O}: \mathrm{C}: 60.87$ (60.83); H: 6.32 (6.20); N: 9.34 (9.25). ${ }^{1} \mathrm{H}$ NMR: $\delta 3.27-3.40\left(\mathrm{~m}, \mathrm{CH}_{3},+\mathrm{CH}_{2}, 5 \mathrm{H}\right), \delta$ 3.42-3.47 (m, $\left.\mathrm{CH}_{2}, 4 \mathrm{H}\right), \delta 3.49-3.62\left(\mathrm{CH}_{3}+\mathrm{CH}_{2}, 12 \mathrm{H}\right), \delta 4.55$ (quartet, $\mathrm{CH}, \mathrm{J}=5.6 \mathrm{~Hz}$, 1H), $\delta 5.30(\mathrm{~s}$, benzyl $H, 2 \mathrm{H}), \delta 5.36(\mathrm{~d}$, benzyl $H, \mathrm{~J}=10.8 \mathrm{~Hz}, 1 \mathrm{H}), \delta 5.43$ (d, benzyl $H$, $\mathrm{J}=10.8 \mathrm{~Hz}, 1 \mathrm{H}), \delta 6.59(\mathrm{~d}, \mathrm{HOPO} H, \mathrm{~J}=7.2 \mathrm{~Hz}, 1 \mathrm{H}), \delta 6.95(\mathrm{t}, \mathrm{NH}, \mathrm{J}=5.2 \mathrm{~Hz}, 2 \mathrm{H}), \delta$ $7.01(\mathrm{t}, \mathrm{HOPO} H, \mathrm{~J}=6.8 \mathrm{~Hz}, 2 \mathrm{H}), \delta 7.21-7.25(\mathrm{~m}$, arom. $H, 6 \mathrm{H}), \delta$ 7.36-7.43 (m, arom. $H, 4 \mathrm{H}), \delta 8.27(\mathrm{t}, \mathrm{NH}, \mathrm{J}=6.0 \mathrm{~Hz}, \delta 8.68(\mathrm{~d}, \mathrm{NH}, \mathrm{J}=7.2 \mathrm{~Hz}, 1 \mathrm{H}) .13 \mathrm{C}$ NMR: $\delta 37.71$, $37.74,39.38,41.63,53.76,59.00,69.49,70.24,70.42,71.87,74.06,74.18,104.50$, $105.64,128.50,128.56,128.66,129.04,129.31,129.84,130.27,130.45,131.99,132.10$ $136.02,136.12,146.25,146.40,159.41,159.44,163.71,164.17,169.43 . \mathrm{MS}(\mathrm{FAB}+)$ : $\mathrm{m} / \mathrm{z} 732.5(\mathrm{MH}+)$.

PEG-3Li-Me-3,2-HOPO(Bn), 2-18(Bn)2. Method B; eluent: 15\% MeOH in EtOAc, $\mathrm{R}_{\mathrm{f}}=$ 0.11. Colorless, tacky residue, $44 \% . \mathrm{C}_{39} \mathrm{H}_{47} \mathrm{~N}_{5} \mathrm{O}_{10}$ : C: 62.81 (62.61); H: 6.35 (6.47); $\mathrm{N}: 9.39$ (9.33). ${ }^{1} \mathrm{H}$ NMR: $\delta$ 1.34-1.39 (m, $\left.\mathrm{CH}_{2}, 1 \mathrm{H}\right), \delta 1.67-1.72\left(\mathrm{~m}, \mathrm{CH}_{2}, 1 \mathrm{H}\right), \delta 2.77-$ $2.82\left(\mathrm{~m}, \mathrm{CH}_{2}, 1 \mathrm{H}\right), \delta 3.25\left(\mathrm{~s}, \mathrm{CH}_{3}, 3 \mathrm{H}\right), \delta 3.36-3.52\left(\mathrm{CH}_{3}+\mathrm{CH}_{2}, 19 \mathrm{H}\right), \delta 4.40$ (quartet, $\mathrm{CH}, \mathrm{J}=7.2 \mathrm{~Hz}, 1 \mathrm{H}), \delta 5.32(\mathrm{~d}$, benzyl $H, \mathrm{~J}=10.8 \mathrm{~Hz}, 1 \mathrm{H}), \delta 5.40(\mathrm{~d}$, benzyl $H, \mathrm{~J}=11.6$ $\mathrm{Hz}, 3 \mathrm{H}), \delta 6.56(\mathrm{~d}, \mathrm{HOPO} H, \mathrm{~J}=7.2 \mathrm{~Hz}, 1 \mathrm{H}), \delta 6.61(\mathrm{~d}, \mathrm{HOPO} H, \mathrm{~J}=7.2 \mathrm{~Hz}, 1 \mathrm{H}), \delta$ $7.00(\mathrm{t}, \mathrm{HOPO} H, \mathrm{~J}=6.8 \mathrm{~Hz}, 2 \mathrm{H}), \delta 7.20-7.22(\mathrm{~m}$, arom. $H+\mathrm{N} H, 6 \mathrm{H}), \delta 7.35-7.36(\mathrm{~m}$, arom. $H, 2 \mathrm{H}), \delta 7.42-7.43(\mathrm{~m}$, arom. $H, 3 \mathrm{H}), \delta 8.21(\mathrm{t}, \mathrm{NH}, \mathrm{J}=5.2 \mathrm{~Hz}, 1 \mathrm{H}), \delta 8.59(\mathrm{~d}$, $\mathrm{N} H, \mathrm{~J}=7.2 \mathrm{~Hz}, 1 \mathrm{H}) .{ }^{13} \mathrm{C} \mathrm{NMR}: \delta 33.36,36.17,37.62,37.65,39.30,51.12,53.51,58.89$, $69.46,70.11,70.33,70.40,71.78,74.16,74.37,77.43,104.46,104.64,116.29,128.46$, $128.52,128.59,128.99,129.47,129.92,130.32,131.91,132.05,135.98,136.31,146.29$, 
159.43, 162.85, 163.77, 170.60. MS (FAB+): m/z $746.34(\mathrm{MH}+), 768.32(\mathrm{MNa}+)$, $784.29(\mathrm{MK}+)$.

PEG-4Li-Me-3,2-HOPO(Bn) 2, 2-19(Bn) 2. Method B; eluent: 5\% MeOH in $\mathrm{CH}_{2} \mathrm{Cl}_{2}$, $\mathrm{R}_{\mathrm{f}}=0.08$. Colorless semisolid, $40 \% . \mathrm{C}_{40} \mathrm{H}_{49} \mathrm{~N}_{5} \mathrm{O}_{10}$ : C: 63.23 (63.18); H: $6.50(6.65) ; \mathrm{N}$ : 9.22 (9.03). ${ }^{1} \mathrm{H}$ NMR: $\delta$ 1.10-1.18 (m, $\left.\mathrm{CH}_{2}, 3 \mathrm{H}\right), \delta 1.49$ (s, br, $\left.\mathrm{CH}_{2}, 1 \mathrm{H}\right), \delta 2.92-2.94(\mathrm{~m}$, $\left.\mathrm{CH}_{2}, 1 \mathrm{H}\right), \delta 3.22-3.27\left(\mathrm{~m}, \mathrm{CH}_{2}, 1 \mathrm{H}\right), \delta 3.30\left(\mathrm{~s}, \mathrm{CH}_{3}, 3 \mathrm{H}\right), \delta 3.35-3.36\left(\mathrm{~m}, \mathrm{CH}_{2}, 2 \mathrm{H}\right), \delta$ 3.45-3.49 (m, $\left.\mathrm{CH}_{2}, 4 \mathrm{H}\right), \delta 3.53-3.56\left(\mathrm{~m}, \mathrm{CH}_{2}+\mathrm{CH}_{3}, 12 \mathrm{H}\right), \delta 4.43$ (quartet, $\mathrm{CH}, \mathrm{J}=5.2$ $\mathrm{Hz}, 1 \mathrm{H}), \delta 5.29$ (s, benzyl $H, 2 \mathrm{H}), \delta 5.40$ (s, benzyl $H, 2 \mathrm{H}), \delta 6.61(\mathrm{~s}, \mathrm{br}, \mathrm{N} H, 1 \mathrm{H}), \delta 6.67$ (d, HOPO $H, \mathrm{~J}=7.2 \mathrm{~Hz}, 1 \mathrm{H}), \delta 6.71(\mathrm{~d}, \mathrm{HOPO} H, \mathrm{~J}=7.2 \mathrm{~Hz}, 1 \mathrm{H}), \delta 7.03(\mathrm{~d}, \mathrm{HOPO} H, \mathrm{~J}$ $=7.2 \mathrm{~Hz}, 1 \mathrm{H}), \delta 7.06(\mathrm{~d}, \mathrm{HOPO} H, \mathrm{~J}=7.2 \mathrm{~Hz}, 1 \mathrm{H}), \delta 7.22-7.34(\mathrm{~m}$, arom. $H, 8 \mathrm{H}), \delta$ 7.42-7.44 (m, arom. $H, 2 \mathrm{H}), \delta 7.82(\mathrm{t}, \mathrm{N} H, \mathrm{~J}=5.2 \mathrm{~Hz}, 1 \mathrm{H}), \delta 8.36(\mathrm{~d}, \mathrm{NH}, \mathrm{J}=8.0 \mathrm{~Hz}$, 1H). ${ }^{13} \mathrm{C}$ NMR: $\delta 25.78,30.00,37.90,37.94,38.86,39.48,52.89,59.22,69.90,70.46$, $70.65,70.69,72.10,74.65,75.15,77.43,104.87,105.09,128.86,128.96,129.02,129.15$, $129.26,129.59,129.94,130.57,132.06,132.32,136.22,136.34,146.70,146.85,159.69$, 163.41, 163.62, 171.24. MS (FAB+): m/z $760(\mathrm{MH}+)$.

PEG-5Li-Me-3,2-HOPO(Bn) 2, 2-20(Bn)2. Method B; eluent: 15\% MeOH in $\mathrm{CH}_{2} \mathrm{Cl}_{2}, \mathrm{R}_{\mathrm{f}}=0.12$. Tacky, colorless oil as the dihydrate, $35 \%$. $\mathrm{C}_{41} \mathrm{H}_{51} \mathrm{~N}_{5} \mathrm{O}_{10} \cdot 2 \mathrm{H}_{2} \mathrm{O}$ : C: 60.80 (60.80); H: 6.84 (6.83); N: 8.65 (8.51). ${ }^{1} \mathrm{H}$ NMR: $\delta$ 0.99-1.04 (m, $\left.\mathrm{CH}_{2}, 2 \mathrm{H}\right), \delta 1.08-$ $1.14\left(\mathrm{~m}, \mathrm{CH}_{2}, 2 \mathrm{H}\right), \delta 1.17-1.26\left(\mathrm{~m}, \mathrm{CH}_{2}, 1 \mathrm{H}\right), \delta 1.51-1.60\left(\mathrm{~m}, \mathrm{CH}_{2}, 1 \mathrm{H}\right), \delta 3.03$ (quartet, $\left.\mathrm{CH}_{2}, \mathrm{~J}=6.4 \mathrm{~Hz}, 2 \mathrm{H}\right), \delta 3.28\left(\mathrm{~s}, \mathrm{CH}_{3}, 3 \mathrm{H}\right), \delta 3.33-3.38\left(\mathrm{~m}, \mathrm{CH}_{2}, 2 \mathrm{H}\right), \delta 3.44-3.48(\mathrm{~m}$, $\left.\mathrm{CH}_{2}, 4 \mathrm{H}\right), \delta 3.51-3.54\left(\mathrm{~m}, \mathrm{CH}_{2}+\mathrm{CH}_{3}, 12 \mathrm{H}\right), \delta 4.29$ (quartet, $\left.\mathrm{CH}, \mathrm{J}=7.6 \mathrm{~Hz}, 1 \mathrm{H}\right), \delta 5.24$ (s, benzyl $H, 2 \mathrm{H}), \delta 5.38(\mathrm{dd}, \mathrm{J}=10.8,12.8 \mathrm{~Hz}, 2 \mathrm{H}), \delta 6.60(\mathrm{t}, \mathrm{NH}, \mathrm{J}=6.4 \mathrm{~Hz}, 1 \mathrm{H}), \delta$ $6.64(\mathrm{~d}, \mathrm{HOPO} H, \mathrm{~J}=7.2 \mathrm{~Hz}, 1 \mathrm{H}), \delta 6.68(\mathrm{~d}, \mathrm{HOPO} H, \mathrm{~J}=7.2 \mathrm{~Hz}, 1 \mathrm{H}), \delta$ 7.03-7.06 (m, 
HOPO $H, 2 \mathrm{H}), \delta 7.20-7.34(\mathrm{~m}$, arom. $H, 8 \mathrm{H}), \delta 7.43(\mathrm{~d}$, arom. $H, \mathrm{~J}=6.4 \mathrm{~Hz}, 2 \mathrm{H}), \delta 7.77$ $(\mathrm{t}, \mathrm{N} H, \mathrm{~J}=5.6 \mathrm{~Hz}, 1 \mathrm{H}), \delta 8.33(\mathrm{~d}, \mathrm{~N} H, \mathrm{~J}=7.6 \mathrm{~Hz}, 1 \mathrm{H}) .{ }^{13} \mathrm{C} \mathrm{NMR}: \delta 22.96,28.66,31.64$, $37.71,37.76,39.29,39.37,53.63,59.02,69.67,70.24,70.43,70.49,71.89,74.47,74.89$, $104.59,104.87,128.72,128.82,128.92,129.02,129.33,129.72,130.55,132.07,132.18$ $135.99,136.20,146.40,146.60,159.46,159.60,163.06,163.22,171.06 . \mathrm{MS}(\mathrm{ESI}+): \mathrm{m} / \mathrm{z}$ $774.37(\mathrm{MH}+)$.

PEG-thio-Me-3,2-HOPO(Bn)2, 2-21(Bn)2. Method A; eluent: 10\% $\mathrm{MeOH}$ in EtOAc, $\mathrm{R}_{\mathrm{f}}=0.16$. Brown, viscous oil that solidifies upon standing, $73 \%$. $\mathrm{C}_{46} \mathrm{H}_{56} \mathrm{~N}_{4} \mathrm{O}_{12} \mathrm{~S}_{3}$ : C: 57.96 (57.58); H: 5.92 (5.90); N: 5.88 (5.70); S: 10.09 (9.72). ${ }^{1} \mathrm{H}$ NMR: $\delta 2.87$ (t, $\mathrm{CH}_{2}$, $\mathrm{J}=6.8 \mathrm{~Hz}, 4 \mathrm{H}), \delta 3.36\left(\mathrm{~s}, \mathrm{CH}_{3}, 6 \mathrm{H}\right), \delta 3.50-3.62\left(\mathrm{~m}, \mathrm{CH}_{2}+\mathrm{CH}_{3}, 26 \mathrm{H}\right), \delta 5.55(\mathrm{~s}$, benzyl $H, 4 \mathrm{H}), \delta 6.67(\mathrm{~d}, \mathrm{HOPO} H, \mathrm{~J}=9.6 \mathrm{~Hz}, 2 \mathrm{H}), \delta 7.03(\mathrm{~d}, \mathrm{HOPO} H, \mathrm{~J}=9.6 \mathrm{~Hz}, 2 \mathrm{H}), \delta 7.25-$ 7.26 (m, arom. $H, 6 \mathrm{H}), \delta$ 7.50-7.52 (m, arom. $H, 4 \mathrm{H}), \delta 9.91(\mathrm{~s}, \mathrm{~N} H, 2 \mathrm{H}) .{ }^{13} \mathrm{C}$ NMR: $\delta$ $37.02,37.94,59.23,69.89,70.50,70.67,70.73,72.09,74.16,105.06,127.46,128.60$, 128.66, 129.28, 130.04, 132.08, 133.81, 136.27, 146.79, 159.62, 161.99. MS (FAB+): m/z $953.7(\mathrm{MH}+)$. MP: $80-82{ }^{\circ} \mathrm{C}$.

PEG-o-phen-Me-3,2-HOPO(Bn) $2, \quad$ 2-22(Bn) $2 . \quad$ Method A; eluent: $1: 1$ $\mathrm{MeOH} /$ EtOAc, $\mathrm{R}_{\mathrm{f}}=0.33$. Brown, viscous oil as the monohydrate, $52 \%$. $\mathrm{C}_{48} \mathrm{H}_{58} \mathrm{~N}_{4} \mathrm{O}_{14} \cdot \mathrm{H}_{2} \mathrm{O}$ : C: 61.79 (61.68); H: 6.48 (6.50); N: 6.00 (6.08). ${ }^{1} \mathrm{H}$ NMR: $\delta 3.28$ (s, $\left.\mathrm{CH}_{3}, 6 \mathrm{H}\right), \delta 3.45-3.48\left(\mathrm{~m}, \mathrm{CH}_{2}+\mathrm{CH}_{3}, 10 \mathrm{H}\right), \delta 3.56-3.67\left(\mathrm{~m}, \mathrm{CH}_{2}, 8 \mathrm{H}\right), \delta 3.67-3.69(\mathrm{~m}$, $\left.\mathrm{CH}_{2}, 4 \mathrm{H}\right), \delta 3.78\left(\mathrm{t}, \mathrm{CH}_{2}, \mathrm{~J}=4.8 \mathrm{~Hz}, 4 \mathrm{H}\right), \delta 4.00\left(\mathrm{t}, \mathrm{CH}_{2}, \mathrm{~J}=4.8 \mathrm{~Hz}, 4 \mathrm{H}\right), \delta 6.58(\mathrm{~d}$, HOPO $H, \mathrm{~J}=7.2 \mathrm{~Hz}, 2 \mathrm{H}$ ), $\delta 6.90$ (s, arom. $H, 2 \mathrm{H}), \delta 7.01$ (d, HOPO $H, \mathrm{~J}=7.2 \mathrm{~Hz}, 2 \mathrm{H}$ ), $\delta$ 7.10-7.16 (m, arom. $H, 6 \mathrm{H}), \delta 7.23-7.25$ (m, arom. $H, 4 \mathrm{H}), \delta 9.44(\mathrm{~s}, \mathrm{~N} H, 2 \mathrm{H}) .{ }^{13} \mathrm{C}$ NMR: $\delta 37.57,58.90,68.99,69.46,70.42,70.58,70.72,71.80,74.27,104.56,110.82$, 
$128.15,128.42,128.59,128.92,130.67,132.20,135.71,145.85,146.62,159.24,161.93$. MS (FAB+): m/z $915.8(\mathrm{MH}+)$.

PEG-m-xy-Me-3,2-HOPO(Bn $)_{2}$, 2-23(Bn) $)_{2}$. Method B; eluent: $4 \% \mathrm{MeOH}$ in $\mathrm{CH}_{2} \mathrm{Cl}_{2}, \mathrm{R}_{\mathrm{f}}=0.21$. Pale yellow oil as the monohydrate, $68 \% . \mathrm{C}_{43} \mathrm{H}_{48} \mathrm{~N}_{4} \mathrm{O}_{10} \cdot \mathrm{H}_{2} \mathrm{O}: \mathrm{C}: 64.65$ (64.53); H: 6.31 (6.13); N: 7.06 (7.06). ${ }^{1} \mathrm{H}$ NMR: $\delta 3.35$ (s, $\left.\mathrm{CH}_{3}, 3 \mathrm{H}\right), \delta$ 3.51-3.54 (m, $\left.\mathrm{CH}_{2}, 2 \mathrm{H}\right), \delta 3.59\left(\mathrm{~s}, \mathrm{CH}_{3}, 6 \mathrm{H}\right), \delta 3.62-3.67\left(\mathrm{~m}, \mathrm{CH}_{2}, 4 \mathrm{H}\right), \delta 3.70-3.73\left(\mathrm{~m}, \mathrm{CH}_{2}, 2 \mathrm{H}\right), \delta$ $3.81\left(\mathrm{t}, \mathrm{CH}_{2}, \mathrm{~J}=4.8 \mathrm{~Hz}, 2 \mathrm{H}\right), \delta 4.00\left(\mathrm{t}, \mathrm{CH}_{2}, \mathrm{~J}=4.8 \mathrm{~Hz}, 2 \mathrm{H}\right), \delta 4.32(\mathrm{~d}$, benzyl $H, \mathrm{~J}=5.6$ Hz, 4H), $\delta 4.53$ (s, benzyl $H, 4 \mathrm{H}), \delta 6.57$ (s, arom. $H, 1 \mathrm{H}), \delta 6.64$ (s, arom. $H, 2 \mathrm{H}), \delta 6.80$ (d, HOPO $H, \mathrm{~J}=7.2 \mathrm{~Hz}, 2 \mathrm{H}), \delta 7.12(\mathrm{~d}, \mathrm{HOPO} H, \mathrm{~J}=7.2 \mathrm{~Hz}, 2 \mathrm{H}), \delta$ 7.23-7.25 (m, arom. $H, 10 \mathrm{H}), \delta 8.24(\mathrm{t}, \mathrm{N} H, \mathrm{~J}=5.6 \mathrm{~Hz}, 2 \mathrm{H}) .{ }^{13} \mathrm{C}$ NMR: $\delta 37.90,43.77,59.23,67.58,69.85$, $70.76,70.86,71.01,72.10,74.93,105.09,113.28,119.82,128.90,129.01,129.18$ $130.50,132.32,136.00,139.91,146.71,159.58,159.75,163.26 . \mathrm{MS}$ (FAB+): 781 $(\mathrm{MH}+)$.

Et-thio-Me-3,2-HOPO(Bn $)_{2}$, 2-37(Bn) 2 . Method A; eluent: 5\% MeOH in EtOAc, $\mathrm{R}_{\mathrm{f}}$ $=0.23$. The product was recrystallized by layering $\mathrm{Et}_{2} \mathrm{O}$ on a concentrated $\mathrm{CH}_{2} \mathrm{Cl}_{2}$ solution to yield a beige, crystalline solid, $66 \% . \mathrm{C}_{36} \mathrm{H}_{36} \mathrm{~N}_{4} \mathrm{O}_{6} \mathrm{~S}_{3}$ : C: 60.31 (60.02); H: 5.06 (5.26); N: 7.82 (7.69); S: 13.42 (13.25). ${ }^{1} \mathrm{H}$ NMR: $\delta 1.15$ (t, $\left.\mathrm{CH}_{3}, \mathrm{~J}=7.2 \mathrm{~Hz}, 6 \mathrm{H}\right), \delta 2.65$ (quartet, $\mathrm{CH}_{2}, \mathrm{~J}=7.2 \mathrm{~Hz}, 4 \mathrm{H}$ ), $\delta 3.52\left(\mathrm{~s}, \mathrm{CH}_{3}, 6 \mathrm{H}\right), \delta 5.53$ (s, benzyl $\left.H, 4 \mathrm{H}\right), \delta 6.67$ (d, HOPO $H, \mathrm{~J}=7.2 \mathrm{~Hz}, 2 \mathrm{H}$ ), $\delta 7.02(\mathrm{~d}, \mathrm{HOPO} H, \mathrm{~J}=7.2 \mathrm{~Hz}, 2 \mathrm{H}$ ), $\delta$ 7.22-7.26 (m, arom. $H$, $6 \mathrm{H}), \delta$ 7.48-7.50 (m, arom. $H, 4 \mathrm{H}), \delta 9.90(\mathrm{~s}, \mathrm{~N} H, 2 \mathrm{H}) .{ }^{13} \mathrm{C}$ NMR: $\delta 14.88,31.89,37.79$, 74.04, 104.92, 127.41, 128.47, 128.52, 129.12, 129.97, 132.06, 133.24, 136.16, 146.67, 159.52, 161.82. MS (FAB+): m/z $717(\mathrm{MH}+)$. MP: $172-174{ }^{\circ} \mathrm{C}$. 
Pr-o-phen-Me-3,2-HOPO(Bn) $)_{2}$, 2-38(Bn) $)_{2}$. Method A; eluent: 2\% $\mathrm{MeOH}$ in $\mathrm{CH}_{2} \mathrm{Cl}_{2}, \mathrm{R}_{\mathrm{f}}=0.26\left(4 \% \mathrm{MeOH}\right.$ in $\left.\mathrm{CH}_{2} \mathrm{Cl}_{2}\right)$. The product was recrystallized by layering $\mathrm{Et}_{2} \mathrm{O}$ on a concentrated $\mathrm{CH}_{2} \mathrm{Cl}_{2}$ solution of the crude material. Beige crystals, $70 \%$. $\mathrm{C}_{40} \mathrm{H}_{42} \mathrm{~N}_{4} \mathrm{O}_{8}: \mathrm{C}: 67.97$ (67.58); H: 5.99 (6.19); N: 7.93 (7.80). ${ }^{1} \mathrm{H}$ NMR: $\delta 1.03$ (s, $\mathrm{CH}_{3}$, $6 \mathrm{H}), \delta 1.82$ (sextet, $\left.\mathrm{CH}_{2}, \mathrm{~J}=7.2 \mathrm{~Hz}, 4 \mathrm{H}\right), \delta 3.58\left(\mathrm{~s}, \mathrm{CH}_{3}, 6 \mathrm{H}\right), \delta 5.41$ (s, benzyl $\left.H, 4 \mathrm{H}\right), \delta$ $3.87\left(\mathrm{t}, \mathrm{CH}_{2}, \mathrm{~J}=6.8 \mathrm{~Hz}, 4 \mathrm{H}\right), \delta 6.69(\mathrm{~d}, \mathrm{HOPO} H, \mathrm{~J}=7.2 \mathrm{~Hz}, 2 \mathrm{H}), 6.92(\mathrm{~s}, \operatorname{arom} . H, 2 \mathrm{H})$, $\delta 7.08(\mathrm{~d}, \mathrm{HOPO} H, \mathrm{~J}=7.2 \mathrm{~Hz}, 2 \mathrm{H}), \delta$ 7.18-7.24 (m, arom. $H, 6 \mathrm{H}), \delta 7.31-7.34$ (m, arom. $H, 4 \mathrm{H}), \delta 9.50(\mathrm{~s}, \mathrm{~N} H, 2 \mathrm{H}) .{ }^{13} \mathrm{C}$ NMR: $\delta 10.68,22.70,37.91,71.06,74.57,105.00$, $110.40,123.19,128.69,128.82,129.24,130.95,132.32,135.98,146.21,147.20,159.58$, 162.20. $\mathrm{MS}(\mathrm{FAB}+): \mathrm{m} / \mathrm{z} 707(\mathrm{MH}+) . \mathrm{MP}: 180-182^{\circ} \mathrm{C}$.

2-Benzyloxy-5-methyl-a, $\alpha^{\prime}-m$-xylene-bis-Me-3,2-HOPO(Bn)2, 2-39(Bn) $)_{3}$. Method B; eluent: 4\% MeOH in $\mathrm{CH}_{2} \mathrm{Cl}_{2}, \mathrm{R}_{\mathrm{f}}=0.19$. Colorless solid, $64 \%$. This material was used without performing elemental analysis. ${ }^{1} \mathrm{H}$ NMR: $\delta 2.21\left(\mathrm{~s}, \mathrm{CH}_{3}, 3 \mathrm{H}\right), \delta 3.58\left(\mathrm{~s}, \mathrm{CH}_{3}\right.$, 6H), $\delta 4.44\left(\mathrm{~d}, \mathrm{CH}_{2}, \mathrm{~J}=6.0 \mathrm{~Hz}, 4 \mathrm{H}\right), \delta 4.67$ (s, benzyl H, 2H), $\delta 5.27$ (s, benzyl H, 4H), $\delta$ $6.78(\mathrm{~d}, \mathrm{HOPO} H, \mathrm{~J}=7.2 \mathrm{~Hz}, 2 \mathrm{H}), \delta 6.93(\mathrm{~s}$, arom. $H, 2 \mathrm{H}), \delta 7.10(\mathrm{~d}, \mathrm{HOPO} H, \mathrm{~J}=7.2$ $\mathrm{Hz}, 2 \mathrm{H}), \delta 7.14-7.20(\mathrm{~m}$, arom. $H, 10 \mathrm{H}), \delta 7.30-7.33(\mathrm{~m}$, arom. $H, 5 \mathrm{H}), \delta 8.26(\mathrm{t}, \mathrm{N} H, \mathrm{~J}=$ $6.0 \mathrm{~Hz}) .{ }^{13} \mathrm{C}$ NMR: $\delta 37.91,38.83,74.65,76.46,105.11,128.18,128.47,128.75,128.82$, $128.87,129.08,129.71,130.70,131.21,132.26,134.65,135.99,136.89,146.54,152.95$ 159.76, 163.34. MS (FAB+): m/z $739(\mathrm{MH}+)$. MP: $275-76^{\circ} \mathrm{C}(\mathrm{dec})$.

2-Methoxy-5-methyl- $\alpha, \alpha^{\prime}-m$-xylene-bis-Me-3,2-HOPO(Bn $)_{2}, 2-40(B n)_{2}$. Method B; eluent: $2 \% \mathrm{MeOH}$ in $\mathrm{CH}_{2} \mathrm{Cl}_{2}, \mathrm{R}_{\mathrm{f}}=0.07$. Off-white needles crystallized by layering Et2O on a concentrated $\mathrm{CH} 2 \mathrm{Cl} 2$ solution of the product, $76 \% \mathrm{C}_{38} \mathrm{H}_{38} \mathrm{~N}_{4} \mathrm{O}_{7}$ : C: 68.87 (68.73); H: 5.78 (5.97); N: 8.45 (8.43). ${ }^{1} \mathrm{H}$ NMR: $\delta 2.18\left(\mathrm{~s}, \mathrm{CH}_{3}, 3 \mathrm{H}\right), \delta 3.50$ (s, $\mathrm{CH}_{3}$, 
$3 \mathrm{H}), \delta 3.56\left(\mathrm{~s}, \mathrm{CH}_{3}, 6 \mathrm{H}\right), \delta 4.42\left(\mathrm{~d}, \mathrm{CH}_{2}, \mathrm{~J}=6.0 \mathrm{~Hz}, 4 \mathrm{H}\right), \delta 5.27$ (s, benzyl $\left.H, 4 \mathrm{H}\right), \delta 6.78$ (d, HOPO $H, \mathrm{~J}=7.2 \mathrm{~Hz}, 2 \mathrm{H}$ ), $\delta 6.90$ (s, arom. $H, 2 \mathrm{H}$ ), $\delta 7.09$ (d, HOPO $H, \mathrm{~J}=7.2 \mathrm{~Hz}$, $2 \mathrm{H}), \delta$ 7.19-7.23 (m, arom. $H, 10 \mathrm{H}), \delta 8.33(\mathrm{t}, \mathrm{N} H, \mathrm{~J}=5.2 \mathrm{~Hz}, 2 \mathrm{H}) .{ }^{13} \mathrm{C}$ NMR: $\delta 20.95$, $37.83,38.74,61.61,74.61,104.97,128.68,128.79,129.02,129.89,130.56,130.77$, 132.26, 134.32, 135.93, 146.51, 154.37, 159.65, 163.15. MS (FAB+): m/z $663(\mathrm{MH}+)$. MP: $174-176^{\circ} \mathrm{C}$.

\subsubsection{Benzyl Deprotection of bis-Me-3,2-HOPO ligands}

General deprotection strategy for bis-Me-3,2-HOPO ligands: A solution of 1-10 mmol of benzyl-protected ligand in 5-10 $\mathrm{mL}$ of $1: 1 \mathrm{conc}$. $\mathrm{HCl} / \mathrm{AcOH}$ was stirred at room temperature for 3-5 days, in some cases resulting in a suspension. The majority of the acids and freed benzyl alcohol were removed under vacuum. Unless otherwise indicated, the residue was suspended in cold $\mathrm{MeOH}$, filtered, and washed with more cold $\mathrm{MeOH}$. The resulting solid cake was allowed to dry under aspiration, ground into a fine powder and dried in a desiccators under vacuum over $\mathrm{P}_{2} \mathrm{O}_{5}$. The solid was removed from the desiccator and let stand overnight open to air overnight, during which time mass gain stopped, resulting in amorphous solids.

Thio-Me-3,2-HOPO, 2-6. Beige solid, 99\%. $\mathrm{C}_{18} \mathrm{H}_{16} \mathrm{~N}_{4} \mathrm{O}_{6} \mathrm{~S}$ : C: 51.92 (51.73); H: 3.87 (3.83); N: 13.45 (13.15); S: 7.70 (7.55). ${ }^{1} \mathrm{H}$ NMR (DMSO- $\left.d_{6}\right): \delta 3.53\left(\mathrm{~s}, \mathrm{CH}_{3}, 6 \mathrm{H}\right), \delta 6.60$ (d, HOPO $H, \mathrm{~J}=7.2 \mathrm{~Hz}, 2 \mathrm{H}), \delta 7.27$ (d, HOPO $H, \mathrm{~J}=7.2 \mathrm{~Hz}, 2 \mathrm{H}), \delta 7.83(\mathrm{~s}, \mathrm{CH}, 2 \mathrm{H}), \delta$ $10.08(\mathrm{~s}, \mathrm{~N} H, 2 \mathrm{H}) .{ }^{13} \mathrm{C}$ NMR (DMSO- $\left.d_{6}\right): \delta 36.97,103.69,112.84,117.99,128.10$, 128.43, 145.94, 158.26, 162.53. MS (FAB+): $\mathrm{m} / \mathrm{z} 417(\mathrm{MH}+)$. MP: $>315^{\circ} \mathrm{C}$.

o-Phen-Me-3,2-HOPO, 2-7. Beige powder, 90\%. $\mathrm{C}_{20} \mathrm{H}_{18} \mathrm{~N}_{4} \mathrm{O}_{6}$ : C: 58.53 (58.29); H: 4.42 (4.45); N: 13.65 (13.33). ${ }^{1} \mathrm{H}$ NMR (DMSO- $\left.d_{6}\right): \delta 3.51\left(\mathrm{~s}, \mathrm{CH}_{3}, 6 \mathrm{H}\right), \delta 5.76$ (s, 
benzyl $H, 4 \mathrm{H}$ ), $\delta 6.61$ (d, HOPO $H, 7.2 \mathrm{~Hz}, 2 \mathrm{H}), \delta$ 7.23-7.27 (m, arom. $H$, HOPO $H, 4 \mathrm{H}$ ), $\delta$ 7.79-7.82 (dd, arom. $H, \mathrm{~J}=4.0,5.6 \mathrm{~Hz}, 2 \mathrm{H}), \delta 10.09(\mathrm{~s}, \mathrm{NH}, 2 \mathrm{H}) .{ }^{13} \mathrm{C}$ NMR (DMSO$\left.d_{6}\right): \delta 37.32,103.84,118.34,125.31,126.05,128.28,130.90,147.20,158.64,163.93 . \mathrm{MS}$ (FAB+): m/z $411(\mathrm{MH}+)$. MP: $307-309^{\circ} \mathrm{C}(\mathrm{dec})$.

o-Tol-Me-3,2-HOPO, 2-8. White powder as the partial hydrate, 97\%. $\mathrm{C}_{21} \mathrm{H}_{20} \mathrm{~N}_{4} \mathrm{O}_{6}{ }^{3 / 4} / 4 \mathrm{H}_{2} \mathrm{O}$ : C: 57.60 (57.51); H: 4.95 (4.98); N: 12.97 (12.69). ${ }^{1} \mathrm{H}$ NMR (DMSO-d $\left.d_{6}\right): \delta 3.47\left(\mathrm{~s}, \mathrm{CH}_{3}, 3 \mathrm{H}\right), \delta 3.52\left(\mathrm{~s}, \mathrm{CH}_{3}, 3 \mathrm{H}\right), \delta 4.50\left(\mathrm{~d}, \mathrm{CH}_{2}, \mathrm{~J}=6.0 \mathrm{~Hz}, 2 \mathrm{H}\right), \delta$ $6.50(\mathrm{~d}, \mathrm{HOPO} H, \mathrm{~J}=7.2 \mathrm{~Hz}, 1 \mathrm{H}), \delta 6.58(\mathrm{~d}, \mathrm{HOPO} H, \mathrm{~J}=7.2 \mathrm{~Hz}, 1 \mathrm{H}), \delta$ 7.18-7.23 (m, $\operatorname{arom} H$, HOPO $H, 2 \mathrm{H}), \delta 7.26(\mathrm{~d}, \mathrm{HOPO} H, \mathrm{~J}=7.2 \mathrm{~Hz}, 1 \mathrm{H}), \delta 7.31(\mathrm{t}, \operatorname{arom} H, \mathrm{~J}=6.4$ $\mathrm{Hz}, 1 \mathrm{H}), \delta 7.37(\mathrm{~d}$, arom $H, \mathrm{~J}=8 \mathrm{~Hz}, 1 \mathrm{H}), \delta 7.71(\mathrm{~d}$, $\operatorname{arom} H, \mathrm{~J}=7.6 \mathrm{~Hz}, 1 \mathrm{H}), \delta 8.91$ (t, $\mathrm{N} H, \mathrm{~J}=6.0 \mathrm{~Hz}, 1 \mathrm{H}), \delta 10.22(\mathrm{~s}, \mathrm{~N} H, 1 \mathrm{H}) .{ }^{13} \mathrm{C} \mathrm{NMR}\left(\mathrm{DMSO}-d_{6}\right): \delta 36.86,36.92,102.85$, $103.18,117.24,118.54,124.67,125.64,127.47,127.80,128.08,128.33,131.71,135.08$ 146.60, 147.24, 158.08, 158.16, 163.68, 165.38. MS (FAB+): m/z 425 (MH+). MP: 303$305^{\circ} \mathrm{C}(\mathrm{dec})$.

m-Phen-Me-3,2-HOPO, 2-9. Beige powder as the partial methanol solvate, 73\%. $\mathrm{C}_{20} \mathrm{H}_{18} \mathrm{~N}_{4} \mathrm{O}_{6} \cdot 2 / 3 \mathrm{CH}_{3} \mathrm{OH}: \mathrm{C}: 57.49$ (57.71); $\mathrm{H}: 4.82$ (4.81); N: 12.98 (12.65). ${ }^{1} \mathrm{H}$ NMR (DMSO- $d_{6}$ ): $\delta 3.51(\mathrm{~s}, \mathrm{CH} 3,6 \mathrm{H}), \delta 6.51(\mathrm{~d}, \mathrm{HOPO} H, \mathrm{~J}=7.2 \mathrm{~Hz}, 2 \mathrm{H}), \delta 7.26(\mathrm{~d}, \mathrm{HOPO}$ $H, \mathrm{~J}=7.2 \mathrm{~Hz}, 2 \mathrm{H}), \delta 7.33(\mathrm{t}$, arom. $H, \mathrm{~J}=8.0 \mathrm{~Hz}, 1 \mathrm{H}), \delta 7.46(\mathrm{dd}$, arom. $H, \mathrm{~J}=8.0,1.6$ $\mathrm{Hz}, 2 \mathrm{H}), \delta 8.04$ (s, arom. $H, 1 \mathrm{H}), \delta 10.26(\mathrm{~s}, \mathrm{~N} H, 2 \mathrm{H}) .{ }^{13} \mathrm{C}$ NMR (DMSO- $\left.d_{6}\right): \delta 36.95$, $103.43,111.45,115.69,119.59,128.20,129.29,138.67,145.65,158.23,163.22$. MP: 298-299 ${ }^{\circ} \mathrm{C}(\mathrm{dec}) . \mathrm{MS}(\mathrm{FAB}+): \mathrm{m} / \mathrm{z} 411(\mathrm{MH}+)$.

Py-Me-3,2-HOPO, 2-10. Beige powder as the dihydrate, $62 \%$. $\mathrm{C}_{19} \mathrm{H}_{17} \mathrm{~N}_{5} \mathrm{O}_{6} \cdot 2 \mathrm{H}_{2} \mathrm{O}$ : C: 51.01 (51.40); H: 4.73 (4.69); N: 15.65 (15.64). ${ }^{1} \mathrm{H}$ NMR (DMSO- $d_{6}$ ): $\delta 3.52$ (s, $\mathrm{CH}_{3}$, 
$6 \mathrm{H}), \delta 6.62(\mathrm{~d}, \mathrm{HOPO} H, \mathrm{~J}=7.2 \mathrm{~Hz}, 2 \mathrm{H}), \delta 7.27(\mathrm{~d}, \mathrm{HOPO} H, \mathrm{~J}=7.6 \mathrm{~Hz}, 2 \mathrm{H}), \delta 7.90(\mathrm{t}$, arom. $H, \mathrm{~J}=7.6 \mathrm{~Hz}, 1 \mathrm{H}), \delta 8.03(\mathrm{~d}$, arom. $H, \mathrm{~J}=8.0 \mathrm{~Hz}, 2 \mathrm{H}), \delta 10.45(\mathrm{~s}, \mathrm{~N} H, 2 \mathrm{H})$. The ligand is not soluble enough in DMSO- $d_{6}$ or basic $\mathrm{D}_{2} \mathrm{O}$ to get a ${ }^{13} \mathrm{C}$ spectrum. MS (FAB+): m/z $412(\mathrm{MH}+)$. MP: $332-333{ }^{\circ} \mathrm{C}(\mathrm{dec}$.$) .$

Naphth-Me-3,2-HOPO, 2-11. Light brown powder, 84\%. $\mathrm{C}_{24} \mathrm{H}_{20} \mathrm{~N}_{4} \mathrm{O}_{6}:$ C: 62.60 (62.27); H: 4.38 (4.48); N: 12.17 (11.94). ${ }^{1} \mathrm{H}$ NMR ( $\mathrm{D}_{2} \mathrm{O} / \mathrm{NaOD}, \mathrm{pD} \approx 13$ ): $\delta 3.43$ (s, $\left.\mathrm{CH}_{3}, 6 \mathrm{H}\right), \delta 6.43(\mathrm{~d}, \mathrm{HOPO} H, \mathrm{~J}=7.2 \mathrm{~Hz}, 2 \mathrm{H}), \delta 6.51(\mathrm{~d}, \mathrm{HOPO} H, \mathrm{~J}=7.2 \mathrm{~Hz}, 2 \mathrm{H}), \delta$ $7.46(\mathrm{~d}$, arom. $H, \mathrm{~J}=7.2 \mathrm{~Hz}, 4 \mathrm{H}), \delta 7.62(\mathrm{t}$, arom. $H, \mathrm{~J}=8.0,4 \mathrm{H}), \delta 8.02(\mathrm{~d}$, arom. $H, \mathrm{~J}=$ $8.0 \mathrm{~Hz}) .{ }^{13} \mathrm{C}$ NMR $\left(\mathrm{D}_{2} \mathrm{O} / \mathrm{NaOD}, \mathrm{pD} \approx 13\right): \delta 37.54,81.08,107.29,114.95,119.19$, 126.04, 127.14, 128.74, 131.21, 135.78, 160.54, 163.98, 169.60. MS (FAB+): m/z 461.1 $(\mathrm{MH}+)$. MP: $\sim 350^{\circ} \mathrm{C}(\mathrm{dec})$.

Biph-Me-3,2-HOPO, 2-12. Gray powder, 54\%. $\mathrm{C}_{26} \mathrm{H}_{22} \mathrm{~N}_{4} \mathrm{O}_{6}$ : C: 64.19 (63.99); H: 4.56 (4.66); $\mathrm{N}: 11.52$ (11.29). ${ }^{1} \mathrm{H}$ NMR $\left(\mathrm{D}_{2} \mathrm{O} / \mathrm{NaOD}, \mathrm{pD} \approx 13\right): \delta 3.45$ (s, $\left.\mathrm{CH}_{3}, 6 \mathrm{H}\right), \delta$ $6.61(\mathrm{~d}, \mathrm{HOPO} H, \mathrm{~J}=7.2 \mathrm{~Hz}, 2 \mathrm{H}), \delta 6.67(\mathrm{~d}, \mathrm{HOPO} H, \mathrm{~J}=7.2 \mathrm{~Hz}, 2 \mathrm{H}), \delta$ 7.33-7.37 (m, arom. $H, 4 \mathrm{H}), \delta 7.50-7.57(\mathrm{~m}$, arom. $H, 2 \mathrm{H}), \delta 8.07(\mathrm{~d}$, arom. $H, \mathrm{~J}=8.4 \mathrm{~Hz}, 2 \mathrm{H}) .{ }^{13} \mathrm{C}$ NMR $\left(\mathrm{D}_{2} \mathrm{O} / \mathrm{NaOD}, \mathrm{pD} \approx 13\right): \delta 37.50,106.70,114.90,120.11,123.91,125.35,128.78$, 130.93, 131.95, 135.93, 161.10, 164.25, 168.39. MS (FAB+): m/z 487.3 (MH+). MP: $307-308^{\circ} \mathrm{C}(\mathrm{dec})$.

m-Tol-Me-3,2-HOPO, 2-13. Beige powder, 82\%. $\mathrm{C}_{21} \mathrm{H}_{20} \mathrm{~N}_{4} \mathrm{O}_{6}$ : C: 59.43 (59.07); H: 4.75 (4.93); N: 13.20 (12.81). ${ }^{1} \mathrm{H}$ NMR (DMSO- $\left.d_{6}\right): \delta 3.34$ (s, $\left.\mathrm{CH}_{3}, 3 \mathrm{H}\right), \delta 3.36$ (s, $\mathrm{CH}_{3}$, $3 \mathrm{H}), \delta 4.42\left(\mathrm{~s}, \mathrm{CH}_{2}, 2 \mathrm{H}\right), \delta 6.40(\mathrm{~d}, \mathrm{HOPO} H, \mathrm{~J}=7.2 \mathrm{~Hz}, 1 \mathrm{H}), \delta 6.45(\mathrm{~d}, \mathrm{HOPO} H, \mathrm{~J}=$ $7.2 \mathrm{~Hz}, 1 \mathrm{H}), \delta 6.56(\mathrm{t}, \mathrm{HOPO} H, \mathrm{~J}=7.2 \mathrm{~Hz}, 2 \mathrm{H}), \delta 6.90(\mathrm{~d}$, arom. $H, \mathrm{~J}=7.2 \mathrm{~Hz}, 1 \mathrm{H}), \delta$ $7.20(\mathrm{t}, \operatorname{arom} . H, \mathrm{~J}=7.8 \mathrm{~Hz}, 1 \mathrm{H}), \delta 7.49(\mathrm{~s}$, arom. $H, 1 \mathrm{H}), \delta 7.61(\mathrm{~d}$, arom. $H, \mathrm{~J}=8.4 \mathrm{~Hz}$, 
$1 \mathrm{H}), \delta 11.67(\mathrm{t}, \mathrm{N} H, \mathrm{~J}=5.4 \mathrm{~Hz}, 1 \mathrm{H}), \delta 13.94(\mathrm{~s}, \mathrm{~N} H, 1 \mathrm{H}) .{ }^{13} \mathrm{C}$ NMR (DMSO- $\left.d_{6}\right): \delta$ $36.58,42.03,106.04,106.77,111.75,116.27,117.00,117.48,117.95,121.40,128.80$, $140.27,140.96,162.39,162.63,164.57,166.10,167.81 . \mathrm{MS}(\mathrm{FAB}+): \mathrm{m} / \mathrm{z} 425(\mathrm{MH}+)$. MP: $302-304{ }^{\circ} \mathrm{C}(\mathrm{dec})$.

o-Xy-Me-3,2-HOPO, 2-14. White powder as the sesquihydrate, 62\%. $\mathrm{C}_{22} \mathrm{H}_{22} \mathrm{~N}_{4} \mathrm{O}_{6} \cdot 1.5 \mathrm{H}_{2} \mathrm{O}: \quad \mathrm{C}: 56.77$ (57.17); H: 5.41 (5.22); N: 12.04 (11.95). ${ }^{1} \mathrm{H}$ NMR (DMSO- $\left.d_{6}\right): \delta 3.47\left(\mathrm{~s}, \mathrm{CH}_{3}, 6 \mathrm{H}\right), \delta 4.58\left(\mathrm{~d}, \mathrm{CH}_{2}, \mathrm{~J}=4.8 \mathrm{~Hz}, 4 \mathrm{H}\right), \delta 6.54(\mathrm{~d}, \mathrm{HOPO} H, \mathrm{~J}=$ $7.2 \mathrm{~Hz}, 2 \mathrm{H}), \delta 7.19(\mathrm{~d}, \mathrm{HOPO} H, \mathrm{~J}=7.2 \mathrm{~Hz}, 2 \mathrm{H}), \delta 7.27-7.30$ (m, arom. $H, 4 \mathrm{H}), \delta 8.82$ (s, NH, 2H). ${ }^{13} \mathrm{C}$ NMR (DMSO- $d_{6}$ ): $\delta 36.85,102.71,117.19,127.23,127.79,127.98$, 136.21, 147.48, 158.04, 165.28. MS (FAB+): m/z $460(\mathrm{MNa}+)$. MP: $310-312^{\circ} \mathrm{C}(\mathrm{dec})$.

m-Xy-bis-Me-3,2-HOPO, 2-15. Off-white powder as the methanol solvate, 98\%. $\mathrm{C}_{22} \mathrm{H}_{22} \mathrm{~N}_{4} \mathrm{O}_{6} \cdot \mathrm{MeOH}: \quad \mathrm{C}: 58.72$ (58.52); H: 5.57 (5.47); N: 11.91 (11.87). ${ }^{1} \mathrm{H}$ NMR (DMSO- $\left.d_{6}\right): \delta 3.16\left(\mathrm{~s}, \mathrm{CH}_{3} \mathrm{OH}, 3 \mathrm{H}\right), \delta 3.47\left(\mathrm{~s}, \mathrm{CH}_{3}, 6 \mathrm{H}\right), \delta 4.48\left(\mathrm{~d}, \mathrm{CH}_{2}, \mathrm{~J}=6.0 \mathrm{~Hz}, 4 \mathrm{H}\right)$, $\delta 6.52(\mathrm{~d}, \mathrm{HOPO} H, \mathrm{~J}=7.2 \mathrm{~Hz}, 2 \mathrm{H}), \delta$ 7.18-7.21 (m, arom. $H+\mathrm{HOPO} H, 4 \mathrm{H}), \delta 7.26-$ $7.31(\mathrm{~m}$, arom. $H, 2 \mathrm{H}), \delta 8.90(\mathrm{t}, \mathrm{N} H, \mathrm{~J}=6.0 \mathrm{~Hz}, 2 \mathrm{H}) .{ }^{13} \mathrm{C} \mathrm{NMR}\left(\mathrm{D}_{2} \mathrm{O} / \mathrm{NaOD}, \mathrm{pD} \approx 13\right)$ : $22.20,37.72,42.38,81.36,106.91,115.00,120.04,124.07,125.40,139.08,164.53$, 170.04. MS (FAB): $\mathrm{m} / \mathrm{z} 439.3(\mathrm{MH}+)$. MP: $301-302{ }^{\circ} \mathrm{C}(\mathrm{dec})$.

Fluo-Me-3,2-HOPO, 2-16. Pale yellow powder as the hemihydrate, 93\%. $\mathrm{C}_{27} \mathrm{H}_{22} \mathrm{~N}_{4} \mathrm{O}_{6} \cdot 1 / 2 \mathrm{H}_{2} \mathrm{O}: \quad \mathrm{C}: 63.90$ (63.59); H: 4.57 (4.52); N: 11.04 (10.68). ${ }^{1} \mathrm{H}$ NMR (DMSO- $\left.d_{6}\right): \delta 3.58\left(\mathrm{~s}, \mathrm{CH}_{3}, 6 \mathrm{H}\right), \delta 3.89\left(\mathrm{~s}, \mathrm{CH}_{2}, 2 \mathrm{H}\right), \delta 6.72(\mathrm{~d}$, arom. $H, \mathrm{~J}=7.2 \mathrm{~Hz}$, 2H), $\delta 7.34(\mathrm{~d}$, arom. $H, \mathrm{~J}=7.2 \mathrm{~Hz}, 2 \mathrm{H}), \delta 7.46(\mathrm{t}$, arom. $H, \mathrm{~J}=7.6 \mathrm{~Hz}, 2 \mathrm{H}), \delta 7.74(\mathrm{~d}$, arom. $H, \mathrm{~J}=7.6 \mathrm{~Hz}, 2 \mathrm{H}), \delta 8.26(\mathrm{~d}$, arom. $H, \mathrm{~J}=8.0 \mathrm{~Hz}, 2 \mathrm{H}), \delta 10.30(\mathrm{~s}, \mathrm{NH}, 2 \mathrm{H})$. The 
compound was not soluble enough to obtain a ${ }^{13} \mathrm{C}$ NMR spectrum. MS (FAB+): m/z 499 $(\mathrm{MH}+) . \mathrm{MP}:>315^{\circ} \mathrm{C}$.

PEG-2Li-Me-3,2-HOPO, 2-17. After initial acid removal the residue was coevaporated with $\mathrm{MeOH}$ then $\mathrm{CHCl}_{3}(2 \times 5 \mathrm{~mL}$ each), and the ligand was held under vacuum for at least a day to remove solvent before use. Beige semi-solid isolated as the monohydrate, 86\%. $\mathrm{C}_{24} \mathrm{H}_{33} \mathrm{~N}_{5} \mathrm{O}_{10} \cdot \mathrm{H}_{2} \mathrm{O}: \mathrm{C}: 50.61$ (50.85); H: 6.19 (6.00); N: 12.30 (12.15). ${ }^{1} \mathrm{H}$ NMR (DMSO- $\left.d_{6}\right): \delta 3.22\left(\mathrm{~s}, \mathrm{CH}_{3}, 6 \mathrm{H}\right), \delta 3.38-3.48\left(\mathrm{~m}, \mathrm{CH}_{2}+\mathrm{CH}_{3}, 15 \mathrm{H}\right), \delta$ 3.60-3.70 (m, $\left.\mathrm{CH}_{2}, 2 \mathrm{H}\right), \delta 4.70$ (quartet, $\left.\mathrm{CH}, \mathrm{J}=6.4 \mathrm{~Hz}, 1 \mathrm{H}\right), \delta$ 6.49-6.53 (m, HOPO $H$, 2H), $\delta$ 7.17-7.20 (m, HOPO H, 2H), $\delta 8.29(\mathrm{t}, \mathrm{NH}, \mathrm{J}=5.6 \mathrm{~Hz}, 1 \mathrm{H}), \delta 8.48(\mathrm{t}, \mathrm{N} H, \mathrm{~J}=5.6$ $\mathrm{Hz}, 1 \mathrm{H}), \delta 8.77(\mathrm{~d}, \mathrm{~N} H, \mathrm{~J}=7.6 \mathrm{~Hz}, 1 \mathrm{H}) .{ }^{13} \mathrm{C}\left(\mathrm{DMSO}-d_{6}\right): \delta 36.86,36.88,38.70,52.55$, $58.04,68.76,69.57,69.65,71.25,103.04,103.38,117.17,117.46,127.67,127.72$, 146.53, 147.03, 158.12, 158.21, 163.97, 165.10, 169.09. MS (FAB+): $552(\mathrm{MH}+)$.

PEG-3Li-Me-3,2-HOPO, 2-18. After acid removal, workup of this ligand followed the procedure for 2-17. Beige semi-solid isolated as the monohydrate, $99 \%$. $\mathrm{C}_{25} \mathrm{H}_{35} \mathrm{~N}_{5} \mathrm{O}_{10} \cdot \mathrm{H}_{2} \mathrm{O}: \mathrm{C}: 51.45$ (51.83); H: 6.39 (6.24); N: 12.00 (11.90). ${ }^{1} \mathrm{H}$ NMR: $\delta$ 1.951.99 (m, br, $\left.\mathrm{CH}_{2}, 1 \mathrm{H}\right), \delta 2.17-2.18$ (m, br, $\left.\mathrm{CH}_{2}, 1 \mathrm{H}\right), \delta 3.16-3.18\left(\mathrm{~m}, \mathrm{br}, \mathrm{CH}_{2}, 1 \mathrm{H}\right), \delta 3.27$ (s, $\left.\mathrm{CH}_{3}, 3 \mathrm{H}\right), \delta 3.36-3.44\left(\mathrm{~m}, \mathrm{CH}_{2}, 4 \mathrm{H}\right), \delta 3.51-3.54\left(\mathrm{~m}, \mathrm{CH}_{3}+\mathrm{CH}_{2}, 14 \mathrm{H}\right), \delta 3.80-3.82$ (m, br, $\mathrm{CH}_{2}, 1 \mathrm{H}$ ), $\delta 4.83$ (quartet, $\mathrm{CH}, \mathrm{J}=5.6 \mathrm{~Hz}, 1 \mathrm{H}$ ), $\delta 6.61$ (d, HOPO H, J = 7.2 Hz, 1H), $\delta 6.68(\mathrm{~d}, \mathrm{HOPO} H, \mathrm{~J}=7.2 \mathrm{~Hz}, 1 \mathrm{H}), \delta 6.76(\mathrm{~d}, \mathrm{HOPO} H, \mathrm{~J}=7.2 \mathrm{~Hz}, 1 \mathrm{H}), \delta 6.81(\mathrm{~d}$, HOPO $H, \mathrm{~J}=7.2 \mathrm{~Hz}, 1 \mathrm{H}), \delta 7.96(\mathrm{~s}, \mathrm{br}, \mathrm{N} H, 1 \mathrm{H}), \delta 8.44$ (s, br, NH, 1H), $\delta 6.65$ (d, NH, J $=7.2 \mathrm{~Hz}, 1 \mathrm{H}) .{ }^{13} \mathrm{C} \mathrm{NMR}: \delta 33.61,36.17,37.74,39.50,51.35,58.99,69.48,70.16,70.37$, $70.48,71.86,77.43,104.13,105.15,116.72,117.28,126.78,127.01,146.58,148.41$ 158.75, 158.84, 164.64, 166.10, 171.39. MS (FAB+): m/z $566(\mathrm{MH}+)$. 
PEG-4Li-Me-3,2-HOPO, 2-19. After acid removal, workup of this ligand followed the procedure for 2-17. Beige semi-solid isolated as the hydrochloride adduct, $84 \%$. $\mathrm{C}_{26} \mathrm{H}_{37} \mathrm{~N}_{5} \mathrm{O}_{10} \cdot \mathrm{HCl}: \mathrm{C}: 50.69$ (51.01); H: 6.27 (6.37); N: 11.37 (11.07). ${ }^{1} \mathrm{H}$ NMR: $\delta 1.72-$ $1.74\left(\mathrm{~m}, \mathrm{CH}_{2}, 2 \mathrm{H}\right), \delta 1.84-1.88\left(\mathrm{~m}, \mathrm{CH}_{2}, 1 \mathrm{H}\right), \delta 1.98-2.00\left(\mathrm{~m}, \mathrm{CH}_{2}, 1 \mathrm{H}\right), \delta 3.36\left(\mathrm{~s}, \mathrm{CH}_{3}\right.$, $3 \mathrm{H}), \delta$ 3.44-3.46 (m, $\left.\mathrm{CH}_{2}, 4 \mathrm{H}\right), \delta 3.52-3.60\left(\mathrm{~m}, \mathrm{CH}_{2}+\mathrm{CH}_{3}, 16 \mathrm{H}\right), \delta 4.81$ (quartet, $\mathrm{CH}, \mathrm{J}$ $=4.8 \mathrm{~Hz}, 1 \mathrm{H}), \delta 6.75(\mathrm{~d}, \mathrm{HOPO} H, \mathrm{~J}=7.2 \mathrm{~Hz}, 1 \mathrm{H}), \delta 6.84-6.87(\mathrm{~m}, \mathrm{HOPO} H, 3 \mathrm{H}), \delta$ 7.27 (s, br, NH,1H), $\delta 8.06$ (s, br, NH,1H), $\delta 8.39(\mathrm{~d}, \mathrm{~N} H, \mathrm{~J}=7.6 \mathrm{~Hz}, 1 \mathrm{H}) .{ }^{13} \mathrm{C}$ NMR: $\delta$ $25.64,30.54,37.84,37.90,39.19,39.58,52.98,59.18,69.71,70.35,70.55,70.65,72.05$, $77.43,105.46,105.93,117.49,118.12,127.13,146.29,146.47,159.04,159.08,164.42$ 164.86, 171.79. MS (FAB+): m/z 580.5 (MH+).

PEG-5Li-Me-3,2-HOPO, 2-20. After acid removal, workup of this ligand followed the procedure for 2-17. Beige semi-solid isolated as the dihydrate, quantitative. $\mathrm{C}_{27} \mathrm{H}_{39} \mathrm{~N}_{5} \mathrm{O}_{10} \cdot 2\left(\mathrm{H}_{2} \mathrm{O}\right)$ : C: 51.50 (51.37); H: 6.88 (6.71); N: 11.12 (10.80). ${ }^{1} \mathrm{H}$ NMR: $\delta 1.48$ (m, br, $\left.\mathrm{CH}_{2}, 2 \mathrm{H}\right), \delta 1.64\left(\mathrm{~m}, \mathrm{br}, \mathrm{CH}_{2}, 2 \mathrm{H}\right), \delta 1.485\left(\mathrm{~m}, \mathrm{br}, \mathrm{CH}_{2}, 1 \mathrm{H}\right), \delta 1.96\left(\mathrm{~m}, \mathrm{br}, \mathrm{CH}_{2}\right.$, $1 \mathrm{H}), \delta 3.35\left(\mathrm{~s}, \mathrm{CH}_{3}, 3 \mathrm{H}\right), \delta 3.42-3.44\left(\mathrm{~m}, \mathrm{CH}_{2}, 4 \mathrm{H}\right), \delta 3.51-3.59\left(\mathrm{~m}, \mathrm{CH}_{2}+\mathrm{CH}_{3}, 16 \mathrm{H}\right), \delta$ 4.64 (quartet, $\mathrm{CH}, \mathrm{J}=5.6 \mathrm{~Hz}, 1 \mathrm{H}), \delta 6.74(\mathrm{~d}, \mathrm{HOPO} H, \mathrm{~J}=7.2 \mathrm{~Hz}, 1 \mathrm{H}), \delta 6.84(\mathrm{~s}, \mathrm{br}$, HOPO $H, 3 \mathrm{H}$ ), $\delta 7.18$ (s, br, NH,1H), $\delta 7.99$ (s, br, NH,1H), $\delta 8.34$ (d, NH, J =7.2 Hz, $1 \mathrm{H}), \delta 8.75$ (s, br, $\mathrm{OH}, 2 \mathrm{H}) .{ }^{13} \mathrm{C}$ NMR: $\delta 22.92,28.95,32.14,37.85,37.90,39.48,39.57$, $53.72,59.15,69.65,70.32,70.52,70.62,72.02,77.43,105.56,106.14,117.68,118.43$, $127.10,127.16,146.12,146.44,158.92,164.42,171.81 . \mathrm{MS}(\mathrm{FAB}+): \mathrm{m} / \mathrm{z} 594.3(\mathrm{MH}+)$, $616.3(\mathrm{MNa}+)$.

PEG-thio-Me-3,2-HOPO, 2-21. After acid removal, workup of this ligand followed the procedure for 2-17. Red/brown viscous oil, quantitative. $\mathrm{C}_{32} \mathrm{H}_{44} \mathrm{~N}_{4} \mathrm{O}_{12} \mathrm{~S}_{3}$ : C: 49.73 
(49.35); H: 5.74 (5.70); N: 7.25 (7.13); S: 12.45 (12.80). ${ }^{1} \mathrm{H}$ NMR: $\delta 3.01$ (t, $\mathrm{CH}_{2}, \mathrm{~J}=6.8$ $\mathrm{Hz}, 4 \mathrm{H}), \delta 3.36\left(\mathrm{~s}, \mathrm{CH}_{3}, 6 \mathrm{H}\right), \delta 3.52-3.54\left(\mathrm{~m}, \mathrm{CH}_{2}, 4 \mathrm{H}\right), \delta 3.58-3.67\left(\mathrm{~m}, \mathrm{CH}_{3}+\mathrm{CH}_{2}\right.$, 22H), $\delta 6.76(\mathrm{~d}, \mathrm{HOPO} H, \mathrm{~J}=7.2 \mathrm{~Hz}, 2 \mathrm{H}), \delta 6.86(\mathrm{~d}, \mathrm{HOPO} H, \mathrm{~J}=7.2 \mathrm{~Hz}, 2 \mathrm{H}), \delta 9.77$ (s, NH, 2H). ${ }^{13} \mathrm{C}$ NMR: $\delta 37.27,37.79,59.20,69.94,70.51,70.71,72.12,105.36,117.14$, $127.12,127.62,133.89,146.89,159.05,162.98 . \mathrm{MS}(\mathrm{FAB}+): \mathrm{m} / \mathrm{z} 773(\mathrm{MH}+)$.

PEG-o-phen-Me-3,2-HOPO, 2-22. After acid removal, the residue was recrystallized from $\mathrm{MeOH}$. Yellow solid, 78\%. $\mathrm{C}_{34} \mathrm{H}_{46} \mathrm{~N}_{4} \mathrm{O}_{14}$ : C: 55.58 (55.29); H: 6.31 (6.39); N: 7.63 (7.55). ${ }^{1} \mathrm{H}$ NMR (DMSO- $d_{6}$ ): $\delta 3.22$ (s, $\left.\mathrm{CH}_{3}, 6 \mathrm{H}\right), \delta 3.41-3.43\left(\mathrm{~m}, \mathrm{CH}_{2}\right.$, $4 \mathrm{H}), \delta 3.50-3.54\left(\mathrm{~m}, \mathrm{CH}_{2}+\mathrm{CH}_{3}, 14 \mathrm{H}\right), \delta 3.60-3.62\left(\mathrm{~m}, \mathrm{CH}_{2}, 4 \mathrm{H}\right), \delta 3.75\left(\mathrm{t}, \mathrm{CH}_{2}, \mathrm{~J}=4.4\right.$ Hz, 4H), $\delta 4.09$ (t, $\left.\mathrm{CH}_{2}, \mathrm{~J}=4.4 \mathrm{~Hz}, 4 \mathrm{H}\right), \delta 6.61(\mathrm{~d}, \mathrm{HOPO} H, \mathrm{~J}=7.2 \mathrm{~Hz}, 2 \mathrm{H}), \delta 7.23(\mathrm{~d}$, HOPO $H, \mathrm{~J}=7.2 \mathrm{~Hz}, 2 \mathrm{H}), \delta 7.46$ (s, arom. $H, 2 \mathrm{H}), \delta 9.97$ (s, NH, 2H), $\delta 11.34$ (s, br, $\mathrm{O} H, 2 \mathrm{H}) .{ }^{13} \mathrm{C}$ NMR (DMSO- $\left.d_{6}\right): 36.91,58.01,68.64,68.91,69.62,69.84 .70 .01,71.29$, $103.38,111.09,117.83,123.87,127.34,145.66,146.99,158.28,163.46 . \mathrm{MS}(\mathrm{FAB}+)$ : m/z $735(\mathrm{MH}+)$. MP: $184-186^{\circ} \mathrm{C}$.

PEG-m-xy-Me-3,2-HOPO, 2-23. After acid removal, the residue was dissolved in warm $\mathrm{MeOH}$ and added dropwise to rapidly-stirred $\mathrm{Et}_{2} \mathrm{O}$ in an ice bath. The precipitated solid was filtered, washed with $\mathrm{Et}_{2} \mathrm{O}$, and dried as the general procedure describes. White solid as the monohydrate, $94 \%$. $\mathrm{C}_{29} \mathrm{H}_{36} \mathrm{~N}_{4} \mathrm{O}_{10} \cdot \mathrm{H}_{2} \mathrm{O}$ : C: 56.30 (56.65); $\mathrm{H}: 6.19$ (6.14); N: 9.06 (8.96). ${ }^{1} \mathrm{H}$ NMR (DMSO- $\left.d_{6}\right): \delta 3.21\left(\mathrm{~s}, \mathrm{CH}_{3}, 3 \mathrm{H}\right), \delta 3.40\left(\mathrm{t}, \mathrm{CH}_{2}, \mathrm{~J}=4.4 \mathrm{~Hz}, 4 \mathrm{H}\right), \delta$ 3.47-3.52 (m, $\left.\mathrm{CH}_{2}+\mathrm{CH}_{3}, 10 \mathrm{H}\right), \delta 3.55-3.56\left(\mathrm{~m}, \mathrm{CH}_{2}, 2 \mathrm{H}\right), \delta 3.71\left(\mathrm{t}, \mathrm{CH}_{2}, \mathrm{~J}=4.4 \mathrm{~Hz}\right.$, $2 \mathrm{H}), \delta 4.04\left(\mathrm{t}, \mathrm{CH}_{2}, \mathrm{~J}=4.4 \mathrm{~Hz}, 2 \mathrm{H}\right), \delta 4.45\left(\mathrm{~d}, \mathrm{CH}_{2}, \mathrm{~J}=5.6 \mathrm{~Hz}, 4 \mathrm{H}\right), \delta 6.52(\mathrm{~d}, \mathrm{HOPO} H$, $\mathrm{J}=7.2 \mathrm{~Hz}, 2 \mathrm{H}), \delta 6.79(\mathrm{~s}$, arom. $H, 2 \mathrm{H}), \delta 6.84(\mathrm{~s}$, arom. $H, 1 \mathrm{H}), \delta 7.19(\mathrm{~d}, \mathrm{HOPO} H, \mathrm{~J}=$ $7.2 \mathrm{~Hz}, 2 \mathrm{H}), \delta 8.88(\mathrm{t}, \mathrm{N} H, \mathrm{~J}=5.6 \mathrm{~Hz}, 2 \mathrm{H}) .{ }^{13} \mathrm{C}$ NMR $\left(\mathrm{DMSO}-d_{6}\right): \delta 36.90,42.49,58.05$, 
$67.08,68.92,69.61,69.79,69.94,71.28,102.69,111.91,117.91,127.84,140.65,147.55$, 158.08, 158.73, 165.44. MS (FAB+): $601(\mathrm{MH}+)$. MP: $116-118^{\circ} \mathrm{C}$.

Et-thio-Me-3,2-HOPO, 2-37. Beige, microcrystalline solid recrystallized from $\mathrm{MeOH}, 74 \% . \mathrm{C}_{22} \mathrm{H}_{24} \mathrm{~N}_{4} \mathrm{O}_{6} \mathrm{~S}_{3}:$ C: 49.24 (48.99); H: 4.51 (4.40); N: 10.44 (10.17); S: 17.93 (17.58). ${ }^{1} \mathrm{H}$ NMR (DMSO- $\left.d_{6}\right): \delta 1.18\left(\mathrm{t}, \mathrm{CH}_{3}, \mathrm{~J}=7.2 \mathrm{~Hz}, 6 \mathrm{H}\right.$ ), $\delta 2.83$ (quartet, $\mathrm{J}=7.2 \mathrm{~Hz}$, $4 \mathrm{H}), \delta 3.48\left(\mathrm{~s}, \mathrm{CH}_{3}, 6 \mathrm{H}\right), \delta 6.58(\mathrm{~d}, \mathrm{HOPO} H, \mathrm{~J}=7.2 \mathrm{~Hz}, 2 \mathrm{H}), \delta 7.21(\mathrm{~d}, \mathrm{HOPO} H, \mathrm{~J}=$ 7.2, 2H), $\delta 9.94(\mathrm{~s}, \mathrm{~N} H, 2 \mathrm{H}) .{ }^{13} \mathrm{C}$ NMR (DMSO- $\left.d_{6}\right): \delta 14.66,30.88,36.91,103.41$, $117.13,126.82,127.97,134.32,146.83,158.14,162.93 . \mathrm{MS}(\mathrm{FAB}+): \mathrm{m} / \mathrm{z} 537(\mathrm{MH}+)$. MP: $217-219^{\circ} \mathrm{C}$.

Pr-o-phen-Me-3,2-HOPO, 2-38. Yellow powder that analysis showed was the hemihydrate, 85\%. $\mathrm{C}_{26} \mathrm{H}_{30} \mathrm{~N}_{4} \mathrm{O}_{8} \cdot 1 / 2 \mathrm{H}_{2} \mathrm{O}$ : C: 58.31 (58.27); H: 5.83 (6.09); N: 10.46 (10.33). ${ }^{1} \mathrm{H}$ NMR (DMSO- $\left.d_{6}\right): \delta 0.99\left(\mathrm{t}, \mathrm{CH}_{3}, \mathrm{~J}=7.2 \mathrm{~Hz}, 6 \mathrm{H}\right), \delta 1.74\left(\right.$ sextet, $\mathrm{CH}_{2}, \mathrm{~J}=$ $7.2 \mathrm{~Hz}, 4 \mathrm{H}), \delta 3.50\left(\mathrm{~s}, \mathrm{CH}_{3}, 6 \mathrm{H}\right), \delta 3.92\left(\mathrm{t}, \mathrm{CH}_{2}, \mathrm{~J}=6.4 \mathrm{~Hz}, 4 \mathrm{H}\right), \delta 6.61(\mathrm{~d}, \mathrm{HOPO} H, \mathrm{~J}=$ $7.6 \mathrm{~Hz}, 2 \mathrm{H}), \delta 7.23$ (d, HOPO $H, \mathrm{~J}=7.6 \mathrm{~Hz}, 2 \mathrm{H}), \delta 7.42$ (s, arom. $H, 2 \mathrm{H}), \delta 9.96(\mathrm{~s}, \mathrm{~N} H$, 2H). ${ }^{13} \mathrm{C}$ NMR (DMSO- $\left.d_{6}\right): \delta 10.43,22.15,36.93,70.19,103.40,110.60,117.87,123.55$, 127.84, 145.84, 146.89, 158.23, 163.46. MS (FAB+): m/z 527 (MH+). MP: $241-243{ }^{\circ} \mathrm{C}$ (dec.).

2-OH-5-Me-m-xy-Me-3,2-HOPO, 2-39. Beige solid isolated as the methanolic hemihydrate (78\%). $\mathrm{C}_{23} \mathrm{H}_{24} \mathrm{~N}_{4} \mathrm{O}_{7^{1}} \frac{1}{2} \mathrm{H}_{2} \mathrm{O} \cdot \mathrm{MeOH}: \mathrm{C}: 56.58$ (56.95); $\mathrm{H}: 5.74$ (5.57); N: 11.00 (10.84). ${ }^{1} \mathrm{H}$ NMR (DMSO- $\left.d_{6}\right): \delta 3.17\left(\mathrm{~s}, \mathrm{CH}_{3}, 3 \mathrm{H}\right), \delta 3.48\left(\mathrm{~s}, \mathrm{CH}_{3}, 6 \mathrm{H}\right), \delta 4.44(\mathrm{~d}$, $\left.\mathrm{CH}_{2}, \mathrm{~J}=6.0 \mathrm{~Hz}, 4 \mathrm{H}\right), \delta 6.55(\mathrm{~d}, \mathrm{HOPO} H, \mathrm{~J}=7.2 \mathrm{~Hz}, 2 \mathrm{H}), \delta 6.96(\mathrm{~s}$, arom. $H, 2 \mathrm{H}), \delta$ $7.20(\mathrm{~d}, \mathrm{HOPO} H, \mathrm{~J}=7.2 \mathrm{~Hz}, 2 \mathrm{H}), \delta 8.90(\mathrm{t}, \mathrm{N} H, \mathrm{~J}=6.0 \mathrm{~Hz}, 2 \mathrm{H}), \delta 9.47(\mathrm{~s}, \mathrm{OH}, 1 \mathrm{H}) .{ }^{13} \mathrm{C}$ 
NMR (DMSO- $\left.d_{6}\right): \delta 20.27,36.92,48.64,103.08,117.26,125.52,127.78,127.89,129.15$, 147.00, 150.61, 158.15, 165.34. MS (FAB+): m/z $469(\mathrm{MH}+)$. MP: $283-285^{\circ} \mathrm{C}$ (dec.).

2-OMe-5-Me-m-xy-Me-3,2-HOPO, 2-40. Beige solid isolated as the monohydrate. $\mathrm{C}_{24} \mathrm{H}_{26} \mathrm{~N}_{4} \mathrm{O}_{7} \cdot \mathrm{H}_{2} \mathrm{O}: \mathrm{C}: 57.59$ (57.37); H: 5.64 (5.94); N: 11.19 (10.96). ${ }^{1} \mathrm{H}$ NMR (DMSO$\left.d_{6}\right): \delta 2.20\left(\mathrm{~s}, \mathrm{CH}_{3}, 3 \mathrm{H}\right), \delta 3.48\left(\mathrm{~s}, \mathrm{CH}_{3}, 6 \mathrm{H}\right), \delta 3.76\left(\mathrm{~s}, \mathrm{CH}_{3}, 3 \mathrm{H}\right), \delta 4.52(\mathrm{~d}, \mathrm{CH} 2, \mathrm{~J}=5.2$ $\mathrm{Hz}, 4 \mathrm{H}$ ), $\delta 6.56$ (d, HOPO H, J = 7.2 Hz, 2H), $\delta 7.01$ (s, arom. $H, 2 \mathrm{H}$ ), $\delta 7.20$ (d, HOPO $H, \mathrm{~J}=7.2 \mathrm{~Hz}, 2 \mathrm{H}), \delta 8.81(\mathrm{t}, \mathrm{N} H, \mathrm{~J}=5.6 \mathrm{~Hz}, 2 \mathrm{H}) .{ }^{13} \mathrm{C}$ NMR (DMSO-d 6 ): $\delta 20.67,36.90$, $37.63,61.09,102.85,117.38,127.90,128.15,131.32,133.04,147.34,153.42,158.13$, 165.20. MS (FAB+): $\mathrm{m} / \mathrm{z} 483(\mathrm{MH}+)$. MP: $263-265^{\circ} \mathrm{C}(\mathrm{dec}$.

\subsubsection{Synthesis/Crystallization Techniques for Uranyl complexes}

General. Unless otherwise stated, one equivalent of bis-Me-3,2-HOPO was suspended in $10 \mathrm{~mL}$ of $\mathrm{MeOH}$ and two equivalents of methanolic $\mathrm{KOH}(0.5051 \mathrm{M}$, Aldrich) was added. The suspension was stirred for three minutes, in most cases becoming a homogeneous solution. This ligand solution was added to a stirred solution of 1-1.2 equivalents of $\mathrm{UO}_{2}\left(\mathrm{NO}_{3}\right)_{2} \cdot 6 \mathrm{H}_{2} \mathrm{O}$ in $4 \mathrm{~mL}$ of $\mathrm{MeOH}$. The resulting reddish suspension was brought to reflux and allowed to stir overnight. The solution was cooled, filtered and washed with $\mathrm{MeOH}$. The solid is dried under vacuum to remove residual $\mathrm{MeOH}$ and then exposed to atmosphere until the sample mass stabilizes.

$\mathrm{UO}_{2}$ (2Li-Me-3,2-HOPO), $\mathbf{U O}_{2}$ (2-2). Synthesis of this complex is described in the literature. ${ }^{1}$ In order to grow X-ray quality crystals, 2-2 (68 mg, $\left.0.19 \mathrm{mmol}\right)$ was dissolved in $10 \mathrm{~mL}$ with 15 drops of $\mathrm{Et}_{3} \mathrm{~N}$. This blue solution was added to a stirred solution of $\mathrm{UO}_{2}\left(\mathrm{NO}_{3}\right)_{2} \cdot 6 \mathrm{H}_{2} \mathrm{O}(86 \mathrm{mg}, 0.17 \mathrm{mmol})$. After a short-lived precipitate re-dissolved, the red solution was stirred at room temperature overnight, followed by removal of the 
solvent under vacuum. The residue was stirred in $1 \mathrm{~mL}$ of 1:1 DMF:DMSO and insoluble material was removed by filtration through a small plug of celite. Diffusion of $\mathrm{MeOH}$ into this solution at room temperature yielded very small, X-ray quality crystals.

$\mathrm{UO}_{\mathbf{2}}$ (thio-Me-3,2-HOPO), $\mathrm{UO}_{2}(\mathbf{2}-6)$. The $\mathrm{UO}_{2}(\mathbf{2 - 6})(\mathrm{DMF})$ complex was crystallized by diffusing $\mathrm{MeOH}$ at room temperature into a solution of 2-6 (23 $\mathrm{mg}, 0.055 \mathrm{mmol})$, $\mathrm{UO}_{2}\left(\mathrm{NO}_{3}\right)_{2} \cdot 6 \mathrm{H}_{2} \mathrm{O}(28 \mathrm{mg}, 0.056 \mathrm{mmol})$ and two drops of $\mathrm{Et}_{3} \mathrm{~N}$ in $5 \mathrm{~mL}$ of DMF, yielding $38 \mathrm{mg}$ of a dark red crystalline solid, $92 \%$. These crystals were suitable for single crystal X-ray diffraction. $\mathrm{C}_{18} \mathrm{H}_{14} \mathrm{~N}_{4} \mathrm{O}_{8} \mathrm{SU} \cdot \mathrm{C}_{3} \mathrm{H}_{7} \mathrm{NO}: \mathrm{C}: 33.30$ (33.21); $\mathrm{H}: 2.79$ (2.57); N: 9.25 (9.15); S: 4.23 (3.85). ${ }^{1} \mathrm{H}$ NMR (DMSO- $d_{6}$ ): $\delta 2.73$ (s, DMF $\mathrm{CH}_{3}, 6 \mathrm{H}$ ), $\delta 2.89$ (s, DMF $\left.\mathrm{CH}_{3}, 6 \mathrm{H}\right), \delta 4.00\left(\mathrm{~s}, \mathrm{CH}_{3}, 6 \mathrm{H}\right), \delta 7.18(\mathrm{~d}, \mathrm{HOPO} H, \mathrm{~J}=7.2 \mathrm{~Hz}, 2 \mathrm{H}), \delta 7.35(\mathrm{~d}, \mathrm{HOPO} H$, $\mathrm{J}=7.2 \mathrm{~Hz}, 2 \mathrm{H}), \delta 7.95(\mathrm{~s}, \mathrm{DMF} \mathrm{CH}, 2 \mathrm{H}), \delta 8.24(\mathrm{~s}, \mathrm{CH}, 2 \mathrm{H}), \delta 13.05(\mathrm{~s}, \mathrm{~N} H, 2 \mathrm{H}) .{ }^{13} \mathrm{C}$ NMR (DMSO- $\left.d_{6}\right): \delta 30.77,35.78,37.66,109.06,110.02,120.27,125.00,127.93,159.50$ 161.97, 162.31, 168.18. MS (FAB+): m/z $685(\mathrm{MH}+)$.

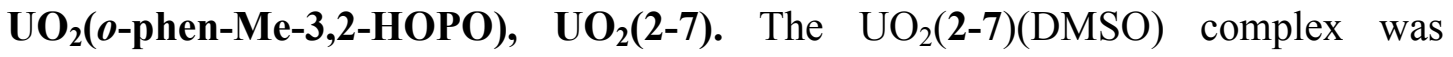
isolated cleanly by diffusing $\mathrm{Et}_{2} \mathrm{O}$ into a filtered solution of $\mathbf{2 - 7}(21.2 \mathrm{mg}, 0.0515 \mathrm{mmol})$, $\mathrm{UO}_{2}\left(\mathrm{NO}_{3}\right)_{2} \cdot 6 \mathrm{H}_{2} \mathrm{O}(26.7 \mathrm{mg}, 0.0531 \mathrm{mmol})$ and two drops of $\mathrm{Et}_{3} \mathrm{~N}$ in $4 \mathrm{~mL}$ of DMF and 2 $\mathrm{mL}$ of DMSO, yielding a crop of red crystals, $23 \mathrm{mg}(59 \%)$. These crystals were used for single crystal X-ray diffraction characterization. $\mathrm{C}_{20} \mathrm{H}_{16} \mathrm{~N}_{4} \mathrm{O}_{8} \mathrm{U} \cdot \mathrm{C}_{2} \mathrm{H}_{6} \mathrm{OS}: \quad \mathrm{C}: 34.93$ (34.62); H: 2.93 (2.68); N: 7.41 (7.07); S: 4.24 (4.30). ${ }^{1} \mathrm{H}$ NMR (DMSO-d $\left.d_{6}\right): \delta 2.54$ (s, DMSO $\left.\mathrm{CH}_{3}, 6 \mathrm{H}\right), \delta 3.99\left(\mathrm{~s}, \mathrm{CH}_{3}, 6 \mathrm{H}\right), \delta 7.18-7.20$ (m, arom. + HOPO H, 4H), $\delta 7.32(\mathrm{~d}$, HOPO $H, 6.4 \mathrm{~Hz}, 2 \mathrm{H}), \delta 8.74(\mathrm{dd}$, arom. $H, \mathrm{~J}=4.0,6.4 \mathrm{~Hz}, 2 \mathrm{H}), \delta 12.38(\mathrm{~s}, \mathrm{NH}, 2 \mathrm{H}) .{ }^{13} \mathrm{C}$ NMR (DMSO- $\left.d_{6}\right): \delta 40.42,110.38,120.54,122.07,123.82,124.82,128.18,158.84$, 162.87, 167.65. MS (FAB+): m/z $679(\mathrm{MH}+)$. 
UO $\mathbf{O}_{2}$ (o-tol-Me-3,2-HOPO), $\quad \mathrm{UO}_{2}(\mathbf{2 - 8})$. The $\mathrm{UO}_{2}(\mathbf{2 - 8})(\mathrm{DMSO})$ complex was crystallized by diffusing $\mathrm{MeOH}$ at room temperature into a solution of $\mathbf{2 - 8} \cdot \mathbf{3} / \mathbf{4} \mathbf{H}_{\mathbf{2}} \mathbf{O}(16.5$ $\mathrm{mg}, 0.0377 \mathrm{mmol}), \mathrm{UO}_{2}\left(\mathrm{NO}_{3}\right)_{2} \cdot 6 \mathrm{H}_{2} \mathrm{O}(20.9 \mathrm{mg}, 0.0416 \mathrm{mmol})$ and three drops of $\mathrm{Et}_{3} \mathrm{~N}$ in $1 / 2 \mathrm{~mL}$ of DMSO, yielding $23 \mathrm{mg}$ of a dark red crystalline solid which analysis showed to be the monohydrate, $77 \%$. Crystals suitable for X-ray diffraction were isolated in a similar manner but from a different batch of uranyl complex. $\mathrm{C}_{21} \mathrm{H}_{18} \mathrm{~N}_{4} \mathrm{O}_{8} \mathrm{U} \cdot \mathrm{C}_{2} \mathrm{H}_{6} \mathrm{OS} \cdot \mathrm{H}_{2} \mathrm{O}: \mathrm{C}: 35.03$ (34.68); H: 3.32 (3.08); N: 7.10 (6.87); S: 4.07 (4.32). ${ }^{1} \mathrm{H}$ NMR (DMSO-d $\left.)_{6}\right): \delta 2.54$ (s, DMSO H, 6H), $\delta 3.94\left(\mathrm{~s}, \mathrm{CH}_{3}, 3 \mathrm{H}\right), \delta 4.00(\mathrm{~s}$, $\mathrm{CH}_{3}, 3 \mathrm{H}$ ), $\delta 4.65$ (s, very broad, $\mathrm{CH}_{2}, 2 \mathrm{H}$ ), $\delta 7.03$ (d, HOPO $H, \mathrm{~J}=7.2 \mathrm{~Hz}, 1 \mathrm{H}$ ), $\delta 7.21-$ $7.26(\mathrm{~m}, \mathrm{HOPO}+$ arom. $H, 3 \mathrm{H}), \delta 7.31-7.36(\mathrm{~m}, \mathrm{HOPO}+\operatorname{arom} . H, 2 \mathrm{H}), \delta 7.54(\mathrm{dd}$, arom. $H, \mathrm{~J}=7.6,1.2 \mathrm{~Hz}, 1 \mathrm{H}), \delta 7.68$ (d, arom. $H, \mathrm{~J}=7.6 \mathrm{~Hz}), \delta 11.40(\mathrm{t}, \mathrm{N} H, \mathrm{~J}=6.8 \mathrm{~Hz}$, $1 \mathrm{H}), \delta 11.62(\mathrm{~s}, \mathrm{~N} H, 1 \mathrm{H}) .{ }^{13} \mathrm{C} \mathrm{NMR}\left(\mathrm{DMSO}-d_{6}\right): \delta 37.46,37.74,40.42,109.84,110.86$, $120.57,120.98,124.07,124.93,125.48,126.81,127.98,131.47,132.51,136.79 . \mathrm{MS}$ $(\mathrm{FAB}+): \mathrm{m} / \mathrm{z} 693(\mathrm{MH}+)$.

$\mathbf{U O}_{2}$ (m-tol-Me-3,2-HOPO), $\mathbf{U O}_{2}(\mathbf{2 - 1 3})$. Red powder isolated as the dihydrate, $92 \%$. $\mathrm{C}_{21} \mathrm{H}_{18} \mathrm{~N}_{4} \mathrm{O}_{8} \mathrm{U} \cdot 2 \mathrm{H}_{2} \mathrm{O}: \mathrm{C}: 34.62$ (34.56); H: 3.04 (2.88); N: 7.69 (7.55). ${ }^{1} \mathrm{H}$ NMR (DMSO$\left.d_{6}\right): \delta 3.97\left(\mathrm{~s}, \mathrm{CH}_{3}, 3 \mathrm{H}\right), \delta 3.98\left(\mathrm{~s}, \mathrm{CH}_{3}, 3 \mathrm{H}\right), \delta 4.56\left(\mathrm{~d}, \mathrm{CH}_{2}, \mathrm{~J}=6.0 \mathrm{~Hz}, 2 \mathrm{H}\right), \delta 6.64(\mathrm{~d}$, HOPO $H, \mathrm{~J}=6.8 \mathrm{~Hz}, 1 \mathrm{H}), \delta 7.03(\mathrm{~d}$, arom. $H, \mathrm{~J}=7.6 \mathrm{~Hz}, 1 \mathrm{H}), \delta 7.10(\mathrm{~d}, \mathrm{HOPO} H, \mathrm{~J}=$ $7.2 \mathrm{~Hz}, 1 \mathrm{H}), \delta 7.21(\mathrm{~d}, \mathrm{HOPO} H, \mathrm{~J}=6.8 \mathrm{~Hz}, 1 \mathrm{H}), \delta 7.32(\mathrm{~d}, \mathrm{HOPO} H, \mathrm{~J}=7.2 \mathrm{~Hz}, 1 \mathrm{H}), \delta$ $7.38(\mathrm{t}, \operatorname{arom} H, 7.6 \mathrm{~Hz}, 1 \mathrm{H}), \delta 7.99$ (s, arom. $H, 1 \mathrm{H}), \delta 8.21(\mathrm{~d}$, arom. $H, \mathrm{~J}=8.0 \mathrm{~Hz}, 1 \mathrm{H})$, $\delta 9.14(\mathrm{t}, \mathrm{N} H, \mathrm{~J}=6.0 \mathrm{~Hz}, 1 \mathrm{H}), \delta 13.35(\mathrm{~s}, \mathrm{~N} H, 1 \mathrm{H}) .{ }^{13} \mathrm{C} \mathrm{NMR}\left(\mathrm{DMSO}-d_{6}\right): \delta 37.61$, $37.67,41.05,109.21,111.43,115.25,117.04,120.87,121.18,123.98,124.95,128.39$, $129.30,139.17,139.85,157.07,160.38,161.73,166.68,168.31,168.36 . \mathrm{MS}(\mathrm{FAB}+)$ : 
m/z $693(\mathrm{MH}+)$. X-ray quality crystals were grown by diffusing $\mathrm{MeOH}$ into a 1:1 DMF:DMSO solution of this material at $4{ }^{\circ} \mathrm{C}$.

$\mathrm{UO}_{\mathbf{2}}(\boldsymbol{o}-\mathrm{xy}-\mathrm{Me-3,2-HOPO}), \mathrm{UO}_{\mathbf{2}}(\mathbf{2 - 1 4})$. A solution of $\mathbf{2 - 1 4} \cdot \mathbf{1 . 5} \mathrm{H}_{\mathbf{2}} \mathrm{O}(22 \mathrm{mg}, 0.047$ mmol) in $4 \mathrm{~mL}$ of 1:1 DMF:DMSO and four drops of $\mathrm{Et}_{3} \mathrm{~N}$ was added to a stirred solution of $\mathrm{UO}_{2}\left(\mathrm{NO}_{3}\right)_{2} \cdot 6 \mathrm{H}_{2} \mathrm{O}(27 \mathrm{mg}, 0.053 \mathrm{mmol})$ in $2 \mathrm{~mL}$ of DMF. The red solution was stirred ten minutes and then $\mathrm{MeOH}$ was diffused into the solution at room temperature, resulting in a crop of red crystals. These were filtered, washed with $\mathrm{MeOH}$ and dried by aspiration to give the $\mathrm{UO}_{2}(\mathbf{2 - 1 4}) \cdot \mathrm{H}_{2} \mathrm{O} \cdot \mathrm{CH}_{3} \mathrm{OH}$ in quantitative yields. $\mathrm{C}_{22} \mathrm{H}_{20} \mathrm{~N}_{4} \mathrm{O}_{8} \mathrm{U} \cdot \mathrm{H}_{2} \mathrm{O} \cdot \mathrm{CH}_{3} \mathrm{OH}: \mathrm{C}: 36.52$ (36.56); H: 3.46 (3.37); N: 7.41 (7.09). ${ }^{1} \mathrm{H} \mathrm{NMR}$ (DMSO-d $): \delta 3.17\left(\mathrm{~d}, \mathrm{CH}_{3} \mathrm{OH}, \mathrm{J}=5.2 \mathrm{~Hz}, 3 \mathrm{H}\right), \delta 3.95\left(\mathrm{~s}, \mathrm{CH}_{3}, 6 \mathrm{H}\right), \delta 4.11$ (quartet, $\left.\mathrm{CH}_{3} \mathrm{OH}, \mathrm{J}=5.2 \mathrm{~Hz}, 1 \mathrm{H}\right), \delta 4.95\left(\mathrm{~d}, \mathrm{CH}_{2}, \mathrm{~J}=6.0 \mathrm{~Hz}, 4 \mathrm{H}\right), \delta 7.18(\mathrm{~d}, \mathrm{HOPO} H, \mathrm{~J}=7.2 \mathrm{~Hz}$, $2 \mathrm{H}), \delta$ 7.27-7.29 (m, HOPO + arom. $H, 4 \mathrm{H}), \delta 7.51(\mathrm{dd}$, arom. $H, \mathrm{~J}=5.6,3.6 \mathrm{~Hz}, 2 \mathrm{H}), \delta$ $10.12(\mathrm{t}, \mathrm{N} H, \mathrm{~J}=6.0 \mathrm{~Hz}, 2 \mathrm{H}) .{ }^{13} \mathrm{C}$ NMR $\left(\mathrm{DMSO}-d_{6}\right): \delta 37.71,38.23,48.61,110.75$, $121.06,124.26,128.40,131.22,138.06,159.84,163.59,168.39 . \mathrm{MS}(\mathrm{FAB}+): \mathrm{m} / \mathrm{z} 707$ $(\mathrm{MH}+)$. X-ray quality crystals were grown following a similar procedure, although from a different batch as that described here.

$\mathrm{UO}_{2}(m-x y-M e-3,2-H O P O), \mathbf{U O}_{2}(2-15) . \mathrm{Red} /$ orange solid isolated as the trihydrate, 80\%. $\mathrm{C}_{22} \mathrm{H}_{20} \mathrm{~N}_{4} \mathrm{O}_{8} \mathrm{U} \cdot 3 \mathrm{H}_{2} \mathrm{O}: \mathrm{C}: 34.75$ (34.90); H: 3.45 (3.12); N: 7.37 (7.18). ${ }^{1} \mathrm{H}$ NMR (DMSO-d $): \delta 3.97\left(\mathrm{~s}, \mathrm{CH}_{3}, 6 \mathrm{H}\right), \delta 4.71\left(\mathrm{~d}, \mathrm{CH}_{2}, \mathrm{~J}=5.2 \mathrm{~Hz}, 4 \mathrm{H}\right), \delta 7.12(\mathrm{~d}, \mathrm{HOPO} H, \mathrm{~J}=$ $7.2 \mathrm{~Hz}, 2 \mathrm{H}), \delta 7.28(\mathrm{~d}, \mathrm{HOPO} H, \mathrm{~J}=7.2 \mathrm{~Hz}, 2 \mathrm{H}), \delta 7.37(\mathrm{~s}$, arom. $H, 3 H), \delta 7.95(\mathrm{~s}$, arom. $H, 1 \mathrm{H}), \delta 9.46(\mathrm{t}, \mathrm{N} H, \mathrm{~J}=5.2 \mathrm{~Hz}, 2 \mathrm{H}) .{ }^{13} \mathrm{C}$ NMR (DMSO-d $\left.{ }_{6}\right): \delta 37.71,43.07$, $110.69,121.52,124.09,125.98,127.29,128.99,139.05,160.18,163.69,168.25 . \mathrm{MS}$ 
$(\mathrm{FAB}+): \mathrm{m} / \mathrm{z} 689[(\mathrm{M}-\mathrm{O})+]$. X-ray quality crystals of $\mathrm{UO}_{2}(\mathbf{2}-\mathbf{1 5})(\mathrm{DMF})$ were grown by diffusing $\mathrm{MeOH}$ into a DMF solution of the compound at $4{ }^{\circ} \mathrm{C}$.

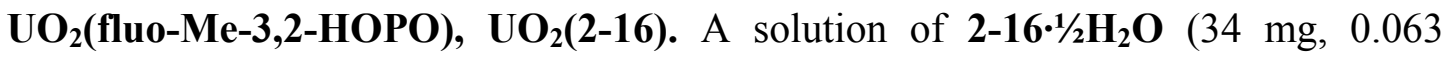
$\mathrm{mmol})$ in $4 \mathrm{~mL}$ of DMF and 3 drops of $\mathrm{Et}_{3} \mathrm{~N}$ was added to a solution of $\mathrm{UO}_{2}\left(\mathrm{NO}_{3}\right)_{2} \cdot 6 \mathrm{H}_{2} \mathrm{O}$ (35 mg, $0.070 \mathrm{mmol}$ ) in $2 \mathrm{~mL}$ of DMF. The red solution quickly became turbid, and after stirring overnight at room temperature, the DMF was removed under vacuum. The residue was suspended in $\mathrm{MeOH}$, filtered, washed with $\mathrm{MeOH}$, and dried by aspiration, yielding $50 \mathrm{mg}$ of an orange solid as the $\mathrm{UO}_{2}(\mathbf{2 - 1 6}) \cdot \mathrm{DMF} \cdot 2 \mathrm{H}_{2} \mathrm{O}$ adduct, $83 \%$. $\mathrm{C}_{27} \mathrm{H}_{20} \mathrm{~N}_{4} \mathrm{O}_{8} \mathrm{U} \cdot \mathrm{DMF} \cdot 2 \mathrm{H}_{2} \mathrm{O}: \mathrm{C}: 41.15$ (41.33); $\mathrm{H}: 3.57$ (3.39); N: 8.00 (7.67). This isolated solid was too insoluble in DMSO for NMR analysis. MS (FAB+): m/z $767(\mathrm{MH}+)$. A small crop of X-ray quality crystals were grown by slow cooling of a near-boiling

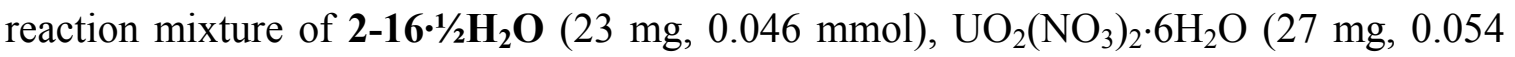
$\mathrm{mmol}$ ) and two drops of $\mathrm{Et}_{3} \mathrm{~N}$ in $6 \mathrm{~mL}$ of DMSO.

$\mathbf{U O}_{2}$ (Et-thio-Me-3,2-HOPO), $\mathbf{U O}_{2}$ (2-37). A homogeneous solution of 2-37 (11.8 $\mathrm{mg}, 0.0220 \mathrm{mmol}), \mathrm{UO}_{2}\left(\mathrm{NO}_{3}\right)_{2} \cdot 6 \mathrm{H}_{2} \mathrm{O}(11.1 \mathrm{mg}, 0.0221 \mathrm{mmol})$, and 1 drop of $\mathrm{Et}_{3} \mathrm{~N}$ in 0.5 $\mathrm{mL}$ of DMSO was layered with $\mathrm{MeOH}$ and allowed to diffuse at $4{ }^{\circ} \mathrm{C}$. Crystals formed after three weeks and were of two distinctly different habits, one orange and one red. One crystal of each habit was mounted for single crystal X-ray diffraction. Dark red blocks were revealed to be a $\left[\mathrm{UO}_{2}(\mathbf{2 - 3 7})(\mathrm{DMSO})\right]_{2}$ dimer and the orange crystals which suffered from rapid desolvation were found to be the same $\left[\mathrm{UO}_{2}(\mathbf{2 - 3 7})(\mathrm{DMSO})\right]_{2}$ dimer but with several methanol inclusions in the unit cell. NMR was performed on the filtered, crystalline samples, although their solubility was found to be quite low. ${ }^{1} \mathrm{H}$ NMR (DMSO- $d_{6}$ ): $\delta 1.23\left(\mathrm{t}, \mathrm{CH}_{3}, \mathrm{~J}=7.2 \mathrm{~Hz}, 6 \mathrm{H}\right.$ ), $\delta 2.54$ (s, DMSO CH$H_{3}, 6 \mathrm{H}$ ), $\delta 2.92$ (quartet, 
$\left.\mathrm{CH}_{2}, \mathrm{~J}=7.2 \mathrm{~Hz}, 4 \mathrm{H}\right), \delta 3.96\left(\mathrm{~s}, \mathrm{CH}_{3}, 6 \mathrm{H}\right), \delta 7.06(\mathrm{~d}, \mathrm{HOPO} H, \mathrm{~J}=6.8 \mathrm{~Hz}, 2 \mathrm{H}), \delta 7.28$ (d, HOPO $H, \mathrm{~J}=6.8 \mathrm{~Hz}, 2 \mathrm{H}), \delta 12.31(\mathrm{~s}, \mathrm{NH}, 2 \mathrm{H})$. The complex was not soluble enough for ${ }^{13} \mathrm{C}$ NMR. MS (FAB+): m/z 804 (MH+), $1307.3\left(\left[2 \mathrm{M}-\mathrm{UO}_{2}-2 \mathrm{O}\right]+\right)$.

$\mathrm{UO}_{2}\left(\mathbf{P r}-\boldsymbol{o}\right.$-phen-Me-3,2-HOPO), $\mathrm{UO}_{2}(\mathbf{2 - 3 8})$. The $\mathrm{UO}_{2}(\mathbf{2 - 3 8})(\mathrm{DMSO})$ complex was

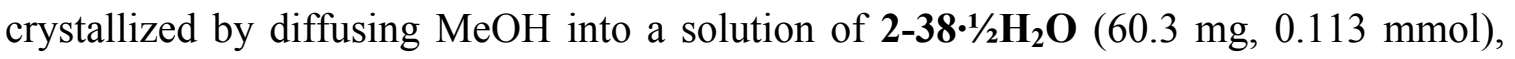
$\mathrm{UO}_{2}\left(\mathrm{NO}_{3}\right)_{2} \cdot 6 \mathrm{H}_{2} \mathrm{O}(58.7 \mathrm{mg}, 0.117 \mathrm{mmol})$ and five drops of $\mathrm{Et}_{3} \mathrm{~N}$ in $3 \mathrm{~mL}$ of DMSO at room temperature, yielding $91 \mathrm{mg}$ of a dark red crystalline solid, $92 \%$. These crystals were suitable for single crystal X-ray diffraction. ${ }^{1} \mathrm{H}$ NMR (DMSO- $\left.d_{6}\right): \delta 1.03(\mathrm{t}, \mathrm{CH}, \mathrm{J}$ $=7.2 \mathrm{~Hz}, 6 \mathrm{H}), \delta 1.78\left(\right.$ sextet, $\left.\mathrm{CH}_{2}, \mathrm{~J}=6.8 \mathrm{~Hz}, 4 \mathrm{H}\right), \delta 2.54\left(\mathrm{~s}, \mathrm{DMSO} \mathrm{CH}_{3}, 6 \mathrm{H}\right), \delta 3.96-$ $3.99\left(\mathrm{~m}, \mathrm{CH}_{3}+\mathrm{CH}_{2}, 10 \mathrm{H}\right), \delta 7.19(\mathrm{~d}, \mathrm{HOPO} H, \mathrm{~J}=7.2 \mathrm{~Hz}, 2 \mathrm{H}), \delta 7.32(\mathrm{~d}, \mathrm{HOPO} H, \mathrm{~J}=$ $7.2 \mathrm{~Hz}, 2 \mathrm{H}$ ), $\delta 8.54$ (s, arom. $H, 2 \mathrm{H}$ ), $\delta 12.32$ (s, NH,2H). ${ }^{13} \mathrm{C}$ NMR (DMSO- $d_{6}$ ): $\delta$ $10.50,22.26,37.64,40.42,70.16,108.23,110.34,120.72,121.72,124.83,143.96$, 158.74, 162.52, 167.65. $\mathrm{C}_{26} \mathrm{H}_{28} \mathrm{~N}_{4} \mathrm{O}_{10} \mathrm{U} \cdot \mathrm{C}_{2} \mathrm{H}_{6} \mathrm{OS}: \mathrm{C}: 38.54$ (38.25); $\mathrm{H}: 3.93$ (3.73); N: 6.42 (6.40); S: 3.67 (3.90). MS (FAB+): m/z $794(\mathrm{MH}+)$.

$\mathrm{UO}_{2}(2-\mathrm{OH}-5-\mathrm{Me}-\mathrm{m}-\mathrm{xy}-\mathrm{Me}-3,2-\mathrm{HOPO}), \mathrm{UO}_{2}(2-39)$. Orange solid isolated as the tetrahydrate, $87 \%$. $\mathrm{C}_{23} \mathrm{H}_{22} \mathrm{~N}_{4} \mathrm{O}_{9} \mathrm{U} \cdot 4 \mathrm{H}_{2} \mathrm{O}$ : C: 34.17 (34.12); $\mathrm{H}: 3.75$ (3.55); $\mathrm{N}: 6.93$ (6.61). ${ }^{1} \mathrm{H}$ NMR (DMSO-d $\left.\mathrm{d}_{6}\right): \delta 2.21\left(\mathrm{~s}, \mathrm{CH}_{3}, 3 \mathrm{H}\right), \delta 3.96\left(\mathrm{~s}, \mathrm{CH}_{3}, 6 \mathrm{H}\right), \delta 4.64\left(\mathrm{~s}, \mathrm{CH}_{2}, 4 \mathrm{H}\right), \delta$ 7.10-7.25 (m, HOPO $H+$ arom. $H, 6 \mathrm{H}), \delta 9.51(\mathrm{~s}, \mathrm{NH}, 2 \mathrm{H})$. The complex was not soluble enough for ${ }^{13} \mathrm{H}$ NMR. MS (FAB+): m/z $737(\mathrm{MH}+)$. X-ray quality crystals were grown by diffusion of $\mathrm{MeOH}$ into a solution of the isolated solid in DMSO at room temperature.

$\mathrm{UO}_{2}$ (2-OH-5-Me-m-xy-Me-3,2-HOPO), $\mathrm{UO}_{2}(\mathbf{2 - 4 0 )}$. Orange solid isolated as the polyhydrate, 94\%. $\mathrm{C}_{24} \mathrm{H}_{24} \mathrm{~N}_{4} \mathrm{O}_{9} \mathrm{U} \cdot 4.5 \mathrm{H}_{2} \mathrm{O}: \mathrm{C}: 34.66$ (34.32); H: 4.00 (3.64); N: 6.74 (6.49). ${ }^{1} \mathrm{H}$ NMR (DMSO- $d 6$ ): $\delta 2.28$ (s, $\left.\mathrm{CH}_{3}, 3 \mathrm{H}\right), \delta 3.89$ (s, $\left.\mathrm{CH}_{3}, 3 \mathrm{H}\right), \delta 3.96$ (s, $\mathrm{CH}_{3}$, 
$6 \mathrm{H}), \delta 4.52\left(\mathrm{~d}, \mathrm{CH}_{2}, \mathrm{~J}=14.0 \mathrm{~Hz}, 2 \mathrm{H}\right), \delta 4.85-4.90\left(\mathrm{~m}, \mathrm{CH}_{2}, 2 \mathrm{H}\right), \delta$ 7.17-7.27 (m, HOPO + arom. $H, 6 \mathrm{H}), \delta 9.66(\mathrm{~d}, \mathrm{~N} H, \mathrm{~J}=7.2 \mathrm{~Hz}, 2 \mathrm{H}) .{ }^{13} \mathrm{C}$ NMR (DMSO- $\left.d_{6}\right): \delta 20.20,37.76$, $40.58,60.22,110.79,121.07,123.95,130.89,131.09,134.04,155.89,160.53,163.43$, 168.32. MS $(\mathrm{FAB}+): \mathrm{m} / \mathrm{z} 751(\mathrm{MH}+), 789(\mathrm{MK}+)$. X-ray quality crystals were grown by diffusion of acetone into a solution of the isolated uranyl complex in DMSO at room temperature.

\subsubsection{X-ray Diffraction Data Collection}

Uranyl complex crystals were mounted on captan loops with oil and cooled under a controlled temperature stream of liquid nitrogen boil-off during data collection. X-ray diffraction data were collected using either Bruker SMART 1000 or APEX I detectors with Mo Ka radiation at the UC Berkeley X-ray crystallographic facility or with a Bruker Platinum 200 or APEX II detector with synchrotron radiation $(\mathrm{hv}=16 \mathrm{keV})$ at Endstation 11.3.1 at the Advanced Light Source (ALS) at LBNL. All data were integrated by the program SAINT. ${ }^{31,32}$ The data were corrected for Lorentz and polarization effects. Data were analyzed for agreement and possible absorption using XPREP and an empirical absorption correction was applied in SADABS. ${ }^{33,34}$ Equivalent reflections were merged without an applied decay correction. All structures were solved using direct methods and were expanded with Fourier techniques using the SHELXL package. ${ }^{35}$ Least squares refinement of $\mathrm{F}^{2}$ against all reflections was carried out to convergence with $\mathrm{R}[\mathrm{I}>2 \sigma(\mathrm{I})]$. Further Details on the crystallographic refinement of the crystal structures are provided in the Appendix. 


\subsubsection{Titrations}

Titration Solutions and Equipment. Corning high performance combination glass electrodes (response to $[\mathrm{H}+]$ was calibrated before each titration ${ }^{36}$ ) were used together with either an Accumet $\mathrm{pH}$ meter or a Metrohm Titrino to measure the $\mathrm{pH}$ of the experimental solutions. Metrohm autoburets (Dosimat or Titrino) were used for incremental additions of acid or base standard solution to the titration cell. The titration instruments were fully automated and controlled using LabVIEW software. ${ }^{37}$ Titrations $^{2}$ were performed in $0.1 \mathrm{M} \mathrm{KCl}$ supporting electrolyte under positive Ar gas pressure. The temperature of the experimental solution was maintained at $25^{\circ} \mathrm{C}$ by an externally circulating water bath. UV-visible spectra for incremental titrations were recorded on a Hewlett-Packard 8452a spectrophotometer (diode array). Solid reagents were weighed on a Metrohm analytical balance accurate to $0.01 \mathrm{mg}$. All titration solutions were prepared using distilled water that was purified by passing through a Millipore Milli-Q reverse osmosis cartridge system and degassed by boiling for $1 \mathrm{~h}$ while being purged under Ar. Carbonate-free $0.1 \mathrm{M} \mathrm{KOH}$ was prepared from Baker Dilut-It concentrate and was standardized by titrating against potassium hydrogen phthalate using phenolphthalein as an indicator. Solutions of $0.1 \mathrm{M} \mathrm{HCl}$ were similarly prepared and were standardized by titrating against sodium tetraborate decahydrate to Methyl Red endpoint.

Spectrophotometric and Potentiometric Titrations: Ligand stock solutions were made by dissolving a weighed amount of ligand accurate to $0.01 \mathrm{mg}$ in DMSO in a volumetric flask. These stock solutions were frozen when not in use to prevent ligand decomposition. A stock uranyl solution in $1.2 \mathrm{wt} \%$ nitric acid was purchased from Aldrich (4.22 mM) and used as received. All titrations were performed with a $c a .5 \%$ 
starting concentration of DMSO added to the $\mathrm{KCl}$ solution to promote the solvation of protonated ligands and their neutral uranyl complexes. Potentiometric titrations were carried out at 150-200 $\mu \mathrm{M}$ ligand concentrations. Spectrophotometric titrations were carried out in the presence of 10-20 equivalents (as compared to ligand concentration) of $\mathrm{NH}_{4} \mathrm{Cl}$, MES, and HEPES buffers in order to dampen the $\mathrm{pH}$ change between incremental additions of titrant. Each addition of acid or base was followed by an equilibration period before $\mathrm{pH}$ and absorbance data were collected. For potentiometric titrations this delay was 300 seconds and for spectrophotometric titrations was 600 seconds for free ligand and 600-1200 seconds for titrations in the presence of $\mathrm{UO}_{2}{ }^{2+}$. Spectra were recorded between $250-550 \mathrm{~nm}$. The UV-silent region above $c a .420 \mathrm{~nm}$ was monitored for baseline drift as an indication of precipitated material.

Ligand concentrations for spectrophotometric titrations with a $6.6 \mathrm{~cm}$ path length cell and incremental addition of titrant were approximately $6 \mu \mathrm{M}$. All mid-pH titrations were repeated a minimum of three times and was run forwards and backwards (from acid to base and reverse) within limits set by the reversibility of the titration. Low $\mathrm{pH}$ titrations were performed and electrodes were calibrated as described in a previous publication. ${ }^{38}$

Titration Data Treatment: Potentiometric data were analyzed using Hyperquad ${ }^{39,40}$ software, while spectrophotometric titration data were analyzed using the program pHab, ${ }^{41}$ both utilizing nonlinear least squares regression to determine formation constants. Values for the hydrolysis product of the uranyl cation were taken from a recent literature publication. ${ }^{42}$ Wavelengths between $250-400 \mathrm{~nm}$ were typically used for data refinement. The number of absorbing species to be refined upon was determined by factor analysis within the pHab program suite. ${ }^{41}$ Reversibility of the titrations was 
determined by comparison of the species- and concentration-independent value $A^{*} v$ (absorbance*volume) at selected wavelengths for the forward and reverse titrations. Speciation diagrams were generated using $\mathrm{HYSS}^{43,44}$ titration simulation software and the protonation and metal complex formation constants determined by potentiometric and spectrophotometric titration experiments.

\subsection{References}

(1) Xu, J.; Raymond, K. N. Inorg. Chem. 1999, 38, 308-315.

(2) Rammo, N. N.; Hamid, K. R.; Ibrahim, T. K. J. Alloys. Compd. 1994, 210, 319324.

(3) Degetto, S.; Marangoni, G.; Bombieri, G.; Forsellini, E.; Baracoo, L.; Gaziani, R. J. Chem. Soc. Dalton Trans. 1974, 1933-1939.

(4) Sitran, S.; Fregona, D.; Casellato, U.; Vigato, P. A.; Graziani, R.; Faraglia, G. Inorg. Chim. Acta. 1986, 121, 103-111.

(5) Alcock, N. W.; Flander, D. J. Acta Cryst. C 1987, C43, 1480-1483.

(6) Drouza, C.; Gramlich, V.; Sigalas, M. P.; Pashalidis, I.; Keramidas, A. D. Inorg. Chem. 2004, 43, 8336-8354.

(7) Casellato, U.; Vigato, P. A.; Tamburini, S.; Graziani, R.; Vidali, M. Inorg. Chim. Acta. 1983, 72, 141-147.

(8) Borovik, A. S.; Dewey, T. M.; Raymond, K. N. Inorg. Chem. 1993, 32, 413-421.

(9) Borovik, A. S.; Du Bois, J.; Raymond, K. N. Angew. Chem. Int. Ed. 1995, 34, $1359-1362$.

(10) HostDesigner was developed by B. P. Hay and T. K. Firman at the Pacific Northwest National Laboratory under partial sponsorship of the Chemical 
Sciences, Office of basic Energy Sciences, Office of Science, U.S. Department of Energy under Contract No. DE-AC06-76RL01830. The software can be obtained at no charge from the following website: http://hostdesigner.emsl.pnl.gov.

(11) Hay, B. P.; Firman, T. K. Inorg. Chem. 2002, 41, 5502-5512.

(12) Xu, J.; Durbin, P. W.; Kullgren, B.; Raymond, K. N. J. Med. Chem. 1995, 38, 2606-2614.

(13) Garrett, T. M.; Cass, M. E.; Raymond, K. N. J. Coord. Chem. 1992, 25, 241-253.

(14) Kajigaeshi, S.; Kobayashi, K.; Kurata, S.; Kitajima, A.; Nakahara, F.; Nago, H.; Nishida, A.; Fujisaki, S. Nippon Kagaku Kaishi 1989, 12, 2052-2058.

(15) Lai, L.-L.; Reid, D. H.; Wang, S.-L.; Liao, F.-L. Heteroat. Chem. 1994, 5, 479486.

(16) Madden, H.; Hemmi, G.; Mody, T.; Pharmacyclics, I., USA, Ed. UK, 2007.

(17) Gallant, A. J.; Hui, J. K.-H.; SZahariev, F. E.; Wang, Y. A.; MacLachlan, M. J. J. Org. Chem. 2005, 70, 7936-7946.

(18) Erker, T. Monatshefte für Chemie 1998, 129, 679-687.

(19) Hao, M.-H.; Haq, O.; Muegge, I. J. Chem. Inf. Model. 2007, 47, 2242-2252.

(20) Mullen, L.; Gong, C.; Czerwinski, K. R. J. Rad. Nucl. Chem. 2007, 273, 683-688.

(21) Durbin, P. W.; Kullgren, B.; Ebbe, S. N.; Xu, J.; Raymond, K. N. Health Phys. 2000, $78,511-521$.

(22) Franczyk, T. S.; Czerwinski, K. R.; Raymond, K. N. J. Am. Chem. Soc. 1992, $114,8138-8146$.

(23) Walton, P. H.; Raymond, K. N. Inorg. Chim. Acta. 1995, 240, 593-601.

(24) Arnold, P. L.; Blake, A. J.; Wilson, C.; Love, J. B. Inorg. Chem. 2004, 43, 8206. 
(25) Arnold, P. L.; Patel, D.; Blake, A. J.; Wilson, C.; Love, J. B. J. Am. Chem. Soc. 2006, 128, 9610-9611.

(26) Schmidt, M.; Amstutz, R.; Crass, G.; Seebach, D. Chem. Ber. 1980, 113, 16911707.

(27) Steinkopf, Q.; Jacop, H.; Penz, H. Liebigs Annalen der Chemie 1934, 512, 136.

(28) Snow, A. W.; Foos, E. E. Synthesis 2003, 4, 509-12.

(29) Sawicki, M.; Lecercle, D.; Grillon, G.; Le Gall, B.; Serandour, A.-L.; Poncy, J.L.; Bailly, T.; Burganda, R.; Lecouvey, M.; Challeix, V.; Leydier, A.; PelletRostaing, S.; Ansoborlo, E.; Taran, F. Eur. J. Med. Chem. 2008, 43, 2768-2777.

(30) Sharghi, H.; Nasseri, M. A.; Niknam, K. J. Org. Chem. 2001, 66, 7287-7293.

(31) SAINT: SAX Area-Detector Integration Program, V.6.40; Bruker Analytical Xray Systems, Inc.: Madison, WI, 2003.

(32) SAINT: SAX Area-Detector Integration Program, V.4.024; Siemens Industrial Automation, Inc, Madison, WI, 1995.

(33) XPREP (V.6.12), Part of SHELXTL Crystal Structure Determination Package; Bruker Analytical X-ray Systems, Inc.: Madison, WI, 2001.

(34) SADABS: Bruker Nonius Area Detector Scaling and Absorption V. 2.05; Bruker Analytical X-ray Systems, Inc.: Madison, WI, 2003.

(35) SHELXTL (V.5.10), SHELXTL Crystal Structure Determination Package; Bruker Analytical X-ray Systems, Inc.: Madison, WI, 1997.

(36) Gans, P.; O'Sullivan, B. Talanta 2000, 51, 33-37.

(37) LABVIEW, version 8.0; National Instruments Corp.: 11500 N Mopac Expwy, Austin, TX 78759-3504. 
(38) Johnson, A. R.; O'Sullivan, B.; Raymond, K. N. Inorg. Chem. 2000, 39, 26522660.

(39) Gans, P.; Sabatini, A.; Vacca, A. Talanta 1996, 43, 1739-1753.

(40) Gans, P.; Sabatini, A.; Vacca, A., HYPERQUAD2003; University of Leed and University of Florence: Leeds, U.K., and Florence, Italy, 2003.

(41) Gans, P.; Sabatini, A.; Vacca, A. Annali Di Chimica 1999, 89, 45-49.

(42) Brown, P. L. Radiochimica Acta 2002, 90, 589-93.

(43) Alderighi, L.; Gans, P.; Ienco, A.; Peters, D.; Sabatini, A.; Vacca, A. Coord. Chem. Rev. 1999, 184, 311-318.

(44) Gans, P., HySS2; University of Leeds, Leeds, U.K., 2000. 


\section{Chapter 3:}

\section{Design, Structure, and Solution Thermodynamics of $\mathrm{UO}_{2}\left[\mathrm{TAM}(\mathrm{HOPO})_{2}\right]$ Complexes}

\subsection{Introduction}

As the work in Chapter 2 illustrates, bis-Me-3,2-HOPO ligands (and by structural analogy any tetradentate bis-CAM, -TAM, or -1,2-HOPO ligand) do not saturate the uranyl coordination plane, allowing for partial hydrolysis or the possible interaction of another ligand with the chelated uranyl cation. The selective binding of actinyl cations is typically performed in the presence of many other potentially coordinating ligands, whether it be in an industrial separations application (excess nitrate), or in vivo chelation therapy (carboxylates, alcohols, inorganic oxides, etc.), which could feasibly also interact with the actinyl center, hindering effective separation or decorporation. In order to remove the possibility of ternary complex formation and solvent/substrate dependence on coordinative saturation of the actinyl center, saturation of the coordination plane is desirable, but this requires a different ligand geometry than the bis-bidentate architecture explored in Chapter 2.

One method for saturating the actinyl coordination plane is to use macrocyclic ligands; such scaffolds would provide optimal chelate effects, kinetic inertness, and thermodynamic stability, while also discouraging ternary complex formation. Macrocycle design for the $f$-elements must take into account the relatively large ionic radii of these species, and a wide variety of macrocylic ligands incorporating coordinating oxygen, nitrogen and sulfur donor atoms have been developed and explored for $f$-element chelation. ${ }^{1}$ Structural investigation of the uranyl complexes with many of these 
macrocycles reveals a saturated, pentagonal planar coordination geometry about the uranyl cation, although the macrocyclic binding moieties are seldom fully utilized and can in some cases form heterodinuclear complexes. Sessler and co-workers have developed conjugated, expanded porphyrin molecules that bind cations in an equatorial fashion, providing a hexagonal equatorial coordination mode to both $\mathrm{UO}_{2}{ }^{2+}$ and $\mathrm{NpO}_{2}{ }^{+}$, while also utilizing all the possible coordinating nitrogens in the complex. ${ }^{2}$ The differences in the amount of ligand ruffling seen in the crystal structures with these two actinyls (Figure 3-1) attest to the sensitivity of carefully-tailored macrocyles to ionic size differences, and makes macrocyclic ligands attractive as selective chelating/extraction agents.

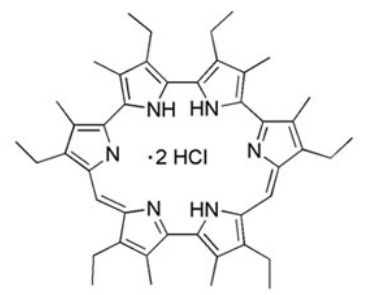

Hexaphyrin, "Hex"

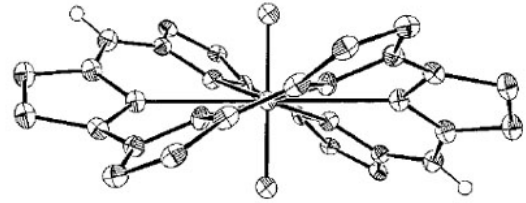

$\mathrm{UO}_{2}[\mathrm{Hex}]$

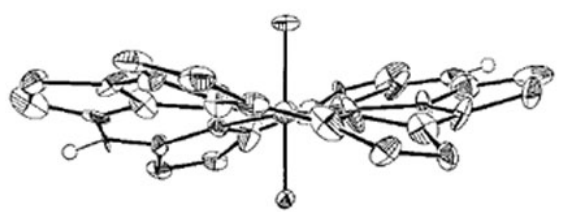

$\mathrm{NpO}_{2}[\mathrm{Hex}]^{-}$

Figure 3-1. Expanded porphyrin Hexaphyrin and its structures with $\mathrm{UO}_{2}{ }^{2+}$ and $\mathrm{NpO}_{2}{ }^{+}$, from Sessler et al. ${ }^{2}$

If actinyl coordinative saturation is to be achieved using Raymond group moieties, three bidentate moieties must be incorporated into the uranyl coordination plane. However, Raymond group ligands have never been observed to form hexagonal planar coordination geometries about the uranyl cation, whether they be HOPO ligands or catechol derivatives such as catecholamides (CAM) or terephthalamides (TAM) (Figure 3-2). Me-3,2-HOPO and 1,2-HOPO moieties chelate $\mathrm{UO}_{2}{ }^{2+}$ at four points of a pentagonal planar coordination geometry, ${ }^{3,4}$ due to the $c a .66^{\circ}$ bite angle these ligands exhibit towards uranyl, making three such moieties difficult to fit in the uranyl coordination 
plane. Typically, Raymond group ligand macrocycles and macropolycycles have been designed for spherical ion chelation, ${ }^{5}$ but such an effort has not been made towards actinyl chelation, and it was hypothesized that hexagonal planar coordination of uranyl using siderophore binding moieties would be feasible if an appropriate ligand geometry could be designed.<smiles>[R]NC(=O)c1ccn(C)c(=O)c1O</smiles>

Me-3,2-HOPOamide (Me-3,2-HOPO)<smiles>[R]NC(=O)c1cccc(=O)n1O</smiles>

1,2-HOPOamide (1,2-HOPO)<smiles>[R]NC(=O)c1cccc(O)c1O</smiles>

Catecholamide

(CAM)<smiles>[R]NC(=O)c1ccc(C(=O)N[R])c(O)c1O</smiles>

Terephthalamide

(TAM)

Figure 3-2. Hydroxypyridinone (Me-3,2- and 1,2-HOPO) and catechol-analog (CAM, TAM) chelating moieties.

Inspiration for this effort was found in the work by Nabeshima and co-workers, who described a salophen-type Schiff base macrocycle containing three catechol derivatives in a central coordinating pocket (Figure 3-3a). ${ }^{6}$ Although this rigid ligand binds transition metals in a salen-type coordination mode, subsequent work with a more flexible macrocycle (Figure 3-3b) and a linear tricatecholate ligand (Figure 3-3c) of similar chemical behavior illustrated that such ligands could preferentially bind larger lanthanide elements in the larger interior catecholate-lined binding pocket (Figure 3-3). ${ }^{7,8}$

The linear ligand design in Figure 3-3(c) suggested that hexagonal planar coordinative saturation of the actinyl coordination plane could be possible by utilizing a properly-designed, tris-bidentate moiety. Such an approach relies on the chelate effect to encourage coordinative saturation instead of the thermodynamics of monobidentate group chelation. A linear ligand also introduces more flexibility than a macrocycle, which is an 
attractive property in our first attempts with this ligand design. This chapter describes the efforts towards achieving actinyl coordinative saturation using tris-bidentate ligands, detailing their design, synthesis, structural investigations, and solution thermodynamics.

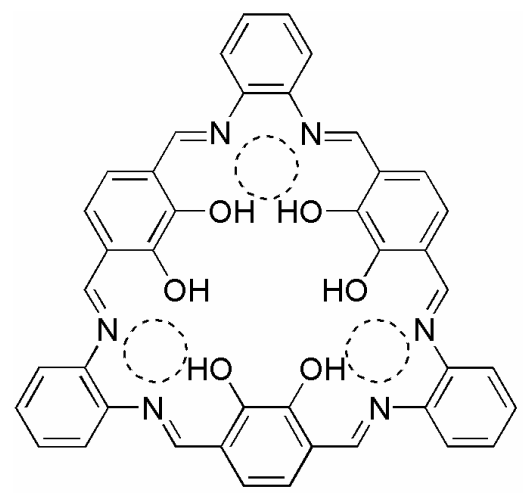

(a)

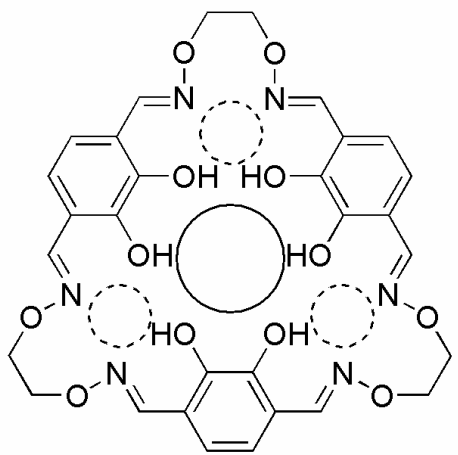

(b)

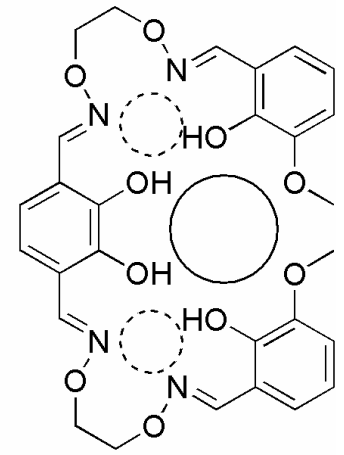

(c)

Figure 3-3. Macrocyclic and linear ligands developed by Akine et. al. ${ }^{6-8}$ Transition metal and lanthanide binding pockets are illustrated by dashed and solid circles respectively.

\subsection{Results and Discussion}

\subsubsection{Hexadentate Ligand Design and Synthesis}

Several poly-bidentate ligands have been developed in the Raymond group that employ a linear polyamine linker upon which to anchor several chelating moieties, but very few ligands utilize the chelating moieties as the structural backbone of the ligand. ${ }^{5}$ In order to minimize steric bulk and encourage near-planar coordination modes about the uranyl cation, this latter approach was chosen in the pursuit of developing linear, trisbidentate ligands. If a chelating moiety is to be used as a linker in the middle of a ligand, it must have the capability to be functionalized in at least two positions, but the Me-3,2HOPO moieties employed in Chapter 2 cannot serve this purpose, as they can only be linked through the one amide functionality, relegating them to the role of terminal binding groups. A chelating unit of similar coordination geometry to the HOPO moieties 
is the TAM unit (Figure 3-2) that the Raymond group employs in much of its chemistry. TAM is a diprotic catechol analog and is known to form strong complexes with hard Lewis acidic ions such as $\mathrm{Fe}(\mathrm{III}){ }^{9}$ Significantly, TAM moieties contain two amide groups ortho to the coordinating phenols, giving it two attachment points through which it can be incorporated into higher denticity ligands. As in HOPO moieties, these amide groups provide well-established hydrogen bond stabilization of the deprotonated and metal-chelated phenolates. ${ }^{9,10}$

A series of $\mathrm{UO}_{2}$ (bis-Me-3,2-HOPO) complexes were presented in Chapter 2 which all exhibited pentagonal planar coordination about the uranyl center. Although bite angles of the Me-3,2-HOPO moieties remained essentially constant across the series, the geometry of the backbone has a significant effect on the solvent-accessible area at the uranyl center by directly changing the $\mathrm{O}_{\text {phenolate }}-\mathrm{U}-\mathrm{O}_{\text {phenolate }}$ (or "ligand bite") angle in the uranyl coordination plane. Specifically, decreasing the length of the linker increases the fraction of the uranyl center available towards solvent coordination (the $\mathrm{O}_{\text {amide }}-\mathrm{U}-\mathrm{O}_{\text {amide }}$ angle). If a third binding moiety with an approximately $66^{\circ}$ bite angle is to bind in an uranyl coordination plane already occupied by two such moieties, it is necessary to maximize the accessible area at the uranyl center, supporting the use of short linkers between the chelating moieties (Figure 3-4).

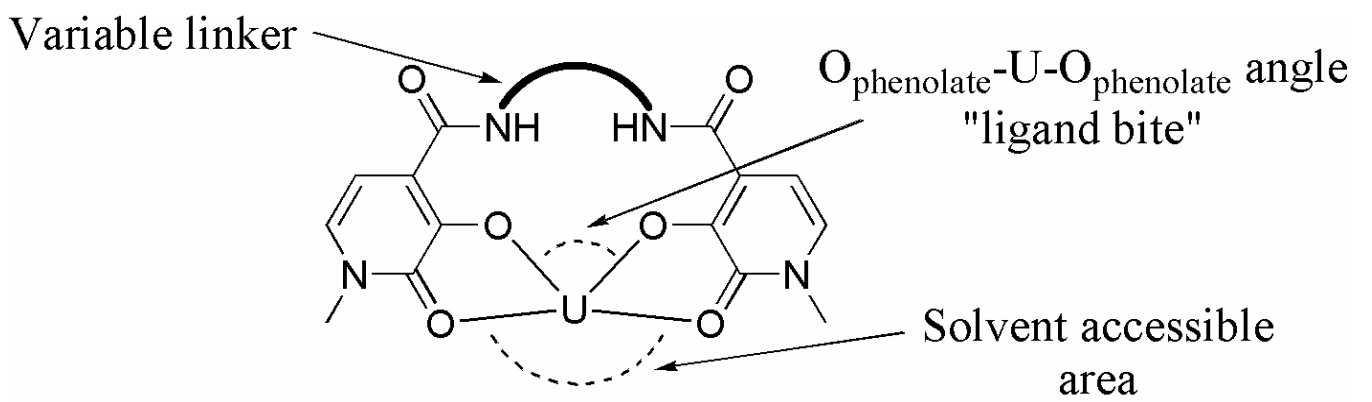

Figure 3-4. Solvent accessible area in $\mathrm{UO}_{2}$ (bis-Me-3,2-HOPO) complexes. Uranyl oxo atoms are omitted for clarity. 

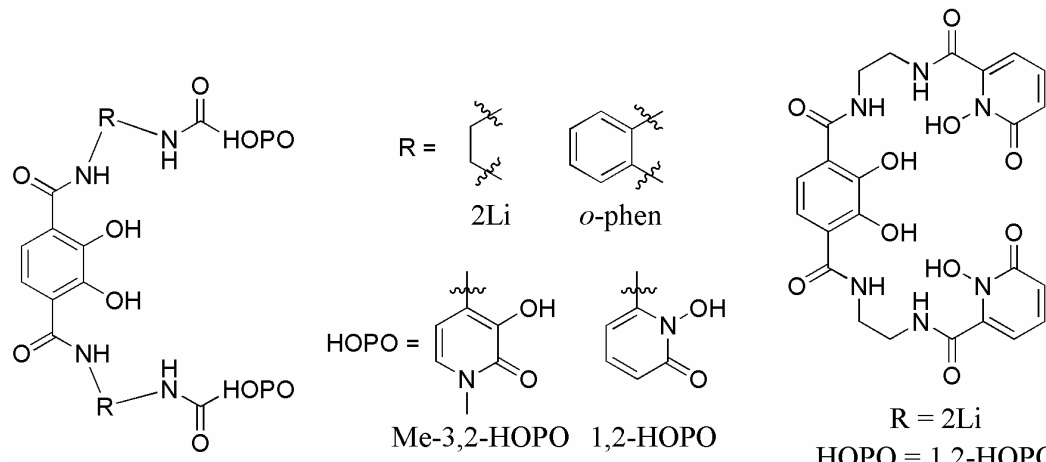

$$
\mathrm{R}=2 \mathrm{Li}
$$

$\mathrm{HOPO}=1,2-\mathrm{HOPO}$

TAM(2Li-1,2-HOPO)

3-1

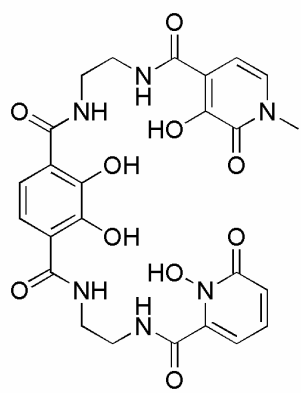

$$
\mathrm{R}=2 \mathrm{Li}
$$

$\mathrm{HOPO}=1,2-\mathrm{HOPO}, \mathrm{Me}-3,2-\mathrm{HOPO}$

TAM(2Li-1,2-HOPO)(2Li-Me-3,2-HOPO) 3-3

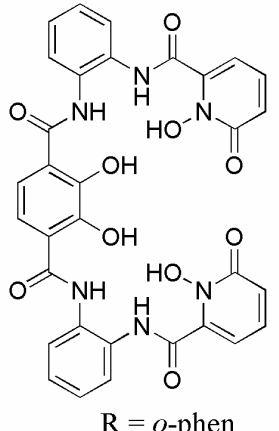

$\mathrm{HOPO}=1,2-\mathrm{HOPO}$

TAM(o-phen-1,2-HOPO) ${ }_{2}$

3-4

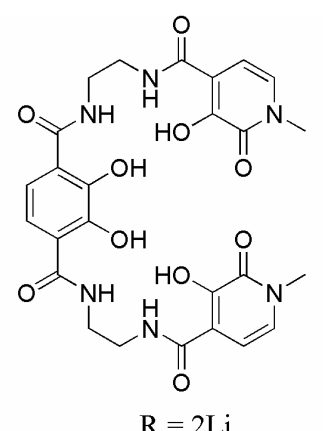

$\mathrm{HOPO}=\mathrm{Me}-3,2-\mathrm{HOPO}$

TAM(2Li-Me-3,2-HOPO)

3-2

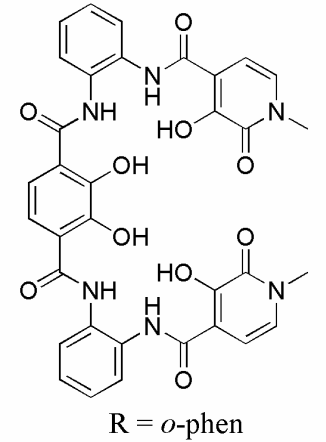

$\mathrm{HOPO}=\mathrm{Me}-3,2-\mathrm{HOPO}$

TAM(o-phen-Me-3,2-HOPO $)_{2}$

3-5

Figure 3-5. General design for $\mathrm{TAM}(\mathrm{HOPO})_{2}$ ligands and initial synthesis targets.

Crystallographic studies in Chapter 2 revealed the 3,4-thiophene-, o-phenylene-, and ethylene-diamine linkers provide the largest solvent accessible area in bis-Me-3,2-HOPO structures $\left(\sigma_{3}+\sigma_{4}\right.$, Table 2-3), and are thus optimal linkers for linear, hexadentate ligands. Unfortunately, 3,4-diaminothiophene is not commercially available, nor does it lend itself to large scale synthesis, making it an impractical synthetic choice. Thus, only the ethylene and $o$-phenylene linkers were utilized in linear, tris-bidentate ligands. Employing a TAM moiety as a center bidentate unit and 1,2- or Me-3,2-HOPO moieties as the terminal bidentate units, a general TAM(HOPO $)_{2}$ ligand scaffold shown in Figure 3-5 was designed, with ligands 3-1 through 3-5 as initial synthetic targets. This general ligand design is very similar to $\mathrm{TAM}(\mathrm{HOPO})_{2}$ ligands pursued in the Raymond group as 
Fe(III) chelators; ${ }^{11}$ the main difference is that the linkers in $\mathbf{3 - 1}$ through $\mathbf{3 - 5}$ are shorter than the linear 3-through 6-carbon alkyl spacers used in designing ligands for octahedral coordination geometries.

The syntheses of ligands 3-1 through 3-5 were performed as illustrated in Scheme 31. Asymmetrically substituted compounds 3-6a,b, 3-8, and 3-10 were synthesized using slow addition amide coupling reactions which take advantage of non-stoichiometric reactant concentrations to afford asymmetric reaction products. In reactions with primary amine linkers the protected TAM and HOPO moieties were activated using the thiazolidine (thiaz) functionality. Because the thiaz derivative is selectively reactive towards primary amines, amide coupling using o-phenylenediamine required the use of the more reactive TAM-diacid-chloride. The large excesses of ethylenediamine and $o$ phenylenediamine were removed from the slow addition reaction mixtures by prolonged high-vacuum exposure and warm water washes respectively.

Benzyl deprotection of $\mathrm{TAM}(\mathrm{HOPO})_{2}$ ligands was performed in 1:1 concentrated $\mathrm{HCl}: \mathrm{AcOH}$ at room temperature. The deprotected ligands were occasionally insoluble in the acidic solution, and TAM moieties generally require longer to deprotect than HOPO moieties, so the reactions were allowed to stir for 10 days before workup to ensure complete ligand deprotection. The TAM(HOPO $)_{2}$ ligands 3-1 through 3-5 are all amorphous, beige solids that are insoluble in most organic solvents with the exception of DMF and DMSO. Deprotonation of the ligands make them significantly more soluble in polar, organic solvents, although the o-phen-linked ligands 3-4 and 3-5 exhibit much lower solubility than the 2Li-linked ligands 3-1, 3-2, and 3-3. 

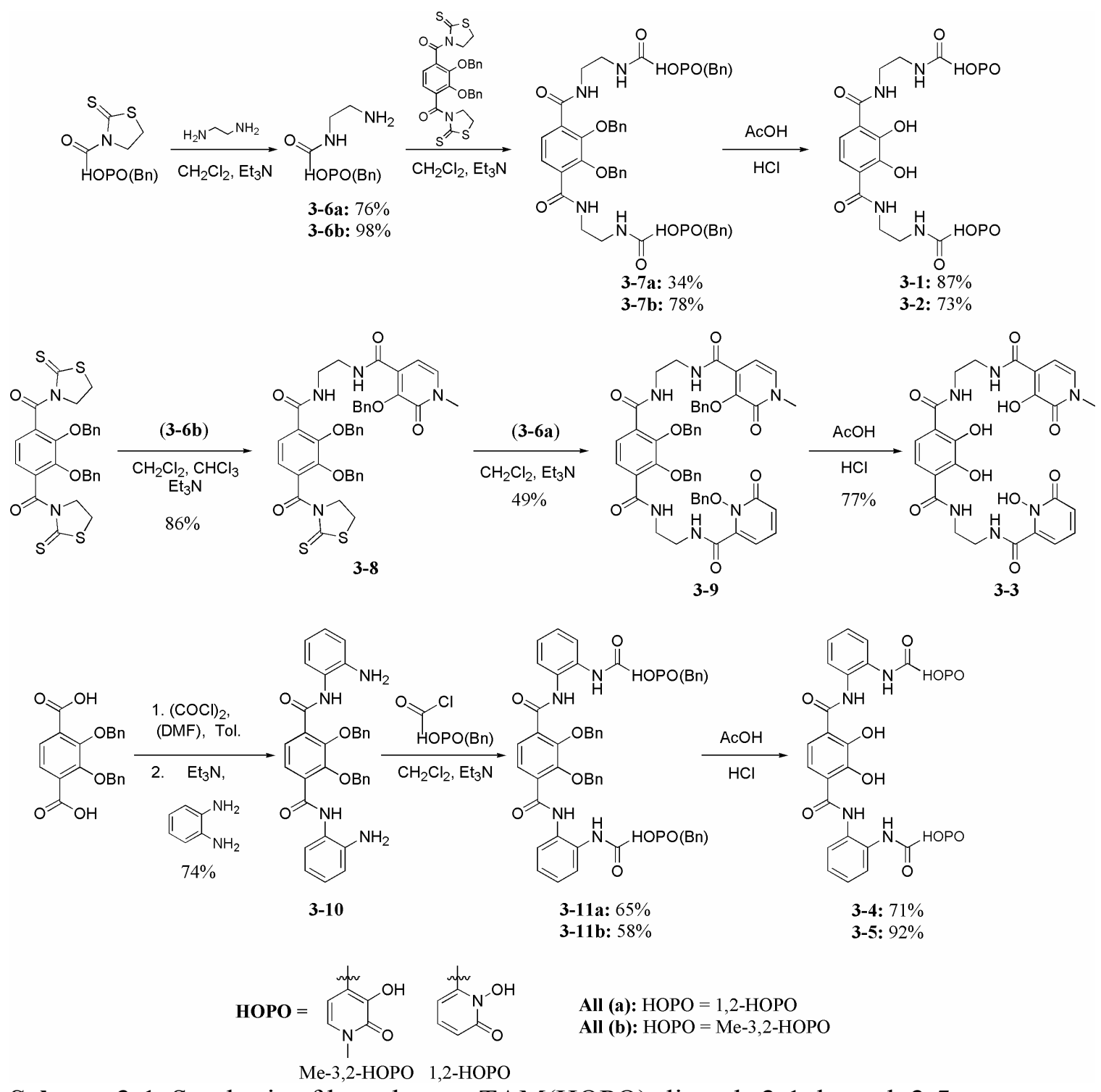

Scheme 3-1. Synthesis of hexadentate TAM(HOPO $)_{2}$ ligands 3-1 through 3-5.

\subsubsection{Synthesis and Structural Comparison of $\mathrm{UO}_{2}{ }^{2+}$ Complexes}

$\mathrm{UO}_{2}\left[\mathrm{TAM}(\mathrm{HOPO})_{2}\right]$ complexes were typically synthesized in either DMF or methanol using a combination of $\mathrm{KOH}$ and $\mathrm{NMe}_{4} \mathrm{OH}$ to deprotonate the ligand. The use of hydroxide bases was necessary to ensure complete ligand deprotonation, since alkylsubstituted TAM moieties have $\mathrm{pK}_{\mathrm{a}}$ values of $c a \cdot 6.0-6.5$ and 10.3-11.0. ${ }^{9}$ Unlike the orange, red, or even maroon color of the $\mathrm{UO}_{2}$ (bis-Me-3,2-HOPO) complexes, the $\mathrm{UO}_{2}\left[\mathrm{TAM}(\mathrm{HOPO})_{2}\right]$ complex solutions are dark brown, independent of the HOPO 
moiety or linker geometry used in the ligand. Because they are dianionic and thus highly soluble, the uranyl complexes are difficult to separate from the mixture of potassium, tetramethylammonium and nitrate salts that are byproducts of the complexation reactions. Clean isolation of the $\mathrm{UO}_{2}\left[\mathrm{TAM}(\mathrm{HOPO})_{2}\right]$ complexes was afforded in most cases by solvent layering or vapor diffusion techniques that selectively deposited the uranyl complexes, often in crystalline form. Uranyl complexes with ligands 3-1 and 3-3 were never successfully isolated, but uranyl complex samples suitable for analysis and single crystal X-ray diffraction could be isolated with ligands 3-2, 3-4, and 3-5. The isolation of these complexes were assisted by their poorer solubility imparted upon them by the Me3,2-HOPO moieties and the o-phenylene linkers (as compared to the ligands incorporating the 1,2-HOPO moiety and linear linkers). Six crystal structures were collected from these samples, one each using 3-2 and 3-5, and four using 3-4. The crystal structures of these complexes are shown in Figure 3-6 and their crystallographic parameters are listed in Table 3-1. 

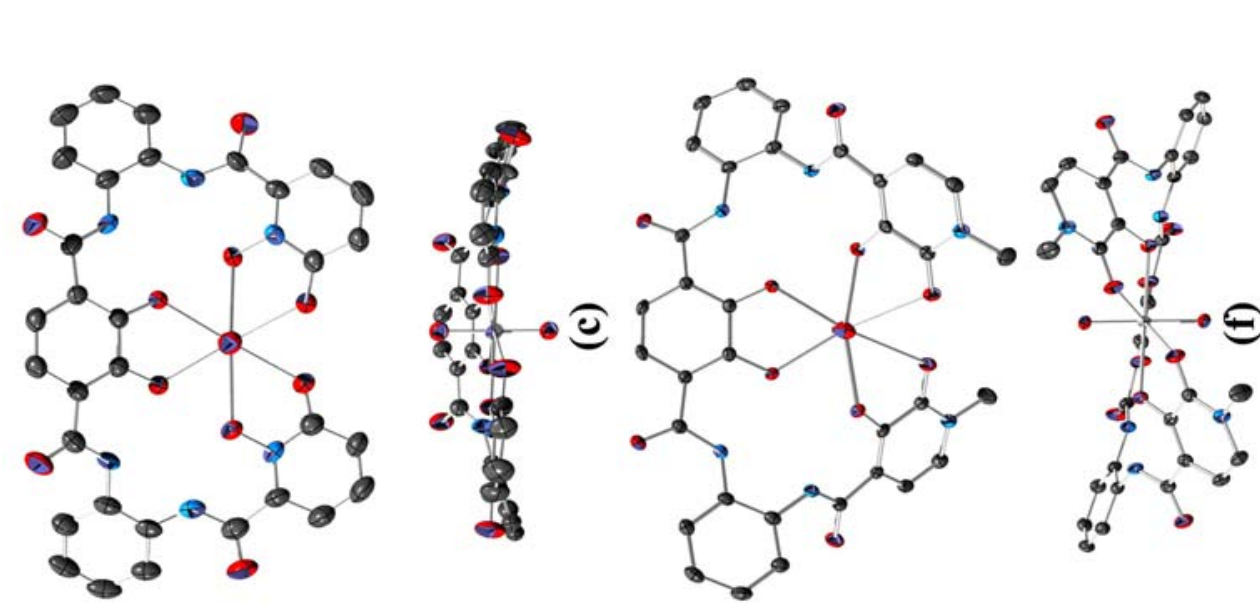

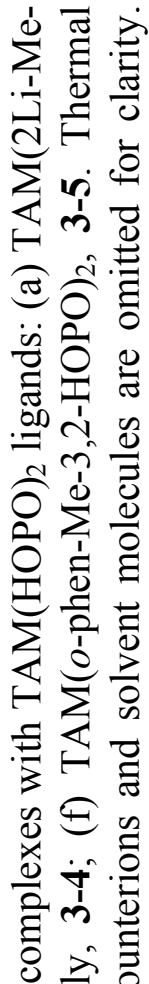
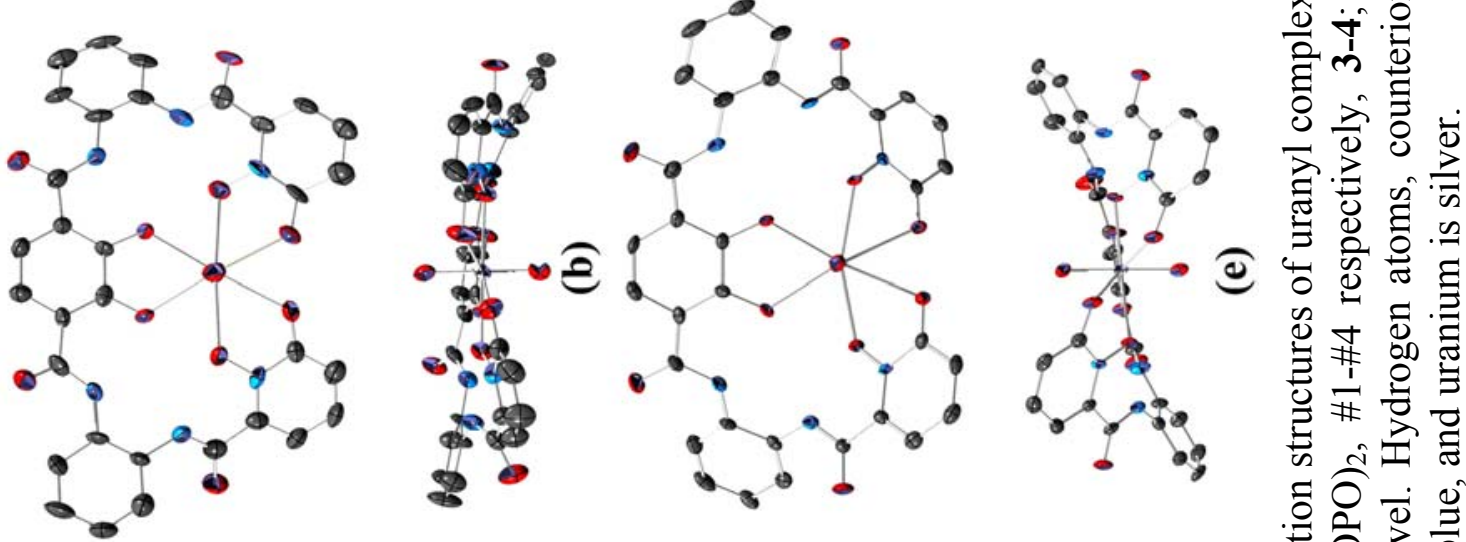

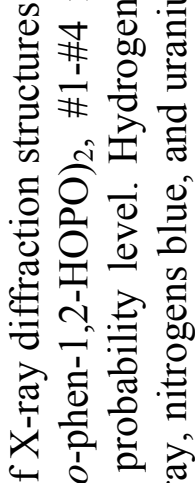
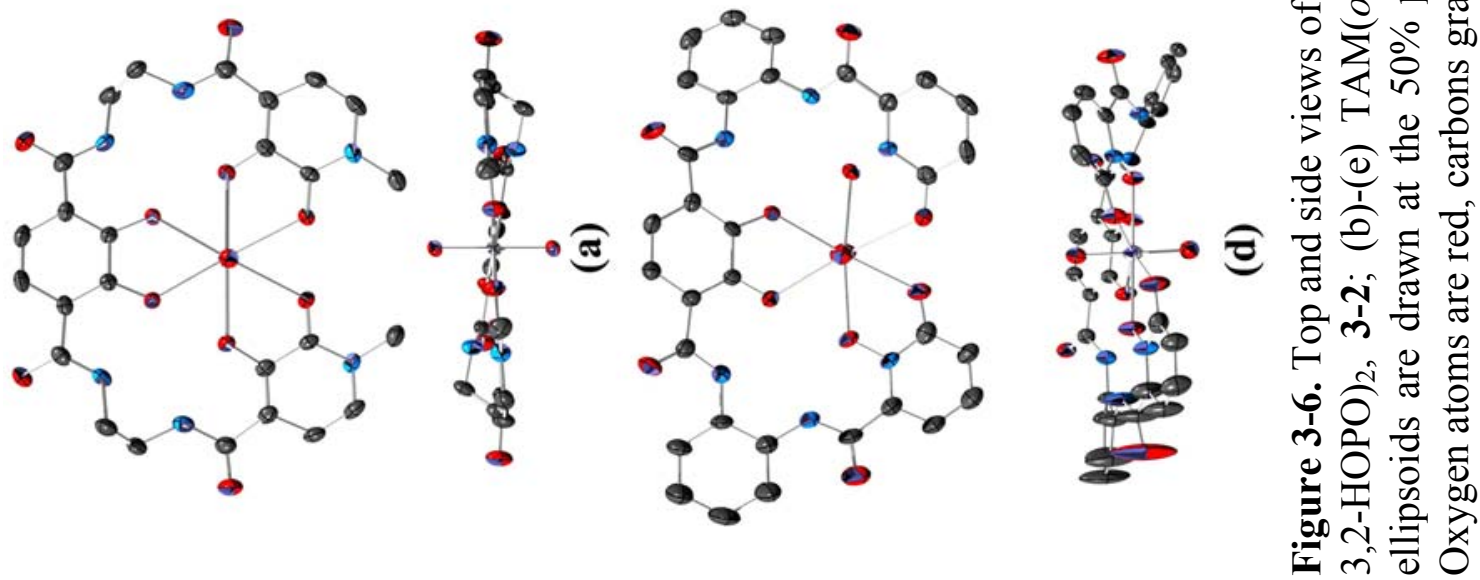
Table 3-1. Crystallographic parameters for $\mathrm{UO}_{2}\left[\mathrm{TAM}(\mathrm{HOPO})_{2}\right]$ and $\mathrm{UO}_{2}(o-$ phen-1,2-HOPO) complexes.

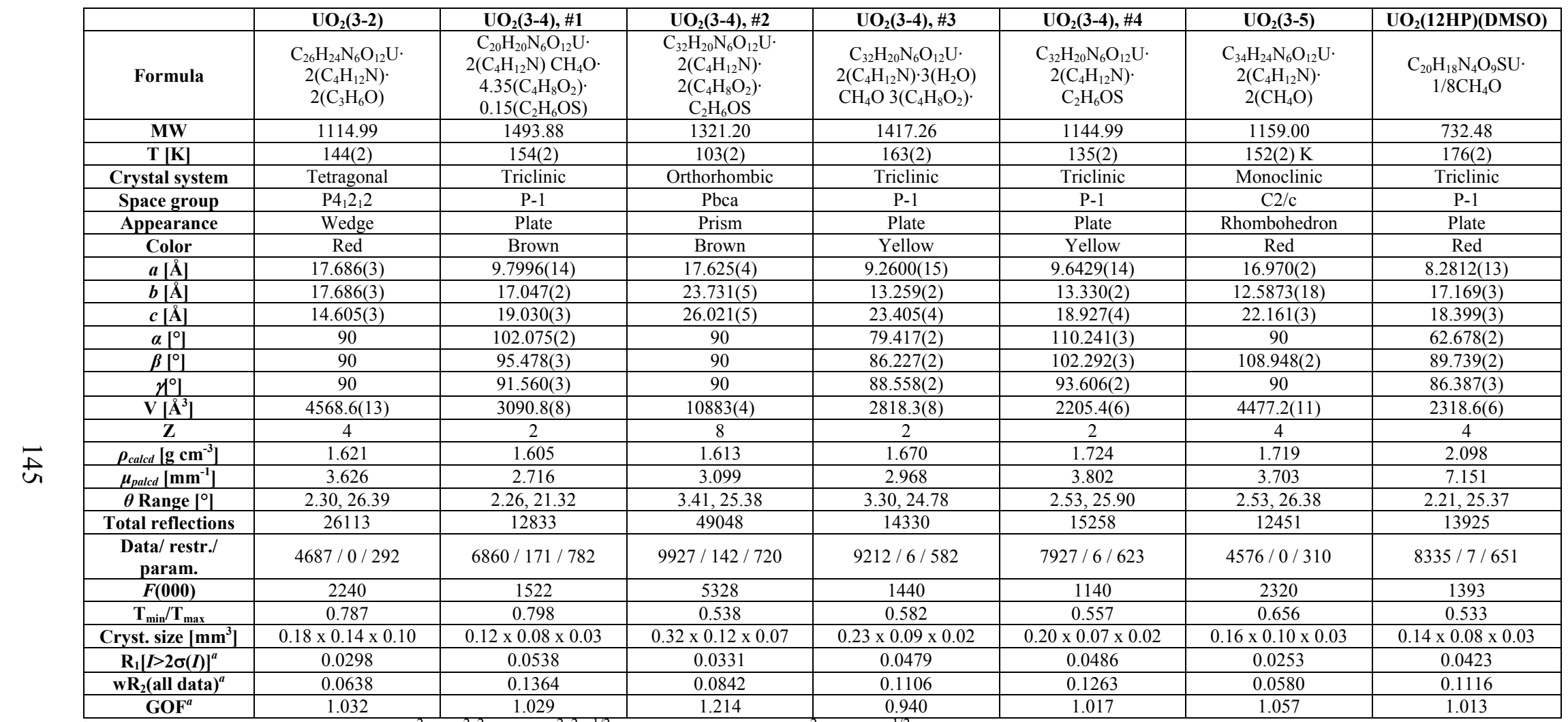

$\mathrm{R}_{1}=\Sigma|| \mathrm{F}_{\mathrm{o}}|-| \mathrm{F}_{\mathrm{c}}|/ \Sigma| \mathrm{F}_{\mathrm{o}} \mid ; \mathrm{wR}_{2}=\left[\Sigma\left[w\left(\mathrm{~F}_{\mathrm{o}}^{2}-\mathrm{F}_{\mathrm{c}}^{2}\right)^{2}\right] / \Sigma\left[w\left(\mathrm{~F}_{\mathrm{o}}^{2}\right)^{2}\right]\right]^{1 / 2} ; \mathrm{GOF}=\left[\Sigma w\left(\left|\mathrm{~F}_{\mathrm{o}}\right|-\left|\mathrm{F}_{\mathrm{c}}\right|\right)^{2} /(n-m)\right]^{1 / 2}$ 
As Figure 3-6 shows, the TAM(HOPO $)_{2}$ ligands complex the uranyl cation in a hexadentate fashion, with all coordinating oxygens binding in the equatorial coordination plane. This result is apparently independent of HOPO moiety identity or linker rigidity, although there is in some cases a noticeable amount of ligand ruffling out of the coordination plane. The amount of this distortion varies significantly between the linearand aromatically-linked ligands, with a wide variety of distortion between the $\mathrm{UO}_{2}(\mathbf{3 - 1 4})$ structures alone. In contrast, the $\mathrm{UO}_{2}($ bis-Me-3,2-HOPO) complexes in Chapter 2 utilizing ethylene- and o-phenylene linkers exhibit very similar arrangement of the HOPO rings about the uranyl cation. In the $\mathrm{UO}_{2}(\mathbf{3 - 2})$ structure, the ethylene linkers adopt the expected staggered alkane geometry, and thus the TAM and HOPO rings need not distort out of the uranyl coordination plane to as severe a degree as they do in the $O$ phenylene linked $\mathrm{UO}_{2}(\mathbf{3 - 4})$ and $\mathrm{UO}_{2}(\mathbf{3 - 5})$ complexes. Apparently, the effect of the rigid aromatic linker is magnified when two such linkers are used in the same ligand scaffold.

To better understand the U-O bond distance differences in the crystal structures above, comparison against analogous tetradentate bis-HOPO uranyl complexes was desired. Such analogs for $\mathrm{UO}_{2}(\mathbf{3 - 2})$ and $\mathrm{UO}_{2}(\mathbf{3 - 5})$ were presented in Chapter 2 [using 2Li-Me-3,2-HOPO (2-2) and o-phen-Me-3,2-HOPO (2-7) respectively], but none existed for $\mathrm{UO}_{2}(\mathbf{3 - 4})$. The tetradentate bis-1,2-HOPO analog to ligand 3-4 is the previouslyreported o-phen-1,2-HOPO (12HP, Figure 3-7). ${ }^{12}$ The uranyl complex with $\mathbf{1 2 H P}$ was made following standard synthetic procedures ${ }^{4}$ and crystallized by diffusion of $\mathrm{MeOH}$ into a DMSO solution of the complex to yield the $\mathrm{UO}_{2}(\mathbf{1 2 H P})(\mathrm{DMSO})$ complex crystal structure shown in Figure 3-8, whose crystallographic parameters are listed in Table 3-1. As with the bis-Me-3,2-HOPO structures in Chapter 2, the uranyl binds four ligand 
oxygens in the equatorial plane, with a fifth coordination site occupied by a DMSO oxygen.

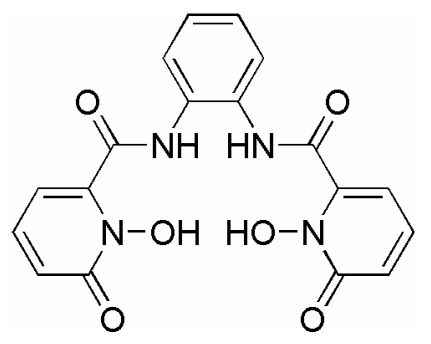

Figure 3-7. $o$-Phen-1,2-HOPO (12HP) ${ }^{12}$
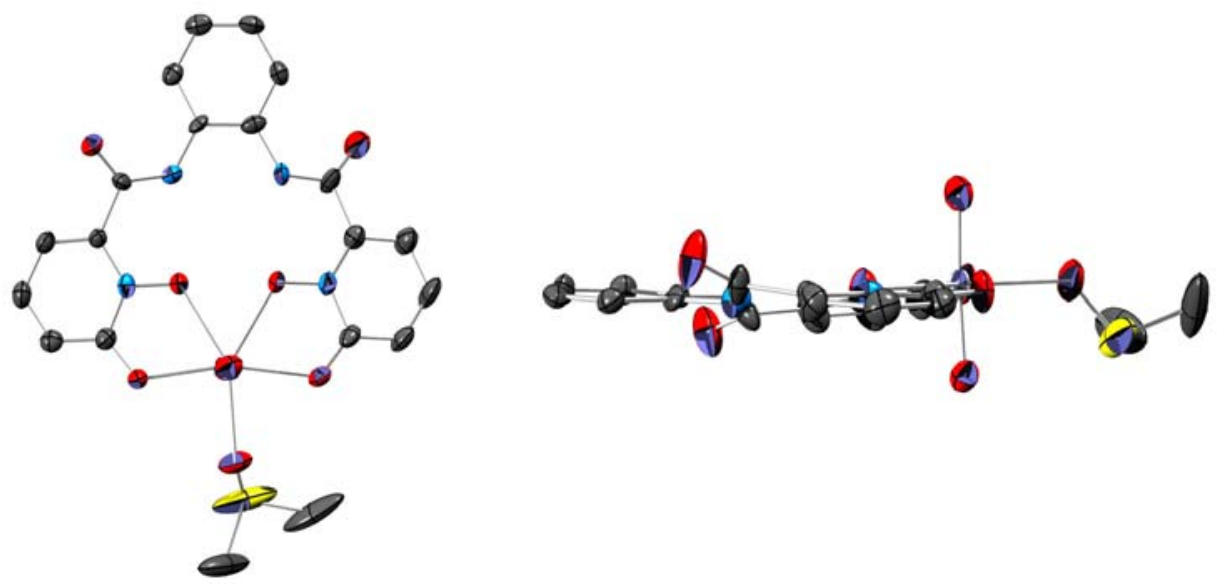

Figure 3-8. Top and side views of $\mathrm{X}$-ray diffraction structures of $\mathrm{UO}_{2}(\mathbf{1 2 H P})(\mathrm{DMSO})$. Thermal ellipsoids are drawn at the $50 \%$ probability level. Only one of two crystallographically unique $\mathrm{UO}_{2}(\mathbf{1 2 H P})(\mathrm{DMSO})$ complexes are shown. Hydrogen atoms and solvent inclusions are omitted for clarity. Oxygen atoms are red, carbons gray, nitrogens blue, sulfurs yellow, and uranium is silver.

The equatorial $\mathrm{U}-\mathrm{O}$ bond lengths in the $\mathrm{UO}_{2}\left[\mathrm{TAM}(\mathrm{HOPO})_{2}\right]$ and $\mathrm{UO}_{2}$ (bis-HOPO) complexes are labeled schematically in Figure 3-9 and are listed in Table 3-2. Although the bond lengths vary significantly between the several structures listed, one constant readily observed is that the $\mathrm{U}-\mathrm{O}_{\mathrm{TAM}}$ distances in $\mathrm{UO}_{2}\left[\mathrm{TAM}(\mathrm{HOPO})_{2}\right]$ structures are very similar, with an average of ca. 2.40(3) $\AA$ despite the different linkers and HOPO moieties employed. This bond distance corresponds very closely to the 2.39-2.49 $\AA \mathrm{M}-\mathrm{O}$ bond lengths in $\left[\mathrm{ML}_{4}\right]^{4-}$ complexes where $\mathrm{M}$ is $\mathrm{U} / \mathrm{Th}(\mathrm{IV})$ and $\mathrm{L}$ is a monobidentate, untethered 
TAM or catechol ligand. ${ }^{13,14}$ This invariance and bond length similarity suggests that the TAM moiety is a dominating influence on the structure of the uranyl complexes.
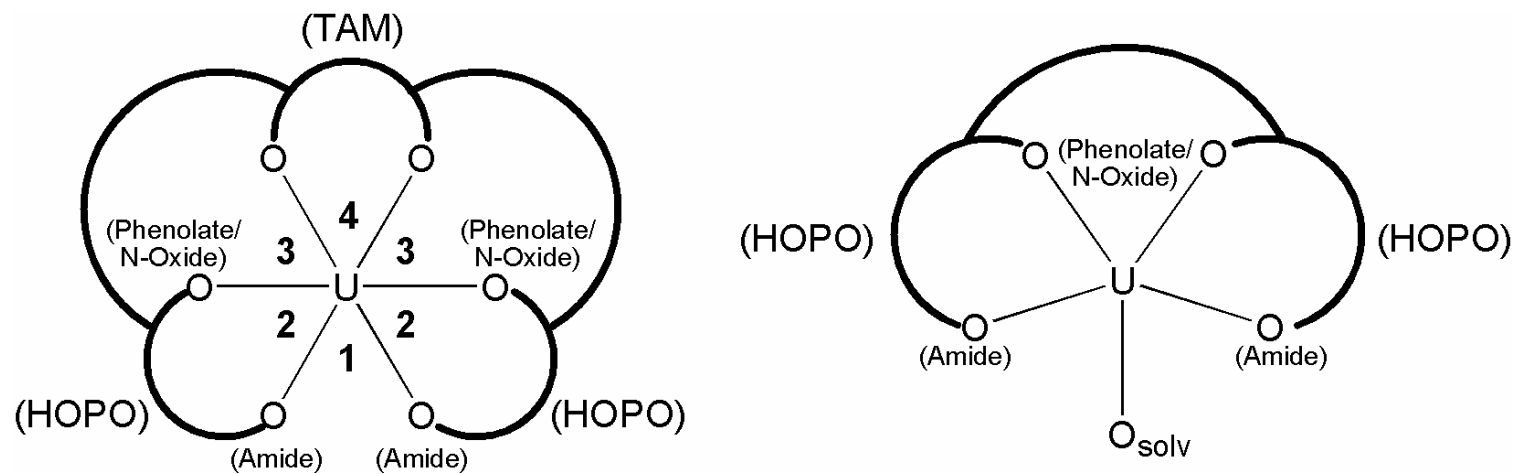

Figure 3-9. Schematic of equatorial U-O bond lengths and binding pocket layout in uranyl complexes with TAM(HOPO $)_{2}$ and bis-Me-3,2-HOPO ligands as referred to in Tables 3-2 and 3-3 and Figure 3-10 below.

Table 3-2. Equatorial U-O bond lengths in $\mathrm{UO}_{2}\left[\mathrm{TAM}(\mathrm{HOPO})_{2}\right]$ and analogous $\mathrm{UO}_{2}$ (bisHOPO) complexes determined by X-ray crystallography and labeled according to Figure 3-9. Hexadentate complexes are in unshaded cells, while tetradenate complexes are listed in gray cells.

\begin{tabular}{|c|c|c|c|}
\hline Ligand & $\begin{array}{c}\text { U-O (HOPO amide), } \\
{[\AA \AA]}\end{array}$ & $\begin{array}{c}\text { U-O (HOPO phenolate/ } \\
\text { N-oxide), }[\AA \AA]\end{array}$ & U-O (TAM), [̊̊] \\
\hline $\begin{array}{c}\text { TAM(2Li-Me-3,2- } \\
\text { HOPO })_{2}, \mathbf{3 - 2}^{\text {a }}\end{array}$ & $2.544(3)$ & $2.528(3)$ & $2.405(3)$ \\
\hline $\begin{array}{l}\text { 2Li-Me-3,2-HOPO, } \\
\mathbf{2 - 2}\end{array}$ & $2.442(8), 2.471(8)$ & $2.301(7), 2.383(7)$ & -- \\
\hline $\begin{array}{l}\text { TAM }(o-p h e n-1,2- \\
\text { HOPO })_{2}, \mathbf{3 - 4}, \# 1\end{array}$ & $2.460(7), 2.508(7)$ & $2.533(7), 2.551(7)$ & $2.384(6), 2.428(7)$ \\
\hline $\begin{array}{l}\text { TAM }(o-\text { phen-1,2- } \\
\text { HOPO })_{2}, \mathbf{3 - 4}, \# 2\end{array}$ & $2.416(3), 2.449(3)$ & $2.590(3), 2.607(3)$ & $2.387(3), 2.407(3)$ \\
\hline $\begin{array}{l}\text { TAM }(o-\text { phen-1,2- } \\
\mathrm{HOPO})_{2}, \mathbf{3 - 4}, \# 3\end{array}$ & $2.443(5), 2.535(4)$ & $2.484(4), 2.517(4)$ & $2.370(4), 2.446(4)$ \\
\hline $\begin{array}{l}\text { TAM }(o-p h e n-1,2- \\
\text { HOPO })_{2}, \mathbf{3 - 4} \# 4\end{array}$ & $2.552(6), 2.558(5)$ & $2.410(5), 2.417(5)$ & $2.353(5), 2.403(5)$ \\
\hline $\begin{array}{c}o-P h e n-1,2-H O P O \\
\mathbf{1 2 H P}^{b}\end{array}$ & $\begin{array}{l}2.341(6), 2.361(6), \\
2.370(6), 2.371(6)\end{array}$ & $\begin{array}{l}2.345(6), 2.367(6), \\
2.374(5), 2.375(5)\end{array}$ & -- \\
\hline $\begin{array}{c}\text { TAM(o-phen-Me-3,2- } \\
\text { HOPO })_{2}, \mathbf{3 - 5}\end{array}$ & $2.730(2)$ & $2.419(2)$ & $2.403(2)$ \\
\hline $\begin{array}{c}\text { o-Phen-Me-3,2-HOPO, } \\
\mathbf{2 - 7}\end{array}$ & $2.446(3), 2.458(3)$ & $2.330(3), 2.349(3)$ & -- \\
\hline
\end{tabular}

${ }^{a}$ The two halves of the molecule are crystallographically identical, giving rise to single values for U-O bonds.

${ }^{b}$ Two unique $\mathrm{UO}_{2} \mathrm{~L}(\mathrm{DMSO})$ complexes exist in the asymmetric unit.

Addressing the HOPO moieties, the $\mathrm{U}-\mathrm{O}_{\text {phenolate }}$ bond lengths in the tetradentate $\mathrm{UO}_{2}(\mathbf{2 - 2})$ structure are shorter than the $\mathrm{U}-\mathrm{O}_{\text {amide }}$ bonds by ca. $0.1 \AA$, which is consistent with a stronger U-O bond to the more negatively charged phenol oxygen compared to the 
neutral amide oxygen. This relative bond order trend is consistent with all the $\mathrm{UO}_{2}$ (bisMe-3,2-HOPO) complexes examined in Chapter 2. However, despite the relative flexibility of the $2 \mathrm{Li}$ linker, the $\mathrm{U}-\mathrm{O}_{\mathrm{HO} O}$ distances in the $\mathrm{UO}_{2}(\mathbf{3 - 2})$ structure are quite nearly equal and both are longer than the $\mathrm{U}-\mathrm{O}_{\text {amide }}$ bonds in $\mathrm{UO}_{2}(\mathbf{2 - 2})$ by ca. $0.1 \AA$. This suggests that the geometric constraints of hexadentate coordination require a sacrifice in bond strength to the HOPO oxygens. The constraints of uranyl chelation also impose upon the coordinated ligand 3-2 a mild helical twist, indicating that fully planar coordination is not quite achievable. This, accompanied by the relative invariance of the U-O $\mathrm{O}_{\text {TAM }}$ bond distances reinforces the hypothesis that the TAM moiety dominates the bonding in the $\mathrm{UO}_{2}\left[\mathrm{TAM}(\mathrm{HOPO})_{2}\right]$ complexes.

In contrast to the mild helical twist observed in $\mathrm{UO}_{2}(3-2)$, the four $\mathrm{UO}_{2}(\mathbf{3 - 4})$ crystal structures exhibit a variety of ligand orientations about the uranyl cation. In the $\mathrm{UO}_{2}(\mathbf{3 - 4})$ structures \#1 and \#4 (Figure 3-6b and e), helical twists similar to that seen in $\mathrm{UO}_{2}(\mathbf{3 - 2}$ ) are observed. However, in $\mathrm{UO}_{2}(3-4)$ structures \#2 and \#3 (Figure 3-6c and d), the TAM moiety does not exhibit a twist with respect to the uranyl coordination plane, but instead bends completely out and to one side of said plane. This amount of conformational variability explains the observed range in the $\mathrm{U}-\mathrm{O}_{\mathrm{HOPO}}$ bond distances in these four structures (intermolecular $\Delta \mathrm{d}_{\max }=0.20 \AA$ ). Surprisingly, $\mathrm{UO}_{2}(\mathbf{3 - 4})$ structures \#1 through \#3 display $\mathrm{U}-\mathrm{O}_{\mathrm{N}-\mathrm{Oxide}}$ bond lengths that are actually longer than their $\mathrm{U}-\mathrm{O}_{\text {amide }}$ bond, Precedent for this relative bond length order exists in the unsubstituted bidentate 1,2HOPO complexes with $\mathrm{Fe}(\mathrm{III}), \mathrm{Co}(\mathrm{III}), \mathrm{UO}_{2}{ }^{2+}$ and $\mathrm{Th}(\mathrm{IV})$ in which the $\mathrm{M}-\mathrm{O}_{\text {phenolate } \mathrm{N}-\text {-oxide }}$ bonds are all comparable within each complex, with the difference in $\mathrm{M}-\mathrm{O}_{\text {phenolate } \mathrm{N}-\text {-oxide }}$ bond lengths ranging from zero to only $0.03 \AA^{3,15,16}$ This is caused by a resonance form 
available in the 1,2-HOPO moiety that populates the formally amide oxygen with negative charge and establishes aromaticity; a resonance form obviously not significant in the Me-3,2-HOPO moiety. However, the intramolecular $\mathrm{U}-\mathrm{O}_{\text {phenolate/ } \mathrm{N}-\text {-oxide }}$ bond length differences in the $\mathrm{UO}_{2}(3-4)$ structures vary a maximum of $0.18 \AA$ and are without exception longer than those in $\mathrm{UO}_{2}(\mathbf{1 2 H P})(\mathrm{DMSO})$. This must again be a result of steric constraints imposed on the hexadentate ligand by the short, and therefore relatively strong $\mathrm{U}-\mathrm{O}_{\text {TAM }}$ bonds in the complex.

The $\mathrm{UO}_{2}(3-5)$ structure displays a severe helical ligand configuration about the uranyl center similar to that in $\mathrm{UO}_{2}(\mathbf{3 - 4})$ structure \#4 and far more pronounced than that in $\mathrm{UO}_{2}(3-2)$. The $\mathrm{U}-\mathrm{O}_{\text {phenolate }}$ bond length is comparable to those in the other hexadentate uranyl structures, but the U- $\mathrm{O}_{\text {amide }}$ distance is very long at $2.7 \AA$, which is even longer than those of coordinated DMSO or DMF molecules in the $\mathrm{UO}_{2}$ (bis-Me-3,2-HOPO) structures presented in Chapter 2 (typically ca. $2.4 \AA$ ). The HOPO amide oxygens therefore present only very weak interactions with the uranyl center as compared to the other $\mathrm{HOPO}$ amide interactions seen in hexadentate $\mathrm{TAM}(\mathrm{HOPO})_{2}$ or tetradentate bisHOPO uranyl complexes. This great disparity in $\mathrm{U}_{-} \mathrm{O}_{\text {HOPO }}$ bond lengths along with the still-short $\mathrm{U}-\mathrm{O}_{\mathrm{TAM}}$ bonds are again evidence that the TAM moiety is the most important binding unit in the $\mathrm{TAM}(\mathrm{HOPO})_{2}$ ligands and dominates the binding interaction with the uranyl cation, despite it being outnumbered by the HOPO moieties two to one.

The tendency for o-phenylene-linked ligands 3-4 and 3-5 complexes to exhibit more pronounced ligand distortions than those with ethylene-linked 3-2 suggests that the ophenylene linker imposes too much steric constraint on the molecule for comfortable uranyl chelation. The staggered conformation of the linear linkers in 3-2 may be the 
reason for the relatively small deviation from planarity in the $\mathrm{UO}_{2}(\mathbf{3 - 2})$ structure; this natural tendency of the 2Li linker allows the close approach of the HOPO moieties to the uranyl center, which the distortions seen in the o-phenylene-linked ligands attempt to mimic, but are unable to achieve because of the rigidity imposed by the linker.

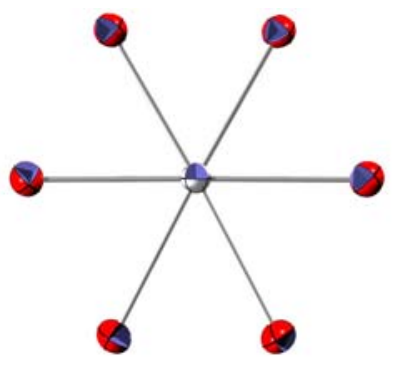

(a)

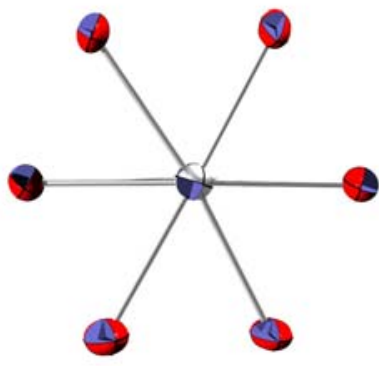

(d)

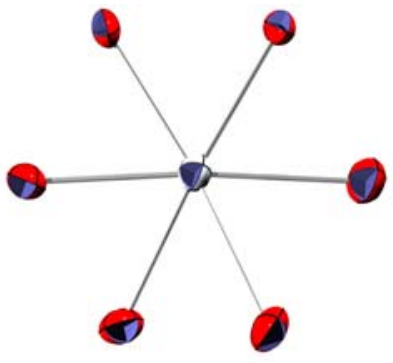

(b)

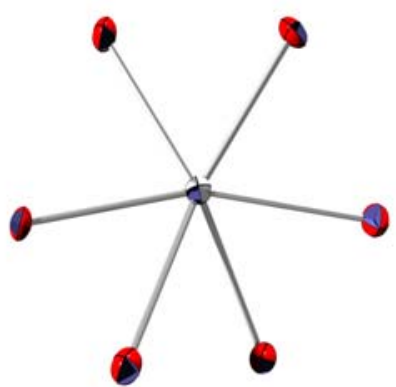

(e)

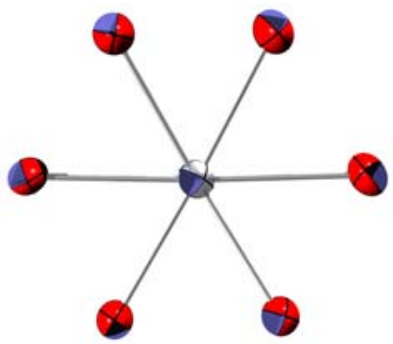

(c)

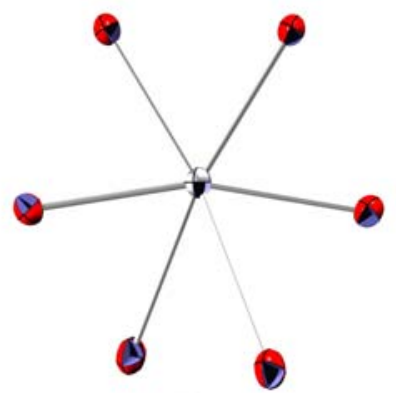

(f)

Figure 3-10. Uranyl binding pockets in the $\mathrm{UO}_{2}\left[\mathrm{TAM}(\mathrm{HOPO})_{2}\right]$ crystal structures: (a) TAM(2Li-Me-3,2-HOPO $)_{2}, \quad 3-2 ; \quad$ (b)-(e) TAM(o-phen-1,2-HOPO) $)_{2}, \quad 3-4, \quad \# 1-\# 4$ respectively; (f) TAM(o-phen-Me-3,2-HOPO) $)_{2}$, 3-5. Structures are viewed down the uranyl $\mathrm{O}=\mathrm{U}=\mathrm{O}$ vector, although uranyl oxo atoms have been removed for clarity, and oxygens are oriented as illustrated in Figure 3-9. Thermal ellipsoids are drawn at the 50\% probability level. Oxygen atoms are red and uraniums are silver.

A visual comparison of the uranyl binding pocket in the $\mathrm{UO}_{2}$ [TAM(HOPO $\left.)_{2}\right]$ complexes is presented in Figure 3-10. These images very clearly show the position of the uranyl center in the hexadentate coordination pocket provided by the TAM(HOPO $)_{2}$ ligands. Clearly the ethylene-linked ligand 3-2 binds the uranyl most equally as compared to the $\boldsymbol{O}$-phenylene linked ligands 3-4 and 3-5, in which the uranyl center sits farther back in the coordination pocket towards the TAM moiety. In fact, the more helical the twist 
seen in the uranyl complex, the more unequal are the U-O bond lengths between the TAM and HOPO moieties.

Table 3-3. In-plane O-U-O bite angles in $\mathrm{UO}_{2}\left[\mathrm{TAM}(\mathrm{HOPO})_{2}\right]$ complexes determined by $\mathrm{X}$-ray crystallography. Angle labels refer to those in Figure 3-9.

\begin{tabular}{|c|c|c|c|c|c|}
\hline Ligand & Angle 1, $\left[^{\circ}\right]$ & Angle 2, $\left[^{\circ}\right]$ & Angle 3, $\left[^{\circ}\right]$ & Angle 4, $\left[{ }^{\circ}\right]$ & Angle Sum, $\left[{ }^{\circ}\right]$ \\
\hline $\begin{array}{l}\text { TAM(2Li-Me-3,2- } \\
\text { HOPO })_{2}, \mathbf{3 - 2}^{a}\end{array}$ & $57.9(1)$ & $60.9(1)$ & $59.6(1)$ & $63.3(1)$ & $362.2(2)$ \\
\hline 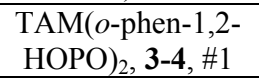 & $59.0(3)$ & $\begin{array}{l}59.8(2) \\
60.7(2) \\
\end{array}$ & $\begin{array}{l}59.4(2) \\
60.8(2)\end{array}$ & $65.0(2)$ & $364.7(5)$ \\
\hline $\begin{array}{l}\text { TAM(o-phen-1,2- } \\
\text { HOPO })_{2}, \mathbf{3 - 4 ,} \# 2\end{array}$ & $60.5(1)$ & $\begin{array}{l}59.3(1), \\
59.9(1)\end{array}$ & $\begin{array}{l}58.87(9), \\
59.25(9)\end{array}$ & $64.4(1)$ & $362.2(2)$ \\
\hline $\begin{array}{l}\text { TAM }(o-\text { phen-1,2- } \\
\text { HOPO })_{2}, \mathbf{3 - 4}, \# 3\end{array}$ & $60.0(2)$ & $\begin{array}{l}60.5(1), \\
61.1(2)\end{array}$ & $\begin{array}{l}60.2(1) \\
62.9(1)\end{array}$ & $64.3(1)$ & $369.0(3)$ \\
\hline $\begin{array}{l}\text { TAM(o-phen-1,2- } \\
\text { HOPO })_{2}, \mathbf{3 - 4}, \# 4\end{array}$ & $64.3(2)$ & $\begin{array}{l}59.4(2), \\
60.7(2)\end{array}$ & $\begin{array}{l}66.6(2), \\
67.5(2)\end{array}$ & $65.2(2)$ & $383.7(5)$ \\
\hline $\begin{array}{l}\text { TAM(o-phen-Me- } \\
3,2-\mathrm{HOPO})_{2}, \mathbf{3 - 5},{ }^{a}\end{array}$ & $59.58(9)$ & $60.71(6)$ & $65.93(7)$ & $65.40(9)$ & $378.3(2)$ \\
\hline
\end{tabular}

${ }^{a}$ The two halves of the molecule are crystallographically identical, giving rise to single values for angles 2 and 3 .

Additional information on the interaction between $\mathrm{UO}_{2}{ }^{2+}$ and TAM(HOPO $)_{2}$ ligands can be gleaned by investigating both the equatorial $\mathrm{O}-\mathrm{U}-\mathrm{O}$ angles as well as the interplanar TAM/HOPO/uranyl plane angles listed in Tables 3-3 and 3-4 respectively. Angles 2 and 4 are the HOPO and TAM bite angles about the uranyl, respectively. The $\mathrm{HOPO}$ bite angle is almost constant at $c a .60^{\circ}$ in all the $\mathrm{UO}_{2}\left[\mathrm{TAM}(\mathrm{HOPO})_{2}\right]$ structures, which is about $6^{\circ}$ smaller than that in the $\mathrm{UO}_{2}$ (bis-Me-3,2-HOPO) structures presented in Chapter 2 and the previously published $\mathrm{UO}_{2}(1,2-\mathrm{HOPO})_{2}$ complex. ${ }^{3}$ The decrease in bite angle is caused by the HOPO moieties backing away from the uranyl cation. Further evidence for this is the distance between the uranium atom and a centroid between the HOPO chelating oxygen atoms, which increases by $0.16-0.21 \AA$ between tetradentate bisHOPO and hexadentate TAM(HOPO $)_{2}$ ligands. This is most assuredly caused by the steric constraints on the hexadentate ligands, resulting in weaker $\mathrm{U}-\mathrm{O}_{\mathrm{HOPO}}$ bonds. In contrast, the TAM bite angles range between $63-65^{\circ}$, which are much closer to the $65^{\circ}$ TAM bite angle in the untethered $\left[\mathrm{Th}(\mathrm{ETAM})_{4}\right]^{4-}$ complex, ${ }^{13}$ demonstrating that the 
TAM moiety sacrifices little bond strength in the $\mathrm{UO}_{2}\left[\mathrm{TAM}(\mathrm{HOPO})_{2}\right]$ complexes compared to its unconstrained binding modes.

As in Chapter 2, the total equatorial angle sum in the uranyl complexes can be measured and represents a metric by which coordinative crowding can be ascertained; the higher the angle sum is above $360^{\circ}$, the more distorted is the planar coordination about the uranyl cation. The $\mathrm{UO}_{2}(\mathbf{3 - 2})$ complex exhibits the smallest angle sum of $362^{\circ}$, but when the linker is changed from the flexible ethylene linker in 3-2 to the rigid $O$ phenylene linker in 3-4 and 3-5, the total angle sums in general increase, with a maximum of $384^{\circ}$ observed in $\mathrm{UO}_{2}(3-4)$ structure \#4. These deviations show quite conclusively that the $o$-phenylene linkers adds a significant geometric constraint to the $\mathrm{TAM}(\mathrm{HOPO})_{2}$ ligands that necessitates coordinative distortion about the uranyl cation. In contrast, the flexible ethylene linkers in 3-2 compensate for potential steric strain, allowing a more planar coordination mode for the hexadentate ligand by adopting the staggered alkane conformation.

Table 3-4. Interplanar angles in $\mathrm{UO}_{2}\left[\mathrm{TAM}(\mathrm{HOPO})_{2}\right]$ complex crystal structures

\begin{tabular}{|c|c|c|c|c|}
\hline Ligand & $\begin{array}{c}\text { HOPO- } \\
\text { HOPO, }\left[{ }^{\circ}\right]\end{array}$ & $\begin{array}{l}\text { HOPO- } \\
\text { TAM, }\left[{ }^{\circ}\right]\end{array}$ & $\begin{array}{c}\text { HOPO- } \\
\text { Uranyl, }\left[{ }^{\circ}\right]\end{array}$ & $\begin{array}{c}\text { TAM- } \\
\text { Uranyl, }\left[{ }^{\circ}\right]\end{array}$ \\
\hline $\begin{array}{l}\text { TAM(2Li-me-3,2- } \\
\text { HOPO })_{2}, \mathbf{3 - 2}^{a}\end{array}$ & $2.5(3)$ & $12.3(2)$ & $3.8(2)$ & $8.7(2)$ \\
\hline $\begin{array}{l}\text { TAM(o-phen-1,2- } \\
\text { HOPO })_{2}, \mathbf{3 - 4 , \# 1}\end{array}$ & $30.8(5)$ & $\begin{array}{l}16.4(5), \\
25.9(5)\end{array}$ & $\begin{array}{l}13.9(5) \\
17.0(4) \\
\end{array}$ & $16.9(4)$ \\
\hline $\begin{array}{l}\text { TAM(o-phen-1,2- } \\
\text { HOPO })_{2}, \mathbf{3 - 4 , \# 2}\end{array}$ & $14.9(2)$ & $\begin{array}{l}23.2(1), \\
37.5(1)\end{array}$ & $\begin{array}{l}5.1(1), \\
13.1(2) \\
\end{array}$ & $24.54(8)$ \\
\hline $\begin{array}{l}\text { TAM(o-phen-1,2- } \\
\text { HOPO })_{2}, \mathbf{3 - 4 , \# 3}\end{array}$ & $45.3(2)$ & $\begin{array}{l}11.6(3), \\
56.6(2)\end{array}$ & $\begin{array}{l}17.8(2), \\
27.6(2) \\
\end{array}$ & $29.0(2)$ \\
\hline $\begin{array}{l}\text { TAM(o-phen-1,2- } \\
\text { HOPO })_{2}, \mathbf{3 - 4 , \# 4}\end{array}$ & $81.8(2)$ & $\begin{array}{l}46.0(3), \\
49.0(2)\end{array}$ & $\begin{array}{l}41.9(2), \\
44.5(2)\end{array}$ & $11.4(3)$ \\
\hline $\begin{array}{l}\text { TAM(o-phen-Me- } \\
3,2-\mathrm{HOPO})_{2}, \mathbf{3 - 5}^{a}\end{array}$ & $71.49(8)$ & $48.3(1)$ & $41.1(1)$ & $13.1(2)$ \\
\hline
\end{tabular}

${ }^{a}$ The two halves of the molecule are crystallographically identical, giving rise to only one HOPO-HOPO and HOPO-uranyl angle.

The interplanar angles listed in Table 3-4 are calculated using the mean squared planes defined by the six ring atoms in the TAM and HOPO moieties and the six 
coordinating oxygens from the $\mathrm{TAM}(\mathrm{HOPO})_{2}$ ligands in the uranyl coordination plane. Assuming that the unconstrained coordination geometry of a HOPO or TAM moiety is co-planar with the uranyl coordination plane (see Chapter 2), the ideal values for all the angles in Table 3-4 are $0^{\circ}$. The interplanar angles are necessarily a result of a combination of twists through and bends out of the uranyl coordination plane, depending on the ligand conformation, and as the table illustrates, can vary significantly between and within the complexes measured.

A trend that is readily noticeable by visual inspection of the structures in Figure 3-6, is that ligand 3-2 imposes the least amount of distortion from ideal planar coordination, with a maximum interplanar angle of $12.3^{\circ}$ between the HOPO and TAM planes. This value is only slightly larger than interplanar angles observed in $\mathrm{UO}_{2}$ (bis-Me-3,2-HOPO) complexes in Chapter 2. In sharp contrast to this are the interplanar angles in uranyl complexes with the o-phenylene-linked ligands 3-4 and 3-5, whose values range from $5.1^{\circ}$ to $81.8^{\circ}$. This again shows that the aromatic linker imposes significant steric constraints on the uranyl complex, consistent with the disparate $\mathrm{U}_{-} \mathrm{O}_{\text {Hоро }}$ distances and larger equatorial angle sums also exhibited in those structures. Although such a result may not be particularly surprising, the variety of interplanar angles seen in the $o$ phenylene-linked ligands is significant; it suggests that the crystal structures do not necessarily represent the lowest energy conformations of the complexes, and that in solution the uranyl complexes with 3-4 and 3-5 exhibit a significant amount of flexibility in solution despite the rigid, aromatic linker. Such flexibility can also be assumed in complexes with 3-2 due to the greater flexibility of the ethylene linker. 
The variety in ligand conformation and bond distances seen in the $\mathrm{UO}_{2}\left[\mathrm{TAM}(\mathrm{HOPO})_{2}\right]$ complexes raises the question of which molecule most strongly binds the uranyl cation. The relatively long $\mathrm{U}_{-} \mathrm{O}_{\mathrm{HOPO}}$ bonds in the hexadentate complexes suggest that individual bonds in the complex may be weaker than those in comparable tetradentate bis-Me-3,2-HOPO complexes, but the increased chelate effect suggests the hexadentate ligands should exhibit a significantly higher uranyl affinity.

\subsubsection{Soluble Hexadentate Ligand Design and Synthesis}

As in Chapter 2, the desired method by which to determine the uranyl affinity of $\mathrm{TAM}(\mathrm{HOPO})_{2}$ ligands was aqueous solution thermodynamics. Although ligands 3-1, 3-2, and 3-3 were soluble enough for protonation constant measurements at $c a .50 \mu \mathrm{M}$ concentrations, the introduction of the o-phenylene linkers makes ligands 3-4 and 3-5 too insoluble when fully protonated to measure protonation constants even at $2 \mu \mathrm{M}$ concentrations. Thus, more soluble versions of these ligands were needed to enable the desired solution thermodynamic measurements. In order to impart sufficient aqueous solubility to $\mathrm{TAM}(\mathrm{HOPO})_{2}$ ligands for solution phase measurements, the methylprotected triethyleneglycol moiety (referred to hereon as PEG) was utilized as a solubilizing group. As before, this moiety was chosen because PEG groups are known to impart increased water solubility to organic molecules, and its lack of charge and hydrogen-bond donors makes purification of intermediates in multiple-step syntheses straightforward.

Because solution thermodynamic measurements are intended in this case to elucidate the effect of geometric and binding moiety differences, the PEG solubilizing groups needed to be attached as far from the uranyl binding pocket as possible so as to avoid 
significantly changing the coordination geometry of the ligands as compared to the unsubstituted ligands 3-1 through 3-5. Additionally, substitution onto binding moiety rings themselves was also unwanted because this would undoubtedly change the electronics of the binding ring, introducing an unknown electronic effect to the thermodynamic studies. Thus, for both the ethylene- and o-phenylene-linked ligands, only the backbones were suitable locations for the PEG groups to be appended. It was expected that electronically inductive effects upon substitution on the ethylene linkers would be negligible, although possible interactions with the HOPO amides may cause small differences in geometry or proton affinity, as was seen with the PEG-nLi-Me-3,2HOPO ligands described in Chapter 2. The o-phenylene linker provides several positions for substitution, with the extremities of the aryl bridge placing substituents farther from the ligand binding pocket than the ethylene linkers. However, the fully conjugated system in o-phenylene-linked ligands makes the possibility of substituent-induced electronic effects on proton or uranyl affinities unavoidable.

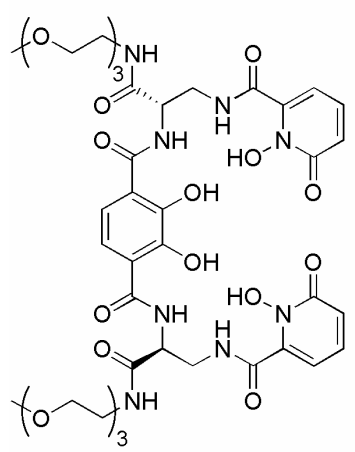

PEG-TAM(2Li$1,2-\mathrm{HOPO})_{2}$

3-12

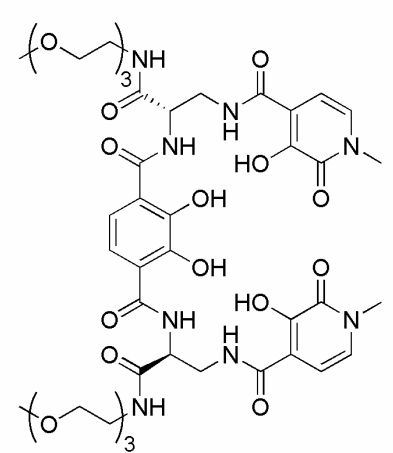

PEG-TAM(2Li$\mathrm{Me}-3,2-\mathrm{HOPO})_{2}$

3-13

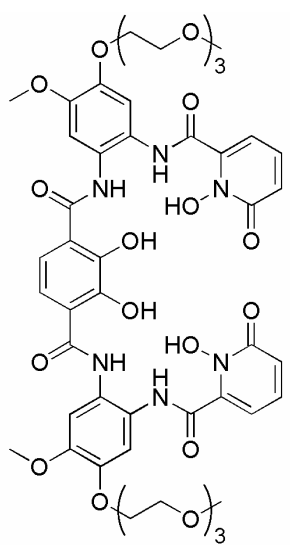

PEG-TAM $(o-$-phen$1,2-\mathrm{HOPO})_{2}$

3-14

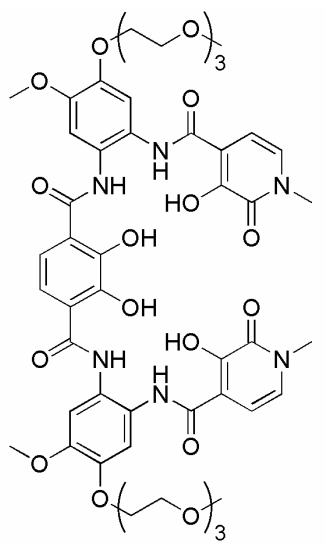

PEG-TAM $(o$-phen$\mathrm{Me}-3,2-\mathrm{HOPO})_{2}$

3-15

Figure 3-11. Soluble PEG-TAM(HOPO $)_{2}$ ligands. 
With these considerations in mind, four PEG-containing TAM(HOPO $)_{2}$ ligands 3-12 through 3-15 were designed and are illustrated in Figure 3-11 below. The linkers in these ligands are no longer symmetric as in 3-1 through 3-5, and each must be asymmetrically linked via amide bonds to both a TAM and a HOPO moiety. This architecture therefore rules out the slow addition techniques employed in the synthesis of 3-1 through 3-5, and requires that the amide linker syntheses include selective protection/deprotection strategies to impart the desired asymmetry to the amide couplings.

The syntheses of ligands 3-12 through 3-15 are illustrated in Scheme 3-2. The linker used for ligands 3-12 and 3-13 was the PEG-subsituted L-diaminopropionic acid (LDAP). The use of enantiopure starting material was preferred over the racemate because two of these linkers are used in each ligand, creating a potential for diasteromeric $\mathrm{TAM}(2 \mathrm{Li}-\mathrm{HOPO})_{2}$ products if using the racemic linker. The ligand flexibility observed in the $\mathrm{UO}_{2}\left[\mathrm{TAM}(\mathrm{HOPO})_{2}\right]$ crystal structures above suggests that the uranyl binding constants of the $\operatorname{TAM}(2 \mathrm{Li}-\mathrm{HOPO})_{2}$ diastereomers would not be significantly different, but targeting one diastereomer makes synthetic characterization far simpler, and the syntheses were approached with this in mind. Retention of stereochemical information throughout the reactions in Scheme 3-2 was not verified, although the cleanliness of the NMR spectra for compounds 3-18a,b suggest the presence of only one diastereomer.

The method of Jiang et al. ${ }^{17}$ was used to facilitate concerted Fmoc-deprotection and amide coupling to the benzyl-protected, thiaz-activated 1,2- and Me-3,2-HOPO moieties to make 3-17a,b. Subsequent Boc-deprotection was performed using $\mathrm{TsOH}$, but the resultant amine was too sterically hindered to react with another thiaz-activated amide, prompting the use of the benzyl-protected TAM-diacid chloride to generate 3-18a,b. 


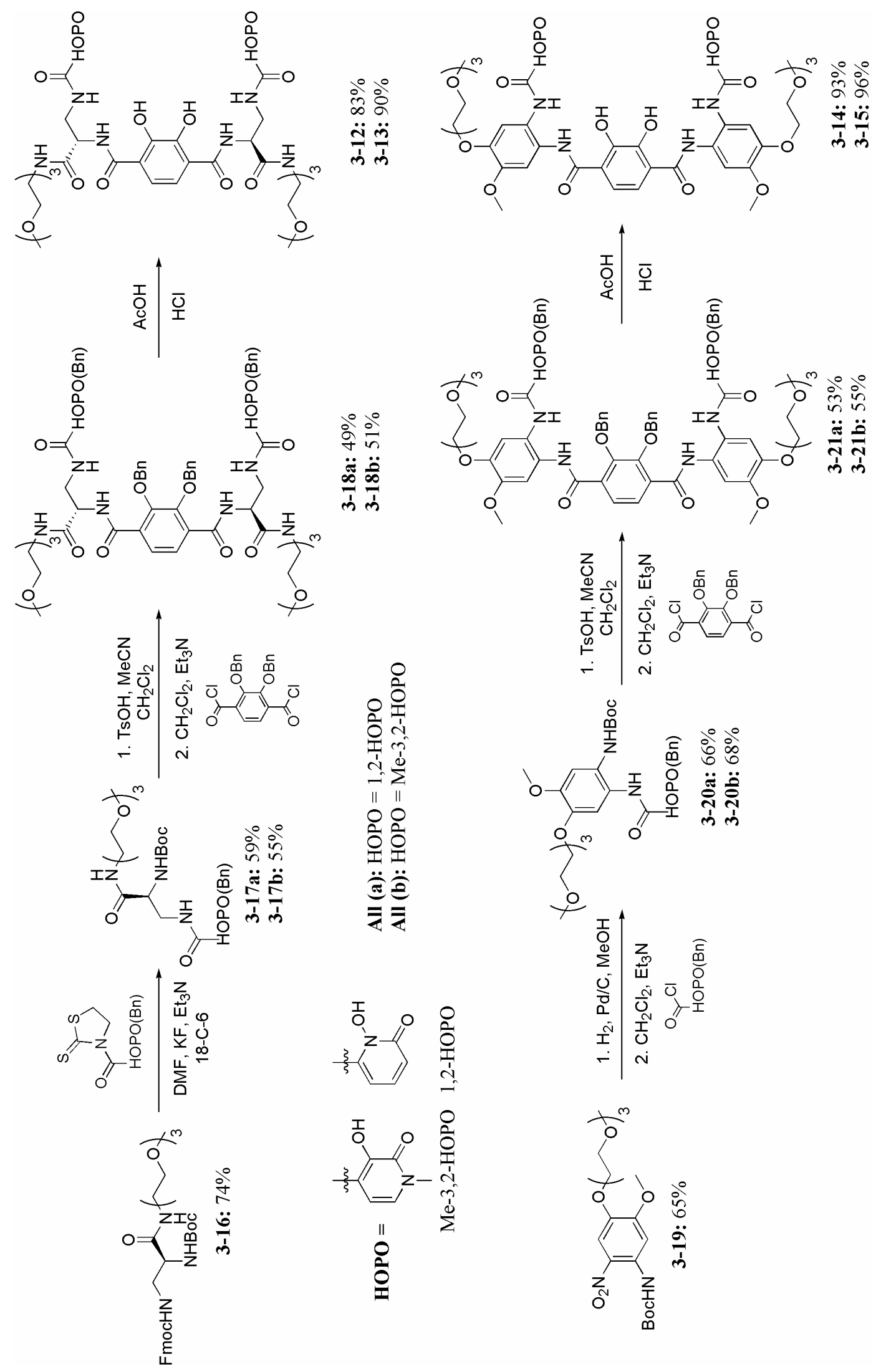

年 
Acid deprotections to generate ligands 3-12 and 3-13 were carried out for 10 days to ensure complete TAM deprotection. Final ${ }^{1} \mathrm{H}$ NMR analysis indicated there had been a small amount of diastereomer formed during deprotection. The diastereomeric ratio (desired all-L vs. L/S) in the product was determined by ${ }^{1} \mathrm{H}$ NMR analysis to be $c a .8 .1: 1$ for 3-12 and 4.6:1 for 3-13. Although the solutions were never heated above $40^{\circ}$ during the reaction or workup, it appears that these conditions are sufficient to switch the chirality of enantiomeric carbon in the linker. As mentioned above, the flexibility in the $\mathrm{UO}_{2}\left[\mathrm{TAM}(\mathrm{HOPO})_{2}\right]$ complexes was assumed to make the diastereomeric mixture in these finished ligands inconsequential, and the ligands were used without further purification in solution thermodynamic measurements.

The PEG-functionalized o-phenylene diamine linker used to make ligands 3-14 and 3-15 was chosen primarily on grounds of synthetic ease, as the precursor molecule to 319 is a literature preparation reported by Toke et al. and can be synthesized on a multigram scale. ${ }^{18,19}$ Additionally, after Boc protection to form 3-19, the two amines for amide coupling can be generated selectively first by palladium-catalyzed reduction of the nitrate group and then TsOH-mediated Boc deprotection before amide coupling to generate 320a,b and 3-21a,b respectively. In both of these steps, however, the free amine generated is unstable; the deprotected species, if exposed to air or solvents that were not thoroughly degassed, generated a strongly-colored product that ranged from dark purple to black. It was assumed that this involved the oxidation of the electron-rich aniline to the azaquinone, as has been observed in work by Heyduk and co-workers. ${ }^{20}$ To avoid extensive decomposition, the intermediate amines were never isolated cleanly or characterized,and were reacted immediately with the appropriate acid chloride to 
generate intermediates 3-20a,b and 3-21a,b, whose yields were rather low because of this decomposition. Acid deprotection to form ligands 3-14 and 3-15 proceeded cleanly.

Ligands 3-12 through 3-15 were isolated as hydroscopic, beige solids. Because of their hydroscopic nature and significant solubility in aqueous and polar organic solvents, they could not be isolated by precipitation as the parent $\mathrm{TAM}(\mathrm{HOPO})_{2}$ ligands were, but instead were isolated by rigorous evaporation of the acid deprotection solution, followed by co-evaporation using methanol and then chloroform.

The uranyl affinities of the $\mathrm{TAM}(\mathrm{HOPO})_{2}$ ligands were expected to be significantly greater than those of the bis-Me-3,2-HOPO moieties reported in Chapter 2. However, whether this effect would be due to increased denticity of the $\mathrm{TAM}(\mathrm{HOPO})_{2}$ ligands or due to the inclusion of the strongly binding TAM moiety could not be determined by titrations using PEG-TAM(HOPO $)_{2}$ ligands alone. To determine which of these two options is the cause of the expected uranyl affinity increase, two tetradentate Pr-TAM2Li-Me-3,2-HOPO ligands (3-22 and 3-23) were designed, one with and one without the PEG solubilizing functionality appended to the ethylene backbone (Figure 3-12). Ligands 3-22 and 3-23 should provide the same ligand geometry about the uranyl cation as the 2Li-Me-3,2-HOPO ligands studied in Chapter 2, but contain the TAM binding moiety to make their electrostatic properties more similar to the $\mathrm{TAM}(\mathrm{HOPO})_{2}$ ligands than any bis-Me-3,2-HOPO ligand. Comparing the uranyl affinity of these ligands with those of PEG-2Li-Me-3,2-HOPO (2-17) and the PEG-TAM(HOPO) $)_{2}$ ligands, it can be determined whether higher denticity or the TAM moiety is the primary cause for any increased uranyl affinity in $\mathrm{TAM}(\mathrm{HOPO})_{2}$ ligands. The syntheses of ligands 3-22 and 323 are illustrated in Scheme 3-3. 


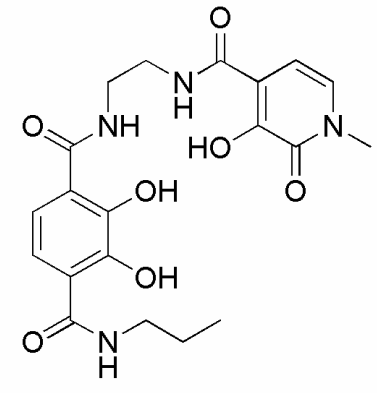

Pr-TAM-2Li-Me-3,2-HOPO 3-22<smiles>CCCNC(=O)c1ccc(C(=O)N[C@@H](CNC(=O)c2ccn(C)c(=O)c2O)C(=O)NCCOC(C)(C)C)c(O)c1O</smiles>

PEG-Pr-TAM-2Li-Me-3,2-HOPO

Figure 3-12. Mixed tetradentate ligands Pr-TAM-2Li-Me-3,2-HOPO (3-22) and PEG-Pr2Li-Me-3,2-HOPO (3-23).

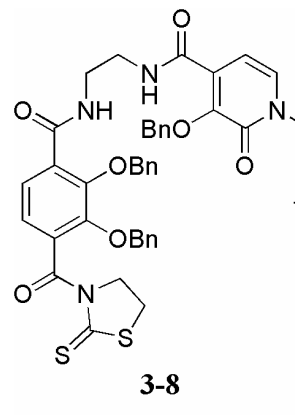<smiles>CCCNC(=O)c1c(O)c(C(=O)NCCNC(=O)c2ccn(C)c(=O)c2O)c(O)c(C(=O)O)c1OCCN</smiles><smiles>COC(O)(Cl)Cl</smiles>

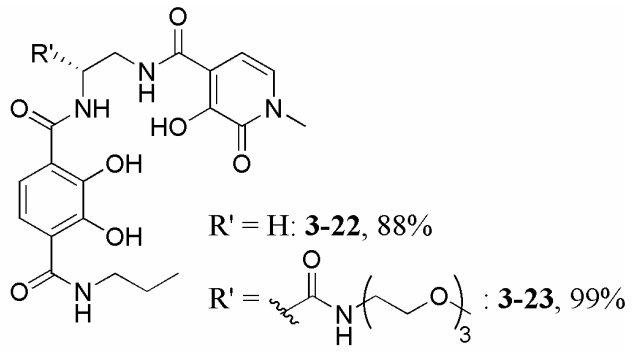

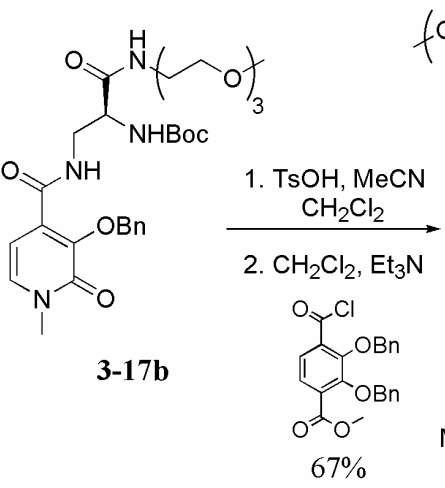<smiles>CCOCCNC(=O)C(CNC(=O)CCOCCOC)NC(=O)c1ccc(C(=O)OCC)c(Br)c1OCCO</smiles>

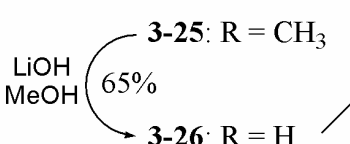

Scheme 3-3. Syntheses of ligands 3-22 and 3-23.

The synthetic procedures for 3-22 and 3-23 utilized starting materials already developed for the synthesis of ligands 3-3 and 3-13 and incorporated similar synthetic procedures and reactivity considerations. The final acid deprotection to make ligand 3-23 may have suffered some loss of chirality as seen in the deprotection of ligands 3-12 and 3-13, but quantification of this effect was not pursued, as it only results in chemically- 
equivalent enantiomers. The final products are beige solids, with 3-22 isolable by filtration, and 3-23 isolated by co-evaporation techniques as with 3-12 through 3-15. It was hoped that a crystalline sample of the $\mathrm{UO}_{2}(\mathbf{3 - 2 2})$ complex could be isolated and analyzed using single crystal X-ray diffraction, but no success was had in these efforts. However, both ligands were soluble enough for solution thermodynamic measurements.

\subsubsection{Solution Thermodynamics}

Because of their low solubilities, protonation constants with $\mathrm{TAM}(\mathrm{HOPO})_{2}$ and $\mathrm{Pr}$ 2Li-Me-3,2-HOPO ligands were determined using spectrophotometric techniques at ligand concentrations of 4-6 $\mu \mathrm{M}$ with a starting DMSO concentration of $c a .5 \%$. A small amount of baseline drift was observed in titrations with ligands 3-14 and 3-15, so their ligand concentrations were lowered to $2 \mu \mathrm{M}$, which still produced significant absorbance signal using a $6.6 \mathrm{~cm}$ path length cell due to their increased conjugation and resultant higher molar absorption coefficients. Ligand titrations were typically performed between $\mathrm{pH} 3-11$, sometimes going as low as $\mathrm{pH} 2.5$ in the presence of 1,2-HOPO moieties and as high as $\mathrm{pH} 11.5$ in order to cover the second $\mathrm{pK}_{\mathrm{a}}$ value of the TAM moiety. Titrations were performed from acidic to basic $\mathrm{pH}$, but attempts to reverse the titration revealed poor reversibility which only worsened with increased equilibration time (see Appendix for reversibility graphs). It was assumed that the irreversibility is caused by the oxidation of the TAM moiety at very basic $\mathrm{pH}$ by trace oxygen in the cell to form the quinone, so no reverse titrations (basic to acidic $\mathrm{pH}$ ) were used to determine the ligand protonation constants. The data from these titrations were combined to give the $\mathrm{pK}_{\mathrm{a}}$ values listed in Table 3-5 below. The $\mathrm{pK}_{\mathrm{a}}$ sums $\left(\Sigma \mathrm{pK}_{\mathrm{a}}\right)$ are also listed, which are an overall measure of ligand acidity, with lower values indicating decreased proton affinity. 
Table 3-5. $\mathrm{pK}_{\mathrm{a}}$ values for TAM-containing ligands.

\begin{tabular}{|c|c|c|c|c|c|}
\hline & $\mathbf{p K _ { \mathrm { a } , 1 }}$ & $\mathbf{p K}_{\mathrm{a}, 2}$ & $\mathbf{p K}_{\mathrm{a}, 3}$ & $\mathbf{p K}_{\mathrm{a}, 4}$ & $\Sigma$ pK $_{\mathrm{a}}$ \\
\hline $\begin{array}{c}\text { TAM }(2 \mathrm{Li}-1,2-\mathrm{HOPO})_{2}, \\
\mathbf{3 - 1}\end{array}$ & 4.91(3) & $6.56(8)$ & $8.7(1)$ & $10.7(2)$ & $30.9(2)$ \\
\hline $\begin{array}{c}\text { TAM(2Li-Me-3,2- } \\
\text { HOPO })_{2}, \mathbf{3 - 2}\end{array}$ & $5.62(8)$ & $6.65(8)$ & $8.12(6)$ & $11.16(9)$ & $31.6(2)$ \\
\hline $\begin{array}{c}\text { TAM(2Li-1,2-HOPO) } \\
(2 \mathrm{Li}-\mathrm{Me}-3,2-\mathrm{HOPO}), \mathbf{3 - 3}\end{array}$ & 4.94(1) & $6.48(1)$ & $8.44(3)$ & $11.12(4)$ & $30.98(5)$ \\
\hline $\begin{array}{c}\text { PEG-TAM(2Li-1,2- } \\
\text { HOPO })_{2}, \mathbf{3 - 1 2}\end{array}$ & $3.90(5)$ & $5.53(3)$ & $7.78(1)$ & $9.95(1)$ & $27.16(6)$ \\
\hline $\begin{array}{c}\text { PEG-TAM }(2 \mathrm{Li}-\mathrm{Me}-3,2- \\
\mathrm{HOPO})_{2}, \mathbf{3 - 1 3}\end{array}$ & $4.5(1)$ & $6.35(2)$ & $9.22(2)$ & $10.89(4)$ & $30.96(5)$ \\
\hline $\begin{array}{c}\text { PEG-TAM }(o-p h e n-1,2- \\
\text { HOPO })_{2}, \mathbf{3 - 1 4}\end{array}$ & $3.20(8)$ & $5.10(7)$ & $7.1(1)$ & $9.8(2)$ & $25.2(2)$ \\
\hline $\begin{array}{l}\text { PEG-TAM }(o \text {-phen-Me- } \\
3,2-\mathrm{HOPO})_{2}, \mathbf{3 - 1 5}\end{array}$ & $4.7(1)$ & $6.7(1)$ & 7.13(9) & $10.07(5)$ & $28.6(2)$ \\
\hline $\begin{array}{c}\text { Pr-TAM-2Li-Me-3,2- } \\
\text { HOPO, 3-22 }\end{array}$ & $5.89(5)$ & $6.8(1)$ & $10.6(1)$ & -- & $23.3(2)$ \\
\hline $\begin{array}{c}\text { PEG-Pr-TAM-2Li-Me- } \\
\text { 3,2-HOPO, } \mathbf{3 - 2 3}\end{array}$ & $4.3(1)$ & $6.15(5)$ & $10.46(6)$ & -- & $20.9(1)$ \\
\hline
\end{tabular}

The first two protonation constants $\left(\mathrm{pK}_{\mathrm{a}, 1}\right.$ and $\left.\mathrm{pK}_{\mathrm{a}, 2}\right)$ for $\mathrm{TAM}(\mathrm{HOPO})_{2}$ ligands correspond to deprotonation of the more acidic HOPO moieties, while the latter two protonation constants are those of the two TAM phenolic protons. Similarly, the last two protonation constants for ligands 3-22 and 3-23 belong to the TAM phenols, while the first corresponds to the Me-3,2-HOPO moiety.

In comparison to the bis-Me-3,2-HOPO ligands discussed in Chapter 2, the TAMcontaining ligands have significantly lower acidities due to the more basic TAM moiety. ${ }^{9}$ As expected, ${ }^{21}$ the 1,2-HOPO containing ligands 3-1, 3-12 and 3-14 are more acidic than their structurally analogous Me-3,2-HOPO analogs 3-2, 3-13 and 3-15. Although the drop in acidity is in large part due to the depression of $\mathrm{pK}_{\mathrm{a}, 1}$ and $\mathrm{pK}_{\mathrm{a}, 2}$, Table 3-5 indicates the 1,2-HOPO-containing ligands are more acidic at every deprotonation step.

Table 3-5 also indicates that the presence of the PEG group in ligands 3-12, 3-13 and 3-23 lowers each $\mathrm{pK}_{\mathrm{a}}$ value and thus the $\Sigma \mathrm{pK}_{\mathrm{a}}$ as compared to their unsubstituted analogs 3-1, 3-2, and 3-22. This effect was seen in the bis-Me-3,2-HOPO ligands described in Chapter 2, and is again most likely caused by the close proximity of a hydrogen-bond 
donating PEG-amide functionality to the HOPO and TAM phenols or N-hydroxides, stabilizing their deprotonated forms.

Another notable trend already observed with bis-Me-3,2-HOPO ligands, is that the presence of the $o$-phenylene linker in ligands 3-14 and 3-15 lowers the overall $\mathrm{pK}_{\mathrm{a}}$ sum compared to their flexible analogs 3-1, 3-2, 3-12 and 3-13. This effect can again be attributed to the unique electronic properties of the o-phenylene linker which creates extensive conjugation. Because no solution thermodynamic data could be collected for bis-Me-3,2-HOPO or TAM(HOPO $)_{2}$ ligands utilizing the unsubstituted $o$-phenylene linker due to their insolubility, it is impossible to discern from these measurements if the $\mathrm{pK}_{\mathrm{a}}$ drop arises from the aromatic linker or the presence of the PEG moieties thereon.

In TAM(HOPO $)_{2}$ ligands the protonation constants of the TAM moiety $\left(\mathrm{pK}_{\mathrm{a}, 3}\right.$ and $\mathrm{pK}_{\mathrm{a}, 4}$ ) also display an interesting trend if compared to the published TAM $\mathrm{pK}_{\mathrm{a}}$ values of 6.0-6.5 and 10.3-11.0. ${ }^{9}$ Namely, while one TAM proton in TAM(HOPO) 2 ligands exhibits the expected higher $\mathrm{pK}_{\mathrm{a}}$ value of $c a .11$, the other TAM proton is less acidic than the published values would lead one to expect. In contrast, the tetradentate ligands 3-22 and 3-23 exhibit TAM $\mathrm{pK}_{\mathrm{a}}$ values $\left(\mathrm{pK}_{\mathrm{a}, 2}\right.$ and $\left.\mathrm{pK}_{\mathrm{a}, 3}\right)$ very close to published values. The HOPO moieties in the tetradenate and hexadentate ligands are in similar environments, so a significant difference in the hydrogen-bonding stabilization of the deprotonated HOPO moiety is unlikely. However, the TAM moieties in 3-22 and 3-23 are asymmetrically substituted unlike those in $\mathrm{TAM}(\mathrm{HOPO})_{2}$ ligands. Results in Chapter 2 demonstrated that short linkers allow for increased intramolecular phenolate hydrogen bond stabilization, utilizing the hydrogen-bonding capabilities of both liker amides as well as the PEGamide group on the linker. TAM(HOPO $)_{2}$ ligands reported here utilize very short linkers 
and thus probably experience this cooperative charge stabilization which explains the very low HOPO $\mathrm{pK}_{\mathrm{a}}$ values in ligands 3-12, 3-13, and 3-23. However, the tetradentate ligands 3-22 and 3-23 both contain one TAM amide that is dedicated solely to stabilizing the TAM phenolate farthest from the HOPO moiety, and it is hypothesized that this dedicated hydrogen-bonding interaction along with the lack of geometric hindrance of the terminal propyl amide is responsible for the lowered $\mathrm{pK}_{\mathrm{a}}$ values of the TAM moiety in those ligands.

Spectrophotometric titrations of TAM-containing ligands with the uranyl cation were performed using a 1:1 metal to ligand ratio to avoid both the formation of polynuclear species as well as the decomposition of the TAM moieties at high $\mathrm{pH}$ as observed in the ligand-only titrations. Metal to ligand ratios were controlled by careful addition of a ligand solution of known concentration in DMSO and a standardized uranyl solution in 1.2 wt. \% nitric acid to the titration apparatus. Uranyl titrations were performed at similar ligand concentrations and with a starting DMSO concentration of $c a .5 \%$ to be consistent with the ligand-only titrations.

Measurements with unsubstituted ligands 3-1 through 3-3 using serial additions of titrant and a 10 minute equilibration time between data points were performed from acidic to basic $\mathrm{pH}$ and reverse without any precipitation observed. These titrations all showed terrible reversibility, but cycling the same solution back and forth between acid and base and then back to base again indicated that the poor reversibility was a kinetic effect, and not one of ligand or complex decomposition (Figure 3-13). Uranyl titrations with 3-1 using a two hour equilibration time indicated that between $\mathrm{pH} 2.5$ and $5.5 \mathrm{a}$ kinetic hysteresis occurs. It was estimated that the equilibration time needed at each $\mathrm{pH}$ 
point between $\mathrm{pH} 2.5$ and 5.5 was about $12-18 \mathrm{hrs}$, so batch titrations were performed with all $\mathrm{TAM}(\mathrm{HOPO})_{2}$ ligands using $48-72$ hour equilibration times. In batch titrations, $\left[\mathrm{UO}_{2}{ }^{2+}\right]=[\mathrm{L}]=1.3-2.0 \mu \mathrm{M}$ to accommodate for the longer 10-cm path length quartzwindow UV/Vis cell used for measurement of the aliquots. With the extended equilibration time, titrations with 3-2 and 3-3 resulted in precipitation of either the protonated ligand or a neutral uranyl complex at low $\mathrm{pH}$, necessitating the use of the PEG-substituted TAM(HOPO $)_{2}$ ligands 3-12 through 3-15 for titration measurements.

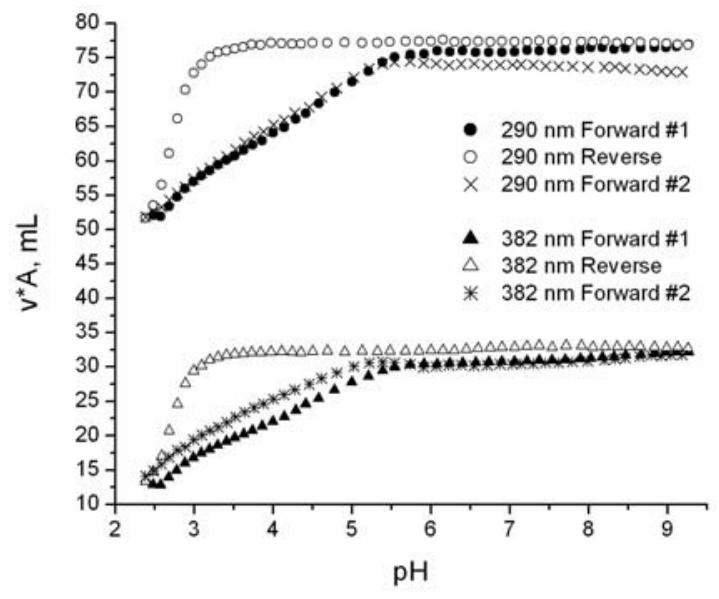

(a)

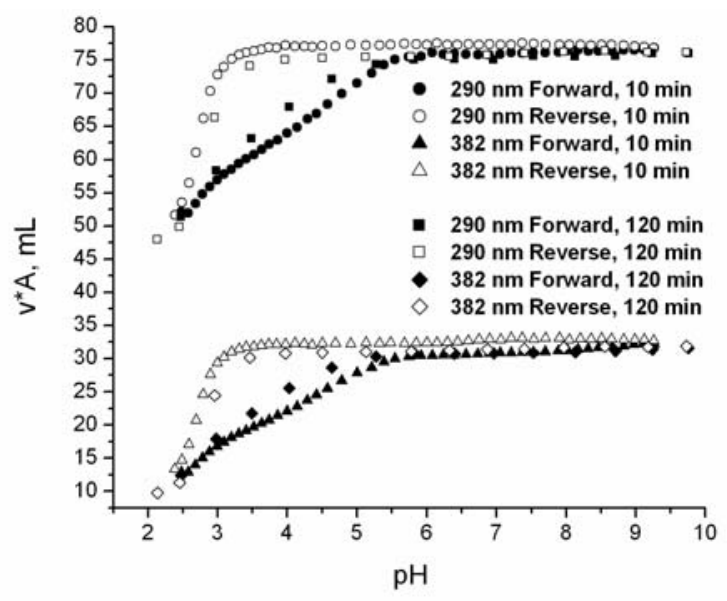

(b)

Figure 3-13. Spectrophotometric reversibility plots of uranyl titrations with TAM(2Li1,2-HOPO $)_{2}$, 3-1: (a) Forward, reverse, then forward titration, 10 minute equilibration time; (b) Overlay of two separate, identical titrations illustrating hysteresis between 10 minute and 120 minute equilibration times.

In contrast to the slow kinetics with $\mathrm{TAM}(\mathrm{HOPO})_{2}$ ligands, uranyl titrations with tetradentate ligands 3-22 and 3-23 exhibited excellent reversibility with equilibration times of 10-20 minutes, so their titrations were performed using incremental titrant addition methods with $\left[\mathrm{UO}_{2}{ }^{2+}\right]=[\mathrm{L}]=6 \mu \mathrm{M}$ and a $6.6 \mathrm{~cm}$ path length cell. In all the uranyl titrations with $\mathrm{TAM}(\mathrm{HOPO})_{2}$ ligands, little change in the UV-Visible spectrum was observed above $\mathrm{pH}$ 8-9 regardless of equilibration time, so these were only carried out up to $\mathrm{pH}$ ca. 9. Early uranyl titrations with 3-1 through 3-3 also showed very good 
reversibility above ca. $\mathrm{pH}$ 6. This behavior is unlike the bis-Me-3,2-HOPO ligands in Chapter 2, whose partial uranyl hydrolysis caused spectrum changes well into the high $\mathrm{pH}$ range, suggesting that in $\mathrm{TAM}(\mathrm{HOPO})_{2}$ complexes there is hydrolysis event, and that the complex is fully formed by about $\mathrm{pH} 9$.

In the case of the tetradentate ligands 3-22 and 3-23, the UV-Visible spectrum continued to change above $\mathrm{pH}$ 9, so uranyl titrations were performed as high as $\mathrm{pH}$ 11.4. Because the coordination mode of the tetradentate ligands is most assuredly similar to that seen in bis-Me-3,2-HOPO ligands, the change at high $\mathrm{pH}$ was assumed to be the partial hydrolysis of the uranyl cation. The excellent reversibility observed at these high $\mathrm{pH}$ values indicated that the spectrum change was not due to ligand decomposition, illustrating that uranyl chelation stabilizes the deprotonated TAM moiety against oxidation to the quinone. Because 3-22 and 3-23 are expected to behave like bis-Me-3,2HOPO ligands, low pH titrations between $\mathrm{pH} 3.0$ and 1.6 were also performed to help more fully characterize the complex formation.

The different equilibration times for uranyl complex formation with hexadentate $\mathrm{TAM}(\mathrm{HOPO})_{2}$ and tetradentate TAM-2Li-Me-3,2-HOPO ligands cannot be dependent on the identity of the chelating moieties used because they both contain similar TAM and HOPO groups; in the absence of uranyl cation, the HOPO moieties in both ligand classes deprotonate at a lower $\mathrm{pH}$ than the TAM moiety. However, the different titration reversibilities illustrate this similarity does not persist in the presence of the uranyl cation, which in turn suggests that the details of initial uranyl chelation with tetradentate and hexadentate TAM-containing ligands is significantly different despite their similar chelating groups. 
It is hypothesized that a step-wise chelation event is responsible for the long equilibration times required in $\mathrm{TAM}(\mathrm{HOPO})_{2}$ titrations, and that the kinetic barrier lies in the rotation of the TAM moiety upon deprotonation at increased $\mathrm{pH}$ (Figure 3-14). Thus, at low $\mathrm{pH}$ both $\mathrm{HOPO}$ moieties bind the uranyl cation, mostly likely with solvent coordinated where the TAM moiety would normally bind. The protonated TAM phenols are rotated away from the uranyl cation and are hydrogen bound to the ortho amide oxygen atoms as prior crystallographic studies would suggest. ${ }^{9,10,22}$ Upon $\mathrm{pH}$ increase, the TAM moiety deprotonates in a stepwise manner, disrupting the hydrogen-bonding network and allowing slow rotation and coordinated solvent displacement at the uranyl center. Upon full deprotonation and uranyl coordination, the TAM phenolates regain a favorable hydrogen-bonding interaction with the amide protons, leading to the kinetic barrier for the reverse titration. Following this reasoning, uranyl titration data with $\mathrm{TAM}(\mathrm{HOPO})_{2}$ ligands were refined using $\mathrm{UO}_{2} \mathrm{LH}_{2}, \mathrm{UO}_{2} \mathrm{LH}$, and $\mathrm{UO}_{2} \mathrm{~L}$ species, assuming that the HOPO protons are of such similar $\mathrm{pK}_{\mathrm{a}}$ values that they simultaneously deprotonate upon initial uranyl chelation.

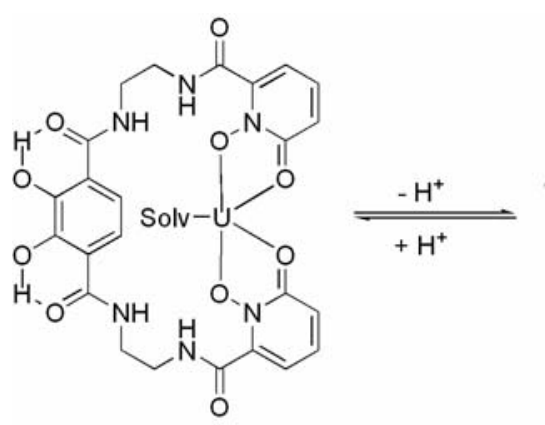

$\mathrm{UO}_{2} \mathrm{LH}_{2}$

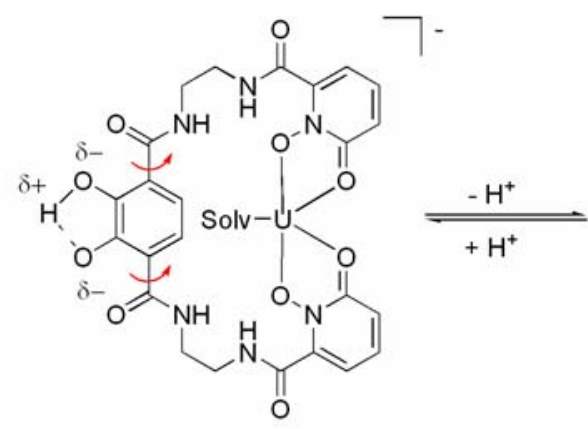

$\mathrm{UO}_{2} \mathrm{LH}$

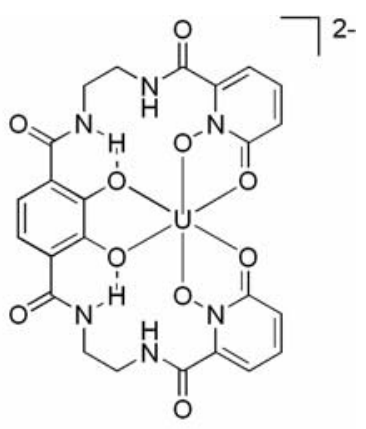

$\mathbf{U O}_{2} \mathbf{L}$

Figure 3-14. Hypothesized deprotonation and TAM rotation in uranyl complex formation with TAM(HOPO) $)_{2}$ ligands (3-1 shown as example). Uranyl oxo atoms are omitted for clarity and hydrogen bonding interactions are only indicated for the TAM moiety.

Titrations with ligand 3-23 required a 20 minute equilibration time as compared to 10 minutes for 3-22, both of which are much shorter than the equilibration times needed 
with hexadentate TAM(HOPO $)_{2}$ ligands. This suggests that there is little steric hindrance in the binding of the TAM moiety to the uranyl cation, although the introduction of the PEG group on the 2Li-linker slightly slows the kinetics of the initial uranyl binding event. The relatively low value of one $\mathrm{TAM} \mathrm{pK}_{\mathrm{a}}$ in both of these ligands is comparable to the $\mathrm{pK}_{\mathrm{a}}$ of parent Pr-Me-3,2-HOPO $\left(\mathrm{pK}_{\mathrm{a}}=6.12\right),{ }^{23}$ and because of its similarity to bisMe-3,2-HOPO ligands, it is reasonable to suppose that initial uranyl chelation occurs via simultaneous and complete deprotonation of the HOPO and TAM moieties to generate an anionic $\mathrm{UO}_{2} \mathrm{~L}$ (solv.) complex. Because uranyl chelation with bis-bidentate Raymond group ligands allows for partial hydrolysis of the metal center without ligand displacement, it was expected that a $\mathrm{UO}_{2} \mathrm{~L}(\mathrm{OH})$ species would form at high $\mathrm{pH}$. Following this reasoning, uranyl titration data with ligands 3-22 and 3-23 were refined using $\mathrm{UO}_{2} \mathrm{~L}$ and $\mathrm{UO}_{2} \mathrm{~L}(\mathrm{OH})$ species as illustrated in Figure 3-15.

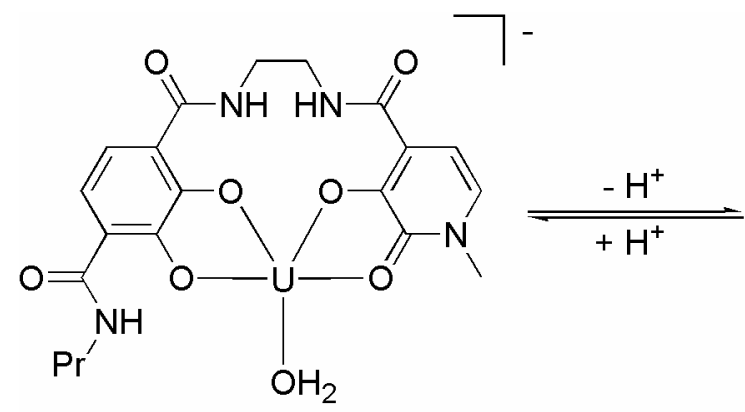

$\mathbf{U} \mathbf{O}_{2} \mathbf{L}$

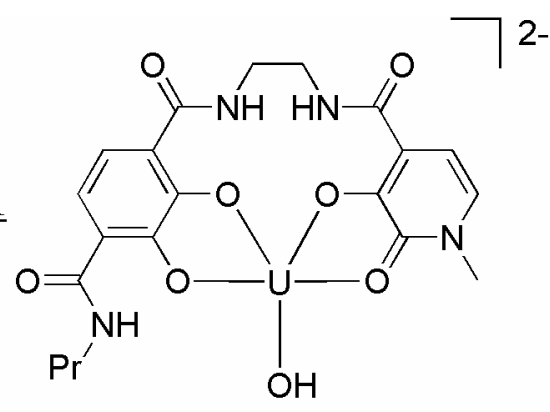

$\mathbf{U} \mathbf{O}_{2} \mathbf{L}(\mathbf{O H})$

Figure 3-15. Proposed speciation in uranyl titrations with ligands 3-22 and 3-23. Uranyl oxo atoms are omitted for clarity.

The uranyl formation constants $\left(\log \beta_{m l h}\right)$ for the TAM(HOPO $)_{2}$ and TAM-2Li-Me3,2-HOPO ligands are reported in Table 3-6. As in Chapter 2, $\log \beta_{m l h}$ values correspond to the concentration- and $\mathrm{pH}-\mathrm{dependent}$ formation constants described by Equations 3-1 and 3-2. The species-independent uranyl affinities $\left(\mathrm{pUO}_{2}\right.$, where $\mathrm{pUO}_{2}=-\log \left[\mathrm{UO}_{2}{ }^{2+}\right.$ free $]$ at the standard conditions of $\left[\mathrm{UO}_{2}{ }^{2+}\right]=1 \mu \mathrm{M}$ and $\left.[\mathrm{L}]=10 \mu \mathrm{M}\right)$ are calculated at low, 
physiological, and high $\mathrm{pH}$ and are compared in Table 3-6. As in Chapter 2, "UO ${ }_{2}^{2+}$ free" refers to solvated uranyl ion unbound by ligand or hydroxides. Also like Chapter 2, further discussion on $\mathrm{pUO}_{2}$ calculations and additional analysis is presented in the Appendix. The large errors in $\mathrm{pUO}_{2}$ values are a result of a combination of the ca. $0.1-0.2$ error in $\mathrm{pK}_{\mathrm{a}}$ values and the 0.1-0.6 errors on $\log \beta_{m l h}$ values, which are themselves a consequence of the low concentrations and large volumes required in the uranyl titrations to achieve aqueous solubility and allow UV-Visible measurements in long path length cells respectively.

$$
\begin{gathered}
m \mathrm{M}^{\mathrm{a}^{+}}+l \mathrm{~L}^{\mathrm{b}-}+h \mathrm{H}^{+} \stackrel{\beta_{m l h}}{=} \mathrm{M}_{m} \mathrm{~L}_{l} \mathrm{H}_{h}^{(m \mathrm{a}-l \mathrm{~b}+h)+} \\
\log \beta_{m l h}=\log \left(\frac{\left[\mathrm{M}_{m} \mathrm{~L}_{l} \mathrm{H}_{h}\right]}{[\mathrm{M}]^{m}[\mathrm{~L}]^{l}[\mathrm{H}]^{h}}\right)
\end{gathered}
$$

\begin{tabular}{|c|c|c|c|c|c|c|c|}
\hline \multirow{2}{*}{ Ligand } & \multirow{2}{*}{$\log \beta_{11-1}$} & \multirow{2}{*}{$\log \beta_{110}$} & \multirow{2}{*}{$\log \beta_{111}$} & \multirow{2}{*}{$\log \beta_{112}$} & \multicolumn{3}{|c|}{$\mathrm{pUO}_{2}{ }^{2+}$} \\
\hline & & & & & pH 3.0 & pH 7.4 & pH 9.0 \\
\hline $\begin{array}{c}\text { TAM(2Li-1,2-HOPO })_{2} \text {, } \\
\mathbf{3 - 1}\end{array}$ & - & $21.95(4)$ & $26.86(8)$ & $30.79(2)$ & $6.9(3)$ & 18.2(3) & $21.0(3)$ \\
\hline $\begin{array}{c}\text { PEG-TAM(2Li-1,2- } \\
\text { HOPO })_{2}, \mathbf{3 - 1 2}\end{array}$ & - & $17.9(3)$ & $24.8(2)$ & $29.4(2)$ & $9.2(3)$ & $15.9(3)$ & $17.8(3)$ \\
\hline $\begin{array}{c}\text { PEG-TAM(2Li-Me-3,2- } \\
\text { HOPO })_{2}, \mathbf{3 - 1 3}\end{array}$ & - & $21.5(5)$ & $28.7(4)$ & $32.0(4)$ & $8.1(5)$ & $17.3(5)$ & $20.1(6)$ \\
\hline $\begin{array}{c}\text { PEG-TAM }(o-\text { phen-1,2- } \\
\text { HOPO })_{2}, \mathbf{3 - 1 4}\end{array}$ & - & $19.1(6)$ & $25.0(4)$ & $30.0(3)$ & $11.5(4)$ & $17.5(8)$ & $19.2(8)$ \\
\hline $\begin{array}{c}\text { PEG-TAM }(o-p h e n-M e- \\
\text { 3,2-HOPO })_{2}, \mathbf{3 - 1 5}\end{array}$ & - & $19.0(5)$ & $25.7(2)$ & $29.5(4)$ & $7.9(5)$ & $17.1(5)$ & $18.8(6)$ \\
\hline $\begin{array}{c}\text { Pr-TAM-2Li-Me-3,2- } \\
\text { HOPO, 3-22 }\end{array}$ & $11.92(6)$ & $19.75(1)$ & - & - & $6.56(8)$ & $17.5(1)$ & $20.3(2)$ \\
\hline $\begin{array}{c}\text { PEG-Pr-TAM-2Li-Me- } \\
\text { 3,2-HOPO, 3-23 }\end{array}$ & $10.31(9)$ & $17.9(1)$ & - & - & $7.0(2)$ & $16.0(2)$ & $18.8(1)$ \\
\hline
\end{tabular}

Table 3-6. $\log \beta_{m l h}$ and $\mathrm{pUO}_{2}$ values for TAM-containing ligands.

Representative speciation diagrams of the uranyl complexes with ligands 3-1 and 322 are illustrated in Figure 3-16; those of all TAM-containing ligands are provided in the Appendix, along with their UV-Visible titration spectra. What is notable is that the initial uranyl complex formation occurs at a lower $\mathrm{pH}$ (well below 2.5) for hexadentate 
$\mathrm{TAM}(\mathrm{HOPO})_{2}$ ligands, while for tetradentate TAM-2Li-Me-3,2-HOPO ligands this only begins between $\mathrm{pH} 2.0-2.5$, and by $\mathrm{pH} 3$ is still incompletely formed. The reason for this is that initial complex formation with the hexadentate ligands involves only the low-pK HOPO units, while those with tetradentate ligands require deprotonation of the more basic TAM moiety. In fact, the complete deprotonation of the TAM moiety in the tetradentate ligands represents an effective maximum $\mathrm{pK}_{\mathrm{a}}$ shift of $c a .8 \log$ units in the presence of the uranyl cation as compared to the free ligand in solution. This illustrates the strong inductive effect of the uranium(VI) dioxo dication that is known to cause effective $\mathrm{pK}_{\mathrm{a}}$ shifts as high as 13 orders of magnitude in chelating ligands. ${ }^{24}$ Such an extreme inductive effect is not observed with hexadentate $\mathrm{TAM}(\mathrm{HOPO})_{2}$ ligands because of the geometric consequences discussed above and is not necessary to form a tetradentate complex between $\mathrm{TAM}(\mathrm{HOPO})_{2}$ ligands and the uranyl cation.

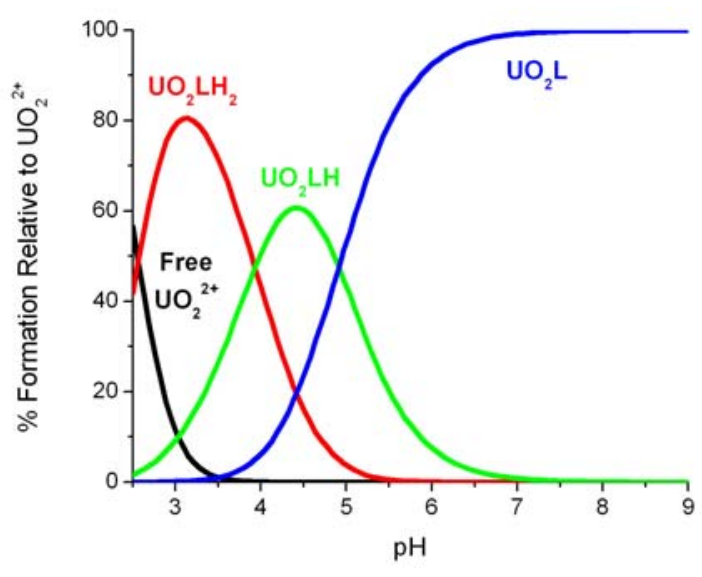

(a)

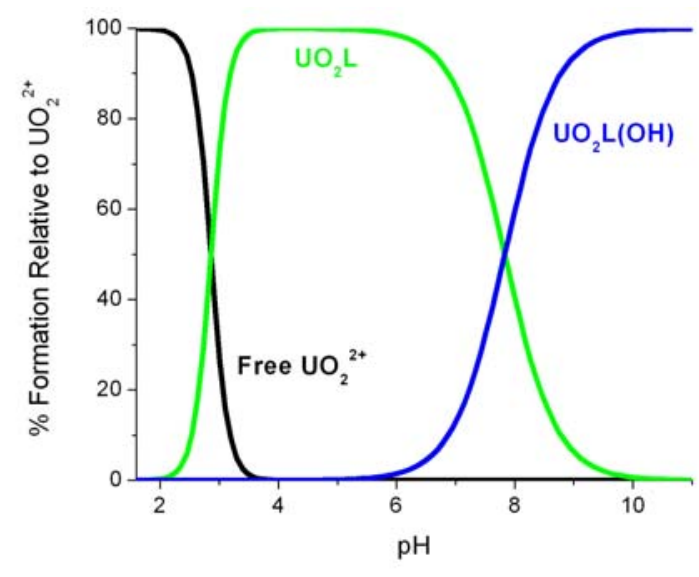

(b)

Figure 3-16. Representative speciation diagrams for uranyl complexes at standard $\mathrm{pUO}_{2}$ conditions of $\left[\mathrm{UO}_{2}{ }^{2+}\right]=1 \mu \mathrm{M},[\mathrm{L}]=10 \mu \mathrm{M}$ : (a) TAM(2Li-1,2-HOPO) $)_{2}, \mathbf{3 - 1}$; (b) PrTAM-2Li-Me-3,2-HOPO, 3-22.

The behavior in basic medium of these ligands is also notable; the tetradentate ligands exhibit the expected partial hydrolysis of the uranyl cation, while the hexadentate ligands inhibit uranyl hydrolysis. Early reversibility studies of the $\mathrm{UO}_{2}\left[\mathrm{TAM}(\mathrm{HOPO})_{2}\right]$ systems 
indicated that the ligands prevent hydrolysis even up through $\mathrm{pH} 11$, which is evidence for a very stable hexacoordinate geometry. This was somewhat surprising in the case of ligand 3-5 because the $\mathrm{UO}_{2}(3-5)$ crystal structure exhibited two very long $\mathrm{U}-\mathrm{O}_{\mathrm{HO} O}$ bonds due to geometric ligand constraints. Although it was expected these might displace in basic conditions, it seems even these weak bonds prevent uranyl hydrolysis when in the $\mathrm{TAM}(\mathrm{HOPO})_{2}$ ligand scaffold.

The $\mathrm{pUO}_{2}$ values in Table 3-6 are significantly higher that those of the bis-Me-3,2HOPO ligands in Chapter 2. This was expected because the $\mathrm{TAM}(\mathrm{HOPO})_{2}$ ligands are both hexadentate and contain the TAM moiety, which is known to form very strong complexes with $\mathrm{Fe}(\mathrm{III}){ }^{9}$ As before, an increase in $\mathrm{pUO}_{2}$ is expected upon increasing $\mathrm{pH}$ due to reduction of $\left[\mathrm{UO}_{2}{ }^{2+}\right.$ free $]$ via cation hydrolysis at higher $\mathrm{pH}$, so comparison between ligands at each $\mathrm{pH}$ is more appropriate than over-interpretation of $\Delta \mathrm{pUO}_{2}$ upon changing $\mathrm{pH}$. The change of 2 to $3 \log$ units in $\mathrm{pUO}_{2}$ at 7.4 compared to 9.0 can be considered to be primarily caused by ion hydrolysis, but the $\Delta \mathrm{pUO}_{2}$ that accompanies changing $\mathrm{pH}$ from 3 to 7.4 (typically 6 to $10 \log$ units) is most likely caused by a combination of hydrolysis and deprotonation of TAM moieties, as the speciation diagrams in Figure 3-16 suggest.

The $\mathrm{pUO}_{2}$ values of most $\mathrm{TAM}(\mathrm{HOPO})_{2}$ ligands at $\mathrm{pH} 7.4$ and 9.0 are typically within experimental error of each other, so the effect of subtle changes in ligand geometry are indiscernible using the measurement techniques employed here. It was expected that the o-phenylene backbone in ligands 3-14 and 3-15 would at the very least cause a measurable difference in $\mathrm{pUO}_{2}$ compared to ligands 3-12 and 3-13 because of the significant geometric variations observed in the structures of their respective uranyl 
complexes discussed above. However, this geometric difference seems to make little more difference than a $\Delta \mathrm{pUO}_{2}$ of 0.5 orders of magnitude, which is below the error of the batch titration measurements.

In contrast, the $\mathrm{pUO}_{2}$ values at $\mathrm{pH} 3.0$ do show significant variation between $\mathrm{TAM}(\mathrm{HOPO})_{2}$ ligands. As mentioned above, the TAM moiety does not coordinate the uranyl at low $\mathrm{pH}$, leaving only the HOPO moieties available to bind the uranyl cation. In support of this theory are the differences in $\mathrm{pUO}_{2}$ values at $\mathrm{pH} 3.0$ for 3-12 versus 3-13 and 3-14 versus 3-15, the only difference between which is the choice of 1,2- or Me-3,2HOPO moieties. Ligands containing 1,2-HOPO moieties show higher $\mathrm{pUO}_{2}$ values than their Me-3,2-HOPO analogs, indicating that the lower $\mathrm{pK}_{\mathrm{a}}$ of 1,2-HOPO moieties makes ligands that incorporate this group more effective at lowered $\mathrm{pH}$. 3-14 demonstrates the highest $\mathrm{pUO}_{2}$ at $\mathrm{pH} 3$ due to a combination of the intrinsically low $\mathrm{pK}_{\mathrm{a}}$ of 1,2-HOPO moieties coupled with the tendency for the PEG-substituted o-phenylene linkers to lower the $\mathrm{pK}_{\mathrm{a}}$ of their ligand moieties compared to their linearly-linked analogs, as illustrated in Table 3-5 and in Chapter 2.

The similarity in the $\mathrm{pUO}_{2}$ values at physiological and basic $\mathrm{pH}$ of ligands 3-22 and 3-23 compared to the $\mathrm{TAM}(\mathrm{HOPO})_{2}$ ligands is a very interesting result which suggests that the most important chelating moiety in these ligands in the TAM moiety. Namely, bis-bidentate ligands are not expected to exhibit such high $\mathrm{pUO}_{2}$ values if their chelating moieties individually exhibit the same uranyl affinity as those in tris-bidentate ligands; the fact that 3-22 and 3-23 exhibit such high $\mathrm{pUO}_{2}$ values reveals that the change in geometry from tetradentate to hexadentate is not as significant as the inclusion of the more basic and strongly binding TAM moiety into the ligand scaffold. 
At $\mathrm{pH}$ 3, however, the $\mathrm{pUO}_{2}$ of ligands 3-22 and 3-23 are generally lower than those of TAM(HOPO $)_{2}$ ligands and similar to those of bis-Me-3,2-HOPO ligands in Chapter 2. These differences are due to the need of TAM-2Li-Me-3,2-HOPO ligands to lose three protons (including those from the more basic TAM moiety) to achieve initial uranyl chelation, while $\mathrm{TAM}(\mathrm{HOPO})_{2}$ and bis-Me-3,2-HOPO ligands need only loose two protons belonging to more acidic HOPO moieties. These comparisons make it clear that if a ligand is to be applicable over a wide $\mathrm{pH}$ range such as that found in the body and its excretory systems, a design that combines chelating moieties of low and high $\mathrm{pK}_{\mathrm{a}}$ values as well as one that can adopt various chelation modes may present the most resilient and highest affinity ligand compared to ligands that are homoleptic and contain inflexible binding pockets.

However, as in Chapter 2, the titration experiments described above cannot address the relative selectivity of these ligands. While the TAM(HOPO $)_{2}$ ligands 3-14 and 3-15 exhibit little difference in $\mathrm{pUO}_{2}$ values despite increased rigidity of 3-12 and 3-13, the effect this has on selectivity may be far more pronounced. Taking selectivity into account, the TAM-2Li-Me-3,2-HOPO ligands may be far less comparable to the TAM(HOPO $)_{2}$ ligands. Additionally, because the TAM moiety is obviously the strongest contributor to the uranyl affinity of the ligands discussed in this chapter, the affinity of the TAM moiety towards other biologically-available ions may be the deciding factor of whether the TAM(HOPO $)_{2}$ or TAM-2Li-Me-3,2-HOPO ligands are feasible extractants in biological or industrial applications. 


\subsection{Conclusions and Future Directions}

The original goal of developing hexadentate $\mathrm{TAM}(\mathrm{HOPO})_{2}$ ligands was to impart increased uranyl affinity over the bis-Me-3,2-HOPO ligands and remove the dependence on solvent for coordinative saturation of the uranyl cation. While the first goal was achieved, and the high $\mathrm{pH}$ titration data and crystal structures indicate that upon complete ligand deprotonation solvent is not present in the uranyl coordination plane, there still remains the question what is happening at low $\mathrm{pH}$. At low $\mathrm{pH}$ the TAM moiety is not bound to the metal, as evidenced by titration data with $\mathrm{TAM}(\mathrm{HOPO})_{2}$ ligands and the very slow kinetics thereof. Without the chelating TAM moiety, the coordination plane of the uranyl is unsaturated, and it is presumed that some solvent must fill this site, constrained though the binding pocket may be. Thus, coordinative saturation of the uranyl cation in the presence of $\operatorname{TAM}(\mathrm{HOPO})_{2}$ ligands is not free from solvent dependence at low $\mathrm{pH}$.

While the study described here could not provide data sufficient to establish a definitive structure/affinity relationship, it has succeeded in demonstrating the relative contribution to uranyl binding that the TAM moiety imparts to poly-bidentate ligands. While the TAM moiety imparts an increased uranyl affinity, the speciation of its complexes with the uranyl cation is highly $\mathrm{pH}$-dependent due to its higher $\mathrm{pK}_{\mathrm{a}}$ values. This does, however, illustrate the importance of the choice of HOPO moiety to tailor ligands for high affinity chelation at low $\mathrm{pH}$.

From the measured ligand acidities it is obvious that substituting solubilizing groups to either the ethylene or o-phenylene linkers cannot be done without affecting the electronics of the ligand, and thus their uranyl affinities. Although this complicates the 
solution thermodynamic comparisons discussed here, it also provides a synthetically accessible method by which the ligand electronics can be modified and through which the ligands could be tethered to larger supports, be they discrete or polymeric, to enhance their applicability in extraction/chelation applications.

Another future outlook to the work presented here is to apply these ligands toward selectivity measurements. The similarity in uranyl affinities revealed here between the numerous $\mathrm{TAM}(\mathrm{HOPO})_{2}$ and Pr-TAM-2Li-Me-3,2-HOPO ligands towards uranyl at neutral and basic $\mathrm{pH}$ may be very useful if the geometric changes between the ligands are found to impart high selectivity for uranyl as compared to another metal cations, whether in biological or separations applications.

Another future direction involving hexadentate $\mathrm{TAM}(\mathrm{HOPO})_{2}$ ligands is the investigation of their structures and thermodynamics with the neptunyl cation $\left(\mathrm{Np}_{2} \mathrm{O}^{+}\right)$. This would explore the selectivity of these ligands within the actinyl species, and it is expected that binding to the neptunyl cation would be favorable, since the neptunium atom therein has an ionic radius $0.08 \AA$ larger than the uranium in $\mathrm{UO}_{2}{ }^{2+}$. The strained geometries and long $\mathrm{U}-\mathrm{O}_{\mathrm{HOPO}}$ bonds in the crystal structures of $\mathrm{UO}_{2}\left[\mathrm{TAM}(\mathrm{HOPO})_{2}\right]$ suggest that binding to a larger cation may in fact allow for a more relaxed ligand geometry with more cooperative binding by all the chelating moieties. Such an effect has been seen with Sessler and co-workers' expanded porphyrins, ${ }^{2}$ and whether such an effect exists here would be of great interest and could expand the future applicability of these ligands. 


\subsection{Experimental}

General. Unless otherwise noted, all chemicals were purchased from commercial sources and used as received or synthesized using literature procedures. Solvents indicated as "dry" were made so by passing them through anhydrous alumina columns or by storage over molecular sieves. The syntheses of 1,2-HOPO-(Bn)-COOH, Me-3,2HOPO-(Bn)-COOH, TAM(Bn) $)_{2}-\mathrm{COOH}$ and their respective thiaz starting materials are described in earlier Raymond group publications. ${ }^{23,25,26}$ All reactions brought to reflux were done so with an efficient condenser attached to the reaction flask. NMR spectra were collected using Bruker AMX-400 and AM-400 spectrometers $\left({ }^{1} \mathrm{H} 400 \mathrm{MHz},{ }^{13} \mathrm{C}\right.$ $100 \mathrm{MHz}$ ) in $\mathrm{CDCl}_{3}$ unless otherwise noted. Mass spectrometry and elemental analyses were performed at the Microanalytical Facility, College of Chemistry, University of California, Berkeley. Melting points are uncorrected. Elemental analyses are reported in a “calculated (found)” format. Reactions were monitored by TLC on 60 mesh $\mathrm{F}_{254}$ silica gel from EMD Chemicals, Inc. Silica gel chromatography was performed on EcoChrom Silica (32-63 D $60 \AA$ ) and $\mathrm{R}_{\mathrm{f}}$ values correspond to the solvent used for chromatographic elution unless otherwise noted. Organic solutions were dried using anhydrous sodium sulfate and solvents were removed on a rotary evaporator or under high vacuum on a Schlenk line. Yields indicate the amount of isolated compound and reactions are unoptimized.

\subsubsection{Synthesis of Benzyl-Protected TAM(HOPO) $)_{n}$ Ligands}

1,2-HOPO(2Li-NH2)(Bn), 3-6a. A solution of 1,2-HOPO-Thiaz(Bn) (2.00 g, 6.80 mmol) in $250 \mathrm{~mL}$ of $\mathrm{CHCl}_{3}$ was added dropwise over one day to a stirred solution of ethylenediamine $(2.0 \mathrm{~mL}, 30 \mathrm{mmol})$ in $10 \mathrm{~mL}$ of $\mathrm{CH}_{2} \mathrm{Cl}_{2}$. The resultant solution was 
washed with $1 \mathrm{M} \mathrm{NaOH}$ in $20 \%$ sat. brine $(3 \times 100 \mathrm{~mL})$ to removed free thiazoline, sat. brine, dried with $\mathrm{Na}_{2} \mathrm{SO}_{4}$ and the solvent was removed under vacuum for two days to yield an off-white, tacky residue that was used in subsequent reactions without further purification (1.26 g, 76\%). ${ }^{1} \mathrm{H}$ NMR: $\delta 1.86\left(\mathrm{~s}, \mathrm{br}, \mathrm{NH}_{2}, 2 \mathrm{H}\right), \delta 2.73\left(\mathrm{t}, \mathrm{CH}_{2}, \mathrm{~J}=6.0 \mathrm{~Hz}\right.$, $2 \mathrm{H}$ ), $\delta 3.30$ (quartet, $\mathrm{CH}_{2}, \mathrm{~J}=6.0 \mathrm{~Hz}, 2 \mathrm{H}$ ), $\delta 5.22$ (s, benzyl $\mathrm{H}, 2 \mathrm{H}$ ), $\delta 6.37$ (dd, HOPO H, $\mathrm{J}=6.8,1.6 \mathrm{~Hz}, 1 \mathrm{H}), \delta 6.60(\mathrm{dd}, \mathrm{HOPO} H, \mathrm{~J}=8.8,1.6 \mathrm{~Hz}, 1 \mathrm{H}), \delta$ 7.17-7.41 (m, arom. $H$ + NH, + HOPO H, 7H). ${ }^{13} \mathrm{C}$ NMR: $\delta 40.88,42.71,79.55,106.64,124.21,128.82,129.71$, $130.41,133.34,138.30,142.60158 .21,160.50$.

Me-3,2-HOPO(2Li-NH $)(\mathbf{B n})$, 3-6b. A solution of Me-3,2-HOPO-Thiaz(Bn) (2.60 $\mathrm{g}, 7.21 \mathrm{mmol}$ ) in $450 \mathrm{~mL}$ of $\mathrm{CHCl}_{3}$ was added dropwise over 3 days to a rapidly stirred solution of ethylenediamine $(2.5 \mathrm{~mL}, 37 \mathrm{mmol})$ and $\mathrm{Et}_{3} \mathrm{~N}(1.0 \mathrm{~mL}, 7.2 \mathrm{mmol})$ in $15 \mathrm{~mL}$ of $\mathrm{CH}_{2} \mathrm{Cl}_{2}$. The solution was washed with $1 \mathrm{M} \mathrm{NaOH}$ in $20 \%$ sat. brine $(4 \times 150 \mathrm{~mL})$ until TLC indicated total removal of free 2-mercaptothiazoline. After washing with sat. brine and drying with $\mathrm{Na}_{2} \mathrm{SO}_{4}$, the sovent and excess amines were removed under vacuum to yield $2.13 \mathrm{~g}$ of a yellow oil $(98 \%)$. The product was used without mass spectrometry or elemental analysis in further steps. ${ }^{1} \mathrm{H}$ NMR $\left(\mathrm{CDCl}_{3}\right)$ : $\delta 1.34$ (s, br, $\mathrm{NH}_{2}$, $2 \mathrm{H}$ ), $\delta 2.64\left(\mathrm{t}, \mathrm{CH}_{2}, \mathrm{~J}=8.0 \mathrm{~Hz}, 2 \mathrm{H}\right), \delta 3.25$ (quartet, $\left.\mathrm{CH}_{2}, \mathrm{~J}=8.0 \mathrm{~Hz}, 2 \mathrm{H}\right), \delta 3.60\left(\mathrm{~s}, \mathrm{CH}_{3}\right.$, $3 \mathrm{H}), \delta 5.41$ (s, benzyl $H, 2 \mathrm{H}), \delta 6.79(\mathrm{~d}, \mathrm{HOPO} H, \mathrm{~J}=9.6 \mathrm{~Hz}, 1 \mathrm{H}), \delta 7.12(\mathrm{~d}, \mathrm{HOPO} H, \mathrm{~J}$ $=9.6 \mathrm{~Hz}, 1 \mathrm{H}), \delta 7.37-7.42(\mathrm{~m}$, arom. $H, 3 \mathrm{H}), \delta 7.45-7.48(\mathrm{~m}$, arom. $H, 2 \mathrm{H}), \delta 8.13(\mathrm{~s}, \mathrm{br}$, $\mathrm{NH}, 1 \mathrm{H}) .{ }^{13} \mathrm{C}$ NMR: $\delta 37.90,58.27,105.21,127.17,128.47,128.65,128.82,129.00$, $129.18,129.26,132.27,139.08$.

TAM(2Li-1,2-HOPO $)_{2}(\mathbf{B n})_{4}$, 3-7a. A solution of 3-6a $(0.426 \mathrm{~g}, 1.48 \mathrm{mmol})$, TAMThiaz(Bn) $2(4.30 \mathrm{~g}, 0.740 \mathrm{mmol})$, and $\mathrm{Et}_{3} \mathrm{~N}(0.40 \mathrm{~mL}, 2.9 \mathrm{mmol})$ in $100 \mathrm{~mL}$ of $\mathrm{CH}_{2} \mathrm{Cl}_{2}$ 
was stirred overnight at room temperature. The solution was washed with $1 \mathrm{M} \mathrm{HCl}$ in $20 \%$ sat. brine $(2 \times 50 \mathrm{~mL})$, sat. brine, dried with $\mathrm{Na}_{2} \mathrm{SO}_{4}$ and the solvent was removed under vacuum. The residue was dissolved in $10 \mathrm{~mL}$ of $\mathrm{CH}_{2} \mathrm{Cl}_{2}$ and eluted on a silica column with $4 \% \mathrm{MeOH}$ in $\mathrm{CH}_{2} \mathrm{Cl}_{2}$. Fractions with $\mathrm{R}_{\mathrm{f}}=0.13$ were collected and the solvent removed to yield a white solid. This solid was recrystallized by dissolving in $\mathrm{CH}_{2} \mathrm{Cl}_{2}$ and layering with $\mathrm{Et}_{2} \mathrm{O}$, producing a white powder as the hemihydrate $(0.234 \mathrm{~g}$, 34\%). $\mathrm{C}_{52} \mathrm{H}_{48} \mathrm{~N}_{6} \mathrm{O}_{10} \cdot \frac{1}{2} \mathrm{H}_{2} \mathrm{O}: \mathrm{C}: 67.45$ (67.36); H: 5.33 (5.14); N: 9.08 (9.10). ${ }^{1} \mathrm{H}$ NMR: $\delta$ $3.38\left(\mathrm{t}, \mathrm{CH}_{2}, \mathrm{~J}=5.2 \mathrm{~Hz}, 8 \mathrm{H}\right), \delta 5.05(\mathrm{~s}$, benzyl $H, 4 \mathrm{H}), \delta 5.26(\mathrm{~s}$, benzyl $H, 4 \mathrm{H}), \delta 6.28$ (dd, HOPO H, J = 6.8, 1.6 Hz, 2H), $\delta 6.62(\mathrm{dd}, \mathrm{HOPO} H, \mathrm{~J}=5.2,1.2 \mathrm{~Hz}, 2 \mathrm{H}), \delta 7.20$ (dd, HOPO $H, \mathrm{~J}=9.2,6.8 \mathrm{~Hz}, 2 \mathrm{H}), \delta 7.27-7.42(\mathrm{~m}$, arom. $H+\mathrm{NH}, 22 \mathrm{H}), \delta 7.68(\mathrm{~s}, \mathrm{NH}, 2 \mathrm{H})$, $\delta 7.92(\mathrm{t}, \mathrm{TAM} H, \mathrm{~J}=5.2 \mathrm{~Hz}, 2 \mathrm{H}) .{ }^{13} \mathrm{C} \mathrm{NMR}: \delta 39.24,40.73,77.42,79.32,106.02$, $124.21,126.27,128.73,128.78,129.11,129.30,129.53,130.38,130.76,133.45,135.75$, 138.16, 142.76, 150.52, 158.71, 160.72, 165.60. MS (FAB+): m/z $917(\mathrm{MH}+) . \mathrm{MP}: 183-$ $185^{\circ} \mathrm{C}$.

TAM(2Li-Me-3,2-HOPO)(Bn) ${ }_{4}$, 3-7b. This compound was synthesized following the procedure for 3-7a, but using 3-6b instead of 3-6a. Eluent: $4 \% \mathrm{MeOH}$ in $\mathrm{CH}_{2} \mathrm{Cl}_{2}, \mathrm{R}_{\mathrm{f}}$ $=0.24$. White solid isolated as the monohydrate, 78\%. $\mathrm{C}_{54} \mathrm{H}_{52} \mathrm{~N}_{6} \mathrm{O}_{10} \cdot \mathrm{H}_{2} \mathrm{O}: \mathrm{C}$ : 67.35(67.47); H: 5.65(5.38); N: 8.73(8.72). ${ }^{1} \mathrm{H} \mathrm{NMR}: \delta 3.31\left(\mathrm{~s}, \mathrm{CH}_{2}, 8 \mathrm{H}\right), \delta 3.58(\mathrm{~s}$, $\left.\mathrm{CH}_{3}, 6 \mathrm{H}\right), \delta 5.05$ (s, benzyl $\left.H, 4 \mathrm{H}\right), \delta 5.36$ (s, benzyl $\left.H, 4 \mathrm{H}\right), \delta 6.71(\mathrm{~d}, \mathrm{HOPO} H, \mathrm{~J}=7.2$ $\mathrm{Hz}, 2 \mathrm{H}), \delta 7.08(\mathrm{~d}, \mathrm{HOPO} H, \mathrm{~J}=7.2 \mathrm{~Hz}, 2 \mathrm{H}), \delta$ 7.27-7.30 (m, arom. $H, 16 \mathrm{H}), \delta$ 7.37-7.38 (m, arom. $H, 4 \mathrm{H}), \delta 7.85(\mathrm{~s}, \mathrm{br}, \mathrm{TAM} H+\mathrm{NH}, 4 \mathrm{H}), \delta 8.07(\mathrm{~s}, \mathrm{br}, \mathrm{NH}, 2 \mathrm{H}) .{ }^{13} \mathrm{C} \mathrm{NMR}: \delta$ $37.92,39.51,39.74,74.90,77.32,104.92,126.59,128.77,129.00,129.11,129.15$, 
$129.20,130.39,130.82,132.21,135.71,136.20,146.66,150.51,159.72,164.00,164.85$. MS (FAB+): m/z $945.5(\mathrm{MH}+)$. MP: $113-115^{\circ} \mathrm{C}$.

TAM(2Li-Me-3,2-HOPO)Thiaz(Bn) 3, 3-8. A solution of 3-6b (2.14 g, 7.09 mmol) and $\mathrm{Et}_{3} \mathrm{~N}(1.0 \mathrm{~mL}, 14 \mathrm{mmol})$ in $500 \mathrm{~mL}$ of $\mathrm{CHCl}_{3}$ was added dropwise over 2 days to a solution of TAM-Thiaz(Bn) $)_{2}(41.2 \mathrm{~g}, 70.9 \mathrm{mmol})$ and $\mathrm{Et}_{3} \mathrm{~N}(1.0 \mathrm{~mL}, 7.2 \mathrm{mmol})$ in 100 $\mathrm{mL}$ of $\mathrm{CH}_{2} \mathrm{Cl}_{2}$. The solution was washed with $1 \mathrm{M} \mathrm{HCl}(2 \times 100 \mathrm{~mL}), 1 \mathrm{M} \mathrm{NaOH}$ in $20 \%$ brine $(3 \times 100 \mathrm{~mL})$, brine, then dried with $\mathrm{Na}_{2} \mathrm{SO}_{4}$ and the solvent was removed under vacuum. The residue was dissolved in $125 \mathrm{~mL}$ of $\mathrm{CH}_{2} \mathrm{Cl}_{2}$ and eluted on a silica plug with $\mathrm{CH}_{2} \mathrm{Cl}_{2}$ to remove unreacted TAM-Thiaz $(\mathrm{Bn})_{2}$ and then with $2 \% \mathrm{MeOH} / \mathrm{CH}_{2} \mathrm{Cl}_{2}$ to remove the desired yellow band with $\mathrm{R}_{\mathrm{f}}=0.19$. The yellow residue from these later fractions was further purified on another short silica column using only $2 \%$ $\mathrm{MeOH} / \mathrm{CH}_{2} \mathrm{Cl}_{2}$, which after removal of solvent yielded $4.68 \mathrm{~g}$ of a yellow solid (86\%). This compound was used in further reactions without performing elemental analysis. ${ }^{1} \mathrm{H}$ NMR: $\delta 2.94\left(\mathrm{t}, \mathrm{CH}_{2}, \mathrm{~J}=7.2 \mathrm{~Hz}, 2 \mathrm{H}\right), \delta 3.27\left(\mathrm{t}, \mathrm{CH}_{2}, \mathrm{~J}=2.8 \mathrm{~Hz}, 4 \mathrm{H}\right), \delta 3.57\left(\mathrm{~s}, \mathrm{CH}_{3}\right.$, $3 \mathrm{H}), \delta 4.38\left(\mathrm{t}, \mathrm{CH}_{2}, \mathrm{~J}=7.2 \mathrm{~Hz}, 2 \mathrm{H}\right), \delta 5.06$ (s, benzyl $\left.\mathrm{H}, 2 \mathrm{H}\right), \delta 5.07$ (s, benzyl $\left.H, 2 \mathrm{H}\right), \delta$ 5.36 (s, benzyl $H, 2 \mathrm{H}$ ), $\delta 6.71(\mathrm{~d}, \mathrm{HOPO} H, \mathrm{~J}=7.2 \mathrm{~Hz}, 1 \mathrm{H}$ ), $\delta 7.08$ (d, HOPO $H, \mathrm{~J}=7.2$ $\mathrm{Hz}, 1 \mathrm{H}), \delta 7.21(\mathrm{~d}, \mathrm{TAM} H, \mathrm{~J}=8.4 \mathrm{~Hz}, 1 \mathrm{H}), \delta$ 7.27-7.30 (m, arom. $H, 7 \mathrm{H}), \delta$ 7.34-7.38 (m, arom. $H, 8 \mathrm{H}), \delta 7.85-7.87(\mathrm{~m}, \mathrm{TAM} H+\mathrm{NH}, 2 \mathrm{H}), \delta 8.04$ (s, br, NH, 1H). ${ }^{13} \mathrm{C}$ NMR: $\delta 37.87,39.47,39.59,53.63,55.71,74.81,76.21,77.11,104.94,124.53,126.89,128.08$, $128.59,128.80,128.97,129.03,129.06,129.11,129.18,130.07,130.47,132.15,133.77$, $135.69,136.20,137.09,146.59,149.43,150.14,159.71,163.86,164.75,166.93,201.52$. MS (FAB+): m/z $763(\mathrm{MH}+)$. MP: $75-77{ }^{\circ} \mathrm{C}$. 
TAM(2Li-3,2-HOPO)(2Li-1,2-HOPO)(Bn)4, 3-9. A solution of 3-6a (0.365 g, 1.27 mmol), 3-8 (0.969 g, $1.27 \mathrm{mmol})$, and $\mathrm{Et}_{3} \mathrm{~N}(0.25 \mathrm{~mL}, 1.79 \mathrm{mmol})$ in $100 \mathrm{~mL} \mathrm{CH}_{2} \mathrm{Cl}_{2}$ was stirred overnight. The solution was washed with $1 \mathrm{M} \mathrm{HCl}$ in $20 \%$ sat. brine $(2 \times 50 \mathrm{~mL})$, sat. brine, dried with $\mathrm{Na}_{2} \mathrm{SO}_{4}$ and the solvent was removed under vacuum. The residue was dissolved in $20 \mathrm{~mL}$ of $\mathrm{CH}_{2} \mathrm{Cl}_{2}$ and eluted on a silica column with $4 \% \mathrm{MeOH} / \mathrm{CH}_{2} \mathrm{Cl}_{2}$. Fractions with $\mathrm{R}_{\mathrm{f}}=0.11$ were collected, dried and the solvent was removed under vacuum to yield the product as a beige solid $(0.578 \mathrm{~g}, 49 \%)$. $\mathrm{C}_{53} \mathrm{H}_{50} \mathrm{~N}_{6} \mathrm{O}_{10}$ : C: 68.37 (68.25); H: 5.41 (5.30); N: 9.03 (8.72). ${ }^{1} \mathrm{H}$ NMR: $\delta 3.31-3.40$ (m, $\left.\mathrm{CH}_{2}, 8 \mathrm{H}\right), \delta 3.56$ (s, $\left.\mathrm{CH}_{3}, 3 \mathrm{H}\right), \delta 5.05$ (s, benzyl $H, 2 \mathrm{H}$ ), $\delta 5.06$ (s, benzyl $\left.H, 2 \mathrm{H}\right), \delta 5.29$ (s, benzyl $\left.H, 2 \mathrm{H}\right), \delta$ $5.34(\mathrm{~s}$, benzyl $H, 2 \mathrm{H}), \delta 6.30(\mathrm{dd}, \mathrm{HOPO} H, \mathrm{~J}=6.8,1.2 \mathrm{~Hz}, 1 \mathrm{H}), \delta 6.68(\mathrm{~d}, \mathrm{HOPO} H, \mathrm{~J}$ $=7.2 \mathrm{~Hz}, 2 \mathrm{H}), \delta 7.06(\mathrm{~d}, \mathrm{HOPO} H, 7.2 \mathrm{~Hz}, 1 \mathrm{H}), \delta 7.21-7.45(\mathrm{~m}$, arom. $H+\mathrm{NH}, 23 \mathrm{H}), \delta$ $7.78(\mathrm{~s}, \mathrm{TAM} H, 2 \mathrm{H}), \delta 7.83(\mathrm{t}, \mathrm{NH}, \mathrm{J}=4.8 \mathrm{~Hz}, 1 \mathrm{H}), \delta 7.94(\mathrm{t}, \mathrm{NH}, \mathrm{J}=4.8 \mathrm{~Hz}, 1 \mathrm{H}), \delta$ $8.07(\mathrm{t}, \mathrm{NH}, \mathrm{J}=4.8 \mathrm{~Hz}, 1 \mathrm{H}) .{ }^{13} \mathrm{C}$ NMR: $\delta 37.85,39.16,39.45,39.78,40.98,74.87,77.31$, $77.44,79.30,104.68,106.92,124.37,126.44,128.68,128.73,128.86,128.95,129.00$ $129.09,129.17,129.31,129.54,130.22,130.41,131.31,132.23,133.49,135.68,135.77$, $136.19,138.10,142.71,146.57,150.40,150.64,158.72,159.66,160.68,164.03,164.82$ 165.66. MS (FAB+): m/z $931(\mathrm{MH}+)$. MP: $198-200{ }^{\circ} \mathrm{C}$.

TAM(o-phen- $\left.\mathrm{NH}_{2}\right)_{2}(\mathrm{Bn})_{2}, \mathbf{3 - 1 0}$. To a suspension of $\mathrm{TAM}(\mathrm{COOH})_{2}(\mathrm{Bn})_{2}(4.529 \mathrm{~g}$, $12.1 \mathrm{mmol}$ ) in $100 \mathrm{~mL}$ of dry toluene and 15 drops DMF was added oxalyl chloride (3.2 $\mathrm{mL}, 36.7 \mathrm{mmol}$ ), turning the suspension into a yellow, homogeneous solution. This solution was stirred for three hours at room temperature under nitrogen, then the solvent and residual oxalyl chloride were removed under vacuum with gentle heating. The light yellow acid chloride was held under vacuum for four hours, and dissolved in $400 \mathrm{~mL}$ of 
dry THF. This solution was added dropwise to a solution of o-phenylenediamine (13.06 $\mathrm{g}, 121 \mathrm{mmol})$ and $\mathrm{Et}_{3} \mathrm{~N}(4.5 \mathrm{~mL}, 32.3 \mathrm{mmol})$ in $150 \mathrm{~mL}$ of dry THF over the course of three hours, and the resulting solution was stirred overnight. The $\mathrm{Et}_{3} \mathrm{~N} \cdot \mathrm{HCl}$ salt was filtered off, and the THF was removed from the filtrate under vacuum to yield a yellowish solid. This was ground in a mortar and washed twice in $80{ }^{\circ} \mathrm{C} \mathrm{H}_{2} \mathrm{O}$ to remove excess diamine. The solid was held under aspiration for one hour then recrystallized from hot $\mathrm{MeOH}$ to yield $5.00 \mathrm{~g}(74 \%)$ of pale yellow solid in two batches. $\mathrm{C}_{34} \mathrm{H}_{30} \mathrm{~N}_{4} \mathrm{O}_{4}$ : C: 73.10 (73.01); H: 5.41 (5.59); N: 10.03 (9.93). ${ }^{1} \mathrm{H}$ NMR: $\delta 3.80$ (s, br, $\left.\mathrm{NH}_{2}, 4 \mathrm{H}\right), \delta 5.32$ (s, benzyl $H, 4 \mathrm{H}), \delta 6.75(\mathrm{t}$, arom. $H, \mathrm{~J}=7.6 \mathrm{~Hz}, 2 \mathrm{H}), \delta 6.72(\mathrm{~d}$, arom. $H, \mathrm{~J}=7.6 \mathrm{~Hz}, 2 \mathrm{H}$ ), $\delta 6.95(\mathrm{~d}$, arom. $H, \mathrm{~J}=7.6 \mathrm{~Hz}, 2 \mathrm{H}), \delta 7.07$ (t, arom. $H, \mathrm{~J}=7.6 \mathrm{~Hz}, 2 \mathrm{H}), \delta$ 7.34-7.41 (m, arom. $H, 10 \mathrm{H}), \delta 8.06$ (s, TAM $H, 2 \mathrm{H}), \delta 9.52(\mathrm{~s}, \mathrm{NH}, 2 \mathrm{H}) .{ }^{13} \mathrm{C}$ NMR: $\delta 77.93,118.37$, $119.56,124.37,124.96,127.33,129.15,129.24,129.41,131.10,135.36,140.81,150.58$ 162.28. MS (FAB+): m/z 559.3 (MH+). MP: $176-178^{\circ} \mathrm{C}$.

TAM(o-phen-1,2-HOPO $)_{2}(\mathbf{B n})_{4}$, 3-11a. To a suspension of 1,2-HOPO(COOH)Bn (0.900 g, $3.67 \mathrm{mmol})$ in $50 \mathrm{~mL}$ of dry toluene and 5 drops DMF was added oxalyl chloride (0.65 mL, $7.45 \mathrm{mmol})$, making the mixture homogeneous. This solution was stirred for four hours under nitrogen, then the solvent and residual oxalyl chloride were removed under vacuum and the residue was dissolved in $50 \mathrm{~mL}$ of dry THF. A solution of 3-10 (1.02 g, $1.83 \mathrm{mmol})$ and $\mathrm{Et}_{3} \mathrm{~N}(0.55 \mathrm{~mL}, 3.95 \mathrm{mmol})$ in $50 \mathrm{~mL}$ of dry THF was added to the acid chloride solution via cannula and allowed to stir overnight. Precipitated $\mathrm{Et}_{3} \mathrm{~N} \cdot \mathrm{HCl}$ was filtered off and the THF was removed from the filtrate under vacuum. The residue was dissolved in $25 \mathrm{~mL}$ of $2 \% \mathrm{MeOH}$ in $\mathrm{CH}_{2} \mathrm{Cl}_{2}$ and eluted on a silica plug with the same. Fraction with $\mathrm{R}_{\mathrm{f}}=0.50\left(4 \% \mathrm{MeOH} / \mathrm{CH}_{2} \mathrm{Cl}_{2}\right)$ were collected, dried, and the 
solvent removed. The residue was recrystallized by layering a concentrated solution in $\mathrm{CH}_{2} \mathrm{Cl}_{2}$ with $\mathrm{Et}_{2} \mathrm{O}$, resulting in $1.20 \mathrm{~g}(65 \%)$ of a white, crystalline solid. $\mathrm{C}_{60} \mathrm{H}_{48} \mathrm{~N}_{6} \mathrm{O}_{10}$ : C: 71.14 (71.20); H: 4.78 (4.59); N: 8.30 (8.25). ${ }^{1} \mathrm{H}$ NMR: $\delta 5.11$ (s, benzyl $\left.H, 4 \mathrm{H}\right), \delta 5.30$ (s, benzyl H, 4H), $\delta 6.40(\mathrm{dd}, \mathrm{HOPO} H, \mathrm{~J}=6.8,1.2 \mathrm{~Hz}, 2 \mathrm{H}), \delta 6.69(\mathrm{dd}, \mathrm{HOPO} H, \mathrm{~J}=$ 9.2, $1.2 \mathrm{~Hz}, 2 \mathrm{H}), \delta$ 7.26-7.41 (m, HOPO + arom. $H, 22 \mathrm{H}), \delta$ 7.44-7.47 (m, TAM + arom. $H, 6 \mathrm{H}), \delta 7.60(\mathrm{~d}$, arom. $H, \mathrm{~J}=7.6 \mathrm{~Hz}, 2 \mathrm{H}), \delta 7.78(\mathrm{~d}$, arom. $H, \mathrm{~J}=7.6 \mathrm{~Hz}, 2 \mathrm{H}), \delta 9.94(\mathrm{~s}$, $\mathrm{NH}, 2 \mathrm{H}), \delta 10.40(\mathrm{~s}, \mathrm{NH}, 2 \mathrm{H}) .{ }^{13} \mathrm{C}$ NMR: $\delta 76.21,78.46,104.54,123.19,124.52,124.72$, $125.42,126.38,128.39,128.49,128.71,128.93,129.13,129.64,131.08,133.24,133.73$, 135.97, 138.67, 143.29, 149.52, 157.46, 159.10, 163.52. MS (FAB+): m/z $1013(\mathrm{MH}+)$. MP: $127-129^{\circ} \mathrm{C}$.

TAM(o-phen-Me-3,2-HOPO $)_{2} \mathbf{B n}_{4}, 3-11 b$. This compound was synthesized in an analogous way to 3-11a, but using $\mathrm{Me}-3,2-\mathrm{HOPO}(\mathrm{COOH}) \mathrm{Bn}$ as the starting acid. White, crystalline solid, 58\%. Eluent: EtOAc, $\mathrm{R}_{\mathrm{f}}=0.14$ in $\mathrm{CH}_{2} \mathrm{Cl}_{2} . \mathrm{C}_{62} \mathrm{H}_{52} \mathrm{~N}_{6} \mathrm{O}_{10}$ : C: 71.53 (71.18); H: 5.03 (5.07); N: 8.07 (7.97). ${ }^{1} \mathrm{H}$ NMR: $\delta 3.41$ (s, $\left.\mathrm{CH}_{3}, 6 \mathrm{H}\right), \delta 5.05$ (s, benzyl $H$, 4H), $\delta 5.43$ (s, benzyl H, 4H), $\delta 6.67$ (d, HOPO H, J = 7.2 Hz, 2H), $\delta 6.92(\mathrm{~d}, \mathrm{HOPO} H, \mathrm{~J}$ $=7.2 \mathrm{~Hz}, 2 \mathrm{H}), \delta 7.12-7.24(\mathrm{~m}$, arom. $H, 22 \mathrm{H}), \delta 7.03(\mathrm{~d}$, arom $H, \mathrm{~J}=6.8 \mathrm{~Hz}, 4 \mathrm{H}), \delta 7.82$ (d, arom. $H, \mathrm{~J}=8.0 \mathrm{~Hz}, 2 \mathrm{H}), \delta 7.81(\mathrm{~s}$, TAM $H, 2 \mathrm{H}), \delta 9.59(\mathrm{~s}, \mathrm{NH}, 2 \mathrm{H}), \delta 9.78(\mathrm{~s}, \mathrm{NH}$, 2H). ${ }^{13} \mathrm{C}$ NMR: $\delta 37.72,75.01,77.66,104.59,124.59,124.97,125.83,126.61,126.75$, $128.69,128.92,129.03,129.13,129.19,129.35,130.13,130.88,131.70,132.16,135.16$, 135.70, 146.47, 150.20, 159.30, 162.08, 162.86. MS (FAB+): m/z 1041.7 (MH+). MP: $208-210^{\circ} \mathrm{C}$.

Pr-TAM-2Li-Me-3,2-HOPO(Bn $)_{3}$, 3-24. A mixture of 3-8 (0.500 g, $\left.0.655 \mathrm{mmol}\right)$, proplyamine $(0.10 \mathrm{~mL}, 1.2 \mathrm{mmol})$, and $\mathrm{Et}_{3} \mathrm{~N}(0.15 \mathrm{~mL}, 1.1 \mathrm{mmol})$ in $50 \mathrm{~mL}$ of $\mathrm{CH}_{2} \mathrm{Cl}_{2}$ 
was stirred for four hours. The reaction mixture was washed with $1 \mathrm{M} \mathrm{HCl}$ in $20 \%$ sat. brine $(2 \times 25 \mathrm{~mL})$, sat. brine, dried with $\mathrm{Na}_{2} \mathrm{SO}_{4}$, and the solvent was removed under vacuum. The residue was dissolved in $6 \mathrm{~mL}$ of $\mathrm{CH}_{2} \mathrm{Cl}_{2}$ and eluted on a silica plug with EtOAc to remove free thiazolidine and then with $4 \% \mathrm{MeOH}$ in $\mathrm{CH}_{2} \mathrm{Cl}_{2}$ to remove colorless fractions with $\mathrm{R}_{\mathrm{f}}=0.09$ (EtOAc), which were dried and the solvent was removed under vacuum. This residue was dissolved in a minimum amount of $\mathrm{CH}_{2} \mathrm{Cl}_{2}$ and layered to $\mathrm{Et}_{2} \mathrm{O}$ to yield $0.359 \mathrm{~g}$ of the product as a fluffy, white solid (78\%). $\mathrm{C}_{41} \mathrm{H}_{42} \mathrm{~N}_{4} \mathrm{O}_{7}$ : C: 70.07 (70.02); H: 6.02 (6.11); N: 7.97 (7.88). ${ }^{1} \mathrm{H}$ NMR: $\delta 0.84$ (t, $\mathrm{CH}_{3}, \mathrm{~J}$ $=7.2 \mathrm{~Hz}, 3 \mathrm{H}), \delta 1.39\left(\right.$ sextet, $\left.\mathrm{CH}_{2}, \mathrm{~J}=7.2 \mathrm{~Hz}, 2 \mathrm{H}\right), \delta 3.23-3.31\left(\mathrm{~m}, \mathrm{CH}_{2}, 6 \mathrm{H}\right), \delta 3.58(\mathrm{~s}$, $\mathrm{CH}_{3}, 3 \mathrm{H}$ ), $\delta 5.09$ (s, benzyl $\left.H, 4 \mathrm{H}\right), \delta 5.36$ (s, benzyl $\left.H, 2 \mathrm{H}\right), \delta 6.71$ (d, HOPO H, J $=7.2$ $\mathrm{Hz}, 1 \mathrm{H}), \delta 7.07(\mathrm{~d}, \mathrm{HOPO} H, \mathrm{~J}=7.2 \mathrm{~Hz}, 1 \mathrm{H}), \delta 7.28-7.40(\mathrm{~m}$, arom. $H, 15 \mathrm{H}), \delta 7.23(\mathrm{t}$, $\mathrm{NH}, \mathrm{J}=5.6 \mathrm{~Hz}, 1 \mathrm{H}), \delta 7.81(\mathrm{~s}, \mathrm{br}, \mathrm{NH}, 1 \mathrm{H}), \delta 7.83(\mathrm{~d}, \mathrm{TAM} H, \mathrm{~J}=8.4 \mathrm{~Hz}, 1 \mathrm{H}), \delta 7.86$ $(\mathrm{d}, \mathrm{TAM} H, \mathrm{~J}=8.4 \mathrm{~Hz}, 1 \mathrm{H}), \delta 6.08$ (s, br, $\mathrm{N} H, 1 \mathrm{H}) .{ }^{13} \mathrm{C}$ NMR: $\delta 11.69,22.66,37.87$, $39.51,39.71,41.75,74.87,77.37,104.90,126.59,126.90,128.75,128.97,129.01$, $129.04,129.11,129.15,129.18,130.39,130.72,130.89,132.18,135.75,135.86,136.22$ $146.65,150.46,150.60,152.75,159.70,163.97,164.17,164.90 . \mathrm{C}_{41} \mathrm{H}_{42} \mathrm{~N}_{4} \mathrm{O}_{7}: \mathrm{C}: 70.07$ (70.02); H: 6.02 (6.11); N: 7.97 (7.88). MS (FAB+): m/z 703.4 (MH+). MP: $165-167{ }^{\circ} \mathrm{C}$.

\subsubsection{Synthesis of Benzyl-Protected PEG-TAM(HOPO) $)_{n}$ Ligands}

PEG-Boc-(Fmoc)-L-DAP, 3-16. A solution of Boc-(Fmoc)-L-DAP-OH (3.00 g, 7.03 mmol), NHS (0.811 g, $7.05 \mathrm{mmol})$, and a catalytic amount of DMAP was stirred in 100 $\mathrm{mL}$ of $\mathrm{CH}_{2} \mathrm{Cl}_{2}$ in an ice bath under argon. DCC (1.45 g, $\left.7.03 \mathrm{mmol}\right)$ was added and the resultant suspension solution was stirred at $0{ }^{\circ} \mathrm{C}$ for four hours. A solution of 3,6,9trioxa-1-aminodecane (1.15 g, $7.03 \mathrm{mmol})$ in $10 \mathrm{~mL}$ of $\mathrm{CH}_{2} \mathrm{Cl}_{2}$ was added and the 
solution was allowed to warm to room temperature and stirred for four hours more. The suspension was again cooled to $0{ }^{\circ} \mathrm{C}$ and precipitated DCU was filtered off. The filtrate solvent was removed under vacuum and the residue was suspended in a minimum amount EtOAc, and filtered once more. After removal of the EtOAc under vacuum, the oily residue was re-dissolved in EtOAc and eluted on a silica column with 1:4 acetone:EtOAc, collecting fractions with $\mathrm{R}_{\mathrm{f}}=0.30$, which were dried and the solvent removed under vacuum to yield $2.98 \mathrm{~g}$ of white solid (74\%). $\mathrm{C}_{30} \mathrm{H}_{41} \mathrm{~N}_{3} \mathrm{O}_{8}$ : C: 63.03 (62.76); H: 7.23 (7.57); N: 7.35 (7.16). ${ }^{1} \mathrm{H}$ NMR: 1.43 (s, $\left.\mathrm{CH}_{3}, 9 \mathrm{H}\right), \delta 3.33\left(\mathrm{~s}, \mathrm{CH}_{3}, 3 \mathrm{H}\right)$, $\delta 3.43\left(\mathrm{t}, \mathrm{CH}_{2}, \mathrm{~J}=4.8 \mathrm{~Hz}, 2 \mathrm{H}\right), \delta 3.49-3.56\left(\mathrm{~m}, \mathrm{CH}_{2}, 12 \mathrm{H}\right), \delta 4.18(\mathrm{t}, \mathrm{CH}, \mathrm{J}=6.8 \mathrm{~Hz}$, $1 \mathrm{H}), \delta 4.25(\mathrm{~s}, \mathrm{br}, \mathrm{CH}, 1 \mathrm{H}), \delta 4.40\left(\mathrm{~d}, \mathrm{CH}_{2}, \mathrm{~J}=6.8 \mathrm{~Hz}, 2 \mathrm{H}\right), \delta 5.79-5.85(\mathrm{~m}, \mathrm{NH}, 2 \mathrm{H}), \delta$ 6.95 (s, br, NH, 1H), 7.29 (t, arom. $H, \mathrm{~J}=7.2 \mathrm{~Hz}, 2 \mathrm{H}$ ), $\delta 7.37$ (t, arom. $H, \mathrm{~J}=7.2 \mathrm{~Hz}$, $2 \mathrm{H}), \delta 7.58(\mathrm{~d}$, arom. $H, \mathrm{~J}=7.2 \mathrm{~Hz}, 2 \mathrm{H}), \delta 7.74(\mathrm{~d}$, arom. $H, \mathrm{~J}=7.2 \mathrm{~Hz}, 2 \mathrm{H}) .{ }^{13} \mathrm{C}$ NMR: $\delta$ $28.43,39.38,43.33,47.29,55.27,59.02,66.91,69.57,70.25,70.43,70.56,71.93,80.30$, $120.06,125.19,127.18,127.80,141.41,143.91,143.99,157.53,170.62 . \mathrm{MS}(\mathrm{FAB}+)$ : m/z 572.3 (MH+), $594.3(\mathrm{MNa}+)$. MP: $99-101{ }^{\circ} \mathrm{C}$.

PEG-2Li-Boc-1,2-HOPO(Bn), 3-17a. Following a procedure similar to that of Joullié et al., ${ }^{17}$ a mixture of 3-16 (1.00 g, $\left.1.75 \mathrm{mmol}\right), \mathrm{KF}(0.457 \mathrm{~g}, 7.86 \mathrm{mmol}), \mathrm{Et}_{3} \mathrm{~N}$ (0.40 mL, $2.9 \mathrm{mmol}), 1,2-\mathrm{HOPO}-\mathrm{Thiaz}(\mathrm{Bn})(0.610 \mathrm{~g}, 1.76 \mathrm{mmol})$, and a catalytic amount of 18-crown-6 in $8 \mathrm{~mL}$ of DMF was stirred for one day under argon, monitoring the reaction by TLC (4:1 EtOAc:acetone). The mixture was diluted with $100 \mathrm{~mL}$ of EtOAc and was washed successively with $1 \mathrm{M} \mathrm{HCl}(2 \times 25 \mathrm{~mL}), 1 \mathrm{M} \mathrm{NaOH}$ in $20 \%$ sat. brine (2 x $25 \mathrm{~mL}$ ), sat. brine, $\mathrm{H}_{2} \mathrm{O}$, and brine. The organic layer was dried over $\mathrm{Na}_{2} \mathrm{SO}_{4}$ and the solvent was removed under vacuum. The residue was dissolved in $5 \mathrm{~mL}$ of $\mathrm{CH}_{2} \mathrm{Cl}_{2}$ and 
eluted on a silica column with $5 \% \mathrm{MeOH}$ in $\mathrm{CH}_{2} \mathrm{Cl}_{2}$, collecting fractions with $\mathrm{R}_{\mathrm{f}}=0.18$, which were dried and the solvent removed to yield $0.619 \mathrm{~g}$ of a pale yellow, tacky semisolid monohydrate (59\%). $\mathrm{C}_{28} \mathrm{H}_{40} \mathrm{~N}_{4} \mathrm{O}_{9} \cdot \mathrm{H}_{2} \mathrm{O}$ : C: 56.55 (56.83); H: 7.12 (7.33); N: 9.42 (9.47). ${ }^{1} \mathrm{H}$ NMR: $\delta 1.34\left(\mathrm{~d}, \mathrm{CH}_{3}, 9 \mathrm{H}\right), \delta 3.25-3.28\left(\mathrm{~m}, \mathrm{CH}_{3}+\mathrm{CH}_{2}, 4 \mathrm{H}\right), \delta 3.37\left(\mathrm{t}, \mathrm{CH}_{2}, \mathrm{~J}\right.$ $=4.8 \mathrm{~Hz}, 2 \mathrm{H}), \delta 3.43-3.45\left(\mathrm{~m}, \mathrm{CH}_{2}, 7 \mathrm{H}\right), \delta 3.49-3.64(\mathrm{~m}, \mathrm{CH} 2,4 \mathrm{H}), \delta 4.28(\mathrm{~s}, \mathrm{br}, \mathrm{CH}$, 1H), $\delta 5.96(\mathrm{~d}, \mathrm{NH}, \mathrm{J}=7.2 \mathrm{~Hz}, 1 \mathrm{H}), \delta 6.29(\mathrm{~d}, \mathrm{HOPO} H, \mathrm{~J}=6.0 \mathrm{~Hz}, 1 \mathrm{H}), \delta 6.63(\mathrm{dd}$, HOPO $H, \mathrm{~J}=9.2,1.2 \mathrm{~Hz}, 1 \mathrm{H}), \delta 6.73(\mathrm{~s}, \mathrm{br}, \mathrm{NH}, 1 \mathrm{H}), \delta 7.19$ (dd, HOPO H, J = 9.2, 6.8 $\mathrm{Hz}, 1 \mathrm{H}), \delta 7.30-7.31(\mathrm{~m}$, arom. $H, 3 \mathrm{H}), \delta 7.46(\mathrm{~s}, \mathrm{br}$, arom. $H, 2 \mathrm{H}), \delta 7.70(\mathrm{~s}, \mathrm{br}, \mathrm{NH}$, 1H). ${ }^{13} \mathrm{C}$ NMR: $\delta 28.39,39.10,42.49,54.42,58.95,69.25,69.93,70.39,70.49,71.85$, $79.25,80.25,105.81,124.08,128.75,129.56,130.55,133.38,138.07,142.78,155.85$, 158.68, 161.49, 170.20. MS (FAB+): m/z 577.5 (MH+).

PEG-2Li-Boc-Me-3,2-HOPO(Bn), 3-17b. This synthesis followed an analogous procedure to that of 3-17a, but using Me-3,2-HOPO-Thiaz(Bn) as the starting material. Eluent: $5 \% \mathrm{MeOH}$ in $\mathrm{CH}_{2} \mathrm{Cl}_{2}, \mathrm{R}_{\mathrm{f}}=0.19$. Pale yellow oil; isolated as a methanol solvate, 55\%. $\mathrm{C}_{29} \mathrm{H}_{42} \mathrm{~N}_{4} \mathrm{O}_{9} \cdot \mathrm{CH}_{3} \mathrm{OH}: \mathrm{C}: 57.86$ (58.29); H: 7.45 (7.47); N: 9.00 (8.96). ${ }^{1} \mathrm{H}$ NMR: $\delta$ $1.34\left(\mathrm{~s}, \mathrm{CH}_{3}, 9 \mathrm{H}\right), \delta 3.28\left(\mathrm{~s}, \mathrm{CH}_{3}, 3 \mathrm{H}\right), \delta 3.33\left(\mathrm{~s}, \mathrm{br}, \mathrm{CH}_{2}, 2 \mathrm{H}\right), \delta 3.42-3.54\left(\mathrm{~m}, \mathrm{CH}_{2}+\right.$ $\left.\mathrm{CH}_{3}, 15 \mathrm{H}\right), \delta 4.18(\mathrm{~s}, \mathrm{br}, \mathrm{CH}, 1 \mathrm{H}), \delta 5.22-5.36(\mathrm{~m}$, benzyl $H, 2 \mathrm{H}), \delta 5.83(\mathrm{~d}, \mathrm{NH}, \mathrm{J}=6.4$ Hz, 1H), $\delta 6.61(\mathrm{~d}$, HOPO H, J = 7.2 Hz, 1H), $\delta 6.97$ (s, br, NH, 1H), $\delta 7.03$ (d, HOPO H, $\mathrm{J}=7.2 \mathrm{~Hz}, 1 \mathrm{H}), \delta 7.27-7.29(\mathrm{~m}$, arom. $H, 3 \mathrm{H}), \delta 7.38-7.40(\mathrm{~m}$, arom. $H, 2 \mathrm{H}), \delta 8.27(\mathrm{~s}$, br, $\mathrm{NH}, 1 \mathrm{H}) .{ }^{13} \mathrm{C}$ NMR: $\delta 28.23,37.67,39.21,41.80,55.09,58.90,69.49,70.17,70.35$, $70.38,71.81,74.33,79.98,104.57,128.64,128.78,129.17,130.27,132.14,135.94$, 146.23, 155.87, 159.42, 164.56, 170.21. MS (FAB+): m/z 591.3 (MH+), $613.3\left(\mathrm{MNa}^{+}\right)$. 
PEG-TAM(2Li-1,2-HOPO) $)_{2}(\mathbf{B n})_{4}$, 3-18a. A solution of 3-17· $\mathbf{H}_{2} \mathbf{O}(0.346 \mathrm{~g}, 0.582$ $\mathrm{mmol})$ and $\mathrm{TsOH} \cdot \mathrm{H}_{2} \mathrm{O}(0.682 \mathrm{~g}, 3.58 \mathrm{mmol})$ in $10 \mathrm{~mL}$ of $1: 1 \mathrm{CH}_{2} \mathrm{Cl}_{2}: \mathrm{MeCN}$ was stirred until TLC indicated consumption of the starting material. The solution was diluted with $20 \mathrm{~mL} \mathrm{CH} \mathrm{Cl}_{2}$ and $\mathrm{Et}_{3} \mathrm{~N}(1.0 \mathrm{~mL}, 7.2 \mathrm{mmol})$ was added. Separately, $\mathrm{SOCl}_{2}(2.0 \mathrm{~mL}, 28$ mmol) was added to a suspension of TAM(Bn)-diacid $(0.113 \mathrm{~g}, 0.300 \mathrm{mmol})$ in $3 \mathrm{~mL}$ of benzene with two drops of DMF. The resulting homogenous solution was stirred under nitrogen at room temperature for four hours. The solvent was removed under vacuum and the residue was co-evaporated twice each with toluene and $\mathrm{CHCl}_{3}$. The white acid chloride was dissolved in $20 \mathrm{~mL}$ of $\mathrm{CH}_{2} \mathrm{Cl}_{2}$ and the $\mathrm{HOPO}$ solution was added. Stirring was continued overnight and the solution was washed with $1 \mathrm{M} \mathrm{HCl}(2 \times 25 \mathrm{~mL}), 1 \mathrm{M}$ $\mathrm{NaOH}$ in $20 \%$ sat. brine, sat. brine, dried with $\mathrm{Na}_{2} \mathrm{SO}_{4}$, and the solvent was removed under vacuum. The residue was dissolved in $5 \mathrm{~mL}$ of $\mathrm{CH}_{2} \mathrm{Cl}_{2}$ an eluted on a silica column with $5 \% \mathrm{MeOH}$ in $\mathrm{CH}_{2} \mathrm{Cl}_{2}$, collecting fractions with $\mathrm{R}_{\mathrm{f}}=0.16$. After drying and removing solvent, these yielded $0.191 \mathrm{~g}$ of the product trihydrate as a solid, white residue (49\%). $\mathrm{C}_{68} \mathrm{H}_{78} \mathrm{~N}_{8} \mathrm{O}_{10} \cdot 3\left(\mathrm{H}_{2} \mathrm{O}\right): \mathrm{C}: 60.52$ (60.56); H: 6.27 (6.37); N: 8.30 (8.11). ${ }^{1} \mathrm{H}$ NMR: $\delta$ 3.16-3.22 (m, $\left.\mathrm{CH}_{2}, 2 \mathrm{H}\right), \delta 3.29\left(\mathrm{~s}, \mathrm{CH}_{3}, 6 \mathrm{H}\right), \delta 3.44-3.52\left(\mathrm{~m}, \mathrm{CH}_{2}, 26 \mathrm{H}\right), \delta 4.68$ (quartet, $\mathrm{J}=6.4 \mathrm{~Hz}, 2 \mathrm{H}), \delta 5.11(\mathrm{dd}$, benzyl $H, \mathrm{~J}=17.2,10.8 \mathrm{~Hz}, 4 \mathrm{H}), \delta 5.28(\mathrm{~d}$, benzyl $H, \mathrm{~J}=8.4$ Hz, 2H), $\delta 5.36$ (d, benzyl H, J = 8.4 Hz, 2H), $\delta 6.25$ (dd, HOPO H, J = 6.4, 1.6 Hz, 2H), $\delta 6.65(\mathrm{dd}, \mathrm{HOPO} H, \mathrm{~J}=5.2,1.6 \mathrm{~Hz}, 2 \mathrm{H}), \delta 6.91(\mathrm{t}, \mathrm{NH}, \mathrm{J}=5.6 \mathrm{~Hz}, 2 \mathrm{H}), \delta 7.17(\mathrm{dd}$, HOPO $H, \mathrm{~J}=9.6,6.4 \mathrm{~Hz}, 2 \mathrm{H}), \delta$ 7.22-7.31 (m, arom. $H, 16 \mathrm{H}), \delta$ 7.47-7.49 (m, arom. $H$, $4 \mathrm{H}), \delta 7.54(\mathrm{t}, \mathrm{NH}, \mathrm{J}=5.6 \mathrm{~Hz}, 2 \mathrm{H}), \delta 7.74(\mathrm{~s}, \mathrm{TAM} H, 2 \mathrm{H}), \delta 8.60(\mathrm{~d}, \mathrm{NH}, \mathrm{J}=7.2 \mathrm{~Hz}$, 2H). ${ }^{13} \mathrm{C}$ NMR: $\delta 39.30,42.54,53.17,58.97,69.17,69.95,70.37,70.47,71.83,77.13$, $79.27,105.55,124.16,126.01,128.66,128.75,128.90,129.18,129.53,130.51,130.76$ 
133.44, 135.35, 138.04, 142.83, 150.58, 158.65, 161.03, 164.64, 169.44. MS (FAB+): m/z 1295.7 (MH+). MP: Broad melt between $160-165^{\circ} \mathrm{C}$.

PEG-TAM(2Li-Me-3,2-HOPO) $)_{2}(\mathbf{B n})_{4}$, 3-18b. This synthesis followed an analogous procedure to that of 3-18a, but using $\mathbf{3 - 1 7} \cdot \mathbf{M e O H}$ as the starting material. White solid monohydrate, $51 \%$. Eluent: 5\% MeOH in $\mathrm{CH}_{2} \mathrm{Cl}_{2}, \mathrm{R}_{\mathrm{f}}=0.16 . \mathrm{C}_{70} \mathrm{H}_{82} \mathrm{~N}_{8} \mathrm{O}_{18} \cdot \mathrm{H}_{2} \mathrm{O}: \mathrm{C}: 62.67$ (62.66); H: 6.31 (6.53); N: 8.35 (8.38). ${ }^{1} \mathrm{H}$ NMR: $\delta 3.53$ (s, $\left.\mathrm{CH}_{3}, 6 \mathrm{H}\right), \delta$ 3.29-3.60 (m, $\mathrm{CH}_{2}+\mathrm{CH}_{3}, 34 \mathrm{H}$ ), $\delta 4.52$ (quartet, $\mathrm{CH}, \mathrm{J}=5.6 \mathrm{~Hz}, 2 \mathrm{H}$ ), $\delta 5.02$ (dd, benzyl $\mathrm{H}, \mathrm{J}=19.2$, $10.8 \mathrm{~Hz}, 4 \mathrm{H}), \delta 5.32$ (s, benzyl H, 4H), $\delta 6.54(\mathrm{~d}, \mathrm{HOPO} H, \mathrm{~J}=7.2 \mathrm{~Hz}, 2 \mathrm{H}), \delta 6.92(\mathrm{~d}$, HOPO $H, \mathrm{~J}=7.2 \mathrm{~Hz}, 2 \mathrm{H}), \delta 7.04(\mathrm{t}, \mathrm{NH}, \mathrm{J}=5.2 \mathrm{~Hz}, 2 \mathrm{H}), \delta$ 7.17-7.21 (m, arom. $H, 16 \mathrm{H})$, $\delta$ 7.35-7.37 (m, arom. $H, 4 \mathrm{H}), \delta 7.67(\mathrm{~s}, \mathrm{TAM} H, 2 \mathrm{H}), \delta 8.31(\mathrm{t}, \mathrm{NH}, \mathrm{J}=6.0 \mathrm{~Hz}, 2 \mathrm{H}), \delta$ $8.52(\mathrm{~d}, \mathrm{NH}, \mathrm{J}=6.8 \mathrm{~Hz}, 2 \mathrm{H}) .{ }^{13} \mathrm{C}$ NMR: $\delta 37.68,39.42,41.77,54.12,58.99,69.48$, $70.27,70.42,70.47,71.86,74.27,104.53,125.89,128.51,128.62,128.69,128.75$, $129.08,129.13,130.19,130.69,132.03,135.27,136.10,146.31,150.43,159.43,164.32$ 164.66, 169.69. MS (FAB+): m/z 1323.58 (MH+), $1345.56(\mathrm{MNa}+)$. MP: $134-136{ }^{\circ} \mathrm{C}$.

5-Methoxy-4-(1-oxo-3,6,9-trioxa-decane)-2-nitro-phenyl-NHBoc, 3-19. A solution of 5-methoxy-4-(1-oxo-3,6,9-trioxa-decane)-2-nitroaniline ${ }^{18,19}(2.00 \mathrm{~g}, 6.05 \mathrm{mmol}), \mathrm{Et}_{3} \mathrm{~N}$ $(0.93 \mathrm{~mL}, 6.67 \mathrm{mmol}), \mathrm{Boc}_{2} \mathrm{O}(1.45 \mathrm{~g}, 6.64 \mathrm{mmol})$, and a catalytic amount of DMAP in $50 \mathrm{~mL}$ of $\mathrm{CH}_{2} \mathrm{Cl}_{2}$ was stirred overnight at room temperature. The solution was washed with $1 \mathrm{M} \mathrm{HCl}(2 \times 25 \mathrm{~mL})$, sat. brine, dried with $\mathrm{Na}_{2} \mathrm{SO}_{4}$ and the solvent was removed under vacuum. The yellow residue was dissolved in $8 \mathrm{~mL}$ of EtOAc and eluted on a silica column with EtOAc. Fractions with $\mathrm{R}_{\mathrm{f}}=0.43$ were collected, dried and the solvent was removed to yield $1.68 \mathrm{~g}$ of a yellow solid (65\%). $\mathrm{C}_{19} \mathrm{H}_{30} \mathrm{~N}_{2} \mathrm{O}_{9}$ : C: 53.02 (52.70); H: 7.02 (7.33); N: 6.51 (6.32). ${ }^{1} \mathrm{H}$ NMR: $\delta 1.53\left(\mathrm{~s}, \mathrm{CH}_{3}, 9 \mathrm{H}\right), \delta 3.36\left(\mathrm{~s}, \mathrm{CH}_{3}, 3 \mathrm{H}\right), \delta 3.52-3.55$ (m, 
$\left.\mathrm{CH}_{2}, 2 \mathrm{H}\right), \delta 3.63-3.68\left(\mathrm{~m}, \mathrm{CH}_{2}, 4 \mathrm{H}\right), \delta 3.72-3.74\left(\mathrm{~m}, \mathrm{CH}_{2}, 2 \mathrm{H}\right), \delta 3.88\left(\mathrm{t}, \mathrm{CH}_{2}, \mathrm{~J}=4.8\right.$ $\mathrm{Hz}, 2 \mathrm{H}), \delta 3.97\left(\mathrm{~s}, \mathrm{CH}_{3}, 3 \mathrm{H}\right), \delta 4.18\left(\mathrm{t}, \mathrm{CH}_{2}, \mathrm{~J}=4.8 \mathrm{~Hz}, 2 \mathrm{H}\right), \delta 7.74(\mathrm{~s}$, arom. $H, 1 \mathrm{H}), \delta$ 8.20 (s, arom. $H, 1 \mathrm{H}), \delta 10.06(\mathrm{~s}, \mathrm{NH}, 1 \mathrm{H}) .{ }^{13} \mathrm{C}$ NMR: $\delta 28.41,56.68,59.23,69.18$, $69.66,70.77,70.84,71.08,72.11,81.88,101.78,109.57,127.91,133.68,142.98,152.63$, 156.65. $\mathrm{MS}(\mathrm{FAB}+): \mathrm{m} / \mathrm{z} 453.2(\mathrm{MNa}+)$. MP: $76-78^{\circ} \mathrm{C}$.

PEG-o-phen-Boc-1,2-HOPO(Bn), 3-20a. To a suspension of 1,2-HOPO-acid(Bn) $(0.284 \mathrm{~g}, 1.16 \mathrm{mmol})$ in $10 \mathrm{~mL}$ of benzene and two drops of DMF was added oxalyl chloride $(0.30 \mathrm{~mL}, 3.44 \mathrm{mmol})$. The resulting homogenous solution was stirred under nitrogen for eight hours. The solvent was removed under vacuum and the residue coevaporated with toluene and $\mathrm{CHCl}_{3}(2 \times 5 \mathrm{~mL}$ each). The residue was dissolved in $25 \mathrm{~mL}$ of $\mathrm{CH}_{2} \mathrm{Cl}_{2}$. Separately, a mixture of 3-19 $(0.500 \mathrm{~g}, 1.16 \mathrm{mmol})$ and $50 \mathrm{mg}$ of $10 \% \mathrm{Pd} / \mathrm{C}$ in $25 \mathrm{~mL}$ of $\mathrm{MeOH}$ was stirred under 500 psi of $\mathrm{H}_{2}$. The solution was filtered quickly through a pad of celite, which was washed with more $\mathrm{MeOH}(2 \times 25 \mathrm{~mL})$. The filtrate was evaporated under vacuum, re-dissolved in $25 \mathrm{~mL}$ of $\mathrm{CH}_{2} \mathrm{Cl}_{2}$ and $\mathrm{Et}_{3} \mathrm{~N}(0.35 \mathrm{~mL}, 2.51$ mmol), and added to the stirred acid chloride solution. After stirring overnight, the purple solution was washed with $1 \mathrm{M} \mathrm{HCl}(2 \times 25 \mathrm{~mL}), 1 \mathrm{M} \mathrm{NaOH}$ in $20 \%$ sat. brine $(2 \times 25$ $\mathrm{mL}$ ), sat. brine, dried with $\mathrm{Na}_{2} \mathrm{SO}_{4}$ and the solvent was removed under vacuum. The purple/black solid was dissolved in $5 \mathrm{~mL}$ of EtOAc and eluted on a silica column with $10 \%$ acetone/EtOAc, collecting fractions with $\mathrm{R}_{\mathrm{f}}=0.22$, which were dried and evaporated to yield $0.498 \mathrm{~g}$ of the gray product as the monohydrate $(66 \%)$. $\mathrm{C}_{32} \mathrm{H}_{41} \mathrm{~N}_{3} \mathrm{O}_{10} \cdot \mathrm{H}_{2} \mathrm{O}$ : C: 59.52 (59.66); H: 6.71 (6.82); N: 6.51 (6.25). ${ }^{1} \mathrm{H}$ NMR: $\delta 1.35$ (s, $\left.\mathrm{CH}_{3}, 9 \mathrm{H}\right), \delta 3.23\left(\mathrm{~s}, \mathrm{CH}_{3}, 3 \mathrm{H}\right), \delta 3.41-3.42\left(\mathrm{~m}, \mathrm{CH}_{2}, 2 \mathrm{H}\right), \delta 3.51-3.55\left(\mathrm{~m}, \mathrm{CH}_{2}, 4 \mathrm{H}\right), \delta$ 3.61-3.62 (m, $\left.\mathrm{CH}_{2}, 2 \mathrm{H}\right), \delta 3.75-3.78\left(\mathrm{~m}, \mathrm{CH}_{2}+\mathrm{CH}_{3}, 5 \mathrm{H}\right), \delta 4.00\left(\mathrm{t}, \mathrm{CH}_{2}, \mathrm{~J}=4.8 \mathrm{~Hz}, 2 \mathrm{H}\right)$, 
$\delta 5.22$ (s, benzyl H, 2H), $\delta 6.38$ (dd, HOPO H, J = 9.2, $1.2 \mathrm{~Hz}, 1 \mathrm{H}$ ), $\delta 6.51$ (dd, HOPO H, $\mathrm{J}=9.2,1.2 \mathrm{~Hz}, 1 \mathrm{H}), \delta$ 7.08-7.32 (m, NH+ arom. $H+\mathrm{HOPO} H, 9 \mathrm{H}) .{ }^{13} \mathrm{C}$ NMR: $\delta 28.28$, $56.05,58.91,68.66,69.47,70.42,70.51,70.72,71.83,79.17,80.56,106.58,108.03$, $110.04,121.45,123.95,124.27,128.51,129.33,130.37,133.09,138.09,142.90,144.99$, 147.62, 153.96, 158.61, 158.73. $\mathrm{MS}(\mathrm{FAB}+): \mathrm{m} / \mathrm{z} 628.3(\mathrm{MH}+), 650.3(\mathrm{MNa}+)$. The hydroscopic nature of the product made melting point determination impossible using standard capillary tubes and apparatuses.

PEG-o-phen-Boc-Me-3,2-HOPO(Bn), 3-20b. This synthesis followed an analogous procedure to that of 3-20a, but using $\mathrm{Me}-3,2-\mathrm{HOPO}(\mathrm{COOH})(\mathrm{Bn})$ as the starting material. Beige solid, 68\%. Eluent: $10 \%$ acetone in EtOAc, $\mathrm{R}_{\mathrm{f}}=0.12 . \mathrm{C}_{33} \mathrm{H}_{43} \mathrm{~N}_{3} \mathrm{O}_{10}$ : C: 61.77 (61.39); H: 6.75 (7.09); N: 6.55 (6.36). ${ }^{1} \mathrm{H}$ NMR: $\delta 1.45$ (s, $\left.\mathrm{CH}_{3}, 9 \mathrm{H}\right), \delta 3.36$ (s, $\mathrm{CH}_{3}$, $3 \mathrm{H}), \delta 3.51-3.55\left(\mathrm{~m}, \mathrm{CH}_{3}+\mathrm{CH}_{2}, 5 \mathrm{H}\right), \delta 3.64-3.69\left(\mathrm{~m}, \mathrm{CH}_{2}, 4 \mathrm{H}\right), \delta$ 3.72-3.75 (m, $\mathrm{CH}_{2}$, 2H), $\delta 3.85-3.87\left(\mathrm{~m}, \mathrm{CH}_{3}+\mathrm{CH}_{2}, 5 \mathrm{H}\right), \delta 4.07\left(\mathrm{t}, \mathrm{CH}_{2}, \mathrm{~J}=4.8 \mathrm{~Hz}, 2 \mathrm{H}\right), \delta 5.50$ (s, benzyl $H, 2 \mathrm{H}), \delta 6.69(\mathrm{~d}, \mathrm{HOPO} H, \mathrm{~J}=7.2 \mathrm{~Hz}, 1 \mathrm{H}), \delta 7.00(\mathrm{~s}, \mathrm{br}$, arom. $H, 2 \mathrm{H}), \delta 7.10(\mathrm{~d}$, HOPO $H, \mathrm{~J}=7.2 \mathrm{~Hz}, 1 \mathrm{H}), \delta$ 7.28-7.29 (m, arom. $H, 3 \mathrm{H}), \delta$ 7.36-7.38 (m, arom. $H, 2 \mathrm{H}), \delta$ $9.82(\mathrm{~s}, \mathrm{NH}, 1 \mathrm{H}) .{ }^{13} \mathrm{C}$ NMR: $\delta 28.21,37.50,55.93,58.81,68.73,69.34,70.34,70.45$, $70.63,71.75,74.53,80.10,104.43,108.53,109.53,124.24,128.60,128.76,129.09$, $130.68,132.29,135.57,145.02,145.75,147.41,153.67,159.23,161.63 . \mathrm{MS}(\mathrm{FAB}+)$ : m/z $642(\mathrm{MH}+)$. MP: $70-72{ }^{\circ} \mathrm{C}$.

PEG-TAM(o-phen-1,2-HOPO $)_{2}(\mathrm{Bn})_{4}$, 3-21a. To a suspension of TAM-COOH(Bn $)_{2}$ $(0.129 \mathrm{~g}, 0.344 \mathrm{mmol})$ in $2 \mathrm{~mL}$ of benzene and 2 drops of DMF was added $\mathrm{SOCl}_{2}(2.0$ $\mathrm{mL}, 27 \mathrm{mmol}$ ) and the resulting homogenous solution was stirred under nitrogen at room temperature for four hours and the solvent was then removed under vacuum. The residue 
was co-evaporated with toluene and $\mathrm{CHCl}_{3}(2 \times 5 \mathrm{~mL}$ each $)$ and re-dissolved in $20 \mathrm{~mL}$ of $\mathrm{CH}_{2} \mathrm{Cl}_{2}$. Separately, a solution of 3-20a $\cdot \mathbf{H}_{2} \mathbf{O}(0.432 \mathrm{~g}, 0.669 \mathrm{mmol})$ and $\mathrm{TsOH} \cdot \mathrm{H}_{2} \mathrm{O}$ $(0.792 \mathrm{~g}, 4.16 \mathrm{mmol})$ in $10 \mathrm{~mL}$ of $1: 1 \mathrm{MeCN}: \mathrm{CH}_{2} \mathrm{Cl}_{2}$ was stirred under nitrogen for 1 hour until TCL analysis indicated total consumption of the starting material. $\mathrm{Et}_{3} \mathrm{~N}(1.0$ $\mathrm{mL}, 7.2 \mathrm{mmol}$ ) and $20 \mathrm{~mL}$ more $\mathrm{CH}_{2} \mathrm{Cl}_{2}$ was added and the solution was added to the stirred acid chloride solution. After stirring overnight, the solution was washed with $1 \mathrm{M}$ $\mathrm{HCl}(2 \times 25 \mathrm{ml}), 1 \mathrm{M} \mathrm{NaOH}$ in $20 \%$ sat. brine $(2 \times 25 \mathrm{~mL})$, sat. brine, dried with $\mathrm{Na}_{2} \mathrm{SO}_{4}$ and evaporated under vacuum. The residue was dissolved in $4 \mathrm{~mL}$ of $\mathrm{CH}_{2} \mathrm{Cl}_{2}$ and eluted on a silica column with $1: 1$ acetone: $\mathrm{CH}_{2} \mathrm{Cl}_{2}$, collecting fractions with $\mathrm{R}_{\mathrm{f}}=0.14$, which were dried and evaporated to yield $0.250 \mathrm{~g}$ of product as a beige, solid monohydrate (53\%). $\mathrm{C}_{76} \mathrm{H}_{80} \mathrm{~N}_{6} \mathrm{O}_{20} \cdot \mathrm{H}_{2} \mathrm{O}: \mathrm{C}: 64.49$ (64.46); $\mathrm{H}: 5.84$ (6.03); N: 5.94 (5.95). ${ }^{1} \mathrm{H}$ NMR: $\delta$ $3.34\left(\mathrm{~s}, \mathrm{CH}_{3}, 6 \mathrm{H}\right), \delta 3.51-3.54\left(\mathrm{~m}, \mathrm{CH}_{2}, 4 \mathrm{H}\right), \delta 3.62-3.69\left(\mathrm{~m}, \mathrm{CH}_{2}+\mathrm{CH}_{3}, 14 \mathrm{H}\right), \delta 3.72-$ $3.74\left(\mathrm{~m}, \mathrm{CH}_{2}, 4 \mathrm{H}\right), \delta 3.87\left(\mathrm{t}, \mathrm{CH}_{2}, \mathrm{~J}=4.8 \mathrm{~Hz}, 4 \mathrm{H}\right), \delta 4.11\left(\mathrm{t}, \mathrm{CH}_{2}, \mathrm{~J}=5.2 \mathrm{~Hz}, 4 \mathrm{H}\right), \delta 5.10$ (s, benzyl $H, 4 \mathrm{H}), \delta 5.28(\mathrm{~s}$, benzyl $H, 4 \mathrm{H}), \delta 6.27$ (dd, HOPO $H, \mathrm{~J}=6.8,1.2 \mathrm{~Hz}, 2 \mathrm{H}), \delta$ $6.62(\mathrm{dd}, \mathrm{HOPO} H, \mathrm{~J}=9.2,1.6 \mathrm{~Hz}, 2 \mathrm{H}), \delta 6.86(\mathrm{~s}$, arom. $H, 2 \mathrm{H}), \delta 7.14-7.18(\mathrm{~m}$, arom. $H$ + HOPO H, 8H), $\delta 7.22-7.30(\mathrm{~m}$, arom. $H, 12 \mathrm{H}), \delta 7.33-7.35(\mathrm{~m}$, arom. $H, 4 \mathrm{H}), \delta 7.80(\mathrm{~s}$, TAM $H, 2 \mathrm{H}), \delta 8.49(\mathrm{~s}, \mathrm{NH}, 2 \mathrm{H}), \delta 9.54(\mathrm{~s}, \mathrm{NH}, 2 \mathrm{H}) .{ }^{13} \mathrm{C} \mathrm{NMR}: \delta 56.20,59.11,68.81$, $69.55,70.61,70.74,70.92,72.02,77.48,79.27,105.73,107.45,110.87,121.61,123.80$, $124.58,126.97,128.53,128.57,129.12,129.21,129.41,130.41,130.78,133.23,135.38$, $137.82,142.81,146.31,148.06,150.25,158.49,158.74,162.67 . \mathrm{MS}(\mathrm{FAB}+): \mathrm{m} / \mathrm{z} 1398$ $(\mathrm{MH}+)$. MP: $68-73{ }^{\circ} \mathrm{C}$.

PEG-TAM(o-phen-Me-3,2-HOPO $)_{2}(\mathrm{Bn})_{4}$, 3-21b. This synthesis followed an analogous procedure to that of 3-21a, but using 3-20b as the starting material. Brown 
solid, 55\%. Eluent: 1:1 acetone: $\mathrm{CH}_{2} \mathrm{Cl}_{2}, \mathrm{R}_{\mathrm{f}}=0.12 . \mathrm{C}_{78} \mathrm{H}_{84} \mathrm{~N}_{6} \mathrm{O}_{20}$ : C: 65.72 (65.60); H: 5.94 (6.09); N: 5.90 (5.83). ${ }^{1} \mathrm{H}$ NMR: $\delta 3.28$ (s, $\left.\mathrm{CH} 3,6 \mathrm{H}\right), \delta 3.32\left(\mathrm{~s}, \mathrm{CH}_{3}, 6 \mathrm{H}\right), \delta 3.46(\mathrm{t}$, $\left.\mathrm{CH}_{2}, \mathrm{~J}=4.8 \mathrm{~Hz}, 4 \mathrm{H}\right), \delta 3.57-3.62\left(\mathrm{~m}, \mathrm{CH}_{2}, 8 \mathrm{H}\right), \delta 3.68\left(\mathrm{t}, \mathrm{CH}_{2}, \mathrm{~J}=4.8 \mathrm{~Hz}, 4 \mathrm{H}\right), \delta 3.73$ (s, $\left.\mathrm{CH}_{3}, 6 \mathrm{H}\right), \delta 3.81\left(\mathrm{t}, \mathrm{CH}_{2}, \mathrm{~J}=4.8 \mathrm{~Hz}, 4 \mathrm{H}\right), \delta 4.00\left(\mathrm{t}, \mathrm{CH}_{2}, \mathrm{~J}=4.8 \mathrm{~Hz}, 4 \mathrm{H}\right), \delta 4.97$ (s, benzyl $H, 4 \mathrm{H}), \delta 5.33$ (s, benzyl $H, 4 \mathrm{H}), \delta 6.58(\mathrm{~d}, \mathrm{HOPO} H, \mathrm{~J}=7.2 \mathrm{~Hz}, 2 \mathrm{H}), \delta 6.76(\mathrm{~s}$, arom. $H, 2 \mathrm{H}), \delta 6.85(\mathrm{~d}, \mathrm{HOPO} H, \mathrm{~J}=7.2 \mathrm{~Hz}, 2 \mathrm{H}), \delta 7.06-7.19$ (arom. $H, 18 \mathrm{H}), \delta 7.24$ (d, arom. $H, \mathrm{~J}=8.0 \mathrm{~Hz}, 4 \mathrm{H}), \delta 7.71(\mathrm{~s}, \mathrm{TAM} H, 2 \mathrm{H}), \delta 9.39(\mathrm{~s}, \mathrm{NH}, 2 \mathrm{H}), \delta 9.56(\mathrm{~s}, \mathrm{NH}$, 2H). ${ }^{13} \mathrm{C}$ NMR: $\delta 37.50,56.03,58.96,68.77,69.41,70.48,70.59,70.77,71.86,74.56$, $77.43,104.33,107.93,110.15,121.77,124.22,126.34,128.50,128.70,128.80,128.87$, $128.95,129.04,130.08,131.45,132.07,135.01,135.55,145.62,146.12,147.64,149.93$, 159.08, 161.75, 162.55. MS (FAB+): m/z 1425.8 (MH+). MP: $62-64{ }^{\circ} \mathrm{C}$.

PEG-TAM-2Li-Me-3,2-HOPO-methyl ester(Bn) $)_{3}$, 3-25. To a suspension of TAMacid-methyl ester(Bn) ${ }_{2}^{27}$ (1.04 g, $\left.2.66 \mathrm{mmol}\right)$ and 2 drops of DMF in $20 \mathrm{~mL}$ of benzene was added $\mathrm{SOCl}_{2}(2.0 \mathrm{~mL}, 27 \mathrm{mmol})$, and the resulting homogenous solution was stirred at room temperature under nitrogen overnight. The solvent was removed under vacuum and the residue was co-evaporated with toluene and $\mathrm{CHCl}_{3}(2 \times 10 \mathrm{~mL}$ each $)$. The white acid chloride was dissolved in $30 \mathrm{~mL}$ of $\mathrm{CH}_{2} \mathrm{Cl}_{2}$. Separately, a solution of 3-17b $\cdot \mathbf{M e O H}$ (1.65 g, $2.66 \mathrm{mmol})$ and $\mathrm{TsOH} \cdot \mathrm{H}_{2} \mathrm{O}(3.04 \mathrm{~g}, 16.0 \mathrm{mmol})$ in $20 \mathrm{~mL}$ of $1: 1 \mathrm{MeCN}: \mathrm{CH}_{2} \mathrm{Cl}_{2}$ was stirred for 1 hour, until TLC $\left(5 \% \mathrm{MeOH}\right.$ in $\left.\mathrm{CH}_{2} \mathrm{Cl}_{2}\right)$ indicated consumption of the starting material. The solution was diluted with $20 \mathrm{~mL}$ of $\mathrm{CH}_{2} \mathrm{Cl}_{2}$ and $\mathrm{Et}_{3} \mathrm{~N}(4.5 \mathrm{~mL}, 32$ mmol) and added to the acid chloride solution. After stirring 5 hours, the reaction mixture was washed with $1 \mathrm{M} \mathrm{HCl}(2 \times 50 \mathrm{~mL}), 1 \mathrm{M} \mathrm{NaOH}$ in $20 \%$ sat. brine $(2 \times 50 \mathrm{~mL})$, sat. brine, dried with $\mathrm{Na}_{2} \mathrm{SO}_{4}$ and the solvent was removed under vacuum. The residue was 
dissolved in $15 \mathrm{~mL}$ of EtOAc and eluted on a silica column with $10 \% \mathrm{MeOH}$ in EtOAc. Colorless fractions with $\mathrm{R}_{\mathrm{f}}=0.27$ were collected, dried and the solvent removed to yield $1.54 \mathrm{~g}$ of a colorless, tacky paste (67\%). $\mathrm{C}_{47} \mathrm{H}_{52} \mathrm{~N}_{4} \mathrm{O}_{12}$ : C: 65.27 (64.93); H: 6.06 (6.18); N: 6.48 (6.47). ${ }^{1} \mathrm{H}$ NMR: $\delta 3.35\left(\mathrm{~s}, \mathrm{CH}_{3}, 3 \mathrm{H}\right), \delta 3.38-3.44\left(\mathrm{~m}, \mathrm{CH}_{2}, 3 \mathrm{H}\right), \delta 3.50-3.52(\mathrm{~m}$, $\mathrm{CH}_{2}, 4 \mathrm{H}$ ), $\delta$ 3.56-3.66 (m, $\mathrm{CH}_{2}+\mathrm{CH}_{3}, 10 \mathrm{H}$ ), $\delta 4.57$ (quartet, $\mathrm{CH}, \mathrm{J}=6.0 \mathrm{~Hz}, 1 \mathrm{H}$ ), $\delta 5.08$ (s, benzyl H, 2H), $\delta 5.10(\mathrm{~d}$, benzyl $H, \mathrm{~J}=10.8 \mathrm{~Hz}, 1 \mathrm{H}), \delta 5.18(\mathrm{~d}$, benzyl $H, \mathrm{~J}=10.8 \mathrm{~Hz}$, 1H), $\delta 5.40$ (s, benzyl H, 2H), $\delta 6.65(\mathrm{~d}, \mathrm{HOPO} H, \mathrm{~J}=7.2 \mathrm{~Hz}, 1 \mathrm{H}), \delta 7.01(\mathrm{~d}, \mathrm{HOPO} H+$ $\mathrm{NH}, \mathrm{J}=7.2 \mathrm{~Hz}, 2 \mathrm{H}), \delta$ 7.22-7.29 (m, arom. $H, 7 \mathrm{H}), \delta$ 7.35-7.37 (m, arom. $H, 4 \mathrm{H}), \delta$ 7.39$7.42(\mathrm{~m}$, arom. $H, 4 \mathrm{H}), \delta 7.58(\mathrm{~d}, \mathrm{TAM} H, \mathrm{~J}=8.4 \mathrm{~Hz}, 1 \mathrm{H}), \delta 7.78(\mathrm{~d}, \mathrm{TAM} H, \mathrm{~J}=8.4 \mathrm{~Hz}$, $1 \mathrm{H}), \delta 8.36(\mathrm{t}, \mathrm{NH}, \mathrm{J}=6.0 \mathrm{~Hz}, 1 \mathrm{H}), \delta 6.61(\mathrm{~d}, \mathrm{NH}, \mathrm{J}=6.8 \mathrm{~Hz}, 1 \mathrm{H}) .{ }^{13} \mathrm{C} \mathrm{NMR}: \delta 37.90$, $39.63,41.96,52.66,54.23,59.22,69.71,70.50,70.66,70.69,72.09,74.47,76.68,77.08$, $77.43,104.82,126.02,126.12,128.54,128.69,128.82,128.85,128.93,129.30,129.33$, $130.14,130.34,130.96,132.10,135.62,136.31,136.74,146.59,151.56,152.09,164.48$ 164.81, 165.93, 169.86. MS (FAB+): m/z 865.5 (MH+).

PEG-TAM-2Li-Me-3,2-HOPO-COOH(Bn) 3, 3-26. A solution of 3-25 (1.37 g, 1.58 $\mathrm{mmol})$ in $25 \mathrm{~mL}$ of $\mathrm{MeOH}$ and aqueous $2 \mathrm{M} \mathrm{LiOH}(2.0 \mathrm{~mL}, 4.0 \mathrm{mmol})$ was stirred at room temperature for two days. The solvent was removed under vacuum and the residue was re-dissolved in $50 \mathrm{~mL}$ of water. After washing with $25 \mathrm{~mL}$ of $\mathrm{CHCl}_{3}$, the aqueous layer was acidified to $\mathrm{pH} 2$ with $6 \mathrm{M} \mathrm{HCl}$ and the turbid mixture was extracted with EtOAc (4 x $25 \mathrm{~mL})$. The combined organic fractions were washed with water and sat. brine, dried with $\mathrm{Na}_{2} \mathrm{SO}_{4}$ and the solvent was removed under vacuum to yield $0.896 \mathrm{~g}$ of the product as a white semi-solid monohydrate $(65 \%) \cdot \mathrm{C}_{46} \mathrm{H}_{50} \mathrm{~N}_{4} \mathrm{O}_{12} \cdot \mathrm{H}_{2} \mathrm{O}: \mathrm{C}: 63.58$ (63.71); H: 6.03 (6.03); N: 6.45 (6.41). ${ }^{1} \mathrm{H}$ NMR: $\delta 3.28\left(\mathrm{~s}, \mathrm{CH}_{3}, 3 \mathrm{H}\right), \delta 3.31-3.41(\mathrm{~m}$, 
$\left.\mathrm{CH}_{2}, 4 \mathrm{H}\right), \delta 3.43-3.47\left(\mathrm{~m}, \mathrm{CH}_{2}, 4 \mathrm{H}\right), \delta 3.49\left(\mathrm{~s}, \mathrm{CH}_{3}, 3 \mathrm{H}\right), \delta 3.51-3.54\left(\mathrm{~m}, \mathrm{CH}_{2}+\mathrm{CH}_{3}\right.$, $6 \mathrm{H}), \delta$ 3.78-3.64 (m, $\left.\mathrm{CH}_{2}, 2 \mathrm{H}\right), \delta 4.53$ (quartet, $\mathrm{CH}, \mathrm{J}=5.6 \mathrm{~Hz}, 1 \mathrm{H}$ ), $\delta 5.02(\mathrm{~d}$, benzyl $\mathrm{H}$, $\mathrm{J}=10.8 \mathrm{~Hz}, 1 \mathrm{H}), \delta 5.09-5.15(\mathrm{~m}$, benzyl $H, 3 \mathrm{H}), \delta 5.34(\mathrm{~s}$, benzyl $H, 2 \mathrm{H}), \delta 6.58(\mathrm{~d}$, HOPO $H, \mathrm{~J}=7.2 \mathrm{~Hz}, 1 \mathrm{H}), \delta 6.94(\mathrm{~d}, \mathrm{HOPO} H, \mathrm{~J}=7.2 \mathrm{~Hz}, 1 \mathrm{H}), \delta 7.09$ (s, br, NH, 1H), $\delta$ 7.19-7.27 (m, arom. $H, 12 \mathrm{H}), \delta 7.36-7.38(\mathrm{~m}$, arom. $H, 3 \mathrm{H}), \delta 7.70(\mathrm{~d}, \mathrm{TAM} H, \mathrm{~J}=8.4$ Hz, 1H), $\delta 7.75(\mathrm{~d}, \mathrm{TAM} H, \mathrm{~J}=8.4 \mathrm{~Hz}, 1 \mathrm{H}), \delta 8.35(\mathrm{t}, \mathrm{NH}, \mathrm{J}=6.4 \mathrm{~Hz}, 1 \mathrm{H}), \delta 8.52(\mathrm{~d}$, $\mathrm{NH}, \mathrm{J}=6.8 \mathrm{~Hz}, 1 \mathrm{H}) .{ }^{13} \mathrm{C}$ NMR: $\delta 37.94,39.64,42.06,54.39,59.17,69.64,70.39,70.56$, $70.62,72.01,74.54,77.39,77.43,104.82,126.38,127.37,127.68,128.74,128.81$, $128.97,129.18,129.29,129.35,130.30,132.18,132.34,135.09,135.30,136.23,146.61$, 150.65, 151.66, 159.64, 164.61, 165.81, 169.79. MS (FAB+): m/z 851.6 (MH+), 873.6 $(\mathrm{MNa}+)$.

PEG-Pr-TAM-2Li-Me-3,2-HOPO(Bn $)_{3}$, 3-27. A solution of 3-26· $\mathbf{H}_{2} \mathbf{O}(0.300 \mathrm{~g}$, $0.345 \mathrm{mmol})$, NHS ( $0.060 \mathrm{~g}, 0.521 \mathrm{mmol})$, and a catalytic amount of DMAP in $25 \mathrm{~mL}$ of $\mathrm{CH}_{2} \mathrm{Cl}_{2}$ was stirred in an ice bath under nitrogen. DCC $(0.106 \mathrm{~g}, 0.514 \mathrm{mmol})$ was added and the mixture was allowed to stir cold for 4 hours. Propylamine $(0.050 \mathrm{~mL}, 0.61 \mathrm{mmol})$ was added and stirring was continued at room temperature for 5 hours more. The mixture was cooled in an ice bath and filtered. The filtrate was washed with $1 \mathrm{M} \mathrm{HCl}$ and $1 \mathrm{M}$ $\mathrm{NaOH}$ in $20 \%$ sat. brine ( $25 \mathrm{~mL}$ each), sat. brine, dried with $\mathrm{Na}_{2} \mathrm{SO}_{4}$, and solvent was removed under vacuum. The residue was dissolved in EtOAc, filtered again and the solvent was evaporated again. Re-dissolution in a minimum amount of EtOAc was followed by elution of the material on a silica gel column with $15 \% \mathrm{MeOH}$ in EtOAc. Fractions with $\mathrm{R}_{\mathrm{f}}=0.30$ were collected, dried and the solvent was removed under vacuum to yield $273 \mathrm{mg}$ of a pasty, colorless semi-solid (89\%). $\mathrm{C}_{49} \mathrm{H}_{57} \mathrm{~N}_{5} \mathrm{O}_{11}$ : C: 65.98 
(65.71); H: 6.44 (6.69); N: 7.85 (7.63). ${ }^{1} \mathrm{H}$ NMR: $\delta 0.75$ (t, $\left.\mathrm{CH}_{3}, \mathrm{~J}=7.2 \mathrm{~Hz}, 3 \mathrm{H}\right), \delta 1.30$ (sextet, $\mathrm{CH}_{2}, \mathrm{~J}=7.2 \mathrm{~Hz}, 2 \mathrm{H}$ ), $\delta 3.17$ (quartet, $\mathrm{CH}_{2}, \mathrm{~J}=5.6 \mathrm{~Hz}, 2 \mathrm{H}$ ), $\delta 3.27\left(\mathrm{~s}, \mathrm{CH}_{3}, 3 \mathrm{H}\right.$ ), $\delta$ 3.30-3.39 (m, $\left.\mathrm{CH}_{2}, 3 \mathrm{H}\right), \delta 3.42-3.52\left(\mathrm{~m}, \mathrm{CH}_{2}+\mathrm{CH}_{3}, 13 \mathrm{H}\right), \delta 3.56-3.59\left(\mathrm{~m}, \mathrm{CH}_{2}, 1 \mathrm{H}\right), \delta$ 4.54 (quartet, $\mathrm{CH}, \mathrm{J}=5.6 \mathrm{~Hz}, 1 \mathrm{H}), \delta 4.96(\mathrm{~d}$, benzyl $\mathrm{H}, \mathrm{J}=10.4 \mathrm{~Hz}, 1 \mathrm{H}), \delta 5.02(\mathrm{~d}$, benzyl $H, \mathrm{~J}=12.8 \mathrm{~Hz}, 2 \mathrm{H}), \delta 5.01$ (d, benzyl $H, \mathrm{~J}=10.8 \mathrm{~Hz}, 1 \mathrm{H}), \delta 5.31$ (s, benzyl $H$, 2H), $\delta 6.55(\mathrm{~d}, \mathrm{HOPO} H, \mathrm{~J}=7.2 \mathrm{~Hz}, 1 \mathrm{H}), \delta 6.92(\mathrm{~d}, \mathrm{HOPO} H, \mathrm{~J}=7.2 \mathrm{~Hz}, 1 \mathrm{H}), \delta 7.07$ (t, $\mathrm{NH}, \mathrm{J}=5.2 \mathrm{~Hz}, 1 \mathrm{H}), \delta$ 7.19-7.36 (m, arom. $H, 15 \mathrm{H}), \delta$ 7.69-7.72 (m, TAM $H+\mathrm{NH}, 2 \mathrm{H})$, $\delta 7.86(\mathrm{~d}, \mathrm{TAM} H, \mathrm{~J}=8.4 \mathrm{~Hz}, 1 \mathrm{H}), \delta 8.30(\mathrm{t}, \mathrm{NH}, \mathrm{J}=6.0 \mathrm{~Hz}, 1 \mathrm{H}), \delta 8.49(\mathrm{~d}, \mathrm{NH}, \mathrm{J}=6.8$ $\mathrm{Hz}, 1 \mathrm{H}) .{ }^{13} \mathrm{C}$ NMR: $\delta 11.60,22.54,37.76,39.52,41.64,41.92,54.15,59.08,69.58$, $70.36,70.51,70.57,71.96,74.36,77.14,104.65,126.13,126.65,128.65,128.70,128.80$ $128.90,128.97,129.04,129.20,130.27,130.56,130.84,132.06,135.49,135.74,136.20$, $146.44,150.39,150.71,159.53,164.03,164.43,164.85,169.76$. MS (ESI+): 892.41 $(\mathrm{MH}+), 914.39(\mathrm{MNa}+)$.

\subsubsection{Benzyl Deprotection of TAM(HOPO $)_{n}$ and PEG-TAM(HOPO $)_{n}$ Ligands}

General benzyl deprotection strategy: Benzyl-protected TAM-containing ligands (0.1-0.5 mmol) were stirred in enough 1:1 conc. $\mathrm{HCl} / \mathrm{AcOH}$ to initially dissolve the solid (5-20 mL). Precipitates formed with ligands devoid of PEG solubilizing groups within 124 hours. Regardless of the homogeneity of the solution, the mixtures were stirred at room temperature for 10 days. The acids were removed under vacuum and unless otherwise indicated, the residue was suspended in cold $\mathrm{MeOH}$, filtered, and washed with more cold $\mathrm{MeOH}$. The solids were held under vacuum before use.

TAM(2Li-1,2-HOPO) 2 , 3-1. After acid removal, the residue was co-evaporated with $\mathrm{MeOH}$ and recrystallized from $\mathrm{MeOH}$. Fluffy, beige solid over two crops which analysis 
showed to be the sesquihydrate, $87 \%$. $\mathrm{C}_{24} \mathrm{H}_{24} \mathrm{~N}_{6} \mathrm{O}_{10} \cdot 1.5\left(\mathrm{H}_{2} \mathrm{O}\right)$ : C: 49.40 (49.47); H: 4.66 (4.41); N: 14.40 (14.36). ${ }^{1} \mathrm{H}$ NMR (DMSO-d ${ }_{6}$ ): $\delta 3.41-3.47$ (m, $\mathrm{CH}_{2}, 8 \mathrm{H}$ ), $\delta 6.32$ (dd, HOPO $H, \mathrm{~J}=6.8,1.6 \mathrm{~Hz}, 2 \mathrm{H}), \delta 6.58(\mathrm{dd}, \mathrm{HOPO} H, \mathrm{~J}=9.2,1.6 \mathrm{~Hz}, 2 \mathrm{H}), \delta 7.32(\mathrm{~s}, \mathrm{TAM}$ $H, 2 \mathrm{H}), \delta 7.40(\mathrm{dd}, \mathrm{HOPO} H, \mathrm{~J}=9.2,6.8 \mathrm{~Hz}, 2 \mathrm{H}), \delta 8.91-8.95(\mathrm{~m}, \mathrm{NH}, 4 \mathrm{H}), \delta 12.62(\mathrm{~s}$, br, OH, 2H). ${ }^{13} \mathrm{C}$ NMR (DMSO- $\left.d_{6}\right): \delta 38.41,38.51,103.82,115.93,117.38,119.53$, 137.24, 142.10, 150.01, 157.46, 160.48, 168.89. MS (FAB+): m/z 557 (MH+). MP: 224$226^{\circ} \mathrm{C}(\mathrm{dec})$.

TAM(2Li-Me-3,2-HOPO) 2, 3-2. White solid as the methanol solvate, 73\%. $\mathrm{C}_{26} \mathrm{H}_{28} \mathrm{~N}_{6} \mathrm{O}_{10} \cdot \mathrm{CH}_{3} \mathrm{OH}: \quad \mathrm{C}: 52.60(52.12) ; \mathrm{H}: 5.23(5.00) ; \mathrm{N}: 13.60(13.51) .{ }^{1} \mathrm{H} \quad \mathrm{NMR}$ (DMSO-d $\left.d_{6}\right): \delta 3.16\left(\mathrm{~s}, \mathrm{CH}_{3}, 3 \mathrm{H}\right), \delta 3.46\left(\mathrm{~s}, \mathrm{CH}_{3}, 6 \mathrm{H}\right), \delta 3.49\left(\mathrm{~s}, \mathrm{CH}_{2}, 8 \mathrm{H}\right), \delta 6.51(\mathrm{~d}$, HOPO $H, \mathrm{~J}=7.2 \mathrm{~Hz}, 2 \mathrm{H}), \delta 7.18(\mathrm{~d}, \mathrm{HOPO} H, \mathrm{~J}=7.2 \mathrm{~Hz}, 2 \mathrm{H}), \delta 7.31(\mathrm{~s}, \mathrm{TAM} H, 2 \mathrm{H}), \delta$ $8.67\left(\mathrm{~s}, \mathrm{NH}, 2 \mathrm{H}\right.$ ), $\delta 9.02(\mathrm{~s}, \mathrm{NH}, 2 \mathrm{H}) .{ }^{13} \mathrm{C}$ NMR $\left(\mathrm{DMSO}-\mathrm{d}_{6}\right.$ ): $\delta 36.89,38.61,38.73$, $102.51,115.87,116.98,117.30,127.74,147.97,150.13,158.04,166.12,169.04 . \mathrm{MS}$ (FAB+): m/z $585(\mathrm{MH}+)$. MP: $269-271^{\circ} \mathrm{C}(\mathrm{dec})$.

TAM(2Li-Me-3,2-HOPO)(2Li-1,2-HOPO), 3-3. Beige solid as the hydrochloride hydrate, 77\%. $\mathrm{C}_{25} \mathrm{H}_{26} \mathrm{~N}_{6} \mathrm{O}_{10} \cdot \mathrm{HCl} \cdot \mathrm{H}_{2} \mathrm{O}$ : C: 48.04 (48.32); H: 4.68 (4.50); N: 13.44 (13.04). ${ }^{1} \mathrm{H}$ NMR (DMSO- $\left.d_{6}\right): \delta 3.41-3.48\left(\mathrm{~m}, \mathrm{CH}_{2}+\mathrm{CH}_{3}, 11 \mathrm{H}\right), \delta 6.32(\mathrm{~d}, \mathrm{HOPO} H, \mathrm{~J}=5.6 \mathrm{~Hz}$, 1H), $\delta 6.51(\mathrm{~d}, \mathrm{HOPO} H, \mathrm{~J}=7.2 \mathrm{~Hz}, 1 \mathrm{H}), \delta 6.58(\mathrm{~d}, \mathrm{HOPO} H, \mathrm{~J}=9.2 \mathrm{~Hz}, 1 \mathrm{H}), \delta 7.19(\mathrm{~d}$, HOPO $H, \mathrm{~J}=7.2 \mathrm{~Hz}, 1 \mathrm{H}$ ), $\delta 7.32(\mathrm{~s}, \mathrm{TAM} H, 2 \mathrm{H}$ ), $\delta 7.41$ (dd, HOPO H, J = 9.2, $6.8 \mathrm{~Hz}$, $1 \mathrm{H}), \delta 8.66(\mathrm{~s}, \mathrm{br}, \mathrm{NH}, 1 \mathrm{H}), \delta 8.93-9.01(\mathrm{~m}, \mathrm{NH}, 3 \mathrm{H}) .{ }^{13} \mathrm{C}$ NMR (DMSO-d $\left.\mathrm{d}_{6}\right): \delta 36.84$, $38.42,38.56,38.71,102.49,103.83,115.85,115.93,116.98,117.29,117.37,119.53$, $127.72,137.24,142.11,147.89,150.02,150.07,157.46,158.01,160.48,166.05,168.89$, 169.00. $\mathrm{MS}(\mathrm{FAB}+): \mathrm{m} / \mathrm{z} 571(\mathrm{MH}+)$. MP: Slow decomposition between $150-250^{\circ} \mathrm{C}$. 
TAM(o-phen-1,2-HOPO) 2 , 3-4. Off-white solid as the partial solvate, 71\%. $\mathrm{C}_{32} \mathrm{H}_{24} \mathrm{~N}_{6} \mathrm{O}_{10} \cdot 1 \frac{1}{2} \mathrm{H}_{2} \mathrm{O} \cdot 1 / 2 \mathrm{CH}_{3} \mathrm{OH}$ : C: 57.61 (57.96); H: 4.02 (3.69); N: 12.40 (12.29). ${ }^{1} \mathrm{H}$ NMR (DMSO-d $)_{6}$ ): $\delta 6.61(\mathrm{~d}, \mathrm{HOPO} H, \mathrm{~J}=6.4 \mathrm{~Hz}, 2 \mathrm{H}), \delta 6.67(\mathrm{~d}, \mathrm{HOPO} H, \mathrm{~J}=9.2 \mathrm{~Hz}$, $2 \mathrm{H}), \delta$ 7.32-7.38 (m, arom. $H, 4 \mathrm{H}), \delta 7.46(\mathrm{t}, \mathrm{HOPO} H, \mathrm{~J}=8.0 \mathrm{~Hz}, 2 \mathrm{H}), \delta 7.58(\mathrm{~s}, \mathrm{TAM}$ $H, 2 \mathrm{H}), \delta$ 7.71-7.73 (m, arom. $H, 4 \mathrm{H}), \delta 10.29$ (s, NH, 2H), $\delta 10.75$ (s, NH, 2H). ${ }^{13} \mathrm{C}$ NMR: $\delta$ 104.98, 117.71, 119.20, 120.08, 125.20, 126.13, 126.29, 126.45, 130.25, 130.77, 137.22, 141.62, 148.95, 157.59, 159.22, 166.81. MS (FAB+): m/z $643(\mathrm{MH}+$, minor), 154 (1,2-HOPOH+). MP: $245-247^{\circ} \mathrm{C}(\mathrm{dec})$.

TAM(o-phen-Me-3,2-HOPO $)_{2}$, 3-5. White solid isolated as the partial hydrate, $92 \%$. $\mathrm{C}_{34} \mathrm{H}_{28} \mathrm{~N}_{6} \mathrm{O}_{10} \cdot{ }^{1 / 2} \mathrm{H}_{2} \mathrm{O} \cdot 1 / 2 \mathrm{HCl}$ : C: 57.69 (57.36); H: 4.20 (3.98); N: 11.87 (11.58). ${ }^{1} \mathrm{H}$ NMR (DMSO-d $\left.d_{6}\right): \delta 3.49\left(\mathrm{~s}, \mathrm{CH}_{3}, 6 \mathrm{H}\right), \delta 6.63(\mathrm{~d}, \mathrm{HOPO} H, \mathrm{~J}=7.2 \mathrm{~Hz}, 2 \mathrm{H}), \delta$ 7.23-7.29 (m, $\mathrm{HOPO}+\operatorname{arom} . H, 4 \mathrm{H}), \delta 7.35(\mathrm{t}$, arom. $H, \mathrm{~J}=7.2 \mathrm{~Hz}, 4 \mathrm{H}), \delta 7.59(\mathrm{~d}, \mathrm{TAM}+\operatorname{arom} . H, \mathrm{~J}$ $=7.2 \mathrm{~Hz}, 4 \mathrm{H}), \delta 8.00(\mathrm{~d}$, arom. $H, \mathrm{~J}=7.6 \mathrm{~Hz}, 2 \mathrm{H}), \delta 10.25(\mathrm{~s}, \mathrm{NH}, 2 \mathrm{H}), \delta 10.49(\mathrm{~s}, \mathrm{NH}$, $2 \mathrm{H}), \delta 12.00(\mathrm{~s}, \mathrm{br}, \mathrm{OH}, 2 \mathrm{H}) .{ }^{13} \mathrm{C}$ NMR: $\delta 36.95,103.42,117.57,118.29,118.56,124.06$, $125.23,126.69,128.06,128.94,132.59,146.47,149.33,158.26,162.99,167.45 . \mathrm{MS}$ $(\mathrm{FAB}+): \mathrm{m} / \mathrm{z} 681(\mathrm{MH}+)$. MP: $282-282{ }^{\circ} \mathrm{C}(\mathrm{dec})$.

PEG-TAM(2Li-1,2-HOPO) 2, 3-12. After acid removal the residue was coevaporated with $\mathrm{MeOH}$ and $\mathrm{CHCl}_{3}(2 \times 5 \mathrm{~mL}$ each), then held under vacuum for at least one day before use. Beige solid, isolated as the diacetic acid, trihydrate, $83 \%$. NMR analysis indicated the compounds exists either as a mixture of diastereomers or as a slowly-exchanging mix of conformers, in a ratio of approximately 8.1:1. $\mathrm{C}_{40} \mathrm{H}_{54} \mathrm{~N}_{8} \mathrm{O}_{18} \cdot 2 \mathrm{CH}_{3} \mathrm{CO}_{2} \mathrm{H} \cdot 3 \mathrm{H}_{2} \mathrm{O}: \mathrm{C}: 47.65$ (47.57); H: 6.18 (6.20); N: 10.10 (10.30). ${ }^{1} \mathrm{H}$ NMR (DMSO-d $\left.d_{6}\right): \delta 3.22-3.66\left(\mathrm{~m}, \mathrm{br}, \mathrm{CH}_{2}+\mathrm{CH}_{3}+\mathrm{CH}_{3} \mathrm{CO}_{2} \mathrm{H}, \mathrm{H}_{2} \mathrm{O}, 49 \mathrm{H}\right), \delta 4.69$ 
(quartet, $\mathrm{CH}, \mathrm{J}=5.6 \mathrm{~Hz}, 2 \mathrm{H}), \delta 6.28(\mathrm{~d}, \mathrm{HOPO} H, \mathrm{~J}=6.4 \mathrm{~Hz}, 2 \mathrm{H}), \delta 6.57(\mathrm{~d}, \mathrm{HOPO} H, \mathrm{~J}$ $=8.8 \mathrm{~Hz}, 2 \mathrm{H}), \delta$ 7.37-7.44 (m, HOPO $H+$ TAM $H, 4 \mathrm{H}), \delta 8.28-8.34(\mathrm{~m}, \mathrm{~N} H, 2 \mathrm{H}), \delta$ 8.97-9.02 (m, NH, 3.1H), $\delta$ 9.09-9.17 (m, NH, 0.9H), $\delta$ 12.13-12.27 (m, br, OH, 2H). ${ }^{13} \mathrm{C}$ NMR (DMSO- $\left.d_{6}\right): \delta 38.56,40.69,52.97,58.06,68.81,69.59,69.71,71.27,104.28$, $117.07,118.09,119.59,119.59,137.15,137.20,141.85,149.08,157.47,160.66,168.96$. MS (FAB+): $935.3(\mathrm{MH}+)$. MP: $87-92{ }^{\circ} \mathrm{C}$.

PEG-TAM(2Li-Me-3,2-HOPO)2, 3-13. Workup of the residue from acid deprotection proceeded in the same manner as for 3-12. Beige solid isolated as the polyhydrate, $90 \%$. NMR analysis indicated the compounds exists either as a mixture of diastereomers or as a slowly-exchanging mix of conformers, in a ratio of approximately 4.6:1. $\mathrm{C}_{42} \mathrm{H}_{58} \mathrm{~N}_{8} \mathrm{O}_{18} \cdot 2.5\left(\mathrm{H}_{2} \mathrm{O}\right)$ : C: 50.05 (50.19); H: 6.30 (6.15); N: 11.12 (11.06). ${ }^{1} \mathrm{H}$ NMR (DMSO-d $)_{6}$ ): $\delta 3.17-4.70\left(\mathrm{~m}, \mathrm{CH}_{2}+\mathrm{CH}_{3}+\mathrm{H}_{2} \mathrm{O}, 49 \mathrm{H}\right), \delta 4.68$ (quartet, $\mathrm{CH}, \mathrm{J}=7.2$ $\mathrm{Hz}, 2 \mathrm{H}), \delta 6.52(\mathrm{~d}, \mathrm{HOPO} H, \mathrm{~J}=7.2 \mathrm{~Hz}, 2 \mathrm{H}), \delta 7.18(\mathrm{~d}, \mathrm{HOPO} H, \mathrm{~J}=7.2 \mathrm{~Hz}, 2 \mathrm{H}), \delta$ $7.43(\mathrm{~s}, \mathrm{TAM} H, 2 \mathrm{H}), \delta 8.26(\mathrm{t}, \mathrm{NH}, \mathrm{J}=5.6 \mathrm{~Hz}, 2 \mathrm{H}), \delta 8.61(\mathrm{t}, \mathrm{NH}, \mathrm{J}=5.6 \mathrm{~Hz}, 1.7 \mathrm{H}), \delta$ $8.71(\mathrm{t}, \mathrm{NH}, \mathrm{J}=5.6 \mathrm{~Hz}, 0.3 \mathrm{H}), \delta 9.08(\mathrm{~d}, \mathrm{NH}, \mathrm{J}=7.2 \mathrm{~Hz}, 1.7 \mathrm{H}), \delta 9.22(\mathrm{~d}, \mathrm{NH}, \mathrm{J}=7.2$ $\mathrm{Hz}, 0.3 \mathrm{H}), \delta 12.07$ (s, OH, $1.4 \mathrm{H}) .{ }^{13} \mathrm{C}$ NMR (DMSO-d $\left.\mathrm{d}_{6}\right): \delta 36.86,38.56,38.73,40.75$, 53.16, 58.04, 66.66, 68.79, 69.57, 69.59, 69.67, 71.25, 102.98, 117.02, 117.18, 118.02, 127.77, 147.15, 149.03, 158.12, 165.41, 167.92, 169.13. MS (FAB+): $963.7(\mathrm{MH}+)$. MP: $135-138^{\circ} \mathrm{C}$.

PEG-TAM(o-phen-1,2-HOPO) $)_{2}$, 3-14. Workup of the residue from acid deprotection proceeded in the same manner as for 3-12. Brown solid isolated as the monohydrate, 93\%. $\mathrm{C}_{48} \mathrm{H}_{56} \mathrm{~N}_{6} \mathrm{O}_{20} \cdot \mathrm{H}_{2} \mathrm{O}$ : C: 54.65 (54.42); $\mathrm{H}: 5.54$ (5.56); N: 7.97 (7.89). ${ }^{1} \mathrm{H}$ NMR (DMSO- $\left.d_{6}\right): \delta 3.42-3.45\left(\mathrm{~m}, \mathrm{CH}_{2}, 4 \mathrm{H}\right), \delta 3.52-3.56\left(\mathrm{~m}, \mathrm{CH}_{2}, 8 \mathrm{H}\right), \delta 3.60-3.62$ 
(m, $\left.\mathrm{CH}_{2}, 4 \mathrm{H}\right), \delta 3.77-3.79\left(\mathrm{~m}, \mathrm{CH}_{2}+\mathrm{CH}_{3}, 10 \mathrm{H}\right), \delta 4.08$ (t, br, $\left.\mathrm{CH}_{2}, \mathrm{~J}=4.8 \mathrm{~Hz}, 4 \mathrm{H}\right), \delta$ $6.59(\mathrm{dd}, \mathrm{HOPO} H, \mathrm{~J}=6.8,1.2 \mathrm{~Hz}, 2 \mathrm{H}), \delta 6.60(\mathrm{dd}, \mathrm{HOPO} H, \mathrm{~J}=9.2,1.2 \mathrm{~Hz}, 2 \mathrm{H}), \delta$ 7.30 (s, arom. $H, 2 \mathrm{H}), \delta 7.32$ (s, arom. $H, 2 \mathrm{H}), \delta 7.45(\mathrm{dd}, \mathrm{HOPO} H, \mathrm{~J}=9.4,6.8 \mathrm{~Hz}, 2 \mathrm{H})$, $\delta 7.54$ (s, TAM $H, 2 \mathrm{H}), \delta 10.16(\mathrm{~s}, \mathrm{NH}, 2 \mathrm{H}), \delta 10.59$ (s, NH, $2 \mathrm{H}) .{ }^{13} \mathrm{C}$ NMR: $\delta 55.85$, $58.08,68.31,68.86,69.64,69.83,70.01,71.31,105.19,110.04,117.41,118.88,119.95$ $123.32,123.65,137.01,141.49,145.64,146.72,149.05,157.52,158.92,166.79 . \mathrm{MS}$ $(\mathrm{FAB}+): \mathrm{m} / \mathrm{z} 1037.9(\mathrm{MH}+)$. MP: $250-252{ }^{\circ} \mathrm{C}(\mathrm{dec})$.

PEG-TAM(o-phen-Me-3,2-HOPO) $)_{2}$, 3-15. Workup of the residue from acid deprotection proceeded in the same manner as for 3-12. Brown solid isolated as the dihydrate, $96 \%$. $\mathrm{C}_{50} \mathrm{H}_{60} \mathrm{~N}_{6} \mathrm{O}_{20} \cdot \mathrm{H}_{2} \mathrm{O}$ : C: 54.54 (54.89); $\mathrm{H}: 5.86$ (5.85); N: 7.63 (7.66). ${ }^{1} \mathrm{H}$ NMR (DMSO-d $\left.d_{6}\right): \delta 3.23\left(\mathrm{~s}, \mathrm{CH}_{3}, 6 \mathrm{H}\right), \delta 3.42-3.61\left(\mathrm{~m}, \mathrm{CH}_{2}, 20 \mathrm{H}\right), \delta 3.79\left(\mathrm{~s}, \mathrm{br}, \mathrm{CH}_{2}+\right.$ $\left.\mathrm{CH}_{3}, 10 \mathrm{H}\right), \delta 4.10\left(\mathrm{~s}, \mathrm{br}, \mathrm{CH}_{2}, 4 \mathrm{H}\right), \delta 6.62(\mathrm{~d}, \mathrm{HOPO} H, \mathrm{~J}=7.2 \mathrm{~Hz}, 2 \mathrm{H}), \delta$ 7.19-7.24 (m, HOPO $H+$ arom. $H, 4 \mathrm{H}), \delta 7.58(\mathrm{~d}, \mathrm{TAM} H+\operatorname{arom} . H, 4 \mathrm{H}), \delta 10.10(\mathrm{~s}, \mathrm{NH}, 2 \mathrm{H}), \delta$ 10.39 (s, NH, 2H), $\delta 12.10$ (s, br, OH, 2H). ${ }^{13} \mathrm{C}$ NMR: $\delta 36.99,55.86,58.09,68.24$, $68.90,69.66,69.85,70.02,71.32,103.41,109.64,110.34,117.36,118.21,118.37$, $122.17,125.46,128.04,145.95,146.22,146.60,149.53,158.27,162.96,167.42 . \mathrm{MS}$ (FAB+): m/z $1065.9(\mathrm{MH}+)$. MP: $207-209^{\circ} \mathrm{C}(\mathrm{dec})$.

Pr-TAM-2Li-Me-3,2-HOPO, 3-22. White solid isolated as the hemihydrate, 88\%. $\mathrm{C}_{20} \mathrm{H}_{24} \mathrm{~N}_{4} \mathrm{O}_{7} \cdot 1 / 2 \mathrm{H}_{2} \mathrm{O}$ : C: 54.42 (54.49); $\mathrm{H}: 5.71$ (5.87); N: 12.69 (12.71). ${ }^{1} \mathrm{H}$ NMR (DMSO-d ${ }_{6}$ ): $\delta 0.89$ (t, $\mathrm{CH}_{3}, \mathrm{~J}=7.2 \mathrm{~Hz}, 3 \mathrm{H}$ ), $\delta 1.55$ (sextet, $\mathrm{CH}_{2}, \mathrm{~J}=7.2 \mathrm{~Hz}, 2 \mathrm{H}$ ), $\delta 3.26$ (quartet, $\left.\mathrm{CH}_{2}, \mathrm{~J}=6.4 \mathrm{~Hz}, 2 \mathrm{H}\right), \delta 3.46-3.49\left(\mathrm{~m}, \mathrm{CH}_{2}+\mathrm{CH}_{3}, 7 \mathrm{H}\right), \delta 6.50(\mathrm{~d}, \mathrm{HOPO} H, \mathrm{~J}=$ $7.2 \mathrm{~Hz}, 1 \mathrm{H}), \delta 7.19$ (d, HOPO $H, \mathrm{~J}=7.2 \mathrm{~Hz}, 1 \mathrm{H}), \delta 7.28-7.35$ (m, TAM H, 2H), $\delta 8.46$ (s, br, NH, 1H), $\delta 8.88(\mathrm{t}, \mathrm{NH}, \mathrm{J}=5.6 \mathrm{~Hz}, 1 \mathrm{H}), \delta 8.98(\mathrm{~s}, \mathrm{br}, \mathrm{NH}, 1 \mathrm{H}), \delta 12.57(\mathrm{~s}, \mathrm{OH}$, 
$1 \mathrm{H}), \delta 12.85(\mathrm{~s}, \mathrm{OH}, 1 \mathrm{H}) .{ }^{13} \mathrm{C}$ NMR (DMSO- $\left.d_{6}\right): \delta 11.38,22.05,36.81,38.56,38.69$, $40.78,102.49,115.65,115.75,117.00,117.19,117.28,127.69,147.82,150.07,150.22$, 158.01, 165.99, 168.64, 168.95. MS (FAB+): m/z $622.4(\mathrm{MH}+), 644.4(\mathrm{MNa}+) . \mathrm{MP}:$ $234-236{ }^{\circ} \mathrm{C}(\mathrm{dec})$.

PEG-Pr-TAM-2Li-Me-3,2-HOPO, 3-23. Workup of the residue from acid deprotection proceeded in the same manner as for 3-12. Beige semi-solid isolated as the sesquihydrate, $154 \mathrm{mg}(99 \%) . \mathrm{C}_{28} \mathrm{H}_{39} \mathrm{~N}_{5} \mathrm{O}_{11} \cdot 1.5\left(\mathrm{H}_{2} \mathrm{O}\right)$ : C: 51.85 (51.60); $\mathrm{H}: 6.53$ (6.23); $\mathrm{N}: 10.80$ (10.51). ${ }^{1} \mathrm{H}$ NMR: $\delta 0.88\left(\mathrm{t}, \mathrm{CH}_{3}, \mathrm{~J}=6.8 \mathrm{~Hz}, 3 \mathrm{H}\right), \delta 1.57\left(\mathrm{~d}, \mathrm{br}, \mathrm{CH}_{2}, \mathrm{~J}=6.4 \mathrm{~Hz}\right.$, $2 \mathrm{H}), \delta 3.28-3.63\left(\mathrm{~m}, \mathrm{CH}_{2}+\mathrm{CH}_{3}, 18 \mathrm{H}\right), \delta 3.94\left(\mathrm{~s}, \mathrm{br}, \mathrm{CH}_{2}, 2 \mathrm{H}\right), \delta 4.83(\mathrm{~s}, \mathrm{br}, 1 \mathrm{H}), \delta 6.64-$ $6.73(\mathrm{~m}, \mathrm{br}, \mathrm{HOPO} H+\mathrm{NH}, 2 \mathrm{H}), \delta$ 7.10-7.17 (m, br, HOPO $H+\mathrm{NH}, 2 \mathrm{H}), \delta$ 7.72-7.89 (m, br, TAM H, 2H), $\delta$ 8.61-8.71 (m, br, NH, 2H), $\delta 11.58$ (s, br, OH, $1 \mathrm{H}) .{ }^{13} \mathrm{C}$ NMR: $\delta$ $11.59,22.68,37.77,39.59,41.68,54.79,58.97,69.49,70.17,70.31,70.45,71.69,71.83$, $77.43,104.67,116.07,116.26,116.68,117.02,117.63,127.21,147.88,150.06,158.71$, 167.12, 168.87, 169.43, 170.19. $\mathrm{MS}(\mathrm{FAB}+): 622.4(\mathrm{MH}+), 644.4(\mathrm{MNa}+)$. MP: $75-78^{\circ} \mathrm{C}$.

\subsubsection{Synthesis/Crystallization Techniques for Uranyl Complexes}

$\mathbf{U O}_{2}\left(\boldsymbol{o}\right.$-phen-1,2-HOPO), $\mathbf{U O}_{2}(\mathbf{1 2 H P})$. A solution of $o$-phen-1,2-HOPO ${ }^{12}(31 \mathrm{mg}$, $0.081 \mathrm{mmol}$ ) and 3 drops of pyridine were dissolved in $15 \mathrm{~mL}$ of $\mathrm{MeOH}$, and a solution of $\mathrm{UO}_{2}\left(\mathrm{NO}_{3}\right)_{2} \cdot 6 \mathrm{H}_{2} \mathrm{O}(37 \mathrm{mg}, 0.074 \mathrm{mmol})$ in $2 \mathrm{~mL}$ of $\mathrm{MeOH}$ was added. The mixture was stirred at reflux overnight. After cooling to room temperature, the precipitate was cooled, washed with $\mathrm{MeOH}$, and dried under vacuum to yield $40 \mathrm{mg}$ of an orange solid, 83\%. $\mathrm{C}_{18} \mathrm{H}_{12} \mathrm{~N}_{4} \mathrm{O}_{8} \mathrm{U}:$ C: 33.24 (33.19); H: 1.86 (1.90); N: 8.62 (8.47). ${ }^{1} \mathrm{H}$ NMR (DMSO$\left.d_{6}\right): \delta 7.33(\mathrm{dd}, \mathrm{HOPO} H, \mathrm{~J}=3.6,6.4 \mathrm{~Hz}, 2 \mathrm{H}), \delta 7.38(\mathrm{dd}$, arom. $H, 1.6,8.4 \mathrm{~Hz}, 2 \mathrm{H}), \delta$ 7.73, (dd, arom. $H, \mathrm{~J}=1.6,7.6 \mathrm{~Hz}, 2 \mathrm{H}), \delta 7.96(\mathrm{t}, \mathrm{HOPO} H, \mathrm{~J}=8.0 \mathrm{~Hz}, 2 \mathrm{H}), \delta 8.48$ (dd, 
HOPO $H, \mathrm{~J}=3.6,6.4 \mathrm{~Hz}, 2 \mathrm{H}), \delta 12.51(\mathrm{~s}, \mathrm{NH}, 2 \mathrm{H}) .{ }^{13} \mathrm{C}$ NMR (DMSO- $\left.d_{6}\right): \delta 115.46$, $118.74,123.48,125.15,128.45,136.75,139.13,157.66,163.63 . \mathrm{MS}(\mathrm{FAB}+): \mathrm{m} / \mathrm{z} 651$ $(\mathrm{MH}+)$.

$\mathrm{UO}_{2}\left[\mathrm{TAM}(2 \mathrm{Li}-\mathrm{Me}-3,2-\mathrm{HOPO})_{2}\right] \cdot 2\left(\mathrm{NMe}_{4}\right), \mathrm{UO}_{2}(3-2) \cdot 2\left(\mathrm{NMe}_{4}\right)$. A solution of 32. MeOH (101 mg, $0.164 \mathrm{mmol})$ and $\mathrm{NMe}_{4} \mathrm{OH} \cdot 5 \mathrm{H}_{2} \mathrm{O}(120 \mathrm{mg}, 0.662 \mathrm{mmol})$ in $5 \mathrm{~mL}$ of $\mathrm{MeOH}$ was added to a stirred solution of $82.4 \mathrm{mg}(0.164 \mathrm{mmol})$ of $\mathrm{UO}_{2}\left(\mathrm{NO}_{3}\right)_{2} \cdot 6 \mathrm{H}_{2} \mathrm{O}$ in 2 $\mathrm{mL} \mathrm{MeOH}$. The dark red solution was heated at reflux overnight and the volume was reduced to $3 \mathrm{~mL}$. Insoluble material was removed by filtering through glass wool and the solution was layered with acetone to yield $106 \mathrm{mg}$ of dark red crystals which were filtered and dried by aspiration. These crystals were used for NMR and X-ray crystallographic analysis and were shown to be $\mathrm{UO}_{2}(3-2) \cdot 2 \mathrm{NMe}_{4} \cdot \mathrm{Me}_{2} \mathrm{CO}, 58 \%$. $\mathrm{C}_{26} \mathrm{H}_{24} \mathrm{~N}_{6} \mathrm{O}_{12} \mathrm{U} \cdot 2 \mathrm{~N}\left(\mathrm{CH}_{3}\right)_{4} \cdot 2 \mathrm{C}_{3} \mathrm{H}_{6} \mathrm{O}: \mathrm{C}: 43.09$ (42.87); H: 5.42 (5.33); N: 10.05 (10.00). ${ }^{1} \mathrm{H}$ NMR (DMSO-d $\left.d_{6}\right): \delta 2.08\left(\mathrm{~s}, \mathrm{CH}_{3}, 6 \mathrm{H}\right), \delta 3.05\left(\mathrm{~s}, \mathrm{CH}_{3}, 24 \mathrm{H}\right), \delta 3.36\left(\mathrm{~s}, \mathrm{OH}_{2}, 8 \mathrm{H}\right), \delta 3.59-$ $3.63\left(\mathrm{~m}, \mathrm{CH}_{2}, 8 \mathrm{H}\right), \delta 3.73\left(\mathrm{~s}, \mathrm{CH}_{3}, 6 \mathrm{H}\right), \delta 6.81(\mathrm{~d}, \mathrm{HOPO} H, \mathrm{~J}=7.2 \mathrm{~Hz}, 2 \mathrm{H}), \delta 6.90(\mathrm{~d}$, HOPO $H, \mathrm{~J}=7.2 \mathrm{~Hz}, 2 \mathrm{H}), \delta 6.92(\mathrm{~s}, \mathrm{TAM} H, 2 \mathrm{H}), \delta 11.55(\mathrm{t}, \mathrm{NH}, \mathrm{J}=5.2 \mathrm{~Hz}, 2 \mathrm{H}), \delta$ $11.75(\mathrm{t}, \mathrm{NH}, \mathrm{J}=5.2 \mathrm{~Hz}, 2 \mathrm{H}) .{ }^{13} \mathrm{C}$ NMR (DMSO-d $\left.d_{6}\right): \delta 30.74,36.46,38.79,54.30,54.34$, $54.38,107.11,113.34,116.01,117.30,120.84,161.80,165.51,166.90,167.04,167.49$.

$\mathrm{UO}_{2}\left[\mathrm{TAM}(o-p h e n-1,2-\mathrm{HOPO})_{2}\right] \cdot 2 \mathrm{NMe}_{4}, \mathrm{UO}_{2}(3-4) \cdot 2 \mathrm{NMe}_{4} \cdot$ A solution of 3$4 \cdot 1 / 2 \mathbf{H}_{2} \mathbf{O} \cdot 1 / 2 \mathbf{C H}_{3} \mathrm{OH}(50 \mathrm{mg}, 0.074 \mathrm{mmol})$ and $\mathrm{NMe}_{4}(\mathrm{OH}) \cdot 5 \mathrm{H}_{2} \mathrm{O}(54 \mathrm{mg}, 0.298 \mathrm{mmol})$ in $4 \mathrm{~mL}$ of $\mathrm{MeOH}$ was added to a stirred solution of $\mathrm{UO}_{2}\left(\mathrm{NO}_{3}\right)_{2} \cdot 6 \mathrm{H}_{2} \mathrm{O}(37 \mathrm{mg}, 0.074 \mathrm{mmol})$ in $1 \mathrm{~mL}$ of $\mathrm{MeOH}$. The deep red solution was heated at reflux overnight, cooled to room temperature, and the solvent was removed under vacuum. The residue was dissolved in $0.5 \mathrm{~mL}$ of DMSO, and THF was diffused into the solution at room temperature. Initial 
precipitates were generally colorless, so the crystallization solution was filtered every week to remove what was suspected to be $\mathrm{NMe}_{4} \mathrm{NO}_{3}$. Once dark material began to precipitate, the solution was filtered one last time and allowed to continue to diffuse at 4 ${ }^{\circ} \mathrm{C}$. The crop of dark crystals and amorphous material were filtered, washed with THF and allowed to dry by aspiration for 2 days, yielding $37 \mathrm{mg}$ of crystalline and amorphous, dark solid that elemental and NMR analyses indicated was $\mathrm{UO}_{2}(3-$ 4) $\cdot 2 \mathrm{NMe}_{4} \cdot \mathrm{DMSO} \cdot \mathrm{H}_{2} \mathrm{O} \cdot 1 / 5 \mathrm{THF} \cdot 1 / 3 \mathrm{NMe}_{4} \mathrm{NO}_{3}$. $\mathrm{C}_{32} \mathrm{H}_{20} \mathrm{~N}_{4} \mathrm{O}_{12} \cdot 2 \mathrm{~N}\left(\mathrm{CH}_{3}\right)_{4} \cdot \mathrm{C}_{2} \mathrm{H}_{6} \mathrm{OS} \cdot \mathrm{H}_{2} \mathrm{O} \cdot 1 / 5 \mathrm{C}_{4} \mathrm{H}_{8} \mathrm{O} \cdot 1 / 3 \mathrm{~N}\left(\mathrm{CH}_{3}\right)_{4} \mathrm{NO}_{3}: \mathrm{C}: 43.35$ (43.49); H: 4.75 (4.45); N: 9.93 (9.59); S: 2.62 (2.67). ${ }^{1} \mathrm{H}$ NMR (DMSO-d 6 ): $\delta$ 1.74-1.77 (m, THF $H$, $0.9 \mathrm{H}), \delta 2.54$ (s, DMSO CH $3,6 \mathrm{H}), \delta 3.04\left(\mathrm{~s}, \mathrm{CH}_{3}, 29 \mathrm{H}\right), \delta 3.58-3.61$ (m, THF $\left.H, 0.9 \mathrm{H}\right), \delta$ 7.11-7.22 (m, TAM + HOPO + arom. $H, 8 \mathrm{H}), \delta 7.55(\mathrm{~d}, \mathrm{HOPO} H, \mathrm{~J}=6.8 \mathrm{~Hz}, 2 \mathrm{H}), \delta$ $7.72(\mathrm{t}, \mathrm{HOPO} H, \mathrm{~J}=8.0 \mathrm{~Hz}, 2 \mathrm{H}), \delta 8.43$ (d, arom. $H, \mathrm{~J}=8.4 \mathrm{~Hz}, 2 \mathrm{H}), \delta 8.67$ (d, arom. $H, \mathrm{~J}=8.4 \mathrm{~Hz}, 2 \mathrm{H}), \delta 13.05(\mathrm{~s}, \mathrm{NH}, 2 \mathrm{H}), \delta 14.16(\mathrm{~s}, \mathrm{NH}, 2 \mathrm{H}) .{ }^{13} \mathrm{C}$ NMR (DMSO-d $\left.\mathrm{d}_{6}\right): \delta$ $25.13,40.42,54.30,54.34,54.38,67.03,111.60,114.33,117.95,122.01,122.36,122.51$, $124.46,126.85,130.69,133.45,138.10,158.18,162.78,166.49,166.57$. (MS (ESI-): $459.1\left(\mathrm{M}^{2-}\right)$. X-ray quality crystals could also be grown by layering a similarly-prepared crude DMSO solution of the complex and accompanying salts with dioxane. After diffusion at room temperature, these aliquots yielded three X-ray quality crystals reported above. These other crystals were grown from crude materials and the mixtures of precipitates from which they were isolated were not suitable for NMR or elemental analysis measurements.

$\mathrm{UO}_{2}\left[\mathrm{TAM}\left(o-p h e n-M e-3,2-\mathrm{HOPO}_{2}\right] \cdot 2 \mathrm{NMe}_{4}, \mathrm{UO}_{2}(3-5) \cdot 2 \mathrm{NMe}_{4}\right.$. A solution of 35.1/2 $\mathrm{H}_{2} \mathrm{O} \cdot 1 / 2 \mathrm{HCl}(100 \mathrm{mg}, 0.141 \mathrm{mmol})$ and $\mathrm{NMe}_{4}(\mathrm{OH}) \cdot 5 \mathrm{H}_{2} \mathrm{O}(106 \mathrm{mg}, 0.585 \mathrm{mmol})$ in 10 
$\mathrm{mL}$ of $\mathrm{MeOH}$ was added to a stirred solution of $\mathrm{UO}_{2}\left(\mathrm{NO}_{3}\right)_{2} \cdot 6 \mathrm{H}_{2} \mathrm{O}(73.6 \mathrm{mg}, 0.147 \mathrm{mmol})$ in $5 \mathrm{~mL}$ of $\mathrm{MeOH}$. The resultant red suspension was stirred at reflux overnight, then cooled to room temperature and filtered. The solid was dried under vacuum, yielding 132 mg of brown powder isolated as the methanolic hydrate, $78 \%$. $\mathrm{C}_{34} \mathrm{H}_{24} \mathrm{~N}_{6} \mathrm{O}_{12} \mathrm{U} \cdot 2\left[\mathrm{~N}\left(\mathrm{CH}_{3}\right)_{4}\right] \cdot \mathrm{CH}_{3} \mathrm{OH} \cdot \mathrm{H}_{2} \mathrm{O}: \mathrm{C}: 44.41$ (44.40); H: 4.85 (4.56); N: 9.64 (9.44). Crystals of this complex were formed by diffusion of $\mathrm{MeOH}$ into a DMSO complex solution. X-ray crystallography revealed the crystals to be of the composition $\mathrm{UO}_{2}\left[\mathrm{TAM}(3-5)_{2}\right] \cdot 2 \mathrm{NMe}_{4} \cdot 2 \mathrm{MeOH}$. NMR analysis was performed on these crystals. ${ }^{1} \mathrm{H}$ NMR (DMSO-d $\left.)_{6}\right): \delta 3.02\left(\mathrm{~s}, \mathrm{CH}_{3}, 24 \mathrm{H}\right), \delta 3.18\left(\mathrm{~d}, \mathrm{CH}_{3} \mathrm{OH}, \mathrm{J}=4.8 \mathrm{~Hz}, 6 \mathrm{H}\right), \delta 3.77$ (s, $\mathrm{CH}_{3}, 6 \mathrm{H}$ ), $\delta 4.10$ (quartet, $\mathrm{CH}_{3} \mathrm{OH}, \mathrm{J}=4.8 \mathrm{~Hz}, 2 \mathrm{H}$ ), $\delta 6.97$ (quartet, arom. $H, \mathrm{~J}=7.2 \mathrm{~Hz}$, 4H), $\delta$ 7.07-7.18 (m, arom. + TAM H, 6H), $\delta 12.89(\mathrm{~s}, \mathrm{NH}, 2 \mathrm{H}), \delta 13.24(\mathrm{~s}, \mathrm{NH}, 2 \mathrm{H}) .{ }^{13} \mathrm{C}$ NMR (DMSO-d $\left.)_{6}\right): \delta 36.65,48.61,54.28,54.32,54.36,106.94,114.13,115.98,117.43$, $121.54,121.72,122.04,122.94,123.43,127.84,130.52,161.29,164.31,166.38,166.59$. MS (ESI-): m/z $473.1\left(\mathrm{M}^{2-}\right)$.

\subsubsection{X-ray Diffraction Data Collection}

General collection strategies for uranyl complex crystals and their general crystallographic data refinement are described in detail in Chapter 2. Details on the crystallographic refinement the crystal structures are provided in the Appendix.

\subsubsection{Titrations}

Titration Solutions and Equipment. Solutions and equipment used for solution titration experiments are the same as used for bis-Me-3,2-HOPO ligands and are explained in detail in Chapter 2. Additionally, UV-visible spectra for batch titrations were recorded on a Cary 300 Scan UV-Vis spectrophotometer. 
Spectrophotometric Titration Methods: Spectrophotometric titrations with incremental addition of acid or base were run in the same manner as those with the bisMe-3,2-HOPO complexes in Chapter 2. The exception to this is that batch titrations were allowed to equilibrate for three days with constant agitation.

Ligand concentrations for spectrophotometric titrations with a $6.6 \mathrm{~cm}$ path length cell and incremental addition of titrant were approximately 2-6 $\mu \mathrm{M}$. Ligand concentrations for batch titrations using a $10 \mathrm{~cm}$ path length cell were 1.3-2 $\mu \mathrm{M}$. Uranyl titrations were conducted with a 1:1 ligand:metal ratio to avoid decomposition of TAM-containing ligands at high $\mathrm{pH}$. All titrations were repeated a minimum of three times. Each titration involving incremental addition of titrant was run forwards and backwards (from acid to base and reverse) when the titrations were deemed reversible. Titrations with $\mathrm{TAM}(\mathrm{HOPO})_{2}$ ligands were only performed down to $\mathrm{pH} 2.4$, while those with tetradentate TAM-2Li-Me-3,2-HOPO ligands were performed down to $\mathrm{pH} 1.6$ by performing two strong acid titrations between $\mathrm{pH} 3.0$ and 1.6. Data from these titrations were combined with those from higher $\mathrm{pH}$ titrations to yield the final values.

Titration Data Treatment: Titration data were analyzed using methods described in Chapter 2. Wavelengths between $250-400 \mathrm{~nm}$ were typically used for data refinement, although batch titration data was often truncated to $c a .270-400 \mathrm{~nm}$ due to large errors in the data at the lower wavelengths that typically had much stronger absorbance that the rest of the spectrum. 


\subsection{References}

(1) Alexander, V. Chem. Rev. 1995, 95, 273-342.

(2) Sessler, J. L.; Seidel, D.; Vivian, A. E.; Lynch, V.; Scott, B. L.; Keogh, D. W. Angew. Chem. Int. Ed. 2001, 40, 591-594.

(3) Casellato, U.; Vigato, P. A.; Tamburini, S.; Graziani, R.; Vidali, M. Inorg. Chim. Acta. 1983, 72, 141-147.

(4) Xu, J.; Raymond, K. N. Inorg. Chem. 1999, 38, 308-315.

(5) Gorden, A. E. V.; Xu, J.; Raymond, K. N. Chem. Rev. 2003, 103, 4207-4282.

(6) Akine, S.; Taniguchi, T.; Nabeshima, T. Tet. Lett. 2001, 42, 8861-8864.

(7) Akine, S.; Taniguchi, T.; Nabeshima, T. J. Am. Chem. Soc. 2006, 128, $15765-$ 15774.

(8) Akine, S.; Sunaga, S.; Taniguchi, T.; Miyazaki, H.; Nabeshima, T. Inorg. Chem. 2007, 46, 2959-2961.

(9) Jurchen, K. M. C.; Raymond, K. N. Inorg. Chem. 2006, 45, 2006.

(10) Garrett, T. M.; Cass, M. E.; Raymond, K. N. J. Coord. Chem. 1992, 25, 241-253.

(11) Jurchen, K. M. C.; Raymond, K. N. J. Coord. Chem. 2005, 58, 55-80.

(12) D'Aleo, A.; Xu, J.; Moore, E. G.; Jocher, C. J.; Raymond, K. N. Inorg. Chem. 2008, 47, 6109-6111.

(13) Gramer, C. J.; Raymond, K. N. Inorg. Chem. 2004, 43, 6397-6402.

(14) Sofen, S. R.; Abu-Dari, K.; Freyberg, D. P.; Raymond, K. N. J. Am. Chem. Soc. 1978, 100, 7882-7887.

(15) Scarrow, R. C.; Riley, P. E.; Abu-Dari, K.; White, D. L.; Raymond, K. N. Inorg. Chem. 1985, 24, 954-967. 
(16) Riley, P. E.; Abu-Dari, K.; Raymond, K. N. Inorg. Chem. 1983, 22, 3940-3944.

(17) Jiang, J.; Li, W.-R.; Joullié, M. M. Synth. Comm. 1994, 24, 187-195.

(18) Toke, L.; Bitter, I.; Agai, B.; Hell, Z.; Lindner, E.; Toth, K.; Horvath, M.; Harfouch, S.; Pungor, E. Liebigs Annalen der Chemie 1988, 8, 549-554.

(19) Toke, L.; Bitter, I.; Agai, B.; Hell, Z.; Lindner, E.; Toth, K.; Horvath, M.; Szollosy, A.; Harfouch, S.; Pungor, E. Liebigs Annalen der Chemie 1988, 8, 7613.

(20) Nguyen, A. I.; Blackmore, K. J.; Carter, S. M.; Zarkesh, R. A.; Heyduk, A. F. J. Am. Chem. Soc. 2009, 131, 3307-3316.

(21) Durbin, P. W.; Kullgren, B.; Ebbe, S. N.; Xu, J.; Raymond, K. N. Health Phys. 2000, 78, 511-521.

(22) Karpishin, T. B.; Stack, T. D. P.; Raymond, K. N. J. Am. Chem. Soc. 1993, 115, 182-192.

(23) Xu, J.; Durbin, P. W.; Kullgren, B.; Raymond, K. N. J. Med. Chem. 1995, 38, 2606-2614.

(24) Szabó, Z.; Grenthe, I. Inorg. Chem. 2000, 39, 5036-5043.

(25) Xu, J.; Durbin, P. W.; Kullgren, B.; Shirley, N. E.; Uhlir, L. C.; Raymond, K. N. J. Med. Chem. 2002, 45, 3963-3971.

(26) Doble, D. M. J.; Melchior, M.; O'Sullivan, B.; Siering, C.; Xu, J.; Pierre, V. C.; Raymond, K. N. Inorg. Chem. 2003, 42, 4930-4937.

(27) Pierre, V. C.; Botta, M.; Aime, S.; Raymond, K. N. Inorg. Chem. 2006, 45, 83558364. 


\section{Chapter 4:}

\section{Pu(IV) Coordination Chemistry with HOPO and Hydroxypyrone Ligands ${ }^{1}$}

\subsection{Introduction}

While uranium is the major actinide constituent in nuclear waste, the various isotopes of plutonium compose up to $1 \%$ of irradiated fuel and are significantly more radioactive, yet still with relatively long half-lives $\left({ }^{235} \mathrm{Ut}_{1 / 2}=7.04 \times 10^{8} \mathrm{yr},{ }^{239} \mathrm{Pu} \mathrm{t}_{1 / 2}=2.41 \times 10^{5}\right.$ yr). ${ }^{2,3}$ Therefore, plutonium (which in biological and oxidizing media is typically found in its +4 oxidation state) is a meaningful target for selective chelation and one that has been the focus of Raymond group efforts for decades. ${ }^{4}$ The design of $\mathrm{Pu}(\mathrm{IV})$-specific chelators follows very different design strategies than that for U(VI) discussed in Chapters 2 and 3, because the $\mathrm{Pu}(\mathrm{IV})$ cation - like all other tri- and tetracationic lanthanide and actinide ions - is a spherical ion whose coordination geometry is typically described as primarily ionic in nature and geometrically fluxional, with coordination numbers typically ranging between 8 and 10 depending on the identity of the ligand. ${ }^{5}$

The similarity between the $\mathrm{Pu}(\mathrm{IV})$ and $\mathrm{Fe}(\mathrm{III})$ cations inspired a biomimetic approach for ligand design in the Raymond group that incorporates siderophore analog chelating moieties into polybidentate ligands of various geometries. ${ }^{6}$ Since the inception of this approach, a large library of poly-bidentate ligands has been developed ${ }^{4}$ and the efficacy of many of them for actinide removal in vivo has been investigated. ${ }^{7}$ These studies resulted in the development of the ligands 5LiO-Me-3,2-HOPO and 3,4,3-Li-1,2-HOPO that display good $\mathrm{Pu}(\mathrm{IV})$ decorporation properties as well as low toxicity (Figure 4-1). ${ }^{8,9}$ However, ambiguities as to whether tetrakis-bidentate ligands incorporating catecholate 
analog binding moieties behave as hexadenate or octadentate species towards $\mathrm{Pu}(\mathrm{IV})$ in vivo illustrate our persistent lack of detailed knowledge about $\mathrm{Pu}(\mathrm{IV})$ and its coordination compounds. ${ }^{6}$<smiles>Cn1ccc(C(=O)NCCOCCNC(=O)c2ccn(C)c(=O)c2O)c(O)c1=O</smiles>

5LiO-Me-3,2-HOPO

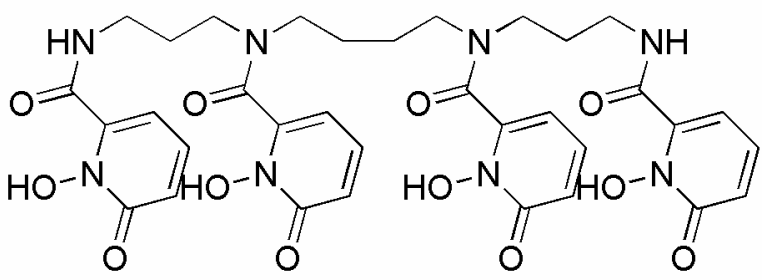

3,4,3-Li-1,2-HOPO

Figure 4-1. High-efficiency Raymond group ligands for $\mathrm{Pu}(\mathrm{IV})$ decorporation.

The structural chemistry of plutonium in its various oxidation states has been widely explored in inorganic solids, ${ }^{3}$ but to date only 45 plutonium coordination complex structures exists in the Cambridge Crystallographic Database compared to 2393 for uranium. Thus, expanding the known coordination chemistry of $\mathrm{Pu}(\mathrm{IV})$ will both increase the fundamental knowledge of how plutonium behaves in its coordination complexes and will assist in rational ligand design for spherical $f$-element ions. The current database of knowledge on $\mathrm{Pu}(\mathrm{IV})$ coordination chemistry encompasses a wide variety of coordination numbers and polyhedra depending on the ligand size and geometry. However, saturated $\mathrm{Pu}(\mathrm{IV})$ complexes with bidentate chelators have the tendency to be octacoordinate; this coordination number is relatively forgiving in terms of ligand bite angle and size, resulting in octacoordinate $\mathrm{PuL}_{4}$ complexes with malonate ${ }^{10}$ and acetylacetonate ${ }^{11}$ ligands. U(IV) and Th(IV) complexes with catechol are also octacoordinate ${ }^{12}$ and suggest that bidentate ligand geometries typical of Raymond group chelators prefer such geometries around spherical, mid-valent actinides. However, in ligands such as trisbidentate desferrioxamine $\mathrm{E}$ that do not provide coordinative saturation to the $\mathrm{Pu}(\mathrm{IV})$ 
cation, higher coordination numbers (nine in this case) can be achieved by coordination of multiple solvent molecules. ${ }^{13}$

Raymond group efforts towards characterizing $\mathrm{Pu}(\mathrm{IV})$ coordination chemistry and associated geometries have utilized catechol amides (CAM), terephthalamides (TAM), and hydroxypyridinones (HOPO), which are all structural analogs to catechol, with the HOPO moieties also acting as electronic analogs to hydroxamic acids. ${ }^{4}$ In 2005 Gorden et al. successfully characterized the neutral $\mathrm{Pu}(5 \mathrm{LiO}-\mathrm{Me}-3,2-\mathrm{HOPO})_{2}$ complex by single crystal X-ray diffraction which revealed the $\mathrm{Pu}(\mathrm{IV})$ to be octacoordinate with a square antiprismatic coordination geometry. ${ }^{14,15}$ The 5LiO-Me-3,2-HOPO ligands bind in a sandwich-type coordination mode, with the $\mathrm{Pu}(\mathrm{IV})$ ion sitting above or below an effective ligand plane of two HOPO moieties (Figure 4-2).
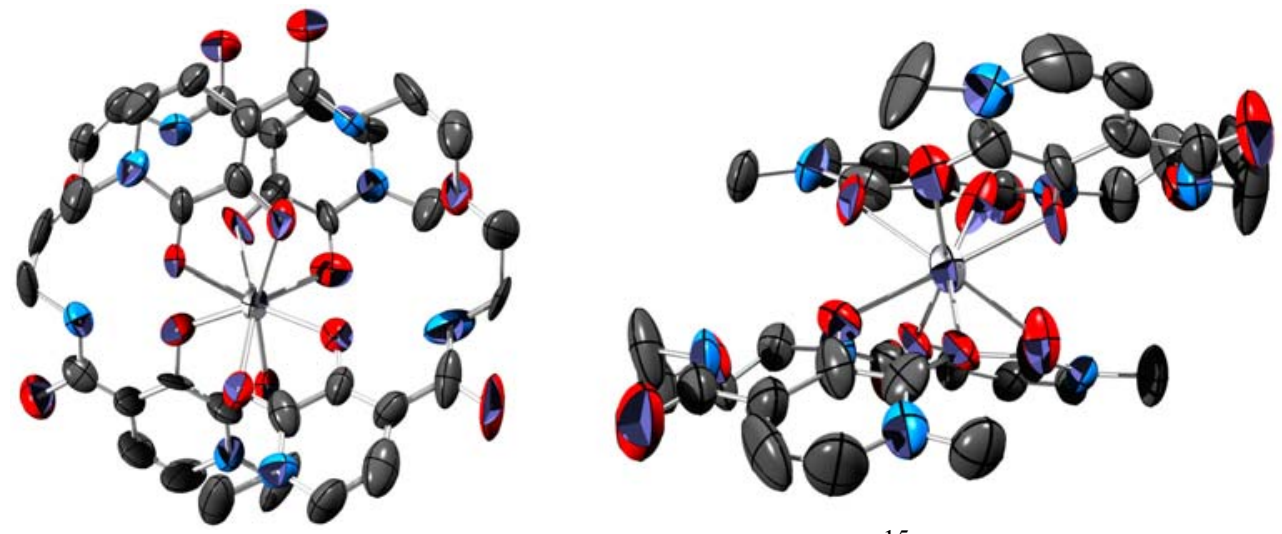

Figure 4-2. Crystal structure of $\mathrm{Pu}(5 \mathrm{LiO}-\mathrm{Me}-3,2-\mathrm{HOPO})_{2}{ }^{15}$

The crystallographic result above was an excellent beginning towards understanding the interaction of $\mathrm{Pu}(\mathrm{IV})$ with siderophore analogs, indicating that if properly designed, they could saturate the $\mathrm{Pu}(\mathrm{IV})$ coordination sphere and produce stable complexes. However, the crystal structure of $\mathrm{Pu}(5 \mathrm{LiO}-\mathrm{Me}-3,2-\mathrm{HOPO})_{2}$ does not necessarily illustrate an unconstrained coordination mode of $\mathrm{Pu}(\mathrm{IV})$, as the complex geometry is influenced by the 5LiO linker's length and the hydrogen bond interactions between the etheric linker 
oxygen and the two amide protons $\left[\mathrm{d}_{\mathrm{O}-\mathrm{N}}=2.90(2) \AA\right]$. Rational design principles would encourage the characterization of $\mathrm{Pu}(\mathrm{IV})$ complexes with untethered bidentate siderophore analogs as a method by which to elucidate the coordination preferences of $\mathrm{Pu}(\mathrm{IV})$ free from constraints imposed by ligand linkers. From such observations ligand development can focus on tailoring linkers that mimic this geometry in poly-bidentate scaffolds. This chapter describes the efforts towards characterizing $\mathrm{Pu}(\mathrm{IV})$ coordination preferences with simple bidentate chelators that are structural analogs of siderophore-type chelators, followed by the application of these design principles in a poly-bidentate ligand design.

\subsection{Results and Discussion}

$\mathrm{Ce}(\mathrm{IV})$ is a generally-accepted structural analog for $\mathrm{Pu}(\mathrm{IV})$ because it has an identical ionic radius and charge as $\mathrm{Pu}(\mathrm{IV})$. In addition, because the bonding interactions of the $f$ elements are typically considered to be governed purely by electrostatic effects, it is generally considered that the ionic charge and radius similarities make Ce(IV) a convenient and non-radioactive structural analog to $\mathrm{Pu}(\mathrm{IV})$. This assumption is supported by the very similar crystal structures of $\mathrm{Pu}(\mathrm{IV})$ and $\mathrm{Ce}(\mathrm{IV})$ complexes with 5LiO-Me-3,2HOPO. ${ }^{14,15} \mathrm{Ce}(\mathrm{IV})$ starting materials organic-soluble Ce(IV) starting materials are more easily accessible, making standard laboratory crystallization techniques more accessible. In contrast, crystallization attempts with $\mathrm{Pu}(\mathrm{IV})$ are relegated to acidic, aqueous media due to the rich redox chemistry available to $\mathrm{Pu}(\mathrm{IV})$ and the practically available chemical forms of plutonium in our laboratory. 


\subsubsection{The Pu(IV)-1,2-Hydroxypyridinone Complex}

Towards our goal of understanding the fundamental coordination chemistry of $\mathrm{Pu}(\mathrm{IV})$ with simple bidentate ligands, crystallization attempts were made using the unsubstituted, monoprotic N-hydroxy-2-pyridinone ligand (1,2-HOPO, 4-1, Figure 4-3). This ligand, like many others studied by the Raymond group, is a structural analog to the catechol binding moiety, an electronic analog to hydroxamic acids, and has been used in structural studies of with $\mathrm{Th}(\mathrm{IV})$ and $\mathrm{UO}_{2}{ }^{2+} \cdot{ }^{16,17}$ The use of 4-1 eliminates the influence of linkers or substituents on the geometry of any resultant coordination complex, providing an unhindered glimpse at preferred coordination geometries.

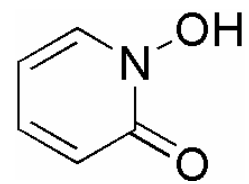

Figure 4-3. N-hydroxy-pyridin-2-one, 1,2-HOPO, 4-1.

Crystals of the $\mathrm{Pu}(\mathrm{IV})$ complex with 4-1 were formed by slow evaporation at room temperature of an acetate-buffered water/methanol solution with a 4.1:1 L:M ratio. Because of their small size and the significant radioactivity of plutonium, X-ray diffraction measurements on Pu-containing crystals were exclusively performed at the Advanced Light Source (ALS) at Lawrence Berkeley National Labs. The structure of the $\mathrm{Pu}(\mathrm{IV})-(4-1)$ complex is shown in Figure 4-4 and its crystallographic parameters are listed in Table 4-1.

Although the ligand to metal ratio in solution was in slight excess of $4: 1$, the crystal used in the data collection was that of a mixed salt that contained one tetrakis bidentate $\mathrm{Pu}(4-1)_{4}$ complex and one $\left[\mathrm{Pu}(4-1)_{3}\left(\mathrm{H}_{2} \mathrm{O}\right)_{2}\right]^{+}$cation whose charge is balanced by the inclusion of a perchlorate anion. The presence of the salt in the crystal structure was a surprise that suggests the solution was either not basic enough for full deprotonation of 4- 
1 or that the $\mathrm{Pu}(4-1)_{4}$ formation constant is lower than expected and requires a larger excess of ligand to favor its formation in solution.

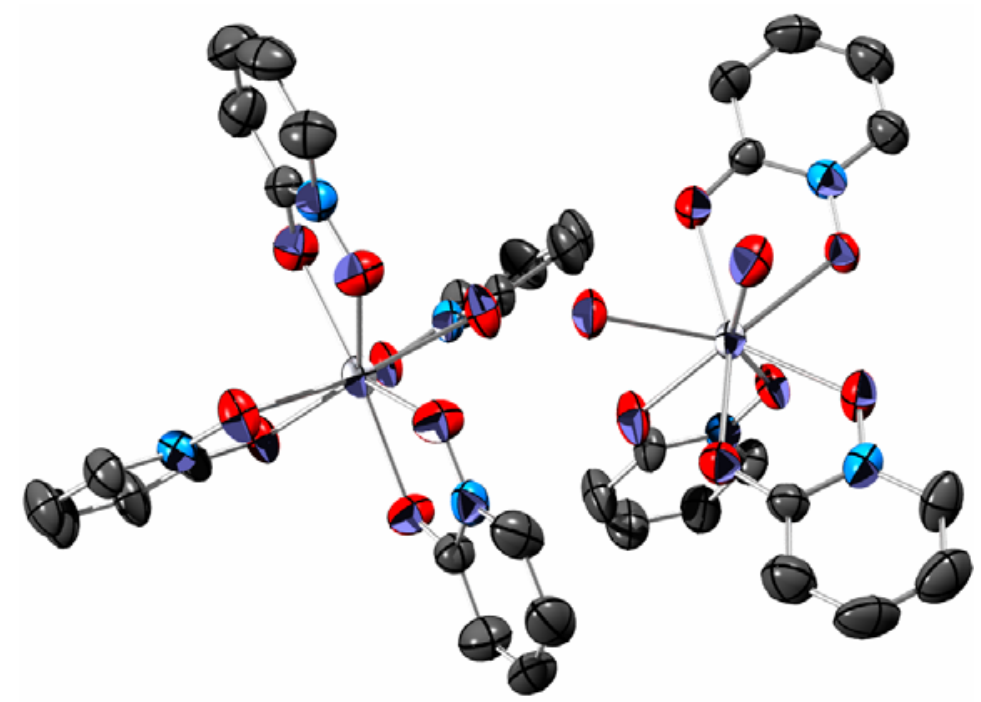

Figure 4-4. X-ray diffraction structure of the $\mathrm{Pu}(\mathrm{IV})-1,2-\mathrm{HOPO}$ mixed salt complex $\mathrm{Pu}(4-1)_{4} \cdot \mathrm{Pu}(4-1)_{3}\left(\mathrm{H}_{2} \mathrm{O}\right)_{2} \cdot \mathrm{ClO}_{4}$. Hydrogen atoms and the perchlorate counterion have been omitted for clarity. Thermal ellipsoids are drawn at the $50 \%$ level. Carbons are gray, oxygens red, and plutonium silver.

Table 4-1. Crystallographic parameters for the $\mathrm{Pu}(\mathrm{IV})-1,2-\mathrm{HOPO}$ structure.

\begin{tabular}{|c|c|c|c|}
\hline Formula & $\begin{array}{c}\mathrm{C}_{20} \mathrm{H}_{16} \mathrm{~N}_{4} \mathrm{O}_{8} \mathrm{Pu} \cdot \\
\mathrm{C}_{15} \mathrm{H}_{16} \mathrm{~N}_{3} \mathrm{O}_{8} \cdot \mathrm{ClO}_{4}\end{array}$ & $\begin{array}{c}\text { Data/ restr./ } \\
\text { param. }\end{array}$ & 7089 / 75 / 596 \\
\hline MW & 1390.13 & $\mathbf{T}[\mathrm{K}]$ & $173(2)$ \\
\hline Crystal system & Monoclinic & $\rho_{\text {calcd }}\left[\mathrm{g} \mathrm{cm}^{-3}\right]$ & 2.241 \\
\hline Space group & $\mathrm{P} 2_{1}$ & $\mu_{\text {palcd }}\left[\mathrm{mm}^{-1}\right]$ & 3.372 \\
\hline Appearance & Block & $\theta_{\min }, \theta_{\max },\left[^{\circ}\right]$ & $1.99,29.00$ \\
\hline Color & Black & Total reflections & 14186 \\
\hline$a[\AA]]$ & $8.8065(14)$ & $\mathrm{Z}$ & 2 \\
\hline$b[\AA]]$ & $20.935(3)$ & $F(000)$ & 1312 \\
\hline$c[\AA]$ & $11.1771(18)$ & $\mathbf{T}_{\min } / \mathbf{T}_{\max }$ & 1.00 \\
\hline$\alpha\left[^{\circ}\right]$ & 90 & Cryst. size $\left[\mathrm{mm}^{3}\right]$ & $0.40 \times 0.30 \times 0.30$ \\
\hline$\beta\left[{ }^{\circ}\right]$ & $91.779(2)$ & $\mathrm{R}_{1}[I>2 \sigma(I)]^{a}$ & 0.0312 \\
\hline$\gamma\left[{ }^{\circ}\right]$ & 90 & $\mathrm{wR}_{2}$ (all data) $^{a}$ & 0.0797 \\
\hline $\mathrm{V}\left[\AA^{3}\right]$ & $2059.7(6)$ & GOF $^{a}$ & 1.034 \\
\hline
\end{tabular}

There are two different $\mathrm{Pu}-\mathrm{O}$ bond types in these complexes: those to the $\mathrm{N}$ hydroxamate oxygens and those to the amide oxygens. In the tetrakis $\mathrm{Pu}(\mathbf{4 - 1})_{4}$ complex both these bond types average 2.33(2) $\AA$. These distances in the $\left[\mathrm{Pu}(4-1)_{3}\left(\mathrm{H}_{2} \mathrm{O}\right)_{2}\right]^{+}$ species have similar values, with the $\mathrm{Pu}-\mathrm{O}_{\mathrm{N} \text {-hydroxamate }}$ bonds averaging 2.31(1) $\AA$ and the 
$\mathrm{Pu}-\mathrm{O}_{\text {amide }}$ bonds averaging 2.30(2) $\AA$. The Pu-O bond equality is consistent with $1,2-$ HOPO complexes with transition metal and f-element hard Lewis acids, and is due to aromatization of the heteroatom ring via resonance forms that place a higher than expected negative charge on the chelating HOPO amide oxygen. ${ }^{16-18}$ In comparison, the $\mathrm{Pu}-\mathrm{O}$ bonds in the $\mathrm{Pu}(5 \mathrm{LiO}-\mathrm{Me}-3,2-\mathrm{HOPO})_{2}$ complex exhibit an average $\mathrm{Pu}-\mathrm{O}_{\text {amide }}$ and Pu- $\mathrm{O}_{\text {phenolate }}$ distances of 2.38(4) $\AA$ and 2.28(4) $\AA$, respectively. While these distances are just within $3 \sigma$ of each other, they indicate that such a resonance form is not as significant in the Me-3,2-HOPO moiety. This result is consistent with U-O bond inequality in complexes with tetra- and hexadentate Me-3,2-HOPO moieties in Chapters 2 and 3 as well as in Ce(IV) complexes with bidentate and tetradentate Me-3,2-HOPO ligands. ${ }^{19}$

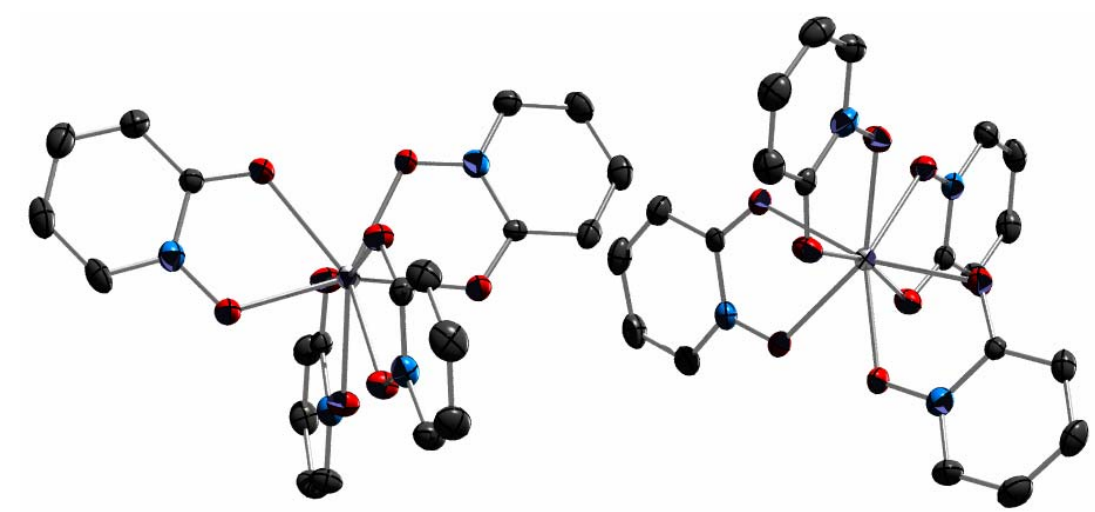

Figure 4-5. Crystal structure of $\mathrm{Ce}(4-1)_{4}$. Hydrogen atoms and solvent inclusions have been omitted for clarity. Thermal ellipsoids are drawn at the 50\% level. Carbons are gray, oxygens red, and cerium is silver.

As a point of structural comparison, the $\mathrm{Ce}(\mathbf{4 - 1})_{4}$ complex was synthesized, crystallized, and X-ray diffraction measurements were collected by Dr. Jide Xu of the Raymond group. The crystal structure of $\mathrm{Ce}(\mathbf{4 - 1})_{4}$ is shown in Figure 4-5 and contains two unique $\mathrm{Ce}(\mathbf{4 - 1})_{4}$ complexes in the asymmetric unit. The average $\mathrm{Ce}-\mathrm{O}_{\mathrm{N}-\text { hydroxamate }}$ and Ce- $\mathrm{O}_{\text {amide }}$ distances are 2.33(2) $\AA$ and 2.35(3) $\AA$ respectively - effectively identical to the 
corresponding $\mathrm{Pu}-\mathrm{O}$ bonds - and exhibit the expected $\mathrm{Ce}-\mathrm{O}$ bond equality typical of 1,2HOPO complexes.

Another method by which the coordination environments of octacoordinate metal centers can be evaluated is by the shape measure metric (S). ${ }^{19}$ The shape measure is a dihedral angle difference minimization according to Equation 4-1 where $m$ is the number of polyhedron edges, and $\delta_{i}$ and $\theta_{i}$ are the dihedral angles between the two polyhedron faces along the $i^{\text {th }}$ edge of the observed and ideal octacoordinate polyhedra, respectively [square antiprism $\left(\mathrm{D}_{4 \mathrm{~d}}\right.$ symmetry), bicapped trigonal prism $\left(\mathrm{C}_{2 \mathrm{v}}\right)$, and trigonal dodecahedral $\left(\mathrm{D}_{2 \mathrm{~d}}\right)$, Figure 4-6].

$$
S(\delta, \theta)=\min \left[\left((1 / m) \sum_{i=1}^{m}\left(\delta_{i}-\theta_{i}\right)^{2}\right)^{1 / 2}\right]
$$

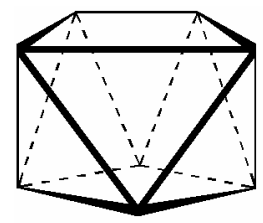

$D_{4 d}$

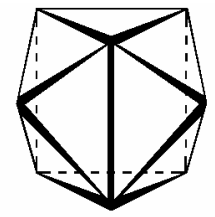

$C_{2 v}$

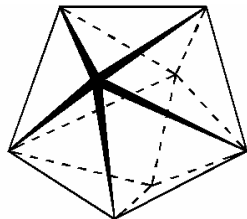

$D_{2 d}$

Figure 4-6. Ideal coordination polyhedra: Square antiprism $\left(\mathrm{D}_{4 \mathrm{~d}}\right)$, bicapped trigonal prism $\left(\mathrm{C}_{2 \mathrm{v}}\right)$, and trigonal dodecahedron $\left(\mathrm{D}_{2 \mathrm{~d}}\right)$.

Table 4-2 lists the shape measure results for the $\mathrm{Pu} / \mathrm{Ce}(\mathrm{IV})$ complexes with 4-1. These indicate the $\left[\mathrm{Pu}(4-1)_{3}\left(\mathrm{H}_{2} \mathrm{O}\right)_{2}\right]^{+}$complex most closely resembles a bicapped trigonal prism $\left(\mathrm{C}_{2 \mathrm{v}}\right)$, while the $\mathrm{Pu}(4-1)_{4}$ complex approaches trigonal dodecahedral geometry $\left(\mathrm{D}_{2 \mathrm{~d}}\right)$. The difference between $\mathrm{C}_{2 \mathrm{v}}$ and $\mathrm{D}_{2 \mathrm{~d}}$ geometries for both species does not exceed $2.3^{\circ}$. Similarly, the coordination geometry of both $\mathrm{Ce}(\mathbf{4 - 1})_{4}$ complexes most closely resembles the trigonal dodecahedron $\left(\mathrm{D}_{2 \mathrm{~d}}\right)$, although the differences in shape measure between this and $\mathrm{C}_{2 \mathrm{v}}$ geometry are $0.2^{\circ}$ and $3.2^{\circ}$. These small differences in shape measure indicate that the coordination geometries of $\mathrm{Pu} / \mathrm{Ce}(\mathrm{IV})$ complexes with 4-1 are intermediates 
between the ideal coordination geometries, although they typically most closely resemble the trigonal dodecahedron. This is in good agreement with the $\mathrm{D}_{2 \mathrm{~d}}$ coordination polyhedron in $\mathrm{Ce}(\mathrm{Me}-3,2-\mathrm{HOPO})_{4},{ }^{19} \mathrm{Ce}(\mathrm{TIRON})_{4},{ }^{20}$ as well as the catecholate complexes with $\mathrm{Th} / \mathrm{U}(\mathrm{IV}){ }^{12}$ which all adopt trigonal dodecahedral coordination polyhedra.

Table 4-2. Shape measure values for $\mathrm{Pu} / \mathrm{Ce}(\mathrm{IV})$ complexes with 4-1. Bold values indicate minimum shape measure value.

\begin{tabular}{|c|c|c|c|}
\hline \multirow{2}{*}{ Metal Ion } & \multicolumn{3}{|c|}{ Shape Measure, $\mathbf{S}\left[^{\circ} \mathbf{I}\right.$} \\
\cline { 2 - 4 } & $\mathbf{D}_{\mathbf{4 d}}{ }^{a}$ & $\mathbf{C}_{2 \mathbf{v}}{ }^{b}$ & $\mathbf{D}_{2 \mathrm{~d}}{ }^{\mathrm{c}}$ \\
\hline \multirow{2}{*}{$\mathrm{Pu}(\mathrm{IV})^{d}$} & 16.3136 & $\mathbf{1 0 . 0 0 6 3}$ & 11.4562 \\
\cline { 2 - 4 } & 18.7766 & 13.8437 & $\mathbf{9 . 3 7 8 1}$ \\
\hline \multirow{2}{*}{$\mathrm{Ce}(\mathrm{IV})^{d}$} & 16.0470 & 13.5587 & $\mathbf{1 3 . 3 9 0 4}$ \\
\cline { 2 - 4 } & 18.9709 & 13.1182 & $\mathbf{9 . 9 1 0 4}$ \\
\hline
\end{tabular}

${ }^{a} \mathrm{D}_{4 \mathrm{~d}}=$ Square antiprism; ${ }^{b} \mathrm{C}_{2 \mathrm{v}}=$ Bicapped trigonal prism; ${ }^{c} \mathrm{D}_{2 \mathrm{~d}}=$ Trigonal dodecahedron

${ }^{d}$ The crystal structure contained two unique $\mathrm{ML}_{4}$ complexes

Another interesting comparison to the $\mathrm{Pu}-(\mathbf{4 - 1})$ crystal structures is that of the $\operatorname{Th}(4-$ 1) $)_{4}\left(\mathrm{H}_{2} \mathrm{O}\right)$ complex. ${ }^{18}$ In contrast to the $\mathrm{Pu} / \mathrm{Ce}(\mathrm{IV})-(\mathbf{4 - 1})$ complexes, the Th(IV) structure is nine-coordinate, with a distorted tricapped trigonal prismatic $\left(\mathrm{D}_{3 \mathrm{~h}}\right)$ coordination geometry. The reason for this structural difference is the larger Th(IV) radius compared to $\mathrm{Pu}(\mathrm{IV})\left(\Delta \mathrm{r}_{\text {ion }}=0.06 \AA\right)$, with the Th- $\mathrm{O}_{\mathrm{HOPO}}$ bonds between $0.07 \AA$ and $0.13 \AA$ longer than the corresponding average $\mathrm{Pu}-\mathrm{O}$ bonds in the $\mathrm{Pu}(\mathbf{4 - 1})_{\mathrm{n}}$ complexes. However, characteristic of 1,2-HOPO complexes, Th-O bonds are very similar to each other, with the average $\mathrm{Th}-\mathrm{O}_{\mathrm{N}-\text { hydroxamate }}$ and $\mathrm{Th}-\mathrm{O}_{\text {amide }}$ distances $2.40(2) \AA$ and $2.48(1) \AA$, respectively.

The structural analyses above reveal that in general, the 1,2-HOPO binding moiety binds $\mathrm{Pu}(\mathrm{IV})$ and $\mathrm{Ce}(\mathrm{IV})$ ions in a coordination environment that most closely resembles trigonal dodecahedral geometry. However, the coordination environments are not as unambiguously $\mathrm{D}_{2 \mathrm{~d}}$ as prior tetracatecholate complex analogs would suggest, indicating that a significant amount of flexibility can be expected in these complexes. 


\subsubsection{Pu(IV)-Hydroxypyrone Complexes}

With the tetrakis-bidentate coordination modes of $\mathrm{Pu} / \mathrm{Ce}(\mathrm{IV})-1,2-\mathrm{HOPO}$ complexes characterized as trigonal dodecahedral, non-HOPO bidentate ligands were investigated to further expand the coordination chemistry of $\mathrm{Pu}(\mathrm{IV})$. One class of $\mathrm{HOPO}$ and catechol analogs are the monoprotic 3-hydroxy-pyran-4-ones, which are precursors to substituted 3,4-HOPO ligands. ${ }^{21}$ One of the simplest compounds of this class, 3-hydroxy-2-methylpyran-4-one (maltol, 4-2, Figure 4-7a) is most commonly used as a food additive, but is a good transition metal chelator and has been considered for applications as a soluble $\mathrm{Fe}$ (III) complex in the treatment of anemia and in iron enriched foods as well as in vanadyl complexes for the treatment of diabetes. ${ }^{22,23}$ The lanthanide complexes with maltol have been characterized and their formation constants determined. ${ }^{24,25}$ Maltol is an attractive ligand for $\mathrm{Pu}(\mathrm{IV})$ coordination chemistry studies because it not only presents a similar chelation mode to bound metals as catechol and HOPO moieties, but is also sterically unconstrained. Bromide substitution on the maltol ring can be carried out following literature procedures ${ }^{26}$ to produce the structurally-similar but electronically modified bromomaltol ligand (4-3, Figure 4-7b) which was also used in structural investigations below.

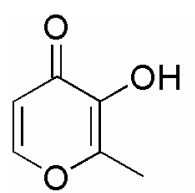

(a)

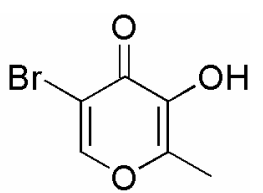

(b)

Figure 4-7. 3-Hydroxy-pyran-4-one ligands: (a) 3-hydroxy-2-methyl-pyran-4-one (maltol, 4-2); (b) 5-bromo-3-hydroxy-2-methyl-pyran-4-one (bromomaltol, 4-3).

The $\mathrm{Pu}(\mathrm{IV})$ complexes with 4-2 and 4-3 were synthesized in an analogous method to 4-1, in which a 4:1 ligand to metal ratio in a buffered water/methanol solution deposited deep red crystals of the plutonium complex upon solvent evaporation at room 
temperature. The analogous $\mathrm{Ce}(\mathrm{IV})$ complexes were synthesized in and crystallized as purple/black crystals from chlorinated solvents by Dr. Jide Xu, and both the Ce(IV) and $\mathrm{Pu}(\mathrm{IV})$ crystals were analyzed by single-crystal X-ray diffraction. The crystal structures of the $\mathrm{Pu}(\mathrm{IV})$ and $\mathrm{Ce}(\mathrm{IV})$ complexes with 4-2 and 4-3 are shown in Figure 4-8 and their crystallographic parameters are listed in Table 4-3.

Table 4-3. Crystallographic parameters for $\mathrm{Pu} / \mathrm{Ce}(4-2 / 4-3)_{4}$ complexes.

\begin{tabular}{|c|c|c|c|c|}
\hline & $\mathrm{Pu}(4-2)_{4}$ & $\mathrm{Pu}(4-3)_{4}$ & $\mathrm{Ce}(4-2)_{4}$ & $\mathrm{Ce}(4-3)_{4}$ \\
\hline Formula & $\mathrm{C}_{24} \mathrm{H}_{20} \mathrm{O}_{12} \mathrm{Pu}$ & $\begin{array}{c}\mathrm{C}_{24} \mathrm{H}_{16} \mathrm{O}_{12} \mathrm{Br}_{4} \mathrm{Pu} \cdot \\
1.7 \mathrm{H}_{2} \mathrm{O}\end{array}$ & $\mathrm{C}_{24} \mathrm{H}_{20} \mathrm{O}_{12} \mathrm{Ce}$ & $\mathrm{C}_{24} \mathrm{H}_{16} \mathrm{O}_{12} \mathrm{Br}_{4} \mathrm{Ce}$ \\
\hline MW & 742.40 & 1073.10 & 640.52 & 956.09 \\
\hline $\mathbf{T}[\mathrm{K}]$ & $223(2)$ & 193(2) & $175(2)$ & $169(2)$ \\
\hline Crystal system & Tetragonal & Triclinic & Tetragonal & Tetragonal \\
\hline Space group & $\mathrm{I} 4_{1} / \mathrm{a}$ & $\mathrm{P}-1$ & $\mathrm{I} 4_{1} / \mathrm{a}$ & $\mathrm{I} 4_{1} / \mathrm{a}$ \\
\hline Appearance & Block & Plate & Block & Block \\
\hline Color & Red & Red & Black & Black \\
\hline$a[\AA \AA]$ & $9.2073(4)$ & $9.1132(19)$ & $9.2036(4)$ & $14.95020(10)$ \\
\hline$b[\AA]$ & $9.2073(4)$ & $9.2739(19)$ & $9.2036(4)$ & $14.95020(10)$ \\
\hline$c[\AA]]$ & $27.068(3)$ & $17.458(4)$ & $27.3801(16)$ & $12.9335(2)$ \\
\hline$\alpha\left[^{\circ}\right]$ & 90 & $76.180(4)$ & 90 & 90 \\
\hline$\beta\left[^{\circ}\right]$ & 90 & $82.495(5)$ & 90 & 90 \\
\hline$\gamma\left[^{\circ}\right]$ & 90 & $88.765(4)$ & 90 & 90 \\
\hline $\mathbf{V}\left[\AA^{3}\right]$ & $2294.7(3)$ & $1420.4(5)$ & $2319.3(2)$ & $2890.75(5)$ \\
\hline $\mathbf{Z}$ & 4 & 2 & 4 & 4 \\
\hline$\rho_{\text {calcd }}\left[\mathrm{g} \mathrm{cm}^{-3}\right]$ & 2.149 & 2.510 & 1.834 & 2.197 \\
\hline$\mu_{\text {palcd }}\left[\mathrm{mm}^{-1}\right]$ & 2.967 & 8.748 & 2.031 & 7.152 \\
\hline$\theta_{\min }, \theta_{\max },\left[^{\circ}\right]$ & $2.55,31.14$ & $2.46,24.20$ & $2.33,26.10$ & $3.43,26.11$ \\
\hline Total reflections & 11541 & 9441 & 5308 & 6616 \\
\hline Data/ restr./ param. & $1442 / 0 / 85$ & $4029 / 685 / 551$ & $1045 / 0 / 85$ & 1304 / 0 / 94 \\
\hline$F(000)$ & 1416 & 997 & 1272 & 1816 \\
\hline $\mathbf{T}_{\min } / \mathbf{T}_{\max }$ & 0.945 & 0.886 & 0.886 & 0.564 \\
\hline Cryst. size $\left[\mathrm{mm}^{3}\right]$ & $0.05 \times 0.03 \times 0.03$ & $0.02 \times 0.02 \times 0.01$ & $0.09 \times 0.08 \times 0.06$ & $0.30 \times 0.20 \times 0.15$ \\
\hline $\mathrm{R}_{1}[I>2 \sigma(I)]^{a}$ & 0.0262 & 0.0890 & 0.0320 & 0.0271 \\
\hline $\mathrm{wR}_{2}$ (all data) $^{a}$ & 0.0642 & 0.2567 & 0.0695 & 0.0669 \\
\hline GOF $^{a}$ & 1.036 & 1.085 & 1.212 & 1.129 \\
\hline
\end{tabular}

\footnotetext{
${ }^{a} \mathrm{R}_{1}=\Sigma|| \mathrm{F}_{\mathrm{o}}|-| \mathrm{F}_{\mathrm{c}}|/ \Sigma| \mathrm{F}_{\mathrm{o}} \mid ; \mathrm{wR}_{2}=\left[\Sigma\left[w\left(\mathrm{~F}_{\mathrm{o}}{ }^{2}-\mathrm{F}_{\mathrm{c}}\right)^{2}\right] / \Sigma\left[w\left(\mathrm{~F}_{\mathrm{o}}{ }^{2}\right)^{2}\right]\right]^{1 / 2} ; \mathrm{GOF}=\left[\Sigma w\left(\left|\mathrm{~F}_{\mathrm{o}}\right|-\left|\mathrm{F}_{\mathrm{c}}\right|\right)^{2} /(n-m)\right]^{1 / 2}$
} 

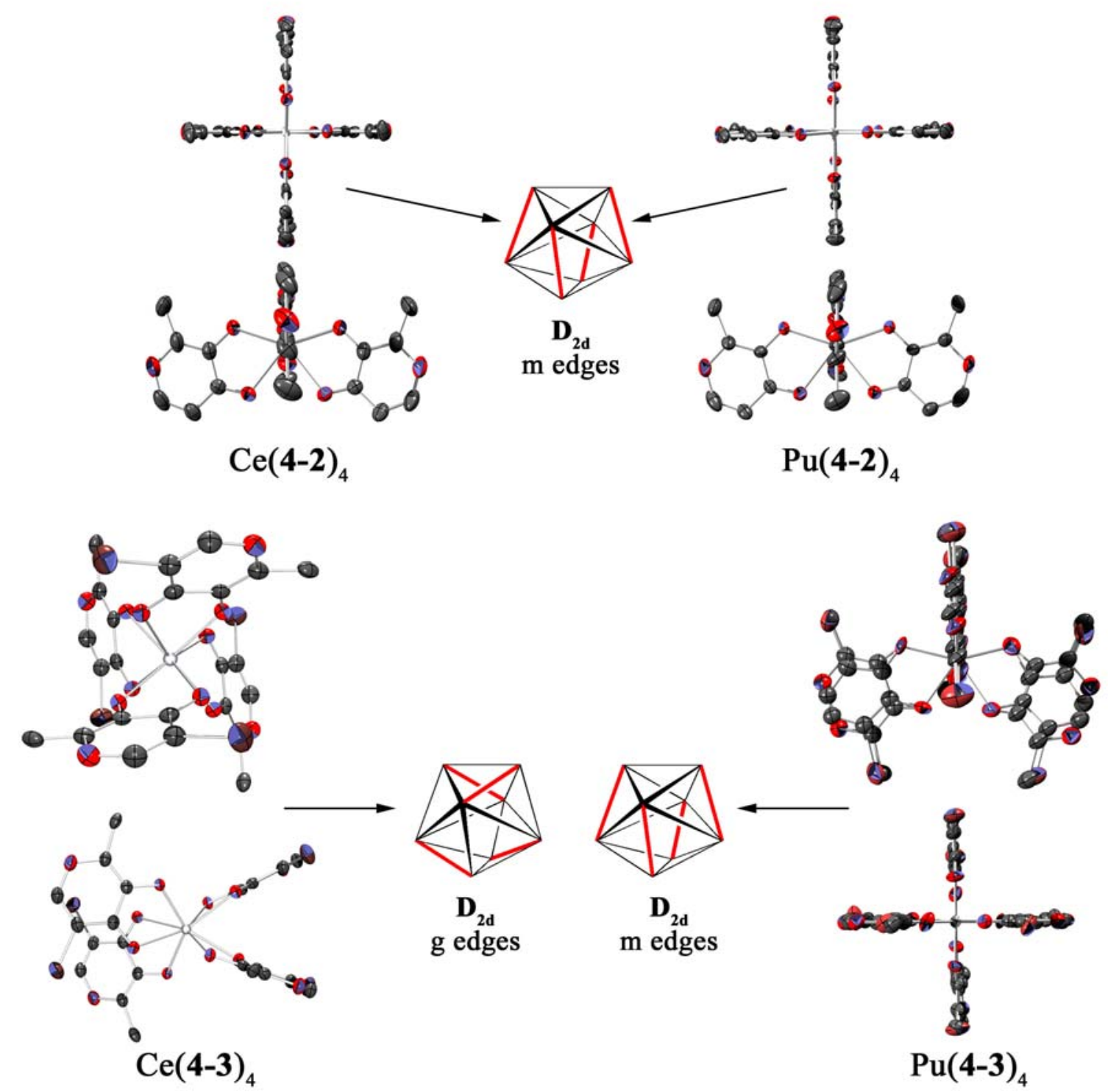

Figure 4-8. Top and side views of crystal structures of $\mathrm{Pu} / \mathrm{Ce}(4-2 / 4-3)_{4}$ complexes along with schematics of their coordination polyhedra. Thermal ellipsoids are drawn at the $50 \%$ probability level. Hydrogen atoms and solvent molecules have been omitted for clarity. Ligand disorder has been included. Carbons are gray, oxygens red, bromines brown and ceriums or plutoniums silver. Edge notation corresponds to that of Hoard and Silverton ${ }^{27}$ and ligand-spanned edges are marked in red.

The complexes in Figure 4-8 are all neutral, tetrakis-bidentate structures in which the hydroxypyrone moiety coordinates in a bidentate fashion through phenolate and carbonyl oxygens. Despite the difference in crystallization techniques used, the $\mathrm{Pu} / \mathrm{Ce}(4-2)_{4}$ complexes are isostructural and crystallize in the tetragonal space group I $4_{1} / \mathrm{a}$ with $\overline{4}$ 
crystallographic symmetry coincident with the central metal ion, with only one ligand present in the asymmetric unit. The $\overline{4}$ crystallographic symmetry generates the other three ligands, imparting approximate (non-crystallographic) $\mathrm{D}_{2 \mathrm{~d}}$ molecular symmetry to the metal complex with the hydroxypyrone ring plane nearly coincident to the crystallographic c-axis. $\mathrm{Ce}(4-3)_{4}$ also crystallizes in the tetragonal space group $\mathrm{I} 4_{1} / \mathrm{a}$, again with crystallographic $\overline{4}$ symmetry coincident with the metal center and only one ligand in the asymmetric unit. However, due to the orientation of the hydroxypyrone ligand with respect to the crystallographic c-axis, generation of the other three ligands by the $\overline{4}$ symmetry operation results in a complex of $\mathrm{S}_{4}$ molecular symmetry. It was anticipated that the $\mathrm{Pu}(4-3)_{4}$ complex would be isostructural to $\mathrm{Ce}(4-3)_{4}$ due to the precedent set in the $\mathrm{Pu} / \mathrm{Ce}(4-2)_{4}$ complexes and the similarities in the $\mathrm{Ce} / \mathrm{Pu}(\mathrm{IV})$ coordination geometries with 1,2- and Me-3,2-HOPO ligands. Unexpectedly, the Pu(43) ${ }_{4}$ complex crystallized in the triclinic space group P-1, includes disordered water molecules in the unit cell, and exhibits a different coordination geometry compared to its $\mathrm{Ce}(\mathrm{IV})$ analog. The $\mathrm{Pu}(\mathbf{4}-3)_{4}$ complex actually more closely resembles the $\mathrm{Pu} / \mathrm{Ce}(\mathbf{4}-\mathbf{2})_{4}$ complexes in its relative ligand orientation about the metal center.

As Figure 4-8 also illustrates, structural elucidation of $\mathrm{Pu}(\mathbf{4}-3)_{4}$ was complicated by a significant amount of total ligand disorder in which each hydroxypyrone moiety can either be in an "up" or "down" position. The structure was modeled using rigid, overlapping 4-3 rings in both of the possible orientations according to a freely-refining ratio. Because of similarities in the degree of disorder observed in each ligand group separately, the extent of ligand disorder was subsequently constrained so that ligand groups opposite (co-planar) to each other expressed the same disorder ratio. 
Consequently there are two ligand pairs about the $\mathrm{Pu}(\mathrm{IV})$ with disordered occupancies of 72:28 and 65:35 in which the bromide (and methyl) substituents of opposing ligands are oriented in opposite directions (Figure 4-9). This coupled disorder can also be described as a "pseudo- $\mathrm{C}_{2}$ " axis oriented vertically between both ligand pairs at a position coincident with the $\mathrm{Pu}(\mathrm{IV})$ center that causes the observed ligand overlap in the disordered crystal structure. This "pseudo- $\mathrm{C}_{2}$ " axis would be coincident with the position of the $\mathrm{S}_{4}$ molecular symmetry axis observed in $\mathrm{Pu} / \mathrm{Ce}(\mathbf{4}-\mathbf{2})_{4}$.

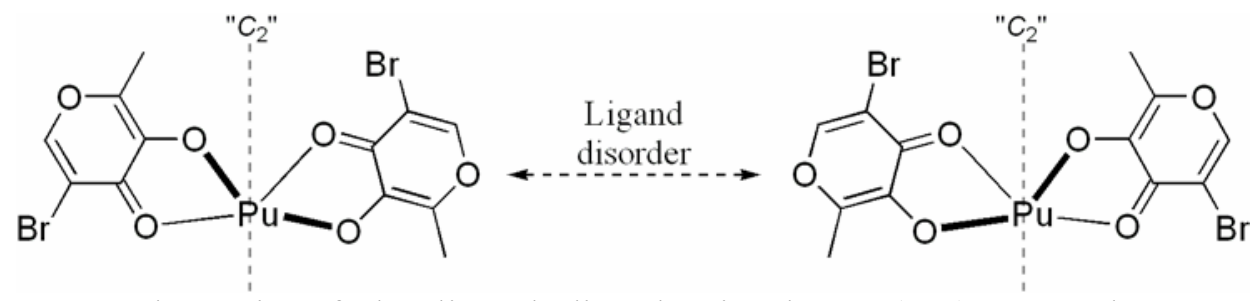

Figure 4-9. Schematic of the ligand disorder in the $\mathrm{Pu}(4-3)_{4}$ crystal structure. The generated "pseudo- $\mathrm{C}_{2}$ " axis is indicated and the shorter $\mathrm{Pu}-\mathrm{O}_{\text {phenolate }}$ bonds in each configuration are indicated in bold. Only one ligand pair is shown here, but the same disorder is seen in the other pair of ligands perpendicular to those shown.

Assuming the trans-coupled disorder in the $\mathrm{Pu}(4-3)_{4}$ structure, two overall molecular geometries result, depending on whether each ligand pair is in its major or minor disorder conformation. In both of these overall geometries there is a $\mathrm{C}_{2}$ axis of molecular symmetry dihedral to the hydroxypyrone planes and perpendicular to the position of the $\mathrm{S}_{4}$ axis in $\mathrm{Pu} / \mathrm{Ce}(4-2)_{4}$ complexes. This relationship is illustrated in Figure 4-10 in which the bold edges represent those spanned by the ligand, the arrow heads point in the direction of the methyl substituents, and arrows related by the indicated symmetry axis have the same color. Thus, from the $\mathrm{D}_{2 \mathrm{~d}}$ molecular geometry present in the $\mathrm{Pu} / \mathrm{Ce}(\mathbf{4}-\mathbf{2})_{4}$ complexes, the $\mathrm{Pu}(4-3)_{4}$ symmetry has dropped to $\mathrm{C}_{2}$ with the loss of the formal $\mathrm{S}_{4} / \mathrm{C}_{2}$ axis and the removal of approximate mirror planes coincident to the hydroxypyrone rings. 


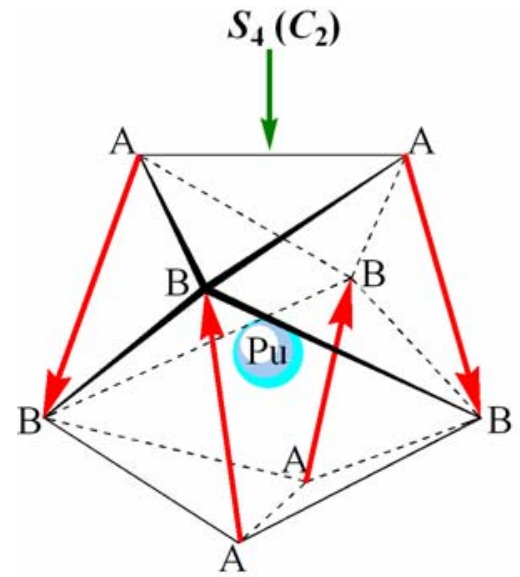

$\mathrm{Pu}(\text { 4-2 })_{4}$

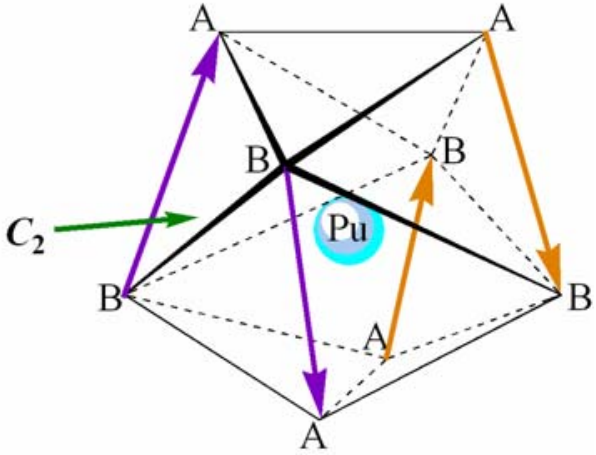

$\mathrm{Pu}(4-3)_{4}$

Figure 4-10. Coordination polyhedra of the $\mathrm{Pu}(4-2)_{4}$ and $\mathrm{Pu}(4-3)_{4}$ complexes with spanned edges indicated by arrows whose heads point towards the methyl substituents. Arrows related by symmetry have the same color, with primary molecular symmetry axes indicated with green arrows. Coordination polyhedra are represented as trigonal dodecahedra using vertex notation of Hoard and Silverton. ${ }^{27}$

The coordinating oxygen atoms in the $\mathrm{Pu}(4-3)_{4}$ complex were not disordered over two positions along with the rest of the ligand because the positions are not sufficiently displaced from each other for the crystallographic data to discern the positional difference. A result of this treatment, however, is an averaging of the observed $\mathrm{Pu}-\mathrm{O}_{\text {phenolate }}$ and $\mathrm{Pu}-\mathrm{O}_{\text {carbonyl }}$ bond distances. Equations 4-2 and 4-3 describe this averaging effect, assuming identical overlay of the disordered phenolate and carbonyl oxygen atoms, in which $\mathrm{Pu}-\mathrm{O} 1$ and $\mathrm{Pu}-\mathrm{O} 2$ are the short and long $\mathrm{Pu}-\mathrm{O}$ distances observed for each ligand group in the crystal structure, $\mathrm{z}$ is the freely-refined variable describing the extent of disorder, and $\mathrm{x}$ and $\mathrm{y}$ are the calculated $\mathrm{Pu}-\mathrm{O}_{\text {phenolate }}$ and $\mathrm{Pu}-\mathrm{O}_{\text {carbonyl }}$ distances respectively. The values of $\mathrm{x}$ and $\mathrm{y}$ determined by this treatment are shown in Table 4-4 and compared against the $\mathrm{Pu} / \mathrm{Ce}-\mathrm{O}$ distances in the three other hydroxypyrone structures in Table 4-5.

$$
\begin{aligned}
& \mathrm{Pu}-\mathrm{O} 1=(\mathrm{z}) \mathrm{x}+(1-\mathrm{z}) \mathrm{y} \\
& \mathrm{Pu}-\mathrm{O} 2=(1-\mathrm{z}) \mathrm{x}+(\mathrm{z}) \mathrm{y}
\end{aligned}
$$


Table 4-4. Calculated $\mathrm{Pu}-\mathrm{O}$ distances in the $\mathrm{Pu}(4-3)_{4}$ crystal structure for each disordered ligand group.

\begin{tabular}{|c|c|c|c|c|c|}
\hline & Group 1 & Group 2 & Group 3 & Group 4 & Average \\
\hline $\mathrm{Pu}-\mathrm{O}_{\text {phenolate, }}[\AA]$ & $2.28(4)$ & $2.25(5)$ & $2.22(4)$ & $2.22(5)$ & $2.24(3)$ \\
\hline $\mathrm{Pu}-\mathrm{O}_{\text {carbonyl }},[\AA]$ & $2.34(4)$ & $2.34(5)$ & $2.40(4)$ & $2.37(5)$ & $2.36(3)$ \\
\hline
\end{tabular}

Table 4-5. $\mathrm{Pu} / \mathrm{Ce}-\mathrm{O}$ bond distances and bite angles from the $\mathrm{Pu} / \mathrm{Ce}(4-2 / 4-3)_{4}$ crystal structures.

\begin{tabular}{|c|c|c|c|}
\hline & M-O ${ }_{\text {phenolate }},[\AA]$ & M-O carbonyl $_{1}[\AA]$ & Bite angle, $\left[{ }^{\circ}\right]$ \\
\hline $\mathrm{Pu}(4-2)_{4}$ & $2.286(3)$ & $2.419(3)$ & $67.9(1)$ \\
\hline $\mathrm{Pu}(4-3)_{4}{ }^{a}$ & $2.24(3)$ & $2.36(3)$ & $67.0(7)$ \\
\hline $\mathrm{Ce}(4-2)_{4}$ & $2.276(3)$ & $2.441(3)$ & $67.6(1)$ \\
\hline $\mathrm{Ce}(4-3)_{4}$ & $2.245(3)$ & $2.503(3)$ & $67.5(1)$ \\
\hline
\end{tabular}

${ }^{a}$ Values reported are averages of the four disordered ligand groups.

The deconvoluted $\mathrm{Pu}-\mathrm{O}$ bond lengths in Table 4-4 cannot be known with the accuracy seen in the ordered hydroxypyrone crystal structures, with the Pu-O bonds within ligand groups 1 and 2 statistically indistinct. However, in all ligand groups the $\mathrm{Pu}-\mathrm{O}_{\text {phenolate }}$ distance is shorter than the $\mathrm{Pu}-\mathrm{O}_{\text {carbonyl }}$ distance, a result that supports the validity of the ligand disorder model used in the crystal structure of $\mathrm{Pu}(4-3)_{4}$; these relative bond lengths are not preserved if the oxygen atoms are disordered over two positions. As expected from atom charge differences, the $\mathrm{M}-\mathrm{O}_{\text {carbonyl }}$ bonds are always longer than the $\mathrm{M}-\mathrm{O}_{\text {phenolate }}$ bonds (ca. $0.13 \AA$ ), with only slight variation between the $\mathrm{Ce}-\mathrm{O}$ and $\mathrm{Pu}-\mathrm{O}$ values $\left(\Delta \mathrm{d}_{\mathrm{Pu} / \mathrm{Ce}-\mathrm{O}}\right.$ ca. $\left.0.04 \AA\right)$. The exception to these trends is the $\mathrm{Ce}(4-$ 3) 4 complex, in which the $\mathrm{Ce}-\mathrm{O}_{\text {carbonyl }}$ and $\mathrm{Ce}-\mathrm{O}_{\text {phenolate }}$ bonds differ by $0.26 \AA$. And while the $\mathrm{Ce} / \mathrm{Pu}(4-3)_{4} \mathrm{M}-\mathrm{O}_{\text {phenolate }}$ distances only differ by $0.04 \AA$, the $\mathrm{M}-\mathrm{O}_{\text {carbonyl }}$ distances differ by $0.14 \AA$. Interestingly, despite the longer bonds in $\mathrm{Ce}(4-3)_{4}$, the bite angles for all four structures are the same within $1^{\circ}$.

To further explore the relatively long $\mathrm{Ce}-\mathrm{O}_{\text {carbonyl }}$ bond distance in $\mathrm{Ce}(4-3)_{4}$, shape measure analysis was performed on the hydroxypyrone complexes, the results of which are listed in Table 4-6. All $\mathrm{Ce} / \mathrm{Pu}(4-2 / 4-3)_{4}$ complexes exhibit unambiguously trigonal dodecahedral $\left(\mathrm{D}_{2 \mathrm{~d}}\right)$ coordination polyhedra, as the schematics in Figures 4-8 and 4-10 
illustrate. However, the unusual molecular structure of the $\mathrm{Ce}(4-3)_{4}$ complex seems out of line with the similar shape value results. Closer inspection of the four crystal structures reveals that the ligands in $\mathrm{Ce}(4-3)_{4}$ span a different set of edges on the trigonal dodecahedron than in the other hydroxypyrone crystal structures; According to the notation of Hoard and Silverton, ${ }^{27}$ the ligands in $\mathrm{Ce}(4-3)_{4}$ span the $g$ edges of the trigonal dodecahedron, while the other hydroxypyrone complexes span $m$ edges as shown in Figure 4-11.

Table 4-6. Shape measure (S) values for $\mathrm{Pu} / \mathrm{Ce}(\mathbf{4 - 2 / 4 - 3})_{4}$ complexes. Bold values indicate minimum shape measure value.

\begin{tabular}{|c|c|c|c|}
\hline \multirow{2}{*}{ Complex } & \multicolumn{3}{|c|}{ Shape Measure, ${ }^{\circ} \mathbf{]}$} \\
\cline { 2 - 4 } & $\mathbf{D}_{\mathbf{4 d}}{ }^{a}$ & $\mathbf{C}_{\mathbf{2 v}}{ }^{b}$ & $\mathbf{D}_{\mathbf{2 d}}{ }^{c}$ \\
\hline $\mathrm{Ce}(\text { Maltol })_{4}$ & 15.1909 & 13.2243 & $\mathbf{3 . 6 6 7 6}$ \\
\hline $\mathrm{Ce}(\text { BrMaltol })_{4}$ & 15.3911 & 13.8823 & $\mathbf{5 . 2 8 5 8}$ \\
\hline $\mathrm{Pu}(\text { Maltol })_{4}$ & 15.0645 & 13.3768 & $\mathbf{3 . 3 7 4 0}$ \\
\hline $\mathrm{Pu}(\text { BrMaltol })_{4}{ }^{d}$ & 16.1235 & 12.6109 & $\mathbf{3 . 8 4 6 5}$ \\
\hline
\end{tabular}

${ }^{a} \mathrm{D}_{4 \mathrm{~d}}=$ Square antiprism; ${ }^{b} \mathrm{C}_{2 \mathrm{v}}=$ Bicapped trigonal prism; ${ }^{c} \mathrm{D}_{2 \mathrm{~d}}=$ Trigonal dodecahedron

${ }^{d}$ Shape measure values calculated using average oxygen positions in the disordered structure.
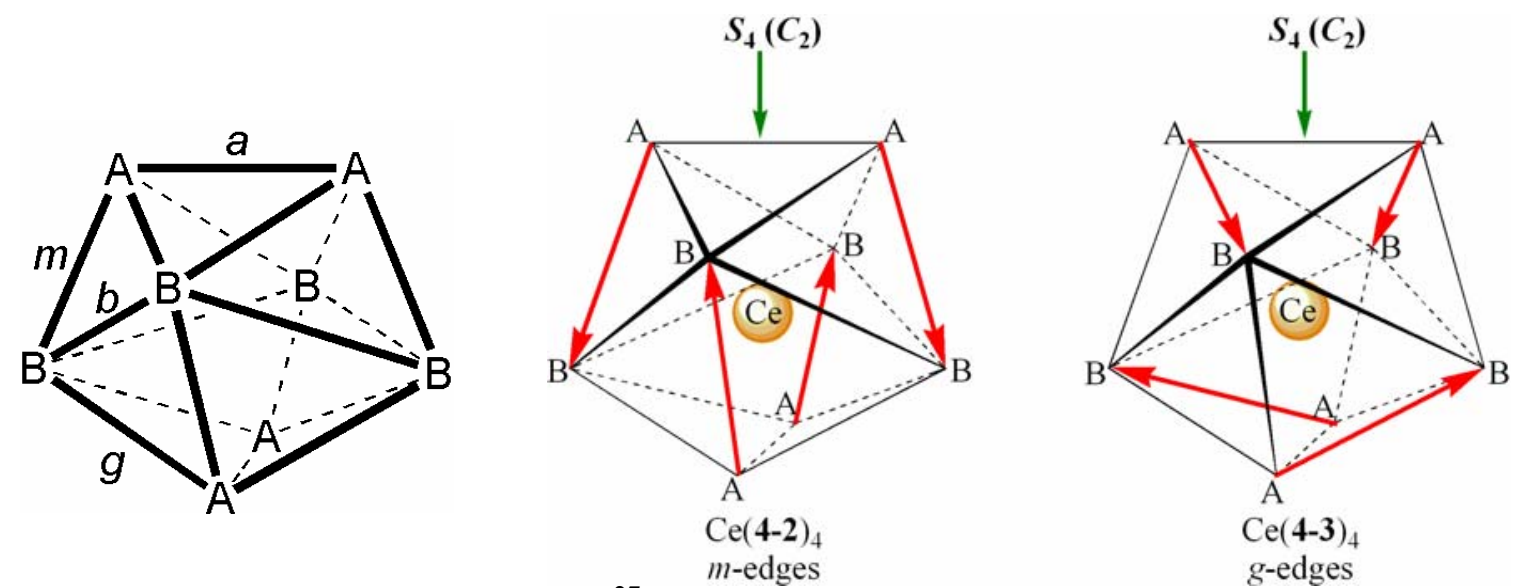

Figure 4-11. Hoard and Silverton ${ }^{27}$ edge notation of trigonal dodecahedral geometry (left) and coordination polyhedra of the $\mathrm{Ce}(4-2)_{4}$ (middle) and $\mathrm{Ce}(4-3)_{4}$ (right) complexes. Ligand-spanned edges are indicated by arrows whose heads point towards the methyl substituents, with primary molecular symmetry axes indicated.

Because there are twice as many $g$ edges as $m$ edges in a trigonal dodecahedron, there are two ways in which four independent bidentate ligands may span these edges. Interestingly, the geometry observed in the structure of $\mathrm{Ce}(4-3)_{4}$ is the one not addressed by Kepert as an intermediate between pure $\mathrm{D}_{2 \mathrm{~d}}$ (trigonal dodecahedral) and $\mathrm{D}_{4 \mathrm{~d}}$ (square 
antiprismatic) symmetries, ${ }^{28}$ and theoretical calculations on U(IV)-catecholate complexes by Hay et al. indicate that this geometry is not an energy minimum for a tetrakisbidentate complexes with symmetric ligands. ${ }^{29}$ In undistorted trigonal dodecahedra, the $g$ edges are longer than the $m$ edges, but to accommodate the fixed $\mathrm{O}_{\text {phenolate }}-\mathrm{O}_{\text {carbonyl }}$ distance of 4-3 on $g$ edges the coordination polyhedron of $\mathrm{Ce}(4-3)_{4}$ distorts along the molecular $\mathrm{S}_{4}$ axis. Specifically, the $m$ edges are ca. $0.36 \AA$ longer in $\mathrm{Ce}(4-3)_{4}$ than in $\mathrm{Ce}(4-2)_{4}$ and the $g$ edges are an average of $0.36 \AA$ shorter despite an $\mathrm{O}_{\text {phenolate }}-\mathrm{O}_{\text {carbonyl }}$ distance difference of only $0.02 \AA$ between 4-2 and 4-3.

What the above hydroxypyrone structures seem to indicate is that despite different molecular geometry, these ligands seem to prefer a trigonal bipyramidal coordination mode about $\mathrm{Ce}(\mathrm{IV})$ and $\mathrm{Pu}(\mathrm{IV})$. However, one caveat of crystal structure analysis is that it is not necessarily an accurate depiction of the solution state behavior of the complex, and seemingly small intermolecular interactions (even less than $1 \mathrm{kcal} / \mathrm{mol}$ ) can have a significant effect on crystal structures. ${ }^{30}$ This raises the question as to whether the observed difference in the $\mathrm{Ce}(\mathrm{IV})$ and $\mathrm{Pu}(\mathrm{IV})$ structures with $4-3$ is a result of the different crystallization conditions used for each crystal or if it is due to the population of expanded $5 f$ orbitals in $\mathrm{Pu}(\mathrm{IV})$ which may affect the bonding in the complex [compared to the unoccupied, contracted $4 f$ orbitals in Ce(IV)]. To determine the cause of this difference, attempts were made to crystallize $\mathrm{Ce}(4-3)_{4}$ by methods to those for $\mathrm{Pu}(\mathrm{IV})$, using $\left(\mathrm{NH}_{4}\right)_{2} \mathrm{Ce}\left(\mathrm{NO}_{3}\right)_{6}$ as a water-soluble metal salt. These attempts were unsuccessful, with $\mathrm{Ce}(\mathrm{IV})$ solutions exhibiting color changes that were indicative of a reduction of $\mathrm{Ce}(\mathrm{IV})$ to $\mathrm{Ce}(\mathrm{III})$, which is favored by $1.72 \mathrm{eV}$ at the acidic, buffered $\mathrm{pH}$ at which the $\mathrm{Pu}(\mathrm{IV})$ complex must be formed to avoid hydrolysis. Theoretical calculations are 
currently being undertaken by the research group of Dr. Andrew Canning at LBNL to address the question of relative energetics in the crystal structures of $\mathrm{Pu} / \mathrm{Ce}(\mathrm{IV})$ with hydroxypyrones.

\subsubsection{Additional Ce(IV)-Hydroxypyrone Complexes}

Because Ce(IV) complexes cannot be synthesized using similar conditions to those in $\mathrm{Pu}(\mathrm{IV})$ crystallizations, an alternative approach to explore the reason for the coordinative variations of $\mathrm{Ce}(\mathrm{IV})$ seen above was to synthesize and structurally characterize several more Ce(IV)-hydroxypyrone complexes. Many hydroxypyrones with a variety of steric, hydrogen bonding, and electronic properties are accessible via commercial sources or published synthetic procedures and Figure 4-12 illustrates those investigated in this structural comparison. The 3-hydroxy-pyran-4-ones explored are of two general classes, namely those with 2-alkyl substitution (maltol derivatives) and those with 6-alkyl substitution (kojic acid derivatives).

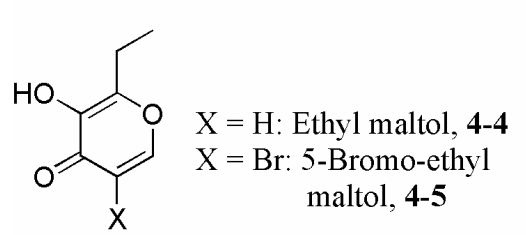

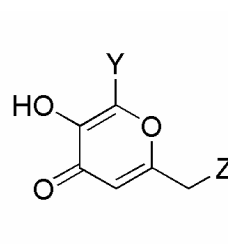

$\mathrm{Y}=\mathrm{H}, \mathrm{Z}=\mathrm{OH}:$ Kojic acid, 4-6

$\mathrm{Y}=\mathrm{H}, \mathrm{Z}=\mathrm{Cl}$ : Chlorokojic acid, 4-7

$\mathrm{Y}=\mathrm{H}, \mathrm{Z}=\mathrm{I}$ : Iodokojic acid, 4-8

$\mathrm{Y}=\mathrm{H}, \mathrm{Z}=\mathrm{H}$ : Alomaltol, 4-9

$\mathrm{Y}=\mathrm{NO}_{2}, \mathrm{Z}=\mathrm{H}:$ 2-Nitro-alomaltol, 4-10

$\mathrm{Y}=\mathrm{CH}_{2} \mathrm{OH}, \mathrm{Z}=\mathrm{H}$ : 2-Hydroxymethyl-alomaltol, 4-11

$\mathrm{Y}=\mathrm{Br}, \mathrm{Z}=\mathrm{OH}$ : 2-Bromo-kojic acid, 4-12

$\mathrm{Y}=\mathrm{Br}, \mathrm{Z}=\mathrm{Cl}$ : 2-Bromo-chlorokojic acid, 4-13

Figure 4-12. Hydroxypyrone ligands used in exploring Ce(IV) coordination chemistry.

Ethyl maltol (4-4) and it brominated derivative 5-bromo-ethyl maltol (4-5) bind $\mathrm{Ce}(\mathrm{IV})$ quickly in organic solution when combined in a L:M ratio of 4:1, precipitating $\mathrm{CeL}_{4}$ complexes from organic solution. In contrast, the $\mathrm{CeL}_{4}$ complexes with kojate ligands 4-6 through 4-13 (although equally insoluble as complexes with 4-4 and 4-5) typically only precipitate out of solution when a L:M ratio greater than 4:1 was used. The ligand to metal ratio used in these studies was $c a$. 10:1, and the resultant Ce(IV)-kojate compounds exhibit terrible solubility in most organic solvents, with reduction of $\mathrm{Ce}(\mathrm{IV})$ 
to $\mathrm{Ce}(\mathrm{III})$ occurring when dissolved in DMSO or DMF accompanied with a change in color from the typically brown/purple $\mathrm{Ce}(\mathrm{IV})$ to a light yellow/orange $\mathrm{Ce}(\mathrm{III})$ complex. Using short reaction times and appropriately chosen solvents to eliminate decomposition, Ce(IV)-hydroxypyrone complexes with ligands 4-4 through 4-13 with the exception of 411 were isolated by filtration and are shelf-stable in their solid state.

Table 4-7. Crystallographic parameters for Ce(IV)-hydroxypyrone crystal structures.

\begin{tabular}{|c|c|c|c|c|c|}
\hline & $\mathrm{Ce}(4-4)_{4}$ & $\mathrm{Ce}(4-5)_{4}$ & $\mathrm{Ce}(4-6)_{4}$ & $\mathrm{Ce}(4-7)_{4}$ & $\mathrm{Ce}(4-11)_{4}$ \\
\hline Formula & $\mathrm{C}_{28} \mathrm{H}_{28} \mathrm{O}_{12} \mathrm{Ce}$ & $\mathrm{C}_{28} \mathrm{H}_{24} \mathrm{O}_{12} \mathrm{Br}_{4} \mathrm{Ce}$ & $\mathrm{C}_{24} \mathrm{H}_{20} \mathrm{O}_{16} \mathrm{Ce}$ & $\begin{array}{c}\mathrm{C}_{24} \mathrm{H}_{16} \mathrm{O}_{12} \mathrm{Cl}_{4} \mathrm{C} \cdot \\
2.25\left(\mathrm{C}_{4} \mathrm{H}_{8} \mathrm{O}_{2}\right)\end{array}$ & $\begin{array}{c}\mathrm{C}_{28} \mathrm{H}_{28} \mathrm{O}_{16} \mathrm{Ce} \cdot \\
\mathrm{C}_{4} \mathrm{H}_{8} \mathrm{O}_{2}\end{array}$ \\
\hline MW & 696.62 & 1012.23 & 704.52 & 966.44 & 848.73 \\
\hline $\mathbf{T}[\mathrm{K}]$ & $158(2)$ & $150(2)$ & $150(2)$ & $150(2)$ & $146(2)$ \\
\hline Crystal system & Monoclinic & Orthorhombic & Monoclinic & Monoclinic & Monoclinic \\
\hline Space group & $\mathrm{P} 2_{1} / \mathrm{c}$ & Pna2 1 & $\mathrm{C} 2 / \mathrm{c}$ & $\mathrm{C} 2 / \mathrm{c}$ & $\mathrm{P} 2_{1} / \mathrm{n}$ \\
\hline Appearance & Plate & Plate & Plate & Plate & Wedge \\
\hline Color & Red & Red & Red & Red & Red \\
\hline$a[\AA ̊]$ & $9.392(4)$ & $18.042(4)$ & $19.024(5)$ & $26.520(10)$ & $7.858(3)$ \\
\hline$b[\AA]$ & $27.969(12)$ & $10.134(2)$ & $9.523(2)$ & $16.134(6)$ & $27.046(11)$ \\
\hline$c[\AA \AA]$ & $10.399(4)$ & $17.449(4)$ & $15.713(4)$ & $17.590(6)$ & $16.351(7)$ \\
\hline$\alpha\left[^{\circ}\right]$ & 90 & 90 & 90 & 90 & 90 \\
\hline$\beta\left[^{\circ}\right]$ & $91.217(8)$ & 90 & $121.084(4)$ & $92.489(6)$ & $100.840(7)$ \\
\hline$\gamma\left[^{\circ}\right]$ & 90 & 90 & 90 & 90 & 90 \\
\hline $\mathrm{V}\left[\AA^{3}\right]$ & $2731(2)$ & $3190.3(11)$ & $2437.8(11)$ & $7519(5)$ & $3413(2)$ \\
\hline $\mathrm{Z}$ & 4 & 4 & 4 & 8 & 4 \\
\hline$\rho_{\text {calcd }}\left[\mathrm{g} \mathrm{cm}^{-3}\right]$ & 1.694 & 2.107 & 1.920 & 1.708 & 1.652 \\
\hline$\mu_{\text {palcd }}\left[\mathrm{mm}^{-1}\right]$ & 1.733 & 8.037 & 2.440 & 1.964 & 1.415 \\
\hline$\theta_{\min }, \theta_{\max },\left[^{\circ}\right]$ & $1.46,25.42$ & $2.46,25.53$ & $2.70,27.18$ & $2.75,26.57$ & $2.59,24.81$ \\
\hline $\begin{array}{c}\text { Total } \\
\text { reflections }\end{array}$ & 14517 & 36008 & 7128 & 32614 & 17074 \\
\hline $\begin{array}{c}\text { Data/ restr./ } \\
\text { param. }\end{array}$ & $5025 / 3 / 383$ & $4582 / 73 / 406$ & $2096 / 1 / 193$ & $6020 / 218 / 497$ & $5834 / 0 / 468$ \\
\hline$F(000)$ & 1400 & 1944 & 1400 & 3840 & 1720 \\
\hline $\mathbf{T}_{\min } / \mathbf{T}_{\max }$ & 0.725 & 0.746 & 0.868 & 0.814 & 0.873 \\
\hline $\begin{array}{l}\text { Cryst. size } \\
{\left[\mathrm{mm}^{3}\right]}\end{array}$ & $\begin{array}{c}0.21 \times 0.10 \times \\
0.01\end{array}$ & $\begin{array}{c}0.05 \times 0.03 \times \\
0.01\end{array}$ & $\begin{array}{c}0.07 \times 0.04 \times \\
0.01\end{array}$ & $\begin{array}{c}0.12 \times 0.05 \times \\
0.01\end{array}$ & $\begin{array}{c}0.12 \times 0.07 \times \\
0.02\end{array}$ \\
\hline $\mathrm{R}_{1}[I>2 \sigma(I)]^{a}$ & 0.0441 & 0.0535 & 0.0451 & 0.0507 & 0.0550 \\
\hline $\mathrm{wR}_{2}$ (all data) ${ }^{a}$ & 0.0889 & 0.1228 & 0.0993 & 0.1329 & 0.1272 \\
\hline GOF $^{a}$ & 0.979 & 1.039 & 1.028 & 1.119 & 0.998 \\
\hline
\end{tabular}

The Ce(IV)-hydroxypyrone precipitates were typically microcrystalline in nature and thus inappropriate for single crystal X-ray diffraction studies. The solids could not be recrystallized due to their poor solubility, so suitably large crystals of $\mathrm{CeL}_{4}$ complexes had to be grown in situ, often employing a three-solvent layering technique illustrated in the Appendix. Ce(IV) crystals with ligands 4-7, 4-9, 4-10, and 4-11 were grown by this 
method, but despite forming beautiful crystals, $\mathrm{Ce}(\mathbf{4 - 9})_{4}$ and $\mathrm{Ce}(\mathbf{4 - 1 0})_{4}$ complexes showed no diffraction even with long exposure times using the intense ALS light source. Reasonably-sized crystals (larger than $10 \mu \mathrm{m} /$ edge) of the Ce(IV) complexes with 4-4, 45, 4-6, 4-7, and 4-11 were successfully grown using a combination of solvent evaporation, in situ precipitation, and three-solvent layering techniques and were investigated using single crystal X-ray diffraction. The resultant structures are illustrated in Figure 4-13. Crystallographic details for these structures are listed in Table 4-7.

The Ce(IV)-hydroxypyrone complexes in Figure 4-13 exhibit a wide range of coordination geometries, with the $\mathrm{Ce}(4-4)_{4}$ and $\mathrm{Ce}(\mathbf{4 - 6})_{4}$ complexes adopting molecular geometries similar to those seen in the $\mathrm{Ce} / \mathrm{Pu}(4-2 / 4-3)_{4}$ complexes. The $\mathrm{Ce}-\mathrm{O}_{\text {phenolate }}$ bond lengths in Figure 4-13 only vary a maximum of $0.06 \AA$ between complexes and the Ce$\mathrm{O}_{\text {carbonyl }}$ bond lengths are the same within $0.11 \AA$, without a noticeable correlation to the presence or location of electron donating/withdrawing groups. Shape measure analysis (Table 4-8) reveals that the coordination geometries about the $\mathrm{Ce}(\mathrm{IV})$ ion vary significantly throughout the structures, with each of the three ideal coordination polyhedra represented. Interestingly, the $\mathrm{D}_{2 \mathrm{~d}}$ coordination polyhedra in $\mathrm{Ce}(4-4)_{4}$ and $\mathrm{Ce}(4-6)_{4}$ are of both the $m$ - and $g$-edge spanning variety respectively, which replicates the two $\mathrm{D}_{2 \mathrm{~d}}$ coordination modes observed in the structures in Figure 4-8. Thus, by the crystal structures alone it is not possible to explain why these coordination changes occur, nor why in some cases different edges of the trigonal dodecahedron are spanned, as was the initial goal of this structural study. 


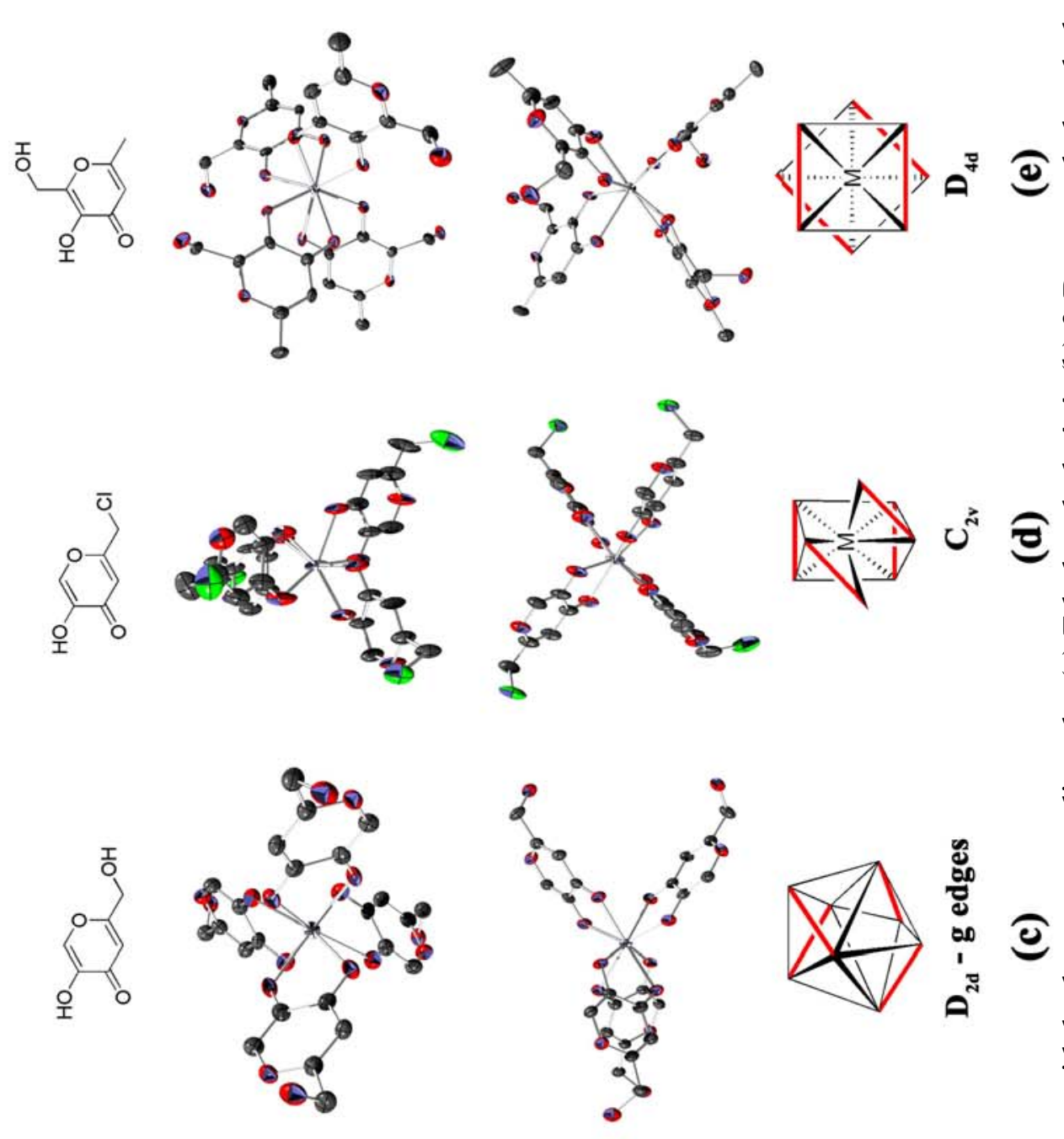

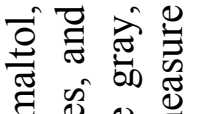

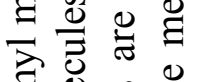

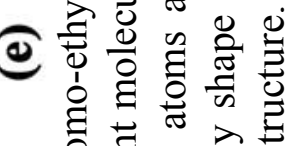

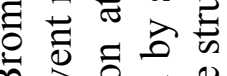

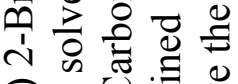

อิ

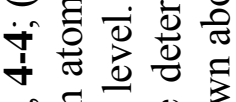

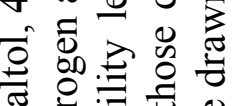

窟芯芯

ج供

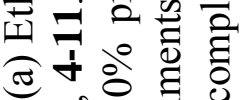

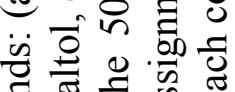

政

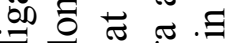

需 要 要

일

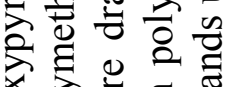

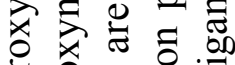

홀을

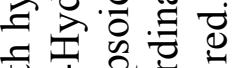

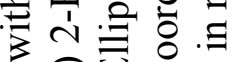

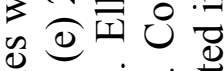

শัへ

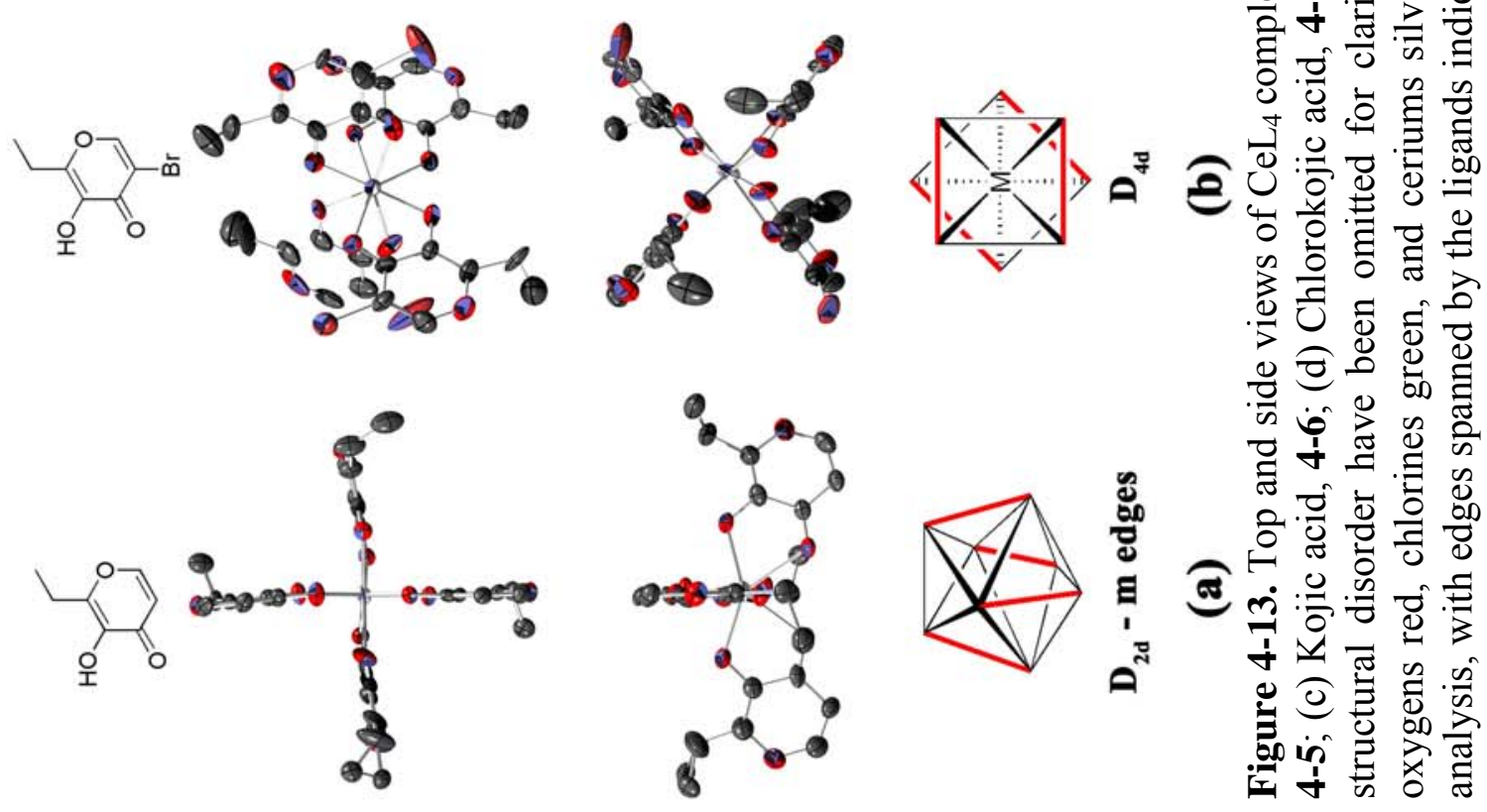


Table 4-8. Shape measure (S) values for Ce(IV)-hydroxypyrone complexes in Figure 413. Bold values indicate minimum shape measure value.

\begin{tabular}{|c|c|c|c|}
\hline \multirow{2}{*}{ Complex } & \multicolumn{3}{|c|}{ Shape Measure, ${ }^{\circ} \mathbf{]}$} \\
\cline { 2 - 4 } & $\mathbf{D}_{\mathbf{4 d}}{ }^{a}$ & $\mathbf{C}_{\mathbf{2 v}}{ }^{b}$ & $\mathbf{D}_{\mathbf{2 d}}{ }^{c}$ \\
\hline $\mathrm{Ce}(\mathbf{4 - 4})_{4}$ & 14.1061 & 11.3168 & $\mathbf{3 . 8 3 1 1}$ \\
\hline $\mathrm{Ce}(\mathbf{4 - 5})_{4}$ & $\mathbf{6 . 8 6 3 1}$ & 12.2233 & 15.8745 \\
\hline $\mathrm{Ce}(\mathbf{4 - 6})_{4}$ & 11.4534 & 11.9993 & $\mathbf{6 . 8 3 5 4}$ \\
\hline $\mathrm{Ce}(\mathbf{4 - 7})_{4}$ & 13.3611 & $\mathbf{8 . 0 7 3 1}$ & 13.3207 \\
\hline $\mathrm{Ce}(\mathbf{4 - 1 1})_{4}$ & $\mathbf{6 . 8 6 3 1}$ & 12.2233 & 15.8745 \\
\hline
\end{tabular}

${ }^{a} \mathrm{D}_{4 \mathrm{~d}}=$ Square antiprism; ${ }^{b} \mathrm{C}_{2 \mathrm{v}}=$ Bicapped trigonal prism; ${ }^{c} \mathrm{D}_{2 \mathrm{~d}}=$ Trigonal dodecahedron

It may be, however, that the energy surface described by the numerous octacoordinate hydroxypyrone structures is simply very shallow, and lattice energies may be responsible for the packing of the Ce(IV) complexes. To explore this possibility, Dr. Benjamin Hay at Oak Ridge National Laboratory performed MM3 minimizations on model $\mathrm{Ce}(4-2 / 4-3)_{4}$ complexes to determine the relative energies of the coordination modes. Previous gas phase calculations by Hay et al. suggested that the $\mathrm{C}_{2 \mathrm{v}}$ and $\mathrm{D}_{2 \mathrm{~d}}$ (g-edge) geometries were not energy minima for complexes with symmetric catecholate ligands, ${ }^{29}$ but the 3hydroxy-pyran-4-ones are not symmetric, perhaps making these conclusions inapplicable. Dr. Hay's results are summarized in Table 4-9, along with indication as to which of the Ce(IV)-hydroxypyrone complexes the coordination mode corresponds to and their relative energies.

Table 4-9. MM3 calculation results on the Ce(4-2/4-3) ${ }_{4}$ structures. Edge notation corresponds to those shown in Figure 4-14.

\begin{tabular}{|c|c|c|c|c|c|}
\hline \multicolumn{3}{|c|}{ Maltol Results } & \multicolumn{3}{|c|}{ BrMaltol Results } \\
\hline $\begin{array}{c}\text { Coord. } \\
\text { Geom./edges } \\
\text { spanned } \\
\end{array}$ & $\begin{array}{l}\text { Relative E } \\
\text { (kcal/mol) }\end{array}$ & $\begin{array}{l}\text { Structures of } \\
\text { same geom. }\end{array}$ & $\begin{array}{c}\text { Coord. } \\
\text { Geom./edges } \\
\text { spanned } \\
\end{array}$ & $\begin{array}{l}\text { Relative E } \\
\text { (kcal/mol) }\end{array}$ & $\begin{array}{l}\text { Structures of } \\
\text { same geom. }\end{array}$ \\
\hline$D_{4 d}-$ ssss & 0.000 & $\begin{array}{l}\mathrm{Ce}(4-5)_{4} \\
\mathrm{Ce}(\mathbf{4 - 1 1})_{4}\end{array}$ & $D_{4 d}-$ SSSS & 0.000 & $\begin{array}{l}\mathrm{Ce}(4-5)_{4} \\
\mathrm{Ce}(\mathbf{4 - 1 1})_{4}\end{array}$ \\
\hline$D_{2 d}-$ gggg & 0.327 & $\begin{array}{l}\mathrm{Ce}(4-3)_{4} \\
\mathrm{Ce}(4-6)_{4}\end{array}$ & $D_{2 d}$-gggg & 0.255 & $\begin{array}{l}\mathrm{Ce}(4-3)_{4} \\
\mathrm{Ce}(4-6)_{4}\end{array}$ \\
\hline$D_{2 d}-$ mmgg & 0.332 & $\begin{array}{c}\mathrm{Ce}(4-7)_{4} \\
\text { (close) }\end{array}$ & $D_{2 d}$-mmgg & 1.160 & -- \\
\hline$D_{2 d}-\mathrm{mmmm}$ & 0.551 & $\begin{array}{l}\mathrm{Ce}(4-2)_{4}, \\
\mathrm{Ce}(4-4)_{4}\end{array}$ & $D_{2 d}-\mathrm{mmmm}$ & 2.409 & $\begin{array}{l}\mathrm{Ce}(4-2)_{4} \\
\mathrm{Ce}(4-4)_{4}\end{array}$ \\
\hline$D_{4 d}-$ ssss & 1.946 & -- & $D_{2 d}-\mathrm{mmmm}$ & 2.658 & $\begin{array}{c}\mathrm{Ce}(4-7)_{4} \\
\text { (close) }\end{array}$ \\
\hline
\end{tabular}




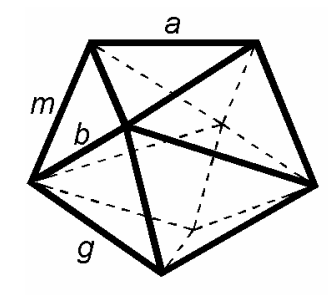

$D_{2 \mathrm{~d}}$

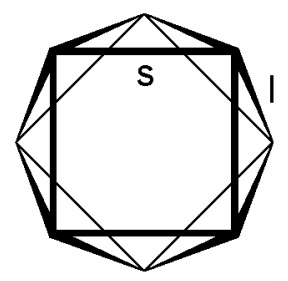

$D_{4 \mathrm{~d}}$

Figure 4-14. Edge notation for $\mathrm{D}_{2 \mathrm{~d}}$ and $\mathrm{D}_{4 \mathrm{~d}}$ coordination polyhedra used in Table 4-9.

Dr. Hay's results, while performed for only two of the ligand types used in this study, indicate that the energy differences between the different coordination modes of the Ce(IV)-hydroxypyrone complexes are very small, with the majority of the observed structural geometries within $0.3-0.5 \mathrm{kcal} / \mathrm{mol}$ of each other. This suggests that the deciding factor in the $\mathrm{Ce}(\mathrm{IV})$ coordination geometry may be in very large part influenced by the crystal packing available based on the interaction between its substituents and incorporated solvent, since something as weakly interacting as two phenyl rings can contribute $2 \mathrm{kcal} / \mathrm{mol} ;{ }^{30}$ some Ce(IV)-hydroxypyrone crystals show some degree of intercomplex $\pi$-stacking interactions, and parent kojic acid has H-bonding capabilities, which can have a stronger influence over the crystallographic packing in the solid state. Interestingly, however, the results from Table 4-9 indicate that the most favorable coordination geometry (albeit by only $0.2-0.3 \mathrm{kcal} / \mathrm{mol}$ and independent of crystal packing influences) is the s-edge-spanned square antiprism, which was not a common geometric outcome in $\mathrm{Pu} / \mathrm{CeL}_{4}$ hydroxypyrone complexes discussed above, but is observed in $\mathrm{Pu} / \mathrm{Ce}(5 \mathrm{LiO}-\mathrm{Me}-3,2-\mathrm{HOPO})_{2}$ complexes. Thus, ambiguities for the "preferred" coordination geometry of $\mathrm{Pu}(\mathrm{IV})$ with bidentate, siderophore analog moieties is still very debatable. However, the trend observed in crystallographic evidence is that the $\mathrm{Pu} / \mathrm{Ce}(\mathrm{IV})$ complexes tend towards the m-edge-spanned trigonal dodecahedron, although continued structural study with unconstrained ligands would be enlightening. 


\subsubsection{Linear Octadentate $\mathrm{TAM}_{4}$ and $\mathrm{TAM}_{2} \mathrm{HOPO}_{2}$ Ligands}

Although many coordination polyhedra geometries were observed in both the $\mathrm{Pu} / \mathrm{Ce}(\mathrm{IV})-\mathrm{HOPO} /$ hydroxypyrone structures examined above, the trigonal dodecahedron appears to be slightly favored over bicapped trigonal prism and square antiprism. With the fundamental coordination studies in hand, the next step in rational ligand design is to tether bidentate moieties together into poly-bidentate moieties designed to mimic the coordination environments seen in the unconstrained coordination complexes. Efforts towards developing high-denticity, poly-bidentate moieties for $f$-element chelation has been ongoing in the Raymond group. ${ }^{4}$ One application of these high-denticity, coordinatively-saturating ligands has been the efficient luminescent sensitization of lanthanide cations, which is highly dependent on the exclusion of water molecules from the primary metal coordination sphere to avoid non-radiative quenching of $\mathrm{Ln}(\mathrm{III})$ excited states. $^{31,32}$ Such ligands designed by the Raymond group for coordinative saturation of the metal center typically consist of either a linear backbone with appended bidentate moieties or a branched polyamine backbone with each branch terminating in another bidentate moiety. ${ }^{4}$

Using the $m$-edge-spanned trigonal dodecahedral coordination polyhedron common to $\mathrm{Ce} / \mathrm{Pu}(\mathrm{IV})-\mathrm{HOPO} /$ hydroxypyrone complexes as a theoretical target geometry, efforts towards designing a new class of linear, tetrakis-bidentate ligands was undertaken. Unlike previous linear octadenate ligands investigated, the bidentate moieties would not be appended to a linear polyamine, but would instead serve as spacers in the ligand, much like the TAM(HOPO $)_{2}$ ligands studied in Chapter 3. Ideally, each chelating moiety would span the $m$-edges of the trigonal dodecahedron, with a central linker spanning one of two 
typically short $a$-edges and two of the eight longer $g$-edges. With these considerations in mind, the $\mathrm{TAM}_{4}$ and $\mathrm{TAM}_{2} \mathrm{HOPO}_{2}$ ligands 4-17a-d were synthesized as shown in Scheme 4-1.

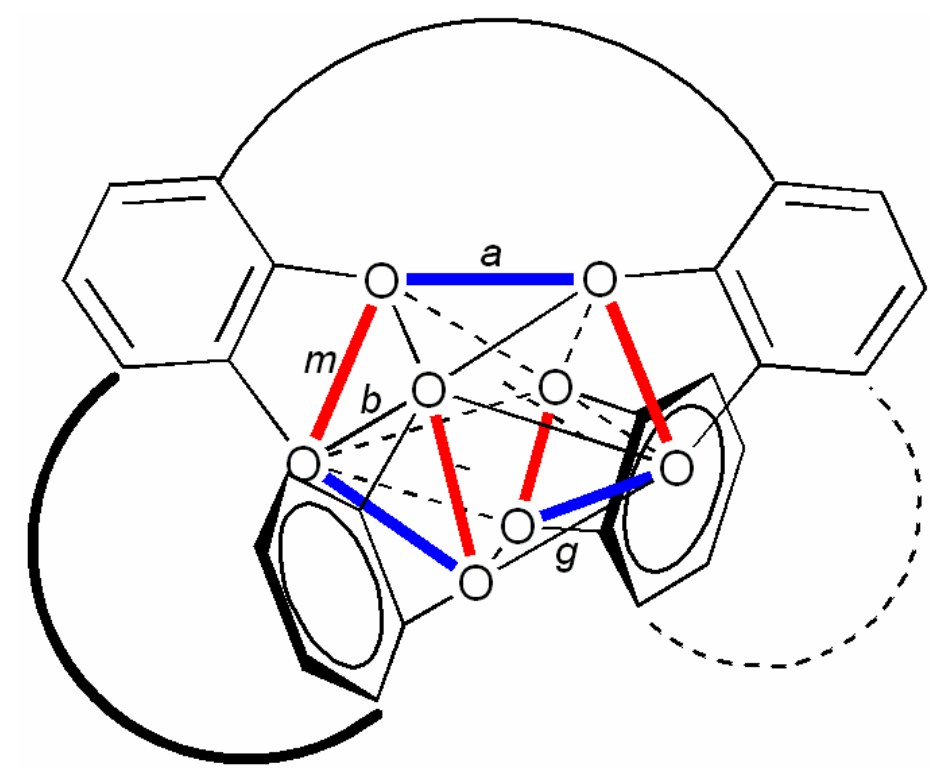

Figure 4-15. Design strategy of linear tetrakis-bidentate ligands for coordinative saturation of $f$-element cations. Chelating moieties are illustrated as catecholates. Ligandand linker-spanned edges are drawn in red and blue respectively.

Ligands 4-17a-d are symmetric and proceeded by a series of slow addition reactions starting with the center linker and appending ligand units sequentially to the ends of the molecule. Each ligand contains a tetradentate, bis-TAM core that can be considered structurally analogous to the $\mathrm{nLi}-\mathrm{Me}-3,2-\mathrm{HOPO}$ ligands $^{33}$ discussed in Chapter 2. The terminal chelating moieties are varied between Me-3,2-HOPO and propyl-substituted TAM moieties. These variations change the overall charge of the ligand when deprotonated (and thus the final charge of a complex at basic $\mathrm{pH}$ ), as well as the ease of deprotonation, as the Me-3,2-HOPO moiety is more acidic than TAM. 


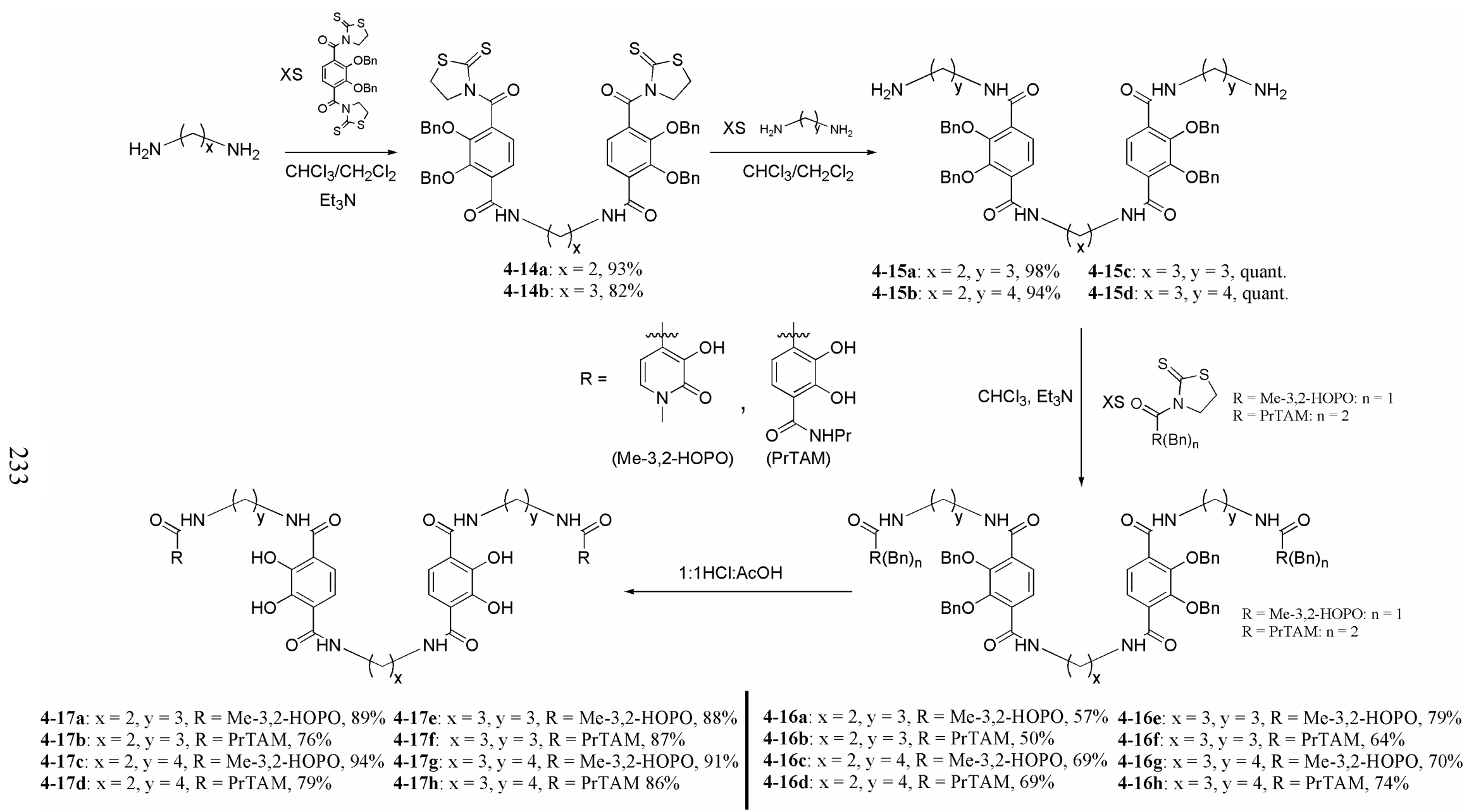

Scheme 4-1. Synthesis of $\mathrm{TAM}_{4}$ and $\mathrm{TAM}_{2} \mathrm{HOPO}_{2}$ ligands. 
It was expected that substitution of Me-3,2-HOPO for terminal TAM moieties would lower the formation constant affinity of these octadentate ligands with $f$-element cations as the results in Chapter 3 would suggest. However, because each ligand 4-17a-h has a tetradentate bis-TAM core, it was still expected that the Me-3,2-HOPO-terminated ligands would exhibit high formation constants nonetheless. Based on the kinetic inertness of the $\mathrm{TAM}(\mathrm{HOPO})_{2}$ ligands in Chapter 3, ligands 4-17a-h are expected to also show slow kinetics and strong chelate effects.

Because of the fluxional coordination chemistry of the spherical $f$-elements and the subtle variations in ionic radius described by the lanthanide and actinide contractions, ${ }^{5}$ the alkyl linker lengths were varied to find hopefully an optimal geometry among the eight octadenate ligands synthesized. The central bridge was typically shorter than the outer linear linkers according to the design strategies discussed above, and because the diamines used for the syntheses of 4-15a-d had reasonable vapor pressures, asymmetric protection strategies were not required, allowing for removal under vacuum of the excess $\alpha, \omega$-proplyene- and -butylene-diamines used in slow addition reactions. Compounds 416a-h were the first major species in Scheme 1 to be isolated cleanly via column chromatography, and were typically mildly hydroscopic, white/beige solids. After aqueous acidic benzyl deprotection ligands 4-17a-h were isolated as beige, amorphous solids that display very poor solubility in organic and aqueous solvents in their neutral, protonated forms, similar to the TAM-containing ligands discussed in Chapter 3.

Drs. Evan Moore and Anthony D'Aléo have in the past several years explored Eu(III) sensitization utilizing 1,2-HOPO chelating moieties in the Raymond Group. ${ }^{34,35}$ In contrast, the Me-3,2-HOPO and TAM moieties can sensitize the near-IR emitting Yb(III) 
ion, and thus ligands 4-17a-h are currently being explored as $\mathrm{Yb}(\mathrm{III})$-sensitizing ligands. To make the $\mathrm{Yb}(\mathrm{III})-(\mathbf{4 - 1 7 a - h})$ complexes with these ligands, aromatic and alkyl amines were used as base, resulting in isolation of only the monoanionic $[\mathrm{YbL}]^{-}$complex, indicating that the ligand only deprotonates one proton per binding moiety on average, independent of whether they are $\mathrm{TAM}_{4}$ or $\mathrm{TAM}_{2} \mathrm{HOPO}_{2}$ ligands. $\mathrm{TAM}_{2} \mathrm{HOPO}_{2}$ ligands 4-17a,c,e,g are hexaprotic and $\mathrm{TAM}_{4}$ ligands 4-17b,d,f,h are octaprotic, and both require full deprotonation if they are to bind in the catecholate binding mode for which these ligands are designed (Figure 4-15). Incomplete deprotonation raises questions as to what coordination mode is being employed in the complex, as the TAM moieties could theoretically bind in a salicylate mode to the $\mathrm{Yb}(\mathrm{III})$ center upon incomplete deprotonation as shown in Figure 4-16 (Me-3,2-HOPO moieties most likely deprotonate fully in the presence of amine bases). ${ }^{36}$ Because the isolated complexes are not polycationic, their solubilities are quite low thanks to the TAM moiety's propensity for poor solubility as observed in Chapter 3. However, because photoluminescence measurements can be carried out at sub-micromolar concentrations, sufficient solubilities of $\mathrm{Yb}(\mathrm{III})-(\mathbf{4 - 1 7 a}-\mathbf{h})$ complexes could be achieved in aqueous media.

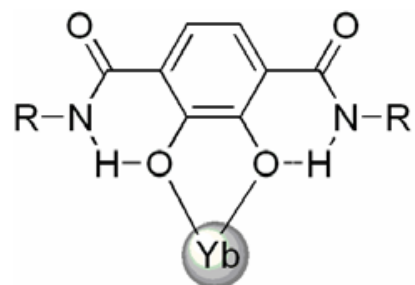

(a)

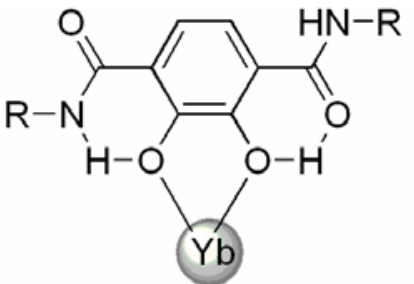

(b)

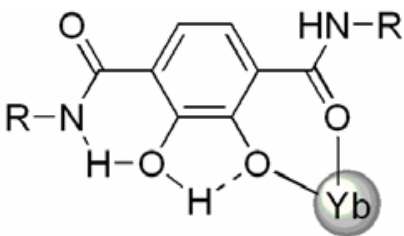

(c)

Figure 4-16. Possible coordination modes for the TAM moiety upon mono and bisdeprotonation: (a) catecholate; (b) protonated catecholate; (c) salicylate.

Despite the binding mode ambiguity that necessarily exists in the absence of crystallographic evidence, ligands $\mathbf{4 - 1 7 a - h}$ are found to efficiently sensitize $\mathrm{Yb}$ (III) 
emission because they effectively block inner sphere water coordination to the $\mathrm{Yb}$ (III) and are also found to be kinetically inert. Necessary equilibration times between the setup and spectrophotometric measurement of the $\mathrm{Yb}(\mathbf{4 - 1 7 a}-\mathbf{h})$ complex solutions are on the order of nine days. ${ }^{37}$ This very slow kinetic behavior is expected for a poly-bidentate ligand and is consistent with the TAM-containing, poly-bidentate ligands explored in Chapter 3.

Competition batch titrations are being carried out to determine the relative affinity of ligands 4-17a-h. These measurements involve monitoring the decrease in photoluminescence of the $\mathrm{Yb}(\mathbf{4 - 1 7 a - h})$ complexes upon incremental addition of known amounts of diethylenetriaminepentaacetic acid (DTPA) to a buffered solution at $\mathrm{pH}$ 6.1, 7.4, and 8.5. By monitoring this decay the relative shift in $\mathrm{pYb}(\Delta \mathrm{pYb})$ compared to the known $\mathrm{pYb}$ value for DTPA can be calculated as described in the Appendix. An example of these analyses is illustrated in Figure 4-17. This work is currently ongoing, but preliminary results for these structures indicate a $\Delta \mathrm{pYb}^{7.4}$ of $c a .0 .9-1.5$ for $\mathrm{TAM}_{2} \mathrm{HOPO}_{2}$ ligands 4-17a,c,e,g and $c a$. 3.3-4.0 for $\mathrm{TAM}_{4}$ ligands $\mathbf{4 - 1 7 b , d , f , h}$. The higher $\Delta \mathrm{pYb}$ for $\mathrm{TAM}_{4}$ ligands is consistent with the stronger metal affinity of TAM compared to Me-3,2HOPO that was established in Chapter 3. The effect of coordination geometry on complex stability is as yet unclear, but the emission spectra of the $\mathrm{Yb}(\mathbf{4 - 1 7 a - h})$ complexes do exhibit structural changes upon changing the terminal binding moieties (HOPO vs. TAM) as well as upon linker length variation. Significantly, however, these linear, octadentate ligands exhibit affinities for the $\mathrm{Yb}(\mathrm{III})$ cation that bests DTPA by between one and four orders of magnitude and also demonstrate high kinetic stability, making them interesting candidates for future study with other lanthanide and actinide 
cations. Their structural variability (linker length/identity/electronics, terminal chelating moiety identity, etc.) allows for many variations to their properties to be easily incorporated in the ligand design.

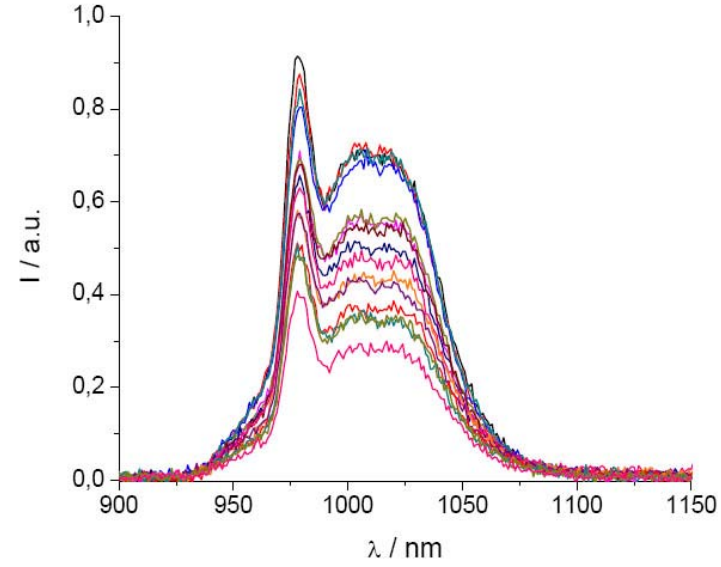

(a)

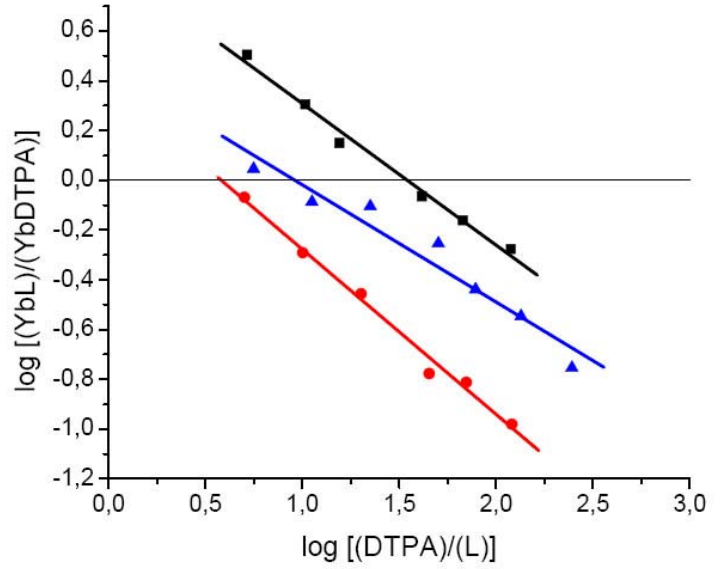

(b)

Figure 4-17. (a) Evolution of the luminescence spectrum of $\mathrm{Yb}(\mathbf{4 - 1 7 g})$ upon addition of DTPA; (b) Competition titration $\log / \log$ plot for $\mathrm{Yb}(\mathbf{4 - 1 7 g})$ against DTPA at varying $\mathrm{pH}$ $(\mathrm{x} \text {-intercepts indicate the difference in } \mathrm{pYb} \text { between ligands and DTPA [pYb(DTPA })^{7.4}=$ 19.40]).

\subsection{Conclusions and Future Directions}

Through a series of crystallographic studies, the coordination behavior of $\mathrm{Pu}(\mathrm{IV})$ and its more accessible, non-radioactive structural analog Ce(IV) have been explored with a variety of simple bidentate ligands that are structurally analogous to catechol and bind in a similar manner. The results of these studies illustrate that $\mathrm{Pu}(\mathrm{IV})$ tends towards a trigonal dodecahedral coordination environment, although studies with $\mathrm{Ce}(\mathrm{IV})$ hydroxypyrone complexes suggest that the precise coordination environment may be easily perturbed and adopt square antiprismatic and bicapped trigonal dodecahedral geometries. Modeling studies suggest that the gas-phase energetic differences between these different conformations are less than $1 \mathrm{kcal} / \mathrm{mol}$, making even weak interactions in 
the crystal lattice capable of bringing about changes in coordination polyhedra. However, in both $\mathrm{Pu}(\mathrm{IV})$ and $\mathrm{Ce}(\mathrm{IV})$ complexes illustrated here, a trigonal-dodecahedral coordination geometry in which the ligands span m-edges seems to be the more commonly-occurring coordination complex geometry. However, MM3 calculations by Dr. Ben Hay raise doubts as to whether this is always the case, or whether what observed in merely a solid state effect. However, we have achieved our goal of expanding the fundamental coordination chemistry knowledge of $\mathrm{Pu}(\mathrm{IV})$, although much more is still needed.

Taking the $m$-edge-spanned trigonal dodecahedral geometry as a target for rational ligand design, a new class of tetrakis-bidentate $\mathrm{TAM}_{4}$ and $\mathrm{TAM}_{2} \mathrm{HOPO}_{2}$ ligands was designed and synthesized. These ligands are currently undergoing analysis as potential $\mathrm{Yb}(\mathrm{III})$ luminescence sensitizers. Early results of these studies indicate that these ligands may exhibit an unanticipated and as yet uncharacterized coordination mode about the $\mathrm{Yb}(\mathrm{III})$ center, but that they exhibit excellent kinetic stability and also have excellent thermodynamic stability, binding $\mathrm{Yb}(\mathrm{III})$ anywhere from one to four orders of magnitude stronger than DTPA.

With many simple bidentate $\mathrm{Pu}(\mathrm{IV})$ structures now characterized, the next step in rational ligand design is to continue the efforts of Gorden et al. ${ }^{14,15}$ by investigating the structure of $\mathrm{Pu}(\mathrm{IV})$ with bis-bidentate ligands that mimic the $m$-edge-spanned trigonal dodecahedral coordination polyhedron observed in the $\mathrm{Pu}(\mathbf{4 - 1 / 4 - 2 / 4 - 3})_{4}$ complexes. Additionally, the thermodynamic titration measurements need to be performed to evaluate the relative stability of the HOPO and hydroxypyrone ligands (and the subsequent bis-bidentate versions thereof) with $\mathrm{Pu}(\mathrm{IV})$ and $\mathrm{Ce}(\mathrm{IV})$, along with 
investigating whether these ligands do indeed show selectivity for $\mathrm{Pu}(\mathrm{IV})$ over other cations.

The development of the new class of $\mathrm{TAM}_{4}$ and $\mathrm{TAM}_{2} \mathrm{HOPO}_{2}$ ligands has opened up a wide variety of ligands for future work. One question that must be addressed is what coordination mode is achieved by incomplete ligand deprotonation and does this change upon full deprotonation? Additionally, the way in which this ligand binds to other lanthanide/actinide cations remains to be investigated. The design of 4-17a-h is also rather simple, with functionalization possible at the chelating moiety and the linkers, thereby potentially varying chelating moiety electronics, ligand solubility, ligand symmetry, and possibly tethering the ligand to solid supports for extraction applications

\subsection{Experimental}

General. Unless otherwise noted, all chemicals were purchased from commercial sources and used as received or synthesized using literature procedures. Me-3,2-HOPOThiaz(Bn) and TAM-Thiaz(Bn $)_{2}$ were synthesized as described in earlier Raymond group publications. ${ }^{9,38,39}$ All reactions brought to reflux were done so with an efficient condenser attached to the reaction flask. NMR spectra were collected using Bruker AMX-400 and AM-400 spectrometers $\left({ }^{1} \mathrm{H} 400 \mathrm{MHz},{ }^{13} \mathrm{C} 100 \mathrm{MHz}\right)$ in $\mathrm{CDCl}_{3}$. Mass spectrometry and elemental analyses were performed at the Microanalytical Facility, College of Chemistry, University of California, Berkeley. Elemental analyses are reported in a "calculated (found)" format. Yields indicate the amount of isolated compound. Purification and synthetic procedures with ${ }^{242} \mathrm{Pu}$ were conducted in a glove box under negative pressure designed for the safe handling of radionuclides. Liquid scintillation counting was 
performed with a Wallac Guardian 1414 liquid scintillation counter, and the scintillation cocktail was Eco-Lume (ICN). Bulk electrolysis using a $\mathrm{Ag} / \mathrm{AgCl}$ reference electrode was conducted in a scintillation vial fitted with a stir bar, a platinum mesh working electrode, an $\mathrm{Ag} / \mathrm{AgCl}$ reference electrode, and a platinum counter electrode. An IBM Voltammic analyzer was used to adjust the potential. Water was distilled and further purified by a Millipore cartridge system (resistivity $18 \times 10^{6} \Omega$ ).

\subsubsection{Pu(IV) Stock Solution Preparation}

${ }^{242} \mathrm{Pu}$ was received from Oak Ridge National Laboratory as $\mathrm{PuO}_{2}$ (lot $\mathrm{Pu}-242-327 \mathrm{~A}$, 99.93 wt. \% of metal ${ }^{242} \mathrm{Pu}$ ). The solid was dissolved in concentrated nitric acid with heating. The ${ }^{242} \mathrm{Pu}$ stock solution was loaded onto a nitrate-activated 400 mesh Dowex anion exchange resin column and washed with several column volumes of $7.5 \mathrm{M} \mathrm{HNO}_{3}$

to remove any daughter products; the ${ }^{242} \mathrm{Pu}$ was isolated as the nitrate. The plutonium was then eluted with $0.4 \mathrm{M} \mathrm{HCl}$ with a trace of $\mathrm{HF}$ to strip the $\mathrm{Pu}(\mathrm{IV})$ from the column. The plutonium eluent was then transferred to a round-bottomed boiling flask fitted with a condensing arm and $\mathrm{KOH}$ traps to collect acid vapors, and boiled to dryness. The remaining salt was dissolved in concentrated $\mathrm{HNO}_{3}$ and boiled for three hours to digest any organic material present in the sample. The solution was concentrated by evaporation. Concentrated perchloric acid was added to the solution, and it was boiled for three hours. Fresh perchloric acid was continually added to maintain the solution volume until the nitrate was removed. The resulting characteristically yellow $\mathrm{Pu}(\mathrm{VI})$ solution in concentrated perchloric acid was diluted with water to reach a final concentration of approximately $1 \mathrm{M}$ perchloric acid. This solution was adjusted to $\mathrm{Pu}(\mathrm{III})$ by electrochemical reduction and then oxidized to $\mathrm{Pu}(\mathrm{IV})$ before use in reactions. An 
aliquot of this solution was diluted with deionized water to $1.0 \mathrm{M} \mathrm{HClO}_{4}$ and the $\mathrm{Pu}$ concentration was determined by alpha liquid scintillation to be $0.006 \mathrm{M}$.

\subsubsection{Bidentate Ligand Synthesis}

5-Bromo-2-ethyl-3-hydroxy-pyran-4-one, (5-Bromo-ethyl maltol), 4-5. This synthesis followed a method analogous to the published synthesis of 5-bromo-2-methyl-

3-hydroxypyran-4-one (bromomaltol, 4-3). ${ }^{26}$ A suspension of ethyl maltol (Aldrich, 5.00 g, $35.7 \mathrm{mmol})$, NBS (8.92 g, $50.1 \mathrm{mmol})$, and benzoyl peroxide (10 mg, cat.) was stirred in $45 \mathrm{~mL}$ of $\mathrm{CCl}_{4}$ for 45 minutes in a $90{ }^{\circ} \mathrm{C}$ water batch while being irradiated by a 500 W incandenscent bulb. The mixture was filtered while still hot and the filtrate was cooled at $4{ }^{\circ} \mathrm{C}$ for 20 hours and the solution was decanted away from an orange oil that had formed. The solution was put into a freezer for a day, and the light yellow precipitate that formed was recrystallized from $\mathrm{CCl}_{4}$, yielding $2.01 \mathrm{~g}$ of a white, crystalline solid, $26 \%$. $\mathrm{C}_{7} \mathrm{H}_{7} \mathrm{O}_{3} \mathrm{Br}: \mathrm{C}: 38.38$ (38.09); $\mathrm{H}: 3.22$ (3.19). ${ }^{1} \mathrm{H}$ NMR: $\delta 1.23$ (t, $\left.\mathrm{CH}_{3}, \mathrm{~J}=7.6 \mathrm{~Hz}, 3 \mathrm{H}\right), \delta$ 2.75 (quartet, $\left.\mathrm{CH}_{2}, \mathrm{~J}=7.6 \mathrm{~Hz}, 2 \mathrm{H}\right), \delta 6.67(\mathrm{~s}, \mathrm{br}, \mathrm{OH}, 1 \mathrm{H}), \delta 8.06(\mathrm{~s}, \mathrm{CH}, 1 \mathrm{H}) .{ }^{13} \mathrm{C}$ NMR: $\delta 10.93,21.88,110.66,141.14,153.06,154.12,169.29 . \mathrm{MS}(\mathrm{FAB}+): \mathrm{m} / \mathrm{z} 219,221$ (MH+). MP: $120-122^{\circ} \mathrm{C}$.

3-Hydroxy-6-iodomethyl-pyran-4-one, (Iodokojic acid), 4-8. Chlorokojic acid $(2.00 \mathrm{~g}, 12.4 \mathrm{mmol})$ and $\mathrm{NaI}(4.69 \mathrm{~g}, 31.2 \mathrm{mmol})$ were stirred in acetone at $45^{\circ} \mathrm{C}$ for 24 hours. The solvent was removed from the dark solution under vacuum and the residue was suspended in $100 \mathrm{~mL}$ of water and filtered. The solids were rapidly stirred in $100 \mathrm{~mL}$ of 1:1 water:EtOAc at $50{ }^{\circ} \mathrm{C}$ until no solids were left in solution. The layers were separated, and the volume of the EtOAc was reduced to recrystallize $0.816 \mathrm{~g}$ of iodokojic acid as a beige solid, $26 \% .{ }^{1} \mathrm{H}$ NMR (DMSO- $\left.d_{6}\right): \delta 4.38\left(\mathrm{~s}, \mathrm{CH}_{2}, 2 \mathrm{H}\right), \delta 6.54(\mathrm{~s}, \mathrm{CH}, 1 \mathrm{H})$, 
$\delta 8.08(\mathrm{~s}, \mathrm{CH}, 1 \mathrm{H}) .{ }^{13} \mathrm{C}$ NMR (DMSO-d 6 ): $\delta-1.22,111.38,139.90,145.91,164.42$, 173.85. The compound was used in complexation reactions without further analysis.

\subsubsection{Synthesis/Crystallization of Ce-Hydroxypyrone Complexes}

General Synthesis: Unless otherwise noted, Ce(IV)-hydroxypyrone complexes were synthesized in the following manner: $15-50 \mathrm{mg}$ of $\mathrm{Ce}(\mathrm{acac})_{4}(\mathrm{acac}=$ acetylacetonate $)(1$ equivalent) was dissolved in $1.5-2 \mathrm{~mL}$ of $\mathrm{MeOH}$ under nitrogen, and a solution of hydroxypyrone (approximately 10 equivalents) dissolved in $4 \mathrm{~mL}$ of $\mathrm{MeOH}$ was added, stirred rapidly to combine thoroughly, then allowed to stand at room temperature under nitrogen without stirring for 1 hour. The precipitated solids were filtered on a membrane filter (0.45 micron), washed with generous amounts of $\mathrm{MeOH}$ to remove excess ligand, and the solids were allowed to dry under aspiration and then under vacuum. All the solids isolated in this manner were very dark microcrystalline solids and ranged in color from deep red to purple/black. NMR data could not be collected for these compounds because of their very low solubility and their instability in solvent that would actually dissolve them (DMF, DMSO). Mass spectrometry data (when collectable) typically showed only the presence of $\mathrm{Ce}(\mathrm{III})$ decomposition products.

Ce(Maltol) $)_{4}, \mathbf{C e}(4-2)_{4}$. A solution of $\mathrm{Ce}(\mathrm{acac})_{4}(50 \mathrm{mg}, 0.093 \mathrm{mmol})$ in $2.5 \mathrm{~mL}$ of $\mathrm{MeOH}$ was added to a stirred solution of maltol (4-2, Aldrich, $48 \mathrm{mg}, 0.38 \mathrm{mmol})$ in 2.5 $\mathrm{mL}$ of $\mathrm{MeOH}$. Stirring was stopped and the solution was allowed to stand in the freezer for 2 days, precipitating out a dark microcrystalline solid which was filtered, washed with cold $\mathrm{MeOH}$. The solid was dried under vacuum yielding $52 \mathrm{mg}$ of a purple/black powder, 87\%. C: ${ }_{24} \mathrm{H}_{20} \mathrm{O}_{12} \mathrm{Ce}: \mathrm{C}: 45.00$ (44.78); H: 3.15 (3.32). ${ }^{1} \mathrm{H}$ NMR: $\delta 2.37$ (s, $\left.\mathrm{CH}_{3}, 12 \mathrm{H}\right), \delta$ $6.45(\mathrm{~d}$, arom. $H, \mathrm{~J}=4.8 \mathrm{~Hz}, 4 \mathrm{H}), \delta 7.69(\mathrm{~d}$, arom. $H, \mathrm{~J}=4.8 \mathrm{~Hz}, 4 \mathrm{H})$. This compound is 
too insoluble for ${ }^{13} \mathrm{C}$ NMR. MS $(\mathrm{FAB}+)$ : $\mathrm{m} / \mathrm{z} 766\left(\mathrm{CeL}_{5}+\right)$. X-ray quality crystals were grown by slow evaporation of a $\mathrm{CH}_{2} \mathrm{Cl}_{2}$ solution of this product.

Ce(Bromo-maltol $)_{4}, \mathbf{C e}(4-3)_{4} \cdot \mathrm{Ce}(\mathrm{acac})_{4}(100 \mathrm{mg}, 0.19 \mathrm{mmol})$ and bromo-maltol ${ }^{26}$ (4$3,153 \mathrm{mg}, 0.75 \mathrm{mmol}$ ) were dissolved in $5 \mathrm{~mL}$ of $\mathrm{MeOH}$ at room temperature. A purple/black microcrystalline solid quickly formed, and after stirring for three hours and cooling in a freezer the solid product was filtered and washed with cold $\mathrm{MeOH}$. The solid was dried under vacuum yielding $157 \mathrm{mg}$ of a purple/black powder which was the methanol solvate, $85 \%$. $\mathrm{C}_{24} \mathrm{H}_{16} \mathrm{O}_{12} \mathrm{Br}_{4} \mathrm{Ce} \cdot \mathrm{CH}_{3} \mathrm{OH}$ : C: 30.39 (30.51); H: 2.04 (2.00). ${ }^{1} \mathrm{H}$ NMR: $\delta 2.37\left(\mathrm{~s}, \mathrm{CH}_{3}, 12 \mathrm{H}\right), \delta 3.49\left(\mathrm{~s}, \mathrm{CH}_{3} \mathrm{OH}, 3 \mathrm{H}\right), \delta 8.01$ (s, arom. $\left.H, 4 \mathrm{H}\right)$. This compound is too insoluble for ${ }^{13} \mathrm{C}$ NMR. MS (FAB+): m/z $548\left(\mathrm{CeL}_{2}+\right), 1300\left(\mathrm{Ce}_{2} \mathrm{~L}_{5}+\right)$. $\mathrm{X}$-ray quality crystals were grown by slow evaporation of a $\mathrm{CH}_{2} \mathrm{Cl}_{2}$ solution.

Ce(Ethyl-maltol $)_{4}, \mathrm{Ce}(4-4)_{4}$. A solution of $\mathrm{Ce}(\mathrm{acac})_{4}(0.106 \mathrm{~g}, 0.198 \mathrm{mmol})$ and ethylmaltol (Aldrich, $0.111,0.792 \mathrm{mmol}$ ) in $5 \mathrm{~mL}$ of $\mathrm{CH}_{2} \mathrm{Cl}_{2}$ was stirred overnight at room temperature under nitrogen. The purple solution was filtered through glass wool, and the solvent was removed on a rotary evaporator. The residue was dissolved in $2.5 \mathrm{~mL}$ of $\mathrm{CH}_{2} \mathrm{Cl}_{2}$, placed in a vial, allowed to evaporate to $1 \mathrm{~mL}$, then put in the freezer. The resultant crystals were filtered and allowed to dry in air to yield $91 \mathrm{mg}$ of purple/black crystals (66\%). $\mathrm{C}_{28} \mathrm{H}_{28} \mathrm{O}_{12} \mathrm{Ce}: \mathrm{C}: 48.28$ (48.40); $\mathrm{H}: 4.05$ (3.97). ${ }^{1} \mathrm{H}$ NMR: $\delta 1.03$ (t, $\mathrm{CH}_{3}$, $\mathrm{J}=7.2 \mathrm{~Hz}, 12 \mathrm{H}$ ), $\delta 2.71$ (quartet, $\left.\mathrm{CH}_{2}, \mathrm{~J}=7.2 \mathrm{~Hz}, 8 \mathrm{H}\right), \delta 6.47(\mathrm{~d}, \mathrm{CH}, \mathrm{J}=5.2 \mathrm{~Hz}, 4 \mathrm{H}), \delta$ $7.71(\mathrm{~d}, \mathrm{CH}, \mathrm{J}=5.2 \mathrm{~Hz}, 4 \mathrm{H}) .{ }^{13} \mathrm{C}$ NMR: $\delta$ 11.27, 21.37, 111.28, 153.24, 153.76 (two ring carbons were not visible). MS (FAB+): m/z $418\left(\mathrm{CeL}_{2}+\right), 975\left(\mathrm{Ce}_{2} \mathrm{~L}_{5}+\right)$. MP: 204-206 ${ }^{\circ} \mathrm{C}$. X-ray quality crystals were grown from evaporation of a solution of the complex in $\mathrm{MeOH}$ at room temperature. 
Ce(5-Bromo-ethyl maltol $)_{4}, \mathrm{Ce}(4-5)_{4}$. A solution of $\mathrm{Ce}(\mathrm{acac})_{4}(0.152 \mathrm{~g}, 0.283$ mmol) and bromo-ethylmaltol $(0.249 \mathrm{~g}, 1.14 \mathrm{mmol})$ in $6 \mathrm{~mL}$ of $\mathrm{MeOH}$ was stirred overnight at room temperature under nitrogen. The solvent was removed under vacuum and the residue was re-dissolved in $10 \mathrm{~mL}$ of $\mathrm{CH}_{2} \mathrm{Cl}_{2}$, filtered through glass wool, then slowly evaporated to approximately $3 \mathrm{~mL}$ and put in a freezer to crystallize. The resultant crystals were filtered and dried in air to yield $156 \mathrm{mg}$ of purple/black crystals (54\%). $\mathrm{C}_{28} \mathrm{H}_{24} \mathrm{O}_{12} \mathrm{Br}_{4} \mathrm{Ce}: \mathrm{C}: 33.22$ (33.13); H: 2.39 (2.11). ${ }^{1} \mathrm{H}$ NMR: $\delta 1.14$ (s, br, $\left.\mathrm{CH}_{3}, 12 \mathrm{H}\right), \delta$ 2.76 (s, br, $\left.\mathrm{CH}_{2}, 8 \mathrm{H}\right), \delta 8.03$ (s, $\left.\mathrm{CH}, 4 \mathrm{H}\right) .{ }^{13} \mathrm{C}$ NMR: $\delta 11.13,21.49,152.32,154.04$ (three ring carbons were not visible by ${ }^{13} \mathrm{C}$ NMR). $\mathrm{MS}(\mathrm{FAB}+): \mathrm{m} / \mathrm{z} 576\left(\mathrm{CeL}_{2}+\right), 1370$ $\left(\mathrm{Ce}_{2} \mathrm{~L}_{5}+\right)$. X-ray quality crystals were grown by evaporation of a solution of the complex in $\mathrm{MeOH}$ at room temperature.

Ce(Kojate) $)_{4}$, Ce(4-6)4. Purple solid, 75\%. $\mathrm{C}_{24} \mathrm{H}_{20} \mathrm{O}_{16} \mathrm{Ce}$ : C: 40.92 (40.69); H: 2.86 (3.03). X-ray quality crystals could be found in the precipitate from the $\mathrm{MeOH}$ solution described above. Crystals could also be grown using a U-tube setup in which a nearlysaturated solution of kojic acid and a solution of $\mathrm{Ce}(\mathrm{acac})_{4}$, both in THF, were allowed to diffuse slowly through a clean layer of dioxane at room temperature. Crystals grown in this latter method had the same unit cell as those precipitated from $\mathrm{MeOH}(\mathrm{L}: \mathrm{M} \approx 10: 1)$.

$\mathrm{Ce}(\text { Chlorokojate })_{4}, \mathrm{Ce}(4-7)_{4}$. (Chlorokojic acid was made by the method of Hider et al. ${ }^{40}$ ) Brown solid, 86\%. $\mathrm{C}_{24} \mathrm{H}_{16} \mathrm{Cl}_{4} \mathrm{O}_{12} \mathrm{Ce}$ : C: 37.04 (36.75); $\mathrm{H}: 2.07$ (2.07). X-ray quality crystals of this complex were grown from a 3-layer setup in which a nearly-saturated solution of chlorokojic acid in THF was layered on a clean layer of dioxane, which was layered over a solution of $\mathrm{Ce}(\mathrm{acac})_{4}$ in $\mathrm{CHCl}_{3}$ at $4{ }^{\circ} \mathrm{C}(\mathrm{M}: \mathrm{L} \approx 1: 10)$. 
Ce(Iodokojate) $)_{4}, \mathbf{C e}(4-8)_{4}$. Brown solid, 75\%. $\mathrm{C}_{24} \mathrm{H}_{16} \mathrm{I}_{4} \mathrm{O}_{12} \mathrm{Ce}: \mathrm{C}: 25.19$ (25.28); H: $1.41(1.46)$.

Ce(Alomaltol) 4 , Ce(4-9) 4. (Alomaltol was made by the method of Hider et al. ${ }^{40}$ ) Red solid, 93\%. $\mathrm{C}_{24} \mathrm{H}_{20} \mathrm{O}_{12} \mathrm{Ce}: \mathrm{C}: 45.00$ (44.64); $\mathrm{H}: 3.15$ (3.36).

Ce(2-Nitro-alomaltol) $)_{4}, \mathbf{C e}(4-10)_{4}$. (2-Nitroalomaltol was made by the method of Eiden et al. ${ }^{41}$ ) Brown solid, 81\%. $\mathrm{C}_{24} \mathrm{H}_{16} \mathrm{~N}_{4} \mathrm{O}_{20} \mathrm{Ce}: \mathrm{C}: 35.13$ (35.42); H: 1.97 (2.16); N: $6.83(6.65)$.

Ce(2-Hydroxymethyl-alomaltol) $4, \quad \mathbf{C e}(4-11)_{4} \cdot \quad$ (2-Hydroxymethylalomaltol was made by the method of Hider et $a l^{42}$ ) This complex was not isolated for elemental analysis due to its higher solubility as compared to the other Ce(IV)-hydroxypyrone complexes. X-ray quality crystals of this complex were grown from a 3-layer setup in which a nearly-saturated solution of hydroxymethylalomaltol in THF was layered on a clean layer of dioxane, which was layered over a solution of $\mathrm{Ce}(\mathrm{acac})_{4}$ in $\mathrm{CHCl}_{3}$ at $4{ }^{\circ} \mathrm{C}$ $(\mathrm{M}: \mathrm{L} \approx 1: 10)$.

Ce(2-Bromo-kojate) $)_{4}, \mathbf{C e}(4-12)_{4} \cdot$ (2-Bromokojic acid was made by the method of Kagan et al. ${ }^{43}$ ) Black solid, 74\%. $\mathrm{C}_{24} \mathrm{H}_{16} \mathrm{Br}_{4} \mathrm{O}_{16} \mathrm{Ce}: \mathrm{C}: 28.26$ (27.98); H: 1.58 (1.70).

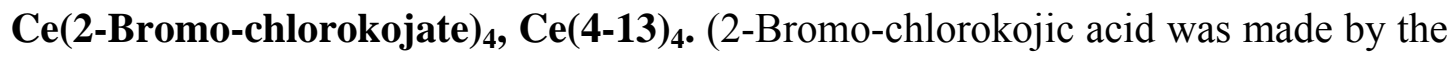
method of Kagan et al. ${ }^{43}$ ) Purple/brown solid, 74\%. $\mathrm{C}_{24} \mathrm{H}_{12} \mathrm{Br}_{4} \mathrm{Cl}_{4} \mathrm{O}_{12} \mathrm{Ce}: \mathrm{C}: 26.35$ (26.37); H: 1.11 (0.97).

\subsubsection{Crystallization of Pu(IV) Complexes}

$\mathbf{P u}(\mathbf{1 , 2 - H O P O})_{4}, \mathbf{P u}(4-1)_{4}$. To a solution of 1,2-HOPO (4-1, $0.5 \mathrm{mg}, 5.5 \times 10^{-3}$ mmol) in $100 \mu \mathrm{L}$ of deionized $\mathrm{H}_{2} \mathrm{O}$ at $40{ }^{\circ} \mathrm{C}$ was added $6 \mathrm{mM} \mathrm{Pu}(\mathrm{IV})$ in $c a .1 \mathrm{M} \mathrm{HClO} 4$ $\left(200 \mu \mathrm{L}, 1.2 \times 10^{-3} \mathrm{mmol}, 1: 4.1 \mathrm{M}: \mathrm{L}\right)$ and $2 \mu \mathrm{L}$ of $0.2 \mathrm{M} \mathrm{NaOH}$ was added. This solution 
was sealed in a plastic tube, and after a period of forty-eight hours, X-ray quality crystals formed as dark blocks and were removed for structural analysis.

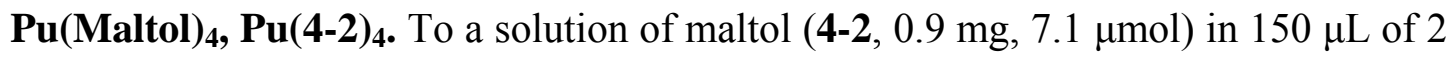
$\mathrm{M} \mathrm{NH}_{4} \mathrm{OAc}$ and $250 \mu \mathrm{L}$ of $\mathrm{MeOH}$ was added $298 \mu \mathrm{L}(1.8 \mu \mathrm{mol})$ of $6 \mathrm{mM} \mathrm{Pu}(\mathrm{IV})$ in $c a .1$ $\mathrm{M} \mathrm{HClO}_{4}(\mathrm{M}: \mathrm{L}=1: 3.9)$. The solution turned orange and was allowed to evaporate slowly over three days at room temperature, depositing red crystals from which X-ray quality samples were removed for structural analysis.

$\mathbf{P u}(\text { Bromo-maltol) })_{4}, \mathbf{P u}(4-3)_{4}$. To a solution of bromo-maltol ${ }^{26}$ (4-3, $1.6 \mathrm{mg}, 7.8$ $\mu \mathrm{mol})$ in $230 \mu \mathrm{L}$ of $2 \mathrm{M} \mathrm{NH}_{4} \mathrm{OAc}$ and $500 \mu \mathrm{L}$ of $\mathrm{MeOH}$ was added $327 \mu \mathrm{L}(1.9 \mu \mathrm{mol})$ of $6 \mathrm{mM} \mathrm{Pu}(\mathrm{IV})$ in ca. $1 \mathrm{M} \mathrm{HClO}_{4}(\mathrm{M}: \mathrm{L}=1: 4.1)$. The solution turned orange and was allowed to evaporate slowly over three days, depositing red crystal clusters from which X-ray quality crystals were cut for structural analysis.

\subsubsection{Synthesis of Octadentate $\mathrm{TAM}_{4}$ and $\mathrm{TAM}_{2} \mathrm{HOPO}_{2}$ Ligands}

Pr-TAM-Thiaz(Bn) 2 . A solution of propylamine $(0.80 \mathrm{~mL}, 9.7 \mathrm{mmol})$ and $\mathrm{Et}_{3} \mathrm{~N}(1.4$ $\mathrm{mL}, 10 \mathrm{mmol}$ ) in $300 \mathrm{~mL}$ of $\mathrm{CHCl}_{3}$ was added dropwise over 1 day to a stirred solution of TAM-Thiaz(Bn $)_{2}$ and $\mathrm{Et}_{3} \mathrm{~N}(1.4 \mathrm{~mL}, 10 \mathrm{mmol})$ in $80 \mathrm{~mL}$ of $\mathrm{CH}_{2} \mathrm{Cl}_{2}$. The solution was washed with $1 \mathrm{M} \mathrm{HCl}(2 \times 100 \mathrm{~mL}), 1 \mathrm{M} \mathrm{NaOH}$ in $20 \%$ sat. brine, sat. brine, dried with $\mathrm{Na}_{2} \mathrm{SO}_{4}$, and the solvent was removed under vacuum. The residue was dissolved in 70 $\mathrm{mL}$ of $\mathrm{CH}_{2} \mathrm{Cl}_{2}$ and eluted on a silica column with $\mathrm{CH}_{2} \mathrm{Cl}_{2}$ to remove unreacted TAMThiaz $(\mathrm{Bn})_{2}$, then $5 \% \mathrm{MeOH}$ in $\mathrm{CH}_{2} \mathrm{Cl}_{2}$ to remove the desired yellow product with $\mathrm{R}_{\mathrm{f}}=$ 0.28. After solvent removal this yielded $4.50 \mathrm{~g}, 89 \%$. ${ }^{1} \mathrm{H}$ NMR: $\delta 0.83$ (t, $\mathrm{CH}_{3}, \mathrm{~J}=7.2$ $\mathrm{Hz}, 3 \mathrm{H}), \delta 1.37$ (sextet, $\left.\mathrm{CH}_{2}, \mathrm{~J}=7.2 \mathrm{~Hz}, 2 \mathrm{H}\right), \delta 2.95\left(\mathrm{t}, \mathrm{CH}_{2}, \mathrm{~J}=7.2 \mathrm{~Hz}, 2 \mathrm{H}\right), \delta 3.23$ (quartet, $\mathrm{CH}_{2}, \mathrm{~J}=7.2 \mathrm{~Hz}, 2 \mathrm{H}$ ), $\delta 4.39\left(\mathrm{t}, \mathrm{CH}_{2}, \mathrm{~J}=7.2 \mathrm{~Hz}, 2 \mathrm{H}\right), \delta 5.11$ (s, benzyl $H, 2 \mathrm{H}$ ), $\delta$ 
5.14 (s, benzyl $H, 2 \mathrm{H}), \delta 7.23(\mathrm{~d}$, TAM $H, \mathrm{~J}=8.0 \mathrm{~Hz}, 1 \mathrm{H}), \delta$ 7.35-7.39 (m, arom. $H$, $10 \mathrm{H}), \delta 7.78(\mathrm{~s}, \mathrm{br}, \mathrm{N} H, 1 \mathrm{H}), \delta 7.95(\mathrm{~d}, \mathrm{TAM} H, \mathrm{~J}=8.0 \mathrm{~Hz}, 1 \mathrm{H}) .{ }^{13} \mathrm{C}$ NMR: $\delta 11.72$, $22.64,28.87,41.72,55.74,76.28,77.13,124.56,127.16,128.10,128.61,128.83,128.99$, $129.09,130.30,133.50,135.88,137.15,149.43,150.25,164.16,167.03,201.54$. This compound was used without further characterization in subsequent reactions.

General Synthesis of En/Pr(TAM-Thiaz) $)_{2}(B n)_{4}$, 4-14a,b: A solution of 5-7.5 mmol ( 1 equivalent) of the ethylene or propylene diamine and 2 equivalents of $\mathrm{Et}_{3} \mathrm{~N}$ dissolved in at least $150 \mathrm{~mL}$ of $\mathrm{CHCl}_{3}$ was added dropwise over 1 day to a stirred solution of $10-15$ equivalents of TAM-Thiaz $(\mathrm{Bn})_{2}$ and another 2 equivalents of $\mathrm{Et}_{3} \mathrm{~N}$ dissolved in at most $100 \mathrm{~mL}$ of $\mathrm{CH}_{2} \mathrm{Cl}_{2}$. The resulting solution was washed with $1 \mathrm{M} \mathrm{HCl}(2 \times 100 \mathrm{~mL}), 1 \mathrm{M}$ $\mathrm{NaOH}$ in $20 \%$ sat. brine $\left(4 \times 100 \mathrm{~mL}\right.$ ), sat. brine, dried with $\mathrm{Na}_{2} \mathrm{SO}_{4}$ and the solvent was removed under vacuum. The residue was dissolved in a minimum amount of $\mathrm{CH}_{2} \mathrm{Cl}_{2}$ and eluted with $\mathrm{CH}_{2} \mathrm{Cl}_{2}$ to remove unreacted TAM-Thiaz $(\mathrm{Bn})_{2}$ and residual free thiazoline. The product was then collected as a yellow band using $2 \% \mathrm{MeOH}$ in $\mathrm{CH}_{2} \mathrm{Cl}_{2}$ for 4-14a and 3:1 EtOAc/hexanes for 4-14b. These products were pure by NMR and used without further characterization in subsequent reactions.

En(TAM-Thiaz) $)_{\mathbf{2}}(\mathbf{B n})_{4}$, 4-14a. Yellow solid, 93\%. ${ }^{1} \mathrm{H}$ NMR: $\delta 2.93\left(\mathrm{t}, \mathrm{CH}_{2}, \mathrm{~J}=7.2\right.$ $\mathrm{Hz}, 4 \mathrm{H}), \delta 3.32-3.33\left(\mathrm{~m}, \mathrm{CH}_{2}, 4 \mathrm{H}\right), \delta 4.37\left(\mathrm{t}, \mathrm{CH}_{2}, \mathrm{~J}=7.2 \mathrm{~Hz}, 4 \mathrm{H}\right), \delta 5.03$ (s, benzyl $H$, 4H), $\delta 5.06$ (s, benzyl H, 4H), $\delta 7.21$ (d, TAM $H, \mathrm{~J}=8.4 \mathrm{~Hz}, 2 \mathrm{H}), \delta$ 7.25-7.30 (m, arom. $H, 10 \mathrm{H}), \delta$ 7.31-7.38 (m, arom. $H, 10 \mathrm{H}), \delta 7.88(\mathrm{~d}, \mathrm{TAM} H, \mathrm{~J}=8.4 \mathrm{~Hz}, 2 \mathrm{H}), \delta 7.94(\mathrm{~s}, \mathrm{br}$, $\mathrm{NH}, 2 \mathrm{H})$.

$\operatorname{Pr}\left(\right.$ TAM-Thiaz) 2 (Bn) 4 , 4-14b. Yellow solid, 82\%. ${ }^{1} \mathrm{H}$ NMR: $\delta 1.25$ (quintet, $\mathrm{CH}_{2}, \mathrm{~J}$ $=7.2 \mathrm{~Hz}, 2 \mathrm{H}$ ), $\delta 2.94\left(\mathrm{t}, \mathrm{CH}_{2}, \mathrm{~J}=7.2 \mathrm{~Hz}, 4 \mathrm{H}\right.$ ), $\delta 3.16$ (quartet, $\mathrm{CH}_{2}, \mathrm{~J}=6.0 \mathrm{~Hz}, 4 \mathrm{H}$ ), $\delta$ 
$4.37\left(\mathrm{t}, \mathrm{CH}_{2}, \mathrm{~J}=7.2 \mathrm{~Hz}, 4 \mathrm{H}\right.$ ), $\delta 5.12$ (s, benzyl $H, 4 \mathrm{H}$ ), $\delta 5.16$ (s, benzyl $H, 4 \mathrm{H}$ ), $\delta 7.22$ $(\mathrm{d}, \mathrm{TAM} H, \mathrm{~J}=8.4 \mathrm{~Hz}, 2 \mathrm{H}), \delta$ 7.32-7.39 (m, arom. $H, 20 \mathrm{H}), \delta$ 7.85-7.88 (m, NH + TAM $H, 4 \mathrm{H})$.

General Synthesis of En/Pr(TAM-pr/bu- $\left.\mathbf{N H}_{2}\right)_{2}(\mathbf{B n})_{4}$, 4-15a-d: A solution of 1-2 g of of 4-14a,b (1-2 mmol, 1 equivalent) dissolved in 300-400 $\mathrm{mL}$ of $\mathrm{CHCl}_{3}$ was added dropwise over 1 day to a solution of 10 equivalents of the appropriate $\alpha, \omega$-diamine dissolved in $5 \mathrm{~mL}$ of $\mathrm{CH}_{2} \mathrm{Cl}_{2}$. The resulting solution was washed with $1 \mathrm{M} \mathrm{NaOH}$ in $20 \%$ sat. brine $(4 \times 50 \mathrm{~mL})$, sat. brine, dried with $\mathrm{Na}_{2} \mathrm{SO}_{4}$ and the solvent and excess diamine were removed under vacuum to yield the diamine product. These compounds were used in subsequent reactions without further purification.

En(TAM-pr-NH $)_{2} \mathbf{2}_{\mathbf{2}}(\mathbf{B n})_{4}$, 4-15a. Beige solid, 98\%. ${ }^{1} \mathrm{H}$ NMR: $\delta 1.47$ (quintet, br, $\mathrm{CH}_{2}$ $\left.+\mathrm{NH}_{2}, \mathrm{~J}=6.4 \mathrm{~Hz}, 8 \mathrm{H}\right), \delta 2.61\left(\mathrm{t}, \mathrm{CH}_{2}, \mathrm{~J}=6.4 \mathrm{~Hz}, 4 \mathrm{H}\right), \delta 3.33-3.38\left(\mathrm{~m}, \mathrm{CH}_{2}, 8 \mathrm{H}\right), \delta 5.06$ (s, benzyl $H, 4 \mathrm{H}$ ), $\delta 5.10$ (s, benzyl $H, 4 \mathrm{H}$ ), $\delta 7.30-7.38$ (m, arom. $H, 20 \mathrm{H}), \delta 7.82-7.90$ (m, TAM $H+\mathrm{NH}, 6 \mathrm{H}), \delta 7.95(\mathrm{~s}, \mathrm{NH}, 2 \mathrm{H}) .{ }^{13} \mathrm{C}$ NMR: $\delta 32.90,37.62,39.78,77.43$, $126.50,126.72,128.69,128.89,129.05,129.15,129.25,130.63,130.93,135.57,135.83$, 150.43, 150.58, 164.38, 165.03. MS (FAB+): m/z 893.5 (MH+). MP: $158-159^{\circ} \mathrm{C}$.

En(TAM-bu-NH $\left.)_{2}\right)_{2}(\mathbf{B n})_{4}$, 4-15b. Pasty, beige solid, 94\%. ${ }^{1} \mathrm{H}$ NMR: $\delta 1.34(\mathrm{t}, \mathrm{br}$, $\left.\mathrm{CH}_{2}+\mathrm{NH}_{2}, \mathrm{~J}=3.2 \mathrm{~Hz}, 10 \mathrm{H}\right), \delta 2.60\left(\mathrm{t}, \mathrm{CH}_{2}, \mathrm{~J}=6.4 \mathrm{~Hz}, 4 \mathrm{H}\right), \delta 3.27\left(\mathrm{~d}, \mathrm{CH}_{2}, \mathrm{~J}=5.6 \mathrm{~Hz}\right.$, $4 \mathrm{H}), \delta 3.37\left(\mathrm{~d}, \mathrm{CH}_{2}, \mathrm{~J}=4.8 \mathrm{~Hz}, 4 \mathrm{H}\right), \delta 5.06(\mathrm{~s}$, benzyl $H, 4 \mathrm{H}), \delta 5.10$ (s, benzyl $\left.H, 4 \mathrm{H}\right), \delta$ 7.28-7.34 (m, arom. $H, 14 \mathrm{H}), \delta$ 7.36-7.38 (m, arom. $H, 6 \mathrm{H}), \delta 7.78(\mathrm{t}, \mathrm{NH}, \mathrm{J}=5.6 \mathrm{~Hz}$, 2H), $\delta 7.83(\mathrm{~d}, \mathrm{TAM} H, \mathrm{~J}=8.4 \mathrm{~Hz}, 2 \mathrm{H}), \delta 7.91(\mathrm{~d}, \mathrm{TAM} \mathrm{H}, \mathrm{J}=8.4 \mathrm{~Hz}, 2 \mathrm{H}), \delta 7.94(\mathrm{~s}, \mathrm{br}$, $\mathrm{NH}, 2 \mathrm{H}) .{ }^{13} \mathrm{C}$ NMR: $\delta 26.73,31.25,39.81,39.84,41.94,77.44,126.51,126.79,128.64$, 
$128.87,129.04,129.05,129.14,129.24,130.68,130.82,135.59,135.84,150.42,150.61$, 164.14, 165.02. MS (FAB+): m/z $921(\mathrm{MH}+)$.

$\operatorname{Pr}\left(\text { TAM-pr- } \mathrm{NH}_{2}\right)_{2}(\mathrm{Bn})_{4}, 4$ 4-15c. Slightly yellow residue, quantitative yield. ${ }^{1} \mathrm{H}$ NMR: $\delta 1.41-1.51\left(\mathrm{~m}, \mathrm{CH}_{2}+\mathrm{NH}_{2}, 10 \mathrm{H}\right), \delta 2.61\left(\mathrm{t}, \mathrm{CH}_{2}, \mathrm{~J}=6.4 \mathrm{~Hz}, 4 \mathrm{H}\right), \delta 3.21$ (quartet, $\mathrm{CH}_{2}, \mathrm{~J}$ $=6.4 \mathrm{~Hz}, 4 \mathrm{H}$ ), $\delta 3.36$ (quartet, $\left.\mathrm{CH}_{2}, \mathrm{~J}=6.4 \mathrm{~Hz}, 4 \mathrm{H}\right), \delta 5.14(\mathrm{~d}$, benzyl $\mathrm{H}, \mathrm{J}=1.6 \mathrm{~Hz}, 8 \mathrm{H}$ ), $\delta$ 7.34-7.39 (m, arom. $H, 20 \mathrm{H}), \delta$ 7.82-7.92 (m, TAM $H+\mathrm{NH}, 8 \mathrm{H}) .{ }^{13} \mathrm{C}$ NMR: $\delta 29.44$, $32.91,37.04,37.60,39.77,77.32,77.38,126.52,126.66,128.69,128.72,128.99,129.04$, $129.08,129.11,130.81,130.88,135.87,135.93,150.49,150.54,164.45,164.74 . \mathrm{MS}$ (FAB+): m/z $907.5(\mathrm{MH}+)$. MP: 52-54 ${ }^{\circ} \mathrm{C}$. IR: $3374.70(w), 2935.09$ (w), 1731.73 (w), $1640.12(\mathrm{~s}), 1525.96(\mathrm{~s}), 1424.23(\mathrm{~m}), 1367.04(\mathrm{~m}), 1288.34(\mathrm{~m}), 1217.88(\mathrm{~m}), 993.83$ (m), $911.35(w), 846.71(w), 741.31(\mathrm{~s}), 696.24(\mathrm{~s})$.

$\operatorname{Pr}\left(\text { TAM-bu- } \mathrm{NH}_{2}\right)_{2}(\mathbf{B n})_{4}, 4$ 4-15d. Slightly yellow semi-solid, quantitative yield. ${ }^{1} \mathrm{H}$ NMR: $\delta 1.27$ (s, br, $\left.\mathrm{NH}_{2}, 4 \mathrm{H}\right), \delta 1.34\left(\mathrm{~d}, \mathrm{br}, \mathrm{CH}_{2}, \mathrm{~J}=2.4 \mathrm{~Hz}, 8 \mathrm{H}\right), \delta 1.46\left(\mathrm{t}, \mathrm{CH}_{2}, \mathrm{~J}=5.6\right.$ $\mathrm{Hz}, 2 \mathrm{H}), \delta 2.60\left(\mathrm{~s}, \mathrm{CH}_{2}, 4 \mathrm{H}\right), \delta 3.20-3.28\left(\mathrm{~m}, \mathrm{CH}_{2}, 8 \mathrm{H}\right), \delta 5.14$ (s, benzyl $\left.H, 8 \mathrm{H}\right), \delta$ 7.34$7.38(\mathrm{~m}$, arom. $H, 20 \mathrm{H}), \delta$ 7.82-7.92 (m, NH + TAM $H, 8 \mathrm{H}) .{ }^{13} \mathrm{C}$ NMR: $\delta 26.73,29.46$, $31.27,37.05,39.83,41.94,77.32,77.40,126.54,126.75,128.65,128.71,129.00,129.03$, $129.08,130.71,130.93,135.89,135.93,150.47,150.57,164.22,164.74 . \mathrm{MS}(\mathrm{FAB}+)$ : $\mathrm{m} / \mathrm{z} 936(\mathrm{MH}+)$.

General synthesis of $\operatorname{TAM}_{2} \mathrm{HOPO}_{2}(\mathrm{Bn})_{6}$ and $\mathrm{TAM}_{4}(\mathrm{Bn})_{8}$, 4-16a-h: A solution of 0.5-1.0 mmol (1 equivalent) of 4-15a-d, approximately 2.2 equivalents of either PrTAMThiaz(Bn $)_{2}$ or Me-3,2-HOPO-Thiaz(Bn), and up to 3 equivalents of $\mathrm{Et}_{3} \mathrm{~N}$ was stirred for 1 day in $50-75 \mathrm{~mL}$ of $\mathrm{CHCl}_{3}$. The solution was then washed with $1 \mathrm{M} \mathrm{HCl}(2 \times 25 \mathrm{~mL})$ to remove $\mathrm{Et}_{3} \mathrm{~N} \cdot \mathrm{HCl}$, then $1 \mathrm{M} \mathrm{NaOH}$ in $20 \%$ sat. brine $(4 \times 25 \mathrm{~mL})$ to remove the majority 
of free 2-mercaptothiazolidine. After a wash with saturated brine and drying of the organic layer over $\mathrm{Na}_{2} \mathrm{SO}_{4}$, the solvent was removed under vacuum. The residue was redissolved in a minimum amount of $\mathrm{CH}_{2} \mathrm{Cl}_{2}$ and loaded on a silica gel column. The column was eluted with EtOAc to remove remaining 2-mercaptothiazolidine as well as unreacted TAM- or HOPO-Thiaz, and the desired compound was recovered from the column by elution with $4 \% \mathrm{MeOH}$ in $\mathrm{CH}_{2} \mathrm{Cl}_{2}$. Fractions of the desired $\mathrm{R}_{\mathrm{f}}$ (reported for $4 \% \mathrm{MeOH}$ in $\mathrm{CH}_{2} \mathrm{Cl}_{2}$ ) were collected and solvent was removed under vacuum.

En(TAM-pr-Me-3,2-HOPO) ${ }_{2}(\mathrm{Bn})_{6}$, 4-16a. Beige solid; $\mathrm{R}_{\mathrm{f}}=0.10, \quad 57 \%$. $\mathrm{C}_{80} \mathrm{H}_{78} \mathrm{~N}_{8} \mathrm{O}_{14}: \mathrm{C}: 69.85$ (69.51); H: 5.72 (6.06); N: 8.15 (8.02). ${ }^{1} \mathrm{H}$ NMR: $\delta 1.37$ (quintet, $\mathrm{CH}_{2}, \mathrm{~J}=6.4 \mathrm{~Hz}, 4 \mathrm{H}$ ), $\delta 3.14$ (quintet, $\mathrm{CH}_{2}, \mathrm{~J}=6.4 \mathrm{~Hz}, 8 \mathrm{H}$ ), $\delta 3.38$ (s, br, $\mathrm{CH}_{2}, 4 \mathrm{H}$ ), $\delta$ 3.57 (s, $\left.\mathrm{CH}_{3}, 6 \mathrm{H}\right), \delta 5.06$ (s, benzyl H, 4H), $\delta 5.10$ (s, benzyl $H, 4 \mathrm{H}$ ), $\delta 5.39$ (s, benzyl $H$, 4H), $\delta 6.73(\mathrm{~d}, \mathrm{HOPO} H, \mathrm{~J}=7.2 \mathrm{~Hz}, 2 \mathrm{H}), \delta 7.09(\mathrm{~d}, \mathrm{HOPO} H, \mathrm{~J}=7.2 \mathrm{~Hz}, 2 \mathrm{H}), \delta 7.31(\mathrm{~s}$, br, arom. $H, 26 \mathrm{H}), \delta 7.40(\mathrm{~d}$, arom. $H, \mathrm{~J}=6.4 \mathrm{~Hz}, 4 \mathrm{H}), \delta 7.77(\mathrm{t}, \mathrm{NH}, \mathrm{J}=5.6 \mathrm{~Hz}, 2 \mathrm{H}), \delta$ $7.84(\mathrm{~s}, \mathrm{TAM} H, 2 \mathrm{H}) \delta 7.98(\mathrm{~d}, \mathrm{NH}, \mathrm{J}=5.2 \mathrm{~Hz}, 4 \mathrm{H}) .{ }^{13} \mathrm{C}$ NMR: $\delta 29.22,37.11,37.17$, $37.89,39.83,75.04,77.32,77.41,105.01,126.51,126.58,128.76,128.94,129.01$, $129.03,129.09,129.11,129.25,130.55,130.62,131.12,132.21,135.64,135.83,136.43$, 146.68, 150.49, 150.57, 159.76, 163.64, 164.54, 165.04. MS (FAB+): m/z 1375.4 (MH+). MP: $178-180^{\circ} \mathrm{C}$.

En(TAM-pr-TAM-pr) ${ }_{2}(\mathbf{B n})_{8}, 4-16 b$. Off-white solid which analysis showed to be the monohydrate; $\mathrm{R}_{\mathrm{f}}=0.18,50 \%$. $\mathrm{C}_{102} \mathrm{H}_{102} \mathrm{~N}_{8} \mathrm{O}_{16} \cdot \mathrm{H}_{2} \mathrm{O}: \mathrm{C}: 71.48$ (71.63); H: 6.22 (6.53); $\mathrm{N}: 6.54(6.51) .{ }^{1} \mathrm{H}$ NMR: $\delta 0.83\left(\mathrm{t}, \mathrm{CH}_{3}, \mathrm{~J}=7.6 \mathrm{~Hz}, 6 \mathrm{H}\right), \delta 1.34-1.49\left(\mathrm{~m}, \mathrm{CH}_{2}, 8 \mathrm{H}\right), \delta$ 3.19-3.28 (m, $\left.\mathrm{CH}_{2}, 12 \mathrm{H}\right), \delta 3.39\left(\mathrm{~d}, \mathrm{CH}_{2}, \mathrm{~J}=4.8 \mathrm{~Hz}, 4 \mathrm{H}\right), \delta 5.08$ (s, benzyl $\left.H, 4 \mathrm{H}\right), \delta$ 5.11 (s, benzyl $H, 4 \mathrm{H}), \delta 5.15$ (s, benzyl $H, 8 \mathrm{H}), \delta 7.31-7.41$ (m, arom. $H, 40 \mathrm{H}), \delta 7.74(\mathrm{t}$, 
$\mathrm{NH}, \mathrm{J}=5.6 \mathrm{~Hz}, 2 \mathrm{H}), \delta$ 7.83-7.88 (m, NH + TAM H, 10H), $7.92(\mathrm{~d}, \mathrm{TAM} H, \mathrm{~J}=8.4 \mathrm{~Hz}$, 2H), $\delta 7.98$ (s, br, NH, 2H). ${ }^{13} \mathrm{C}$ NMR: $\delta 11.68,14.37,22.63,29.48,37.06,39.85,41.74$, $77.31,77.41,126.46,126.53,126.56,126.83,128.75,128.93,129.01,129.05,129.07$, $129.10,129.16,129.25,130.72,130.79,130.88,131.06,135.63,135.84,135.92,150.50$ $150.58,150.59,164.20,164.66,164.77,165.04 . \mathrm{MS}(\mathrm{FAB}+): \mathrm{m} / \mathrm{z} 1696.7(\mathrm{MH}+), 1718.7$ $(\mathrm{MNa}+)$. MP: $180-182^{\circ} \mathrm{C}$.

En(TAM-bu-Me-3,2-HOPO) ${ }_{2}(\mathrm{Bn})_{6}$, 4-16c. Beige solid which analysis showed to be the sesquihydrate; $\mathrm{R}_{\mathrm{f}}=0.15,69 \% . \mathrm{C}_{82} \mathrm{H}_{82} \mathrm{~N}_{8} \mathrm{O}_{14} \cdot 3 / 2 \mathrm{H}_{2} \mathrm{O}: \mathrm{C}: 68.84$ (68.82); H: 5.99 (5.93); N: 7.83 (7.83). ${ }^{1} \mathrm{H}$ NMR: $\delta 1.19$ (s, br, $\left.\mathrm{CH}_{2}, 8 \mathrm{H}\right), \delta 3.11$ (s, br, $\left.\mathrm{CH}_{2}, 4 \mathrm{H}\right), \delta 3.18$ (s, br, $\left.\mathrm{CH}_{2}, 4 \mathrm{H}\right), \delta 3.38\left(\mathrm{~s}, \mathrm{CH}_{2}, 4 \mathrm{H}\right), \delta 3.59\left(\mathrm{~s}, \mathrm{CH}_{3}, 6 \mathrm{H}\right), \delta 5.04$ (s, benzyl H, 4H), $\delta 5.10$ (s, benzyl $H, 4 \mathrm{H}), \delta 5.35$ (s, benzyl $H, 4 \mathrm{H}), \delta 6.77(\mathrm{~d}, \mathrm{HOPO} H, \mathrm{~J}=7.2 \mathrm{~Hz}, 2 \mathrm{H}), \delta 7.11$ (d, HOPO H, J = 7.2 Hz, 2H), $\delta$ 7.26-7.40 (m, arom. H, $30 \mathrm{H}), \delta 7.67(\mathrm{~s}, \mathrm{br}, \mathrm{NH}, 2 \mathrm{H}), \delta$ 7.84-7.86 (m, NH + TAM $H, 4 \mathrm{H}), \delta 7.91-7.93(\mathrm{~m}, \mathrm{NH}+\mathrm{TAM} H, 4 \mathrm{H}) .{ }^{13} \mathrm{C} \mathrm{NMR}: \delta$ $26.74,37.89,39.48,39.58,39.84,75.17,77.34,105.06,126.55,126.83,128.67,128.89$, $128.98,129.09,129.20,129.27,130.50,130.74,132.26,135.60,135.74,136.36,146.73$, $150.45,150.64,159.78,163.25,164.13,165.03 . \mathrm{MS}(\mathrm{FAB}+): \mathrm{m} / \mathrm{z} 1403.5(\mathrm{MH}+) . \mathrm{MP}:$ $218-220^{\circ} \mathrm{C}$.

En(TAM-bu-TAM-pr) 2 (Bn) $)_{\mathbf{8}}$, 4-16d. Slightly yellow solid which analysis indicated was the monohydrate; $\mathrm{R}_{\mathrm{f}}=0.07,69 \% . \mathrm{C}_{104} \mathrm{H}_{106} \mathrm{~N}_{8} \mathrm{O}_{16} \cdot \mathrm{H}_{2} \mathrm{O}: \mathrm{C}: 71.71$ (71.64); H: 6.25 (6.59); N: 6.43 (6.38). ${ }^{1} \mathrm{H}$ NMR: $\delta 0.83\left(\mathrm{t}, \mathrm{CH}_{3}, \mathrm{~J}=7.6 \mathrm{~Hz}, 6 \mathrm{H}\right), \delta 1.26\left(\mathrm{~s}, \mathrm{br}, \mathrm{CH}_{2}, 8 \mathrm{H}\right)$, $\delta 1.38$ (sextet, $\left.\mathrm{CH}_{2}, \mathrm{~J}=7.2 \mathrm{~Hz}, 4 \mathrm{H}\right), \delta 3.19-3.28\left(\mathrm{~m}, \mathrm{CH}_{2}, 12 \mathrm{H}\right), \delta 3.38\left(\mathrm{~d}, \mathrm{CH}_{2}, \mathrm{~J}=4.8\right.$ $\mathrm{Hz}, 4 \mathrm{H}), \delta 5.04$ (s, benzyl $H, 4 \mathrm{H}), \delta 5.10$ (s, benzyl $H, 4 \mathrm{H}), \delta 5.13$ (s, benzyl $H, 4 \mathrm{H}), \delta$ 5.14 (s, benzyl $H, 4 \mathrm{H}), \delta 7.28-7.41$ (m, arom. $H, 40 \mathrm{H}), \delta 7.70-7.72(\mathrm{~m}, \mathrm{NH} 2,6 \mathrm{H}), \delta 7.84$ 
$(\mathrm{d}, \mathrm{TAM} H, \mathrm{~J}=8.4 \mathrm{~Hz}, 2 \mathrm{H}), \delta$ 7.90-7.96 (m, $\mathrm{NH}+\mathrm{TAM} H, 8 \mathrm{H}) .{ }^{13} \mathrm{C}$ NMR: $\delta 11.68$, $22.64,26.93,26.96,39.59,39.62,39.85,41.74,77.36,77.44,77.47,126.53,126.82$, $126.90,128.70,128.88,129.07,129.09,129.18,129.23,129.24,129.27,130.50,130.67$ $130.77,130.90,135.57,135.72,135.81,135.84,150.45,150.52,150.56,150.65,164.15$, 164.18, 164.27, 164.31, 165.01. MS (TOF+): m/z 1746.7 (MNa+). MP: $240-42{ }^{\circ} \mathrm{C}$.

Pr(TAM-pr-Me-3,2-HOPO $)_{2}(\mathbf{B n})_{6}$, 4-16e. Off-white residue which analysis indicated was the monohydrate; $\mathrm{R}_{\mathrm{f}}=0.11,79 \% . \mathrm{C}_{81} \mathrm{H}_{80} \mathrm{~N}_{8} \mathrm{O}_{14} \cdot \mathrm{H}_{2} \mathrm{O}$ : C: 69.12 (68.79); H: 5.87 (5.76); N: 7.96 (7.89). ${ }^{1} \mathrm{H}$ NMR: $\delta 1.38$ (quintet, $\mathrm{CH}_{2}, \mathrm{~J}=6.4 \mathrm{~Hz}, 4 \mathrm{H}$ ), $\delta 1.46$ (quintet, $\left.\mathrm{CH}_{2}, \mathrm{~J}=6.4 \mathrm{~Hz}, 2 \mathrm{H}\right), \delta 3.12-3.24\left(\mathrm{~m}, \mathrm{CH}_{2}, 12 \mathrm{H}\right), \delta 3.56\left(\mathrm{~s}, \mathrm{CH}_{3}, 6 \mathrm{H}\right), \delta 5.14(\mathrm{~s}$, benzyl $H, 4 \mathrm{H}), \delta 5.15$ (s, benzyl $H, 4 \mathrm{H}), \delta 5.39$ (s, benzyl $H, 4 \mathrm{H}), \delta 6.72(\mathrm{~d}, \mathrm{HOPO} H, \mathrm{~J}=$ $7.2 \mathrm{~Hz}, 2 \mathrm{H}), \delta 7.09$ (d, HOPO H, J = 7.2 Hz, 2H), $\delta .27-7.38$ (m, arom. H, 26H), $\delta 7.41$ (dd, arom. $H, \mathrm{~J}=8.0,1.4 \mathrm{~Hz}, 4 \mathrm{H}), \delta 7.78(\mathrm{t}, \mathrm{NH}, \mathrm{J}=6.0 \mathrm{~Hz}, 2 \mathrm{H}), \delta 7.84(\mathrm{~s}, \mathrm{TAM} H, 4 \mathrm{H})$, $\delta 7.87(\mathrm{t}, \mathrm{NH}, \mathrm{J}=6.0 \mathrm{~Hz}, 2 \mathrm{H}), \delta 7.99(\mathrm{t}, \mathrm{NH}, \mathrm{J}=6.0 \mathrm{~Hz}, 2 \mathrm{H}) .{ }^{13} \mathrm{C} \mathrm{NMR}: \delta 29.22,29.49$, $37.06,37.13,37.19,37.85,75.02,77.37,104.99,126.54,128.76,128.78,128.92,129.01$, $129.09,129.23,130.57,130.90,130.98,132.18,135.92,135.95,136.44,146.66,150.53$, 159.75, 163.63, 164.61, 164.76. MS (FAB+): m/z $1389(\mathrm{MH}+)$. MP: $75-77^{\circ} \mathrm{C}$.

Pr(TAM-pr-TAM-pr $)_{2}(\mathbf{B n})_{8}$, 4-16f. White solid; $\mathrm{R}_{\mathrm{f}}=0.10,64 \% . \mathrm{C}_{103} \mathrm{H}_{104} \mathrm{~N}_{8} \mathrm{O}_{16}$ : C: 72.35 (72.02); H: 6.13 (6.13); N: 6.55 (6.66). ${ }^{1} \mathrm{H}$ NMR: $\delta 0.83$ (t, $\left.\mathrm{CH}_{3}, \mathrm{~J}=8.0 \mathrm{~Hz}, 6 \mathrm{H}\right), \delta$ 1.36-1.48 (m, $\left.\mathrm{CH}_{2}, 10 \mathrm{H}\right), \delta 3.20-3.28\left(\mathrm{~m}, \mathrm{CH}_{2}, 16 \mathrm{H}\right), \delta$ 5.15-5.16 (m, benzyl $\left.H, 16 \mathrm{H}\right), \delta$ 7.28-7.43 (m, arom. $H, 40 \mathrm{H}), \delta 7.76(\mathrm{t}, \mathrm{NH}, \mathrm{J}=5.6 \mathrm{~Hz}, 2 \mathrm{H}), \delta 7.82-7.89(\mathrm{~m}, \mathrm{NH}+\mathrm{TAM}$ $H, 14 \mathrm{H}) .{ }^{13} \mathrm{C}$ NMR: $\delta 11.66,22.60,29.46,37.04,41.71,77.63,126.47,126.51,126.78$, $128.72,128.75,128.85,128.89,128.99,129.01,129.07,129.13,130.73,130.89,130.94$ 
$135.81,135.89,150.47,150.54,150.56,164.19,164.72,164.73,164.75 . \mathrm{MP}: 133-135{ }^{\circ} \mathrm{C}$ MS (FAB+): m/z 1711.3 (MH+).

$\operatorname{Pr}(\text { TAM-bu-Me-3,2-HOPO })_{2}(\mathrm{Bn})_{6}$, 4-16g. White solid that analysis showed to be the monohydrate; $\mathrm{R}_{\mathrm{f}}=0.13,70 \% . \mathrm{C}_{83} \mathrm{H}_{84} \mathrm{~N}_{8} \mathrm{O}_{14} \cdot \mathrm{H}_{2} \mathrm{O}: \mathrm{C}: 69.44$ (69.37); $\mathrm{H}: 6.10(6.13) ; \mathrm{N}$ : 7.81 (7.79). ${ }^{1} \mathrm{H}$ NMR: $\delta 1.19$ (t, $\mathrm{CH}_{2}, \mathrm{~J}=3.2 \mathrm{~Hz}, 8 \mathrm{H}$ ), $\delta 1.46$ (quintet, $\mathrm{CH}_{2}, \mathrm{~J}=6.4 \mathrm{~Hz}$, $2 \mathrm{H}), \delta 3.11\left(\mathrm{~d}, \mathrm{CH}_{2}, \mathrm{~J}=5.6 \mathrm{~Hz}, 4 \mathrm{H}\right), \delta 3.17-3.24\left(\mathrm{~m}, \mathrm{CH}_{2}, 8 \mathrm{H}\right), \delta 3.58\left(\mathrm{~s}, \mathrm{CH}_{3}, 6 \mathrm{H}\right), \delta$ 5.12 (s, bezyl $H, 4 \mathrm{H}$ ), $\delta 5.15$ (s, benzyl $H, 4 \mathrm{H}$ ), $\delta 5.35$ (s, benzyl $H, 4 \mathrm{H}$ ), $\delta 6.77$ (d, HOPO $H, \mathrm{~J}=7.2 \mathrm{~Hz}, 2 \mathrm{H}), \delta 7.11(\mathrm{~d}, \mathrm{HOPO} H, \mathrm{~J}=7.2 \mathrm{~Hz}, 2 \mathrm{H}), \delta 7.30-7.41(\mathrm{~m}$, arom. $H, 30 \mathrm{H})$, $\delta 7.69(\mathrm{t}, \mathrm{NH}, \mathrm{J}=5.6 \mathrm{~Hz}, 2 \mathrm{H}), \delta$ 7.84-7.87 (m, NH + TAM H, 6H), $\delta 7.92(\mathrm{~d}, \mathrm{TAM} H, \mathrm{~J}$ $=8.0 \mathrm{~Hz}) .{ }^{13} \mathrm{C}$ NMR: $\delta 26.73,26.77,29.49,75.13,77.38,77.41,105.03,126.57,126.77$, $128.66,128.71,128.96,129.00,129.06,129.09,129.18,130.49,130.58,130.99,132.23$, $135.82,135.88,136.34,146.70,150.48,150.59,159.75,163.22,164.18,164.72$. MS $(\mathrm{FAB}+): \mathrm{m} / \mathrm{z} 1417.6(\mathrm{MH}+) . \mathrm{MP}: 71-73{ }^{\circ} \mathrm{C}$.

Pr(TAM-bu-TAM-pr $)_{2}(\mathbf{B n})_{8}$, 4-16h. Light solid; $\mathrm{R}_{\mathrm{f}}=0.11,74 \% . \mathrm{C}_{105} \mathrm{H}_{108} \mathrm{~N}_{8} \mathrm{O}_{16}: \mathrm{C}$ : 72.56 (72.61); H: 6.55 (6.26); N: 6.45 (6.41). ${ }^{1} \mathrm{H}$ NMR: $\delta 0.83\left(\mathrm{t}, \mathrm{CH}_{3}, \mathrm{~J}=7.2 \mathrm{~Hz}, 6 \mathrm{H}\right), \delta$ 1.27 (s, br, $\mathrm{CH}_{2}, 8 \mathrm{H}$ ), $\delta 1.40$ (sextet, $\mathrm{CH}_{2}, \mathrm{~J}=7.8 \mathrm{~Hz}, 4 \mathrm{H}$ ), $\delta 1.48$ (quintet, $\mathrm{CH}_{2}, \mathrm{~J}=6.4$ $\mathrm{Hz}, 2 \mathrm{H}), \delta 3.12-3.28\left(\mathrm{~m}, \mathrm{CH}_{2}, 16 \mathrm{H}\right), \delta$ 5.13-5.15 (m, benzyl $H$ 16H), $\delta$ 7.35-7.39 (m, arom. $H, 40 \mathrm{H}), \delta$ 7.70-7.75 (m, NH, 6H), $\delta 7.83-7.87$ (m, NH + TAM $H, 4 \mathrm{H}), \delta$ 7.90-7.96 (m, TAM H, 6H). ${ }^{13} \mathrm{C}$ NMR: $\delta 11.67,22.64,26.95,29.50,37.06,39.60,41.74,77.43$, $126.56,126.76,128.80,126.89,128.69,128.71,129.02,129.05,129.09,129.17,129.22$, $130.55,130.58,130.92,131.05,135.82,135.83,135.85,135.89,150.51,150.55,150.61$, 164.19, 164.24, 164.27, 164.73. MS (FAB+): m/z $1760.9(\mathrm{MNa}+)$. MP: $163-165^{\circ} \mathrm{C}$. 
General synthesis of $\mathrm{TAM}_{2} \mathrm{HOPO}_{2}$ and $\mathrm{TAM}_{4}$ ligands 4-17a-h: A solution of 0.5$0.7 \mathrm{mmol}$ of 4-16a-h in $10 \mathrm{~mL}$ of $1: 1 \mathrm{conc} . \mathrm{HCl} / \mathrm{AcOH}$ was stirred at room temperature for 4 to 8 days (the longer times being used in cases in which precipitation occurred). The acids and the produced benzyl alcohol were removed under vacuum and the resulting residue was held under vacuum for several more hours. The residue was suspended in cold $\mathrm{MeOH}$ and filtered. The solid was washed with cold $\mathrm{MeOH}$, ground up into a powder and dried under vacuum over $\mathrm{P}_{2} \mathrm{O}_{5}$. The powder was allowed to stand open to air overnight until no more gain in mass was observed. The finished ligands were isolated as white or beige powders.

En(TAM-pr-Me-3,2-HOPO) $)_{2}$, 4-17a. Beige powder which analysis indicated was the trihydrate, $89 \%$. $\mathrm{C}_{38} \mathrm{H}_{42} \mathrm{~N}_{8} \mathrm{O}_{14} \cdot 3 \mathrm{H}_{2} \mathrm{O}:$ C: 51.35 (51.10); H: 5.44 (5.29); N: 12.61 (12.51). ${ }^{1} \mathrm{H}$ NMR (DMSO-d $): \delta 1.79\left(\mathrm{t}, \mathrm{CH}_{2}, \mathrm{~J}=6.4 \mathrm{~Hz}, 4 \mathrm{H}\right), \delta 3.34\left(\mathrm{~d}, \mathrm{CH}_{2}+\mathrm{H}_{2} \mathrm{O}, \mathrm{J}=\right.$ $5.6 \mathrm{~Hz}, 11 \mathrm{H}), \delta 3.46\left(\mathrm{~s}, \mathrm{CH}_{3}, 6 \mathrm{H}\right), \delta 3.51\left(\mathrm{~s}, \mathrm{br}, \mathrm{CH}_{2}, 8 \mathrm{H}\right), \delta 6.50(\mathrm{~d}, \mathrm{HOPO} H, \mathrm{~J}=7.2$ Hz, 2H), $\delta 7.18$ (d, HOPO H, J = 7.2 Hz, 2H), $\delta 7.31$ (s, TAM H, 4H), $\delta 8.55$ (s, br, NH, $2 \mathrm{H}), \delta 8.93(\mathrm{~s}, \mathrm{br}, \mathrm{NH}, 2 \mathrm{H}), \delta 9.05(\mathrm{~s}, \mathrm{br}, \mathrm{NH}, 2 \mathrm{H}), \delta 12.69$ (s, br, 4H). ${ }^{13} \mathrm{C}$ NMR $\left(\right.$ DMSO-d $\left.{ }_{6}\right): \delta 28.76,36.88,36.90,38.59,102.55,115.68,115.80,115.84,117.14$, $117.25,127.75,147.76,150.25,158.07,165.68,168.77,169.14 . \mathrm{MS}(\mathrm{FAB}+): \mathrm{m} / \mathrm{z} 835$ $(\mathrm{MH}+)$. MP: $237-39^{\circ} \mathrm{C}(\mathrm{dec})$.

En(TAM-pr-TAM-pr) 2, 4-17b. White solid which analysis indicated was the trihydrate, 76\%. $\mathrm{C}_{46} \mathrm{H}_{54} \mathrm{~N}_{8} \mathrm{O}_{16} \cdot 3 \mathrm{H}_{2} \mathrm{O}$ : C: 53.69 (53.97); H: 5.88 (5.89); N: 10.89 (10.62). ${ }^{1} \mathrm{H}$ NMR (DMSO- $d_{6}$ ): $\delta 0.89$ (t, $\mathrm{CH}_{3}, \mathrm{~J}=7.2 \mathrm{~Hz}, 6 \mathrm{H}$ ), $\delta 1.57$ (sextet, $\mathrm{CH}_{2}, \mathrm{~J}=7.2 \mathrm{~Hz}$, $4 \mathrm{H}), \delta 1.84\left(\mathrm{t}, \mathrm{CH}_{2}, \mathrm{~J}=6.8 \mathrm{~Hz}, 4 \mathrm{H}\right), \delta 2.50\left(\mathrm{~d}, \mathrm{CH}_{2}, \mathrm{~J}=1.6 \mathrm{~Hz}, 4 \mathrm{H}\right), \delta 3.26$ (quartet, $\left.\mathrm{CH}_{2}, \mathrm{~J}=6.4 \mathrm{~Hz}, 4 \mathrm{H}\right), \delta 3.56\left(\mathrm{~s}, \mathrm{br}, \mathrm{CH}_{2}+\mathrm{H}_{2} \mathrm{O}, 14 \mathrm{H}\right), \delta 3.50\left(\mathrm{~s}, \mathrm{CH}_{2}, 4 \mathrm{H}\right), \delta 7.32-7.35$ 
(m, TAM H, 8H), $\delta 8.87-8.92(\mathrm{~m}, \mathrm{NH}, 6 \mathrm{H}), \delta 9.04(\mathrm{~s}, \mathrm{NH}, 2 \mathrm{H}), \delta$ 12.67-12.71 (m, OH, $6 \mathrm{H}), \delta 12.85(\mathrm{~s}, \mathrm{OH}, 2 \mathrm{H}) .{ }^{13} \mathrm{C}$ NMR $\left(\mathrm{DMSO}_{-} \mathrm{d}_{6}\right): \delta 11.40,22.07,28.61,36.94,38.57$, $40.79,115.64,115.69,115.78,117.17,117.24,117.25,117.36,150.15,150.23,150.30$, 168.66, 168.69, 169.10. MS (FAB+): m/z 975.6 (MH+). MP: 259-61 ${ }^{\circ} \mathrm{C}(\mathrm{dec})$.

En(TAM-bu-Me-3,2-HOPO) 2 , 4-17c. Beige solid which analysis indicated was the 4-17c $\cdot 3 \mathrm{H}_{2} \mathrm{O} \cdot 1 / 2 \mathrm{MeOH}$ adduct, 94\%). $\mathrm{C}_{40} \mathrm{H}_{46} \mathrm{~N}_{8} \mathrm{O}_{14} \cdot 3 \mathrm{H}_{2} \mathrm{O} \cdot 1 / 2 \mathrm{MeOH}$ : C: 52.14 (52.27); $\mathrm{H}$ : 5.83 (5.64); N: 12.01 (11.85). ${ }^{1} \mathrm{H}$ NMR (DMSO-d 6 ): $\delta 1.57$ (s, br, $\left.\mathrm{CH}_{2}, 8 \mathrm{H}\right), \delta 3.32$ (s, $\left.\mathrm{CH}_{2}+\mathrm{CH}_{3} \mathrm{OH}, 9.5 \mathrm{H}\right), \delta 3.46\left(\mathrm{~s}, \mathrm{CH}_{3}, 6 \mathrm{H}\right), \delta 3.51\left(\mathrm{~s}, \mathrm{CH}_{2}, 4 \mathrm{H}\right), \delta 6.51(\mathrm{~d}, \mathrm{HOPO} H, \mathrm{~J}=$ $6.8 \mathrm{~Hz}, 2 \mathrm{H}), \delta 7.18(\mathrm{~d}, \mathrm{HOPO} H, \mathrm{~J}=6.8 \mathrm{~Hz}, 2 \mathrm{H}), \delta 7.32(\mathrm{~s}, \mathrm{TAM} H, 4 \mathrm{H}), \delta 8.49(\mathrm{~s}, \mathrm{NH}$, 2H), $\delta 8.90(\mathrm{~s}, \mathrm{NH}, 2 \mathrm{H}), \delta 9.05(\mathrm{~s}, \mathrm{NH}, 2 \mathrm{H}), \delta 12.65$ (s, br, OH, 2H), $\delta 12.84$ (s, br, OH, 2H). ${ }^{13} \mathrm{C}$ NMR (DMSO- $d_{6}$ ): $\delta 26.27,26.47,36.84,38.57,38.77,102.38,115.63,115.75$, $116.95,117.23,127.70,148.00,150.24,150.33,158.02,165.73,168.71,169.11 . \mathrm{MS}$ $(\mathrm{FAB}+): \mathrm{m} / \mathrm{z} 863(\mathrm{MH}+) . \mathrm{MP}: 228-30{ }^{\circ} \mathrm{C}(\mathrm{dec})$.

En(TAM-bu-TAM-pr) 2, 4-17d. White solid which analysis indicated was the 417d $\cdot 2 \mathrm{H}_{2} \mathrm{O} \cdot 1 / 4 \mathrm{MeOH} \cdot \mathrm{HCl}$ adduct, $79 \%$. $\mathrm{C}_{48} \mathrm{H}_{58} \mathrm{~N}_{8} \mathrm{O}_{16} \cdot 2 \mathrm{H}_{2} \mathrm{O} \cdot 1 / 4 \mathrm{MeOH} \cdot \mathrm{HCl}: \quad \mathrm{C}: 53.48$ (53.64); H: 5.95 (5.91); N: 10.34 (10.06). ${ }^{1} \mathrm{H}$ NMR (DMSO-d $)_{6}$ ): $\delta 0.89$ (t, $\mathrm{CH}_{3}, \mathrm{~J}=7.6$ $\mathrm{Hz}, 6 \mathrm{H}), \delta$ 1.51-1.60 (m, $\left.\mathrm{CH}_{2}, 12 \mathrm{H}\right), \delta 3.17\left(\mathrm{~s}, \mathrm{CH}_{3} \mathrm{OH}, 0.75 \mathrm{H}\right), \delta 3.25$ (quartet, $\mathrm{CH}_{2}, \mathrm{~J}=$ $6.4 \mathrm{~Hz}, 4 \mathrm{H}), \delta 3.34(\mathrm{~d}, \mathrm{br}, \mathrm{J}=4.8 \mathrm{~Hz}, 8 \mathrm{H}), \delta 3.50\left(\mathrm{~s}, \mathrm{br}, \mathrm{CH}_{2}, 4 \mathrm{H}\right), \delta$ 7.29-7.34 (m, TAM $H, 8 \mathrm{H}), \delta 8.86-8.89(\mathrm{~m}, \mathrm{NH}, 6 \mathrm{H}), \delta 9.04(\mathrm{~s}, \mathrm{NH}, 2 \mathrm{H}), \delta 12.64$ (s, br, OH, 2H), $\delta 12.82$ (s, br, $\mathrm{OH}, 6 \mathrm{H}$ ). ${ }^{13} \mathrm{C}$ NMR (DMSO-d $)$ ): $\delta 11.40,22.07,26.31,38.57,38.74,40.78,115.58$, $115.74,117.10,117.18,117.24,150.21,150.30,168.64,168.70,169.08$. MS (FAB+): m/z 1003.5 (MH+). MP: 272-74 ${ }^{\circ} \mathrm{C}(\mathrm{dec})$. IR: $3375.16(\mathrm{~m}), 2938.00(\mathrm{w}), 1601.07$ (s), 
1541.09 (s), $1428.24(\mathrm{~s}), 1333.01(\mathrm{~s}), 1254.77$ (s), $1231.58(\mathrm{~s}), 1190.32(\mathrm{~s}), 792.45(\mathrm{~m})$, $729.51(\mathrm{~s})$.

Pr(TAM-pr-Me-3,2-HOPO) 2 , 4-17e. Beige solid which analysis indicated was the 4-17e $\cdot \mathrm{H}_{2} \mathrm{O} \cdot 1 \frac{1}{2} \mathrm{MeOH} \cdot 5 / 2 \mathrm{HCl}$ adduct, $88 \%$. $\mathrm{C}_{39} \mathrm{H}_{44} \mathrm{~N}_{8} \mathrm{O}_{14} \cdot \mathrm{H}_{2} \mathrm{O} \cdot \frac{1}{2} \mathrm{MeOH} \cdot 5 / 2 \mathrm{HCl}: \mathrm{C}: 48.71$ (48.50); H: 5.23 (5.46); N: 11.50 (11.30). ${ }^{1} \mathrm{H}$ NMR (DMSO- $\left.d_{6}\right): \delta 1.78-1.85\left(\mathrm{~m}, \mathrm{CH}_{2}\right.$, $6 \mathrm{H}), \delta 3.16\left(\mathrm{~s}, \mathrm{CH}_{3} \mathrm{OH}, 1.5 \mathrm{H}\right), \delta 3.34-3.35\left(\mathrm{~m}, \mathrm{CH}_{2}, 12 \mathrm{H}\right), 3.46\left(\mathrm{~s}, \mathrm{CH}_{3}, 6 \mathrm{H}\right), \delta 6.53(\mathrm{~d}$, HOPO $H, \mathrm{~J}=7.2 \mathrm{~Hz}, 2 \mathrm{H}), \delta 7.18(\mathrm{~d}, \mathrm{HOPO} H, 2 \mathrm{H}), \delta 7.33-7.38$ (m, TAM $H, 4 \mathrm{H}$ ), $\delta 8.61$ $(\mathrm{t}, \mathrm{NH}, \mathrm{J}=5.2 \mathrm{~Hz}, 2 \mathrm{H}), \delta$ 8.99-9.03 (m, NH, 4H). ${ }^{13} \mathrm{C}$ NMR (DMSO-d $\left.d_{6}\right): \delta 28.61,28.75$, $36.86,36.91,102.52,115.73,117.05,117.20,117.24,127.76,147.99,150.29,150.33$, 158.08, 165.86, 168.85. MS (FAB+): m/z $849(\mathrm{MH}+)$. MP: $224-226{ }^{\circ} \mathrm{C}(\mathrm{dec})$.

Pr(TAM-pr-TAM-pr) 2 , 4-17f. White powder that analysis showed to be the 417f· $3 \mathrm{H}_{2} \mathrm{O} \cdot 1 / 2 \mathrm{MeOH}$ adduct, $87 \%$. $\mathrm{C}_{47} \mathrm{H}_{56} \mathrm{~N}_{8} \mathrm{O}_{16} \cdot 3 \mathrm{H}_{2} \mathrm{O} \cdot 1 / 2 \mathrm{MeOH}$ : C: 53.87 (54.06); H: 6.09 (6.02); N: 10.58 (10.29). ${ }^{1} \mathrm{H}$ NMR (DMSO- $\left.d_{6}\right): \delta 0.89\left(\mathrm{t}, \mathrm{CH}_{3}, \mathrm{~J}=7.2 \mathrm{~Hz}, 6 \mathrm{H}\right), \delta 1.55$ (sextet, $\mathrm{CH}_{2}, \mathrm{~J}=7.2 \mathrm{~Hz}, 4 \mathrm{H}$ ), $\delta 1.84$ (quintet, $\mathrm{CH}_{2}, \mathrm{~J}=5.2 \mathrm{~Hz}, 6 \mathrm{H}$ ), $\delta 3.25$ (quartet, $\mathrm{CH}_{2}$, $\mathrm{J}=6.4 \mathrm{~Hz}, 4 \mathrm{H}), \delta 3.36\left(\mathrm{~s}, \mathrm{br}, \mathrm{CH}_{2}, 12 \mathrm{H}\right), \delta 7.33(\mathrm{~s}, \mathrm{TAM} H, 8 \mathrm{H}), \delta 8.87-8.94(\mathrm{~m}, \mathrm{NH}$, $8 \mathrm{H}), \delta 12.72(\mathrm{~s}, \mathrm{br}, \mathrm{OH}, 8 \mathrm{H}) .{ }^{13} \mathrm{C}$ NMR (DMSO- $\left.d_{6}\right): \delta 11.40,22.08,28.62,36.95,40.79$, $115.64,115.69,117.17,117.26,150.18,150.30,150.60,168.06,168.71$. MP: $253-255{ }^{\circ} \mathrm{C}$ (dec.). MS (FAB+): m/z $989.4(\mathrm{MH}+)$.

Pr(TAM-bu-Me-3,2-HOPO) 2 , 4-17g. Beige solid which analysis indicated was the 4-17g.5/2 $\mathrm{H}_{2} \mathrm{O} \cdot 1 / 2 \mathrm{MeOH}$ adduct, $91 \%$. $\mathrm{C}_{41} \mathrm{H}_{48} \mathrm{~N}_{8} \mathrm{O}_{14} \cdot 5 / 2 \mathrm{H}_{2} \mathrm{O} \cdot 1 / 2 \mathrm{MeOH}$ : C: 53.14 (53.05); H: 5.91 (6.31); N: 11.95 (11.86). ${ }^{1} \mathrm{H}$ NMR (DMSO-d 6 ): $\delta 1.57$ (s, br, $\left.\mathrm{CH}_{2}, 8 \mathrm{H}\right), \delta 1.84(\mathrm{t}$, $\left.\mathrm{CH}_{2}, \mathrm{~J}=6.8 \mathrm{~Hz}, 2 \mathrm{H}\right), \delta 3.16\left(\mathrm{~s}, \mathrm{CH}_{3} \mathrm{OH}, 1.5 \mathrm{H}\right), \delta 3.32-3.37\left(\mathrm{~m}, \mathrm{CH}_{2}, 14 \mathrm{H}\right), \delta 3.45(\mathrm{~s}$, $\left.\mathrm{CH}_{3}, 6 \mathrm{H}\right), \delta 6.51(\mathrm{~d}, \mathrm{HOPO} H, \mathrm{~J}=7.2 \mathrm{~Hz}, 2 \mathrm{H}), \delta 7.17(\mathrm{~d}, \mathrm{HOPO} H, \mathrm{~J}=7.2 \mathrm{~Hz}, 2 \mathrm{H}), \delta$ 
$7.33(\mathrm{~s}, \mathrm{TAM} H, 4 \mathrm{H}), \delta 8.50(\mathrm{t}, \mathrm{NH}, \mathrm{J}=5.2 \mathrm{~Hz}, 2 \mathrm{H}), \delta 8.91-8.96(\mathrm{~m}, \mathrm{NH}, 4 \mathrm{H}) .{ }^{13} \mathrm{C}$ NMR (DMSO-d $\left.d_{6}\right): \delta 26.34,26.53,28.66,36.91,36.96,38.83,48.67,102.45,115.72,117.01$, $117.19,117.33,127.76,148.06,150.22,150.38,158.08,165.81,168.77 . \mathrm{MS}(\mathrm{FAB}+)$ : m/z $877(\mathrm{MH}+)$. MP: $149-51{ }^{\circ} \mathrm{C}(\mathrm{dec})$.

Pr(TAM-bu-TAM-pr)2, 4-17h. Beige solid, that analysis indicated was the monohydrate, $86 \%$. $\mathrm{C}_{49} \mathrm{H}_{60} \mathrm{~N}_{8} \mathrm{O}_{16} \cdot \mathrm{H}_{2} \mathrm{O}:$ C: 56.86 (56.86); H: 6.02 (6.22); N: 10.83 (10.63). ${ }^{1} \mathrm{H}$ NMR (DMSO-d $\left.)_{6}\right): \delta 0.89\left(\mathrm{t}, \mathrm{CH}_{3}, \mathrm{~J}=7.2 \mathrm{~Hz}, 6 \mathrm{H}\right), \delta 1.51-1.60\left(\mathrm{~m}, \mathrm{CH}_{2}\right.$, $12 \mathrm{H}$ ), $\delta 1.45$ (quintet, $\mathrm{CH}_{2}, \mathrm{~J}=6.4 \mathrm{~Hz}, 2 \mathrm{H}$ ), $\delta 3.25$ (doublet, $\mathrm{CH}_{2}, \mathrm{~J}=6.4 \mathrm{~Hz}, 4 \mathrm{H}$ ), $\delta 3.35$ (s, br, $\mathrm{CH}_{2}, 12 \mathrm{H}$ ), $\delta 7.34$ (s, TAM H, 8H), $\delta 8.87-8.93$ (m, $\mathrm{NH}_{2}, 8 \mathrm{H}$ ), $\delta 12.83$ (s, br, $\mathrm{OH}$, $6 \mathrm{H}) .{ }^{13} \mathrm{C}$ NMR (DMSO- $\left.d_{6}\right): \delta 11.41,22.10,26.35,28.65,36.94,38.77,40.81,115.58$, 115.64, 117.11, 117.18, 117.27, 150.22, 150.35, 168.67, 168.73. MP: $256-58^{\circ} \mathrm{C}(\mathrm{dec})$.

\subsubsection{X-ray Diffraction Data Collection}

Ce(IV) crystals were mounted on captan loops with oil and X-ray diffraction data were collected using either Bruker SMART 1000 or APEX I detectors with Mo K $\alpha$ radiation at the UC Berkeley X-ray crystallographic facility or with Bruker Platinum 200 or APEX II detectors with synchrotron radiation $(\mathrm{hv}=16 \mathrm{keV})$ at Endstation 11.3.1 at the Advanced Light Source (ALS) at LBNL. The Pu crystals were mounted in oil inside a quartz capillary which was sealed by epoxy and coated with nail polish to prevent shattering. $\mathrm{Pu}(\mathrm{IV})$ crystal data was collected exclusively with a Bruker Plantinum 200 detector with synchrotron radiation $(\mathrm{hv}=16 \mathrm{keV})$ at Endstation 11.3.1 at the ALS at

LBNL. All data were integrated by the program SAINT. ${ }^{44,45}$ The data were corrected for Lorentz and polarization effects. Data were analyzed for agreement and possible absorption using XPREP and an empirical absorption correction was applied in 
SADABS ${ }^{46,47}$ Equivalent reflections were merged without an applied decay correction. $\mathrm{Ce}$ structures and the $\mathrm{Pu}(\mathrm{maltol})_{4}$ structure were solved by direct methods with SHELXS,${ }^{48}$ while the Pu(bromo-maltol $)_{4}$ structure was solved by Patterson methods, ${ }^{49,50}$ and all structures were expanded using Fourier techniques using the SHELXTL package. ${ }^{51}$ Least squares refinement of $\mathrm{F}^{2}$ against all reflections was carried out to convergence with $\mathrm{R}[\mathrm{I}>2 \sigma(\mathrm{I})]$.

\subsection{References}

(1) Portions of this chapter are published in J. Am. Chem. Soc., 2007, 129 (21), 66746675 and Eur. J. Inorg. Chem., 2008, 13, 2143-2147.

(2) "Disposition of High-Level Waste and Spent Nuclear Fuel; The Continuing Societal and Technical Challenges,” National Research Council, 2001.

(3) Clark, D. L.; Hecker, S. S.; Jarvinen, G. D.; Neu, M. P. In The Chemistry of the Actinide and Transactinide Elements; $3^{\text {rd }}$ ed.; Morss, L. R., Edelstein, N. M., Fuger, J., Eds.; Springer: Dordrecht, The Netherlands, 2006; Vol. 2, p 813-1264.

(4) Gorden, A. E. V.; Xu, J.; Raymond, K. N. Chem. Rev. 2003, 103, 4207-4282.

(5) Greenwood, N. N.; Earnshaw, A. Chemistry of the Elements; Pergamon Press: New York, 1997.

(6) Raymond, K. N.; Freeman, G. E.; Kappel, M. J. Inorg. Chim. Acta. 1984, 94, 193204.

(7) Durbin, P. W. Health Phys. 2008, 95, 465-492.

(8) Durbin, P. W.; Kullgren, B.; Raymond, K. N. Rad. Prot. Dosim. 1998, 79, 433443. 
(9) Xu, J.; Durbin, P. W.; Kullgren, B.; Shirley, N. E.; Uhlir, L. C.; Raymond, K. N. J. Med. Chem. 2002, 45, 3963-3971.

(10) Grigoriev, M. S.; Krot, N. N.; Bessonov, A. A.; Lyssenko, K. A. Acta Cryst. E 2006, E62, m2889-m2890.

(11) Brown, D.; Whittaker, B.; Tacon, J. J. Chem. Soc. Dalton Trans. 1975, 34-39.

(12) Sofen, S. R.; Abu-Dari, K.; Freyberg, D. P.; Raymond, K. N. J. Am. Chem. Soc. 1978, 100, 7882-7887.

(13) Neu, M. P.; Matonic, J. H.; Ruggiero, C. E.; Scott, B. L. Angew. Chem. Int. Ed. 2000, 39, 1442-1444.

(14) Gorden, A. E. V.; Shuh, D. K.; Tiedemann, B. E. F.; Wilson, R. E.; Xu, J.; Raymond, K. N. Chem. Eur. J. 2005, 11, 2842-2848.

(15) Gorden, A. E. V.; Shuh, D. K.; Tiedemann, B. E. F.; Wilson, R. E.; Xu, J.; Raymond, K. N. Chem. Eur. J. 2007, 13, 378.

(16) Riley, P. E.; Abu-Dari, K.; Raymond, K. N. Inorg. Chem. 1983, 22, 3940-3944.

(17) Scarrow, R. C.; Riley, P. E.; Abu-Dari, K.; White, D. L.; Raymond, K. N. Inorg. Chem. 1985, 24, 954-967.

(18) Casellato, U.; Vigato, P. A.; Tamburini, S.; Graziani, R.; Vidali, M. Inorg. Chim. Acta. 1983, 72, 141-147.

(19) Xu, J.; Radkov, E.; Ziegler, M.; Raymond, K. N. Inorg. Chem. 2000, 39, 41564164.

(20) Haddad, S. F.; Raymond, K. N. Inorg. Chim. Acta. 1986, 122, 111-118. 
(21) Veeck, A. C.; White, D. J.; Whisenhunt, D. W. J.; Xu, J.; Gorden, A. E. V.; Romanovski, V.; Hoffman, D. C.; Raymond, K. N. Solv. Extr. Ion Exch. 2004, 22, 1037-1068.

(22) Thompson, K. H.; Barta, C. A.; Orvig, C. Chem. Soc. Rev. 2006, 35, 545-556.

(23) Ahmet, M. T.; Frampton, C. S.; Silver, J. J. Chem. Soc., Dalton Trans. 1988, 1159-1163.

(24) Dutt, N. K.; Sarma, U. U. M. J. Inorg. Nucl. Chem. 1975, 37, 1801-1802.

(25) Dutt, N. K.; Sharma, U. V. M. J. Inorg. Nucl. Chem. 1970, 32.

(26) Looker, J. H.; Prokop, R. J.; Serbousek, W. E.; Cliffton, M. D. J. Org. Chem. 1979, 44, 3408-10.

(27) Hoard, J. L.; Silverton, J. V. Inorg. Chem. 1963, 2, 235-242.

(28) Kepert, D. L. Inorganic Stereochemistry; Springer-Verlag: New York, 1982.

(29) Hay, B. P.; Uddin, J.; Firman, T. K. Polyhedron 2004, 23, 145-54.

(30) Dance, I. Mol. Cryst. Liq. Cryst. 2005, 440, 265-293.

(31) Beeby, A.; Clarkson, I. M.; Dickins, R. S.; Faulkner, S.; Parker, D.; Sousa, A. S. d.; Williams, J. A. G.; Woods, M. J. Chem. Soc. Perkin Trans. 2 1999, 493-503.

(32) Beeby, A.; Dickins, R. S.; Faulkner, S.; Williams, J. A. G. Chem. Comm. 1997, 1401-1402.

(33) Xu, J.; Raymond, K. N. Inorg. Chem. 1999, 38, 308-315.

(34) Moore, E. G.; Jocher, C. J.; Xu, J.; Werner, E. J.; Raymond, K. N. Inorg. Chem. 2007, 46, 5468-5470.

(35) Moore, E. G.; Xu, J.; Jocher, C. J.; Werner, E. J.; Raymond, K. N. J. Am. Chem. Soc. 2006, 128, 10648-10649. 
(36) Abergel, R. J.; Warner, J. A.; Shuh, D. K.; Raymond, K. N. J. Am. Chem. Soc. 2006, 128, 8920-8931.

(37) Personal communication with Dr. Anthony D'Aleo, June, 2009.

(38) Xu, J.; Durbin, P. W.; Kullgren, B.; Raymond, K. N. J. Med. Chem. 1995, 38, 2606-2614.

(39) Doble, D. M. J.; Melchior, M.; O'Sullivan, B.; Siering, C.; Xu, J.; Pierre, V. C.; Raymond, K. N. Inorg. Chem. 2003, 42, 4930-4937.

(40) Ma, Y.; Luo, W.; Quinn, P. J.; Liu, Z.; Hider, R. C. J. Med. Chem. 2004, 47, 6349-6362.

(41) Eiden, F.; Plueckhan, J. Archiv der Pharmazie und Berichte der Deutschen Parmazeutischen Gasellschaft 1969, 302, 622-627.

(42) Fakih, S.; Podinovsdaia, M.; Kong, X.; Collins, H. L.; Schaible, U. E.; Hider, R. C. J. Med. Chem. 2008, 51, 4539-4552.

(43) Tolentino, L.; Kagan, J. J. Org. Chem. 1974, 39, 2308-2309.

(44) SAINT: SAX Area-Detector Integration Program, V.6.40; Bruker Analytical Xray Systems, Inc.: Madison, WI, 2003.

(45) SAINT: SAX Area-Detector Integration Program, V.4.024; Siemens Industrial Automation, Inc, Madison, WI, 1995.

(46) XPREP (V.6.12), Part of SHELXTL Crystal Structure Determination Package; Bruker Analytical X-ray Systems, Inc.: Madison, WI, 2001.

(47) SADABS: Bruker Nonius Area Detector Scaling and Absorption V. 2.05; Bruker Analytical X-ray Systems, Inc.: Madison, WI, 2003.

(48) Sheldrick, G. M. Acta Cryst. 2008, A64, 112-122. 
(49) SHELXTL (V.5.10), SHELXTL Crystal Structure Determination Package; Bruker Analytical X-ray Systems, Inc.: Madison, WI, 1997.

(50) SIR92: Altomare, A., Burla, M. C., Camalli, M., Cacarano, M., Giacavazzo, C., Guagliardi, A., Polidori, G., J. Appl. Cryst, 1994.

(51) Bartusek, M.; Sommer, L. J. Inorg. Nucl. Chem. 1965, 27, 2397-2412. 


\section{Chapter 2 Appendix}

\section{A2.1 Crystallographic Refinement Details}

General. Unless otherwise noted, all non-hydrogen atoms were refined anisotropically. Hydrogen atoms, although usually visible in the Fourier maps, were generated in calculated positions and their positions were refined using the riding model.

Hydrogen atoms on methyl groups attached to $\mathrm{sp}^{2}$-hybridized atoms were refined again with the riding model, but the rotation of the $\mathrm{C}-\mathrm{X}$ bond (where $\mathrm{X}$ represents the $\mathrm{sp}^{2}$ hybridized atom) was allowed to refine freely. Instances in which such refinement did not lead to reliable results were addressed by omission of the hydrogens in question. Relative occupancies of disordered solvent or ligand conformations are determined by free refinement of free variables. Data to resolution of 0.80 to $0.83 \AA$ were used for crystal structure analysis unless otherwise noted. Any lower resolution limits were imposed because of lack of significantly intense reflections beyond those resolutions. The diffraction location and detector used to collect each structure is indicated after it title in parentheticals (ALS = Advanced Light Source at LBNL, Bruker Plantinum 200 or APEX II detectors; SMART $=$ UC Berkeley, Bruker SMART 1000 detector; APEX $=$ UC Berkeley, Bruker APEX I detector).

$\mathbf{U O}_{2}$ (2Li-Me-3,2-HOPO), $\mathbf{U O}_{2}$ (2-2). (ALS). The ML complex was not a discrete species in this structure. Four coordinating oxygens about the uranium were provided by one bis-HOPO ligand, while the fifth, typically occupied by solvent, was provided by an amide oxygen of another $\mathrm{UO}_{2}(2-2)$ complex. The result is a 1-dimensional coordination polymer in the crystal. Mild similarity restraints were used on some atoms in the HOPO moieties due to their erratic refinement. This behavior could not be resolved by re- 
integration or modified absorption correction on the diffraction data, and was attributed to the very small size of the crystal which then caused difficulties with reliable absorption correction.

UO $_{2}$ (thio-Me-3,2-HOPO)(DMF), UO UO $_{2}$ (2-6)(DMF). (APEX). Mild similarity restraints were used on the anisotropic displacement parameters in the thiophene backbone ring due to the abnormal displacement parameter of one of the ring carbons, which was inconsistent with a possible direction of thermal disorder and was not proportionate to its neighboring atoms.

UO $_{2}$ (o-phen-Me-3,2-HOPO)(DMSO), $\quad \mathrm{UO}_{2}$ (2-7)(DMSO). (APEX). Standard refinement.

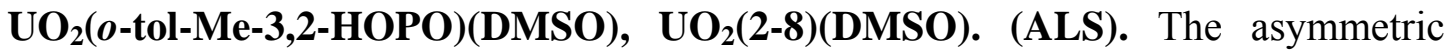
unit of this crystal contained two molecules of the $\mathrm{UO}_{2}(2-8)(\mathrm{DMSO})$ complex. Both coordinated DMSO molecules exhibited rotational disorder about their $\mathrm{U}-\mathrm{O}_{\mathrm{DMSO}}$ bonds, with the oxygen positions shared between the two observed conformations. In one case, both carbons of the DMSO molecules are also shared, with only the sulfur atom exhibiting positional disorder refined to be $85: 15$. The other DMSO had one carbon in a shared position, with one carbon and the sulfur exhibiting positional disorder. The carbon atoms that were not shared were kept isotropic because their anisotropic thermal ellipsoids were unreasonably large if refined as such. This residue's disorder ratio was refined to be $75: 25$. Standard distance restraints were used to model these DMSO residues. One reflection was removed from the refinement due to an abnormally large $\mathrm{F}_{\mathrm{obs}} / \mathrm{F}_{\text {calc }}$ ratio. This reflection was at very low angle, and was most likely contaminated 
with glass scatter from the capillary in which the crystal was mounted during data collection.

$\mathrm{UO}_{2}$ (m-tol-Me-3,2-HOPO)(MeOH), $\mathrm{UO}_{2}(2-13)(\mathrm{MeOH})$. (APEX). The crystal structure contains a free DMSO solvent molecule which displayed positional disorder in a ratio of 78:22. Standard distance restraints were used to model these residues, and the anisotropic displacement parameters of chemically equivalent atoms were restrained to be similar. The oxygen atoms of the disordered positions were found to be 2.49 and 3.56 angstroms from the oxygen atom of the methanol coordinated to the uranyl cation, suggesting significant hydrogen bonding to both disordered positions.

$\mathrm{UO}_{2}\left(\mathrm{o}\right.$-xy-Me-3,2-HOPO), $\mathrm{UO}_{2}(2-14)$. (APEX). Four coordinating oxygens about the uranium were provided by one bis-HOPO ligand, while the fifth, typically occupied by solvent, was provided by an amide oxygen of another $\mathrm{UO}_{2}(2-14)$ complex. The result is a 1-dimensional coordination polymer in the crystal. The other amide oxygen of the complex is located 2.70 angstroms from a free $\mathrm{MeOH}$ solvent atom, suggesting significant hydrogen bonding. The rotation of the methanolic proton in that solvent molecule was refined freely and results in a position that supports the suspected hydrogen bonding.

$\mathrm{UO}_{2}$ (m-xy-Me-3,2-HOPO)(DMF), $\quad \mathrm{UO}_{2}$ (2-15)(DMF). $\quad$ (APEX). $\quad$ Standard refinement.

UO $_{2}$ (fluo-Me-3,2-HOPO)(DMSO), UO $_{2}$ (2-16)(DMSO). (ALS). The coordinated DMSO molecule was disordered over two positions, with relative ratio of 90:10. This disorder is essentially a rotation about the $\mathrm{U}-\mathrm{O}_{\text {DMSO }}$ bond, but also includes a displacement of the coordinated oxygen atom. Standard distance and angle restraints 
were used to maintain reasonable geometries in the disordered residues of this DMSO. For the purpose of calculations of angles, angle sums, and angles between mean square planes used only the $90 \%$ occupancy oxygen atom. There was a disordered water molecule found over two positions in the crystal structure. By varying the size of their thermal ellipsoids, it was determined that each of the water molecules was only present $60 \%$ of the time. Because no $\mathrm{H}$ atom could be seen in these sites as well as their significant disorder, no hydrogen atoms were added to these atoms.

$\left[\mathrm{UO}_{2}(\text { Et-thio-Me-3,2-HOPO)(DMSO) }]_{2}, \mathrm{UO}_{2}(2-37)(\mathrm{DMSO})\right]_{2} \# 1$. (APEX). The asymmetric unit of the crystal contains one bis-HOPO ligand and one uranyl cation. A crystallographic two-fold axis generates the other half of the $\left[\mathrm{UO}_{2}(2-37)(\mathrm{DMSO})\right]_{2}$ dimer. One ethyl substituent on the thiophene backbone was disordered and so was modeled over two positions with a 78:22 disorder ratio. Mild restraints were used to make the disordered C-C and C-S distances the same.

$\left[\mathrm{UO}_{2}(\text { Et-thio-Me-3,2-HOPO)(DMSO) }]_{2}, \quad\left[\mathrm{UO}_{2}(2-37)(\mathrm{DMSO})\right]_{2} \quad \# 2 . \quad\right.$ (SMART). Data collection for this crystal was carried out to $0.83 \AA$. The spots were rather diffuse and weak due to the rapid desolvation of the crystal during the mounting procedure, making integration and scaling difficult. Several attempts were made to find the best scaling for the crystal, but the plate-like shape and diffuse scattering of the crystal, but no significantly better solution was found than that used in the final refinement.

One methyl group on a thiophene alkyl substituent showed positional disorder and so was modeled over two positions with a disorder ratio of 59:41. Both uranium-coordinated DMSO molecules also showed positional disorder. One DMSO was modeled with common carbon atoms and uranyl-coordinated oxygen, but with the sulfur atom 
disordered over two positions in a $66: 34$ ratio. In the other DMSO one carbon and the uranyl-coordinated oxygen are common, but with one methyl group and the sulfur atom disordered over two positions in a 90:10 ratio. Standard bond distance restraints were used in modeling these disorders and chemically equivalent bonds were restrained to be similar.

The crystal structure contains a pocket of solvent molecules that were easily discernable at methanol molecules. One of these had the oxygen atom disordered over two positions in a 74:26 ratio with a common carbon atom. Another molecule was determined to have only $1 / 3$-occupancy due to the relative sizes of its atom volumes as compared to the other methanol molecules. Bond lengths in the methanol molecules were restrained to known $\mathrm{C}-\mathrm{O}$ bond lengths and restrained to be similar. Anisotropic displacement parameters of the atoms in each individual methanol molecule were restrained to be similar. Hydrogen atoms were not placed on the methanol molecules because no obvious residual peaks were found in the Fourier map and possible hydrogen bonding networks makes it difficult to predict their positions.

Mild thermal displacement parameter restrains were used in one HOPO ring to even out the anomalous thermal displacement parameter of $\mathrm{C} 6$, and similar restraints were used on $\mathrm{O7}$. The use of anisotropic displacement parameter restraints was relatively widespread in this structure and was considered necessary to compensate for the necessarily poor scaling of the data that resulted anomalous displacement parameters of some atoms. In one case (C38), a carbon had to be left isotropic because no reasonable combination of restraints could make it refine anisotropically without becoming nonpositive-definite. 


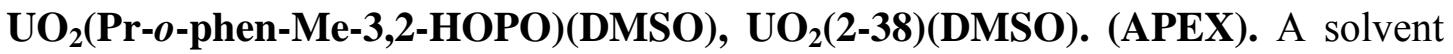
water molecule was found in the structure, and modeled as having $1 / 4$-occupancy based on the size of the original residue peak of approximately 2 electrons. This solvent water was refined isotropically and hydrogens were not assigned to the oxygen because no q-peaks were found and the proximity of the oxygen to a uranyl oxo atom and both HOPO amide protons suggests a variety of hydrogen bonding possibilities. Displacement parameter restraints were used in modeling the ligand one atom in the backbone whose behavior deviated anomalously from those of its neighbors.

$\mathrm{UO}_{2}$ (2-Hydroxy-5-methyl- $\left.\alpha, \alpha^{\prime}-m-x y-M e-3,2-H O P O\right), \mathrm{UO}_{2}$ (2-39). (SMART). Four coordinating oxygens about the uranium were provided by one bis-HOPO ligand, while the fifth, typically occupied by solvent, was provided by an amide oxygen of another $\mathrm{UO}_{2}$ (2-39) complex. The result is a 1-dimensional coordination polymer in the crystal. There was also a methanol molecule in the crystal lattice that is engaged in a hydrogen bonding interaction with the backbone phenol (O-O distance is $2.69 \AA$ ).

$\mathrm{UO}_{2}$ (2-Methoxy-5-methyl- $\left.\alpha, \alpha^{\prime}-m-x y-M e-3,2-H O P O\right)(D M S O)$, $\mathrm{UO}_{2}$ (240)(DMSO). (APEX). One HOPO methyl group exhibited rotational disorder of the methyl hydrogen atoms, so was modeled as a perfectly disordered methyl group displaced at a $60^{\circ}$ interval in a 50:50 distribution. One linking amide oxygen acts as a hydrogen-bond acceptor to a water inclusion in the crystal structures $(\mathrm{O}-\mathrm{O}$ distance of $2.81 \AA$ ). The protons on the water molecule were constrained to typical $\mathrm{O}-\mathrm{H}$ and $\mathrm{H}-\mathrm{-H}$ distances, but were allowed to position themselves about the water oxygen atom freely, generating the observed hydrogen bonding interaction. Symmetry considerations require 
that this water is also hydrogen-bonded to another linking amide oxygen from another $\mathrm{UO}_{2}(\mathbf{2 - 4 0})(\mathrm{DMSO})$ complex.

\section{A2.2 Crystallographic Figures}

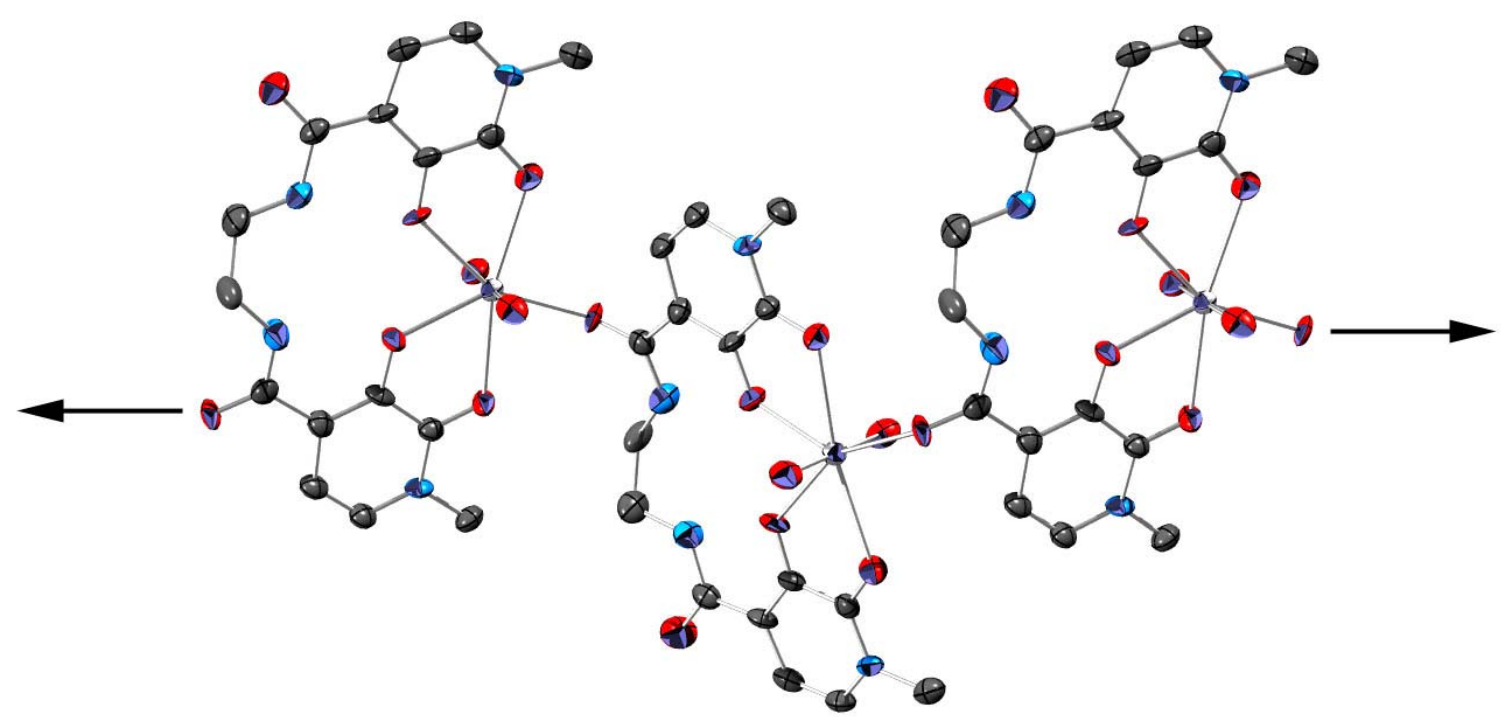

Figure A2-1. Polymeric chain in the crystal structure of $\mathrm{UO}_{2}(2-2)$. Only three subunits are shown here. Hydrogen atoms have been omitted for clarity. Thermal ellipsoids are drawn at the 50\% level. Carbons are gray, oxygens red, nitrogens blue, and uraniums silver.

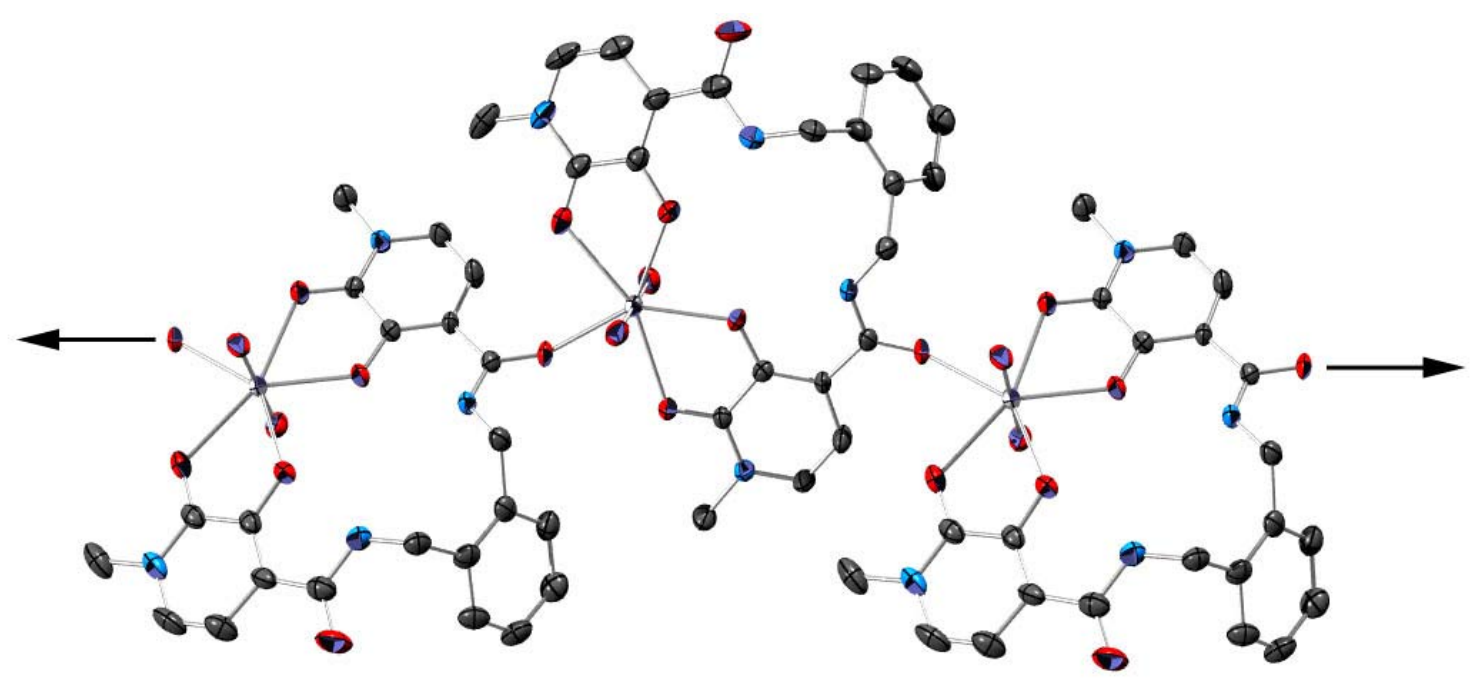

Figure A2-2. Polymeric chain in the crystal structure of $\mathrm{UO}_{2}(2-14)$. Hydrogen atoms and methanol inclusion have been omitted for clarity. Only three subunits are shown here. Thermal ellipsoids are drawn at the 50\% level. Carbons are gray, oxygens red, nitrogens blue, and uraniums silver. 


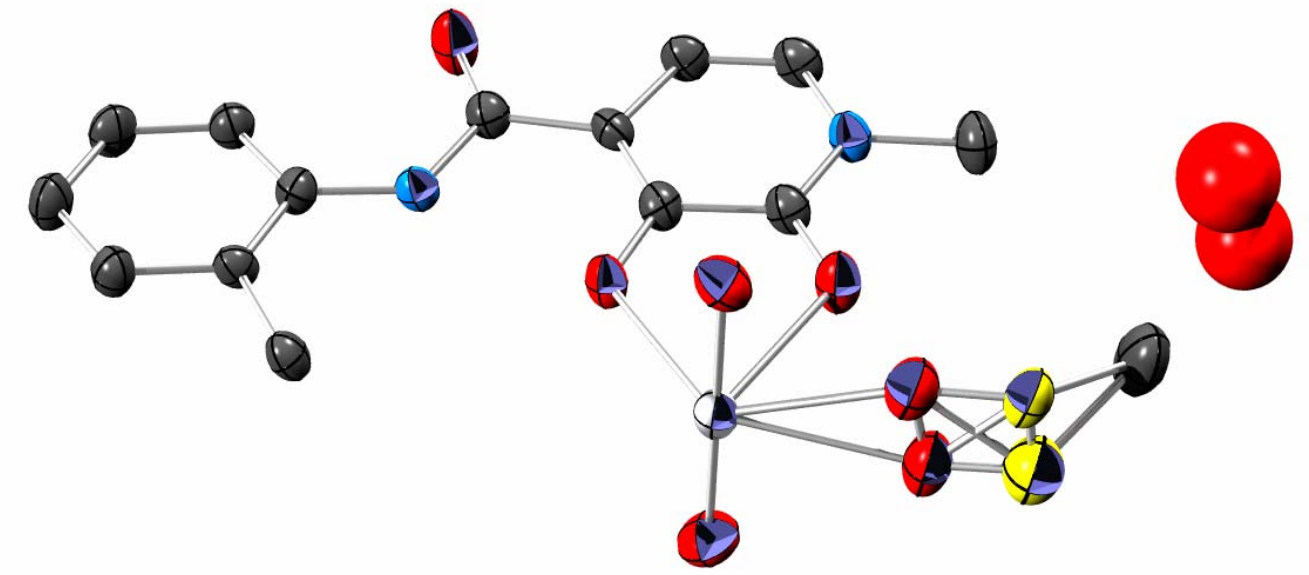

Figure A2-3. Asymmetric unit in the crystal structure of $\mathrm{UO}_{2}(2-16)(\mathrm{DMSO})$. The disordered water molecule and the coordinated DMSO disorder are included. Hydrogen atoms have been omitted for clarity. Thermal ellipsoids are drawn at the $50 \%$ level. Carbons are gray, oxygens red, nitrogens blue, sulfur yellow, and uranium silver.

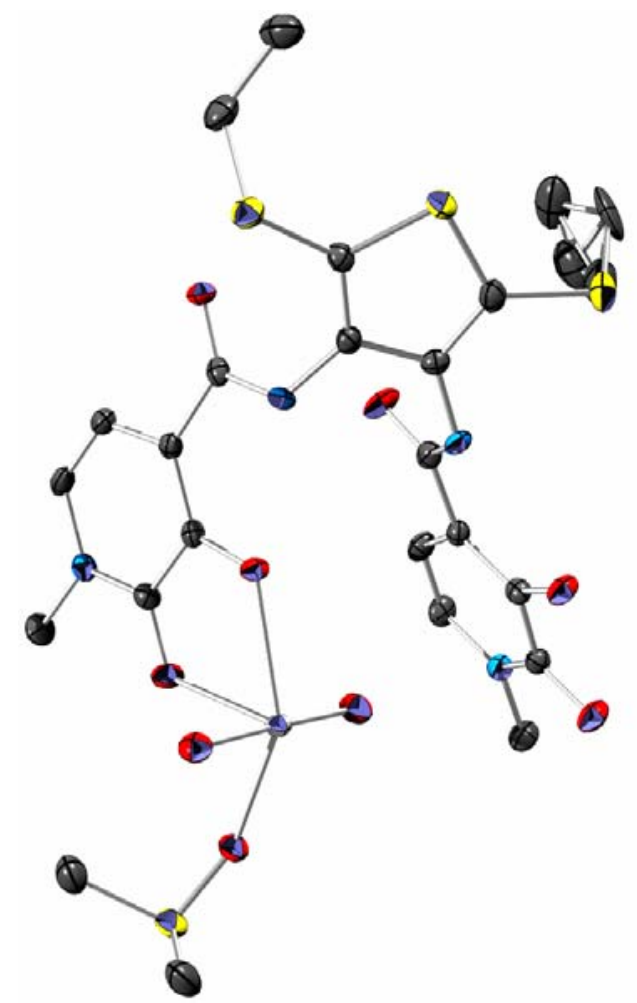

Figure A2-4. Asymmetric unit in the dimeric crystal structure of $\left[\mathrm{UO}_{2}(2-37)(\mathrm{DMSO})\right]_{2}$. Alkyl chain disorder has been included. Hydrogen atoms have been omitted for clarity. Thermal ellipsoids are drawn at the 50\% level. Carbons are gray, oxygens red, nitrogens blue, sulfurs yellow, and uranium silver. 


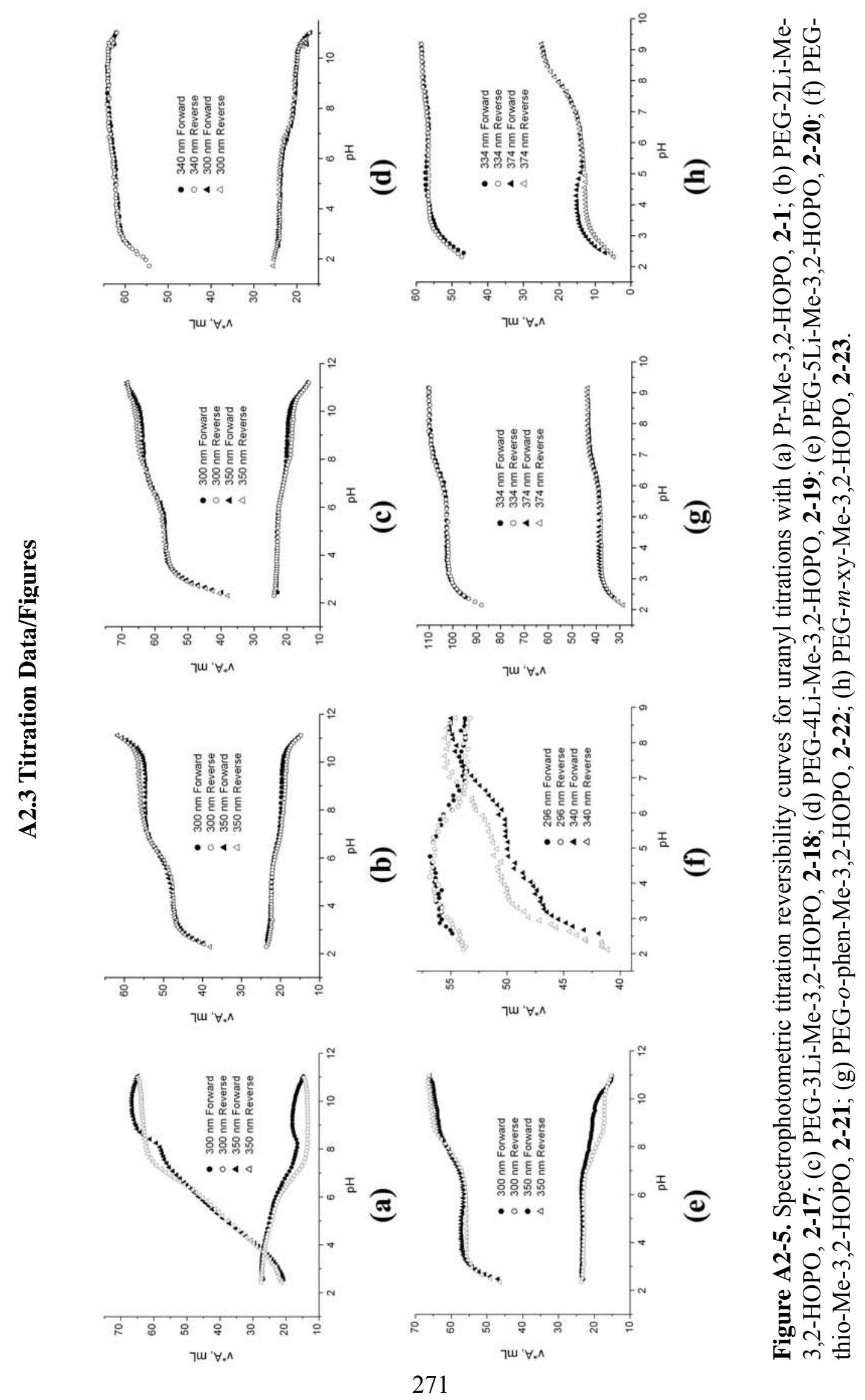



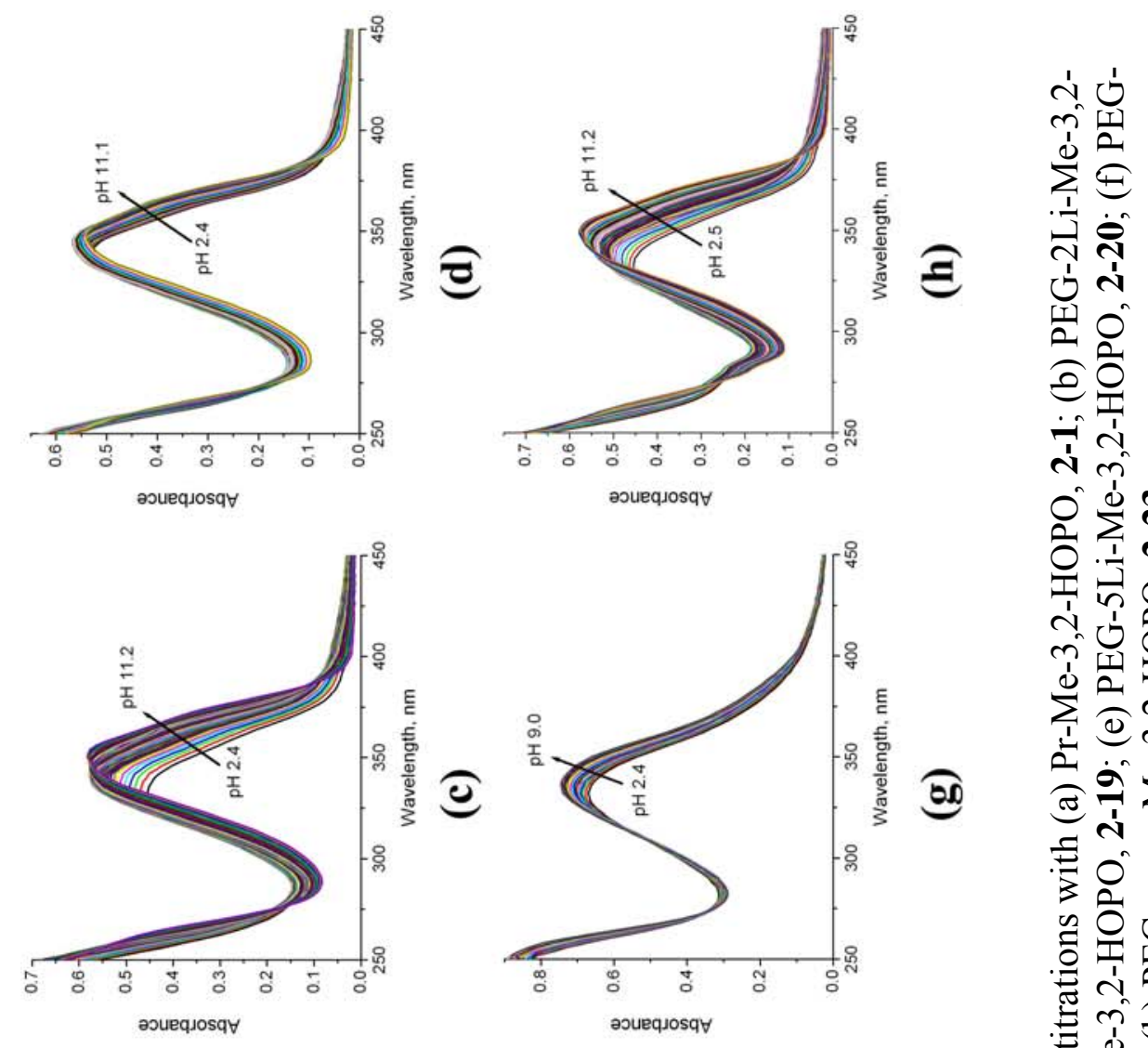

Oิ $\sum_{0}^{0}$ ì

궁

ติ

$\sum_{1} \sqrt{0}$

尚

ฮิ

氠主

ํํㅇำ

兄

预
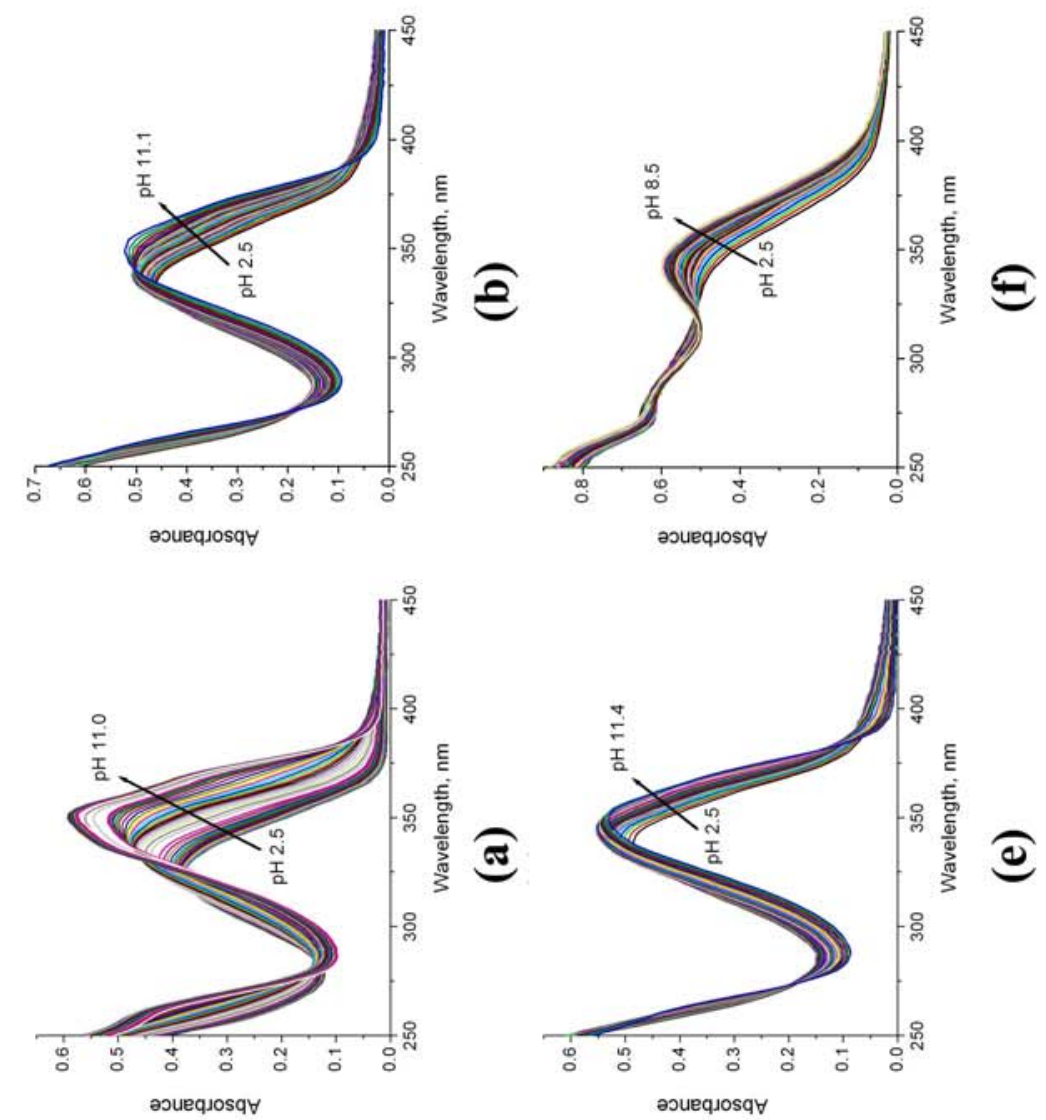

¿ $\sum_{1}$

클 ㅅ․

○े

\& 2

ᄅ्छे

0 क

.ํํㄹㄹ

:

ㅇํㅇ 옹

चै ป่ रा

उ $\sum_{1}^{0}$ ब0

ڤึ

잉

응

\& 00

ம்

ขै

ㅇํㅇ

总跳 

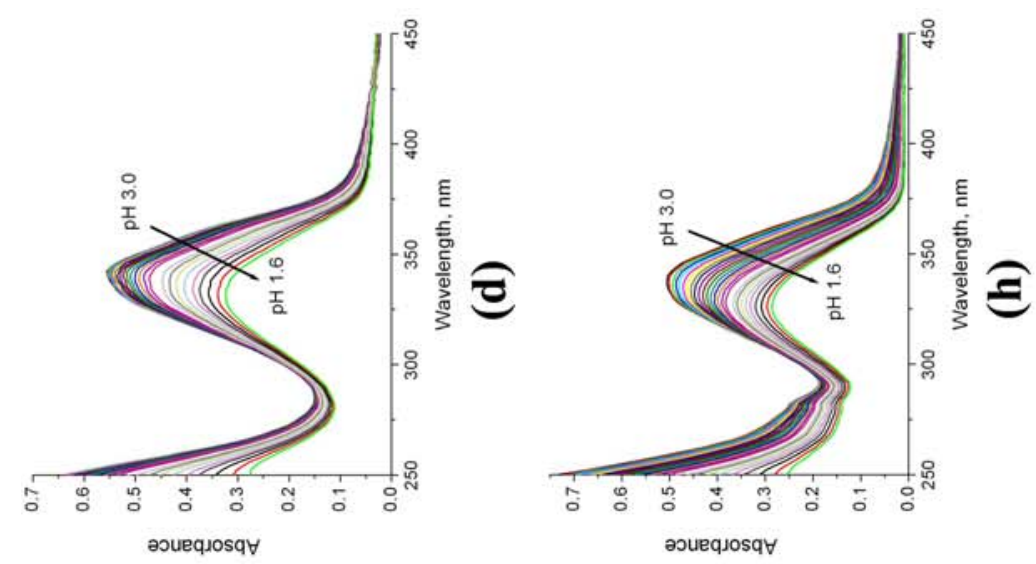

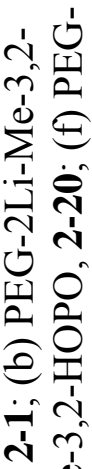
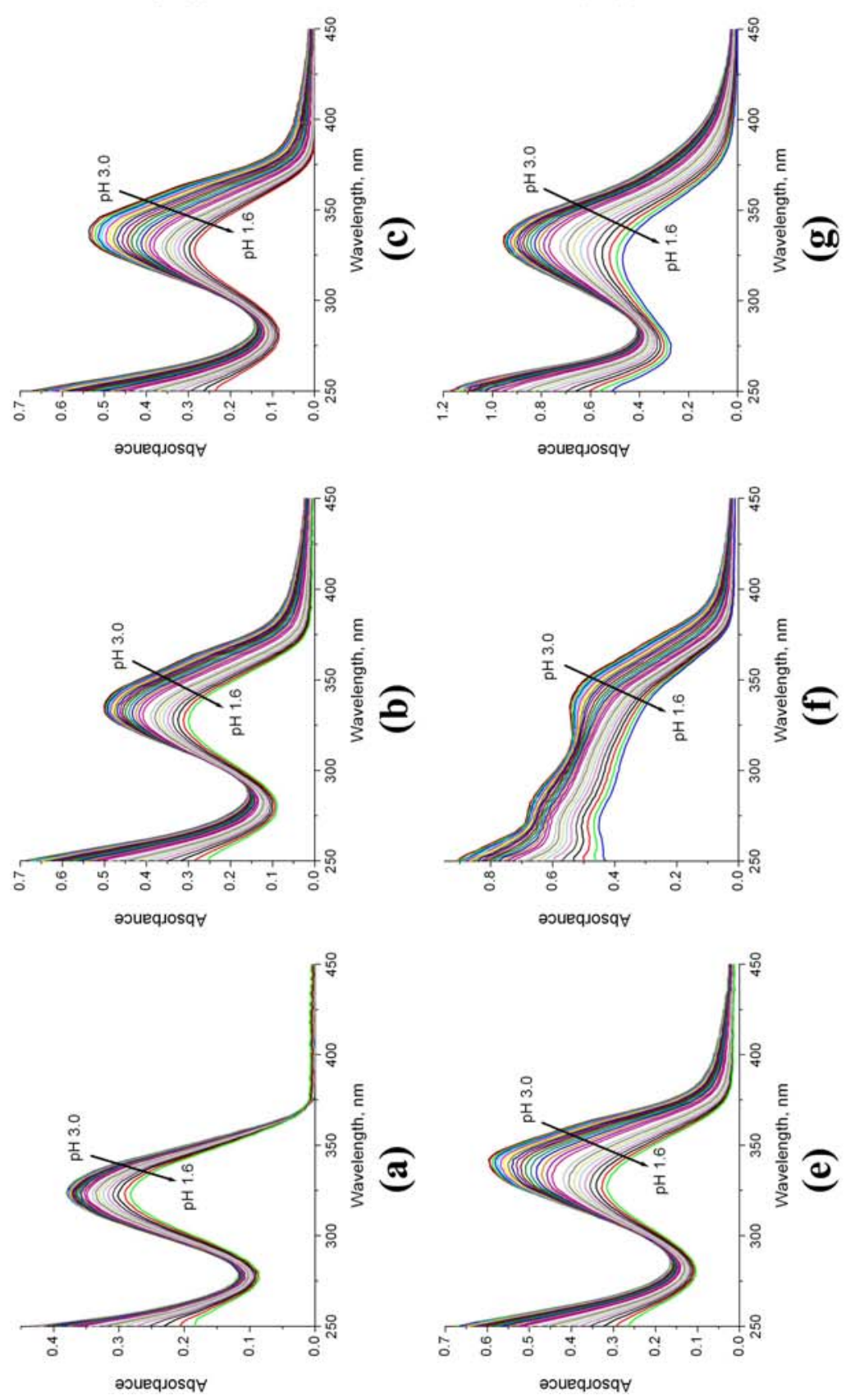

$0 \sum_{1}^{\infty} \stackrel{n}{~}$

봉

ป่

ต펄

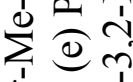

ப்

ฮิ ते

.气

옹

흠

컵

就

入

晃

ํํㅇ

के

है

ธี 기

쿵

断

일 1

든

$1 \stackrel{0}{0}$

$3 \sum_{1}^{0}$ क्ष

- 굮

엠ㄷㅇ

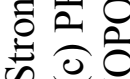

\%

ขे ㄱํํ

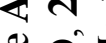

ํํㄹ

몬언을 

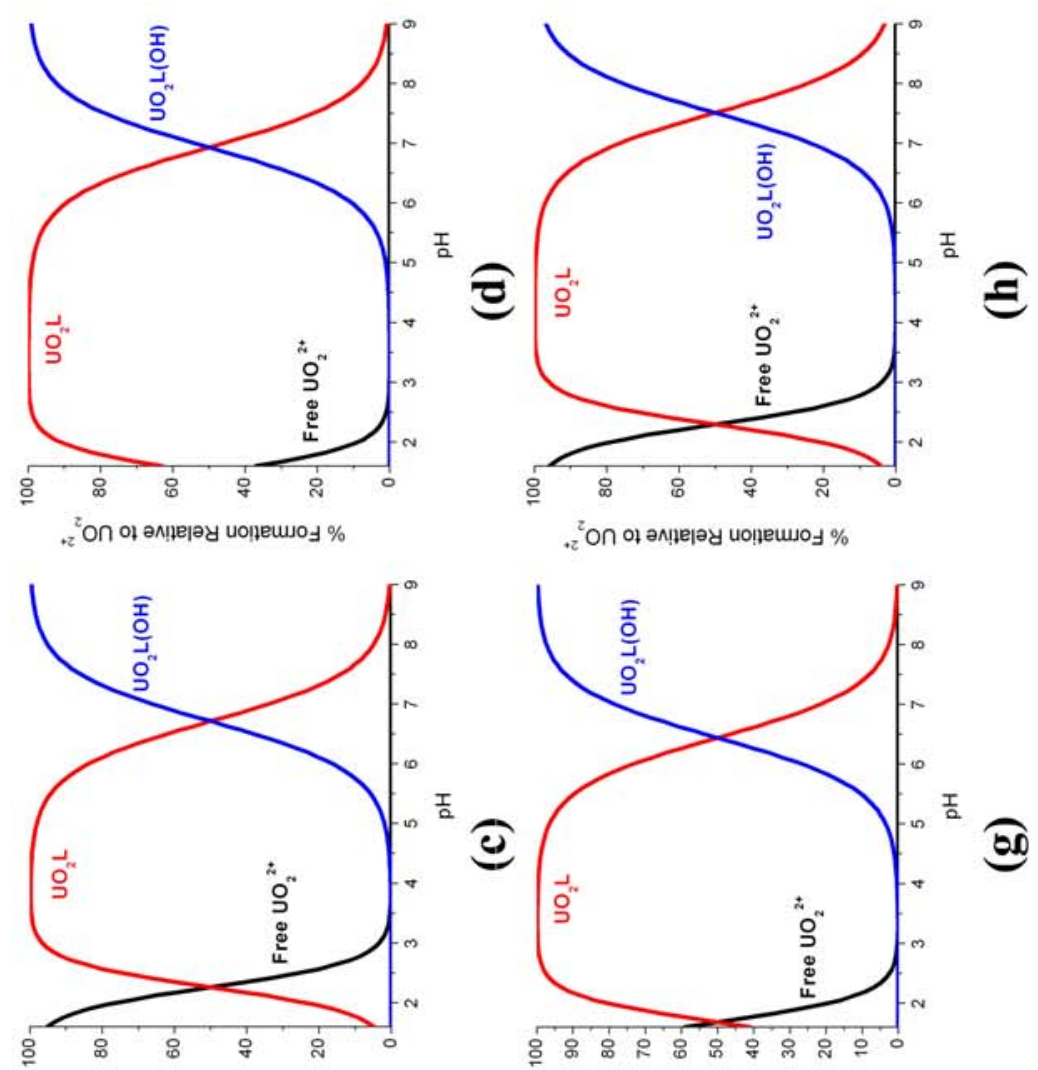

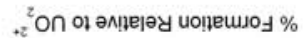

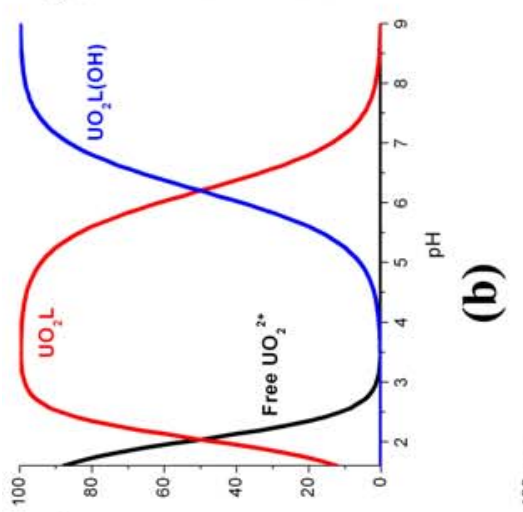

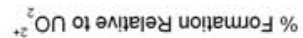

ํำ

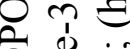

검 $\sum_{-1}^{0}$

ले

$\sum^{\prime} \circlearrowright$

in 1

하

की

3 '

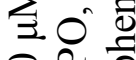

응ㅇㅇ

11 피

ป ำ

चल

$\sum_{\sum}^{0} \sum_{1}^{000}$

二흐

ㅎํ

¿

+ త

ชั่

$Ð \stackrel{1}{\prime}$

แ

응 잉

灭 正

ฮ่

을

○ृ $\sum_{i}^{d} \Theta$

㑒

2 ํํ

氙

50 0

음

뭄

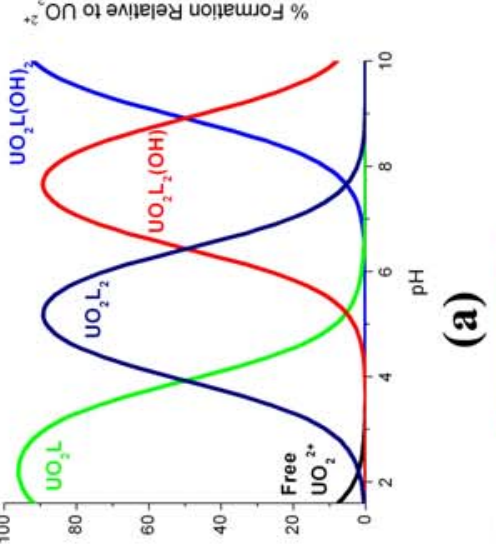

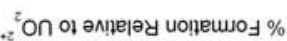

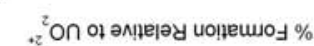

ஸे

- $\bigodot^{\infty}$

负店

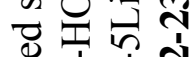

䒕

䓠

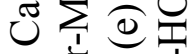

$\infty \dot{\sigma} \dot{n}$

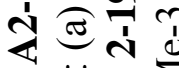

\& $\ddot{\theta} 0 \sum_{1}$

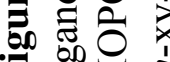

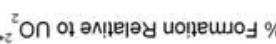




\section{A2.4 Additional $\mathrm{pUO}_{2}$ Analysis}

The classical definition of $\mathrm{pM}$ utilizes the value " $\mathrm{M}_{\text {freee }}$ which represents the free, solvated ion excluding hydrolysis products $\left(\mathrm{pM}=-\log \left[\mathrm{M}_{\text {free }}\right]\right)$. However, an increased $\mathrm{pUO}_{2}$ upon increased $\mathrm{pH}$ (as seen in Chapter 2) can feasibly be the result of two competing processes: metal binding by ligand and hydrolysis of the ion (metal coordination by hydroxide ion). The former phenomenon may be significant if the ligand in question experiences increased deprotonation upon increased $\mathrm{pH}$, perhaps making it a better chelator towards the metal ion. However, if no change in the ligand or the complex increases the ligand's affinity for the metal ion upon increased $\mathrm{pH}$, an increased $\mathrm{pM}$ could result primarily from an increase in hydroxide concentration and a resultant increase in hydrolysis product concentration. This latter option would tend to suggest (falsely) that a ligand's metal affinity increases upon increased $\mathrm{pH}$.

A method by which these effects can be discerned is by adopting an alternative interpretation of " $\mathrm{M}_{\text {freee }}$ " in the $\mathrm{pM}$ formula; namely, " $\mathrm{M}_{\text {free" would need to be defined as }}$ "any metal ion species that is uncomplexed by the ligand in question." This definition of " $M_{\text {free }}$ includes both the standard definition of solvated free ion, but also includes any hydrolysis products that may form at higher $\mathrm{pH}$. The $\mathrm{pM}$ derived using this modified definition of " $\mathrm{M}_{\text {free }}$ " will be referred to here as $\mathrm{pM}^{*}$, or in the case of the current discussion, $\mathrm{pUO}_{2} *$. While $\mathrm{pUO}_{2}{ }^{*}$ is no longer a truly thermodynamic measure like the classical $\mathrm{pUO}_{2}$, it may help clarify the $\mathrm{pUO}_{2}$ results presented in the main text. Following the reasoning described above, an increase in $\mathrm{pUO}_{2} *$ upon increased $\mathrm{pH}$ suggests increased affinity of the ligand for the uranyl cation, while a $\mathrm{pUO}_{2} *$ that stays roughly constant or decreases upon increased $\mathrm{pH}$ indicates that the ligand in question binds the 
uranyl cation with the same or decreased affinity, respectively, at higher $\mathrm{pH}$ as compared to the competing hydroxide ion. The $\mathrm{pUO}_{2}{ }^{*}$ values for the Me-3,2-HOPO ligands investigated in Chapter 2 are listed in Table A2-1.

Table A2-1. Calculated $\mathrm{pUO}_{2}{ }^{*}$ values for Me-3,2-HOPO ligands.

\begin{tabular}{|c|c|c|c|}
\hline \multirow{2}{*}{ Ligand } & \multicolumn{3}{|c|}{$\mathbf{p U O}_{2}$ * $^{\boldsymbol{a}}$} \\
\cline { 2 - 4 } & $\mathbf{p H ~ 2 . 5}$ & $\mathbf{p H}$ 7.4 & $\mathbf{p H ~ 8 . 5}$ \\
\hline $\mathbf{2 - 1}$ & $7.98(3)$ & $12.37(4)$ & $12.57(1)$ \\
\hline $\mathbf{2 - 1 7}$ & $7.0(1)$ & $12.34(8)$ & $12.36(8)$ \\
\hline $\mathbf{2 - 1 8}$ & $6.6(1)$ & $11.95(7)$ & $11.95(6)$ \\
\hline $\mathbf{2 - 1 9}$ & $8.0(1)$ & $13.17(8)$ & $13.10(7)$ \\
\hline $\mathbf{2 - 2 0}$ & $7.1(1)$ & $12.15(6)$ & $11.77(4)$ \\
\hline $\mathbf{2 - 2 1}$ & $6.01(1)$ & $11.10(3)$ & $11.09(2)$ \\
\hline $\mathbf{2 - 2 2}$ & $7.62(4)$ & $12.68(9)$ & $12.7(1)$ \\
\hline $\mathbf{2 - 2 3}$ & $6.55(6)$ & $11.71(3)$ & $11.47(5)$ \\
\hline
\end{tabular}

The $\mathrm{pUO}_{2} *$ values at $\mathrm{pH} 2.5$ are the same as the $\mathrm{pUO}_{2}$ values reported in Table 2-10, since the free uranyl cation is more prominent than its hydrolysis products at this $\mathrm{pH}$. However, at $\mathrm{pH} 7.4$ and 8.5 the hydrolysis products of the uranyl cation are the most common ligand-free species in solution, and become more concentrated as $\mathrm{pH}$ increases. Because the quantity $\mathrm{pUO}_{2} *$ considers these hydrolysis products in its definition of " $\mathrm{UO}_{2}{ }^{2+}$ free", the $\mathrm{pUO}_{2}{ }^{*}$ values above are consistently about 2 to $3 \log$ units lower at $\mathrm{pH}$ 7.4 and 3 to $5 \log$ units lower at $\mathrm{pH} 8.5$ than those reported in Chapter 2.

As with $\mathrm{pUO}_{2}$ values in Table 2-10, all ligands exhibit a dramatic rise in $\mathrm{pUO}_{2}$ (ca. 5 $\log$ units) between $\mathrm{pH} 2.5$ and $\mathrm{pH}$ 7.4, which arises from the need for Me-3,2-HOPO ligands to be deprotonated for metal chelation to occur. The crucial difference between the values in Table 2-10 and Table A2-1 is that the difference in $\mathrm{pUO}_{2}{ }^{*}$ values at $\mathrm{pH} 7.4$ and 8.5 are similar or statistically identical to each other. This indicates that the difference in ligand binding capacity towards the uranyl cation does not actually increase upon increased $\mathrm{pH}$, as the $\mathrm{pUO}_{2}$ values in Table 2-10 may seem to suggest. More importantly, it also indicates that the ligand does not become a better chelator towards 
uranyl upon deprotonation (e.g. in the case of subsequent deprotonation of chelating atoms), and also that the increasing hydroxide ion concentration poses no competition to the bis-Me-3,2-HOPO ligands at least up through $\mathrm{pH} 8.5$.

Another interesting comparison to make between the bis-Me-3,2-HOPO ligands evaluated by solution thermodynamics is to use their calculated $\mathrm{pK}_{\mathrm{a}}$ and $\log \beta_{m l h}$ values to model the uranyl distribution between the various ligands if they were to exist in solution simultaneously. Namely, if $\left[\mathrm{UO}_{2}{ }^{2+}\right]=1 \mu \mathrm{M}$ and $[2-17]=[2-18]=[2-19]=[2-20]=[2-$ $21]=[2-22]=[2-23]=1.43 \mu \mathrm{M}(\Sigma[\mathrm{L}]=10 \mu \mathrm{M})$, which ligand would bind the uranyl cation most prominently, and by how much in comparison to the other ligands? A species distribution for this scenario is shown in Figure A2-9, and selected percentage distributions are listed in Table A2-2.

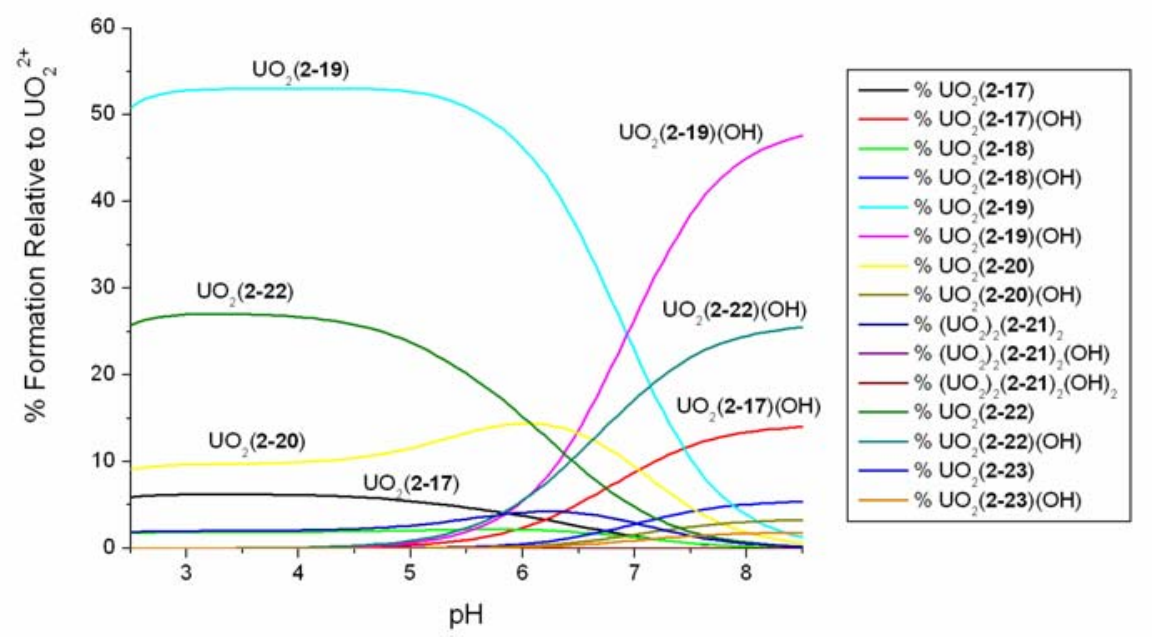

Figure A2-9. Species distribution for uranyl in the presence of seven bis-Me-3,2-HOPO ligands $\left(\left[\mathrm{UO}_{2}{ }^{2+}\right]=1 \mu \mathrm{M}, \Sigma[\mathrm{L}]=10 \mu \mathrm{M}\right)$. 
Table A2-2. Percentage of complexed $\mathrm{UO}_{2}{ }^{2+}$ in the presence of multiple bis-Me-3,2$\mathrm{HOPO}$ ligands $\left(\left[\mathrm{UO}_{2}{ }^{2+}\right]=1 \mu \mathrm{M}\right.$ and $[\mathbf{2 - 1 7}]=[\mathbf{2 - 1 8}]=[\mathbf{2 - 1 9}]=[\mathbf{2 - 2 0}]=[\mathbf{2 - 2 1}]=[2-22]=$ $[2-23]=1.43 \mu \mathrm{M})$.

\begin{tabular}{|c|c|c|c|}
\hline Ligand & \% UO $_{2}{ }^{\mathbf{2 +}}$ & Complexed by Ligand \\
\hline & $\mathbf{p H ~ 2 . 5}$ & $\mathbf{p H ~ 7 . 4}$ & $\mathbf{p H ~ 8 . 5}$ \\
\hline $\mathbf{2 - 1 7}$ & 5.9 & 11.9 & 14.1 \\
\hline $\mathbf{2 - 1 8}$ & 1.7 & 4.6 & 5.4 \\
\hline $\mathbf{2 - 1 9}$ & 50.8 & 48.9 & 48.9 \\
\hline $\mathbf{2 - 2 0}$ & 9.1 & 8.0 & 3.9 \\
\hline $\mathbf{2 - 2 1}$ & 0 & 0 & 0 \\
\hline $\mathbf{2 - 2 2}$ & 25.7 & 23.5 & 25.7 \\
\hline $\mathbf{2 - 2 3}$ & 1.9 & 3.1 & 2.0 \\
\hline
\end{tabular}
\% complexed includes $\mathrm{UO}_{2} \mathrm{~L}(\mathrm{OH})$ species

As with $\mathrm{pUO}_{2}$ or $\mathrm{pUO}_{2}{ }^{*}$ values, the comparison described here does not give any information on the relative selectivities of bis-Me-3,2-HOPO ligands for $\mathrm{UO}_{2}{ }^{2+}$, but the values in Table A2-2 do illustrate the much higher affinity for the uranyl cation of 2-17 over all other bis-Me-3,2-HOPO ligands investigated, with the second best affinity displayed by 2-22. Figure A2-9 illustrates that the affinity of these ligands is dominant at all $\mathrm{pH}$, while lower affinity ligands exchange speciation dominance at different $\mathrm{pH}$ (e.g 2-20 showing more uranyl affinity than 2-17 below pH ca. 7, and switching above pH 7). This behavior in lower affinity ligands is caused by the differing affinity of the hydrolyzed uranyl complexes $\mathrm{UO}_{2} \mathrm{~L}(\mathrm{OH})$ compared to the $\mathrm{UO}_{2} \mathrm{~L}$ complexes. 


\section{Chapter 3 Appendix}

\section{A3.1 Crystallographic Refinement Details}

General. Unless otherwise noted, all non-hydrogen atoms were refined anisotropically. Hydrogen atoms, although usually visible in the Fourier maps, were generated in calculated positions and their positions were refined using the riding model. Hydrogen atoms on methyl groups attached to $\mathrm{sp}^{2}$-hybridized atoms were refined again with the riding model, but the rotation of the $\mathrm{C}-\mathrm{X}$ bond (where $\mathrm{X}$ represents the $\mathrm{sp}^{2}$ hybridized atom) was allowed to refine freely. Instances in which such refinement did not lead to reliable results were addressed by omission of the hydrogens in question. Relative occupancies of disordered solvent or ligand conformations are determined by free refinement of free variables. Data to resolution of 0.80 to $0.83 \AA$ were used for crystal structure analysis unless otherwise noted. Any lower resolution limits were imposed because of lack of significantly intense reflections beyond those resolutions. The diffraction location and detector used to collect each structure is indicated after it title in parentheticals $($ SMART $=$ UC Berkeley, Bruker SMART 1000 detector; APEX $=$ UC Berkeley, Bruker APEX I detector).

$\mathrm{UO}_{2}$ (o-phen-1,2-HOPO)(DMSO), UO $\mathbf{U}_{2}(12 \mathrm{HP})(\mathrm{DMSO})$. (APEX) The asymmetric unit of this crystal contained two $\mathrm{UO}_{2}(\mathbf{1 2 H P})(\mathrm{DMSO})$ complexes. One of the DMSO molecules exhibited positional disorder in which the carbon atoms are shared, and only the sulfur exists in two positions, in a ratio of 77:23. Standard restraints were used on S-C distances in the modeling of this disorder, and the anisotropic thermal displacement parameters of the disordered sulfur atoms were restrained to be similar. There is also a disordered methanol molecule in the crystal structure, which was refined to have 1/8-occupancy. Five reflections 
were omitted from the refinements because their $\mathrm{F}_{\text {obs }}$ were of unreasonably low values. They are all very low angle reflections, so it was assumed that these reflections were lost behind the backstop.

$\mathrm{UO}_{2}\left[\mathrm{TAM}(2 \mathrm{Li}-\mathrm{Me}-3,2-\mathrm{HOPO})_{2}\right] \cdot 2 \mathrm{NMe}_{4}, \mathrm{UO}_{2}(3-2) \cdot 2 \mathrm{NMe}_{4} \cdot(\mathrm{SMART})$ The $\mathrm{UO}_{2}(3-$ 2) complex spans a 2-fold crystallographic axis that passes through the TAM aromatic ring and the uranium atom. Thus, the asymmetric unit is only half of the molecule. In addition to the two tetramethylammonium cations, there is also one acetone solvent molecule in each asymmetric unit, or two per $\mathrm{UO}_{2}(3-2)$ complex. Because it is a chiral space group, Friedel opposites were not merged, causing the data completeness of greater than $100 \%$. The structure was solved in both $\mathrm{P}_{4}{ }_{3}{ }_{1} 2$ and $\mathrm{P} 4_{1} 2_{1} 2$; in the former case the Flack parameter was 1.0 and in the latter it was 0.0 , indicating the correct handedness.

\section{$\mathrm{UO}_{2}\left[\mathrm{TAM}(\text { o-phen-1,2-HOPO) })_{2}\right] \cdot 2 \mathrm{NMe}_{4}, \mathrm{UO}_{2}(3-4) \cdot 2 \mathrm{NMe}_{4}, \# 1 . \quad(\mathrm{SMART})$ Data}

were cut at $0.98 \AA$ becuase beyond that there was essentially no significant diffraction intensity. This low level of diffraction lowered the resultant completeness and redundancy of the data set, but despite the 9:1 data to parameter ratio and significant amount of neccessary solvent disorder modeling, the GooF of 1.029 matches very well with the restrained GooF of 1.033, suggesting the data set is not over-restrained. The crystal structure contained large layers of solvent molecules, requiring extensive disorder modeling:

1.) A molecule of $\mathrm{MeOH}$ was found and modeled to be disordered on itself with $50 / 50$ occupancy of the carbon and oxygen on each of the two atomic coordinates. Neither end of the molecule was considered to be in close contact with a well-defined 
hydrogen bond donor or acceptor and no significant q-peaks could be found to indicate the positions of hydrogen atoms, so none were placed on this molecule.

2.) Two dioxane molecules were found that exhibited reasonable amounts of order so the non-hydrogen atoms were refined anisotropically and hydrogens were added in calculated positions. These solvents showed larger than normal anisotropic displacement parameters due to thermal motion; modeling this motion by positional disorder was not stable, so the ellipsoids were left as they were.

3.) Two dioxane molecules showed significant disorder and were modeled over two positions each, with the geometries of the residues restrained to be similar to one of the ordered dioxane molecules found in the unit cell. Oxygen positions were chosen based on the possible presence of disordered hydrogen atoms seen in the local disorder, but due to the high level of disorder at these sites the hydrogen atoms were not added and the non-hydrogen atoms were refined isotropically. One of these dioxane molecules is disordered over an inversion center, with three atoms of each disordered fragment in the asymmetric unit. These dioxanes exhibit approximately 59:41 and 56:44 occupancy disorder.

4.) One additional dioxane molecule shares a void in the unit cell that is also shared with a partial-occupancy DMSO. These molecules have approximately 85:15 occupancy of the site respectively, and they share a common oxygen atom coordinate. The sulfur atom was refined anisotropically, but the carbon and oxygen atoms were left isotropic, and no hydrogen atoms were added to these partial occupancy atoms.

$\mathrm{UO}_{2}\left[\mathrm{TAM}(o-p h e n-1,2-\mathrm{HOPO})_{2}\right] \cdot 2 \mathrm{NMe}_{4}, \mathrm{UO}_{2}(3-4) \cdot 2 \mathrm{NMe}_{4}$, \#2. (SMART) One tetramethylammonium cation in the crystal was disordered and so modeled as a rigid 
body over two positions with a refined occupancy ratio of 50:50. Of the two dioxane solvent molecules, one is disordered over two position via a chair flip, with a freely refined occupancy ratio of 77:23. The DMSO solvent molecule also showed disorder in which the carbon atoms are shared but the sulfur exists over two positions, with a refined occupancy ratio of 79:21. Modeling of solvent disorder employed mild anisotropic displacement parameter and distance restraints.

\section{$\mathrm{UO}_{2}\left[\mathrm{TAM}(o-p h e n-1,2-\mathrm{HOPO})_{2}\right] \cdot 2 \mathrm{NMe}_{4}, \mathrm{UO}_{2}(3-4) \cdot 2 \mathrm{NMe}_{4}$, \#3. (SMART) Data} were cut at 0.85 angstroms because beyond that resolution the intensity of measured spots dropped precipitously, as did statistical agreement between them.

Some atoms in the $\mathrm{UO}_{2}(3-4)$ complex and the associated cations have relatively large anisotropic thermal displacement parameters, corresponding to very slight ligand disorder or normal thermal motion. Splitting these atoms to model them as positional disorder did not significantly increase the quality of the model, so the atoms were left with large displacement parameters that are all in reasonable directions with respect to expected rotational degrees of freedom.

Two isolated water molecules were found in the Fourier map, with q-peaks suggesting the positions of the hydrogen atoms. These water molecules were found within hydrogen bond distances to electronegative atoms. One water molecule is located 3.1, 2.8, and 2.9 angstroms away from two of the U-coordinated HOPO amide oxygens and one of the linker amide oxygens respectively. The other water molecule is located 2.8 angstroms from a linker amide oxygen as well. These distances support a hydrogen bonding network that dictates their position in the unit cell. Hydrogen positions on these 
water molecules were strictly constrained to have typical $\mathrm{O}-\mathrm{H}$ and $\mathrm{H}--\mathrm{H}$ distances, but their positions were allowed to refine freely otherwise.

The structure contained several disordered regions of solvent close to an inversion center at $(0,0,0.5)$. These regions strongly resembled the shape of dioxane molecules, but the disorder in these molecules could not be modeled satisfactorily enough to justify the severe increase in restraint-to-parameter ratio that resulted. Additionally, in some of the regions could be seen fragments that resembled disordered methanol or water molecules. Thus, the program SQUEEZE ${ }^{1}$ by Spek et. al. in the PLATON ${ }^{2}$ software suite was used to calculate the electron count in these voids of disordered solvent, and these areas were removed from further refinement. The calculation determined there to be 176 electrons per unit cell in these regions, which is approximately the electron count of three dioxane molecules, one methanol and one water, which corresponds roughly to what was seen during refinement and what is represented in the reported atom count for the structure.

$\mathrm{UO}_{2}\left[\mathrm{TAM}(o-p h e n-1,2-\mathrm{HOPO})_{2}\right] \cdot 2 \mathrm{NMe}_{4}, \mathrm{UO}_{2}(3-4) \cdot 2 \mathrm{NMe}_{4}$, \#4. (SMART) This crystal structure was very well ordered with the exception of a disordered DMSO molecule. This molecule was disordered over two positions with a freely-refined disorder ratio of 55:45. The DMSO molecule lies close to an inversion center, and the positional disorder is required because when one molecule of the DMSO is in the major position, the other molecule is in the minor position. If they both existed closer to the inversion center about which they sit, they would exist slightly too close to each other.

The data for this structure was collected and refined to $0.83 \AA$, but because it is a triclinic cell it has poor coverage statistics, especially in the very highest resolution shell 
of weak data. However, the $\mathrm{R}_{\text {sym }}$ and $\mathrm{R}_{\text {shell }}$ values for this shell were in good agreement with the other data, so were kept in the refinement.

$\mathrm{UO}_{2}\left[\mathrm{TAM}\left(o-p h e n-M e-3,2-\mathrm{HOPO}_{2}\right] \cdot 2 \mathrm{NMe}_{4}, \quad \mathrm{UO}_{2}(3-5) \cdot 2 \mathrm{NMe}_{4} \cdot \quad(\mathrm{SMART})\right.$ The $\mathrm{UO}_{2}(3-5)$ complex spans a 2-fold crystallographic axis that passes through the TAM aromatic ring and the uranium atom. Thus, the asymmetric unit is only half of the molecule. In addition to the two tetramethylammonium cations, there is also one $\mathrm{MeOH}$ solvent molecule in each asymmetric unit, or two per $\mathrm{UO}_{2}(3-5)$ complex. The hydrogen atom on the methanol was refined with a constrained $\mathrm{O}-\mathrm{H}$ distance, but the rotation around the $\mathrm{O}-\mathrm{C}$ bond was free to refine, placing the proton directed towards an amide proton on the $\mathrm{UO}_{2}(3-5)$ complex. This, along with the $2.76 \AA$ distance between the methanolic oxygen and the ligand amide oxygen, suggests that hydrogen bonding interactions are responsible for the placement of the methanol molecule in the crystal structure. 


\section{A3.2 Crystallographic Figures}

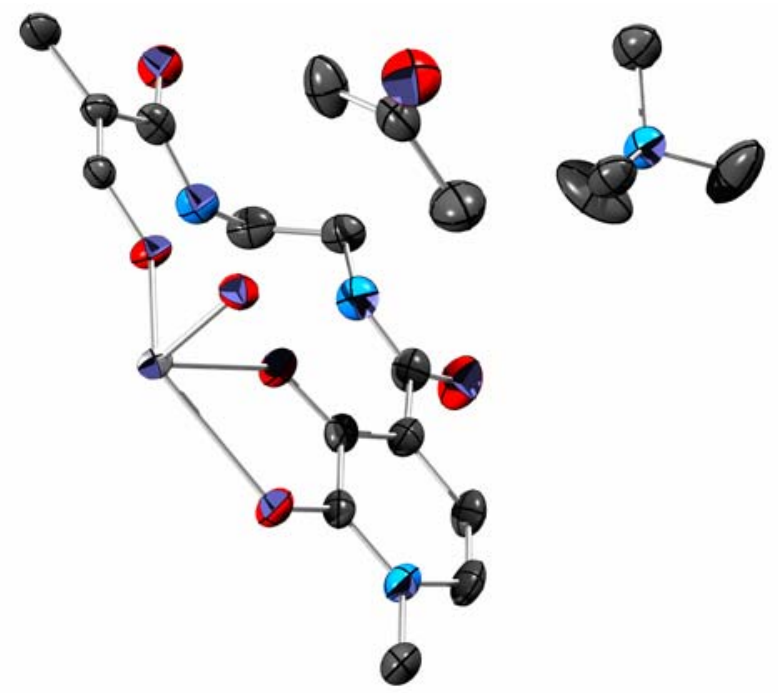

Figure A3-1. Asymmetric unit in the crystal structure of $\mathrm{UO}_{2}[\mathrm{TAM}(2 \mathrm{Li}-\mathrm{Me}-3,2-$ $\left.\mathrm{HOPO})_{2}\right], \mathrm{UO}_{2}(3-2)$. Tetramethylammonium cation and acetone inclusion are included. Hydrogen atoms have been omitted for clarity. Thermal ellipsoids are drawn at the $50 \%$ level. Carbons are gray, oxygens red, nitrogens blue, and uranium silver.

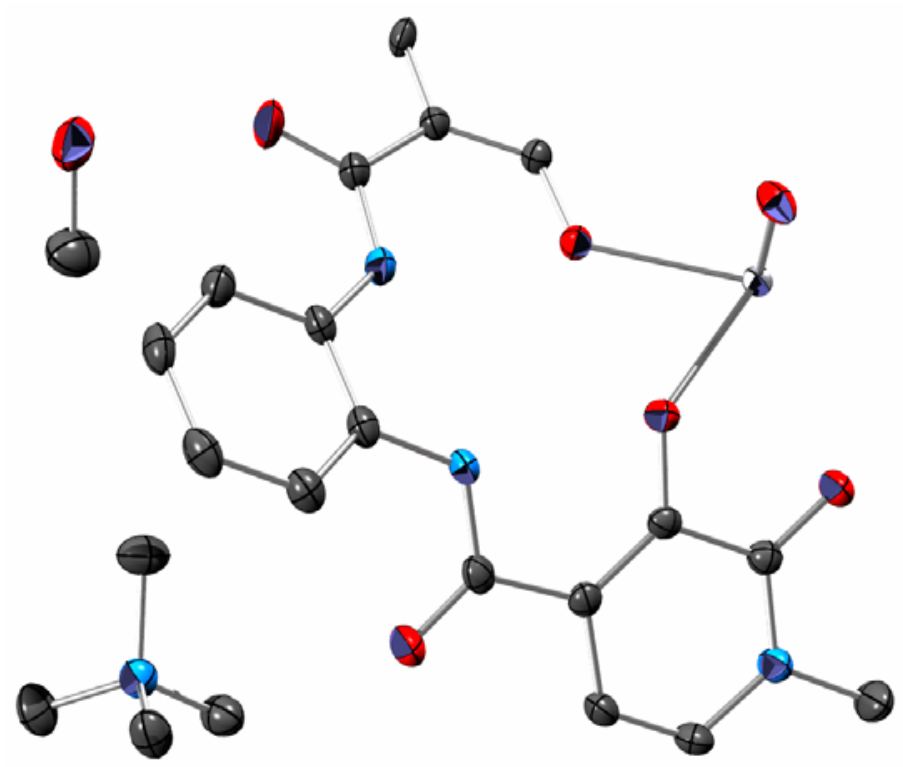

Figure A3-2. Asymmetric unit in the crystal structure of $\mathrm{UO}_{2}[\mathrm{TAM}(o-\mathrm{phen}-\mathrm{Me}-3,2-$ $\left.\mathrm{HOPO})_{2}\right], \mathrm{UO}_{2}(3-5)$. Tetramethylammonium cation and methanol inclusion included. Hydrogen atoms have been omitted for clarity. Thermal ellipsoids are drawn at the 50\% level. Carbons are gray, oxygens red, nitrogens blue, and uranium silver. 


\section{A3.3 Titration Data/Figures}

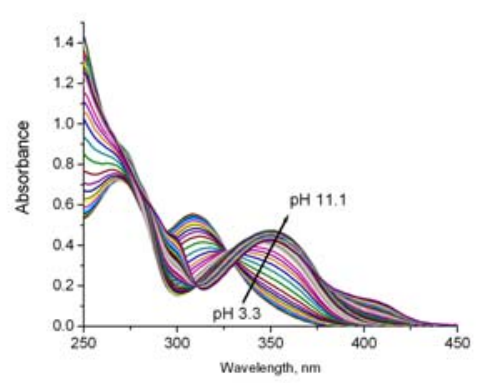

(a)

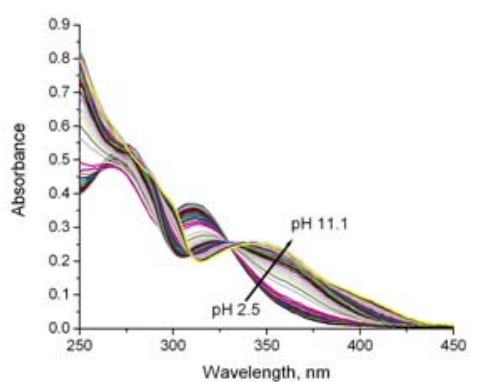

(d)

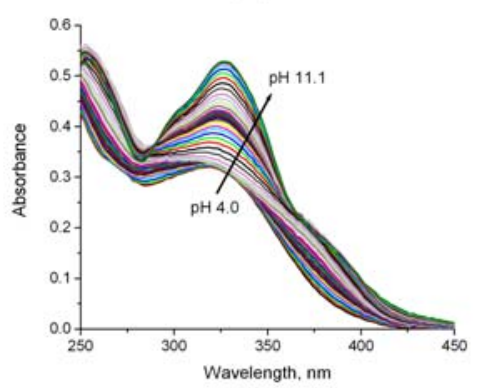

(g)

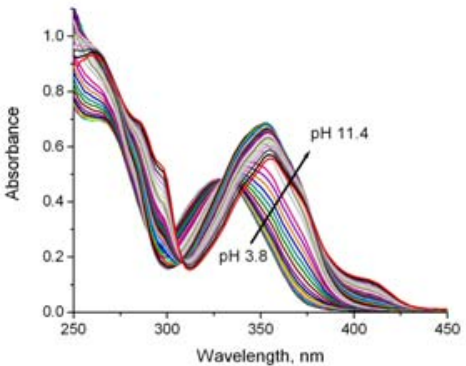

(b)

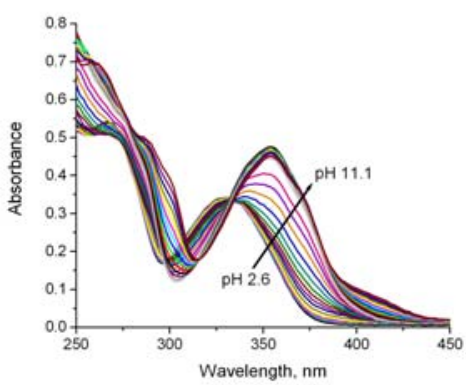

(e)

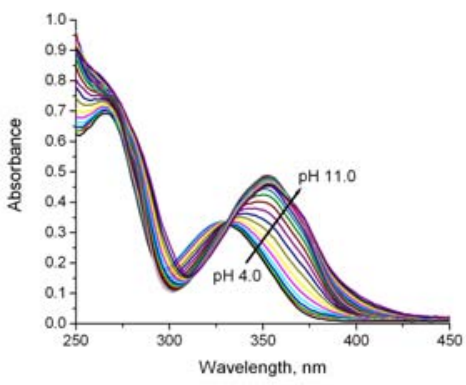

(h)

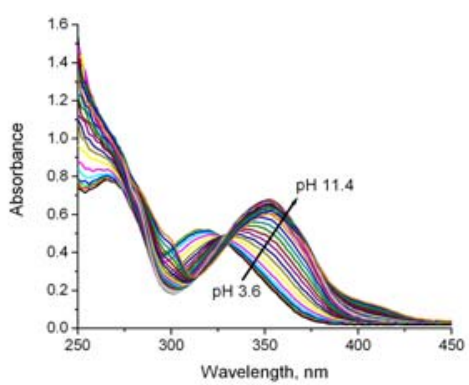

(c)

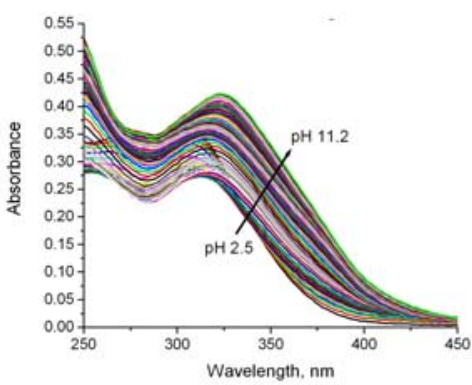

(f)

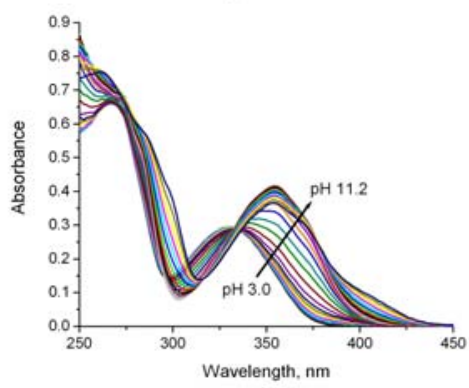

(i)

Figure A3-3. Acid to base UV-Visible titration curves of TAM-containing ligands; (a) TAM(2Li-1,2-HOPO) 2 , 3-1; (b) TAM(2Li-Me-3,2-HOPO) 2 , 3-2; (c) TAM(2Li-1,2HOPO)(2Li-Me-3,2-HOPO), 3-3; (d) PEG-TAM(2Li-1,2-HOPO) 2 , 3-12; (e) PEGTAM(2Li-Me-3,2-HOPO) 2 , 3-13; (f) PEG-TAM(o-phen-1,2-HOPO) $)_{2}$, 3-14; (g) PEGTAM(o-phen-Me-3,2-HOPO) 2 , 3-15; (h) Pr-TAM-2Li-Me-3,2-HOPO, 3-22; (i) PEG-PrTAM-2Li-Me-3,2-HOPO, 3-23. 


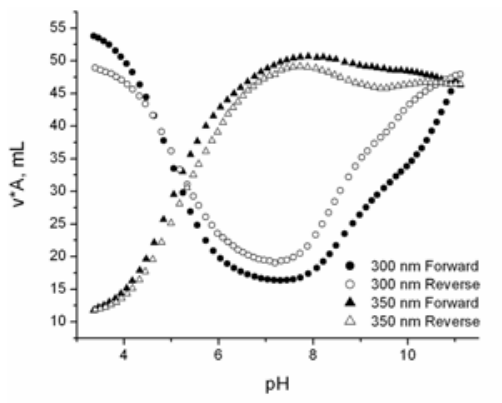

(a)

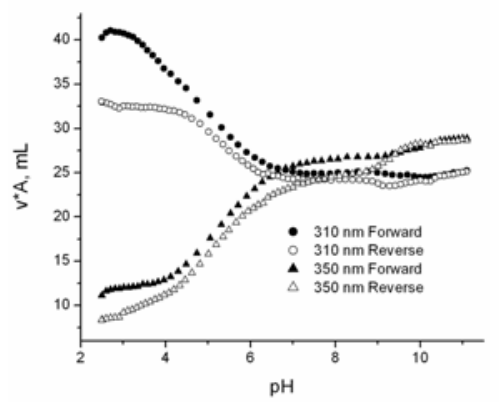

(d)

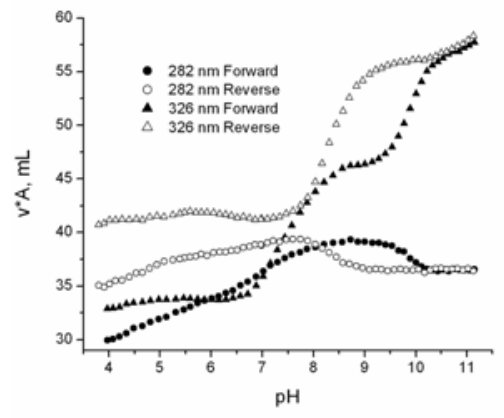

(g)

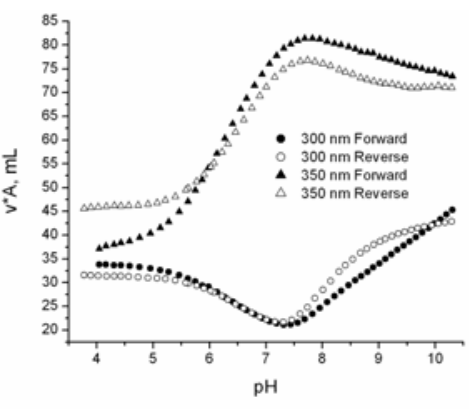

(b)

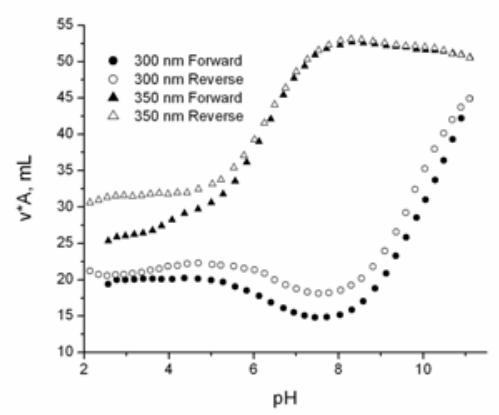

(e)

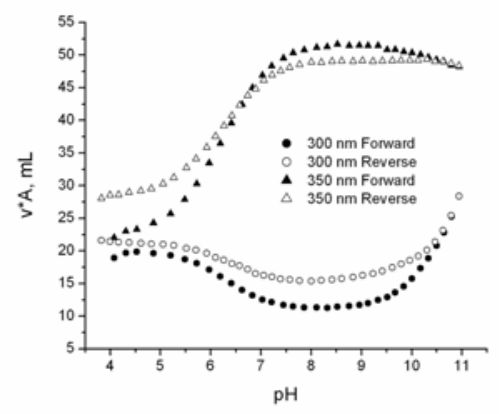

(h)

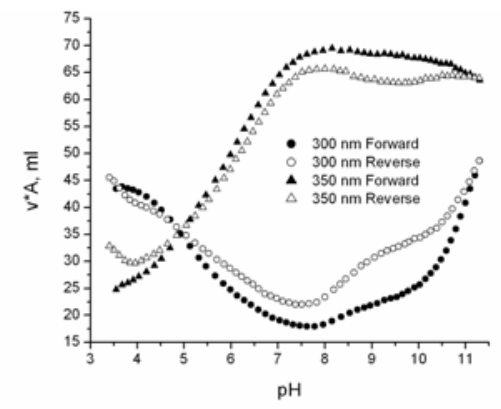

(c)

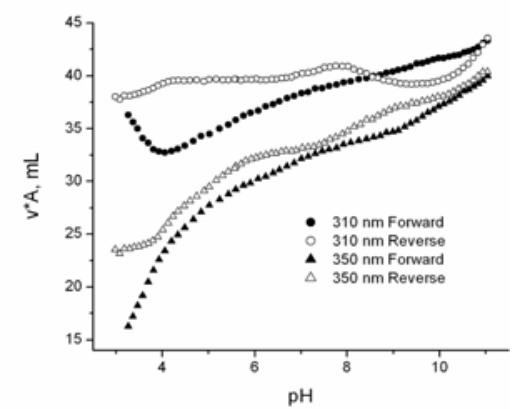

(f)

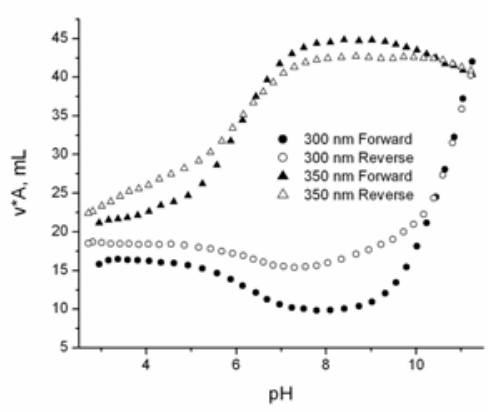

(i)

Figure A3-4. Spectrophotometric titration reversibility curves of TAM-containing ligands; (a) TAM(2Li-1,2-HOPO $)_{2}, \quad 3-1 ;$ (b) TAM(2Li-Me-3,2-HOPO $)_{2}, \quad 3-2$; (c) TAM(2Li-1,2-HOPO)(2Li-Me-3,2-HOPO), 3-3; (d) PEG-TAM(2Li-1,2-HOPO) 2 , 3-12; (e) PEG-TAM(2Li-Me-3,2-HOPO) 2 , 3-13; (f) PEG-TAM(o-phen-1,2-HOPO) 2 , 3-14; (g) PEG-TAM(o-phen-Me-3,2-HOPO) $)_{2}$, 3-15; (h) Pr-TAM-2Li-Me-3,2-HOPO, 3-22; (i) PEG-Pr-TAM-2Li-Me-3,2-HOPO, 3-23. 


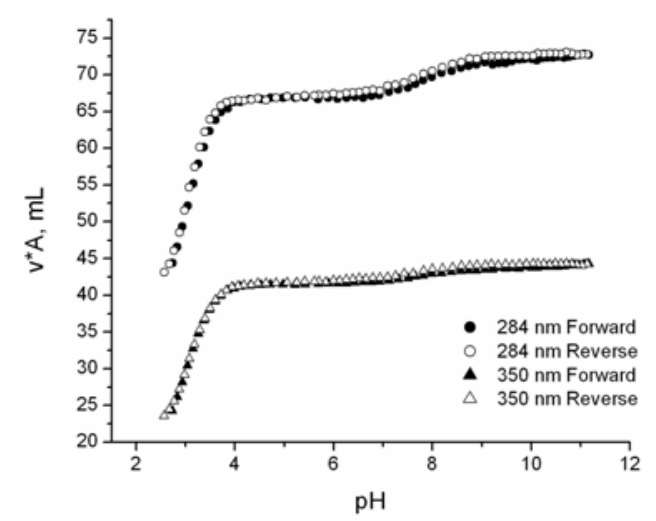

(a)

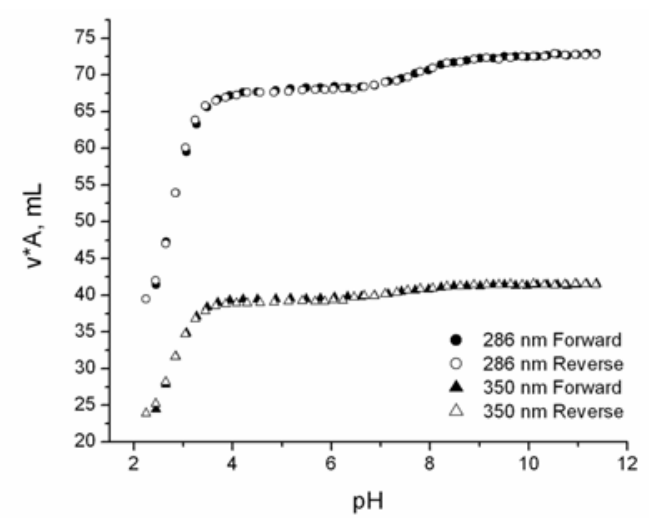

(b)

Figure A3-5. Spectrophotometric titration reversibility curves for uranyl titrations with (a) PrTAM-2Li-Me-3,2-HOPO (3-22, equilibration time: 10 minutes), and (b) PEG-Pr-TAM-2Li-Me3,2-HOPO (3-23, equilibration time: 20 minutes).

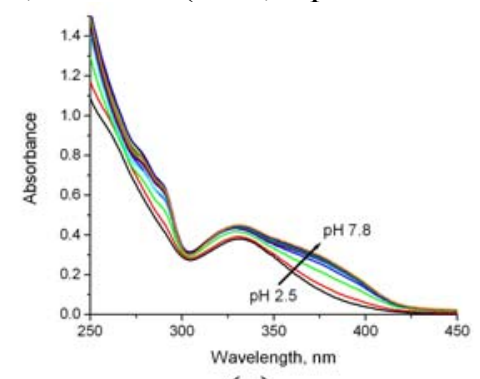

(a)

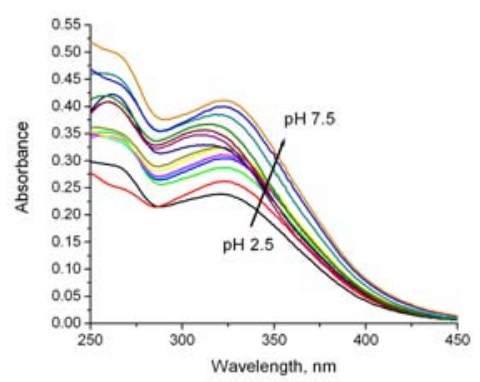

(d)

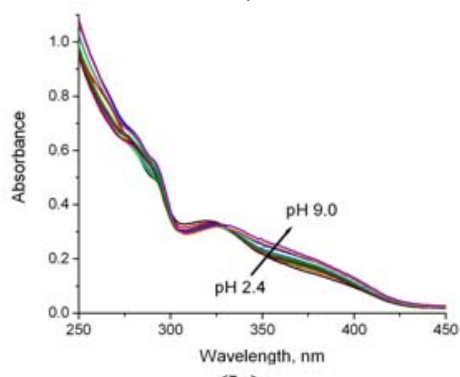

(b)

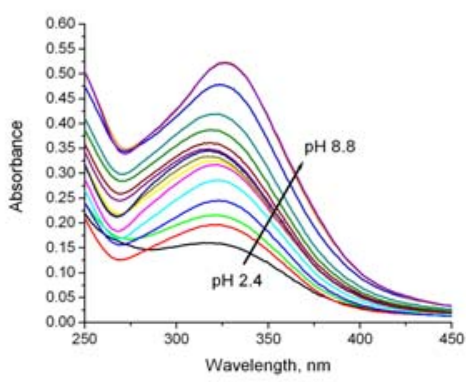

(e)

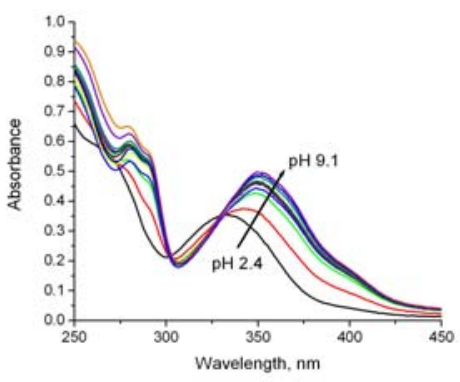

(c)

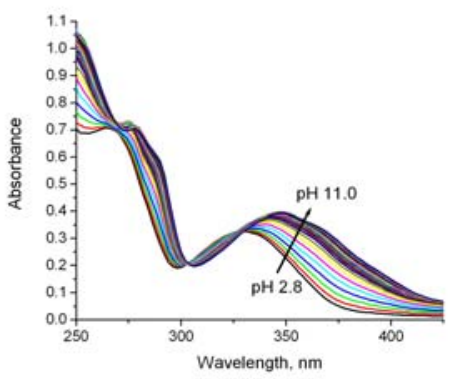

(f)

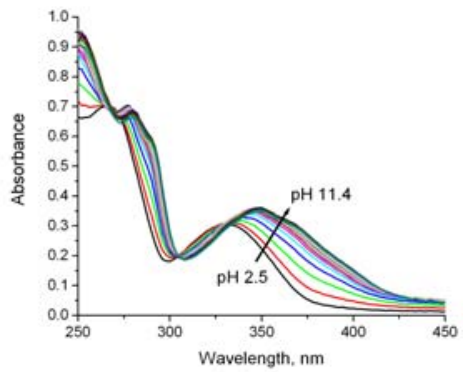

(g)

Figure A3-6. Acid to base UV-Visible titration curves for uranyl titrations with (a) TAM(2Li1,2-HOPO) $)_{2}$, 3-1; (b) PEG-TAM(2Li-1,2-HOPO) ${ }_{2}$, 3-12; (c) PEG-TAM(2Li-Me-3,2-HOPO) 2 , 313; (d) PEG-TAM(o-phen-1,2-HOPO) 2 , 3-14; (e) PEG-TAM(o-phen-Me-3,2-HOPO) 2 , 3-15; (f) Pr-TAM-2Li-Me-3,2-HOPO, 3-22; (g) PEG-Pr-TAM-2Li-Me-3,2-HOPO, 3-23. (a)-(e) are spectra of batch titrations; (f) and (g) are spectra of titrations using incremental addition of titrant. 


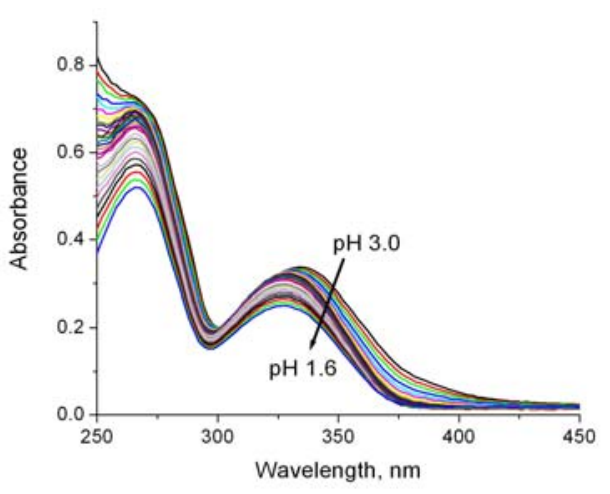

(a)

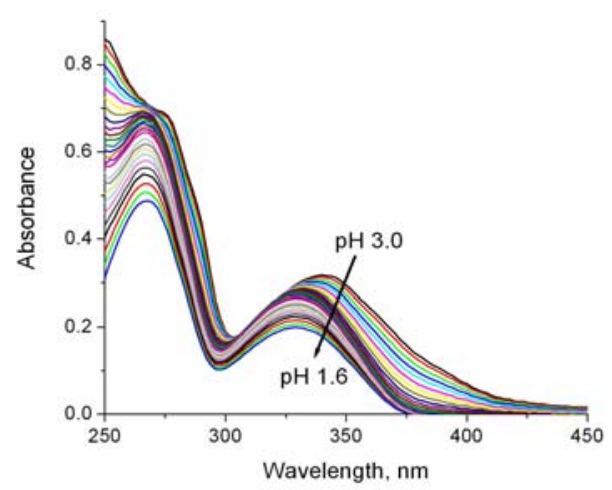

(b)

Figure A3-7. Strong acid UV-Visible titration curves for uranyl titrations with (a) $\mathrm{Pr}$ TAM-2Li-Me-3,2-HOPO, 3-22 and (b) PEG-Pr-TAM-2Li-Me-3,2-HOPO, 3-23.

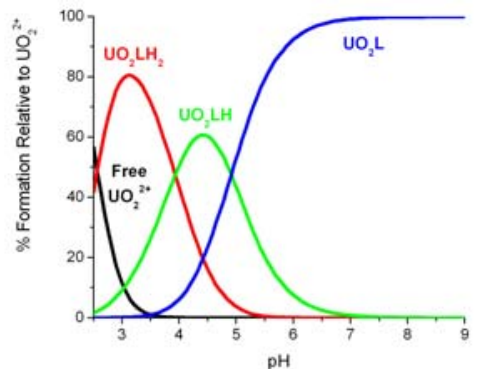

(a)

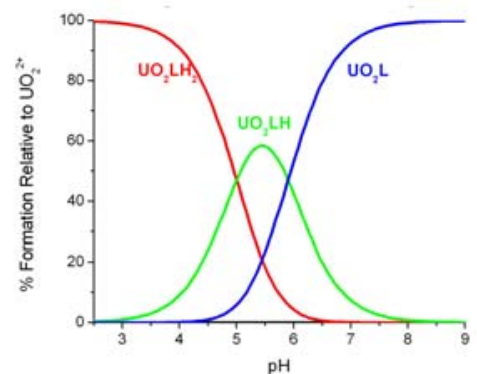

(d)

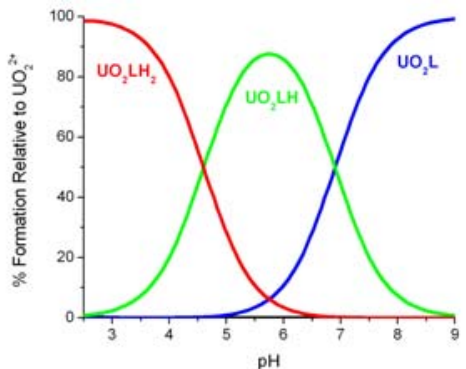

(b)

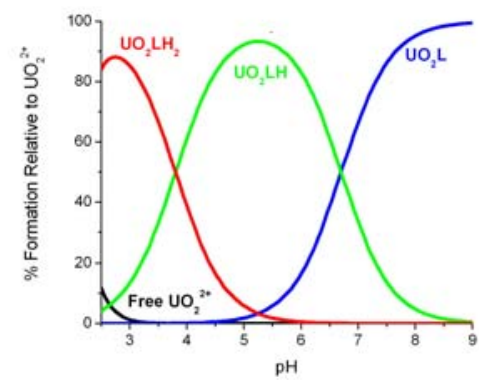

(e)

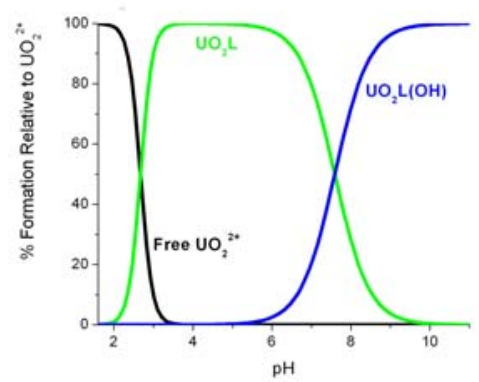

(g)

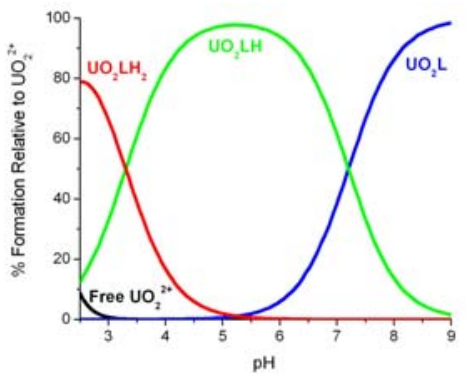

(c)

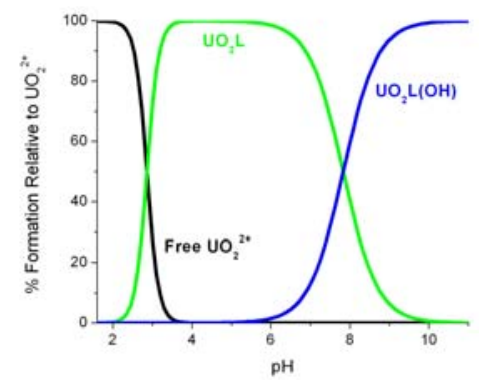

(f)

Figure A3-8. Speciation diagrams for complexation of $\mathrm{UO}_{2}{ }^{2+}$ at $[\mathrm{M}]=1 \mu \mathrm{M},[\mathrm{L}]=10$ $\mu \mathrm{M}$ with TAM-containing ligands: (a) TAM(2Li-1,2-HOPO) 2 , 3-1; (b) PEG-TAM(2Li1,2-HOPO) 2 , 3-12; (c) PEG-TAM(2Li-Me-3,2-HOPO) 2 , 3-13; (d) PEG-TAM(o-phen1,2-HOPO $)_{2}$, 3-14; (e) PEG-TAM(o-phen-Me-3,2-HOPO) $)_{2}$, 3-15; (f) Pr-TAM-2Li-Me3,2-HOPO, 3-22; (g) PEG-Pr-TAM-2Li-Me-3,2-HOPO, 3-23. 


\section{A3.4 Additional $\mathrm{pUO}_{2}$ Analysis}

A discussion on the definition and interpretation of $\mathrm{pUO}_{2}$ versus $\mathrm{pUO}_{2}{ }^{*}$ can be found in the Appendix for Chapter 2. Table A3-1 lists the $\mathrm{pUO}_{2}{ }^{*}$ values for TAM-containing ligands investigated in Chapter 3.

Table A3-1. $\mathrm{pUO}_{2}$ * values for TAM-containing ligands.

\begin{tabular}{|c|c|c|c|}
\hline \multirow{2}{*}{ Ligand } & \multicolumn{3}{|c|}{$\mathrm{pUO}_{2} *^{a}$} \\
\hline & pH 3.0 & pH 7.4 & pH 9.0 \\
\hline TAM $(2 \mathrm{Li}-1,2-\mathrm{HOPO})_{2}, \mathbf{3 - 1}$ & $6.9(3)$ & $15.9(1)$ & $17.1(3)$ \\
\hline PEG-TAM(2Li-1,2-HOPO) $)_{2}, \mathbf{3 - 1 2}$ & $9.2(3)$ & $13.6(3)$ & $13.9(3)$ \\
\hline PEG-TAM(2Li-Me-3,2-HOPO $)_{2}, \mathbf{3 - 1 3}$ & $8.1(5)$ & $15.0(4)$ & $16.2(6)$ \\
\hline PEG-TAM $(o \text {-phen-1,2-HOPO })_{2}, \mathbf{3 - 1 4}$ & $11.5(4)$ & $15.2(6)$ & 15.3(8) \\
\hline PEG-TAM $(o-\text { phen-Me-3,2-HOPO })_{2}, 3-15$ & $7.9(5)$ & $14.8(4)$ & $14.9(6)$ \\
\hline Pr-TAM-2Li-Me-3,2-HOPO, 3-22 & $6.56(8)$ & 15.2(1) & 16.4(2) \\
\hline PEG-Pr-TAM-2Li-Me-3,2-HOPO, 3-23 & $7.0(2)$ & $13.7(2)$ & $14.9(1)$ \\
\hline
\end{tabular}

The $\mathrm{pUO}_{2}{ }^{*}$ values in Table A3-1 are significantly higher that those of the bis-Me-3,2HOPO ligands in Table A2-1 (Chapter 2 Appendix). Unlike the dramatic increase in $\mathrm{pUO}_{2}$ observed between $\mathrm{pH} 7.4$ and 9.0 in Table 3-6, $\mathrm{pUO}_{2}{ }^{*}$ values at $\mathrm{pH} 7.4$ and 9.0 in Table A3-1 are similar, and in many cases within experimental error. This is consistent with the speciation diagrams shown above and in Figure 3-16, because by neutral $\mathrm{pH}$ a uranyl complex has already formed and is merely transitioning between the protonated $\mathrm{UO}_{2} \mathrm{LH}$ and deprotonated $\mathrm{UO}_{2} \mathrm{~L}$ forms. It is reasonable to believe that these tetradentate and hexadentate modes with $\mathrm{TAM}(\mathrm{HOPO})_{2}$ ligands both exhibit high affinity for the uranyl cation and are stable against ligand exchange with hydroxide ions or solvent molecules. In other words, the most of the TAM-containing ligands achieve their maximum uranyl chelating ability by $\mathrm{pH} 7.4$, with only $\mathbf{3 - 1}, \mathbf{3 - 2 2}$, and $\mathbf{3 - 2 3}$ showing higher uranyl affinity upon $\mathrm{pH}$ increase to 9.0.

The fact that at neutral to basic $\mathrm{pH}$ the $\mathrm{TAM}(\mathrm{HOPO})_{2}$ ligands exhibit similar $\mathrm{pUO}_{2}$ * values suggests that the choice of HOPO moiety at these $\mathrm{pH}$ ranges is unimportant, and that the one commonality of all the ligands - namely the TAM moiety - dominates the 
bonding characteristics of each ligand. This is consistent with the observation in the crystal structures of $\mathrm{UO}_{2}\left[\mathrm{TAM}(\mathrm{HOPO})_{2}\right]$ complexes that the $\mathrm{U}-\mathrm{O}_{\mathrm{TAM}}$ bonds remain essentially constant independent on ligand conformation, linker geometry, or HOPO moiety, while those of the HOPO moiety fluctuate between structures.

That the TAM unit is the most dominant chelating moiety in the poly-bidentate ligands is supported by the $\mathrm{pUO}_{2}{ }^{*}$ values for ligands $\mathbf{3 - 2 2}$ and 3-23, which at $\mathrm{pH} 7.4$ and 9.0 are only 1 to 2 orders of magnitude below the hexadentate TAM(HOPO $)_{2}$ ligands, and 3 to 4 orders of magnitude above the bis-Me-3,2-HOPO values. These results are similar to the trends in $\mathrm{pUO}_{2}$ discussed in the main text, but give a more revealing picture as to how important subsequent deprotonation is on uranyl complex stability than the $\mathrm{pUO}_{2}$ values do. These results suggest that the significant increase in $\mathrm{pUO}_{2}$ between the tetradentate bis-Me-3,2-HOPO ligands and the hexadentate $\mathrm{TAM}(\mathrm{HOPO})_{2}$ ligands does not arise from the increased ligand denticity, but is primarily caused by the inclusion of the more basic TAM moiety.

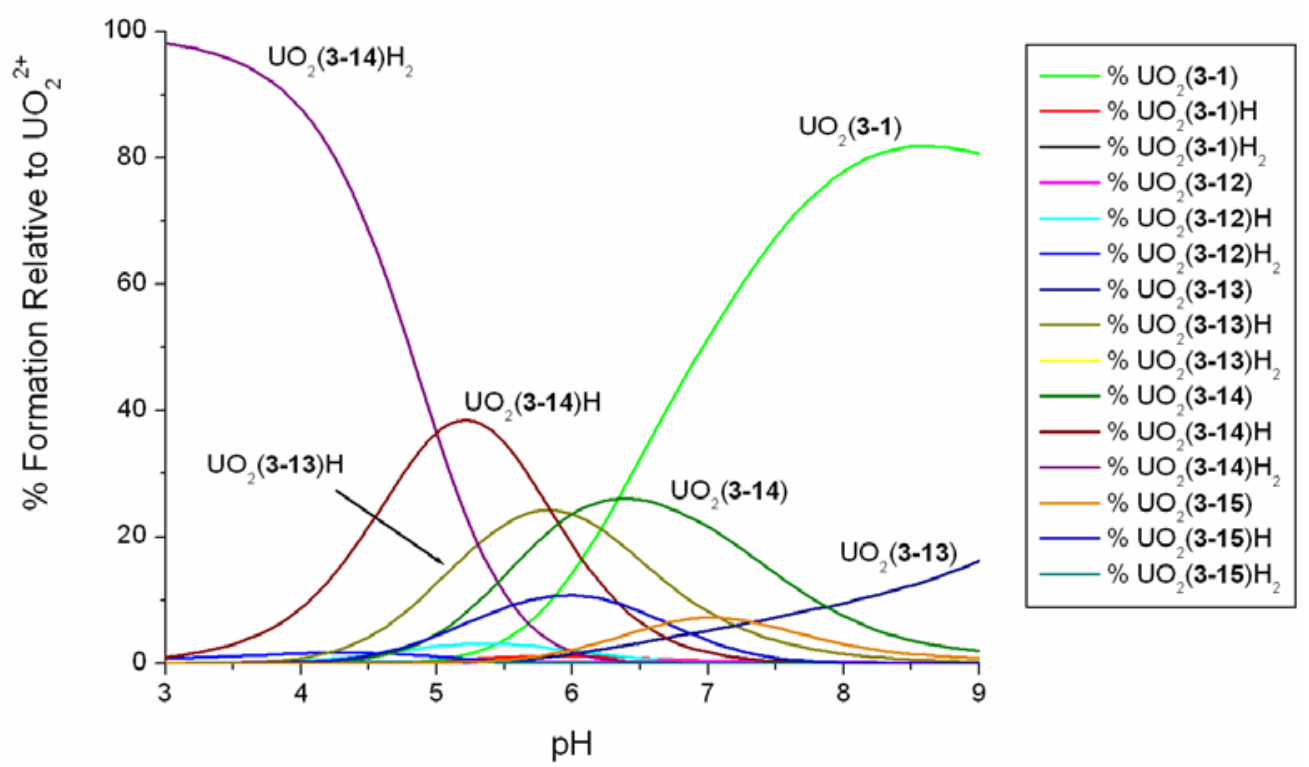

Figure A3-9. Species distribution for uranyl in the presence of five $\mathrm{TAM}(\mathrm{HOPO})_{2}$ ligands $\left(\left[\mathrm{UO}_{2}{ }^{2+}\right]=1 \mu \mathrm{M}, \Sigma[\mathrm{L}]=10 \mu \mathrm{M}\right)$. 
As with bis-Me-3,2-HOPO ligands, an interesting comparison to make between the $\mathrm{TAM}(\mathrm{HOPO})_{2}$ ligands is to use their calculated $\mathrm{pK}_{\mathrm{a}}$ and $\log \beta_{\text {mlh }}$ values to model the uranyl distribution between the various ligands if they were to exist in solution simultaneously. Namely, if $\left[\mathrm{UO}_{2}{ }^{2+}\right]=1 \mu \mathrm{M}$ and $[3-1]=[3-12]=[3-13]=[3-14]=[3-15]$ $=2.0 \mu \mathrm{M}(\Sigma[\mathrm{L}]=10 \mu \mathrm{M})$, which ligand would bind the uranyl cation most prominently, and by how much in comparison to the other ligands? A species distribution for this scenario is shown in Figure A3-9, and percentage distributions are listed in Table A3-2.

Table A3-2. Percentage of complexed $\mathrm{UO}_{2}{ }^{2+}$ in the presence of multiple bis-Me-3,2HOPO ligands $\left(\left[\mathrm{UO}_{2}{ }^{2+}\right]=1 \mu \mathrm{M}\right.$ and $\left.[3-1]=[3-12]=[3-13]=[3-14]=[3-15]=2.0 \mu \mathrm{M}\right)$.

\begin{tabular}{|c|c|c|c|}
\hline Ligand & \% UO $_{2}{ }^{2+}$ & Complexed by Ligand \\
\hline & pH 3.0 & pH 7.4 & pH 9.0 \\
\hline $3-1$ & 0.0 & 64.2 & 80.7 \\
\hline $\mathbf{3 - 1 2}$ & 0.8 & 0.4 & 0.1 \\
\hline $\mathbf{3 - 1 3}$ & 0.1 & 11.2 & 16.4 \\
\hline $\mathbf{3 - 1 4}$ & 99.1 & 16.6 & 2.0 \\
\hline $\mathbf{3 - 1 5}$ & 0.0 & 7.6 & 0.8 \\
\hline
\end{tabular}

The values in Table A3-2 very clearly indicate that ligand 3-14 most strongly bind the uranyl cation at low $\mathrm{pH}$ compared to other TAM(HOPO $)_{2}$ lignds. Figure A3-9 illustrates that the species responsible for this affinity are the protonated forms of the complex which results from the very low pKa values of 3-14 compared to other ligands evaluated here. However, beyond ca. 6.5, the deprotonated complex with 3-1 dominates the uranyl speciation, indicating that the more flexible ligand geometry is superior to the more constrained one of 3-14. Additionally, at middle to high $\mathrm{pH}$ values, ligand 3-13 is also bound to the uranyl cation to a significant degree at various degrees of protonation.

\section{A3.5 References:}

(1) Van Der Sluis, P.; Spek, A. L. Acta Cryst. 1990, A46, 194-201.

(2) Spek, A. L. J. Appl. Cryst. 2003, 36, 7-13. 


\section{Chapter 4 Appendix}

\section{A4.1 Crystallographic Refinement Details}

General. Typical data refinement proceeded as described in Chapter 2. The diffraction location and detector used to collect each structure is indicated after the title in parentheticals (ALS $=$ Advanced Light Source at LBNL, Bruker Plantinum 200 or APEX II detectors; SMART = UC Berkeley, Bruker SMART 1000 detector; APEX = UC Berkeley, Bruker APEX I detector).

Ce(1,2-HOPO) 4 , Ce(4-1)4. (SMART) The crystal structure contained two unique $\mathrm{Ce}(4-1)_{4}$ complexes in the asymmetric unit. There existed in the crystal a pocket of disordered solvent, which was modeled as seven water molecules, the protons of which were allowed to freely refine.

$\mathrm{Ce}(\text { Maltol })_{4} \mathrm{Ce}(4-2)_{4}$. (SMART). The Ce atom sits on a crystallographic $\overline{4}$-axis, and thus only one maltol ligand exists in the asymmetric unit. Symmetry generation results in the complete $\mathrm{Ce}(4-2)_{4}$ complex, in which each ligand is related to each other by a molecular $\mathrm{S}_{4}$ axis.

Ce(Bromomaltol $)_{4}, \mathbf{C e}(4-3)_{4}$ (SMART). As with the $\mathrm{Ce}(\mathrm{Maltol})_{4}$ structure, the Ce atom sits on a crystallographic $\overline{4}$-axis, and thus only one maltol ligand exists in the asymmetric unit. Symmetry generation results in the complete $\mathrm{ML}_{4}$ complex, in which each ligand is related to each other by a molecular $\mathrm{S}_{4}$ axis.

Ce(EtMaltol)4, Ce(4-4)4. (APEX). One ethyl group on the complex exhibited positional disorder. The disordered methyl group was modeled over two positions in a ratio of 54:64. The shared methylene carbons about which the disorder occurs exhibits abnormally large anisotropic thermal ellipsoids, but splitting this atom into two separate 
sites led to a poorer model than that presented here, so was left oblong. Common C-C bond distance restraints were used to model the disordered methyl group.

Ce(5-Bromo-ethyl-maltol) 4 , Ce(4-5) 4 . (ALS) Data were cut at $0.90 \AA$ due to poor internal agreement between high angle reflections. The extremities of the ligands (bromine and ethyl substituents) exhibited larger than normal thermal displacement parameters. Attempts to model these ligand fragments over two positions were unsuccessful, so standard 1-2 and 1-3 distance constraints were utilized in conjunction with anisotropic displacement parameter similarity restraints.

$\mathrm{Ce}(\mathrm{Kojate})_{4}, \mathbf{C e}(4-6)_{4}$. (ALS). Data were cut at 0.85 Angstroms. The Ce atoms sits on a crystallographic 2-fold axis, with only two of the four kojate ligands in the asymmetric unit. One of the two crystallographically unique kojate ligands exhibited positional disorder of the oxygen in the hydroxymethyl ring substituent. This disorder was modeled over two positions with a disorder ratio of 88:22 and employing standard C$\mathrm{O}$ bond distance and thermal displacement parameter restraints. The rotation of the C$\mathrm{O}_{\text {hydroxide }}$ bond (and thus the position of the alcoholic hydrogens) was allowed to freely refine. The final position of these protons (towards the coordinated Ce-coordinated oxygen atoms of other complexes in the crystal) as well as the $\mathrm{O}-\mathrm{O}$ distances between the hydroxide and Ce-coordinated oxygens of 2.74 and $2.80 \AA$ suggest that hydrogenbonding is responsible for the long-range structure in the crystal.

Ce(Chlorolkojate) $)_{4}, \mathrm{Ce}(4-7)_{4 \cdot}$ (ALS). Data were cut at $0.865 \AA$ because beyond this resolution the data had $\mathrm{I}<2 \mathrm{I} / \sigma$. No distance or thermal parameter restraints were used for the main $\mathrm{Ce}(4-7)_{4}$ residue structure. There is a well-ordered dioxane solvent molecule in the unit cell, and one much larger pocket of disordered solvent that spans a 
crystallographic 2-fold axis. This disordered pocket was initially modeled with freelyrefining oxygen atoms, and from that refinement it was determined there were approximately 110 electrons in that disordered pocket (55 electrons per asymmetric unit). The Fourier map in this disordered solvent region revealed a series of chair-shaped forms that were modeled as dioxane molecules using many distance restraints and anisotropic displacement parameter similarity restraints. These dioxane molecules were also restrained to have a similar geometry to that of the well-ordered dioxane molecule in the structure.

The disorder in the asymmetric unit of the solvent pocket contains one dioxane molecule disordered over two positions that freely refined to a 50:50 ratio and were then fixed to that ratio. Another dioxane molecule was found to span the 2-fold axis and also existed in two disordered positions. The occupancy of this molecule was allowed to freely refine to 0.25 , and then fixed to that value. The molecule of dioxane spanning the 2-fold axis is too close to one of the disordered positions of the other dioxane in the solvent pocket. The result of this analysis is shown in Figure A4-1 and illustrates that the central disordered dioxane only exists $50 \%$ of the time the outer dioxane molecules exists in the position farther from the 2-fold axis. When the outer dioxane molecules exist more towards the 2-fold axis, the central dioxane does not have the space necessary to fit into the cell, and is thus absent. No hydrogen atoms were added to these residues due to their high level of disorder, but were taken into account in structure refinement. The relatively close match of the free and restrained GOF of 1.119 and 1.137 suggest that the restraints used in modeling the disorderd solvent regions represent a reasonable model. 


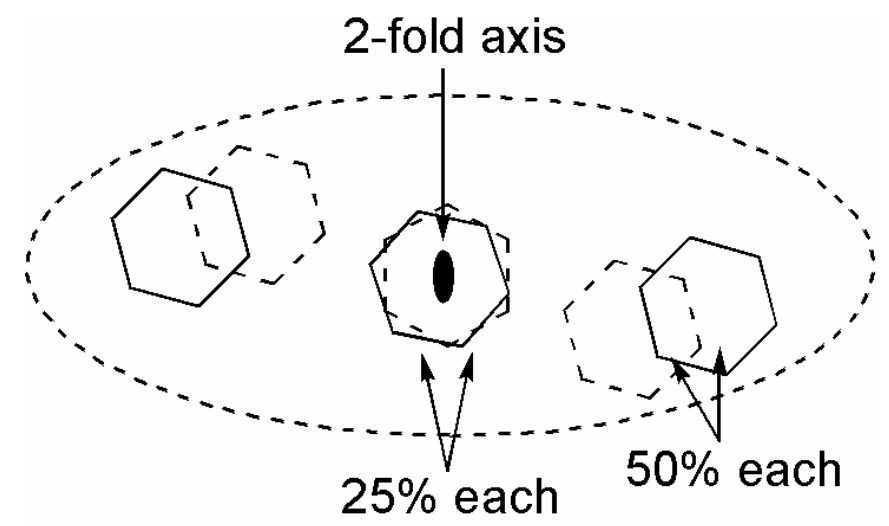

Figure A4-1. Crystallographic refinement of disordered solvent pocket in the crystal structure of $\mathrm{Ce}(4-7)_{4}$.

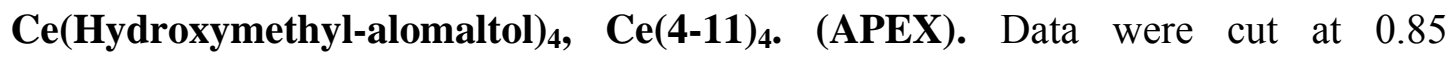
angstroms because beyond this resolution most data had $\mathrm{I}<2 \mathrm{I} / \sigma$. There is one wellordered dioxane in the crystal structure. Hydrogen positions on the free hydroxyide groups were allowed to refine by letting the $\mathrm{C}-\mathrm{O}$ bond rotation refine freely. Many of these hydrogen positions point towards other oxygen atoms in neighboring complexes. Two significantly short distances were found between two free hydroxide oxygens $(2.72$ and $2.82 \AA$ ) and one between a hydroxide oxygen and a Ce-coordinated carbonyl oxygen $(2.70 \AA)$, suggesting that hydrogen bonding interactions dictate the packing of the complex in the crystalline state.

Pu(1,2-HOPO) $)_{x}, \mathbf{P u ( 4 - 1 ) _ { x } \cdot ( A L S ) . ~ A s ~ d e s c r i b e d ~ i n ~ t h e ~ m a i n ~ t e x t , ~ t h e ~ u n i t ~ c e l l ~ o f ~ t h i s ~}$ crystal contained $\mathrm{Pu}(\mathbf{4 - 1})_{4}$ and $\mathrm{Pu}(\mathbf{4 - 1})_{3}\left(\mathrm{H}_{2} \mathrm{O}\right)_{2}$ complexes in the asymmetric unit, with a perchlorate anion present to balance the charge. The perchlorate was disordered and modeled as a rigid body over two positions with a common chlorine atom at the center. The oxygen atoms were refined isotropically, with a refined disorder ratio of 58:42. Standard distance restraints were also used modeling this rigid body disorder. The positions of the hydrogens on the coordinated water molecules were refined freely, using 
standard $\mathrm{O}-\mathrm{H}$ and $\mathrm{H}--\mathrm{H}$ distance restraints. The crystal was also found to exist as an inversion twin, in approximately a 52:48 ratio (BASF).

The relative position of the nitrogen in the 1,2-HOPO rings was determined by examination of the N/C-O and O-Pu distances. Although the relative size of q-peaks in the nitrogen and carbon positions could normally be used, the proximity to the very heavy $\mathrm{Pu}$ made this unreliable on its own, and relative bond lengths were used to finalize the determination.

Pu(Maltol) $)_{4}, \mathbf{P u}(4-2)_{4}$. (ALS). As with the $\mathrm{Ce}(4-2)_{4}$ structure, the $\mathrm{Pu}$ atom sits on a crystallographic $\overline{4}$-axis, and thus only one maltol ligand exists in the asymmetric unit. Symmetry generation results in the complete $\mathrm{Pu}(4-2)_{4}$ complex, in which each ligand is related to each other by a molecular $\mathrm{S}_{4}$ axis.

Pu(Bromomaltol) 4 , Pu(4-3) 4. (ALS). The data were cut at $0.95 \AA$ because above this resolution most data had $\mathrm{I}<2 \mathrm{I} / \sigma$. Using the program Cell_Now ${ }^{1}$, it was determined that the collected crystal contained a separate crystallographic domain approximately $25 \%$ of the measured crystal. Reflections of these two domains were separated and refined together $\mathrm{u}$ using a HKLF5 file format. $\mathrm{R}_{\mathrm{int}}$ was determined by merging all data (9441 reflections, merging to 3421 unique) into a HKLF4 file. The refinement on HKLF5 containing independant data from both domains of the crystal accounts for the excess data in the stated $2 \theta$ cutoff.

Severe total molecule disorder was observed in the structure, stemming from the apparent existence of both an "up" and "down" coordination mode for the bromomaltol at each of the four ligand sites. Because the binding oxygens are chemically different, this ligand flip in accompanied by a shift in the hydroxypyrone ligand which is described 
more thoroughly in the main text of this chapter. Refinement of both the observed ligand positions was unstable due to their very high correlation in the data refinement, so the structure of the 4-3 ligand was imported as a rigid body from the $\mathrm{Ce}(4-3)_{4}$ structure, and the thermal displacement parameters for the atoms in each ring were restrained to be similar. Only under these conditions could the atoms be refined anisotropically. The Pubound oxygen atoms, however, were not split into an "up" and "down" fragment like the rest of the molecule. This was because the difference in the positions was so small that refinement again became unreliable, and in fact the relative lengths of the $\mathrm{Pu}-\mathrm{O}_{\text {phenolate }}$ and $\mathrm{Pu}-\mathrm{O}_{\text {carbonyl }}$ bonds were inverted as compared to the behavior seen in all the other M(IV)-hydroxypyrone structures discussed above.

The degree of ligand disorder was initially freely refined for each ligand site separately, but it was found after refinement that the disorder ratios of ligand groups across from each other were the same within three standard deviations, so these opposing groups' disorder ratios were tied to each other, resulting in final disorder ratios of 72:28 and 65:35.

Because the Pu-bound oxygen atoms in the crystal structure were refined to be common to both disordered ligand orientations, the observed $\mathrm{Pu}-\mathrm{O}$ bond lengths are thus composites of the actual $\mathrm{Pu}-\mathrm{O}$ distances in the crystal. Deconvolution of the observed $\mathrm{Pu}-$ $\mathrm{O}$ bond distances can be achieved utilizing Equations 4-2 and 4-3 described in the main text, in which $\mathrm{Pu}-\mathrm{O} 1$ and $\mathrm{Pu}-\mathrm{O} 2$ are the short and long $\mathrm{Pu}-\mathrm{O}$ distances observed for each ligand group, $\mathrm{z}$ is the free variable describing the extent of disorder, and $\mathrm{x}$ and $\mathrm{y}$ are the calculated Pu- $\mathrm{O}_{\text {phenolate }}$ and $\mathrm{Pu}-\mathrm{O}_{\text {carbonyl }}$ distances respectively. Rearrangement of Equations 4-2 and 4-3 give Equations A4-1 and A4-2 to calculate the individual $\mathrm{Pu}-\mathrm{O}$ 
bond distances $\mathrm{x}$ and $\mathrm{y}$. The values of $\mathrm{x}$ and $\mathrm{y}$ determined by this treatment are shown in Table 4-4 in the main text.

Two small regions in the crystal structure seemed to have disordered solvent in them, which were modeled as water oxygens disordered over two positions each. Using fixed displacement parameters, their occupancies were freely refined to and then fixed at occupancies that total 1.7 oxygens over four sites. The hydrogen atoms on these oxygens could not be seen and due to their disorder were not added in calculated positions. These oxygen atoms were refined isotropically.

$$
\begin{aligned}
& x=\frac{(\mathrm{Pu}-\mathrm{O} 1)(\mathrm{z})-(\mathrm{Pu}-\mathrm{O} 2)(1-\mathrm{z})}{(\mathrm{z})^{2}-(1-\mathrm{z})^{2}} \\
& y=\frac{(\mathrm{Pu}-\mathrm{O} 2)(\mathrm{z})-(\mathrm{Pu}-\mathrm{O} 1)(1-\mathrm{z})}{(\mathrm{z})^{2}-(1-\mathrm{z})^{2}}
\end{aligned}
$$

\section{A4.2 Crystallographic Figures}

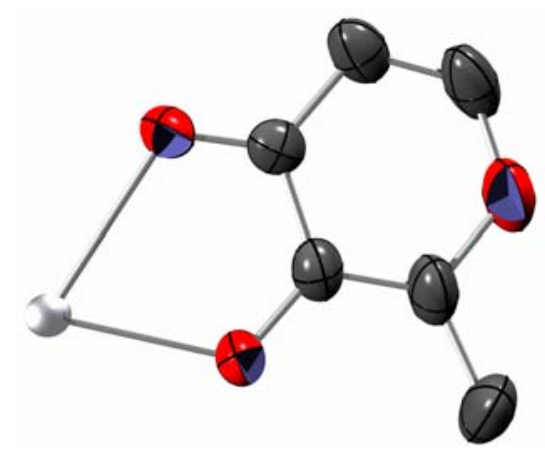

Figure A4-2. Asymmetric unit in the crystal structure of Ce(4-2) 4 . Hydrogen atoms have been omitted for clarity. Thermal ellipsoids are drawn at the $50 \%$ level. Carbons are gray, oxygens red, and cerium silver. 


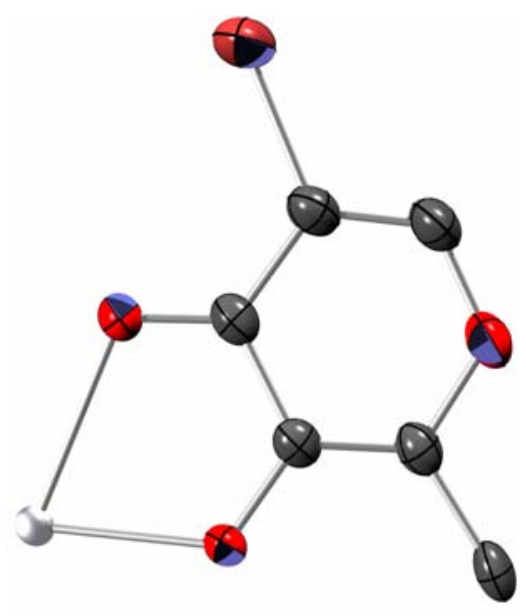

Figure A4-3. Asymmetric unit in the crystal structure of $\mathrm{Ce}(4-3)_{4}$. Hydrogen atoms have been omitted for clarity. Thermal ellipsoids are drawn at the $50 \%$ level. Carbons are gray, oxygens red, bromines brown, and cerium silver.

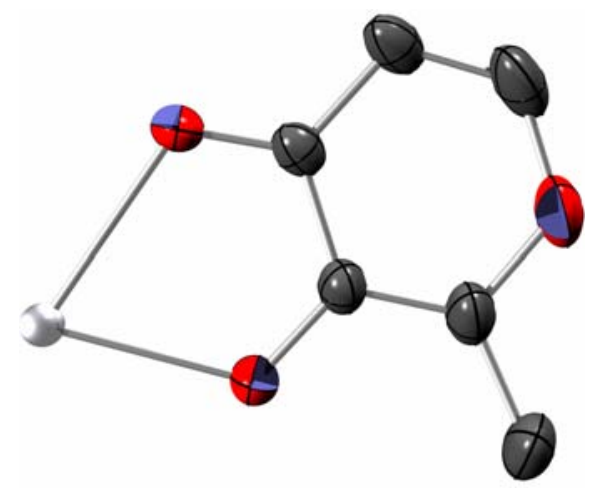

Figure A4-4. Asymmetric unit in the crystal structure of $\mathrm{Pu}(4-2)_{4}$. Hydrogen atoms have been omitted for clarity. Thermal ellipsoids are drawn at the 50\% level. Carbons are gray, oxygens red, and plutonium silver.

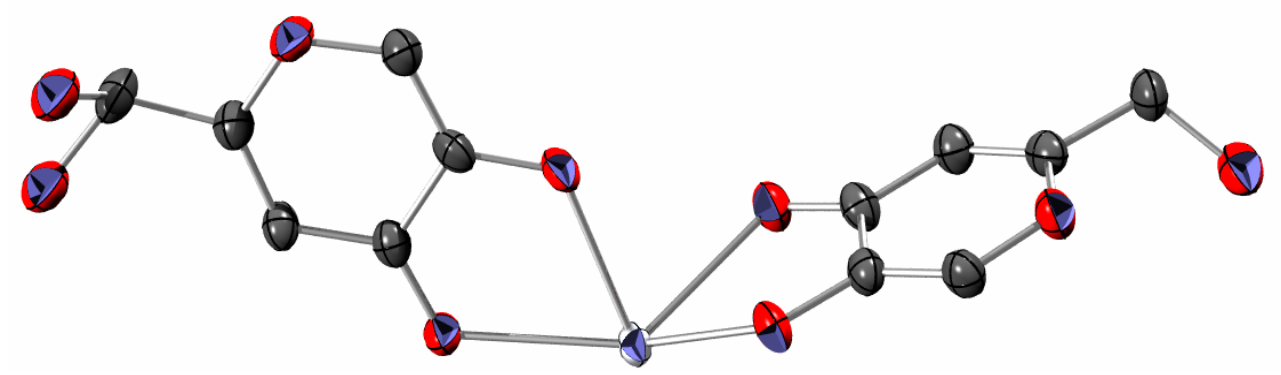

Figure A4-5. Asymmetric unit in the crystal structure of $\mathrm{Ce}(4-6)_{4}$. Hydrogen atoms have been omitted for clarity and hydroxide disorder has been included. Thermal ellipsoids are drawn at the 50\% level. Carbons are gray, oxygens red, and cerium silver. 


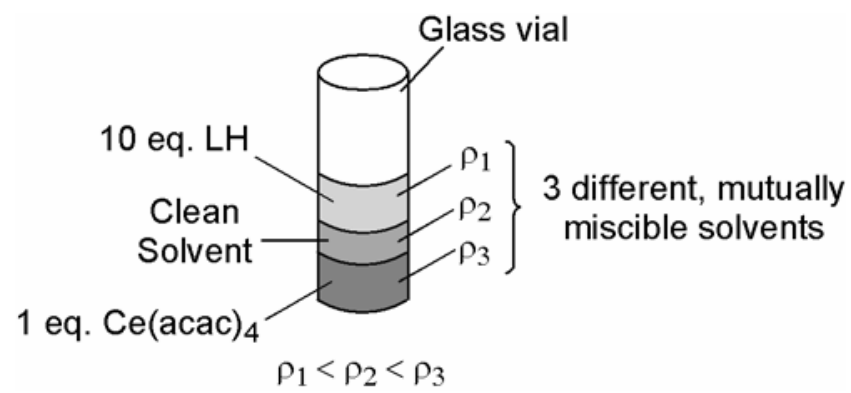

Figure A4-6. Experimental schematic of 3-layer crystallization technique utilized with $\mathrm{Ce}(\mathrm{IV})$-hydroxypyrone complexes. Mixtures were allowed to diffuse undisturbed at either room temperature or $4{ }^{\circ} \mathrm{C}$.

\section{A4.3 Competition Batch Titration Methodology}

In competition batch titration experiments the protonation constants of the ligands being used is not needed due to the constant $\mathrm{pH}$ of measurement. It is necessary, however, that the emission or absorption spectrum of the solutions exhibit some measureable shift between the metal ion binding to one ligand versus the other. In the following equations, " $\mathrm{L}$ " is the ligand under scrutiny and " $\mathrm{C}$ " is the competitor ligand whose protonation constants and formation constants with the metal ion " $\mathrm{M}$ " are known and from which a pM can be calculated.

$$
\begin{array}{ll}
\mathrm{M}+\mathrm{L} \rightleftharpoons \mathrm{ML} & \beta_{\mathrm{ML}}=\frac{[\mathrm{ML}]}{[\mathrm{M}][\mathrm{L}]} \\
\mathrm{M}+\mathrm{C} \rightleftharpoons \mathrm{MC} & \beta_{\mathrm{MC}}=\frac{[\mathrm{ML}]}{[\mathrm{M}][\mathrm{C}]}
\end{array}
$$

These formation constants are conditional upon the $\mathrm{pH}$ at which they are measured. The difference in the $\log \beta$ values from this derivation is equivalent to the difference in $\mathrm{pM}$ values for " $\mathrm{L}$ " and "C". The plot from which $\Delta \mathrm{pM}$ is determined is derived as follows: 


$$
\begin{aligned}
\log \beta_{\mathrm{ML}}-\log \beta_{\mathrm{MC}}=\mathrm{pM}_{\mathrm{ML}}-\mathrm{pM}_{\mathrm{MC}} & =\log \frac{[\mathrm{ML}]}{[\mathrm{M}][\mathrm{L}]}-\log \frac{[\mathrm{MC}]}{[\mathrm{M}][\mathrm{C}]} \\
& =\log \frac{[\mathrm{ML}][\mathrm{C}]}{[\mathrm{L}][\mathrm{MC}]} \\
\Delta \mathrm{pM} & =\log \frac{[\mathrm{ML}]}{[\mathrm{MC}]}+\log \frac{[\mathrm{C}]}{[\mathrm{L}]}
\end{aligned}
$$

The graph generated by plotting $\log ([\mathrm{ML}] /[\mathrm{MC}])$ versus $\log ([\mathrm{C}] /[\mathrm{L}])$ at several independently-measured $\mathrm{C}: \mathrm{L}$ ratios allows the determination of $\Delta \mathrm{pM}$ simply by reading the $\mathrm{x}$-intercept $[\log ([\mathrm{ML}] /[\mathrm{MC}])=0]$ of the linear equation describing the scattered points measured. Formation constants used to derive $\mathrm{pM}(\mathrm{DTPA})^{7.4}$ were taken from Martell. $^{2}$

\section{A4.4 References:}

(1) Sheldrick, G. Cell_Now 2004, Bruker-AXS, Inc., Madison, WI.

(2) Martell, A. E.; Smith, R. M. Critical Stability Constants; Plenum: New York, 1977; Vol. 1. 